Studien aus dem Max-Planck-Institut

für ausländisches und internationales Sozialrecht

Maria Grienberger-Zingerle

\title{
Kooperative Instrumente
}

der Arbeitsverwaltungen

in England und Deutschland

Eine rechtsvergleichende Untersuchung des Jobseeker's

Agreement und der Eingliederungsvereinbarung im

Zweiten und Dritten Buch Sozialgesetzbuch

\section{Nomos}


Studien aus dem Max-Planck-Institut

für ausländisches und internationales Sozialrecht

Band 47 
Maria Grienberger-Zingerle

Kooperative Instrumente der Arbeitsverwaltungen in England und Deutschland

Eine rechtsvergleichende Untersuchung des Jobseeker's Agreement und der Eingliederungsvereinbarung im Zweiten und Dritten Buch Sozialgesetzbuch 
Die Deutsche Nationalbibliothek verzeichnet diese Publikation in der Deutschen Nationalbibliografie; detaillierte bibliografische Daten sind im Internet über http://dnb.d-nb.de abrufbar.

Zugl.: München, Univ., Diss., 2009

ISBN 978-3-8329-5023-1

\begin{abstract}
1. Auflage 2009
(c) Nomos Verlagsgesellschaft, Baden-Baden 2009. Printed in Germany. Alle Rechte, auch die des Nachdrucks von Auszügen, der fotomechanischen Wiedergabe und der Übersetzung, vorbehalten. Gedruckt auf alterungsbeständigem Papier.
\end{abstract}




\section{Vorwort}

Die vorliegende Arbeit wurde im Wintersemester 2008/2009 von der Juristischen Fakultät der Ludwig-Maximilians-Universität München als Dissertation angenommen. Ich habe sie für die Veröffentlichung im Hinblick auf die Rechtslage auf den Stand 31.12.2008 gebracht. Literatur konnte bis Frühjahr 2009 berücksichtigt werden. Auf gesetzliche Änderungen im SGB III und SGB II durch das Gesetz zur Neuausrichtung der arbeitsmarktpolitischen Instrumente (BGBl. I 2008, S. 2917), das zum 1.1.2009 in Kraft getreten ist, wird in Fußnoten hingewiesen.

Die Arbeit entstand am Max-Planck-Institut für ausländisches und internationales Sozialrecht in München. Ich danke dem Direktor dieses Institutes, meinem Doktorvater Herrn Prof. Dr. Ulrich Becker, LL.M. (EHI) für die Gelegenheit, diese Arbeit im Rahmen einer fünfköpfigen Doktorandengruppe zu verfassen und für die Unterstützung, die Freiheit und das Vertrauen, das er mir bei der Anfertigung und Fertigstellung entgegengebracht hat. Ferner danke ich Herrn Prof. Dr. Rudolf Streinz für die Erstellung des Zweitgutachtens.

Ich weiß, dass mein Promotionsvorhaben ohne die Unterstützung meiner Familie, insbesondere meines Ehemannes und meiner Eltern neben der Geburt dreier Kinder und meinem Berufseinstieg am Bayerischen Staatsministerium für Arbeit und Sozialordnung nicht hätte abgeschlossen werden können. Jenen Menschen sei deshalb dieses Buch gewidmet.

Daneben gibt es einige Personen, denen ich ausdrücklich für Anregungen und Korrekturlesen danke. Nennen möchte ich meine Schwester Frau Dr. Regine Grienberger, meinen Kollegen am Institut Herrn Martin Landauer, M.Jur. (Oxon) und meine Freundin Frau Antonia Hoffmann. 


\section{Inhaltsübersicht}

Inhaltsverzeichnis

Abkürzungsverzeichnis

Einführung

A. Aktivierung als Konzept für den Umbau der sozialen Sicherung 28

I. Nationale Konzepte

II. Internationale und supranationale Konzepte

B. Eingrenzung des Themas

C. Methodik und rechtsvergleichender Ansatz der Arbeit

I. Länderauswahl

II. Funktion des Rechtsvergleichs

III. Gang der Untersuchung

Erstes Kapitel: Grundlagen

A. Kooperation 47

I. Kooperation als (politik-, rechts-, verwaltungs-)wissenschaftlicher Begriff 48

II. Ziele von Kooperation $\quad 54$

III. Gefahren und Risiken von Kooperation $\quad 58$

IV. Grenzen kooperativen Verwaltungshandelns 62

V. Kooperation und Aktivierung $\quad 64$

B. Typen und Formen kooperativen Handelns 65

I. Kooperative Elemente einer Entscheidung 65

II. Kooperative Elemente des planenden Verwaltungshandelns 68

$\begin{array}{ll}\text { III. Privatrechtlicher Vertrag } & 70\end{array}$

IV. Vertrag im öffentlichen Recht $\quad 82$

V. Vertragsähnliches Verwaltungshandeln 91

C. Systematisierungskriterien für kooperative Handlungsformen 96

I. Beteiligungszeitpunkt 96

II. An Kooperation Beteiligte: Leistungsempfänger - Leistungserbringer - Dritte 96

III. Beteiligungsform: einseitig - zweiseitig - mehrseitig 97

IV. Förmlichkeit der Beteiligung 97

V. Bindungswirkung des Kooperationsergebnisses 97

VI. Durchsetzbarkeit des Kooperationsergebnisses 98

Zweites Kapitel: Länderberichte $\quad 99$

A. Englisches Recht 99

I. Jobseekers Act 1995 und New Deals 99

1. Jobseekers Act $1995 \quad 99$

2. Jobseeker's allowance 100

3. New Deal Programme 106 
II. Jobseeker's agreement 109

1. Definition 109

2. Inhaltliche Aspekte des JSA 110

3. Formelle Gesichtspunkte 111

4. Rechtliche Einordnung 111

5. Funktion des JSA 116

6. Rechtliche Wirkungen des JSA 121

7. Störungen bei der Abwicklung des vertragsähnlichen Verhältnisses 124

8. Beendigung des JSA 130

9. Rechtsschutz 131

B. Deutsches Recht 140

I. Arbeitsförderungsrecht 140

1. SGB III und Job-AQTIV-Gesetz 140

2. Definition der Eingliederungsvereinbarung und Abgrenzung 145

3. Inhalt der Eingliederungsvereinbarung 147

4. Formelle Gesichtspunkte 150

5. Rechtliche Einordnung 150

6. Funktion der Eingliederungsvereinbarung 162

7. Wirkungen der Eingliederungsvereinbarung 166

8. Störungen bei der Abwicklung des vertragsähnlichen Verhältnisses 176

9. Beendigung der Eingliederungsvereinbarung 178

10. Rechtsschutz 179

Recht der Grundsicherung für Arbeitsuchende 182

1. SGB II/Viertes Gesetz für moderne Dienstleistungen am Arbeitsmarkt 182

2. Definition der Eingliederungsvereinbarung und Abgrenzung 187

3. Inhalt der Eingliederungsvereinbarung 193

4. Form 198

5. Rechtliche Einordnung 198

6. Funktion der Eingliederungsvereinbarung 212

7. Wirkungen der Eingliederungsvereinbarung 214

8. Störungsfälle 226

9. Beendigung der Eingliederungsvereinbarung 237

$\begin{array}{ll}10 . & \text { Rechtsschutz } \\ \end{array}$

Drittes Kapitel: Ergebnisse und Auswertung des Rechtsvergleichs 243

A. Zusammenfassung der Ergebnisse 243

$\begin{array}{ll}\text { I. Jobseeker's agreement } & 243\end{array}$

II. $\quad$ Eingliederungsvereinbarung nach SGB III 243

III. Eingliederungsvereinbarung nach SGB II 245

B. Vergleich und Bewertung 246

I. Kooperation - Ziele erreicht? 246

II. Kooperation - Gefahren gebannt? $\quad 252$

III. Kooperation an ihren Grenzen $\quad 260$

IV. Kooperation und Aktivierung als Rahmen eines Politiktransfers 265

V. Bewährungsprobe und Zukunft der Handlungsinstrumente 266

C. Abschließende Bemerkung 268

$\begin{array}{ll}\text { Literaturverzeichnis } & 269\end{array}$ 


\section{Inhaltsverzeichnis}

Abkürzungsverzeichnis

Einführung

A. Aktivierung als Konzept für den Umbau der sozialen Sicherung 28

I. Nationale Konzepte 28

1. Hintergrund und Entwicklung der Activating Labour Market Policy 28

a) Der welfare-to-work-Ansatz der Regierung Thatcher 28

b) Aktivierung in den Vereinigten Staaten von Amerika 29

c) Elemente aktivierender Politik unter der Regierung Blair $\quad 30$

2. Das deutsche Leitbild vom aktivierenden Sozialstaat $\quad 30$

a) Inhaltliche Beschreibung des Leitbildes $\quad 30$

b) Adressaten aktivierender Maßnahmen $\quad 32$

c) Ziele des aktivierenden Staates 32

aa) Stärkung der Eigenverantwortung 33

bb) Positiver Einfluss auf Arbeitslosigkeit 34

cc) Einsparungen bei den Sozialausgaben 34

3. Vergleichbarkeit der Leitbilder $\quad 35$

a) Staatsverständnis als Grundlage der Reformen $\quad 35$

b) Politische Entwicklung und Akzeptanz sozialpolitischer Maßnahmen 36

c) Rechtssystem als Rahmen für Aktivierung 36

II. Internationale und supranationale Konzepte $\quad 37$

1. Empfehlungen der OECD 37

2. Beschäftigungspolitische Leitlinien der Europäischen Union 38

B. Eingrenzung des Themas 40

C. Methodik und rechtsvergleichender Ansatz der Arbeit 42

I. Länderauswahl 42

II. Funktion des Rechtsvergleichs 43

III. Gang der Untersuchung $\quad 45$

1. Mikro- oder Makrovergleich $\quad 45$

2. Untersuchungsschritte 45

3. Zusammenfassung 46 
A. Kooperation 47

I. Kooperation als (politik-, rechts-, verwaltungs-)wissenschaftlicher Begriff 48

1. Definition 48

2. Wirkungsfelder 49

a) Steuerung verwaltungsinterner Vorgänge 49

b) Steuerung verschiedener Leistungserbringer und freier Träger $\quad 50$

aa) Aufrechterhaltung einer Trägervielfalt $\quad 50$

bb) Am Wettbewerb orientierte Leistungserbringung 51

cc) Gemeinsame Aufgabenerfüllung in Public-Private-Partnerships $\quad 52$

c) Unmittelbare und mittelbare Steuerung des Bürgers 53

II. Ziele von Kooperation 54

1. Steigerung der Effizienz in der Verwaltungsarbeit 54

2. Information der Verwaltung in offenem Miteinander 55

3. Legitimierende Wirkung 56

4. Psychologisch-pädagogischer Effekt: Disziplinierung vs. Chance 57

III. Gefahren und Risiken von Kooperation 58

1. Übervorteilung und Verhandlungsungleichgewicht 58

2. Desintegration durch Kooperation $\quad 59$

3. „Entrechtlichung" und „Verrechtlichung“" 60

4. Defizite bei Haftung und Rechtsschutz 60

5. Kosten-Nutzen-Vergleich 61

IV. Grenzen kooperativen Verwaltungshandelns 62

1. Verfassungsrechtliche Vorgaben $\quad 62$

2. Vorgaben im Verfahrensrecht 62

3. Allgemeine Vorgaben des materiellen Rechts: Bindung inter partes 63

V. Kooperation und Aktivierung $\quad 64$

B. Typen und Formen kooperativen Handelns 65

I. Kooperative Elemente einer Entscheidung 65

1. Verwaltungsakt im deutschen Recht 65

$\begin{array}{ll}\text { a) Begriff } & 65\end{array}$

b) Verwaltungsakte mit kooperativen Elementen 66

aa) Mitwirkungsbedürftiger/zustimmungsbedürftiger Verwaltungsakt 66

bb) Zusage und Zusicherung 66

cc) Verwaltungsakt mit Nebenbestimmungen 66

$\begin{array}{rr}\text { dd) Anhörung } & 67 \\ \text { 2. Decision } & 68\end{array}$

II. Kooperative Elemente des planenden Verwaltungshandelns 68

1. Pläne im deutschen Verwaltungs- und Sozialrecht 68

a) Begriff $\quad 68$

b) Einordnung als kooperative Handlungsform $\quad 69$

2. Back-to-work-plan im englischen Recht 69 
1. Begriff und Bindung an den Vertrag 70

2. Vertragsfreiheit 71

a) Historischer Hintergrund $\quad 71$

b) Begriff und verfassungsrechtliche Garantie der Vertragsfreiheit $\quad 72$

c) Kontrahierungszwang und gestörtes Verhandlungsgleichgewicht 73

3. Die Verwaltung als Vertragspartner eines privatrechtlichen Vertrages $\quad 75$

a) Verwaltungsprivatrecht im deutschen (Sozial-)Recht 75

aa) Wahlfreiheit der Verwaltung 76

bb) Anwendungsbereich im Rahmen der Sozialverwaltung 76

b) Government contracts im englischen Recht 77

aa) Begriff $\quad 77$

bb) Besonderheiten aufgrund der Beteiligung der Krone 78

(1) Doktrin der Ultra vires und Local Government Act $1997 \quad 78$

(2) Doctrine of executive necessity 78

(3) Rule against fettering future executive action $\quad 79$

cc) Judicial review 79

dd) Unterscheidung von private law und public law $\quad 80$

(1) Source of power approach $\quad 81$

(2) Nature of the power approach $\quad 81$

$\begin{array}{ll}\text { (3) Functional approach } & 82\end{array}$

$\begin{array}{ll}\text { (4) Stellungnahme } & 82\end{array}$

IV. Vertrag im öffentlichen Recht $\quad 82$

1. Der verwaltungsrechtliche Vertrag im deutschen Recht 82

a) Hintergrund und historische Entwicklung bis zur gesetzlichen Regelung 82

b) Begriff 84

c) Abgrenzung zwischen öffentlichem Recht und Privatrecht 85

aa) Interessentheorie $\quad 85$

bb) Subordinationstheorie $\quad 85$

cc) Subjektstheorie, Zuordnungstheorie (modifizierte Subjektstheorie) 86

dd) Sonderrechtslehre 86

ee) Kelsens Idee vom Einheitsrechtssystem 87

d) Abgrenzung von verwaltungsrechtlichem und privatrechtlichem Vertrag 87

e) Abgrenzung verwaltungsrechtlicher Vertrag und Verwaltungsakt $\quad 88$

f) Zulässigkeit der Handlungsform $\quad 89$

aa) Handlungsformermächtigung 89

bb) Gebundene Wahlfreiheit der Verwaltung 89

cc) Handlungsformverbote 90

2. Public law contract $\quad 90$

a) Hintergrund und Kritik an der Idee eines public law contract $\quad 90$

b) Inhaltliche Ausgestaltung eines public contract law 91

V. Vertragsähnliches Verwaltungshandeln 91

1. Begriff 91

2. Voraussetzungen $\quad 92$

3. Zulässigkeit vertragsähnlicher Vorgehensweise der Verwaltung 93

a) NHS-contract $\quad 93$

b) Disciplinary contract 94

c) Leistungsabrede, § 12 SGB XII 94

4. Inhaltliche Ausgestaltung und Wirkungen 95 
C. Systematisierungskriterien für kooperative Handlungsformen 96

I. Beteiligungszeitpunkt 96

II. An Kooperation Beteiligte: Leistungsempfänger - Leistungserbringer - Dritte 96

III. Beteiligungsform: einseitig - zweiseitig - mehrseitig 97

IV. Förmlichkeit der Beteiligung 97

V. Bindungswirkung des Kooperationsergebnisses 97

VI. Durchsetzbarkeit des Kooperationsergebnisses 98

Zweites Kapitel: Länderberichte $\quad 99$

A. Englisches Recht 99

I. Jobseekers Act 1995 und New Deals $\quad 99$

1. Jobseekers Act $1995 \quad 99$

2. Jobseeker's allowance 100

a) Grundvoraussetzungen der jobseeker's allowance 101

b) Besond. Voraussetzungen der contribution-based jobseeker's allowance103

c) Besond. Voraussetzungen der income-based jobseeker's allowance 104

d) Beantragung als joint-claim couple 105

e) Höhe der jobseeker's allowance $\quad 105$

3. New Deal Programme 106

a) New Deal for Young People 107

b) New Deal for long-term unemployed people over 25

c) Weitere New Deals 108

$\begin{array}{ll}\text { II. Jobseeker's agreement } & 109\end{array}$

$\begin{array}{ll}\text { 1. Definition } & 109\end{array}$

2. Inhaltliche Aspekte des JSA 110

a) Gesetzlich geregelte Mindestanforderungen 110

b) Prüfungsrecht bzw. Prüfungspflicht des employment officer 111

3. Formelle Gesichtspunkte $\quad 111$

4. Rechtliche Einordnung 111

a) Rechtliche Handlungsfähigkeit auf Seiten des employment officer $\quad 111$

b) Vereinbarung von Pflichten 113

c) Freiwilligkeit - Vertragsfreiheit 113

aa) Abschlussfreiheit des Antragstellers 114

bb) Gestaltungsfreiheit der Verwaltung 115

d) (Gesetzlicher) Ausschluss der Anwendung von contract law 115

e) Vertragsähnliches Verwaltungshandeln 115

5. Funktion des JSA 116

a) Citizen's Charter und Customer's Charter des JCP 116

b) Social citizenship $\quad 117$

c) Social contract $\quad 118$

d) Principal of conditionality 119 
6. Rechtliche Wirkungen des JSA

a) Voraussetzung für den Leistungsbezug 121

aa) Anspruchsvoraussetzung 121

bb) Fiktion eines JSA 121

cc) Rückdatierung (back-dating) 122

b) Verhängung von Sanktionen gegenüber dem Antragsteller 122

c) Auslegung (unbestimmter) Rechtsbegriffe und Ermessensausübung 123

d) Ausschluss weiterer Wirkungen 124

7. Störungen bei der Abwicklung des vertragsähnlichen Verhältnisses 124

a) Veränderung der tatsächlichen Verhältnisse 124

b) Nichteinhaltung der Vereinbarungen aus dem JSA durch das JCP 125

aa) Doctrine of executive necessity 125

bb) Rule against fettering future executive action $\quad 126$

c) Nichteinhaltung der Vereinbarungen aus dem JSA durch Antragsteller 126

d) Restitution 127

aa) Grundsätzliche Zulässigkeit einer Klage auf restitution 127

bb) Ausschluss aufgrund einer Entscheidung des Gesetzgebers $\quad 128$

8. Beendigung des JSA 130

a) Erledigung des Antrags auf jobseeker's allowance 130

b) Einvernehmliche Abänderung des JSA 130

c) Angeordnete Abänderung $\quad 130$

d) Keine einseitige Beendigung 130

9. Rechtsschutz 131

a) Belehrung 131

b) Ausschluss einer private law procedure 131

c) Verwaltungsinterne Kontrolle: decision making, revision, supersession 132

aa) Decision making 132

bb) Revision 133

cc) Supersession 134

dd) Zusammenfassung $\quad 134$

d) Appeal 135

aa) Social Security Appeal Tribunal (SSAT) 135

(1) Entstehung und deren Gründe 135

(2) Zusammensetzung des Entscheidungsgremiums $\quad 135$

(3) Gegenstand des Verfahrens 136

(4) Formfragen 136

(5) Verfahren 136

(6) Entscheidung und Anfechtbarkeit 137

bb) Social Security Commission 138

e) Judicial Review 138

aa) Gegenstand des Verfahrens 138

bb) Zuständiges Gericht und Verfahren 139

f) Kosten 
B. Deutsches Recht 140

I. Arbeitsförderungsrecht 140

1. SGB III und Job-AQTIV-Gesetz 140

a) Arbeitsförderungsgesetz und SGB III 140

b) Job-AQTIV-Gesetz 141

c) Überblick über die Leistungen nach dem SGB III 142

aa) Versicherungspflichtverhältnis 142

bb) Leistungen der aktiven Arbeitsförderung, § 3 Abs. 4 SGB III 143

cc) Entgeltersatzleistungen, $\S \S 116$ ff SGB III 143

2. Definition der Eingliederungsvereinbarung und Abgrenzung 145

a) Definition $\quad 145$

b) Abgrenzung zum Eingliederungsvertrag, §§ 229 ff SGB III a. F. 145

3. Inhalt der Eingliederungsvereinbarung $\quad 147$

a) Niederlegung der ,individuellen Vermittlungsstrategie“ 147

b) Vermittlungsbemühungen der Agentur für Arbeit 147

c) Eigenbemühungen des Arbeitslosen 148

d) Leistungen der aktiven Arbeitsförderung 149

e) Geltungszeitraum der Vereinbarung 149

4. Formelle Gesichtspunkte 150

5. Rechtliche Einordnung $\quad 150$

a) Verwaltungsakt, $\S 31 \mathrm{SGB} X$

aa) Hoheitliche Maßnahme 151

bb) Regelung 154

cc) Zwischenergebnis 155

b) „Eingliederungsplan“ $\quad 155$

c) Verwaltungsrechtlicher Vertrag, $\S \S 53$ ff SGB X 156

aa) Allgemeine Voraussetzungen 156

bb) Abgrenzung zum privatrechtlichen Vertrag 156

cc) Ausschluss verwaltungsrechtlicher Vertrag, § 53 Abs. 2 SGB X 157

dd) Vertragsschluss 157

ee) Begründung, Änderung oder Aufhebung eines Rechtsverhältnisses 158

(1) Rechtsverhältnis 158

(2) Aufhebung eines Rechtsverhältnisses 158

(3) Begründung eines Rechtsverhältnisses 159

(4) Inhaltliche Änderung eines Rechtsverhältnisses 159

(5) Zusammenfassung 161

ff) Zwischenergebnis 161

d) Vertragsähnliches Verwaltungshandeln $\quad 161$

6. Funktion der Eingliederungsvereinbarung 162

a) Grundsatz vom Fördern und Fordern 162

b) Neubegründung von Leistungspflichten 163

c) Mitwirkungspflicht, §38 SGB III 163

d) Auslegung unbestimmter Rechtsbegriffe 164

e) Eigenbemühungen, § 119 Abs. 4 Nr. 1 SGB III 165

f) Qualitätssicherung und Qualitätsprüfung 165

7. Wirkungen der Eingliederungsvereinbarung 166

a) Voraussetzung für Leistungen der Agenturen für Arbeit 166

aa) Vermittlungstätigkeit 166

bb) Entgeltersatzleistungen, insbesondere Arbeitslosengeld 166

cc) Zwischenergebnis 167 
b) Ermessensausübung

aa) Verwaltungsermessen: Begriff und Abgrenzung 167

bb) Ermächtigung 168

cc) Zweck der Ermächtigung 168

dd) Gesetzliche Grenzen bei der Ermessensausübung 169

ee) Grenzen aus der Eingliederungsvereinbarung 169

ff) Gerichtliche Überprüfbarkeit des Ermessens 170

$\begin{array}{ll}\text { c) Verhängung von Sanktionen } & 171\end{array}$

aa) Sperrzeit, § 144 SGB III 171

(1) Versicherungswidriges Verhalten 172

(2) Sperrzeit bei unzureichenden Eigenbemühungen 172

(3) Verhältnis von Sperrzeit und § 2 Abs. 5 SGB III 173

bb) Einstellung der Vermittlung, § 38 Abs. 2 SGB III 174

d) Darlegungs- und Beweislastverteilung 175

aa) Untersuchungsgrundsatz im Verwaltungsverfahren $\quad 175$

bb) Umkehr der Darlegungs- und Beweislast bei einer Sperrzeit $\quad 175$

cc) Beweisfunktion im gerichtlichen Verfahren 176

8. Störungen bei der Abwicklung des vertragsähnlichen Verhältnisses $\quad 176$

a) Veränderung der tatsächlichen Verhältnisse 176

b) Nichteinhaltung von vereinbarten Pflichten durch den Arbeitslosen 177

aa) Kein Erfüllungsanspruch 177

bb) Schadensersatz 177

cc) Vertragsstrafe 177

c) Nichteinhaltung von vereinbarten Pflichten durch Agentur für Arbeit 177

9. Beendigung der Eingliederungsvereinbarung 178

a) Erledigung infolge der Beendigung der Arbeitslosigkeit 178

$\begin{array}{ll}\text { b) Zeitablauf } & 178\end{array}$

c) Abänderung 178

$\begin{array}{ll}\text { d) Kündigung } & 179\end{array}$

10. Rechtsschutz 179

a) Außergerichtlicher Rechtsschutz $\quad 179$

aa) Beratung und Entscheidung des Vorgesetzten 179

bb) Dienstaufsichtsbeschwerde 179

b) Widerspruch gegen einseitige Entscheidung $\quad 180$

c) Gerichtlicher Rechtsschutz $\quad 180$

aa) Rechtsweg 180

bb) Klägerisches Begehren 180

cc) Klage gegen den Sanktionsbescheid 180

dd) Klage gegen die Einstellung der Vermittlung 181

d) Kosten 
II. Recht der Grundsicherung für Arbeitsuchende

1. SGB II und Viertes Gesetz für moderne Dienstleistungen am Arbeitsmarkt 182

a) Zusammenführung von Arbeitslosenhilfe und Sozialhilfe: „Hartz IV“ 182

b) Kommunales Optionsgesetz

c) Überblick über die Leistungen nach dem SGB II

183

aa) Leistungen zur Eingliederung in Arbeit, $\S \S 14$ ff SGB II

185

bb) Leistungen zur Sicherung des Lebensunterhaltes, $\$$ \& 19 ff SGB II

2. Definition der Eingliederungsvereinbarung und Abgrenzung

a) Begriff

187

b) Parteien der Vereinbarung und Einvernehmen des kommunalen Trägers 188 aa) Fallmanager als Bevollmächtigter der Agentur für Arbeit 188

bb) Erwerbsfähiger Hilfebedürftiger

189

cc) Einvernehmen des kommunalen Trägers, § 15 Abs. 1 S. 1 SGB II 190

c) Bedarfsgemeinschaft, § 7 Abs. 3 SGB II 191

d) Abgrenzung 192

aa) Bundessozialhilfegesetz (BSHG) 192

bb) Zwölftes Buch Sozialgesetzbuch (SGB XII) 193

3. Inhalt der Eingliederungsvereinbarung 193

a) Niederlegung „verbindlicher Aussagen zum Fördern und Fordern“ 193

b) Leistungen zur Eingliederung, § 15 Abs. 1 S. 2 Nr. 1, §§ 16 ff SGB II 193 aa) §16 Abs. 1 SGB II 194

bb) § 16 Abs. 2 SGB II 195

cc) §16 Abs. 3 SGB II 195

c) Bemühungen erwerbsfähiger Hilfebedürftiger, § 15 Abs. 1 Nr. 2 SGB II196

d) Schadensersatzpflicht, § 15 Abs. 3 SGB II 197

e) Geltungszeitraum der Vereinbarung 197

4. Form 198

5. Rechtliche Einordnung 198

a) Verwaltungsakt, § $31 \mathrm{SGB} X$

aa) Hoheitliche Maßnahme 198

bb) „Ersatzvornahme“ durch die Verwaltung, § 15 Abs. 1 S. 6 SGB II 200

cc) Zwischenergebnis 201

b) „Eingliederungsplan“, „Hilfeplan“ 201

c) Verwaltungsrechtlicher Vertrag, $\S 53$ ff SGB X 201

aa) Abgrenzung zum privatrechtlichen Vertrag 201

bb) Ausschluss verwaltungsrechtlicher Vertrag, § 53 Abs. 2 SGB X 202

cc) Vertragschluss und Kontrahierungszwang 202

(1) Die Willenserklärung des Bürgers 203

(2) Willenserklärung und Abschlusspflicht der Arbeitsverwaltung 204

(3) Kontrahierungszwang infolge § 2 S. 2 SGB II 206

(4) Fakt. Kontrahierungszwang, $\S \S 2$ S. 2, 31 Abs. 1 Nr. 1a SGB II207

(5) Fakt. Kontrahierungszwang aufgrund der Monopolstellung 208

(6) Fakt. Kontrahierungszwang aufgrund $\S 15$ Abs. 1 S. 6 SGB II 209

(7) Kontrahierungszwang im Rahmen des § 31 Abs. 5 SGB II 209

dd) Begründung, Änderung oder Aufhebung von Rechten und Pflichten210

ee) Einordnung als Vertrag i.S.d. § 53 Abs. 1 S. 2 SGB X 211

d) Zwischenergebnis 212 
a) Zusammenführung von Arbeitslosenhilfe und Sozialhilfe 212

b) Erwerbsfähigkeit und Hilfebedürftigkeit, §§ 8, 9 SGB II 213

c) Unbestimmte Rechtsbegriffe 213

d) Qualitätssicherung und Qualitätsprüfung 213

7. Wirkungen der Eingliederungsvereinbarung 214

a) Voraussetzung für Leistungen der Grundsicherung für Arbeitsuchende 214

aa) Leistungen zur Eingliederung in Arbeit, $\S \S 14$ ff SGB II 214

bb) Leistungen zur Sicherung des Lebensunterhaltes, §§ 19 ff SGB II 215

cc) Zwischenergebnis 215

b) Ermessensausübung $\quad 215$

aa) Ermächtigung 215

bb) Zweck der Ermächtigung 216

cc) Gesetzliche Grenzen 217

dd) Grenzen aus der Eingliederungsvereinbarung 218

ee) Ermessensfehlerlehre und Nichtigkeit gem. § 58 SGB X 218

c) Verhängung von Sanktionen, § 31 SGB II 218

aa) Ablehnung angebotener Vereinbarung, §31 Abs. 1 Nr. 1a SGB II 220

(1) Angebot einer Eingliederungsvereinbarung 220

(2) Abschlussverweigerung 220

(3) Wichtiger Grund für die Abschlussverweigerung 221

(4) Rechtsfolgenbelehrung 222

bb) Nichteinhaltung vereinbarter Pflichten, § 31 Abs. 1 Nr. 1b SGB II 222

(1) Anwendungsbereich der Sanktionsvorschrift 222

(2) Verletzung der Nachweispflicht 223

(3) Wichtiger Grund 223

cc) Weigerung, Arbeit aufzunehmen, § 31 Abs. 1 Nr. 1c SGB II 224

dd) Sonderregelung für Jugendliche, § 31 Abs. 5 SGB II 224

d) Einstellung der Vermittlung 225

e) Darlegungs- und Beweislastverteilung im Verwaltungsverfahren 225

8. Störungsfälle 226

a) Nichtigkeit des verwaltungsrechtlichen Vertrages, §58 SGB X 226

aa) Grundsatz der Gesetzmäßigkeit der Verwaltung 226

bb) Grundsätze des Verwaltungsverfahrensrechts 227

cc) Verstoß gegen Verbotsgesetz, § 58 Abs. 1 SGB X, § 134 BGB 228

dd) Sittenwidrigkeit, §58 Abs. 1 SGB X i.V.m. § 138 BGB 229

ee) Nichtigkeit gem. § 58 Abs. 2 Nr. 1 SGB X i.V.m. § 40 SGB X 230

ff) Nichtigkeit gem. $\S 58$ Abs. 2 Nr. 2 SGB X 230

gg) Nichtigkeit gem. $\S \S 58$ Abs. 2 Nr. 3, 4 SGB X 231

hh) Zwischenergebnis 231

b) Nichterteilung des Einvernehmens des kommunalen Trägers 231

c) Veränderung der tatsächlichen Verhältnisse 233

d) Nichteinhaltung vereinbarter Pflichten durch Hilfebedürftigen 233

aa) Schadensersatz bei Bildungsmaßnahme, § 15 Abs. 3 SGB II 234

bb) Vertragsstrafe, §61 SGB X i.V.m. §§339 ff BGB 235

cc) Schadensersatz, § 61 S. 2 SGB X i.V.m. § 280 BGB 236

e) Nichteinhaltung der vereinbarten Pflichten durch die Agentur für Arbeit236

aa) Erfüllungsanspruch 236

bb) Vertraglicher Schadensersatz/Aufwendungsersatz 236

cc) Amtshaftungsansprüche 237 
9. Beendigung der Eingliederungsvereinbarung

a) Erledigung 237

b) Zeitablauf 237

c) Änderung der Verhältnisse, §59 Abs. 1 S. 1 SGB X 237

d) Kündigung des verwaltungsrechtlichen Vertrags, § 59 SGB X 237

10. Rechtsschutz 238

a) Außergerichtlicher Rechtsschutz 238

aa) Dienstaufsichtsbeschwerde 238

bb) Ombudsrat 238

b) Widerspruchsverfahren 239

c) Gerichtlicher Rechtsschutz 239

aa) Rechtsweg, § 51 SGG 239

bb) Rechtsschutz bei Umsetzung der Vereinbarung 239

cc) Rechtschutz gegen Sanktionen und Einstellung der Vermittlung 240

(1) Klägerisches Begehren 240

(2) Klageart 240

dd) Feststellung der Nichtigkeit der Eingliederungsvereinbarung 240

d) Kosten

Drittes Kapitel: Ergebnisse und Auswertung des Rechtsvergleichs

A. Zusammenfassung der Ergebnisse

I. Jobseeker's agreement

II. Eingliederungsvereinbarung nach SGB III

III. Eingliederungsvereinbarung nach SGB II

B. Vergleich und Bewertung

I. Kooperation - Ziele erreicht? 246

1. Steigerung der Effizienz in der Verwaltungsarbeit 246

2. Information der Verwaltung $\quad 248$

3. Legitimierende Wirkung $\quad 249$

4. Psychologisch-pädagogischer Effekt 250

II. Kooperation - Gefahren gebannt? 252

1. Übervorteilung und Verhandlungsungleichgewicht $\quad 252$

2. Ausschluss aus dem System 253

3. Verrechtlichung 254

4. Haftung und Rechtsschutz $\quad 256$

a) Haftung 256

b) Defizite beim Rechtsschutz 257

5. Kosten - Nutzen -Vergleich 258

III. Kooperation an ihren Grenzen $\quad 260$

1. Verfassungsrechtlicher Rahmen 260

a) Eigentum $\quad 260$

b) Handlungsfreiheit 260

c) Existenzminimum 261

d) Rechtsweggarantie 262 
2. Vorgaben des Verfahrensrechts

a) Fairer Umgang mit dem Bürger 263

b) Untersuchungsgrundsatz

c) Ermessensausübung

3. Bindung inter partes

IV. Kooperation und Aktivierung als Rahmen eines Politiktransfers 265

V. Bewährungsprobe und Zukunft der Handlungsinstrumente 266

C. Abschließende Bemerkung 268

Literaturverzeichnis 


\section{Abkürzungsverzeichnis}

a.A.

a.F.

AB1

Abs.

AC

$\mathrm{AcP}$

Admin LR

AGSG

AiB

Anm

AöR

$\mathrm{APuZ}$

ARGE

Art.

AuA

AuR

Az.

BArbB1

BayVwVfG

BayLSG

Bd.

BGB

BGB1.

BGesundB1

$\mathrm{BGH}$

BGHZ

$\mathrm{BHO}$

BJPIR

BJPS

BJSW

BSHG

BKK

BSG

BSGE

BT-Drucksache

BR-Drucksache

BVerfG

BVerfGE

BVerwG

BVerwGE andere Auffassung

alte Fassung

Amtsblatt

Absatz

Appeal Cases

Archiv für die civilistische Praxis

Administrative Law Review

(Bayer.) Gesetz zur Ausführung der Sozialgesetze

Arbeitsrecht im Betrieb

Anmerkung

Archiv des öffentlichen Rechts

Aus Politik und Zeitgeschichte

Arbeitsgemeinschaft gem. $\S 44 b$ SGB II

Artikel

Arbeit und Arbeitsrecht

Arbeit und Recht

Aktenzeichen

Bundesarbeitsblatt

Bayerisches Verwaltungsverfahrensgesetz

Bayerisches Landessozialgericht

Band

Bürgerliches Gesetzbuch

Bundesgesetzblatt

Bundesgesundheitsblatt

Bundesgerichtshof

Entscheidungen des Bunesgerichtshofs in Zivilsachen

Bundeshaushaltsordnung

British Journal of Politics and International Relations

British Journal of Political Science

British Journal of Social Work

Bundessozialhilfegesetz

Die Betriebskrankenkasse

Bundessozialgericht

Entscheidungen des Bundessozialgerichtes

Bundestagsdrucksache

Bundesratsdrucksache

Bundesverfassungsgericht

Entscheidungen des Bundesverfassungsgerichts

Bundesverwaltungsgericht

Entscheidungen des Bundesverwaltungsgerichts 
bzw.

CA

CDU

CEPA

CJQ

CJSA

CLP

$\mathrm{Cm}$

$\mathrm{CO}$

CS

CS

DAngVers

DB

d.h.

ders.

dies.

DNotZ

DÖV

DRV

DStZ

DuR

DVB1

DWP

EGV

Einl.

EJIR

EJPR

EJSS

EMRK

EuGRZ

EuR

$\mathrm{EuV}$

EWRSP

f, ff

Fisc Stud

FPR

FS

gem.

GG

GK

GKV-WSG beziehungsweise

Court of Appeal

Christlich Demokratische Union

Center for Economic Policy Analysis

Civil Justice Quarterly

Decisions of the Commissioner Jobseekers Allowance

Current Legal Problems

command paper

Crown Office Digest

Critical Social Policy

Christlich Soziale Union

Die Angestelltenversicherung

Der Betrieb

das heißt

Derselbe

dieselbe, dieselben

Deutsche Notarzeitung

Die Öffentliche Verwaltung

Deutsche Rentenversicherung

Deutsche Steuerzeitung

Demokratie und Recht

Deutsches Verwaltungsblatt

Department for Work and Pensions

Vertrag zur Gründung der Europäischen Gemeinschaft, EG-Vertrag

Einleitung

European Journal of Industrial Relations

European Journal of Political Research

European Journal of Social Security

Europäische Menschenrechtskonvention

Europäische Grundrechte Zeitschrift

Europarecht

Vertrag über die Europäische Union (Vertrag von Maastricht); EU-

Vertrag

East-West Review of Social Policy

folgende

Fiscal Studies

Familie, Partnerschaft, Recht

Festschrift

gemäß

Grundgesetz

Gemeinschaftskommentar

Gesetz zur Stärkung des Wettbewerbs in der gesetzlichen Krankenver-

sicherung; GKV-Wettbewerbsstärkungsgesetz 
GLJ

GSP

$\mathrm{GMH}$

h.M.

HansOLG

HMSO

Halbs.

HRA

IAB

IAT-Report

ibv

IJCLLIR

IJSW

ILJ

info also

Info AuslR

i.V.m.

JCP

JESP

JLS

JSA

JSP

JSSL

JSSW

Jura

jurisPR-ArbR

jurisPR-SozR

$\mathrm{JuS}$

JZ

KB

$\mathrm{KJ}$

KOM

LMT

LPK

LQR

MDR

Mitb

MLR

m.w.N.

NDV

NE
German Law Journal

Gesundheits- und Sozialpolitik

Gewerkschaftliche Monatshefte

herrschende Meinung

Hanseatisches Oberlandesgericht

Her Majesty's Stationary Office

Halbsatz

Human Rights Act

Institut für Arbeitsmarkt und Berufsforschung, Nürnberg

Elektronischer Report des Instituts Arbeit und Technik

Informationen für die Beratungs- und Vermittlungsdienste

International Journal of Comparative Labour Law and Industrial Relations

International Journal of Social Welfare

The Industrial Law Journal

Informationen zum Arbeitslosenrecht und Sozialhilferecht

Informationsbrief für das Ausländerrecht

in Verbindung mit

Jobcentre Plus

Journal of European Social Policy

Journal of Law and Society

jobseeker's agreement

Journal of Social Policy

Journal of Social Security Law

Journal of Sociology and Social Welfare

Juristische Ausbildung

juris Praxisreport Arbeitsrecht

juris Praxisreport Sozialrecht

Juristische Schulung

Juristenzeitung

King's Bench

Kritische Justiz

Kommission

Labour Market Trends

Lehr- und Praxiskommentar

The Law Quarterly Review

Monatsschrift des Deutschen Rechts

Die Mitbestimmung

The Modern Law Review

mit weiteren Nachweise

Nachrichtendienst des Deutschen Vereins für öffentliche und private Fürsorge

New Economy 
$\mathrm{NG} / \mathrm{FH}$

NHS

$\mathrm{Nr}$.

NJW

NVwZ

NwUnivLR

NWVBl

NZA

NZS

OECD

OJLS

para.

PL

PM

PP

PPMR

PQ

PSA

PubAdmin

QB

$\mathrm{RdA}$

reg.

RL

$\mathrm{Rn}$.

RsDE

Rspr.

Rz.

$\mathrm{S}$.

SDSRV

sched.

sec.

SEDEC-Network

SG

$\mathrm{SGb}$

SGB

SGG

SI

sog.

SozFort

SozR
Die neue Gesellschaft/Frankfurter Hefte

National Health Service

Nummer

Neue Juristische Wochenschrift

Neue Zeitschrift für Verwaltungsrecht

Northwestern University Law Review

Nordrheinwestfälische Verwaltungsblätter

Neue Zeitschrift für Arbeitsrecht

Neue Zeitschrift für Sozialrecht

Organisation for Economic Co-operation and Development

Oxford Journal of Legal Studies

paragraph

Public Law

Public Management

Policy and Politics

Public Performance and Management Review

The Political Quarterly

Personal Service Agentur

Public Administration Review

Queen's Bench

Recht der Arbeit

regulation

Richtlinie

Randnummer

Beiträge zum Recht der sozialen Dienste und Einrichtungen

Rechtsprechung

Randzeichen

Satz, Seite

Schriftenreihe des Deutschen Sozialrechtsverbandes

schedule

Section

thematisches wissenschaftliches Netzwerk, finanziert durch das TSERProgramm (Targeted Socio-Economic Research) der EU-Kommission zum Thema "Vergleichende Politik der sozialen Eingliederung und der Staatsbürgerschaft in Europa."

Sozialgericht

Die Sozialgerichtsbarkeit

Sozialgesetzbuch

Sozialgerichtsgesetz

Statutory Instrument

sogenannt(e)

Sozialer Fortschritt

Sozialrechtliche Entscheidungen 
SozR akt

SozSich

SPA

SPD

SPR

SpuRt

SSAT

subs.

SuP

TSO

u.a.

UCLA

UK

usw.

$\mathrm{V}$

v.

v.a.

VersR

VerwArch

vgl.

VSSR

VVDStRL

VwGO

VwVfG

WEP

WES

WLR

WiW

WRB

WSI-Mitteilungen

WuM

WzS

ZAR

ZaöRV

z.B.

ZESAR

ZEuP

ZfA

ZfBauR

$\mathrm{ZfF}$

ZfJ

ZfS
Sozialrecht aktuell

Soziale Sicherheit

Social Policy and Administration

Sozialdemokratische Partei Deutschlands

Social Policy Review

Zeitschrift für Sport und Recht

Social Security Administrative Tribunal

subsection

Sozialrecht und Praxis

Theorie und Praxis der Sozialen Arbeit

und andere, unter anderem

United Nations

United Kingdom

und so weiter

versus

vom, von

vor allem

Versicherungsrecht

Verwaltungsarchiv

vergleiche

Vierteljahresschrift für Sozialrecht

Veröffentlichungen der Vereinigung der Deutschen Staatsrechtslehrer

Verwaltungsgerichtsordnung

Verwaltungsverfahrensgesetz

West European Politics

Work, Employment and Society

Weekly Law Reports

Wirtschaft im Wandel

Welfare Rights Bulletin

Monatszeitschrift des Wirtschafts- und Sozialwissen-schaftlichen Instituts in der Hans-Böckler-Stiftung

Wohnungswirtschaft und Mietrecht

Wege zur Sozialversicherung

Zeitschrift für Ausländer- und Asylrecht

Zeitschrift für ausländisches und öffentliches Recht und Völkerrecht zum Beispiel

Zeitschrift für europäisches Sozial- und Arbeitsrecht

Zeitschrift für europäisches Privatrecht

Zeitschrift für Arbeitsrecht

Zeitschrift für deutsches und internationales Bau- und Vergaberecht

Zeitschrift für das Fürsorgewesen

Zentralblatt für Jugendrecht

Zentralblatt für Sozialversicherung, Sozialhilfe und Versorgung 
$\mathrm{ZfSH} / \mathrm{SGB}$

ZfVglRWiss

ZfWU

ZIAS

ZIP

ZRP

ZSR
Zeitschrift für Sozialhilfe und Sozialgesetzbuch

Zeitschrift für Vergleichende Rechtswissenschaften

Zeitschrift für Wirtschafts- und Unternehmensethik

Zeitschrift für ausländisches und internationales Arbeits- und Sozialrecht

Zeitschrift für Wirtschaftsrecht

Zeitschrift für Rechtspolitik

Zeitschrift für Sozialreform 


\section{Einführung}

Der deutsche Gesetzgeber hat im Job-AQTIV-Gesetz ${ }^{1}$ und im Vierten Gesetz für moderne Dienstleistungen am Arbeitsmarkt ${ }^{2}$, aber auch bei der Eingliederung des Bundessozialhilfegesetzes in das Sozialgesetzbuch ${ }^{3}$ ein Handlungsinstrument bei der Gewährung von Sozialleistungen in ein neues Licht gerückt, das bislang im Schatten einseitig-hoheitlichen Verwaltungshandelns stand: Die Vereinbarung zwischen Sozialverwaltung und Leistungsempfänger, in der geregelt wird, ob und wie bestimmte Sozialleistungen und die Gegenleistungen des Leistungsempfängers erbracht werden.

Entsprechend der Ergebnisse der Hartz-Kommission ${ }^{4}$ und dem Schlagwort „Fördern und Fordern" 5 müssen der Leistungsempfänger und die Verwaltung kooperieren, wollen sie den Geist des Gesetzes erfüllen. Hilfe soll auf das im konkreten Einzelfall Erforderliche beschränkt, und gleichzeitig die Einhaltung des von beiden Seiten Vereinbarten sichergestellt werden. Der Staat verzichtet insoweit auf hoheitliche Maßnahmen. Vom Gesetzgeber beabsichtigte Freiräume sind so auszufüllen, dass die staatliche Unterstützung genau auf die Bedürfnisse des Einzelnen ausgerichtet ist. Wird der Einzelne auf diese Weise, soweit er selbst das überhaupt zulässt, in die Entscheidung über Leistungsgewährung und Leistungsabwicklung mit einbezogen, soll er sich stärker für die Einhaltung verantwortlich fühlen. Gerade bei der Bekämpfung der Arbeitslosigkeit erscheint dem Gesetzgeber Kommunikation mit dem Leistungsempfänger und deren schriftliche Fixierung in einer Vereinbarung für die „passgenaue“ (Eingliederungs-)Leistung zielführend.

Das englische Recht, in dem Arbeitslose nur staatliche Unterstützung erhalten, wenn sie vorher mit dem zuständigen Jobcentre ein sogenanntes jobseeker's agreement geschlossen haben, wird oft in pauschaler Weise als Vorbild für den deutschen Gesetzgeber bezeichnet. In dieser Vereinbarung zwischen Arbeitslosem und Arbeitsverwaltung sind die Voraussetzungen für die Sozialleistung detailliert beschrieben. Die gewährte Sozialleistung ist eng verknüpft mit Anforderungen, die vor allem an den Arbeitslosen selbst adressiert sind und die ihm deutlich machen sollen, dass er seine Arbeitslosigkeit, jedenfalls aber den Hilfebezug, zu beenden suchen muss.

1 Gesetz zur Reform der arbeitsmarktpolitischen Instrumente (Job-AQTIV-Gesetz) v. 10.12.2001 (BGBl. I, S. 3443).

2 Gesetz v. 24.12.2003 (BGB1. I, S. 2954).

3 Gesetz zur Eingliederung des Sozialhilferechts in das Sozialgesetzbuch v. 27.12.2003 (BGB1. I, S. 3022).

4 Hartz, Moderne Dienstleistungen am Arbeitsmarkt, 2002.

5 So in der BT-Drucksache 14/6944, S. 25 (Entwurf Job-AQTIV-Gesetz) und der BT-Drucksache 15/1516, S. 65 (Entwurf Viertes Gesetz für moderne Dienstleistungen am Arbeitsmarkt). 
Die Einbeziehung des einzelnen Leistungsempfängers durch Kooperation mit dem Leistungsträger im Rahmen von Vereinbarungen ist nur ein Element in einer ganzen Reihe von Veränderungen, die für das Verwaltungs- und Sozialrecht in England und in der Bundesrepublik Deutschland vorgeschlagen werden und teilweise auch Gesetz geworden sind. Diesen Veränderungen, die auf Aktivierung und mehr Eigenverantwortung des Einzelnen abzielen, liegt das Konzept „activating labour market policy “ beziehungsweise ,aktivierende Arbeitsmarktpolitik“ zugrunde. ${ }^{6}$ Die Vereinbarung als Handlungsinstrument der Arbeits- und Sozialverwaltung zu etablieren, ist zwar nicht die einzige, aber doch die zentrale Voraussetzung für die tatsächliche Aktivierung des Einzelnen. Für diese Arbeit ist es deshalb erforderlich, Inhalt, Hintergründe und Entwicklungen ebenso wie Ziele aktivierender Vorgehensweise der Verwaltung auf nationaler Ebene zu beschreiben, das Handeln des englischen und deutschen Gesetzgebers in Bezug zueinander zu setzen und Verbindungen zwischen den Rechtsordnungen aufzuzeigen.

Dafür spielen Vorgaben und Empfehlungen inter- und supranationaler Institutionen eine wichtige Rolle. Der nationale Gesetzgeber bewegt sich in der bestehenden Verwobenheit von nationaler und inter- und supranationaler Ebene. Die inter- und supranationalen Institutionen beobachten wissenschaftlich fundiert Entwicklungen in den einzelnen sozialen Sicherungssystemen und geben Handlungsempfehlungen ab, z.B. dafür, wie ein wegen hoher Arbeitslosigkeit als erforderlich angesehener Umbau der sozialen Sicherung, insbesondere der Umbau der Arbeitsverwaltung vonstatten gehen soll. Daneben gibt es auf supranationaler Ebene auch verbindliche Vorgaben für den nationalen Gesetzgeber. Mit diesen Ergebnissen und Vorgaben setzen sich die nationalen Gesetzgeber auseinander und passen gegebenenfalls ihre nationalen Politiken an.

\section{Nationale Konzepte}

\section{Hintergrund und Entwicklung der Activating Labour Market Policy im Vereinig-} ten Königreich und in den Vereinigten Staaten von Amerika

a) Der welfare-to-work-Ansatz der Regierung Thatcher

Im Vereinigten Königreich betrieb die Regierung unter Margaret Thatcher gefolgt von der John Majors bereits vor der Regierungszeit Tony Blairs eine Politik, die den Ansatz von welfare-to-work in den Mittelpunkt ihres Kampfes gegen Arbeitslosigkeit stellte. ${ }^{7}$ Die Regelungen zur jobseeker's allowance traten bereits 1996 in Kraft. ${ }^{8}$ Mit

6 Einen Überblick über Staaten, die Konzepte aktivierender Arbeitsmarktpolitik umgesetzt haben, bietet Eichhorst/Kaufmann/Konle-Seidl, Bringing the jobless into work?, 2008.

7 Judge, Evaluating welfare to work in the United Kingdom, in: Gilbert/Van Voorhis, Activating the unemployed, 2001, S. 2; Finn/Schulte, "Employment first": Activating the British welfare state, in: Eichhorst/Kaufmann/Konle-Seidl, Bringing the jobless into work?, 2008, S. $297 \mathrm{ff}$. 
der Zusammenfassung von Versicherungsleistungen und fürsorgenden Leistungen gingen weitere Änderungen einher: Die Leistungen bei Arbeitslosigkeit wurden gesenkt, die Leistungsdauer wurde von zwölf Monaten auf sechs Monate beschränkt und die eigenständige Arbeitsuche (actively seeking work) als Voraussetzung für Leistungen eingeführt. ${ }^{9}$

\section{b) Aktivierung in den Vereinigten Staaten von Amerika}

Das Vorgehen im Vereinigten Königreich war beeinflusst durch die Sozial- und Wirtschaftspolitik in den Vereinigten Staaten ${ }^{10}$ und wird als umfangreicher policy transfer beschrieben. Damit ist gemeint, dass die sozial- und wirtschaftspolitischen Ideen und Strategien, nicht aber deren rechtliche Umsetzung, aus den Vereinigten Staaten übernommen wurde. ${ }^{11}$ Der policy transfer wird mit den engen persönlichen Verbindungen auf politischer Ebene und der nahen Verwandtschaft der sozialen Sicherungssysteme des Vereinigten Königreichs und der Vereinigten Staaten begründet. ${ }^{12}$

In den USA begann mit der Clinton-Regierung und den Schlagworten ,we end welfare as we know it" und "when you can work you must work" eine neue Sozialpolitik, die den amerikanischen Wohlfahrtsstaat grundlegend verändert hat. ${ }^{13}$ Angekündigt mit diesen programmatischen Äußerungen William J. Clintons im Präsidentschaftswahlkampf $1992^{14}$ und einer Wiederholung in der State of the Union Adress vom 17. Februar 199315 wurde die Sozialhilfe im Jahre 1996 neu geregelt. Seither gibt es - und darin besteht die gravierende Änderung - in den USA keinen bundesweit einheitlichen und zeitlich unbegrenzten Rechtsanspruch auf Sozialhilfe mehr. ${ }^{16}$ Hinzukam die Ausrichtung von Sozialleistungen an der „Arbeitsfähigkeit“ und die Verknüpfung von Rechten und Pflichten in Bezug auf Sozialleistungen, die unter dem Stichwort from welfare to

8 Jobseekers Act 1995 (1995 c. 18), daneben Jobseeker's Allowance Regulations 1996 und Jobseeker's Allowance (Transitional Provisions) Regulations 1995.

9 King, In the name of liberalism, Oxford, 1999, S. 248.

10 Quade/O'Leary/Dupper, Activation from income support, in: Eichhorst/Kaufmann/Konle-Seidl, Bringing the jobless into work?, 2008, S. $345 \mathrm{ff}$.

11 Aus politikwissenschaftlicher Sicht Dolowitz, Policy transfer and British social policy, 2000.

12 Deacon, PP 1999, S. 5 ff; Daguerre/Taylor, JESP 2004, S. 25 ff; kritisch unter Hinweis auf Europäisierungstendenzen innerhalb der Labour-Regierung Annesley, BJPIR 2003, S. 143 ff; Heffernan, PQ 2001, S. $180 \mathrm{ff}$.

13 Dazu Quade, Verantwortung und ihre Zuschreibung im Recht der Arbeitsförderung, [erscheint demnächst], S. $124 \mathrm{ff}$.

14 Clinton, Acceptance Speech to the Democratic Convention of July 16, 1992, in: Wolley/Peters, The American Presidency project [online], University of California (hosted), Gerhard Peters (database), http://www.presidency.ucsb.edu/ws/?pid=25958.

15 Clinton, Adress before a joint session of Congress on administration goals of February 17 1993, in: Wolley/Peters, The American Presidency project [online], University of California (hosted), Gerhard Peters (database), http://www.presidency.ucsb.edu/ws/?pid=47232.

16 Personal Responsibility and Work Opportunity Reconciliation Act 1996, ausführlich dazu Quade, Verantwortung und ihre Zuschreibung im Recht der Arbeitsförderung, [erscheint demnächst], S. $192 \mathrm{ff}$. 
work zusammengefasst werden können. ${ }^{17}$ Wichtig ist dabei, dass welfare in einem pejorativen Sinn gebraucht wurde. ${ }^{18}$ Derjenige, der Sozialleistungen beansprucht, muss mit dem Makel leben, kein angesehenes Mitglied der Gesellschaft zu sein, denn nur derjenige ist angesehen, der auch arbeitet und seinen Lebensunterhalt selbst verdient.

\section{c) Elemente aktivierender Politik unter der Regierung Blair}

Die Reformen der vorangegangenen Tory-Regierung führte die Labour-Regierung unter Tony Blair fort, gab ihnen aber eine weitere Note, die dem „europäischen Wertehorizont" der Blairschen Reformen zugeschrieben wird. ${ }^{19}$ Die sog. New Deals (in der Bedeutung von „Neuanfang“") ${ }^{20}$ weisen wesentliche Unterschiede zu den Aktivierungsstrategien in den Vereinigten Staaten auf.

Zwar geht es auch bei ihnen um verstärkte Überwachung der Arbeitslosen bei der Arbeitsuche und bei Trainingsmaßnahmen, um drohenden Leistungsentzug bei fehlender Kooperation, um sog. tax credits und Mindestlöhne zur Bekämpfung der Armut, sowie um die Förderung bezahlter Arbeit. ${ }^{21}$ Doch hat die Förderung der Maßnahmeteilnehmer einen hohen Stellenwert; der Zwang trifft vor allem ungebundene junge Menschen und nicht - überspitzt formuliert - in erster Linie minderjährige, alleinerziehende Mütter. ${ }^{22}$ Im Vereinigten Königreich übernimmt der Staat grundsätzlich weiterhin Verantwortung für die soziale Sicherung seiner Bürger, auch wenn diese mit vom Einzelnen $\mathrm{zu}$ erbringenden Gegenleistungen verknüpft werden. Ein Beispiel dafür ist, dass die Veränderungen im sozialen Sicherungssystem nicht zur zeitlichen Befristung von Arbeitslosenunterstützung oder der Abschaffung einer jedenfalls in Teilen als Sozialversicherung gestalteten Arbeitslosenunterstützung führten. ${ }^{23}$

\section{Das deutsche Leitbild vom aktivierenden Sozialstaat}

\section{a) Inhaltliche Beschreibung des Leitbildes}

Das Leitbild vom aktivierenden (Sozial-)Staat hat in der deutschen wissenschaftlichen Diskussion Konjunktur. ${ }^{24}$ Es wird in der politikwissenschaftlichen Literatur ${ }^{25}$ be-

17 King/Wickham-Jones, PQ 1999, S. 64; vgl. dazu den Überblick bei Levy, SPR 2004, S. 194-197.

18 Annesley, BJPIR 2003, S. 146 spricht deshalb vom moralisierenden amerikanischen Ansatz.

19 Leisering/Hilkert, Von Großbritannien lernen?, 2000, S. 7; Grover/Stewart, JSP 1999, S. 89.

20 Z.B. New Deal Young People (18-24), New Deal 50 plus, New Deal Partners (http://www.jobcentreplus.gov.uk/JCP/Customers/New_Deal).

21 Judge, Evaluating welfare to work in the United Kingdom, in: Gilbert/Van Voorhis, Activating the unemployed, 2001, S. 2.

22 Vgl. ausführlich dazu Brewer/Clark/Wakefield, Fisc Stud 2002, S. 505 ff; Levy, SPR 2004, S. 198201; Nachtwey/Heise, WSI-Mitteilungen 2006, S. 131 ff.

23 Merkel, The third ways of social democracy, in: Cuperus/Duffek/Kandel, Multiple third ways, 2001, S. 57; Heron, SPR 2001, S. 75 ff.

24 Zusammenfassend zur Entwicklung Eichhorst/Grienberger-Zingerle/Konle-Seidl, Activation policies in Germany, in: Eichhorst/Kaufmann/Konle-Seidl, Bringing the jobless into work?, 2008, S. 17 ff. 
schrieben und seine Auswirkungen werden inzwischen auch aus ökonomischer ${ }^{26}$ und rechtlicher Sicht ${ }^{27}$ analysiert. Es handelt sich nicht um ein bereits fertig entwickeltes Konzept. Es ist vielmehr als Gedanken- und Ideengerüst zu verstehen, das sich einerseits aus unterschiedlichen theoretisch-ideologischen Quellen speist und umgekehrt für verschiedene theoretische Ansätze und Staatsverständnisse anschlussfähig ist. ${ }^{28}$ Es macht gerade ein Leitbild aus, dass es ein „dynamisches Element des Reformprozesses" 29 ist, das nicht abgeschlossen zu sein braucht.

Auf der einen Seite steht eine Programmatik der Reorganisation der Verwaltung, die von Staats- und Verwaltungsmodernisierung ${ }^{30}$ handelt, und teilweise vom neuen Steuerungsmodell ${ }^{31}$ gespeist wird. Auf der anderen Seite geht es um eine Programmatik der Reorganisation der staatlichen Politik, welche im Kern auf die Herstellung eines veränderten Beziehungsgeflechts zwischen Individuum und Gesellschaft abzielt. ${ }^{32}$ Schlagworte sind Zivilgesellschaft ${ }^{33}$ und bürgerschaftliches Engagement. ${ }^{34}$ Gleichzeitig übernimmt der aktivierende Staat weiterhin Kernaufgaben, die in seiner alleinigen Verantwortung verbleiben. ${ }^{35}$

Bei der Bestimmung des Verantwortungsbereichs des Einzelnen setzt der aktivierende Staat auf ein Fördern und Fordern, indem nach Möglichkeit Eigenbeiträge der Leistungsempfänger zur Voraussetzung der Unterstützung gemacht werden. Für den Einzelnen bedeutet dies, dass die zu erwartende Hilfe zielgruppenspezifisch, konsequenter und individueller ausgelegt wird und die Motivation, selbst an der eigenen Situation etwas zu ändern, nicht mehr durch pauschale Leistungsversprechen genommen wird. ${ }^{36}$

Das Verhältnis zwischen der Arbeitsverwaltung und dem Leistungsempfänger wird künftig als Vertragsbeziehung verstanden. Beiden Seiten werden auf diese Weise Rechte und Pflichten auferlegt. Im Zusammenspiel mit anderen Elementen aktivierender Po-

25 Damkowski/Rösener, Auf dem Weg zum aktivierenden Staat, 2003; Mezger/West, Aktivierender Sozialstaat und politisches Handeln, 2000; Blanke, Aktivierender Staat - aktive Bürgergesellschaft, 2001; Urban, WSI-Mitteilungen 2004, S. 467 ff; Trube/Wohlfahrt, WSI-Mitteilungen 2001, S. 27 ff; Sutor, Stimmen der Zeit 2005, S. 103 ff.

26 Holzner/Ochel/Werding, Vom OFFENSIV-Gesetz zur "Aktivierenden Sozialhilfe", 2003; Koch/Walwei, GSP 2004, S. 9 ff; Barkhaus/Hollstein, ZfWU 2003, S. 287 ff.

27 Luthe, NDV 2003, S. 167 ff; Kingreen, SDSRV 52 (2004), S. 7 ff; Münder, in: Münder/Armborst, LPK-SGB II, 2005, Einl. Rn. 9.

28 Damkowski/Rösener, Auf dem Weg zum aktivierenden Staat, 2003, S. 43; ebenso Heinze/Strünck, TuP 2001, S. $163 \mathrm{f}$.

29 Stöbe, Verwaltungsreform durch Organisationsentwicklung - Leitbilder als Instrument einer Modernisierungsstrategie, in: Behrens u.a., Den Staat neu denken, 1995, S. 130.

30 Dazu Blanke u.a., Handbuch zur Verwaltungsreform, 2001.

31 Damkowski/Rösener, Auf dem Weg zum aktivierenden Staat, 2003, S. 15.

32 Lessenich, WSI-Mitteilungen 2003, S. 214.

33 Schröder, NG/FH 2000, S. 200 ff.

34 Blanke/Schridde, Bürgerengagement und aktivierender Staat, in: Heinze/Olk, Bürgerengagement in Deutschland, 2001, S. 93 ff; Evers, TuP 2001, S. 83 ff.

35 Damkowski/Rösener, Auf dem Weg zum aktivierenden Staat, 2003, S. 45 f; Heinze/Strünck, TuP 2001, S. $163 \mathrm{f}$.

36 So die Kritik an der früheren Sozialhilfe bei Bartelheimer/Hobusch/Reis, Case Management in der Sozialhilfe, in: Dahme u.a., Soziale Arbeit für den aktivierenden Staat, 2003, S. 312. 
litik entstehen verschiedenste „vertragliche“ Beziehungen, nicht nur im Verhältnis Staat - Bürger wie bei der Eingliederungsvereinbarung, sondern auch im Verhältnis zu Maßnahmeträgern oder zwischen Service-Agenturen bzw. privaten Vermittlungsdiensten und der Arbeitsverwaltung. ${ }^{37}$ Um den Bürger als Träger der Ressourcen Information über und Wissen um den eigenen Bedarf einbeziehen zu können, bedient sich der aktivierende Staat der Vereinbarung. Er erhofft sich, so das Engagement des Einzelnen für die eigenen Belange zu erhöhen und die Entscheidungen der Behörden mit höherer Akzeptanz beim Einzelnen zu versehen. ${ }^{38}$

\section{b) Adressaten aktivierender Maßnahmen}

Aktivierung findet nicht nur in Form von Vereinbarungen im Zwei-PersonenVerhältnis statt. Der Staat selbst wird erfasst, aber auch die Gesellschaft als Ganzes und nur im Besonderen der einzelne Bürger. Auf wen dabei das Hauptaugenmerk gerichtet wird, wird in der Literatur uneinheitlich beurteilt. Teilweise wird Aktivierung schlicht als zweiseitiger Prozess beschrieben. ${ }^{39}$ Andere betonen, dass vor allem die Verwaltung selbst aktiviert werden müsse. ${ }^{40}$ Wieder andere legen den Schwerpunkt auf den Einzelnen ${ }^{41}$ und schreiben von der „,sozialen Hängematte“, die zum „Trampolin“ werden müsse. 42

Bezogen auf die Umgestaltung des Arbeitsmarktes ist Zielgruppe aktivierender Maßnahmen die Gruppe der Arbeitslosen, insbesondere der Langzeitarbeitlosen, also Menschen, die ein Jahr und länger arbeitslos sind.

Auch die ehemalige Bundesanstalt für Arbeit wurde nicht nur in Bundesagentur für Arbeit umbenannt, sondern umfassend reformiert und insbesondere der Auftritt der Behörde gegenüber dem Bürger verändert. ${ }^{43}$

\section{c) Ziele des aktivierenden Sozialstaates}

Das Leitbild vom aktivierenden Sozialstaat lässt sich nicht auf ein Ziel reduzieren. So heißt es von Seiten derjenigen, die sich programmatisch mit dem aktivierenden Sozialstaat befasst haben, in allgemeinen Worten, es gelte im aktivierenden Sozialstaat da-

37 Eichenhofer, VSSR 2004, S. $107 \mathrm{f}$.

38 Spitzer, Bürgeraktivierung und Verwaltungsmodernisierung, in: Blanke u.a., Handbuch zur Verwaltungsreform, 2001, S. $144 \mathrm{f}$.

39 SEDEC-Network, Comparative Social Inclusion Policies and citizenship in Europe: Towards a new European social model, S. 42 (http://www.rwbsocialplanners.com.au/spt2006/Social\%20Inclusion/ Comparative\%20Social\%20Inclusion\%20Models.pdf).

40 Bartelheimer/Hobusch/Reis, in: Dahme u.a., Soziale Arbeit für den aktivierenden Staat, 2003, S. 312.

41 Evers, Aktivierender Staat, in: Mezger/West, Aktivierender Sozialstaat und politisches Handeln, 2000, S. 23 f.

42 Hombach, SozSich 1999, S. 43; aufgegriffen von Graser, GLJ 2003, S. 201-221.

43 Drittes Gesetz für moderne Dienstleistungen v. 23.12.2003 (BGBl. I, S. 2848); dazu Konle-Seidl, BArbBl 2004, S. 4 ff; Konle-Seidl, New delivery forms of employment services in Germany, in: Sol/Westerveld, Contractualism in employment services, 2005, S. 187 ff; Karasch, SozSich 2004, S. 2 ff; Schönig, SozFort 2002, S. 9 ff. 
mit aufzuhören, „staatlicherseits über den Menschen auszuschütten, was ihm qua Norm zugestanden wird, orientiert an den Einschätzungen und Machtinteressen verschiedenster Interessengruppen, nur nicht im Interesse derjenigen, die Hilfe brauchen" “. ${ }^{4}$

Während sich die frühere Sozialpolitik insbesondere mit der Reduzierung des Angebots an Arbeitskräften befasste - Mittel der Wahl waren Frühverrentung und Aufrechterhaltung der Arbeitslosenhilfe als Einkommensersatz - werden diese Lösungsansätze im aktivierenden Staat als überholt betrachtet. ${ }^{45}$ Es wird weder der Vorstellung eines Staates gefolgt, der die gesellschaftliche Wohlfahrt durch immer mehr staatliches Tätigwerden $\mathrm{zu}$ erhöhen beabsichtigt, noch die Idee eines neoliberalen Minimalstaats (,immer weniger Staat“) propagiert. 46 Der aktivierende Staat kann vielmehr als „Dritter Weg“ oder die „Neue Mitte“ zwischen einem möglicherweise entmündigenden Sozialoder Wohlfahrtsstaat und einem Staat verstanden werden, der sich z.B. mittels Privatisierung auf ein Mindestmaß an Aufgaben zurückzieht. ${ }^{47}$

\section{aa) Stärkung der Eigenverantwortung}

Der aktivierende Staat strebt eine Veränderung der gesellschaftlichen Beziehungen zwischen Staat und Einzelnem durch Dialog an und verfolgt dabei das Ziel, Potenziale und Kräfte freizusetzen, mit deren Hilfe innovative Problemlösungs- und Gestaltungsansätze erarbeitet und umgesetzt werden können. Ein Aspekt ist dabei die Hilfe zur Selbsthilfe, die sich nicht mehr wie bisher auf eine „Privatisierung mit anderen Mitteln“48 beschränkt. Die Menschen sollen es leichter haben, Initiative zu entwickeln, Engagement zu zeigen und Verantwortung zu übernehmen. ${ }^{49}$ Ihre Motivation, aktiv nach Arbeit zu suchen, soll durch entsprechende Anreize unterstützt werden.

Dieses (Spannungs-)Verhältnis von Eigenverantwortung und staatlicher Verantwortung ist ein Gedanke, den die Katholische Soziallehre, geprägt von von NellBreuning ${ }^{50}$, durch den Begriff Subsidiarität bereits lange vor der Diskussion um den aktivierenden Sozialstaat beschrieben hat. ${ }^{51}$ Der aktivierende Staat setzt zwar an derselben Stelle wie das Subsidiaritätsprinzip an: Die untere Ebene, der Bürger, organisiert

44 So pointiert Hombach, SozSich 1999, S. 42.

45 Manow/Seils, Adjusting badly: The German welfare state, in: Scharpf/Schmidt, Welfare and work in the open economy, Bd. 2, 2000, S. $264 \mathrm{ff} ;$ Manow/Seils, The employment crisis of the German welfare state, WEP 2000, S. 137-160.

46 Heinze/Strünck, TuP 2001, S. 163 f.

47 Vgl. die politische Diskussion um den Begriff des „schlanken Staats“ in der Koalitionsvereinbarung der CDU/CSU/FDP-Koalition, November 1994; dazu Scholz, Abschlussbericht des Sachverständigenrates „Schlanker Staat“, 1998 und zur Diskussion in England Vincent-Jones, PL 1999, S. 327.

48 Bandemer/Blanke/Hilbert/Schmid, Staatsaufgaben - von der "schleichenden Privatisierung" zum "aktivierenden Staat", in: Behrens u.a., Den Staat neu denken, 1995, S. 52.

49 Groner-Weber, Der aktivierende Staat, in: Mezger/West, Aktivierender Sozialstaat und politisches Handeln, 2000, S. 167.

50 Nell-Breuning, Soziallehre der Kirche, 1977.

51 Bandemer/Blanke/Hilbert/Schmid, Staatsaufgaben - von der "schleichenden Privatisierung" zum "aktivierenden Staat", in: Behrens u.a., Den Staat neu denken, 1995, S. 52; Strünck, Mitb 2000, S. 16. 
und hilft sich selbst, die übergeordnete Institution, der Staat oder ein Sozialleistungsträger, versetzt ihn dazu in die Lage und gibt entsprechende Ressourcen an die Hand.

Das Leitbild des aktivierenden Staates hat gleichwohl eine andere Zielrichtung als das Subsidiaritätsprinzip. Es geht beim aktivierenden Sozialstaat nicht - im Sinn einer „negativen Staatszielbestimmung“52 - um die Eingrenzung der staatlichen Tätigkeit als Kompetenzbegrenzung, sondern vielmehr um die Erweiterung der Initiativen des Einzelnen, also um die Beschreibung seines Verantwortungsbereichs. Der aktivierende Sozialstaat definiert diesen Verantwortungsbereich des Einzelnen neu.

\section{bb) Positiver Einfluss auf Arbeitslosigkeit}

Als weiteres Ziel des aktivierenden Sozialstaates ist die Vorstellung auszumachen, dass durch die Veränderung der Strukturen im sozialen Sicherungssystem Erfolg versprechende Umstände für den Rückgang der Arbeitslosigkeit geschaffen werden können. Der soziale Ausschluss durch Arbeitslosigkeit werde auf diese Weise bekämpft und die Abhängigkeit von Sozialleistungen beendet.

Es ist jedenfalls zweifelhaft, ob dieses Ziel mit Hilfe aktivierender Politik jemals wird erreicht werden können. Gerade die Anknüpfung an der Verantwortung des Einzelnen kann nicht darüber hinweg täuschen, dass strukturelle Arbeitslosigkeit nicht durch ein besonderes Engagement des Einzelnen erfolgreich bekämpft werden kann. ${ }^{53}$

Ob der Ansatz der Aktivierung tatsächlich keine positive Wirkung auf die Arbeitslosigkeit hat, vermag diese Arbeit nicht allgemein zu klären. Sie versucht aber, den Beitrag der Vereinbarung als Handlungsinstrument einzuschätzen.

\section{cc) Einsparungen bei den Sozialausgaben}

In Folge einer konsequenten Aktivierung sollen Einsparungen bei den Ausgaben für die soziale Sicherung erzielt werden, jedenfalls mittel- bis langfristig. Es wurde bereits bei der Beschreibung des Leitbildes dargestellt, dass das Leitbild für Deutschland diesen Aspekt nicht betont. Es ist nicht die Rede von Sozialabbau oder einem Rückzug des Staates.

Zwar wird die finanzielle Krise der Systeme der sozialen Sicherheit als die herausragende Chance des aktivierenden Sozialstaates angesehen ${ }^{54}$, doch dürfte diese Ansicht als Versuch anzusehen sein, „positiv zu denken“. Der Rückzug des Staates aufgrund von Sparzwängen ist vielmehr ein erfolgskritischer Faktor für das Leitbild, und die Instrumentalisierung des Bürgers möglicherweise ein „Feigenblatt für Haushaltskonsolidierung"55. Aktivierung vor dem Hintergrund eines Sparzwanges ist nicht zwangsläufig

52 Bandemer/Hilbert, Vom expandierenden zum aktivierenden Staat, in: Blanke u.a., Handbuch zur Verwaltungsreform, 2001, S. 20.

53 Dazu Trube, NDV 2003, S. 336.

54 Hombach, SozSich 1999, S. 44.

55 Damkowski/Rösener, Auf dem Weg zum aktivierenden Staat, 2003, S. 126. 
gleichzusetzen mit Sozialabbau. Das Leitbild zielt vielmehr auf die Qualität der Leistung, nicht allein auf deren Kosten ab. ${ }^{56}$

\section{Vergleichbarkeit der Leitbilder}

Aus den unterschiedlichen Staatssystemen im Vereinigten Königreich und in Deutschland ergeben sich Besonderheiten für das nationale Verständnis des Leitbildes vom aktivierenden Staat. Ob bei erfolgreicher Verwirklichung des Leitbildes in beiden Staaten diese Unterschiede eingeebnet werden, bleibt in den weiteren Ausführungen zu klären. Hier sollen nur stichpunktartig einige Aspekte angesprochen werden, die die Vergleichbarkeit der Leitbilder beschreiben.

\section{a) Staatsverständnis als Grundlage der Reformen}

Die Bestimmung des Staatsverständnisses legt den Ausgangspunkt für aktivierende Ansätze fest.

Im Vereinigten Königreich ist der Minimalstaat der Orientierungsmaßstab. Der Staat selbst ist zwar zuständig für die soziale Sicherheit, der Eintritt eines sozialen Risikos ist aber nicht immer und unverzüglich durch staatliches Engagement abzumildern, sondern zunächst durch ,private Solidarität“. Gemeint ist damit, dass vorrangig insbesondere institutionalisierte Hilfe durch Wohlfahrtsorganisationen und karitative Einrichtungen erbracht werden soll. ${ }^{57}$ Aktivierung verfolgte im Vereinigten Königreich auch das Ziel, diese staatliche Verantwortung für die soziale Sicherheit wieder in diesem Sinn zu verteilen und Tendenzen, die die Abhängigkeit der Bürger von Sozialleistungen beförderten, zurückzudrängen.

Aus deutscher Perspektive geht es bei der Verwirklichung des Leitbildes nicht in erster Linie um die Abgabe von staatlicher Zuständigkeit für soziale Leistungen z.B. an den Bürger, sondern v.a. darum, diese Leistungen zu optimieren. 58

Das Leitbild vom aktivierenden Staat entwickelte sich aufgrund dieser Ausgangslage unterschiedlich. Die rechtliche Umsetzung und tatsächliche Verwirklichung hängen ab von den jeweils vorhandenen Rahmenbedingungen des Sozialleistungssystems. Während es sich im Vereinigten Königreich um ein System handelt, in dem Entscheidungsund Kontrollkompetenzen zentralstaatlich gebündelt sind, hat Deutschland ein föderales System, in dem Verantwortung auf die Länder verteilt ist, aber auch selbstverwaltete Sozialversicherungsträger ${ }^{59}$ und Wohlfahrtsorganisationen dazwischen geschaltet sind. Auch die kommunale Ebene erfüllt im deutschen System sozialer Sicherung z.B. als Träger der Sozialhilfe gem. § 3 SGB XII wesentliche Aufgaben. Von aktivierender Sozialpolitik werden deshalb unterschiedlichste Organisationseinheiten betroffen sein.

56 So auch Groner-Weber, Der aktivierende Staat, in: Mezger/West, Aktivierender Sozialstaat und politisches Handeln, 2000, S. 169 f.

57 Z.B. Clasen, WEP 2000, S. 96 f; Knuth/Finn, IAT-Report 2004-04, S. 2.

58 Bandemer/Hilbert, Vom expandierenden zum aktivierenden Staat, in: Blanke u.a., Handbuch zur Verwaltungsreform, 2001, S. 18.

59 Dazu im Einzelnen Becker, Staat und autonome Träger im Sozialleistungsrecht, 1996, S. 116 ff. 
b) Politische Entwicklung und die Akzeptanz sozialpolitischer Maßnahmen

Die Akzeptanz einer Reform in der Bevölkerung hängt nicht nur von ihrem Inhalt ab. Abhängig vom Zeitpunkt ihrer Umsetzung wird gerade die Idee vom aktivierenden Staat entweder positiv auf- oder nur schmerzlich hingenommen.

Im Vereinigten Königreich wurden die grundlegenden rechtlichen Veränderungen im sozialen Sicherungssystem bereits vor der Labour-Regierung Blairs durch die Regierungen unter Margaret Thatcher und John Major vorgenommen. ${ }^{60}$ Es fand folglich kein Bruch mit konservativer Politik statt, sondern in Bezug auf aktivierende Elemente eine Fortführung. ${ }^{61}$ Hinzu kam, dass der wirtschaftliche Aufschwung im Vereinigten Königreich Arbeitsplätze brachte. Damit konnten strengere Anforderungen an den Einzelnen eher gerechtfertigt und vor allem zunehmend erfüllt werden.

In Deutschland muss von einem Politikwandel hin zu Aktivierung gesprochen werden. Zunächst lag der Schwerpunkt der Sozialpolitik auf einer Verschlankung des Staates durch Abbau von Bürokratie und Einsparungen im Bereich der Verwaltung. Der Einzelne war also nicht gefordert, sondern es fanden Umstrukturierungen allein im internen Bereich der Verwaltung statt. Erst nach dem Regierungswechsel im Jahr 1998 schlug die Koalition aus SPD und Bündnis 90/Die Grünen den Weg der Aktivierung ein, der den Einzelnen in das Zentrum stellte. In einer Zeit steigender (größtenteils auch struktureller) Arbeitslosigkeit und allgemein schlechter wirtschaftlicher Lage war es nur schwer politisch vermittelbar, dem einzelnen Arbeitslosen eine Mitschuld, jedenfalls eine große Verantwortung für seine Arbeitslosigkeit aufzubürden, wo doch gleichzeitig ungelöst war, wie genau Arbeitsplätze entstehen könnten.

\section{c) Rechtssystem als Rahmen für Aktivierung}

Im englischen Recht steht der Grundsatz supremacy of Parliament im Zentrum. ${ }^{62}$ Dieser erlaubt es dem Parlament, durch Regelungen die Rechtsordnung zu gestalten, ohne durch ein dichtes Netz rechtlicher Einschränkungen gebunden zu sein. So bestanden vor diesem Hintergrund kaum Widerstände, aktivierende Maßnahmen, z.B. neue Pflichten für die Sozialleistungsempfänger vorzusehen. Dabei sah man sich insbesondere nicht durch Garantien der Europäischen Menschenrechtskonvention bzw. dem Human Rights Act behindert. 63

Im deutschen Rechtssystem ist der Teil des Grundsatzes „Fördern und Fordern“, der die staatliche Forderung nach Einsatz der eigenen (Arbeits-)Kraft enthält, keinesfalls neu. Deren grundsätzliche Vereinbarkeit mit der Menschenwürde des Einzelnen war durch die Gerichte festgestellt ${ }^{64}$ und in der Literatur anerkannt ${ }^{65}$. Bereits im Sozialhil-

60 Finn, SPR 1998, S. 105-122.

61 Dazu Scharf, Sozialpolitik in Großbritannien: Vom Armengesetz zum "Dritten Weg", in: Kraus/Geisen, Sozialstaat in Europa, 2001, S. 7 ff; Leisering/Hilkert, Von Großbritannien lernen?, 2000, S. 7.

62 Dazu allgemein Phillips/Jackson, Constitutional and administrative law, 2001, S. 50 ff; Wade/Bradley, Constitutional and administrative law, 1985, S. $60 \mathrm{ff}$.

63 Dazu Craig, LQR 2002, S. 551 ff; Oliver, PL 2000, S. 476 ff.

64 BVerwGE 23, 149 [153]. 
fe- und Arbeitsförderungsrecht führte die Verweigerung zumutbarer Arbeit zu Sanktionen, was grundsätzlich als unproblematisch angesehen wurde. Es stand dabei nicht in Frage, dass die staatliche Verpflichtung zur individuellen Existenzsicherung in Form der Sozialhilfe als letztem Auffangnetz erfüllt wurde. In Bezug auf die Regelungen der Grundsicherung für Arbeitsuchende wird bezweifelt, ob hier der aktivierende Sozialstaat nur einer Rhetorik von Leistung und Gegenleistung verfallen ist und ob das verfassungsrechtlich garantierte Existenzminimum noch sichergestellt ist. 66

\section{Internationale und supranationale Konzepte}

Die eben dargestellten nationalen Konzepte müssen auch im Zusammenhang mit den Vorgaben supra- und Empfehlungen internationaler Institutionen gesehen werden, denn der nationale Gesetzgeber bewegt sich stets in der Verwobenheit von nationaler, internationaler und supranationaler Ebene. Der nationale Gesetzgeber wird von inter- und supranationalen Institutionen wie der OECD und EU mehr oder weniger verbindlich beurteilt. ${ }^{67} \mathrm{Im}$ Folgenden wird herausgearbeitet, wie sich das Leitbild des aktivierenden Sozialstaates in deren breit angelegte wissenschaftliche Beobachtungen und Empfehlungen einfügt.

\section{Empfehlungen der OECD}

Nach Beobachtung der Entwicklung der Weltwirtschaft und der nationalen Arbeitsmärkte veröffentlichte die OECD 1994 die OECD Jobs Strategy68. Diese enthält insgesamt 60 Vorschläge zum Abbau von Arbeitslosigkeit. Nach und nach wurden diese Vorschläge für verschiedene Staaten ausgearbeitet und in auf das jeweilige Land abgestimmte Empfehlungen konkretisiert. ${ }^{69} 2006$ gab die OECD eine vergleichende Evaluierung der Maßnahmen in Deutschland, den Niederlanden und dem Vereinigten Königreich heraus. Darin wurde überprüft, inwiefern die nationalen Arbeitsmarktpolitiken den Empfehlungen der OECD entsprechen und wie sich (deshalb) die Wirtschaft und der Arbeitsmarkt entwickeln. Während die OECD für Deutschland in erster Linie an der organisatorischen Umsetzung der Reform durch die komplexe Zuständigkeitsverteilung zwischen Bundesagentur für Arbeit und Kommunen bzw. der Arbeitsgemeinschaft nach $\S 44$ b SGB II Kritik übt und Verwunderung darüber äußert, dass die Zusammenführung

65 Dazu statt vieler zusammenfassend Neumann, NVwZ 1995, S. 428; Berlit, RsDE 1996, S. 30 ff.

66 Vgl. zweites Kapitel B II 1c) S. 185 f, v.a. im Hinblick auf das Sozialgeld für Kinder und Jugendliche, vgl. Vorlagebeschluss des BSG v. 27.1.2009, B 14/11b AS 9/07 R.

67 Vgl. z.B. Casey, EJIR 2004, S. 329 ff zum Vergleich der OECD jobs strategy und der Europäischen Beschäftigungsleitlinien.

68 OECD, The OECD jobs strategy, 1994.

69 Z.B. $O E C D$, Innovations in labour market policies: the Australian way, 2001. Kritisch dazu, ob die OECD mit ihrer jobs strategy hinter den Entwicklungen steht, z.B. Schmitt/Wadsworth, Is the OECD jobs strategy behind US and British employment and unemployment success in the 1990ies?, in: CEPA Working Papers 2002-06 (April 2002). 
von Arbeitslosenhilfe und Sozialhilfe, die die OECD ausdrücklich begrüßt, zu einer deutlichen Ausweitung des Empfängerkreises und damit einem Kostenanstieg geführt hat, stehen bei den Umsetzungsmängeln für das Vereinigte Königreich die hohe Anzahl von Erwerbsunfähigkeitsrenten (incapability benefits), das Angebot an wiedereingliedernden Maßnahmen zur Verbesserung von Grundkompetenzen und die Ausbildung des Personals der Arbeitsverwaltung im Mittelpunkt. ${ }^{70}$ Alles in allem begrüßt die OECD die Reformaktivitäten in beiden Staaten. ${ }^{71}$ Sie weist aber auch darauf hin, dass der Erfolg der Maßnahmen in Deutschland derzeit aufgrund der Datenlage nicht hinreichend bestimmt werden und deshalb nicht abschließend beurteilt werden kann. ${ }^{72}$

\section{Beschäftigungspolitische Leitlinien der Europäischen Union}

Seit dem Amsterdamer Vertrag von 1997 ist die Beschäftigungspolitik auf europäischer Ebene gemeinsame Aufgabe. Die einzelnen Mitgliedstaaten und die Gemeinschaft arbeiten auf die Entwicklung einer koordinierten Beschäftigungsstrategie und insbesondere auf die Förderung der Qualifizierung, Ausbildung und Anpassungsfähigkeit der Arbeitnehmer sowie der Fähigkeit der Arbeitsmärkte hin, um auf die Erfordernisse des wirtschaftlichen Wandels reagieren zu können (Art. 3 Abs. 1 lit. i, 125 ff EGV). Man einigte sich darauf, in Beschäftigungsleitlinien gemeinsame Standpunkte festzulegen und mit Hilfe jährlich aufzustellender nationaler Aktionspläne deren Verwirklichung in den Mitgliedstaaten voranzutreiben (Art. 128 EGV). Auf dem Gipfel von Lissabon im März 2000 wurde als Ziel beschlossen, dass die Union bis 2010 der wettbewerbsfähigste und dynamischste wissensbasierte Wirtschaftsraum der Welt werden soll. ${ }^{73}$ Dafür wird zutreffenderweise die Entwicklung eines aktiven und dynamischen Wohlfahrtsstaats als Voraussetzung angesehen. Die Verbindung von Wettbewerb und wirtschaftlicher Entwicklung mit sozialen Fragen und die Aussage, dass das beste Mittel gegen sozialen Ausschluss ein Arbeitsplatz sei, machen deutlich, dass damit der „Dritte Weg“ eine europäische Dimension erhalten hat. ${ }^{74}$ Auf der Tagung des Europäischen Rates im März 2003 in Brüssel bekräftigte man diese Vorgehensweise erneut und unterstrich die wichtige Rolle der Beschäftigungspolitik für die supranationale Entscheidungsebene. ${ }^{75}$ Anfang 2005 stand die Europäische Union ernsthaften strukturellen Schwierigkeiten gegenüber, die das „europäische Sozialmodell“ unter Druck setzten: eine alternde Be-

70 OECD, Activation strategies and performance of employment services in the Netherlands, Germany and the United Kingdom, 2006, S. $10 \mathrm{ff}$.

71 OECD, Activation strategies and performance of employment services in the Netherlands, Germany and the United Kingdom, 2006, S. $46 \mathrm{ff}$.

$72 O E C D$, Activation strategies and performance of employment services in the Netherlands, Germany and the United Kingdom, 2006, S. 12.

73 Europäischer Rat (Lissabon), 23./24.3.2000, Nr. 5 (http://www.europarl.europa.eu/summits/lis1_ de.htm). Kritisch zu dieser Entwicklung Lesch, APuZ 2000, B 14-15.

74 Dazu Telò, The European dimension of the third way, in: Cuperus/Duffek/Kandel, Multiple third ways, 2001, S. 178.

75 Europäischer Rat (Brüssel), 20./21.3.2003, Nr. 4, 12, 42 (http://www.consilium.europa.eu/ueDocs/ cms_Data/docs/pressData/de/ec/75146.pdf). 
völkerung und zurückgehende wirtschaftliche Leistungsfähigkeit in den Mitgliedstaaten. Dies veranlasste die europäische Kommission, die Lissabon-Strategie zu überarbeiten und sie in Verbindung zu makro- und mikroökonomischen Leitlinien zu stellen (,,integrierte Leitlinien“). Die europäische Arbeitsmarktpolitik wurde stärker mit wirtschaftspolitischen Strategien verknüpft, um stärkeres und dauerhaftes Wachstum und mehr und bessere Beschäftigung in der Europäischen Union zu erzeugen. ${ }^{76}$

Der für die vorliegende Arbeit interessierende Aspekt der Aktivierung findet sich in den beschäftigungspolitischen Leitlinien der Europäischen Union für 199877 und für 200378. Die Leitlinien enthalten keine verbindliche Rechtssetzung ${ }^{79}$. Doch einigte sich der Europäische Rat, bestehend aus den Staats- und Regierungschefs der Mitgliedstaaten und dem Kommissionspräsidenten (Art. 4 EUV), auf die Leitlinien, sodass eine gemeinsame Strategie zur Bekämpfung der Arbeitslosigkeit beschlossen werden konnte. Es wurde ein Prozess auf den Weg gebracht, den klare politische Verpflichtungen und eine hohe Akzeptanz bei allen Akteuren auszeichnen. Die Leitlinien werden wegen der differenzierten Ausgangslage in den Mitgliedstaaten in unterschiedlicher Weise umgesetzt, aber deren Verwirklichung nach gemeinsamer Bewertung der Ergebnisse überprüft. ${ }^{80}$ Dieses Vorgehen wird als offene Methode der Koordinierung bezeichnet. 81

Inhaltlich sind folgende Punkte hervorzuheben:

Bereits in den Leitlinien für 1998 werden vorbeugende Maßnahmen als bedeutsam angesehen, um der Entwicklung von Jugend- und Langzeitarbeitslosigkeit durch eine frühzeitige Ermittlung der individuellen Bedürfnisse und durch angepasste Reaktionen zu begegnen, bei denen aktiven Maßnahmen der Vorrang vor passiven Unterstützungsmaßnahmen gegeben wird. Aktiv wird in diesem Zusammenhang verstanden als Gegenbegriff zur bloßen Verteilung finanzieller Leistungen. Ziel der Maßnahme ist die Verbesserung der individuellen Beschäftigungsfähigkeit ${ }^{82}$; der Einzelne wird noch nicht auf besondere Weise in die Verantwortung genommen. ${ }^{83}$ Diese Vorgaben wurden

76 ABl. L 205/21 v. 6.8.2005. Vgl. auch den Gemeinsamen Beschäftigungsbericht 2006/2007, KOM (2006) 816 endg.

77 ABl. C 30 v. 28.1.1998, S. 1-5.

78 Beschluss 2003/578/EG des Rates vom 22.7.2003 über die Leitlinien für beschäftigungspolitische Maßnahmen der Mitgliedstaaten, in: ABl. L 197 v. 5.8.2003, S. 22 ff.

79 Dazu Aust, ZSR 2000, S. 21.

80 ABl. C 30 v. 28.1.1998, S. 1 f.

81 Dazu allgemein Karl, Offene Methode der Koordinierung als Gestaltungsgrundsatz europäischen Rechts, SDSRV 53 (2005), S. 7-27; Streinz, Sozialpolitische Zuständigkeit der EU im Rahmen der offenen Methode der Koordinierung, SDSRV 53 (2005), S. 29 ff; sowie zur europäischen Beschäftigungspolitik Eichhorst/Rhein, Die Europäische Beschäftigungsstrategie, SDSRV 53 (2005), S. 53 ff; Kenner, IJCLLIR 1999, S. 33 ff; Porte, The soft open method of co-ordination in social protection, in: European Trade Union Yearbook 2001, S. 339 ff.

82 Anhand der nationalen Aktionspläne analysiert Raveaud, Dynamics of the welfare states regimes and employability, in: Pieters u.a., Confidence and changes, 2001, S. $5 \mathrm{ff}$ die Entwicklung der Idee von der Beschäftigungsfähigkeit angestoßen durch den Luxemburg-Prozess.

83 ABl. C 30 v. 28.1.1998, S. 2 f. 
bei den Reformvorschlägen der Hartz-Kommission bereits anerkannt und deren Umsetzung in Deutschland als Ziel der Reformen benannt. ${ }^{84}$

In den Leitlinien für 2003 werden die von den Mitgliedstaaten vorzunehmenden Maßnahmen dahingehend konkretisiert, dass frühzeitige Beratung, Unterstützung bei der Arbeitsuche und individuelle Aktionspläne zur Verfügung gestellt werden sollen. Ferner wird ein Zeitrahmen von sechs Monaten für Jugendliche und von zwölf Monaten für Erwachsene vorgeschlagen, in dem jedem Arbeitslosen eine Maßnahme zur Beschäftigung angeboten werden soll, gegebenenfalls begleitet von kontinuierlicher Unterstützung bei der Arbeitsuche. Daneben soll die Modernisierung und Stärkung der Arbeitsverwaltungen, insbesondere der örtlichen Einheiten, vorangetrieben werden. Die Umgestaltung der finanziellen Anreizmechanismen für die Arbeitsuche fördert die Beseitigung von Arbeitslosigkeits-, Armuts- und Nichterwerbstätigkeitsfallen und damit die zukünftige Unabhängigkeit von sozialen Leistungen. Die Vorgabe der Europäischen Union lautet, dass die betroffenen Regelungen in den Mitgliedstaaten entsprechend anzupassen sind. ${ }^{85}$

In Nummer 19 der aktuellen Leitlinien für beschäftigungspolitische Maßnahmen der Mitgliedstaaten von $2005^{86}$ werden erneut aktive und präventive Arbeitsmarktmaßnahmen angesprochen. Es wird insbesondere Unterstützung bei der Arbeitsuche und Weiterbildung im Rahmen personalisierter Aktionspläne verlangt und damit Bezug genommen auf die in den Leitlinien von 2003 enthaltenen Ziele.

Schwerpunktmäßig ist festzustellen, dass die selbständige oder von der Arbeitsverwaltung unterstützte Arbeitsuche in den Vorgaben der Europäischen Union an Bedeutung gewinnt, um die Wiedereingliederungschancen zu erhöhen. Es wird betont, dass der einzelne Arbeitslose von der Arbeitsverwaltung wahrzunehmen, seine individuellen Bedürfnisse zu erfassen und anschließend die dabei gewonnenen Erkenntnisse in individuelle Fördermaßnahmen umgesetzt werden sollen.

In jüngsten Papieren der Europäischen Union au dem Jahr 2007 werden sowohl Deutschland als auch dem Vereinigten Königreich gute Fortschritte bei der Umsetzung der nationalen Reformpläne bescheinigt. Auch aus Sicht der Europäischen Union gingen die durchgeführten Reformen in die ,richtige Richtung“. 87

\section{B. Eingrenzung des Themas}

Die Vielgestaltigkeit des Leitbildes vom aktivierenden (Sozial-)Staat lädt dazu ein, grundlegend und allgemein das vorherrschende Verständnis von sozialer Sicherung zu

84 Hartz, Moderne Dienstleistungen am Arbeitsmarkt, 2002, S. $341 \mathrm{f}$.

85 ABl. L 197 v. 5.8.2003, S. 18, 20.

86 ABl. L 205 v. 6.8.2005, S. 25.

87 Vgl. Anlage S. 12 f, 50 f der Empfehlung des Rates zu den 2007 aktualisierten Grundzügen der Wirtschaftspolitik der Mitgliedstaaten und der Gemeinschaft zur Umsetzung der Beschäftigungspolitik der Mitgliedstaaten v. 15.3.2007 (7456/07) (http://ec.europa.eu/employment_social/ employment_strategy/pdf/recomm_adopted_de.pdf). 
diskutieren, es lässt aber genauso zu, konkrete rechtliche Fragen aufzuwerfen und zu beantworten. Diese Arbeit muss sich zwangsläufig beschränken. Im Folgenden wird die betrachtete Fragestellung näher umschrieben und eine Eingrenzung in Bezug auf den Adressaten der Aktivierung, aber auch in Bezug auf das betrachtete soziale Risiko und das Absicherungssystem, innerhalb dessen die Aktivierung stattfindet, vorgenommen:

Die Arbeit greift Vereinbarungen zwischen Staat und Einzelnem heraus und untersucht, wie kooperative Verwaltung mittels Vereinbarungen funktioniert. Kooperation und dabei vor allem Vereinbarungen werden als das entscheidende Instrument aktivierender Politik betrachtet. Aktivierende Politik lenkt den Fokus auf den einzelnen Bürger und will ihn als gleichberechtigten Partner ansehen. Diese Idee in eine vertragliche Ausgestaltung dieser Beziehung umzusetzen, ist nahe liegend. Zudem erscheint sie der Verwaltung bestens geeignet, Verantwortung an den Bürger abzugeben.

Zugleich stellen sich Fragen der Einordnung in Bestehendes: Das Verhältnis Staat Bürger ist bereits verrechtlicht und in ein System von mehr oder weniger dichter rechtlicher Kontrolle eingebettet. Der Leistungsempfänger, eine natürliche Person, die eine soziale Leistung beantragt hat, steht einer Verwaltung gegenüber, die es gewohnt ist, dieses Verhältnis allein zu steuern und auszugestalten. Der Vergleich etablierter Handlungsinstrumente, die in dieser Arbeit keineswegs als überholt angesehen werden, sondern möglicherweise lediglich einen neuen Platz einnehmen müssen, und innovativer Instrumente bestimmt die Untersuchung. Die Abgrenzung zu bereits vorhandenen Instrumenten und die Einordnung der Vereinbarung in das bestehende System staatlicher Handlungsformen ist ein zentrales Anliegen dieser Arbeit.

Eine Eingrenzung erfolgt auch in Bezug auf die Art der sozialen Leistung. Während das Leitbild vom aktivierenden Sozialstaat sich zu verschiedenen Bereichen sozialer Leistungen äußert, ist die Verflechtung von Rechten und Pflichten des Einzelnen mit Ansprüchen und politischen Maßgaben gegenüber der Verwaltung bei der Bekämpfung von Arbeitslosigkeit besonders groß. Untersucht werden deshalb Leistungssysteme, die das Risiko der Arbeitslosigkeit absichern. Für Deutschland sind dies Vereinbarungen im Anwendungsbereich des Dritten Buches Sozialgesetzbuch (SGB III) ${ }^{88}$ und Vereinbarungen im Rahmen der Grundsicherung für Arbeitsuchende gemäß dem Zweiten Buch Sozialgesetzbuch (SGB II) ${ }^{89}$. Nur am Rande werden Vereinbarungen behandelt, die auf dem Gebiet des Zwölften Buchs Sozialgesetzbuch (SGB XII) ${ }^{90}$ geschlossen werden. Das Recht im Vereinigten Königreich kennt nur noch ein einheitliches Leistungsregime im Fall der Arbeitslosigkeit, das Recht der jobseeker's allowance.

Ziel der Arbeit ist es, Erkenntnisse zu gewinnen über Vereinbarungen zwischen Staat und Einzelnem und insbesondere darüber, welche Stellung Vereinbarungen gerade im

88 Drittes Buch Sozialgesetzbuch - Arbeitsförderung (Artikel 1 des Gesetzes v. 24.3.1997 (BGB1. I, S. 594), zuletzt geändert durch Artikel 3 des Gesetzes vom 28.3.2009 (BGBl. I, S. 634).

89 Zweites Buch Sozialgesetzbuch - Grundsicherung für Arbeitsuchende (Artikel 1 des Gesetzes v. 24.12.2003, BGBl. I, S. 2954), Artikel 8 und Artikel 9 des Gesetzes vom 2.3.2009 (BGB1. I, S. 416).

90 Zwölftes Buch Sozialgesetzbuch - Sozialhilfe (Artikel 1 des Gesetzes v. 27.12.2003 (BGB1. I, S. 3022), zuletzt geändert durch Artikel 4 des Gesetzes vom 22.12.2008 (BGB1. I, S. 2955) 
Sozialrecht in Abgrenzung zum Privatrecht einnehmen. Dazu sind die Hintergründe kooperativer (Sozial-)Verwaltung darzustellen und die Zusammenhänge zur aktivierenden Arbeitsmarktpolitik aufzuzeigen. In einem Kapitel über die Grundlagen sind deshalb kooperative Handlungsformen vorab allgemein darzustellen und zu systematisieren und dann im Kontext einer bestimmten sozialen Leistung zu betrachten.

Der Rechtsvergleich zwischen englischem und deutschem Recht verspricht zudem einen zusätzlichen Erkenntnisgewinn, der sich aus der „Vorbildfunktion“ des Rechts der jobseeker's allowance für das deutsche Recht ergibt. Dabei ist auch das jeweilige Vertragsverständnis im public law/öffentlichen Recht zu beleuchten.

\section{Methodik und rechtsvergleichender Ansatz der Arbeit}

\section{Länderauswahl}

Die Arbeit vergleicht Vereinbarungen im Geltungsbereich des Arbeitsförderungsrechts (Arbeitslosenversicherung) und der Grundsicherung für Arbeitsuchende mit einer Vereinbarung, die im Vereinigten Königreich im Jobseekers Act 1995 eingeführt wurde.

Das jobseeker's agreement wird oft als Vorbild für die zu behandelnden deutschen Regelungen gesehen. Dies hat seinen Grund zum einen in der engen politischen $\mathrm{Zu}-$ sammenarbeit der verantwortlichen Regierungen ${ }^{91}$, zum anderen in dem gemeinsamen politikwissenschaftlichen und soziologisch-philosophischen Hintergrund für die Gesetzgebung. Damit ist vor allem die programmatische Reform der Sozialdemokratie gemeint, die mit den Schlagwörtern „Dritter Weg“92 oder third way ${ }^{93}$ umschrieben wurde.

Auch die jüngsten OECD-Evaluierungen entlang der Jobs Strategy ${ }^{94}$ beschreiben im Ergebnis, dass in Deutschland zum Zeitpunkt des Reformbeginns 1998 anders als im Vereinigten Königreich nur wenige der von der OECD vorgeschlagenen Maßnahmen umgesetzt waren, und dass sich Deutschland deshalb insbesondere im Vergleich zum Vereinigten Königreich im „Reformrückstand“ befand. ${ }^{95}$ Der Vergleich zweier ähnlicher Instrumente, die in einer Rechtsordnung bereits in Verwaltungsroutine übergegangen sind, während sie in einer anderen Rechtsordnung noch erprobt werden, lässt interessante Rückschlüsse erwarten.

Neben diesen Aspekten, die die Auswahl vorwiegend aus politikwissenschaftlichen und soziologischen Gründen rechtfertigen, prägen die Länderauswahl folgende Überlegungen:

91 Schröder-Blair-Papier v. 8.6.1999 (http://doku.iab.de/chronik/2x/1999_02_01_21_dass.pdf).

92 Hombach, Aufbruch, 1998.

93 Giddens, The third way, 1998.

$94 O E C D$, Activation strategies and performance of employment services in the Netherlands, Germany and the United Kingdom, 2006, S. $10 \mathrm{ff}$.

95 Elmeskov, Implementing the OECD Jobs Strategy, in: $O E C D$, Education at a glance, 2000, S. $50 \mathrm{f}$. 
Das Recht im Vereinigten Königreich geht von einer stärkeren Betonung der Verantwortung des Einzelnen aus. ${ }^{96}$ Dagegen zeichnet sich Deutschland als sog. ,korporatistischer Wohlfahrtsstaat" durch die Aufgabenteilung zwischen Staat und Verbänden aus. ${ }^{97}$ Beiden Staaten ist zugleich gemeinsam, dass sie grundsätzlich Verantwortung für die Absicherung sozialer Risiken übernehmen und eine entsprechende staatliche Infrastruktur bereitstellen. Diese Gemeinsamkeit ist die notwendige Grundlage für eine Untersuchung des Verhältnisses Staat-Bürger. ${ }^{98}$

Die Betonung der Stellung des Einzelnen im Verständnis von sozialer Sicherung im Vereinigten Königreich hat das deutsche Leitbild vom aktivierenden Sozialstaat beeinflusst. Diese Beeinflussung nachzuvollziehen und auf seine rechtliche Durchsetzung hin zu untersuchen, macht den Rechtsvergleich reizvoll. Das gilt insbesondere für die Frage, ob im deutschen Recht im Rahmen der Reformen typische Handlungsformen aufgegeben werden oder ob das Recht im Vereinigten Königreich sich mit dem dazu gegensätzlichen Ziel - Etablierung von Handlungsformen - verändert hat.

Besonders interessant macht den Vergleich mit dem Vereinigten Königreich, dass eine der zentralen Fragen - welche Rechtsnatur haben die Vereinbarungen? - vor dem Hintergrund eines anderen Verständnisses von öffentlichem Recht/public law und Privatrecht/private law ${ }^{99}$ beantwortet werden muss.

\section{Funktion des Rechtsvergleichs}

Der eben angesprochene Vergleich von deutschem und englischem Recht, den diese Arbeit sich vorgenommen hat, dient allgemein gesprochen dem „besseren Erfassen, Verstehen und Bewerten des Rechts" 100 . Dabei spielt der oftmals im Zentrum stehende rechtspolitische Zweck ${ }^{101}$ in der vorliegenden Arbeit eine unbedeutende Rolle, denn es wird nicht eine Gesetzesreform vorbereitet, sondern nachvollzogen. Dabei kann die Warnung Montesquieus an alle, die Rechtsvergleichung betreiben, sensibilisierend wirken:

«Les lois politiques et civiles de chaque nation [...] doivent être tellement propres au peuple pour lequel elles sont faites, que c'est un grand hazard si celles d'une nation peuvent convenir à une autre.»102

Wie es Montesquieu - vor dem Hintergrund seiner Zeit - als Risiko betrachtet, dass Regelungen, die in eine andere Rechtsordnung eingebracht werden, erfolgreich Rechts-

96 Esping-Andersen, Three worlds of welfare capitalism, 1990, S. 26.

97 Esping-Andersen, Three worlds of welfare capitalism, 1990, S. 27.

98 Zur Kritik an der Typologisierung Esping-Andersens vgl. Arts/Gelissen, JESP 2002, S. 137 ff.

99 Im Bereich des private law sind relevante Besonderheiten der anderen Zivilrechtsordnungen im Vereinigten Königreich, insbesondere Nordirlands und Schottlands, in Bezug auf den betrachteten Gegenstand nicht festgestellt worden. Die Arbeit beschränkt sich insoweit auf englisches Recht.

100 Zacher, Methodische Probleme des Sozialrechtsvergleichs, 1977, S. 22.

101 Vgl. zur Rechtsvergleichung im Staatsrecht Sommermann, DÖV 1999, S. 1020, der ebenfalls von Erkenntniszwecken und rechtspolitischen Zwecken spricht.

102 Montesquieu, De l'esprit des lois, 1749, Bd. 1, Kapitel 3, S. 10. 
probleme lösen, so stellt sich genau diese Frage für die vorliegende Arbeit: Ist das jobseeker's agreement für das deutsche Recht ein legal transplant ${ }^{103}$, ein rechtliches Versatzstück zur Lösung eines bestimmten Problems? Das deutsche Recht übernahm die Reform des englischen Rechts nicht vollständig, z.B. die Vereinheitlichung von Arbeitslosenversicherung und Sozialhilfe als solche, sondern machte sich eine unter vielen Ideen des englischen Rechts zu Eigen, die Einbeziehung des Leistungsempfängers über Vereinbarungen. Oder handelt es sich um einen komplexen Prozess der Übernahme einer bestimmten Rechtskultur? Schließlich könnte es sich auch um „Rezeption auf informeller Ebene" handeln, bei der die Erfahrung eines anderen Landes verarbeitet wird. ${ }^{104}$ Der deutsche Gesetzgeber bereicherte das Sozialgesetzbuch mit einem bislang - jedenfalls in diesem Ausmaß - unbekannten Handlungsinstrument. Montesquieus Anmerkung soll gerade die Vorsicht unterstreichen, mit der in der Arbeit untersucht wird, wie die Übertragung dieser Idee in ein anderes System funktioniert (hat).

Der Rechtssoziologe Kahn-Freund differenzierte den Ansatz Montesquieus und erweiterte ihn dahin, dass neben umweltbedingten Aspekten der politische Hintergrund einer nationalen Regelung verstanden werden muss. Er meint damit ihre Entstehung in einem nationalen Gesetzgebungsverfahren mit all den darin zu berücksichtigenden organisierten Interessen wirtschaftlicher, aber auch kultureller Art (Kirchen, Verbände, usw.). ${ }^{105}$ Für ihn ist Montesquieus Aussage, die sich auf das Zivilrecht bezog, ebenso für das politische Recht, das Verfassungsrecht, Verwaltungsrecht, Gesetzgebungsverfahren, in Kürze das public law, anwendbar ${ }^{106}$. Erfüllt doch der Staat im Bereich des public law öffentliche Aufgaben, spielt dort erst recht die enge Verbindung mit der politischen Kultur in einer Rechtsordnung eine Rolle. 107

Gegen diese Ansicht wandte sich der Rechtshistoriker Watson und führte aus, dass jedenfalls die Kenntnis des politischen Hintergrunds des Vergleichslands unwichtig sei, da sich der Reformer nur darum bemühen müsse zu prüfen, ob die Regelung in das eigene System passten. Die Kenntnis des ausländischen Rechts genüge völlig. ${ }^{108}$

Beide Ansichten haben etwas für sich, wenngleich bei Watson offen bleibt, wie man das ausländische Recht vollständig erfassen soll, ohne sich klarzumachen, wie das Recht gelebt wird und wie es in das bestehende Rechtssystem hineinpasst. ${ }^{109}$ Gerade für das Sozialrecht spielt die Einbettung einer Regelung in den nationalen Kontext eine

103 Dazu Watson, Legal transplants and law reform, LQR 1976, S. 79 ff.

104 Ausführlich m.w.N. zum Begriff der Rechtsrezeption mit all den damit auftretenden Fragen Darimont, Rezeption und die Bedeutung für das deutsche Sozialrecht, in: Becker/Gongcheng/Darimont, Grundfragen und Organisation der Sozialversicherung in China und Deutschland, 2005, S. 127 ff; Darimont, ZIAS 2003, S. 354 ff; grundlegend Rehbinder, Die Rezeption fremden Rechts in soziologischer Sicht, in: Rehbinder/Sonn, Zur Rezeption des deutschen Rechts in Korea, 1990, S. 5 ff; Hänlein, ZIAS 1998, S. 104 ff.

105 Kahn-Freund, MLR 1974 (37), S. 12.

106 Kahn-Freund, MLR 1974 (37), S. 6.

107 Sommermann, DÖV 1999, S. 1022 arbeitet mit den Begriffen „textbezogener Ansatz“ und „kontextualistischer Ansatz" und bevorzugt mit ähnlichen Argumenten wie Kahn-Freund Letzteren.

108 Watson, LQR 1976, S. 82.

109 So die Kritik von Stein, NwUnivLR 1977, S. 209. 
besondere Rolle, da die Systeme oft mit so unterschiedlichen Ansätzen arbeiten, dass das Herausgreifen einer sozialen Leistung ohne, nur um ein Beispiel zu nennen, die steuerrechtlichen Aspekte zu kennen, zu falschen Bewertungen führt. ${ }^{110}$

Die vorliegende Arbeit bereitet nun nicht eine Reform vor, sondern vollzieht eine Gesetzesänderung nach. Gerade wenn es ein rechtliches Vorbild gibt, ist dieses bei der Interpretation der Änderung einzubeziehen. Zudem sollen nicht nur Erkenntnisse über das eigene Recht gefunden werden, sondern das Vergleichsrecht steht - zumindest was das Erfassen und Verstehen angeht - auf gleicher Stufe mit dem eigenen Recht. Infolgedessen ist auch die Entwicklung der Reformen im Vergleichsland von Bedeutung für das Verständnis der Reformen im „eigenen Land“. Rückschlüsse auf das Funktionieren einer Regelung sind ohne diese Zusammenhänge nicht möglich. Insoweit neigt die vorliegende Arbeit einer umfänglichen Betrachtung des Vergleichsrechtssystems zu.

\section{Gang der Untersuchung}

\section{Mikro- oder Makrovergleich}

In der vorwiegend von der zivilrechtlichen Rechtsvergleichung geprägten Methodik wird unterschieden zwischen Mikro- und Makrovergleich ${ }^{111}$, also dem Vergleich einzelner Regelungen einerseits und dem Vergleich ganzer Rechtsordnungen, oft genannt Rechtskreise ${ }^{12}$, andererseits. Die Arbeit könnte die speziellen Regelungen der Vereinbarungen im deutschen und englischen Recht untersuchen (Mikrovergleich). Sie könnte aber auch die Unterscheidung von civil law und common law Rechtskreis sowie die Unterscheidung von private law und public law beziehungsweise Privatrecht und öffentlichem Recht betrachten (Makrovergleich). Letztendlich wird beides im erforderlichen Maße der Fall sein, um den Untersuchungsgegenstand aufzuklären. Es wird sowohl die Abgrenzung zwischen öffentlichem und privatem Recht betrachtet als auch das jeweilige Leistungsrecht beschrieben.

\section{Untersuchungsschritte}

Im Kapitel über die Grundlagen wird zunächst die verwaltungswissenschaftliche Klammer „Kooperation“, ihre Vorteile und Nachteile, Grenzen und Gefahren beschrieben und der Zusammenhang mit dem Leitbild des aktivierenden Sozialstaats hergestellt. Zudem werden Typen und Formen kooperativen Handelns in jeder Vergleichsrechtsordnung beschrieben und daraus Systematisierungskriterien für kooperative Handlungs-

110 Dazu Pieters, Reflections on the methodology of social security law comparison, in: Ruland/Maydell/Papier, Verfassung, Theorie und Praxis des Sozialstaats, FS Zacher, 1998, S. 719.

111 Zweigert/Kötz, Einführung in die Rechtsvergleichung, 1996, S. 4 f.

112 Neuere Kritik zur Rechtskreislehre bei Kötz, ZEuP 1998, 493 ff; Heiss, ZfVglRWiss 2001, S. 396 ff; zum Begriff der families of nations als Einteilungsvorschlag aus dem Bereich der Sozialpolitik Castles, Families of nations, 2000. 
formen entwickelt. So entsteht ein Raster für die sich an die Länderberichte anschlieBende Auswertung.

In den Länderberichten geht es um die konkrete Ausgestaltung der Vereinbarungen im Sozialrecht des jeweiligen Landes. Dabei kommt der rechtlichen Einordnung der Vereinbarungen zwischen Arbeitsverwaltung und Arbeitslosem eine besondere Bedeutung zu. Ist die Bestimmung der Rechtsnatur eines Handlungsinstrumentes im deutschen Recht geradezu ein „Klassiker“, scheint dies für ein Handlungsinstrument der englischen Verwaltung auf den ersten Blick ungewöhnlich zu sein. Das Rechtssystem im Vereinigten Königreich hat im Gegensatz zum deutschen Recht keinen strikt dogmatischen Zugang zu Handlungsformen. Die rechtliche Einordnung ist in einer rechtsvergleichenden Arbeit gleichwohl sinnvoll. Sie ermöglicht, das Funktionieren des Instrumentes in der jeweiligen Rechtsordnung zu beschreiben. Im Übrigen ist in der rechtswissenschaftlichen Literatur im Vereinigten Königreich eine Diskussion um die Frage entbrannt, wie Vereinbarungen abgewickelt werden können, die wegen ihrer Natur oder anderer Gründe nicht dem sog. contract law unterliegen. Gerade diese Frage, wann solche Vereinbarungen vorliegen, ist die Frage nach ihrer rechtlichen Einordnung und damit u.a. das, was die Arbeit im weiteren Verlauf aufzuklären versucht.

In der sich an die Länderberichte anschließenden Auswertung werden zunächst die Vereinbarungen anhand der Systematisierungskriterien eingeordnet und damit die Ergebnisse der Länderberichte zusammengefasst. Am im zweiten Kapitel (Grundlagen) entwickelten Raster für kooperative Verwaltung werden sodann die Unterschiede und Gemeinsamkeiten der jeweiligen Instrumente der Rechtsordnungen herausgearbeitet und kritisch nachvollzogen, wie die Einführung und Anwendung des Handlungsinstrumentes Vereinbarung in der jeweiligen Rechtsordnung wirken kann.

\section{Zusammenfassung}

Ziel der Arbeit ist es, ausgehend von den Hintergründen kooperativer Sozialverwaltung, insbesondere sog. aktivierender Arbeitsmarktpolitik und der Rolle, die danach Vereinbarungen einnehmen sollen, Erkenntnisse zu gewinnen über das Verhältnis zwischen Staat und Bürger. Sie will insbesondere klären, was bei der Gestaltung dieser Beziehung von kooperativem Handeln erwartet werden kann. Der Rechtsvergleich verspricht zudem einen zusätzlichen Erkenntnisgewinn, der die Vorbildfunktion des englischen Rechts für das deutsche Recht im Bereich kooperativer Verwaltung beleuchtet. 


\section{Erstes Kapitel: Grundlagen}

\section{A. Kooperation}

Kooperation und kooperatives Verwaltungshandeln bilden die Grundlage für die durch die aktivierende Arbeitsmarktpolitik angestoßene Diskussion über Vereinbarungen als Instrumente bei der Gewährung von Sozialleistungen im Fall von Arbeitslosigkeit. Es handelt sich dabei um einen Begriff, der in verschiedenen wissenschaftlichen Disziplinen verwendet wird. Insbesondere für von Staats- und Verwaltungsrecht begeisterte Juristen geht von Kooperation eine „Faszination“1 aus, weil sie den Gegensatz zum einseitig hoheitlichen Handeln des Staates darstellt. Einseitiges Handeln wird immer noch als typische Handlungsform der Verwaltung angesehen, obwohl Kooperation in der Verwaltung weit verbreitet ist. Verbleibt es allerdings bei einem Gespräch zwischen Verwaltung und Bürger, das ohnehin stets wegen der Stellung des Bürgers im Staat stattfinden soll ${ }^{2}$, werden lediglich die Möglichkeiten echter Kooperation nicht ausgeschöpft.

Als Einstieg in die Begriffsfindung kann der vom Bundesverfassungsgericht im Brokdorf-Beschluss verwendete Ansatz dienen, der gleichsam hinführt zu einer Thematik, die nicht nur für das deutsche Recht Bedeutung hat. Dort heißt es: „Die Anmeldepflicht des $\S 14$ VersG enthält ein behördliches Angebot zur Kooperation im Interesse einer gedeihlichen Verwirklichung des Versammlungszweckes. Ein frühzeitiger Dialog zwischen Veranstalter und Behörde, eine im gegenseitigen Benehmen vorgenommene Prüfung der wechselseitigen Belastbarkeit sowie eine Zusammenarbeit unter Berücksichtigung der jeweiligen Interessen trügen gerade bei Großdemonstrationen zur Vermeidung späterer Konfliktsituationen bei."3

Was wird unter Kooperation verstanden, warum wird sie für vorteilhaft gehalten, und welche Ziele verfolgt der jeweilige Gesetzgeber mit der gesetzlichen Anordnung kooperativer Handlungsformen ${ }^{4}$ ? Welche Gefahren und Risiken ergeben sich, welche Grenzen kooperativen Verwaltungshandelns sind zu beachten?

1 Schulze-Fielitz, Kooperatives Recht im Spannungsfeld von Rechtsstaatsprinzip und Verfahrensökonomie, in: Dose/Voigt, Kooperatives Recht, 1995, S. 225.

2 So BVerfGE 45, 297 [335]; dazu Schmidt-Aßmann, Das allgemeine Verwaltungsrecht als Ordnungsidee, 2004, S. 341.

3 BVerfGE 69, 315 [351].

4 Als Handlungsformen werden Strukturen verstanden, durch die sich Handlungen unabhängig von ihrem konkreten Inhalt in Verfahrensweise, Funktion und Rechtsfolge entsprechen. Vgl. Krause, Rechtsformen des Verwaltungshandelns, 1974, S. 14. 


\section{Definition}

In der deutschen Rechts- und Politikwissenschaft finden sich Werke, deren Gegen-

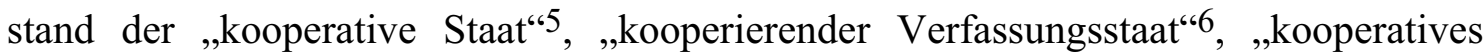
Recht $^{\text {"7 }}$, der „,kooperative Rechtsstaat" ${ }^{\prime 8}$ oder die „,kooperative Verwaltung ${ }^{\text {“9 }}$ war. Auch in der englischen Rechtswissenschaft gibt es seit längerem eine Debatte darüber, ob und wie der Staat, insbesondere bei der Erledigung staatlicher Aufgaben Dritte einbeziehen kann, z.B. als Leistungserbringer. ${ }^{10}$ Es wird von government by contract ${ }^{11}$, von new public contracting 12 und sogar von einem contracting state ${ }^{13}$ gesprochen. Auch die Besonderheiten von Vereinbarungen zwischen dem Einzelnen und dem Staat werden zunehmend thematisiert. ${ }^{14}$

Als Ergebnis dieser Diskussion legt diese Arbeit folgendes Begriffsverständnis zugrunde, ohne dieses im Einzelnen herzuleiten:

„Kooperatives Verwaltungshandeln umfasst die Gesamtheit der Verträge und Absprachen zwischen Verwaltung und nichtstaatlichen Personen/Bürgern, das in rechtlichen oder nichtrechtlichen (informalen ${ }^{15}$ ) Formen ihren Niederschlag gefunden hat. "16

Mit rechtlichen Formen sind förmliche Verfahren, z.B. Anhörung, Erörterungstermin oder Planauslegung, aber auch gesetzlich geregelte Handlungsformen gemeint. Als Kooperation in informalen Handlungsweisen ${ }^{17}$ sind in die Betrachtung gesetzlich nicht geregelte Handlungsformen eingeschlossen.

5 Voigt, Der kooperative Staat, 1995.

6 Vgl. dazu Michael, Rechtsetzende Gewalt im kooperierenden Verfassungsstaat, 2002.

7 Dose/Voigt, Kooperatives Recht, 1995.

8 Treutner, Kooperativer Rechtsstaat, 1998.

9 Benz, Kooperative Verwaltung, 1994.

10 Perri/Kendall, The contract culture in public service, 1997; Deakin/Michie, Contracts, co-operation and competition, 1997.

11 Mather, Government by contract, in: Vibert, Britain's constitutional future, 1991, S. 73 ff; Freedland, PL 1994, S. 86 ff.

12 Vincent-Jones, The new public contracting, 2006.

13 Harden, The contracting state, 1992.

14 Handler, Myth and ceremony in workfare, in: UCLA, School of Law Research Paper No. 02-21; Freedland/King, Client contractualism between the employment service and jobseekers in the United Kingdom, in: Sol/Westerveld, Contractualism in employment services, 2005, S. $119 \mathrm{ff} ; E b$ sen, Contracting between social services and their clients in the German concept of "Fördern und Fordern”, in: dies., S. 231 ff; Eichenhofer/Westerveld, Contractualism - A legal perspective, in: dies., S. $28 \mathrm{ff}$.

15 Informal wird statt informell verwendet. Es bedeutet das Gegenteil von formal, aber nicht wie informell auch informierend, mitteilend. Vgl. dazu erläuternd Neumann, VSSR 1993, S. 119 f; Kautz, Absprachen im Verwaltungsrecht, 2002, S. $32 \mathrm{ff}$.

16 Z.B. Schulze-Fielitz, Kooperatives Recht im Spannungsfeld von Rechtsstaatsprinzip und Verfahrensökonomie, in: Dose/Voigt, Kooperatives Recht, 1995, S. 225.

17 Brohm, Rechtsstaatliche Vorgaben für informelles Verwaltungshandeln, DVBl 1994, S. 138. Gegen diesen Begriff wendet sich Bulling, DÖV 1989, S. 278 „Die Wortwahl "informal/informell", die an 
Nicht als informale Kooperation behandelt werden dagegen Handlungen zur Herbeiführung eines beabsichtigten Erfolges, die in von der Rechtsordnung bereitgestellten öffentlich-rechtlichen oder privatrechtlichen Handlungsformen erfolgen könnten, aber in dieser Form unterbleiben. ${ }^{18}$ Das sind Fälle, in denen die Verwaltung inoffiziell Betroffene zusammenholt und mit diesen Probleme einer Maßnahme bespricht. Dort ergänzt diese Vorgehensweise eine einseitige Entscheidung außerhalb des vorgeschriebenen Verfahrens. Es folgt danach das gesetzlich vorgesehene Verfahren und die einseitige Entscheidung der Verwaltung. Es steht das (ergebnis-)offene Gespräch mit dem Bürger im Mittelpunkt, was nach dem Verständnis dieser Arbeit nicht ausreicht, um von kooperativem Verwaltungshandeln zu sprechen.

Gleichzeitig findet keine Verengung der Perspektive auf informales, das heißt rechtsformunabhängiges Verwaltungshandeln statt, da es auch gesetzlich verankerte Handlungsformen gibt, die kooperative Elemente aufweisen. ${ }^{19}$

\section{Wirkungsfelder}

\section{a) Steuerung verwaltungsinterner Vorgänge}

Die Steuerung verwaltungsinterner Vorgänge durch Kooperation ist ein weites Feld, das mit dem Schlagwort New Public Management ${ }^{20}$ oder Neues Steuerungsmodell ${ }^{21}$ überschrieben werden kann. Im Mittelpunkt steht das sog. Kontraktmanagement. In Zielvereinbarungen, Leistungsvereinbarungen oder Rahmenvereinbarungen wird - in Zweierbeziehungen aufgegliedert - das Vorgehen von hierarchisch organisierten Verwaltungseinheiten vereinbart. Dabei wird entweder ein Netzwerk bestehender Beziehungen zwischen verschiedenen Ebenen einer hierarchisch strukturierten Organisationseinheit ausgestaltet ${ }^{22}$ oder auch eine neue Ebene geschaffen. Dies geschieht in England mit der Bindung der executive agencies über framework-documents an die übergeordneten Ministerien (z.B. Department for Work and Pensions). Durch diese Vereinbarungen

Kungelei, Heimlichkeiten und rechtlich oder ethisch nicht einwandfreie Praktiken denken lässt, ist völlig deplaziert.“

18 Bohne, VerwArch 1984, S. 343 ff.

19 Als Begriffsverengung betrachtet dies auch Schmidt-Aßmann, Das allgemeine Verwaltungsrecht als Ordnungsidee, 2004, S. 342.

20 Oliver, Constitutional reform in the UK, 2003, S. $221 \mathrm{ff}$.

21 Grundlegend Kommunale Gemeinschaftsstelle für Verwaltungsmanagement, Das Neue Steuerungsmodell, KGSt-Bericht 5/1993; Mehde, Neues Steuerungsmodell und Demokratieprinzip, 2000; Dahm, Das neue Steuerungsmodell auf Bundes- und Länderebene, 2004; Schwarting, Effizienz in der Kommunalverwaltung, 2005; innerhalb der Arbeitsverwaltung vgl. Schönig, SozFort 2002, S. 9 ff.

22 Dazu Erfahrungen über die deutsche Gestaltung Tondorf/Bahnmüller/Klages, Steuerung durch Zielvereinbarungen, 2002. In England werden sog. public service agreements zwischen treasury und departments geschlossen. Dazu Oliver, Constitutional reform in the UK, 2003, S. 212 ff; Carmel/Papadopoulos, The new governance of social security in Britain, in: Millar, Understanding social policy, 2003, S. 42-47; Vincent-Jones, The new public contracting, 2006, S. 21. 
entstehen die executive agencies, was auch Bedeutung für die Zuständigkeiten und Kompetenzen der Mitarbeiter hat. ${ }^{23}$

Die Ziele des Handelns mit Hilfe dieser verwaltungsinternen Vereinbarungen sind vielfältig. Sie reichen von der Verschlankung der Ministerialverwaltung bei Absicherung eines gleichmäßigen Gesetzesvollzuges durch Konkretisierung gesetzlicher Ziele über die Vereinbarung von Fehlerfolgen zur besseren Kontrolle der Einhaltung des Vereinbarten bis zur Aktivierung des einzelnen Beamten und Änderungen seines Verhaltens. ${ }^{24}$

b) Steuerung verschiedener Leistungserbringer und freier Träger

Neben den eben beschriebenen rein verwaltungsinternen Vorgängen, wirkt Kooperation steuernd im sozialrechtlichen Leistungserbringungsverhältnis. Der Staat steuert Leistungserbringer und insbesondere freie Träger mit Hilfe kooperativer Vorgehensweisen. Er tritt zur Erfüllung der Aufgabe der sozialen Sicherung in Verhandlungen und arbeitet mit ihnen zusammen. Die Zusammenarbeit kann verschiedene Zielrichtungen haben.

\section{aa) Aufrechterhaltung einer Trägervielfalt}

Das gesetzlich garantierte Wunsch- und Wahlrecht des Leistungsberechtigten gem. § 5 SGB VIII ist ohne die Vielfalt verschiedener Träger (vgl. §§ 3, 4 SGB VIII) nicht denkbar. Um dieses aufrecht zu erhalten, sind stete Bemühungen um deren Einbeziehung und Steuerung erforderlich. Deshalb finden insbesondere vor und bei der Umsetzung von besonderen Sozialleistungsprogrammen intensive Verhandlungen der verantwortlichen Bundes bzw. Landesministerien mit den betroffenen Stellen, mit Verbänden und Interessenvertretungen im sozialen Bereich oder mit Vertretern der freien Berufe statt. ${ }^{25}$ Konkrete Beispiele für kooperatives Zusammenwirken zur Steuerung der Träger sind der Versorgungsvertrag gem. §§ 69 ff SGB XI, der die Zulassung einer Pflegeeinrichtung beinhaltet und zwischen den Landesverbänden der Pflegekassen und den einzelnen Pflegeeinrichtungen abgeschlossen wird, und der Versorgungsvertrag nach § 109 SGB V, der zwischen den Landesverbänden der Krankenkassen und dem Krankenhausträger zustande kommt. ${ }^{26}$ Das Nebeneinander von öffentlichen Aufgabenträgern und privaten oder gemeinnützigen Organisationen prägt die deutsche Sozialpolitik und das

23 Butcher, Managing the welfare state, in: Jones/MacGregor, Social issues and party politics, 1998, S. 26.

24 Hill, NVwZ 2002, S. 1059 ff; Vincent-Jones, The new public contracting, 2006, S. 139 ff.

25 Benz, Kooperative Verwaltung, 1994, S. 25.

26 Quaas, NZS 1995, S. 197 ff; Quaas, Der Versorgungsvertrag nach dem SGB V mit Krankenhäusern und Rehabilitationseinrichtungen, 2000. 
Sozialrecht. ${ }^{27}$ Gerade bei der Gesundheitsversorgung ist ein Ausbau kooperativer Handlungsformen festzustellen, der auch zunehmend gesetzlich verankert ist. ${ }^{28}$

In England findet ebenfalls eine Zusammenarbeit von Staat und verschiedenen Dritten, insbesondere Leistungserbringern statt, die aber bislang keine mit der deutschen Situation vergleichbare rechtlich verankerte Bedeutung für den Einzelnen erreicht. So geht es vor allem um das Vorhandensein der Infrastruktur für ein vielfältiges Angebot auf dem Markt für soziale Dienstleistungen ${ }^{29}$, das wiederum dem Einzelnen zugute kommen soll. ${ }^{30}$

\section{bb) Am Wettbewerb orientierte Leistungserbringung}

Der Staat engagiert Leistungserbringer auf dem (freien) Markt in erster Linie deshalb, um deren Potenziale zu nutzen, aber auch um sich selbst wie in England aus der Leistungserbringung zurückzuziehen. ${ }^{31}$ Es gibt Kooperation im Sinne von Absprachen, die vertraglichen Charakter annehmen kann, wobei an dieser Stelle offen bleiben muss, welches Verständnis von Vertrag zugrunde gelegt wird. Dieses Vorgehen ist getragen von der Idee, dass die Leistungserbringung ohne das Vorhandensein eines (Quasi-)Marktes nicht effizient erfolgen kann. Der Endpunkt der Entwicklung könnte sein, dass soziale Leistungen genauso unkompliziert wie andere Produkte - einem virtuellen Supermarkt vergleichbar - erhältlich sind. ${ }^{32}$

Ein Beispiel für Kooperation zwischen Leistungsträgern und Leistungserbringern mit Bezug zur Arbeitsmarktpolitik ist die Personal-Service-Agentur (PSA). Gem. $\S 37 \mathrm{c}$ SGB III kann jede Agentur für Arbeit eine PSA, die im Normalfall ein privates Unternehmen sein wird, einrichten. Dazu schließt die Agentur für Arbeit nach den Vorgaben des Vergaberechtes einen privatrechtlichen Vertrag mit dem Unternehmen, in dem die in der Regel erfolgsbezogene Honorierung und die Überweisung von Arbeitslosen in eine PSA vereinbart werden. ${ }^{33}$ Die entstehenden Rechtsbeziehungen zwischen PSA und Arbeitslosem (öffentlich-rechtlich überlagerter Maklervertrag gem. §§ 296 SGB III, 652 $\mathrm{BGB})^{34}$, sowie die Beziehung zwischen Bundesagentur für Arbeit und Arbeitslosem

27 Dazu im Einzelnen Schlette, Die Verwaltung als Vertragspartner, 2000, S. 306 ff; Neumann, VSSR 1993, S. $120 \mathrm{f}$.

28 Vgl. Gesetz zur Stärkung des Wettbewerbs in der gesetzlichen Krankenversicherung (GKVWettbewerbsstärkungsgesetz - GKV-WSG) v. 26.3.2007 (BGBl. I, S. 378), zuletzt geändert durch Artikel 5b des Gesetzes v. 19.12.2007 (BGB1. I, S. 3024).

29 Perri/Kendall, The contract culture in public service, 1997; so bereits angesprochen bei Birkinshaw/Harden/Lewis, Government by moonlight, 1990, S. 99 ff.

30 Butcher, Managing the welfare state, in: Jones/MacGregor, Social issues and party politics, 1998, S. $26 \mathrm{f}$.

31 Deakin/Walsh, Pub Admin 1996, S. 33 ff; Bennet/Ferlie, Pub Admin 1996, S. 49 ff; Butcher, Managing the welfare state, in: Jones/MacGregor, Social issues and party politics, 1998, S. 25 ff; Vincent-Jones, The new public contracting, 2006, S. 167 ff; Schlette, Die Verwaltung als Vertragspartner, 2000, S. 308.

32 Deakin/Walsh, Pub Admin 1996, S. 34.

33 Spellbrink, SGb 2004, S. 156; Rixen, NZS 2003, S. 401 ff.

34 Rixen, NZS 2002, S. 469 f; ders., NZS 2003, S. 409. 
aufgrund des Vermittlungsgutscheins gem. § 421g SGB III ${ }^{35}$ sind komplex. Seit Zahlen über Vermittlungsquoten und Qualifikationsmaßnahmen der PSA vorliegen, wird die Weiterführung dieses Instrumentes bezweifelt, denn es zeigen sich keine signifikanten Verbesserungen gegenüber der Leistungserbringung durch die Bundesagentur für Arbeit. 36

In England gewinnt die Auslagerung von Aufgaben des employment service beziehungsweise des $J C P$ auf private Arbeitsvermittler und die Erbringung von Leistungen durch diese an Bedeutung. Die Einordnung jener Verträge ist noch ungeklärt, bleibt aber in dieser Arbeit wegen der Fokussierung auf das Verhältnis Staat-Bürger außen vor. ${ }^{37}$

\section{cc) Gemeinsame Aufgabenerfüllung in Public-Private-Partnerships}

Eine weitere Gestaltungsvariante für die Kooperation zwischen Staat und Leistungserbringer ist die gemeinsame Aufgabenerfüllung mit Hilfe von public-private-partnerships. Es handelt sich um eine Mischform zwischen bloßen Gesprächen zur Aufrechterhaltung der Trägervielfalt und einer Leistungserbringung unter staatlicher Trägerschaft. Darunter werden Vertragsbeziehungen zwischen öffentlichen und privatwirtschaftlich tätigen Leistungserbringern verstanden, bei denen beide Vertragspartner spezifische Beiträge, nicht nur monetäre oder sonstige austauschbare Fähigkeiten, in einen gemeinsamen Leistungsprozess einbringen. ${ }^{38}$ Öffentliche Aufgaben werden nicht mehr (allein) durch öffentliche Stellen erbracht, sondern in Zusammenarbeit mit einem Privaten, meist einem privaten Unternehmen. Diese Zusammenarbeit wird in einem Vertrag geregelt. Es handelt sich damit nicht um eine Privatisierung in dem Sinn, dass die Verantwortung des Staates trotz der zumindest teilweisen Ausführung durch ein privates Unternehmen entfiele. ${ }^{39}$

Gerade im Bereich der lokalen Arbeitsmarktpolitik spielte diese Ausprägung der Kooperation eine große Rolle ${ }^{40}$, bis durch die Einführung der Grundsicherung für Arbeitsuchende die Aufgabenverteilung zwischen Bundesagentur für Arbeit und Kommunen mit einer Trennung der Leistungsträgerschaft neu gestaltet wurde. Inwieweit die Einbeziehung privater Unternehmen innerhalb der vorgegebenen Kompetenzen des SGB II

35 Rixen, NZS 2002, S. $470 \mathrm{f}$.

36 Mosley/Schmid, Evaluation der Maßnahmen zur Umsetzung der Vorschläge der Hartz-Kommission, Implementations- und Wirkungsanalyse der Personal-Service-Agentur, Abschlussbericht, 2006, S. 472 (http://www.wzb.eu/bal/ab/pdf/hartz_endbericht/endbericht_komplett.pdf).

37 Dazu Gray, WES 2002, S. 655 ff; Handler, Social citizenship and workfare in the United States and Western Europe, 2004, S. 149 f; Finn, JESP 2000, S. 47.

38 Wolff, Public-Private Partnerships, in: Schenk, Vom Hoheitsstaat zum Konsensualstaat, 1996, S. 254.

39 Ziekow, Verankerung verwaltungsrechtlicher Kooperationsverhältnisse (Public Private Partnership) im Verwaltungsverfahrensgesetz, 2001, S. 72 ff.

40 Allgemein dazu Stöbe-Blossey, Kooperation in der lokalen Arbeitsmarktpolitik, 1992; im Einzelnen Treutner, Kooperativer Rechtsstaat, 1998, S. 154 ff; Berlit, Hilfe zur Arbeit im Kontext aktueller sozialpolitischer Entwicklungen, in: Fuchs/Schulze-Böing, Hilfe zur Arbeit und kommunale Beschäftigungspolitik, 1999, S. 15 ff; Spindler, Aktivierende Ansätze in der Sozialhilfe, in: Dahme u.a., Soziale Arbeit für den aktivierenden Staat, 2003, S. 230 f. 
noch Platz hat, bleibt abzuwarten. ${ }^{41}$ Eine konfliktfreie, abgestimmte Zusammenarbeit zwischen Bundesagentur für Arbeit und Kommunen zu erreichen, um sog. „Verschiebebahnhöfe" zwischen Arbeitslosenversicherung und Fürsorgesystem ${ }^{42}$ endgültig abzustellen, war jedenfalls Ziel des Gesetzgebers bei der Einführung des SGB II. Insofern werden Zwischenlösungen mit public-private-partnerships einer verrechtlichten Zusammenarbeit weichen müssen, solange sich bereits zwei Träger aufeinander abstimmen müssen. Anders ist die Situation in den sog. Optionskommunen, den zugelassenen kommunalen Trägern, die das SGB II ohne Mitwirkung der Bundesagentur für Arbeit allein vollziehen, denn dort werden teilweise Strukturen und Einrichtungen aus der Zeit der Hilfe zur Arbeit gem. $\S 18$ ff BSHG weitergeführt.

\section{c) Unmittelbare und mittelbare Steuerung des Bürgers}

Neben den eben dargestellten Wirkungsfeldern kooperativer Vorgehensweisen, die nur am Rande beschrieben werden, ist die Steuerung des Bürgers mittels Kooperation ein zentraler Aspekt dieser Arbeit.

Mittelbare Steuerung des Bürgers durch Kooperation bedeutet, dass der Staat mit einer kollektiven Interessenvertretung (Selbsthilfegruppen) kooperiert. Ist das Durchsetzungsvermögen des einzelnen Versicherten/Hilfebedürftigen nicht ausreichend, insbesondere aufgrund fehlender Informationen oder aufgrund vorhandener Strukturen, kann der Zusammenschluss gesellschaftlicher Kräfte zu einer Verbesserung des Verhandlungsgleichgewichts und damit einer Stärkung der Position und Interessen des Einzelnen führen. ${ }^{43}$ Eine Steuerung ist möglich, da diese kollektiven Interessenvertretungen und Selbsthilfegruppen staatliche Förderung erfahren, die mittels Verträgen und Vereinbarungen abgewickelt wird. In diesem Prozess der Aushandlung des Förderzwecke und werden die Interessen des Einzelnen und der Verwaltung aufeinander abgestimmt. Dieses Wirkungsfeld kooperativer Verwaltung wird nicht weiter vertieft. ${ }^{44}$

Unmittelbare Steuerung beruht auf Vereinbarungen oder Absprachen zwischen Staat und Bürger. Dabei kann Kooperation im Vorfeld, bei der Entscheidung oder bei ihrem Vollzug stattfinden. Für die vorliegende Arbeit ist Gegenstand der Entscheidung die Gewährung einer oder mehrerer sozialer Leistungen. Diese Leistungen können wiederum von einer (vertraglich begründeten) Verpflichtung des Einzelnen abhängig gemacht werden. Steuerung bedeutet Disziplinierung ${ }^{45}$, wenn staatlicherseits klare Vorstellungen darüber geäußert werden, wie sich der Einzelne zu verhalten hat, und wenn dann abweichendes Verhalten negative Konsequenzen, z.B. einen Leistungsausschluss, nach sich zieht.

41 Möller/Bornholm/Stöcken, TuP 2004, S. 13 ff, die auf die Organisation der Zusammenarbeit zwischen Arbeits- und Sozialverwaltung nach dem Kieler Modell hinweisen.

42 Z.B. Bauer, DÖV 2004, S. 1019.

43 Bieback, GMH 2003, S. 28 f.

44 Braun, Selbsthilfe und Selbsthilfeunterstützung in der Bundesrepublik Deutschland, 1997; Geis, Die öffentliche Förderung sozialer Selbsthilfe, 1997.

45 Vincent-Jones, The new public contracting, 2006, S. $229 \mathrm{ff}$. 


\section{Steigerung der Effizienz in der Verwaltungsarbeit}

In der Verwaltung ist eine auf Kooperation ausgerichtete Verwaltungspraxis dort verstärkt zu finden, wo Entscheidungen eine hohe Komplexität aufweisen. ${ }^{46}$ Beim Umweltschutz ${ }^{47}$, der Bauleitplanung ${ }^{48}$ und der Vergabe und Abwicklung von Subventionen ${ }^{49}$ kann die Verwaltung ohne Mitwirkung der Betroffenen ihre Aufgaben nur noch mit großem Aufwand und unter Zeitverzögerungen, z.B. wegen politischer Proteste oder langwieriger Gerichtsverfahren, erfüllen. ${ }^{50}$ Es ist allgemein von „Staatsversagen “51 die Rede, weil der Eindruck entsteht, dass es der Staat versäumt, durch geeignete Verfahren und Regeln die eigene Handlungsfähigkeit zu erhalten.

Das eben beschriebene Defizit an effizienten Strategien der Verwaltung führt zu einem Effizienzverlust in der Verwaltungsarbeit. Dieser kann auf unterschiedliche Weise behoben werden.

Möglich wäre es, dass sich der Staat als Akteur zurückzieht. Dies ist für bestimmte Aufgaben der Verwaltung ein Weg, den die Politik eingeschlagen hat. Daneben gibt es verschiedenste sachliche Zwänge in Richtung Steuerung durch kooperatives Vorgehen, wenn sich der Staat nicht jeder Verantwortung begeben und jedenfalls Einfluss auf die Aufgabenerfüllung behalten will. ${ }^{52}$ Privatisierungen im sog. business sector bei Aufgaben im Zusammenhang mit wirtschaftlicher Tätigkeit des Staates ${ }^{53}$ machen ein Festhalten an herkömmlichen Verfahren unmöglich. Es ginge an dieser Stelle zu weit, die Gründe für Privatisierungen im Einzelnen aufzuführen oder deren rechtliche Ausgestaltung darzustellen. ${ }^{54}$ Ein Ergebnis des staatlichen Bemühens um Steuerung ist jedenfalls die Entstehung verschiedenster Verträge und vertragsähnlicher Beziehungen, in denen unter anderem Standards und Kostenniveaus für die Leistungserbringung vereinbart werden, die vormals in staatlicher Hand lag. 55

Für die Aufgaben, die die Verwaltung weiterhin federführend gegenüber dem einzelnen Bürger erfüllt, ist flexibles Verwaltungshandeln erforderlich, um in den Augen der

46 Kunig/Rublack, Jura 1990, S. 1.

47 Kloepfer, JZ 1991, S. 737 ff.

48 Stüer/König, ZfBauR 2000, S. 528 ff; allgemein dazu Schmidt-Aßmann/Krebs, Rechtsfragen städtebaulicher Verträge, 1992.

49 Ziekow, Verankerung verwaltungsrechtlicher Kooperationsverhältnisse, 2001, S. 70; Schlette, Die Verwaltung als Vertragspartner, 2000, S. 264.

50 Brohm, DVBl 1994, S. 137 f.

51 Benz, Kooperative Verwaltung, 1994, S. 59.

52 Hill, DVB1 1993, S. 976; Benz, Kooperative Verwaltung, 1994, S. 15, 59. Kooperation wird deshalb oft als ,dritter Weg“ zwischen hoheitlich-hierarchischer Erledigung öffentlicher Aufgaben und gesellschaftlicher Selbststeuerung ohne staatliche Beteiligung bezeichnet.

53 Lane, PM 1999, S. 180 ff.

54 Dazu im Einzelnen mit weiteren Hinweisen Freedland, Government by contract, in: Craig/Rawlings, Law and administration in Europe, 2003, S. $123 \mathrm{ff}$.

55 Oliver/Drewry, Public service reform, 1996, S. 135; Collins, Regulating contracts, 2002, S. 303. 
Betroffenen Verständnis für die Rechtslage zu schaffen, den Grund für die Verhängung von Sanktionen deutlich zu machen, kurz: die Akzeptanz der Entscheidungen zu steigern. ${ }^{56}$ Zunächst mag der Abschluss eines Vertrages aufwendiger erscheinen als einseitiges Handeln, doch wird sich spätestens bei der Möglichkeit, diese Vereinbarungen einvernehmlich anzupassen oder abzuändern, die Erkenntnis einstellen, dass dies unkomplizierter vonstatten geht als die Abänderung eines Verwaltungsaktes. Hinzu kommt in bestimmten Aufgabenbereichen, z.B. erprobt im Umweltschutz ${ }^{57}$, dass sich im Wege der Verhandlung und Vereinbarung Ziele erreichen lassen, die über gesetzliche (Mindest-)Vorgaben hinausgehen, sodass jedenfalls hier von einer Steigerung der Qualität der Verwaltungsarbeit auszugehen ist. 58

\section{Information der Verwaltung in offenem Miteinander}

Für das Sozialrecht spielt die Kommunikation zwischen Sozialverwaltung und Leistungsberechtigtem eine wichtige Rolle. ${ }^{59}$ Die persönliche Situation des Empfängers sozialer Leistungen ist für die Entscheidung über die Gewährung einer Leistung von großer Bedeutung60, zum Teil als Leistungsvoraussetzung61, zum Teil bei der Ausübung eingeräumten Ermessens ${ }^{62}$. Das setzt voraus, dass der Entscheidungsträger ausreichend Informationen über diese Situation hat. Bei einem Vorgehen, das den Einzelnen nur so weit wie unbedingt nötig einbezieht, entsteht oftmals ein Informationsdefizit bei der Verwaltung in Bezug auf die tatsächlichen Fähigkeiten und Bedürfnisse des Einzelnen. Es ist davon auszugehen, dass Verwaltungsinstrumente, die vorsehen, dass sich der Mitarbeiter der Verwaltung stärker als bisher mit dem Einzelfall beschäftigt (sog. Profiling, § 6 SGB III), ohne ein offenes Miteinander nicht funktionieren können. 63

Kooperatives Vorgehen ist ein möglicher Weg, diese Offenheit zu erreichen und während des Leistungsbezugs aufrechtzuerhalten. Lösungen werden nicht standardisiert vorgegeben, sondern mit dem Betroffenen zusammen erarbeitet, um die Handlungsfähigkeit der Verwaltung zu sichern. Dadurch werden Entscheidungen auf sichererer Informationsgrundlage gemeinsam getroffen. Gerade die Anpassung an die jeweils jüngsten Entwicklungen beim Leistungsempfänger droht sonst unterzugehen.

56 Spannowsky, Grenzen des Verwaltungshandelns durch Verträge und Absprachen, 1994, S. 51.

57 Rengeling, Das Kooperationsprinzip im Umweltrecht, 1988; Mills/Dean /Young, Entwicklung einer Umweltstrategie durch den Rat der Stadt Cardiff, in: Zilleßen/Dienel/Strubelt, Die Modernisierung der Demokratie, 1993, S. 233 ff.

58 Spannowsky, Grenzen des Verwaltungshandelns durch Verträge und Absprachen, 1994, S. 50.

59 Treutner, Kooperativer Rechtsstaat, 1998, S. 100 f.

60 Vgl. Bieback, GMH 2003, S. 26 f.

61 Z.B. Bedürftigkeit gem. § 7 Abs. 1 S. 1 Nr. 3, § 9 SGB II.

62 Vgl. § 3 Abs. 1 S. 2 Nr. 1, 2 SGB II, § 7 S. 2 Nr. 1 SGB III.

63 Eichenhofer/Westerveld, Contractualism, in: Sol/Westerveld, Contractualism in employment services, 2005, S. 32 f. 
Ziel ist stets, dass in Folge der Koordination von Intentionen, Ressourcen und Handlungen der Akteure auch der Zielerreichungsgrad sozialer Leistungen erhöht wird. ${ }^{64}$ Das bedeutet z.B. für einen Bürger, der von Arbeitslosigkeit betroffen ist, dass auf der einen Seite seine persönlichen Entwicklungsschritte, aber auch die Dynamik und die sich stets wandelnden Anforderungen des Arbeitsmarktes an Qualifikation und Mobilität berücksichtigt werden. In Form einer Reaktion werden die vorgeschlagenen und vereinbarten Eingliederungsleistungen verändert.

\section{Legitimierende Wirkung}

Die Verwaltung stützt grundsätzlich ihr Handeln auf eine Rechtsgrundlage, die im Regelfall ausdrücklich normiert ist, und rechtfertigt damit auch mit dem Verwaltungshandeln verbundene Leistungen (Legitimierung). Gesetze/statutes als Rechtsgrundlagen enthalten Tatbestand und Rechtsfolge in allgemeinen Worten. Aufgabe der Verwaltung ist es, diese Anforderungen, die der Gesetzgeber abstrakt formuliert hat, auf einen Einzelfall anzuwenden.

Kooperation geht dabei über den stets erforderlichen Subsumtionsvorgang hinaus. Vor einer Vereinbarung wird gemeinsam erfasst, welche Voraussetzungen die Rechtsgrundlage hat, welche Spielräume sie lässt und was diese im Einzelfall bedeuten. Schlussendlich weiß der Bürger, was von ihm erwartet wird. Verhaltensanforderungen werden genau formuliert. Dann entscheidet der Bürger darüber, ob und wie er sich mit der Verwaltung einigt. Erst im Anschluss an diese Einigung fällt die Entscheidung, ob er sich an das Vereinbarte halten wird oder nicht - im Wissen um die Konsequenzen.

Im kooperativen Prozess wird damit nicht nur der Adressat z.B. bei der Entscheidungsfindung beteiligt und damit die Akzeptanz der Entscheidung erhöht ${ }^{65}$, sondern es wird eine Rechtfertigung für das Verwaltungshandeln erarbeitet und transparent gemacht. Das Ergebnis eines kooperativen Prozesses entfaltet auf diese Weise legitimierende Wirkung. Verträge können sogar vollständig an die Stelle einer Norm als Rechtsgrundlage treten, Vereinbarungen können die Rechtsgrundlage für das Verwaltungshandeln ergänzen und konkretisieren.

In England ist dieses Anliegen Thema der Citizen's Charter. 66 Die Citizen's Charter sieht vor, dass das Miteinander von Verwaltung und Bürger durch Vereinbarungen und Absprachen in Kernbereichen der sozialen Sicherung gestaltet werden soll. ${ }^{67}$ Anlass war die Spannung zwischen der Aufgabe des Staates, welfare services vorzuhalten und damit für ein Netz sozialer Leistungen zu sorgen und der Aufgabe, durch eine Reform des Sozialleistungssystems den Staatshaushalt zu entlasten und die Entstehung eines leistungsfähigen Arbeitsmarktes zu fördern. Ziel war es, durch Reform insbesondere der Sozialverwaltung, diese effizienter und wirtschaftlicher zu machen, ohne den einzelnen

64 Treutner, Kooperativer Rechtsstaat, 1998, S. 51 f; Carney/Ramia, From rights to management, 2002, S. $40 \mathrm{ff}$.

65 Hill, DVBl 1993, S. 975; Bulling, DÖV 1989, S. 278.

66 Barron/Scott, MLR 1992 (55), S. 543 f; Lewis, PQ 1993, S. 323 f.

67 Lane, PM 1999, S. 190 f. 
Bürger und seine Rechte aus dem Auge zu verlieren. Hier sollte die Einbeziehung des Einzelnen ein mögliches Legitimationsdefizit schließen, das durch die organisatorischen Umstrukturierungen entstanden war. ${ }^{68}$

\section{Psychologisch-pädagogischer Effekt: Disziplinierung vs. Chance}

Kooperation kann bedeuten, dass der Empfänger einer sozialen Leistung auf der Grundlage einer Vereinbarung an der Beseitigung des Grundes für die Sozialleistung mitwirkt. Teilweise wird dafür der Ausdruck „Disziplinierung“ (disciplinary contract ${ }^{69}$ ) verwendet, teilweise wird schlicht die Chance gesehen, die Fähigkeiten der insbesondere wegen Arbeitslosigkeit sozial ausgeschlossenen Bürger im Miteinander zu entwickeln, und diese wieder in die Gesellschaft zu integrieren. ${ }^{70}$ Dem neuen Selbstverständnis der Arbeitsverwaltung als Serviceeinrichtung folgend steht der Mensch als „Kunde“ im Vordergrund. ${ }^{71}$ Die Forderung nach kooperativem Vorgehen geht einher mit einer Programmatik der gestärkten Eigenverantwortung des einzelnen Bürgers, die das bisher vorherrschende Menschenbild des Sozialstaats als nicht mehr zeitgemäß betrachtet. ${ }^{72}$ Der „Mensch von heute“ will als Bürger seine Innovationsfähigkeit, Eigeninitiative und Kreativität durch Mitgestaltung seiner Lebens- und Arbeitswelt einbringen. Er erwartet Freiräume, Chancen und mehr Gerechtigkeit und besitzt sowohl die Fähigkeit wie auch die Bereitschaft zur Selbstverantwortung. ${ }^{73}$

Es stellt sich die Frage, wann sich dieser psychologisch-pädagogische Effekt kooperativen Vorgehens einstellt. Dazu bleiben für eine Weile rechtliche Kategorien außen vor. Nachgezeichnet wird vielmehr die Diskussion um die Effekte des sog. social work contract ${ }^{74}$, des „Vertrags“ zwischen Sozialarbeiter und Arbeitslosem. ${ }^{75}$ Dieser weist zwei Elemente auf: das agreement (Einverständnis) und die structure to work (Struktur für den Arbeitsablauf). ${ }^{76}$ In der Praxis ist es schwierig, beiden Elementen gleichermaBen Geltung zu verschaffen und sicherzustellen, dass der rechtliche Kontext eines Ver-

68 Kirkpatrick/Lucio, Pub Admin 1996, S. 1 f; Butcher, Managing the welfare state, in: Jones/MacGregor, Social issues and party politics, 1998, S. 26 f.

69 Freedland/King, Client contractualism between the employment service and jobseekers in the United Kingdom, in: Sol/Westerveld, Contractualism in employment services, 2005, S. $123 \mathrm{ff}$.

70 Handler, Social citizenship and workfare in the United States and Western Europe, 2004, S. 7; Lauxen-Spangenberg, TuP 2000, S. 124.

71 Vgl. Pitschas, Das sozialrechtliche Verwaltungsverfahren im "aktivierenden" Sozialstaat, in: Wulffen, FS 50 Jahre Bundessozialgericht, 2004, S. 767 f; Schönig, SozFort 2002, S. 9; zur „Kundenzentrierung“ in der Bundesversicherungsanstalt für Angestellte Meurer, DAngVers 2000, S. $361 \mathrm{ff}$.

72 Vgl. Einführung A I S. 28 ff.

73 Empter/Esche, Eigenverantwortung und Solidarität, 1997, S. 7 f.

74 Corden, BJSW 1980, S. 143 ff; Nelken, CLP 1987, S. 207 ff; Walsh u.a., Contracts for public services, in: Campbell/Vincent-Jones, Contracts and economic organisation: socio-legal initiatives, 1996, S. 212 ff.

75 So auch Freedland/King, Client contractualism between the employment service and jobseekers in the United Kingdom, in: Sol/Westerveld, Contractualism in employment services, 2005, S. $124 \mathrm{f}$.

76 Rojek/Collins, BJSW 1987, S. 200. 
trages (Bindung an das Vereinbarte, Sanktionierung) ${ }^{77}$ nicht den pädagogischen Prozess behindert. 78

Ein agreement setzt voraus, dass das grundsätzlich bestehende Ungleichgewicht zwischen Klient und Sozialarbeiter (sog. Asymmetrien) im Gespräch verringert werden kann. Zunächst werden die Rahmenbedingungen für eine Vereinbarung genau umschrieben und die Pflichten beider Parteien systematisch vereinbart. Es wird sowohl die Rolle des Sozialarbeiters in Worte gefasst, als auch festgehalten, welche Schritte der Klient unternehmen muss (structure to work). So wird die Wechselseitigkeit der Beziehung betont und die Reihenfolge der Arbeitsschritte beschrieben.

Am Ende steht das agreement, also das freiwillig erteilte Einverständnis jeder Partei mit dem Arbeitsprogramm. In Fällen, in denen der Kontakt mit dem Sozialarbeiter unfreiwillig stattfindet, z.B. auf der Grundlage einer gerichtlichen Anordnung, kann es im Zweifel nicht zu einem Einverständnis kommen. Aus Sicht der Methodik sozialer Arbeit bleibt das Vorgehen mit Hilfe einer Vereinbarung dennoch so lange sinnvoll, wie sich die strukturierende Wirkung des gemeinsamen Vorgehens positiv auswirkt. Das Element des Einverständnisses tritt hingegen wegen der fehlenden Freiwilligkeit des Klienten zurück. ${ }^{79}$

Geht man nun einen Schritt zurück aus der Theorie sozialer Arbeit und taucht in das Verhältnis Arbeitsverwaltung - Arbeitsloser ein, wird deutlich, dass es Aufgabe des Fallmanagers ist, beim Arbeitslosen das Bewusstsein für die Bedeutung des Vereinbarten zu wecken. Nur dann wird die Eingliederungsstrategie letztendlich akzeptiert werden, wird sich entsprechendes Verhalten einstellen. Daraus kann sich eine Chance für den Arbeitslosen entwickeln. Wie aus der Theorie der sozialen Arbeit bekannt, führt Unfreiwilligkeit, z.B. durch die gesetzliche Anordnung einer Vereinbarung, nicht dazu, dass der psychologisch-pädagogische Effekt kooperativen Vorgehens entfällt. Das Prozesshafte des Aushandelns und die Struktur gebenden Elemente einer Strategie wirken trotzdem positiv auf die Erfolgschancen einer Eingliederungsmaßnahme.

\section{Gefahren und Risiken von Kooperation}

\section{1. Übervorteilung und Verhandlungsungleichgewicht}

Soziale Leistungen werden grundsätzlich gewährt, wenn sich Risiken verwirklichen, für deren Eintreten der Einzelne meist nur eingeschränkt, wenn überhaupt, Verantwortung trägt. Es handelt sich dann stets um sozial angespannte Lebenssituationen, in denen auf Seiten des Bürgers eine gewisse Einschränkung der Entscheidungsstärke feststellbar sein kann, während die Verwaltung aufgrund fehlender persönlicher Betroffenheit stets

77 Maluccio/Marlow, Social Work 1974, S. 35.

78 Fargion, BJSW 2003, S. 517.

79 Walsh u.a., Contracts for public services, in: Campbell/Vincent-Jones, Contracts and economic organisation, 1996, S. 212 ff; allgemein dazu Gehrmann/Müller, Aktivierende soziale Arbeit mit nicht-motivierten Klienten, 2005. 
unbeeinflusst agiert. Daraus kann ein Missverhältnis zwischen der Verhandlungsstärke der Sozialverwaltung und der Verhandlungsfähigkeit des einzelnen Bürgers entstehen. Es ist deshalb ein äußerst wirkungsvolles Druckmittel, eine Sozialleistung von der Mitwirkung des Bürgers abhängig zu machen. Eine Sozialleistung nicht oder anders zu gewähren, kann einen Bürger so unter Druck setzen, dass dieser keinen Verhandlungsspielraum sieht, auch wenn jener rechtlich betrachtet gegeben wäre. Es besteht die Gefahr, dass ein Hilfe suchender Bürger, der sich selbst nur eingeschränkt oder gar nicht selbst helfen kann, nicht überdenkt, was ihm von Seiten der Verwaltung vorgeschlagen wird. Er nimmt die angebotene Hilfe entgegen, ohne zu überlegen, ob er auf die staatliche Entscheidung Einfluss nehmen könnte. Formuliert die Verwaltung das Angebot so, dass dem Verhandlungspartner jeder Spielraum zur Verhandlung vorenthalten wird, spricht man von Vereinbarungen, die auf der Grundlage von take it or leave it geschlossen werden und damit mehr als Vereinbarungen auf Unterwerfung anzusehen sind. ${ }^{80}$ Es ist anhand der Vergleichsrechtsordnungen zu prüfen, ob ein solches Vorgehen auch dann nicht zulässig ist, wenn es nachweislich nicht zum Nachteil des Leitungsempfängers war.

Auch die Verwaltung kann übervorteilt werden, denn der Einzelne hat es in der Hand, die Verwaltungstätigkeit ins Leere laufen zu lassen, wenn er nicht im unrechtlichen Sinn „mitwirkt“: Er kann z.B. durch Vorenthaltung von Informationen eine ordnungsgemäße Verwaltung wesentlich erschweren beziehungsweise unmöglich machen.

Es sind auch strukturelle Nachteile der Verwaltung denkbar. Hat der potenzielle Vertragspartner der Verwaltung ein großes Gewicht, z.B. wegen seiner Stellung als wichtiger Arbeitgeber, kann die Verwaltung bei der Aushandlung möglicherweise rechtliche Anforderungen weniger stark in der Vereinbarung verankern, wie das in anderen Konstellationen der Fall wäre. ${ }^{81}$

\section{Desintegration durch Kooperation}

Kooperative Handlungsformen setzen auf beiden Seiten ein gewisses Maß an Selbstüberzeugung voraus. Für einen Teil der Gesellschaft (den „weniger sozial disziplinierten Menschen“"82) kann es deshalb zum Ausschluss aus dem System führen oder zumindest einen strukturellen Nachteil darstellen, wenn sie auch im Zusammenhang mit sozialen Leistungen die eigene Position in einer Verhandlung vertreten müssen. Gerade bei Menschen, die bereits längere Zeit arbeitslos sind, bringt eine ,individualistische Organisation der Lebenswelt desintegrative Folgeprobleme für die Gesellschaft mit sich“. 83 Die Fähigkeit zu verhandeln, hat nicht jeder Bürger gleichermaßen. Gerade von

80 Neumann, VSSR 1993, S. 127.

81 Schlette, Die Verwaltung als Vertragspartner, 2000, S. 373.

82 Treutner, Kooperativer Rechtsstaat, 1998, S. 95.

83 Treutner, Kooperativer Rechtsstaat, 1998, S. 95; Däubler, NZS 2005, S. 225 ff. So bezeichnet Handler, Myth and ceremony in workfare, in: UCLA, School of Law Research Paper No. 02-21, S. 40 die Idee von der Integration des Hilfebedürftigen sogar als „,myth” und betont die desintegrative Wirkung. 
diesen die Mitwirkung bei der Bewältigung ihrer Probleme zu verlangen, kann bedeuten, dass die Schwächsten erneut benachteiligt werden. ${ }^{84}$ Besonders die gesetzliche Verpflichtung zur Verhandlung mit der Perspektive, sonst Rechte und Ansprüche zu verlieren, stellt für diese Menschen ein fast unüberwindliches Problem dar.

Es ist deshalb nicht damit getan, der Rhetorik vom „Ernstnehmen des Einzelnen“ zu vertrauen und seiner Aktivierung folgend ungesteuert Verantwortung zu übertragen, ohne weitere Elemente in der rechtlichen Ausgestaltung der kooperativen Vorgehensweise vorzusehen, die die eben beschriebene desintegrative Komponente abfedern. Eine kritische Prüfung dieses Punktes ist geboten.

\section{3. „Entrechtlichung “ und ,Verrechtlichung“}

Neben Gefahren im Zusammenhang mit den Einflussmöglichkeiten der Verhandlungspartner auf das Verhandlungsergebnis bergen die Schnittstellen zwischen kooperativer Vorgehensweise und Rechtssystem als Ganzes Gefahren für das Funktionieren von Kooperation.

Auf der einen Seite ist die Autorität des gesetzten Rechts gefährdet (Entrechtlichung). ${ }^{85}$ Kooperation ist rechtlich zulässig, wenn Verhandlungsspielräume im gesetzten Recht zu finden sind, beispielsweise als Ermessensspielräume. Sobald Rechtsfragen, und nicht nur die Erhebung von Tatsachen, Verhandlungsmasse sind, wird das Gesetz zum „Droh- und Tauschmittel“"86. Es stellt sich die Frage, ob die Gleichheit vor dem Gesetz dann noch gewahrt ist. Eine Rechtfertigung gibt es, solange es wichtige Gründe für die Ungleichbehandlung gibt, z.B. dass die effiziente Erbringung von Sozialleistungen ohne eine auf den Einzelnen abgestellte Strategie zum Scheitern verurteilt ist.

Auf der anderen Seite kann Einbettung in eine Rechtsordnung bedeuten, dass die zulässigen Verfahren und Instrumente des Rechts den positiven Effekten kooperativen Vorgehens im Weg stehen oder ihnen zu wenig Raum lassen (Verrechtlichung).Sinkt aus diesem Grund die Motivation beider Seiten der Vereinbarung, dieses Instrument zu nutzen, oder werden die positiven Effekte durch den Aufwand, den die Maßgaben der Rechtsordnung vorgeben, aufgewogen, ist die Erfolglosigkeit von Kooperation vorprogrammiert.

\section{Defizite bei Haftung und Rechtsschutz}

Eine Gefahr für das Gelingen von Kooperation besteht darin, dass die Haftung beziehungsweise die Verantwortung für das Verwaltungshandeln durch die Ausgestaltung kooperativen Verwaltungshandelns nicht eindeutig bestimmbar, und dadurch öffentliche

84 Olk, Der "aktivierende" Staat, in: Müller u.a., Soziale Arbeit, 2000, S. 117.

85 Di Fabio, DVB1 1990, S. 338 ff; Dauber, Möglichkeiten und Grenzen kooperativen Verwaltungshandelns, in: Becker-Schwarze u.a., Wandel der Handlungsformen im öffentlichen Recht, 1991, S. 82 f; Spannowsky, Grenzen des Verwaltungshandelns durch Verträge und Absprachen, 1994, S. 53; Vincent-Jones, PL 1999, S. 309 f.

86 Brohm, DVB1 1994, S. 138 f; 
Interessen und Interessen des einzelnen Bürgers nicht mehr gewahrt sein könnten. Werden z.B. wegen vertraulicher Vorgehensweise die genauen Verwaltungsabläufe nicht dokumentiert und sind damit nicht mehr rekonstruierbar, wird ein Nachweis in einem möglicherweise folgenden Verfahren erschwert. ${ }^{87}$

In England wird das beschriebene Haftungsdefizit als ein Aspekt von accountability diskutiert. Die Einschaltung eines Privaten ist in einem Vertrag geregelt. Darin ist vereinbart, wie bestimmte Leistungen zu erbringen sind, aber die Entscheidung, welche Leistungen genau erbracht werden, wird dem Markt überlassen. Damit ist die Auswahl des Angebotes einer direkten Einflussnahme in Ausübung öffentlicher Interessen (public interests) entzogen. ${ }^{88}$ Es stellt sich dann die Frage, wer die politische Verantwortung für diesen Vorgang der Gestaltung des Angebotes übernimmt, vor allem dann, wenn komplexe und mehrfach gestufte vertragliche Beziehungen entstehen. ${ }^{89}$

Ein weiterer Aspekt ist vor der Verankerung kooperativen Vorgehens zu beachten. Scheitert ein kooperatives Vorgehen stellt sich die Frage, aufgrund welcher Verfahren die Verwaltung oder auch der Bürger die Rechtslage klären lassen können. So können z.B. große Unterschiede zwischen der Entscheidungsfindung vor einem common law Gericht/Zivilgericht und einer Entscheidung nach verwaltungsrechtlichen Grundsätzen (z.B. im Sinne einer good governance) bestehen. 90

\section{Kosten-Nutzen-Vergleich}

Es stellt ein Risiko für ein dauerhaftes Festhalten an kooperierender Verwaltung dar, wenn ein Kosten-Nutzen-Vergleich hervorbringt, dass der längere Verständigungsprozess mit ungewissem Ausgang einer zügigen einseitigen Entscheidung ohne kooperative Elemente unterlegen ist. 91

Verglichen werden müssen die höheren Verwaltungskosten, die aus der Aushandlung und dem Gespräch über tatsächliche Fragen und Rechtsfragen, und damit einer insgesamt personalintensiveren Bearbeitung der einzelnen Anträge resultiert. ${ }^{92}$ Auf der anderen Seite steht der erwartete Nutzen von Kooperation, der sich messen und ermitteln lässt. So können die allgemeine Zufriedenheit mit der Verwaltungsarbeit, die Einhal-

87 Dauber, Möglichkeiten und Grenzen kooperativen Verwaltungshandelns, in: Becker-Schwarze u.a., Wandel der Handlungsformen im öffentlichen Recht, 1991, S. 83 f.

88 Cane, Administrative law, 2004, S. 311 ff; Scott, Privatisation, control and accountability, in: McCahery/Picciotto/Scott, Corporate control and accountability, 1993, S. 234 ff; Davies, Accountability: A public law analysis of government by contract, 2001, S. $41 \mathrm{ff}$.

89 Dazu Hunt, Accountability, openness and the Citizen's Charter, in: Chandler, The Cititzen's Charter, 1996, S. 55 ff; Oliver/Drewry, Public service reform, 1996, S. 6 ff; Deakin/Walsh, Pub Admin 1996, S. 42 f.

90 Seddon, Government contracts, 1999, S. 30 f.

91 Dauber, Möglichkeiten und Grenzen kooperativen Verwaltungshandelns, in: Becker-Schwarze u.a., Wandel der Handlungsformen im öffentlichen Recht, 1991, S. 82.

92 Seddon, Government contracts, 1999, S. 31. 
tung der Vereinbarung und weniger Rechtsstreitigkeiten Kriterien sein, die den Nutzen kooperativer Verwaltung prägen. ${ }^{93}$

Dieser Kosten-Nutzen-Vergleich hat Bedeutung für das Handeln des Gesetzgebers. Er muss unabhängig von einem Einzelfall der Überzeugung sein, dass der Vergleich in den überwiegenden Fällen positiv ausfallen wird. Diese Prüfung muss der Gesetzgeber im Wege der Evaluation seiner Handlungsinstrumente vornehmen und eventuell Korrekturen vornehmen.

\section{Grenzen kooperativen Verwaltungshandelns}

\section{Verfassungsrechtliche Vorgaben}

Unabhängig von Gefahren und Risiken von Kooperation, die aufzeigen, welche Faktoren den (dauerhaften) Erfolg von Kooperation beeinflussen, findet kooperatives Verwaltungshandeln dort eine echte Grenze, wo verfassungsrechtliche Vorgaben zu erfüllen sind. Verfassungsrechtlich geschützte Rechtsgüter, wie z.B. die persönliche Handlungsfreiheit, das Existenzminimum, das Eigentum oder die Berufsfreiheit sind bei kooperativer Vorgehensweise der Verwaltung zu wahren. Dies ist unabhängig davon, wie kooperative Verfahren in der jeweiligen Rechtsordnung umgesetzt sind. Dies ist auch unabhängig davon, ob die verfassungsrechtlichen Vorgaben wie in Deutschland im Grundgesetz kodifiziert sind oder sich wie in England aus dem Human Rights Act in Verbindung mit der Europäischen Menschenrechtskonvention ergeben oder gar nicht kodifiziert sind.

\section{Vorgaben im Verfahrensrecht}

Das Verfahrensrecht regelt den Umgang zwischen dem Bürger als Antragsteller und Empfänger der Sozialleistung und der Verwaltung. Grenzen ergeben sich aus dem Verfahrensrecht, wenn die Einhaltung dieser Regeln kooperatives Vorgehen beschränkt.

So stellt es eine Grenze für kooperatives Verwaltungshandeln dar, wenn, wie in der Citizen's Charter ${ }^{94}$ verankert, die Verfahrensposition des Einzelnen gegenüber der Verwaltung abstrakt gestärkt wird (consumer empowerment). Dies stellt eine allgemeine Form der Verhinderung von Machtmissbrauch von Seiten der Verwaltung dar und begrenzt damit, allerdings im positiven Sinn, die Möglichkeiten der Verwaltung. ${ }^{95}$

Begrenzend wirkt sich Verfahrensrecht auch aus, wenn es vorsieht, dass die Verwaltung bestimmte Sachverhalte feststellen muss, bevor sie diese als richtig voraussetzen darf (inquisitorial proceedings $96 /$ Untersuchungsgrundsatz gem. § 20 SGB X). Während des Aushandelns der Vereinbarung müssen demnach bestimmte tatsächliche Fragen

93 Im Einzelnen ist der Nutzen von Kooperation im ersten Kapitel A II S. 54 ff beschrieben.

94 Lewis, PQ 1993, S. 316 ff.

95 Vincent-Jones, The new public contracting, 2006, S. 206-209.

96 Dazu Mesher, Social security law, in: Kiralfy, The burden of proof, 1987, S. $211 \mathrm{ff}$. 
thematisiert und geklärt werden, denn die Verwaltung kann nicht Informationen des Bürgers unbesehen als richtig annehmen und einer Vereinbarung zugrunde legen. ${ }^{97}$ So werden Ressourcen der Verwaltung gebunden. Inwieweit eine Wechselwirkung zwischen den Maßgaben des Verfahrensrechtes und kooperativer Vorgehensweise besteht, die z.B. auch eine Modifizierung des Untersuchungsgrundsatzes rechtfertigen könnten, bleibt in den nationalen Rechtsordnungen zu prüfen.

Kooperation kommt ferner aus verfahrensrechtlichen Gründen dort an ihre Grenzen, wo die Verwaltung auf ein Zusammenwirken mit dem Einzelnen bei der Aufgabenerfüllung zwingend angewiesen ist. Hier ist es erforderlich, dass der Verwaltung bei fehlender Bereitschaft des Bürgers mitzuwirken, auch eine nicht-kooperative Handlungsweise zur Verfügung steht. Folge wäre sonst, dass die Verwaltung handlungsunfähig ist. Es können z.B. Mitwirkungspflichten des Bürgers begründet werden, die die Handlungsfähigkeit der Verwaltung sicherstellen und ihr die Erfüllung eigener Feststellungspflichten erleichtern. Damit wird Kooperation gleichzeitig in einen rechtlichen Kontext gerückt. 98

Verfahrensrecht hat auch in Randbereichen zum materiellen Recht, insbesondere bei der Ausübung von Ermessen, Einfluss darauf, ob und wie sich die Verwaltung durch Vereinbarungen vor festlegen darf.

\section{Allgemeine Vorgaben des materiellen Rechts: Bindung inter partes}

Vereinbarungen wirken grundsätzlich nur inter partes. Einem Dritten, der durch die Vereinbarung in eigenen Rechten beeinträchtigt ist, ist es grundsätzlich nicht möglich, diese Rechte geltend zu machen. ${ }^{99}$ Eine Einbeziehung anderer, von der Vereinbarung Betroffener würde bewirken, dass dieser Personenkreis von der Bindungswirkung der Vereinbarung erfasst ist. Das ist nur möglich, wenn der Personenkreis namentlich bekannt ist oder eine abgrenzbare Gruppe darstellt. Die Verhandlungen in einem Dreiecksverhältnis sind jedoch stets schwieriger und verkomplizieren das Verfahren. ${ }^{100}$

Sind die Dritten nicht abgrenzbar und können deshalb nicht eingebunden werden, beziehungsweise entfaltet die Vereinbarung nicht bereits ohne Einbeziehung ihnen gegenüber eine Bindungswirkung, ist die angestrebte befriedende Wirkung der Vereinbarungen jedenfalls abgeschwächt und begrenzt die Möglichkeiten, kooperativen Vorgehens. Es kann zu Rechtsverletzungen kommen, die dazu führen, dass mittels Dritt-

97 Pitschas, Verwaltungsverantwortung und Verwaltungsverfahren, 1990, S. 729 ff; Dauber, Möglichkeiten und Grenzen kooperativen Verwaltungshandelns, in: Becker-Schwarze u.a., Wandel der Handlungsformen im öffentlichen Recht, 1991, S. 85 f; Wolff/Bachof/Stober, Verwaltungsrecht, Bd. 2, 2000, S. 325 f.

98 Kreikebohm, Ralf/Koch, Friedrich von: Das Sozialleistungsverhältnis - generelle Rechte und Pflichten zwischen Sozialleistungsempfängern und -trägern, in: Maydell/Ruland/Becker, Sozialrechtshandbuch, 2008, § 6 Rn. 148 ff; Steck, BKK 1992, S. 349.

99 Gelegentlich wird in der Beschneidung der Erfolgsaussichten von Drittschutzklagen der eigentliche Sinn informellen Handelns gesehen. Dazu Neumann, VSSR 1993, S. 123.

100 Dauber, Möglichkeiten und Grenzen kooperativen Verwaltungshandelns, in: Becker-Schwarze u.a., Wandel der Handlungsformen im öffentlichen Recht, 1991, S. 83. 
schutzklage Rechtsschutz gesucht wird. ${ }^{101}$ Deren Ausgang ist unsicher, insbesondere seitdem die Rechtsprechung die Zulässigkeit von Drittschutzklagen ausweitet. ${ }^{102}$ Dies führt zu einer deutlichen Schwächung kooperativer Prozesse, da die Bindung inter partes und deren beabsichtigte Ausschlusswirkung gegenüber Dritten ausgehebelt werden.

Die Bindungswirkung inter partes ist ferner eine Grenze für kooperatives Verwaltungshandeln, wenn aufgrund von Zuständigkeitsvorschriften nur eine bestimmte Behörde durch die Vereinbarung gebunden ist, während eine andere Behörde zusätzlich tätig werden und im Zweifel auch anders entscheiden kann. Dann muss der Vertragspartner der Verwaltung mit jener erneut verhandeln und eine Einigung suchen. ${ }^{103}$ Dies ist z.B. problematisch bei einem Umzug des Leistungsberechtigten während der Laufzeit einer Vereinbarung.

\section{Kooperation und Aktivierung}

Kooperation und Aktivierung sind Begriffe, die miteinander zu tun haben, aber auch unabhängig voneinander gedacht werden können.

Kooperieren kann auf Aktivierung abzielen, wenn z.B. durch kooperative Vorgehensweisen Ressourcen einbezogen werden, die vorher nicht genutzt werden konnten. Kooperation kann aber auch andere Ziele verfolgen, z.B. eine Vereinfachung von Verwaltungsabläufen.

Aktivierung kann auch ohne kooperative Vorgehensweise stattfinden. Dann geht es um einseitige Leistungsanreize und Sanktionierung von Fehlverhalten, was auf einseitige Verwaltungsentscheidungen hinausläuft.

In dieser Arbeit geht es darum, die Überschneidungen von Aktivierung und Kooperation in Bezug auf die Reform der Arbeitsmarktpolitik in England und Deutschland zu beleuchten, rechtlich zu systematisieren und die Tragfähigkeit dieser Phänomene beim Kampf gegen (Langzeit-)Arbeitslosigkeit zu analysieren. Das Leitbild des aktivierenden Sozialstaates gibt die Zielrichtung kooperativen Verwaltungshandelns vor. Der aktivierende Staat dynamisiert das, was Partnerschaft zwischen Bürger und Staat erreichen kann, und erweitert die Perspektive hin zu einer neuen Aufgaben- und Verantwortungsverteilung zwischen Bürger und Verwaltung. ${ }^{104}$ Die kooperative Vorgehensweise der Verwaltung ist für diese Arbeit das Mittel zum Zweck und steht im Dienst der Aktivierung und der Reform der Sicherungssysteme, die beim Risiko der Arbeitslosigkeit eingreifen.

101 So hat z.B. ein Insasse eines privatisierten Gefängnisses keine vertragliche Beziehung mit dem Betreiber des Gefängnisses und ist in diesen Vertrag auch nicht einbezogen. Es kommen Rechte aus dem Human Rights Act in Betracht, die eine dem Vertrag entgegenstehende Entscheidung des privaten Betreibers notwendig machen können und damit die Vereinbarung indirekt ergänzen, aber auch die Bindungswirkung des Vereinbarten inter partes auflösen. Dazu Harden, The contracting state 1992, S. 37-51; Cane, Administrative law, 2004, S. 313.

102 Kunig/Rublack, Jura 1990, S. 8.

103 Dazu Klindt, NVwZ 2003, S. 309.

104 Vgl. Ziekow, Verankerung verwaltungsrechtlicher Kooperationsverhältnisse, 2001, S. 2. 


\section{B. Typen und Formen kooperativen Handelns}

\section{Kooperative Elemente einer Entscheidung}

\section{Verwaltungsakt im deutschen Recht}

\section{a) Begriff}

Der Verwaltungsakt ist in seiner Grundform die zentrale Handlungsform im deutschen Verwaltungsrecht, der typischerweise nicht-kooperativ einseitig erlassen wird. 105 Dies gilt insbesondere für das Sozialrecht im Verhältnis zwischen Leistungsträger und Versichertem beziehungsweise Leistungsempfänger. Die Massenvorgänge der Sozialverwaltung können ohne den Verwaltungsakt nicht funktionieren. 106 Der Verwaltungsakt weist dann einen Zusammenhang zu kooperativen Handlungsformen auf, wenn die enthaltene Regelung nicht nur einseitig, sondern im Zusammenwirken mit dem Adressaten formuliert wird.

Bereits vor seiner gesetzlichen Regelung bildete sich ein Begriff heraus, der den Verwaltungsakt zu anderem, gerichtlich nicht nachprüfbarem Verwaltungshandeln abgrenzte. Das Erfordernis gerichtlicher Kontrolle der Verwaltung, das freilich für den formellen Rechtsstaat wesentlich und im Übrigen in Art. 19 Abs. 4 GG normiert ist, beeinflusst die Begriffsbildung des Verwaltungsaktes stark. ${ }^{107}$.

Der Verwaltungsakt ist in $\S 35 \mathrm{~S} .1 \mathrm{VwVfG}$, Art. $35 \mathrm{~S} .1$ BayVwVfG 108 , $\S 31 \mathrm{~S} .1$ SGB X legal definiert. Ein Verwaltungsakt ist jede Verfügung, Entscheidung oder andere hoheitliche Maßnahme, die eine Behörde zur Regelung eines Einzelfalles auf dem Gebiet des öffentlichen Rechts trifft und die auf unmittelbare Rechtswirkung nach auBen gerichtet ist.

Ein Verwaltungsakt muss grundsätzlich nicht in Schriftform erlassen werden. Wegen der besseren Beweisbarkeit seines Inhalts ist dies gleichwohl in der Verwaltungspraxis meist der Fall. Er ist für die Verwaltung bindend, bis er, wenn das rechtlich zulässig ist, aufgehoben ist. ${ }^{109}$ Der Verwaltungsakt ist innerhalb der dem deutschen Recht bekannten Klagearten gerichtlich überprüfbar. Zudem hat er Titelfunktion und ist vollstreckbar, obwohl er einseitig von der Verwaltung erlassen wird.

105 Wolff/Bachof/Stober, Verwaltungsrecht, Bd. 2, 2000, S. 13 f; Kirchhof, Mittel staatlichen Handelns, in: Isensee/Kirchhof, Handbuch des Staatsrechts, Bd. 3, 2004, § 59 Rn. 147.

106 Schmidt-Aßmann, Das allgemeine Verwaltungsrecht als Ordnungsidee, 2004, S. 335.

107 Krause, Rechtsformen des Verwaltungshandelns, 1974, S. 141 spricht deshalb sogar von einer ,Justizförmigkeit der Verwaltung“".

108 Das bayerische VwVfG wird beispielhaft für die deckungsgleich formulierten Verwaltungsverfahrensrechte anderer Bundesländer zitiert.

109 Vgl. Rücknahme und Widerruf, §§ 48-50 VwVfG; $\S ~ 44-49$ SGB X. 


\section{aa) Mitwirkungsbedürftiger/zustimmungsbedürftiger Verwaltungsakt}

Ist es für den rechtmäßigen Erlass eines Verwaltungsaktes erforderlich, dass der Betroffene entweder vor dem Erlass durch Stellung eines entsprechenden Antrags (Mitwirkung verfahrensrechtlicher Natur) oder auf andere Art und Weise, z.B. durch ausdrückliche Zustimmung zur Behördenentscheidung (Mitwirkung materiell rechtlicher Natur) sein Einverständnis mit dem Verwaltungsakt erklärt hat, spricht man von einem mitwirkungsbedürftigen/zustimmungsbedürftigen Verwaltungsakt. ${ }^{110}$

Es bleibt bei einer einseitigen Regelung durch die Behörde. Die Zustimmung des Einzelnen zielt nicht darauf $a b$, ihm eine Mitentscheidungskompetenz zu gewähren, sondern zu verhindern, dass ihm ein Verwaltungsakt gegen seinen Willen aufgedrängt wird. ${ }^{111}$ Die Mitwirkung des Bürgers ist damit Rechtmäßigkeitsvoraussetzung, nicht Entstehungsvoraussetzung. Fehlt sie, wird dies in der Regel zur Anfechtbarkeit des Verwaltungsaktes führen. Die Nichtigkeit des Verwaltungsaktes kommt nur in Betracht, wenn die Mitwirkung aus materiellen Gründen erforderlich ist, z.B. weil der persönliche Status des Bürgers betroffen ist (vgl. Einbürgerung oder Ernennung oder Entlassung eines Beamten). ${ }^{112}$

\section{bb) Zusage und Zusicherung}

Die Zusage ist das verbindliche Versprechen der zuständigen Behörde, eine bestimmte Verwaltungsmaßnahme vorzunehmen oder zu unterlassen. Entscheidend ist der Bindungswille der Behörde. Das BVerwG führte aus, dass es sich bei der Zusage um eine „hoheitliche Selbstverpflichtung mit Bindungswillen zu einem späteren Tun oder Unterlassen" handelt. ${ }^{113}$ Die Zusicherung ist nach der Legaldefinition in $\S 38$ Abs. 1 VwVfG ein Unterfall der Zusage, nämlich diejenige Zusage, die sich auf den Erlass oder Nichterlass eines Verwaltungsaktes bezieht. ${ }^{114}$

Tritt der Bürger nicht an die Verwaltung heran, werden weder Zusage noch Zusicherung erteilt. Gleichwohl sind die Zusage und die Zusicherung als kooperative Handlungsform anzusehen. Es findet eine Absprache zwischen Antragsteller und Behörde statt, wobei der Einfluss des Einzelnen auf den Inhalt der Entscheidung für die Feststellung der Handlungsform unwesentlich ist. Die Beteiligung des Einzelnen ist auf das Vorfeld der Entscheidung reduziert.

\section{cc) Verwaltungsakt mit Nebenbestimmungen}

Weiter ausgestaltet ist das konsensuale Element des mitwirkungsbedürftigen Verwaltungsakts beim Verwaltungsakt mit Nebenbestimmungen, wenn in der Phase der Festlegung der Auflagen diese mit dem Bürger abgestimmt werden. Der einseitigen Regelung

110 Stelkens, in: Stelkens/Bonk/Sachs, VwVfG, 2001, § 35 Rn. 153-155.

111 Maurer, Allgemeines Verwaltungsrecht, 2009, S. 378.

112 Stelkens, in: Stelkens/Bonk/Sachs, VwVfG, 2001, § 35 Rn. 169.

113 BVerwGE 23, 31 [36].

114 Maurer, Allgemeines Verwaltungsrecht, 2009, S. 226. 
im Verwaltungsakt werden weitere, wechselseitige Leistungspflichten beigefügt. Als Folge entfallen rechtliche oder tatsächliche Hindernisse, die einer uneingeschränkten Genehmigung entgegenstehen. Die Nebenbestimmungen dienen einer „elastischen Verwaltung“115, weil so ihre Handlungsmöglichkeiten über ein „,ja“ oder „,nein“ zum Antrag hinausgehen.

Zulässigkeit und inhaltliche Gestaltung sind in $\S 36$ VwVfG, § 32 SGB X geregelt. Für die Wirksamkeit des Verwaltungsakts mit Nebenbestimmungen ist das Einverständnis des Bürgers nicht erforderlich. Es bleibt bei einer einseitigen Regelung der Verwaltung. Das Einverständnis bezieht sich auf die Phase des Nachdenkens über den Inhalt der Nebenbestimmungen. Man darf sich nicht täuschen, denn nicht jeder Verwaltungsakt mit Nebenbestimmungen wird tatsächlich mit dem Bürger abgestimmt. Nicht abgestimmte Verwaltungsakte erfüllen damit nicht die oben beschriebene Definition kooperativen Verwaltungshandelns, weil die Absprache im Vorfeld der Entscheidung fehlt.

\section{dd) Anhörung}

Die Anhörung des Beteiligten gem. § $28 \mathrm{VwVfG}, \S 24$ SGB X ist nur vor Erlass eines Verwaltungsaktes 116 durchzuführen, der in Rechte eines Beteiligten eingreift. Teilweise wird darin eine Form kooperativen Verwaltungshandelns gesehen.

Die Anhörung dient der Information der Behörde über den entscheidungserheblichen Sachverhalt ${ }^{117}$, der Gewährung rechtlichen Gehörs als Ausfluss des Rechtsstaatsprinzips, aber auch der Erhöhung der Akzeptanz der Verwaltungsentscheidung ${ }^{118}$, mithin also den wesentlichen Gründen für kooperatives Verwaltungshandeln allgemein und scheint zunächst von der dieser Arbeit zugrunde liegenden, weiten Definition von Kooperation erfasst zu sein.

Bei der Anhörung fehlt aber ein wesentlicher Aspekt. Ein Verfahrensschritt, der eine spätere einseitige Entscheidung vorbereitet, erfüllt nicht die dieser Arbeit zugrunde gelegte Definition. Es ist der Anhörung immanent, dass sie den Willensbildungsprozess in der Verwaltung vorbereitet, aber keine Bindung der Verwaltung für die sich anschlieBende Verwaltungshandlung erzeugt. Zudem besteht keine Pflicht für den Bürger, der Einladung zu einer Anhörung nachzukommen. Damit hat die Anhörung nicht mit Entscheidung und Verantwortung des Angehörten zu tun. ${ }^{119}$

115 Maurer, Allgemeines Verwaltungsrecht, 2009, S. 331.

116 Hochhuth, NVwZ 2003, S. 30 ff zur analogen Anwendung des $§ 29$ VwVfG auf einen schlichthoheitlichen Verwaltungseingriff. Die entsprechende Anwendung auf verwaltungsrechtliche Verträge wird von der h.M. wegen des eindeutigen Wortlauts und der qualifizierten Form der Beteiligung des Bürgers bei einem Vertrag abgelehnt. So auch Kopp/Ramsauer, VwVfG, 2008, § 28 Rn. 6.

117 Köhler, WzS 2001, S. 129 ff.

118 Wolff/Bachof/Stober, Verwaltungsrecht, Bd. 2, 2000, S. 332; Kopp/Ramsauer, VwVfG, 2008, § 28 Rn. 1 ff; Pickel/Marschner, SGB X, Bd. 1, § 24 Rn. 3 ff; Vogelsang, in: Hauck/Noftz, SGB X, § 24 Rn. 1 ff.

119 So auch Dauber, Möglichkeiten und Grenzen kooperativen Verwaltungshandelns, in: BeckerSchwarze u.a., Wandel der Handlungsformen im öffentlichen Recht, 1991, S. 71. 


\section{Decision}

Das weitaus weniger dogmatisch erforschte Pendant zum deutschen Verwaltungsakt ist die decision. Es handelt sich dabei um eine einseitige Entscheidung der Verwaltung, die entsprechend der rule of law rechtmäßig ergangen sein muss: Voraussetzung ist eine Rechtsgrundlage für das Verwaltungshandeln, sowie eine rechtmäßige Begründung. Decisions sind grundsätzlich gerichtlich überprüfbar. Der jeweilige Rechtsweg richtet sich nach der Art der Entscheidung. ${ }^{120}$

Kooperative Elemente wie beim Verwaltungsakt werden in der im deutschen Recht bekannten Ausführlichkeit nicht diskutiert.

\section{Kooperative Elemente des planenden Verwaltungshandelns}

\section{Pläne im deutschen Verwaltungs- und Sozialrecht}

\section{a) Begriff}

Der Begriff Plan wird zwar in deutschen Rechtsvorschriften verwendet ${ }^{121}$, aber nicht legal definiert. $\mathrm{Ob}$ der Plan wegen seines heterogenen Erscheinungsbildes als eigenständige Handlungsform der Verwaltung betrachtet werden kann, ist umstritten. Teilweise wird dies bejaht ${ }^{122}$, teilweise verneint. ${ }^{123}$ Als Kompromissformel wird der Plan als offene Handlungsform des Verwaltungsrechts angesehen und nach Plantypen und planrelevanten Handlungsformen, die auch bekannte Rechtsformen sein können, unterschieden. 124

Während unter Planung 125 die systematische Vorbereitung und Festlegung rationalen Verhaltens verstanden wird, um unter gegebenen Umständen ein Ziel auf bestmögliche Weise zu erreichen, werden im Plan selbst diese Überlegungen niedergelegt und zusammengefasst. ${ }^{126}$

120 Dazu im zweiten Kapitel A II 9 S. 108 ff.

121 Bebauungsplan, Haushaltsplan, Krankenhausbedarfsplan, usw.

122 Hoppe, Planung und Pläne in der verwaltungsgerichtlichen Kontrolle, in: Erichsen, FS Menger, 1985, S. 750, der die Handlungsform Plan trotz der Vielfalt an Planvarianten als dogmatisch hilfreich ansieht.

123 Danwitz, Verwaltungsrechtliches System und europäische Integration, 1996, S. 68; Maurer, Allgemeines Verwaltungsrecht, 2009, S. 428.

124 Dazu gibt Wolff/Bachof/Stober, Verwaltungsrecht, Bd. 2, 2000, S. 259 f den Diskussionsstand wieder und resümiert, dass es eine eigenständige Handlungsform Plan gibt, die sich aber unterschiedlicher Rechtsformen bedient.

125 Z.B. § 95 SGB X, § 80 SGB VIII.

126 Wolff/Bachof/Stober, Verwaltungsrecht, Bd. 2, 2000, S. 258; zur sozialrechtlichen Planung Engelmann, in: Wulffen, SGB X, 2008, § 95 Rn. 4; Grüner, SGB X, § 95, S. 6; Pickel/Marschner, SGB X, Bd. 1, § 95 Rn. 7 ff. 
b) Einordnung als kooperative Handlungsform

Der Plan ist das Ergebnis einer bestimmten Form staatlicher Aufgabenerfüllung. Wichtig ist, dass den Plan nicht sein Inhalt kennzeichnet, sondern die Art und Weise seiner Entstehung. Die zu untersuchenden kooperativen Elemente sind deshalb im Verfahren der Ausarbeitung zu suchen. ${ }^{127}$ Der Plan weist kooperative Elemente auf, wenn er unter Einbeziehung der Betroffenen entsteht und diese insbesondere bei der Umsetzung des Geplanten, also bei der Aufgabenerfüllung mitwirken, z.B. in Form von public-private-partnerships. ${ }^{128}$ Der Plan kann allerdings trotz der Kooperation während der Ausarbeitung als Sonderform des Verwaltungsakts ergehen. Wird kein Verwaltungsakt erlassen, handelt es sich um die offene Handlungsform Plan. ${ }^{129}$

\section{Back-to-work-plan im englischen Recht}

Auch im englischen Recht wird der Begriff plan verwendet. Seine wesentlichen Merkmale sind den eben herausgearbeiteten Kriterien des deutschen Rechts ähnlich.

Vor der Einführung der jobseeker's allowance mit der Voraussetzung des jobseeker's agreement war der 1990 eingeführte sog. back-to-work-plan Instrument der Arbeitsvermittlung. Im back-to-work-plan wurden Maßnahmen zur Integration des Arbeitslosen in den Arbeitsmarkt vereinbart. Der Abschluss war für beide Seiten freiwillig. ${ }^{130}$ Das unter Mitwirkung des Arbeitslosen entstandene Dokument hatte keine verpflichtende Wirkung. ${ }^{131}$ Es handelte sich um ein pro forma document. Es hatte dann Auswirkungen auf die Rechtsposition des Antragstellers, wenn ihm Sanktionen wegen fehlender aktiver Arbeitsuche auferlegt werden sollten. ${ }^{132}$

Die soziale Arbeit spielt seit jeher in der Sozialverwaltung im Vereinigten Königreich eine größere Rolle als in Deutschland. Es war bereits die Rede vom social work contract. ${ }^{133}$ Dessen zweites Element, das strukturgebende Element einer Vereinbarung, entspricht dem, was den back-to-work-plan ausmacht. Der Arbeitsprozess bei der Ausarbeitung des Vorgehens definiert die Situation zwischen Klient und Sozialarbeiter von Beginn an. Das Vereinbarte ist Hilfsmittel des Sozialarbeiters für ein strukturiertes, für den Einzelnen vorhersehbares Vorgehen. Die Vorgehensweise und die jeweiligen Rollen in der Beziehung zwischen Klient und Sozialarbeiter werden geklärt. Der Sozialarbeiter kann derjenige sein, der die Beziehung mit Hilfe seiner besonderen Kenntnisse

127 Zur Hilfeplanung in der Jugendhilfe (SGB VIII) Gerlach, ZfJ 1998, S. 134 ff; Merchel, Von der psychosozialen Diagnose zur Hilfeplanung, in: Jordan/Schrapper, Hilfeplanung und Betroffenenbeteiligung, 1994, S. 53.

128 Wolff/Bachof/Stober, Verwaltungsrecht, Bd. 2, 2000, S. 256.

129 Luthe, ZSR 1994, S. $853 \mathrm{f}$.

130 Wood u.a., Social security legislation 2006, Bd. 2, 2006, S. 68; vgl. auch HMSO, Jobseeker's Allowance, Cm 2687, 1994, S. 20.

131 Fulbrook, ILJ 1995 (24), S. 400.

132 Wikeley/Ogus, The law of social security, 2002, S. 351; Vincent-Jones, The new public contracting, 2006, S. 241.

133 Vgl. erstes Kapitel A II 4 S. 57. 
steuert, den Plan entwirft und vorlegt. Diese „übergeordnete“ Rolle des Sozialarbeiters wird von ihm sogar teilweise erwartet, wenn der Klient Hilfe sucht und selbst nicht zurechtkommt.

\section{Privatrechtlicher Vertrag}

\section{Begriff und Bindung an den Vertrag}

Im englischen Recht versteht man unter einem Vertrag eine Vereinbarung, die rechtlich durchsetzbar ist oder jedenfalls rechtlich anerkannte Pflichten begründet. ${ }^{134}$ Es findet sich auch häufig folgende Definition, die den Gedanken des Einverständnisses zwischen den Parteien betont, im Ergebnis aber dasselbe bedeutet: A contract is a legally enforceable obligation between two or more people which is created by consent. ${ }^{135}$ Dabei ist die einvernehmliche Übernahme einer Verpflichtung nur wirksam, wenn für das Versprechen eine Gegenleistung (consideration) gewährt wird und wenn es sich um den Austausch von Versprechen handelt. Die bindende Wirkung des Vertrages ergibt sich nicht aus der Willensübereinstimmung allein, sondern erst aus dem Hinzutreten des Elementes der Gegenseitigkeit. ${ }^{136}$ Abzugrenzen ist der Vertrag vom agreement, einer Vereinbarung, bei der die rechtliche Bindungswirkung fehlt. ${ }^{137}$

Das Bürgerliche Gesetzbuch enthält keine Legaldefinition des Vertrages. Es besteht aber Einigkeit über die ihn konstituierenden Elemente. Dazu gehört die Willensübereinstimmung zwischen mindestens zwei Rechtssubjekten, die auf die Herbeiführung eines rechtlichen Erfolges gerichtet ist. 138 Dieser sog. Rechtsbindungswille muss sich auch auf den Konsens über die rechtliche Verbindlichkeit der Abrede erstrecken. ${ }^{139}$

Die Gegenseitigkeit der Leistungspflichten kennzeichnet weder im deutschen noch im englischen Recht den Begriff Vertrag. Es gibt neben dem gegenseitigen (bilateral contract) Vertrag auch einseitig verpflichtende (unilateral contract) und unvollkommen zweiseitige Verträge (formal contract). Dabei zeichnet den gegenseitigen Vertrag aus, dass (zumindest einzelne) Leistungspflichten im Verhältnis von Leistung und Gegenleistung stehen, was bedeutet, dass jede Partei die Pflicht nur übernommen hat, weil sie von der anderen Partei eine Gegenleistung erhalten wird (,do ut des“ beziehungsweise Synallagma). Der einseitig verpflichtende Vertrag (z.B. die Schenkung gem. §§ $516 \mathrm{ff}$ BGB) begründet nur Verpflichtungen einer Partei, während beim unvollkommen zwei-

134 So die Definition bei Atiyah, An introduction to the law of contract, 1995, S. 37; Beatson, Anson's law of contract, 2002, S. 2.

135 Jewell, An introduction to English contract law, 2002, S. 21.

136 Henrich/Huber, Einführung in das englische Privatrecht, 2003, S. 47.

137 Bullinger, Vertrag und Verwaltungsakt, 1962, S. 21.

138 Palandt, Bürgerliches Gesetzbuch, 2009, Einf v § 145, Rn. 1; Kramer, in: Münchener Kommentar, Bürgerliches Gesetzbuch, 2006, v $\S 145$ Rn. 26; Bork, in: Staudinger, Kommentar zum Bürgerlichen Gesetzbuch, 2003, Vorbem $§ 145$ Rn. 1; Flume, Allgemeiner Teil des Bürgerlichen Rechts, Bd. 2, 1975, § 33, 2; Larenz/Wolf, Allgemeiner Teil des Bürgerlichen Rechts, 2004, § 23 Rn. 9.

139 Kramer, in: Münchener Kommentar, Bürgerliches Gesetzbuch, 2006, v § 145 Rn. 26. 
seitigen Vertrag (z.B. Leihe gem. $§ 598$ ff BGB) die Verpflichtung zur Leistung nicht wegen der Gegenleistung eingegangen wird. ${ }^{140}$

Die Bindung an den wirksamen Vertrag wird als Folge der Willensübereinstimmung betrachtet und tritt nur im Ausnahmefall nicht ein. Diesen Zusammenhang nennt das englische Recht sanctity of contract. ${ }^{141}$

Es besteht im englischen und deutschen Recht Übereinstimmung im Hinblick auf das Verständnis der Wirksamkeit eines privatrechtlichen Vertrages. Die Ähnlichkeit der beiden Rechtsordnungen, den privatrechtlichen Vertrag betreffend, lässt sich mit den gemeinsamen Wurzeln erklären. Sowohl das römische Recht als auch die Rechtsprechung englischer Gerichte prägten die frühe Phase der Entstehung des Bürgerlichen Gesetzbuchs und seiner Vorläufer. Besonders die Betonung des freien Willens des Einzelnen ist eine Gemeinsamkeit, die trotz anderer Weiterentwicklungen nie verloren ging. ${ }^{142}$ Dieser freie Wille ist gemeint, wenn von der rechtlichen Figur der Vertragsfreiheit die Rede ist.

\section{Vertragsfreiheit}

\section{a) Historischer Hintergrund}

Der Grundsatz der Vertragsfreiheit (freedom of contract) entwickelte sich im 19. Jahrhundert in Deutschland und England und war Grundkategorie des Liberalismus. ${ }^{143}$

Historisch betrachtet ${ }^{144}$ diente die Idee von der Vertragsfreiheit dazu, bestehende Feudalbeziehungen zurückzudrängen und staatlichen Einfluss im Bereich der Vertragstätigkeit auf ein Mindestmaß zu beschränken. Vertragsfreiheit steht auch für die Anerkennung des Einzelnen und seines freien Willens ${ }^{145}$ und für die Erweiterung seiner Wahlmöglichkeiten. ${ }^{146}$ Vertragsfreiheit im Sinn von „formaler Parität“ der Vertragspartner ist anerkannt, seit die grundsätzlich gleichberechtigte Mitgliedschaft jedes Bür-

140 Emmerich, in: Münchener Kommentar, Bürgerliches Gesetzbuch, 2007, v § 320, Rn. 3 ff; Brownsword, Contract law, 2000, S. 3, 13 f.

141 Mitchell, The contracts of public authorities, 1954, S. $5 \mathrm{f}$.

142 Gordley, The philosophical origins of modern contract doctrine, 1992, S. $1 \mathrm{ff}, 214 \mathrm{ff}$.

143 Sie gehört inzwischen zu den gemeinsamen Prinzipien des Privatrechts aller Mitgliedstaaten der Europäischen Union. Dazu Hartkamp u.a., Towards a European Civil Code, 1998, S. 203 f; Kötz/Flessner, Europäisches Vertragsrecht, 1996, S. 6 f.

Das Prinzip von freedom of contract fand auch Anerkennung in Art. 1.1 der Principles of International Commercial Contracts (UNIDROIT), in Art. 1.102 der Principles of European Contract Law der Lando-Kommission (dazu Zimmermann, JZ 1995, S. 477 ff) und im UN-Kaufrecht (dazu Wasmer, Vertragsfreiheit im UN-Kaufrecht, 2004).

144 Aus vielen Collins, The law of contract, 2003, S. 22; Höfling, Vertragsfreiheit, 1991, S. 1 f; zur rechtshistorischen Forschung Abegg/Thatcher, GLJ 2004, S. 101 ff.

145 Diesen Gedanken formuliert Spieß, DVBl 1994, S. 1222 überspitzt so: „Selbstherrlichkeit und Willkür sind für privatautonome Lebensgestaltung kennzeichnend. Das Menschenbild, das der Privatautonomie vor Augen steht, ist das des frei und gleich gedachten, abstrakten Einzelmenschen, eines homo oeconomicus."

146 Collins, The law of contract, 2003, S. 22; eingehend auch Hönn, Kompensation gestörter Vertragsparität, 1982, S. 5-9. 
gers in der Rechtsgemeinschaft rechtlich verankert ist. Die „materielle“ Vertragsfreiheit ${ }^{147}$ im Sinn von gleichen tatsächlichen Einwirkungschancen auf den Vertrag basiert auf der Idee eines fairen Marktes, wie er in der Realität wohl zu keiner Zeit gegeben war. ${ }^{148}$ Vertragsfreiheit in diesem Sinne ist aufgrund tatsächlicher Entwicklungen (z.B. Standardverträge ${ }^{149}$, Allgemeine Geschäftsbedingungen ${ }^{150}$ ) gefährdet. Auch gesetzgeberische Aktivitäten (z.B. verbraucherschützende Regelungen ${ }^{151}$ ) und Gerichtsurteile (z.B. inhaltliche Kontrolle von Verträgen ${ }^{152}$ ) wirken modifizierend. ${ }^{153}$

\section{b) Begriff und verfassungsrechtliche Garantie der Vertragsfreiheit}

Vertragsfreiheit bedeutet, dass Rechtsbeziehungen zu anderen Personen durch private Vereinbarungen gestaltet werden können. ${ }^{154}$ Sie hat nach herrschender Meinung zwei Elemente ${ }^{155}$, die Abschlussfreiheit (freedom to contract/party freedom) und die Gestaltungs- oder auch Inhaltsfreiheit (freedom of contract/term freedom). Unter Abschlussfreiheit versteht man die Freiheit der Parteien, einen Vertrag abzuschließen oder dies nicht zu tun (sog. positive und negative Abschlussfreiheit). Gestaltungsfreiheit bedeutet, dass die Parteien in der inhaltlichen Gestaltung des Vertrags frei sind. Das Prinzip ,pac-

147 Hönn, Kompensation gestörter Vertragsparität, 1982, S. 9.

148 Zweigert, „Rechtsgeschäft“ und „Vertrag“ heute, in: Caemmerer, FS Rheinstein, 1969, S. 503 f; Adomeit, NJW 1994, S. 2467 ff.

149 Trebilcock, The limits of freedom of contract, 1993, S. 119 f; Beatson, Anson's law of contract, 2002, S. 5 f.

150 Dazu ein Überblick bei Kötz/Flessner, Europäisches Vertragsrecht, 1996, S. 209-234; detailliert Roscher, Vertragsfreiheit als Verfassungsproblem, 1974, S. 78 ff.

151 Zur deutschen und europaweiten Diskussion vgl. Knobel, Wandlungen im Verständnis der Vertragsfreiheit, 2000, S. 30-50; 204-209; zur englischen Rechtslage Brownsword, Contract law, 2000, S. 47 ff; Beatson, Anson's law of contract, 2002, S. 5: Unfair Contract Terms Act 1977, in dem mit Hilfe einer blacklist eine Reihe von Ausschlussklauseln bei Verträgen für unwirksam erklärt wurden. In diese Richtung weisen auch der Sex Discrimination Act 1975, der Race Relations Act 1976 und die Unfair Terms in Consumer Contracts Regulations 1999.

152 Beginnend mit BVerfGE 81, 242 ff (Handelsvertreter); BVerfG, NJW 1994, S. 36 (Bürgschaften einkommens-, vermögensloser Familienangehöriger); vgl. für das englische Recht Hippel, Die Kontrolle der Vertragsfreiheit nach anglo-amerikanischem Recht, 1963, S. $40 \mathrm{ff}$.

153 Allgemein dazu Alexander, The limits of freedom of contract, in: Buckley, The fall and rise of freedom of contract, 1999, S. 103 ff; Posner, The decline of formality in contract law, in: Buckley, The fall and rise of freedom of contract, 1999, S. 61 ff; Brownsword, Contract law, 2000, S. 44 ff; so auch bereits früh Zweigert, „Rechtsgeschäft“ und „Vertrag“ heute, in: Caemmerer, FS Rheinstein, 1969, S. 503 f.

154 Di Fabio, in: Maunz/Dürig, Grundgesetz, Art. 2 Abs. 1 Nr. 101; Höfling, in: Friauf/Höfling, Berliner Kommentar, C Art. 2 Nr. 40; detailliert zu den unterschiedlichen Facetten des Begriffes Enderlein, Rechtspaternalismus und Vertragsrecht, 1996, S. 71; für das englische Recht Harden, The contracting state, 1992, S. 3.

155 Teilweise wird die Auflösungsfreiheit als eigenständiges Element der Vertragsfreiheit genannt (z.B. Bettermann, Grundfragen des Preisrechts für Mieten und Pachten, 1952, S. 2), doch kann diese als Aspekt der Gestaltungsfreiheit aufgefasst werden. So auch Manssen, Privatrechtsgestaltung durch Hoheitsakt, 1994, S. 119. 
ta sunt servanda" gehört zu den wesentlichen Grundstrukturen des Vertragsrechts und bedeutet, dass der Inhalt der Verträge bindend ist. ${ }^{156}$

Die Vertragsfreiheit ist damit eine besondere Ausprägung der wirtschaftlichen Handlungsfreiheit beziehungsweise der Privatautonomie und insofern „Teil der zivilrechtli-

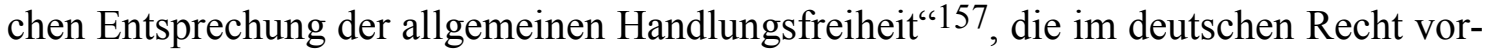
behaltlich speziellerer Grundrechte 158 in Art. 2 Abs. 1 GG enthalten ist. ${ }^{159}$ Im englischen Recht lässt sich eine verfassungsrechtliche Verankerung nicht ausmachen. ${ }^{160}$ Die Handlungsfreiheit scheint dieser Formulierung nach nur im Privatrechtsverkehr Geltung zu haben ${ }^{161}$ und ist anerkannte Grundlage der Marktwirtschaft westlicher Prägung. ${ }^{162}$

\section{c) Kontrahierungszwang und gestörtes Verhandlungsgleichgewicht}

Der Kontrahierungszwang (compulsion to contract) und das gestörte Verhandlungsgleichgewicht (unequal bargaining power) sind zwei Situationen, in denen das Prinzip Vertragsfreiheit beeinträchtigt scheint.

Die Abschlussfreiheit wird durch das Institut des Kontrahierungszwanges ${ }^{163}$ beschränkt. Kontrahierungszwang bedeutet, dass eine Person verpflichtet ist, mit einer anderen Person einen Vertrag abzuschließen. Dieser Zwang kann gesetzlich angeordnet sein, wie z.B. bei der Versorgung mit Strom und Gas ${ }^{164}$, oder sich als sog. ,allgemeiner Kontrahierungszwang“ ohne ausdrückliche gesetzliche Vorschrift aus allgemeinen Rechtsprinzipien 165 ergeben. Beiden Arten von Kontrahierungszwang ist gemeinsam, dass sie nur als zulässig angesehen werden, wenn eine Partei aus einer Monopol- oder marktbeherrschenden Stellung heraus ein höheres Verhandlungsgewicht hat als die andere. Mit Hilfe des Kontrahierungszwangs wird die stärkere Partei zum Schutz der

156 Zum deutschen Recht Palandt, Bürgerliches Gesetzbuch, 2009, Einf v § 145, Rn. 4a, Kramer, in: Münchener Kommentar, Bürgerliches Gesetzbuch, 2006, v § 145 Rn. 26, § 145 Rn. 19; zum englischen Recht Brownsword, Contract law, 2000, S. 36, 38, 43; Harden, The contracting state, 1992, S. 3.

157 Di Fabio, in: Maunz/Dürig, Grundgesetz, Art. 2 Abs. 1 Nr. 101.

158 Dazu m.w.N. Manssen, Privatrechtsgestaltung durch Hoheitsakt, 1994, S. 133-140.

159 Seit BVerfGE 8, 249 ff in ständiger Rspr.; Erichsen, Allgemeine Handlungsfreiheit, in: Isensee/Kirchhof, Handbuch des Staatsrechts, Bd. 6, 2001, § 152 Rn. 56 ff; a.A. Struck, in: DuR 1988, S. 39 ff, der die Vertragsfreiheit nicht als Grundrecht ansehen will. Grundlegend Huber, Die verfassungsrechtliche Bedeutung der Vertragsfreiheit, 1966; Höfling, Vertragsfreiheit, 1991.

160 Beatson, Anson's law of contract, 2002, S. 4.

161 Manssen, Privatrechtsgestaltung durch Hoheitsakt, 1994, S. 183-197 führt aus, dass die Vertragsfreiheit als unbenanntes Grundrecht von ihrer privatrechtlichen Wurzel verselbständigt ist und deshalb nicht von einer privatrechtlichen Ausformung abhängt.

162 Dazu detailliert Spieß, DVBl 1994, S. 1222 f.

163 Dazu bereits Bydlinski, Grundfragen des Kontrahierungszwanges, AcP 180 (1980), S. 1 ff; Kilian, Kontrahierungszwang und Zivilrechtssystem, AcP 180 (1980), S. $47 \mathrm{ff}$.

164 Z.B. für das englische Recht Beatson, Anson's law of contract, 2002, S. 6.

165 Kramer, in: Münchener Kommentar, Bürgerliches Gesetzbuch, 2006, v § 145 Rn. 13; a.A. aus $\S 826$ BGB, weil sich der Schadensersatzanspruch im Wege der Naturalrestitution als Vertragsschluss realisiert; grundlegend Nipperdey, Kontrahierungszwang und diktierter Vertrag, 1920. 
Schwächeren gebunden. Damit wird kein Einfluss auf die inhaltliche Gestaltung des Vertrages ausgeübt.

Anders ist dies bei der Einschränkung der Gestaltungsfreiheit bei gestörtem Verhandlungsgleichgewicht ${ }^{166}$ der Parteien zu sehen. ${ }^{167}$

Gestaltungsfreiheit meint, dass die inhaltliche Freiheit der Vertragsparteien ihre Grenze nur an gesetzlichen Verboten oder der Sittenwidrigkeit sowie anderem zwingenden Recht findet, das aber einen weiten Spielraum bei den Vertragsparteien belässt. ${ }^{168}$ Ferner ist Vertragsfreiheit (als sog. normativ konstituierte Freiheit) nur denkbar, wenn es eine staatliche Garantie der Durchsetzung der versprochenen Vertragsinhalte gibt. Ist nicht vorgesehen, dass die Inhalte auch verbindlich und durchsetzbar sind, fehlt der Vertragsfreiheit und damit auch der Gestaltungsfreiheit ein wesentliches Charakteristikum. ${ }^{169}$ In England wird dieses Element der Vertragsfreiheit als sanctity of contract bezeichnet und damit betont, dass die ausgehandelten, in Ausübung freien Willens gefundenen Vertragsinhalte gerichtlich unantastbar sind, um die Freiheit des Einzelnen nicht über Gebühr einzuschränken. ${ }^{170}$

Die Willensbetätigung in Ausübung der Gestaltungsfreiheit setzt voraus, dass das Verhandlungsgleichgewicht zwischen den Vertragspartnern eine „Richtigkeitsgewähr“ in Bezug auf den Vertragsinhalt hervorbringt. Ein zwischen zwei ebenbürtigen Partnern ausgehandelter Vertrag birgt - so die Theorie - die Gewähr, dass Leistung und Gegenleistung in einem angemessenen Verhältnis zueinander stehen. Situationen, in denen das Verhandlungsgleichgewicht und infolgedessen die Richtigkeitsgewähr gestört sind, entstehen dort, wo ein Vertragspartner aus faktischen (z.B. Informationsdefizit) oder wirtschaftlichen (z.B. Monopolstellung) Gründen weniger Verhandlungsmacht oder Verhandlungsspielraum hat als der andere. Typische Fälle sind das Arbeitsverhältnis ${ }^{171}$, das Verhältnis Unternehmer-Verbraucher ${ }^{172}$, aber auch Situationen, in denen allgemeine Geschäftsbedingungen eines Vertragsteils verwendet werden ${ }^{173}$. Zu nennen sind in diesem Zusammenhang auch Vertragssituationen, in denen die Annahme des Vertragsangebots unter der Prämisse take it or leave it steht. ${ }^{174}$ Das bedeutet, dass ein Vertrags-

166 Wellenhofer-Klein, ZIP 1997, S. 775 schlägt insofern den Begriff „existenzielle Abhängigkeit“ vor, der sich aber nicht durchgesetzt hat und auch, wie sie selbst konstatiert, die allgemeine Unterlegenheit des Verbrauchers nicht erfasst. Diese stellt aber einen einzubeziehenden Anwendungsfall dar.

167 Betrachtet man das Problem gestörten Verhandlungsgleichgewichtes mit verfassungsrechtlichem Blick, ist zunächst zu klären, ob die inhaltliche Gestaltungsfreiheit innerhalb der Grenzen zwingenden Rechts besteht (so Starck, in: Mangoldt/Klein/Starck, Das Bonner Grundgesetz, Bd. 1, Art. 2 Abs. 1 Rn. 136) oder ob zwingendes Recht eine Einschränkung der ansonsten bestehenden Freiheit darstellt (so Enderlein, Rechtspaternalismus und Vertragsrecht, 1996, S. 75).

168 Kramer, in: Münchener Kommentar, Bürgerliches Gesetzbuch, 2006, v § 145 Rn. 19.

169 Höfling, Vertragsfreiheit, 1991, S. 22.

170 Brownsword, Contract law, 2000, S. 37, $39 \mathrm{ff}$.

171 Ferge, EJSS 2000, S. 20 f; Hönn, Kompensation gestörter Vertragsparität, 1982, S. 134 ff.

172 Dazu allgemein Dauner-Lieb, Verbraucherschutz durch Ausbildung eines Sonderprivatrechts für Verbraucher, 1983.

173 Dazu ausführlich Roscher, Vertragsfreiheit als Verfassungsproblem, dargestellt am Beispiel der Allgemeinen Geschäftsbedindungen, 1974.

174 Neumann, VSSR 1993, S. 127 spricht von „Vereinbarungen auf Unterwerfung“. 
partner den Vertragsinhalt bestimmt, ohne eine Verhandlung im Sinne des Wortes zuzulassen. ${ }^{175}$

Für die Beseitigung dieser Defizite gibt es verschiedene Lösungsvarianten. Mögliche Extreme stellen die Ablehnung eines wirksamen und gerichtlich durchsetzbaren Vertrags oder die Ignorierung des gestörten Verhandlungsgleichgewichts dar. Dazwischen liegt die Variante einer regulierenden Tätigkeit des Staates z.B. durch Einführung von zwingenden, die schwächere Partei schützenden Regelungen im Vertragsrecht ${ }^{176}$ oder durch eine gerichtliche Kontrollkompetenz ${ }^{177}$ hinsichtlich des Inhalts des Vertrages zur Vermeidung von Benachteiligungen der schwächeren Partei. ${ }^{178}$

Im englischen Recht wurde der Unfair Contract Terms Act 1977 eingeführt, durch den die oben beschriebenen Situationen eingeschränkten Verhandlungsgleichgewichts abgefedert werden sollten. Eine general doctrine of unequal bargaining power hat sich nicht durchgesetzt, so dass andere Eingriffe in die Bindungswirkung geschlossener Verträge rechtlich nicht zulässig sind. ${ }^{179}$

\section{Die Verwaltung als Vertragspartner eines privatrechtlichen Vertrages}

\section{a) Verwaltungsprivatrecht im deutschen (Sozial-)Recht}

Das Verwaltungsprivatrecht beschreibt im deutschen Recht die Möglichkeiten der (Sozial-)Verwaltung, sich privatrechtlicher Handlungsformen zu bedienen, und beantwortet die Frage, an welche Rechtssätze die Verwaltung in diesem Fall gebunden ist. ${ }^{180}$ Der Bedarf an Handlungsformen des Privatrechts ist vor dem Hintergrund eines sich emanzipierenden Verwaltungsvertragsrechts zu sehen und weniger als „Flucht ins Privatrecht", um öffentlich-rechtlichen Bindungen zu entgehen. ${ }^{181}$

175 Trebilcock, The limits of freedom of contract, 1993, S. 119; Brownsword, Contract law, 2000, S. 61.

176 So z.B. Unfair contract Terms Act 1977 im englischen Recht, vgl. Fn. 151.

177 Wolf, JZ 1976, S. 42 f schlägt ein ,subjektives Recht auf Vertragsschluss zu den Bedingungen des dispositiven Rechts“ vor, um die gerichtliche Kontrolle von Verträgen in Bezug auf Nebenbedingungen zu ersetzen. Problematisch erscheint dieser Vorschlag, weil er sich wegen der Beschränkung auf Nebenbedingungen nicht verallgemeinern lässt und nur greift, wenn dispositives Recht vorhanden ist. Der Vorschlag hat sich deshalb auch nicht durchgesetzt. Dazu Hönn, Kompensation gestörter Vertragsparität, 1982, S. 28-30.

178 Diese Berechtigung zur richterlichen Inhaltskontrolle verortet das Bundesverfassungsgericht in den Generalklauseln des BGB, insbesondere in $\S 242$ BGB, und statuiert eine Pflicht zur Inhaltskontrolle bei Verträgen, die einen der beiden Vertragspartner ungewöhnlich stark belasten und das Ergebnis strukturell ungleicher Verhandlungsstärke sind. Dazu BVerfG, ZIP 1993, S. 1779; Spieß, DVB1 1994, S. 1222 ff; Wellenhofer-Klein, ZIP 1997, S. 774-781.

179 Dazu Brownsword, Contract law, 2000, S. 57 ff, 76, der die Entwicklung der Rechtsprechung ausführlich darstellt und bewertet.

180 Sehr detailliert zum Begriff Stelkens, Verwaltungsprivatrecht, 2005, S. 23-51.

181 So bereits Zezschwitz, NJW 1983, S. 1875 nach der Kodifizierung des verwaltungsrechtlichen Vertrages. 
Gegenstand der Auswahlentscheidung der Verwaltung kann die Organisation des Verwaltungshandelns (z.B. Wahl der Benutzungsform ${ }^{182}$ ) oder die Handlungsform selbst sein. Die Wahl der Organisationsform bleibt in der vorliegenden Arbeit außen vor. Zentral ist dagegen die sog. „Formenwahlfreiheit“"183, auch genannt das „Handlungsformenwahlrecht" 184 .

Der Verwaltung steht kein Wahlrecht zwischen verwaltungsrechtlichem und privatrechtlichem Vertrag zu, sondern grundsätzlich nur ein Wahlrecht bezüglich einseitiger oder vertraglicher Handlungsform, denn die Abgrenzung zwischen verwaltungsrechtlichem und privatrechtlichem Vertrag hängt nach bislang herrschender Meinung nicht von der Beteiligung eines Verwaltungsträgers oder vom Willen der Vertragspartner, insbesondere der Verwaltung ab, sondern wird nach dem Vertragsgegenstand bestimmt (Gegenstandstheorie). ${ }^{185} \mathrm{Da}$ weder geklärt ist, was genau der Vertragsgegenstand ist, noch wann dieser dem öffentlichen Recht angehört, ist diese Abgrenzung ungenau. Sie ist aber immer noch der herrschende Ansatz in Rechtsprechung und Literatur. Folgt man der Gegenstandstheorie, ist es ausgeschlossen, dass ein Wahlrecht der Verwaltung besteht, da das Abgrenzungskriterium, Gegenstand des Vertrages, ein objektives ist, und somit auf den Willen der Vertragsparteien nicht abgestellt wird. Ohne Willensausübung ist aber die Annahme eines Wahlrechts nicht denkbar. ${ }^{186}$

\section{bb) Anwendungsbereich im Rahmen der Sozialverwaltung}

Der Anwendungsbereich privatrechtlicher Verträge der Sozialverwaltung ist beschränkt. Ein ausdrückliches Verbot eines Handelns mit Hilfe privatrechtlicher Verträge ist nicht auffindbar, doch entsteht aus der rechtlichen Vorordnung vieler Anwendungsgebiete von Verträgen im Sozialrecht ein einheitliches Bild: In der Regel werden keine privatrechtlichen Verträge zwischen Bürger und Sozialverwaltung geschlossen.

Grund dafür ist, dass Sozialleistungsansprüche zwar unmittelbar auf Geld- oder Sachleistungen gerichtet sind, aber grundsätzlich in der Massenverwaltung auf der Grundlage eines Verwaltungsaktes erfüllt werden. Werden nun diese Sozialleistungen auf der Grundlage eines Vertrages erbracht, ist die Zuordnung zum öffentlichen Recht eindeutig. Gegenstand des Vertrages ist die Sozialleistung, die im Sozialrecht geregelt ist.

Problematisch ist die Zuordnung in den Fällen, in denen die Leistungserbringung durch Dritte (z.B. Verträge mit Personal-Service-Agenturen gem. § 37c Abs. 2 SGB III) erfolgt und das Verhältnis zwischen Leistungsträger und privatem Leistungserbringer

182 Grundlegend dazu Fischedick, Wahl der Benutzungsform, 1986, S. 7 ff.

183 Dazu m.w.N. Schlette, Die Verwaltung als Vertragspartner, 2000, S. 122 ff.

184 Bonk, in: Stelkens/Bonk/Sachs, VwVfG, 2001, § 54 Rn. 91.

185 Z.B. Maurer, Allgemeines Verwaltungsrecht, 2009, S. 370 f; Pickel/Marschner, SGB X, Bd. 1, § 53 Rn. 14; Engelmann, in: Wulffen, SGB X, 2008, § 53 Rn. 7; dazu im Überblick m.w.N. Schlette, Die Verwaltung als Vertragspartner, 2000, S. $112 \mathrm{f}$.

186 So auch Neumann, DÖV 1992, S. 156; Stelkens, Verwaltungsprivatrecht, 2005, S. 665. 
durch Verträge ausgestaltet wird. Hier ist zu prüfen, was der Vertragsgegenstand ist und ob dieser dem öffentlichen Recht zuzuordnen ist. 187

\section{b) Government contracts im englischen Recht}

aa) Begriff

Bei government contracts handelt es sich um Vereinbarungen, die zwischen der Krone ${ }^{188}$ und einem Einzelnen, meist einem Unternehmen abgeschlossen werden, mit denen eine öffentliche Aufgabe erfüllt wird. 189

Die Krone kann sich wie jeder Private auf dem freien Markt betätigen und vertraglich binden. Sie hat dabei keine Sonderstellung rechtlicher Art. ${ }^{190}$ Sie hat nach den Grundsätzen des common law rechtliche Handlungsfähigkeit, wenn der Vertrag sich innerhalb der Befugnisse des Staates bewegt und durch einen Beamten/Mitarbeiter (crown servant) innerhalb dessen Ermächtigung abgeschlossen wird. ${ }^{191}$ Auch andere Behörden beziehungsweise zum Handeln berechtigte Mitarbeiter können die Krone vertraglich binden, um ihre Aufgaben zu erfüllen oder die Aufgabenerfüllung zu vereinfachen. Ihre rechtliche Handlungsfähigkeit ergibt sich entweder aus dem Gesetz, durch das die Behörden begründet werden, oder aus Einzelermächtigungen. 192

Handelt es sich nicht um Verträge zur Erfüllung öffentlicher Aufgaben (fiskalische Geschäfte), ist das contract law, also das allgemeine Vertragsrecht ohne Besonderheiten anwendbar. Es liegen schlichte common law contracts vor.

Government contracts sind Verträge, die grundsätzlich auch dem contract law unterliegen. 193 Sie kommen durch zwei Willenserklärungen zustande, die freiwillig abgegeben worden sein müssen. Der Inhalt muss eindeutig bestimmt beziehungsweise bestimmbar und alle Aspekte der öffentlichen Aufgabe und deren Erfüllung müssen enthalten sein. ${ }^{194}$ Es gibt bei ihnen aber Besonderheiten, die aus der direkten oder indirekten Beteiligung der Krone resultieren.

187 Hierzu detailliert Stelkens, Verwaltungsprivatrecht, 2005, S. 772-781.

188 Zum Begriff Hogg, Liability of the Crown, 1989, S. 9 f: "Although we now have a "constitutional monarchy", [...], the term "the Crown" has persisted as the name for the executive branch (but not the legislative branch) of government."

189 Seddon, Government contracts, 1999, S. 2 f; Walsh u.a., Contracts for public services, in: Campbell/Vincent-Jones, Contracts and economic organisation, 1996, S. 212, 218 f; Pollard/Hughes, Constitutional and administrative law, 1990, S. 57.

190 Hogg, Liability of the Crown, 1989, S. $159 \mathrm{f}$.

191 Cane, Administrative law, 2004, S. 295; im Einzelnen dazu Hogg, Liability of the Crown, 1989, S. 163-167 bzw. S. 168 f zum Handeln durch crown servants.

192 Foulkes, Administrative law, 1990, S. 414 f.;

193 Probleme von Verträgen, auf die das englische beziehungsweise europäische Vergaberecht Anwendung findet, werden in der vorliegenden Arbeit nicht behandelt. Dazu Turpin, Government procurement and contracts, 1989, S. 61 ff. Handelt es sich bei diesen Verträgen um solche, auf die Europarecht Anwendung findet, ist ein bestimmtes Verfahren einzuhalten, welches mit der European Community Service Directive 92/50/EEC [1992] OJL 209/1, die durch die Public Services Contracts Regulations 1993 (SI 1993/3228) in englisches Recht umgesetzt wurde, übereinstimmt.

194 Selznick, Law, society and industrial justice, 1969, S. 52-62; Sainsbury/Kennedy, Contracting welfare?, in: Lunt/Coyle, Welfare and policy, 1996, S. 117. 
(1) Doktrin der ultra vires und Local Government (Contracts) Act 1997

Das Handeln einer Behörde, deren Handlungsfähigkeit sich aus einem Gesetz ergibt, wird durch die Doktrin der Ultra vires bestimmt. Vereinfacht dargestellt bedeutet dies, dass jedes Handeln, insbesondere jeder Vertrag, der sich nicht im Rahmen der gesetzlichen Ermächtigung hält, unwirksam ist. ${ }^{195}$ Dies dient dem Schutz des Steuerzahlers vor ungesetzlichen (Zahlungs-)Verpflichtungen des Staates. Zum Schutz der privaten Vertragspartner, für die angesichts der Rechtsunsicherheit sonst Verträge mit öffentlichen Stellen zunehmend uninteressant sein könnten, sind im Local Government (Contracts) Act 1997 Regelungen enthalten, die auch Verträge, die ultra vires sind, durchsetzbar (enforceable) machen, aber gleichzeitig vorsehen, dass die Handlungsfähigkeit der $p u b$ lic authority im Wege von judicial review überprüft werden kann. 196

(2) Doctrine of executive necessity

Die Krone kann sich nicht darauf berufen, generell Verträge, die ihre Handlungsfreiheit zu stark einschränken, nicht erfüllen zu müssen. ${ }^{197}$ Es kann jedoch Fälle geben, in denen die Nichteinhaltung eines Vertrages zur Aufrechterhaltung einer funktionierenden Verwaltung notwendig ist: Die doctrine of executive necessity 198 führt dazu, dass die Verwaltung nur dann folgenlos einen government contract nicht einhalten muss, wenn dagegen schwerwiegende Gründe sprechen. ${ }^{199}$ Die Lehre entstammt der Entscheidung Rederiaktiebolaget "Amphitrite“ v. The King, in der ein Gericht entschied, dass der Staat einen Vertrag brechen darf, wenn sonst das Wohlergehen des Staates (welfare of the State), insbesondere in Kriegszeiten, in Gefahr wäre. ${ }^{200}$

Kritisiert wird an dieser Entscheidung, dass die Formulierung welfare of the State sehr weit ist, so dass Verträge mit dem Staat ein hohes Risiko der Nichteinhaltung durch den Staat beinhalten. ${ }^{201}$ Allerdings ist die Krone weiterhin ein sicherer Vertragspartner, da in der großen Masse der Verträge die doctrine of executive necessity schlechthin irrelevant ist. ${ }^{202}$ Die Gerichte gehen ferner davon aus, dass hinter der Nichteinhaltung des Vertrages eine politische Notwendigkeit stehen muss, nicht die bloße Unzufriedenheit mit dem Vertragspartner oder dem Vertragsinhalt. ${ }^{203}$

195 Wade/Bradley, Constitutional and administrative law, 1985, S. 687.

196 Beatson, Anson's law of contract, 2002, S. 212 f.

197 Crown proceedings Act 1947 sec. 1; dazu Philips/Jackson, Constitutional and administrative law, 2001, S. 737 f; Cane, Administrative law, 2004, S. 206-210.

198 Foulkes, Administrative law, 1990, S. 418 ff; Philips/Jackson, Constitutional and administrative law, 2001, S. $739 \mathrm{f}$.

199 Seddon, Government contracts, 1999, S. $169 \mathrm{ff}$.

200 Rederiaktiebolaget Amphitrite v. The King [1920] 3 KB S. 500 [503].

201 Mitchell, The contracts of public authorities, 1954, S. 52-57.

202 Turpin, Government procurement and contracts, 1989, S. 87 ff; Hogg, Liability of the Crown, 1989, S. 169 ff; zu diesem Ergebnis kommt auch Schlette, Die Verwaltung als Vertragspartner, 2000, S. 374.

203 Commissioners of Crown Lands v. Page [1960] 2 QB S. 274 [291]. 
(3) Rule against fettering future executive action

Die rule against fettering future executive action ${ }^{204}$ besagt, dass sich die Verwaltung durch Verträge bei der Ausfüllung von Ermessensspielräumen nicht so stark binden darf, dass sie nicht mehr in Einklang mit ihren öffentlichen Aufgaben oder gesetzlich bestehenden Pflichten handeln kann (incompatibility test ${ }^{205}$ ). Die Rechtsfolgen einer Anwendung dieser Regel sind nicht abschließend geklärt. Verträge werden als (teilweise) unwirksam betrachtet ${ }^{206}$ oder als wirksam, aber nicht durchsetzbar ${ }^{207}$. Sie können auch schlechthin wirksam und durchsetzbar sein mit den Nachteilen, die für den Vertragspartner aus der Nichteinhaltung durch die Verwaltung resultieren, und eine Rückabwicklung wegen unrechtmäßiger Bereicherung (unjust enrichment) nach sich ziehen. ${ }^{208}$

\section{cc) Judicial review}

Judicial review ist das Verfahren, in welchem über die Rechtmäßigkeit (lawfulness) des Verwaltungshandelns entschieden wird. ${ }^{209}$ Überprüft werden können auch Verträge und andere Handlungen der Verwaltung, die in Ausübung einer public function vorgenommen werden. ${ }^{210}$ Die Entscheidung trifft der Administrative Court, eine bestimmte Abteilung des High Court, die nur mit judicial review befasst ist. ${ }^{211}$ Die Besonderheiten dieses Verfahrens gegenüber private law procedures sind vielfältig und können nicht im Einzelnen dargestellt werden. ${ }^{212}$ Es handelt sich um eine gegenüber dem allgemeinen Verfahren eingeschränkte Rechtsschutzmöglichkeit aufgrund von kurzen Klagefristen und Antragsvoraussetzungen (z.B. sufficient interest, issue of public law, availabilitiy of alternative remedies, etc.) ${ }^{213}$ Bei der Prüfung sind stets auch die Vorgaben des Human Rights Act zu beachten. ${ }^{214}$ Wichtig ist, dass im judicial review das Gericht eine Entscheidung nur aufheben, aber nicht durch eine eigene ersetzen kann. Sie kann lediglich eine Anweisung aussprechen, dass die Behörde im Sinn des Gerichts entscheidet (direc-

204 Birkdale District Supply Co Ltd. v. Southport Corp [1926] AC S. 355 [364]; Stringer v. Minister of Housing and Local Government [1979] 1 WLR S. 1281; dazu Feldman/Birks, English public law, 2004, S. 742 ff; Cane, Administrative law, 2004, S. 206 ff; Craig, Administrative Law, 2003, S. 540 ff.

205 Craig, Administrative Law, 2003, S. 541-545.

206 Feldman/Birks, English public law, 2004, S. 742.

207 Wade/Forsyth, Administrative law, 2004, S. 336.

208 Dazu ein Überblick bei Seddon, Government contracts, 1999, S. 186.

209 Civil Procedure Rules Rule 54.1 lautet: "This Section of this Part contains rules about judicial review. In this Section a 'claim for judicial review' means a claim to review the lawfulness of an enactment or a decision, action or failure to act in relation to the exercise of a public function."

210 Arrowsmith, LQR 1990, S. $277 \mathrm{ff}$.

211 Civil Procedure Rules 2.1 Practice Direction part 54, zu dessen Entstehung Cane, Administrative law, 2004, S. 29.

212 Einführend Cane, Administrative law, 2004, S. 28-32; weiterführend Lewis, Judicial remedies in public law, 1992; Woolf/Jowell, Judicial review of administrative action, 2005.

213 Dazu Harlow/Rawlings, Law and administration, 1997, S. $530 \mathrm{ff}$.

214 Vgl. Klug/O'Brien, PL 2002, S. 649 ff zu den ersten Gerichtsentscheidungen seit dem Inkrafttreten des HRA. 
tion). ${ }^{215}$ Ferner darf das Gericht im judicial review die Entscheidung nicht inhaltlich voll überprüfen, sondern nur über die Übereinstimmung mit dem Recht beziehungsweise Gesetz entscheiden (lawfulness). ${ }^{216}$

\section{dd) Unterscheidung von private law und public law}

Die common law courts lehnten vor der gesetzlichen Einführung eines Administrative Court die Unterscheidung zwischen public law und private law ab und nahmen damit die Krone im Rahmen der common law Grundsätze gegen Klagen einzelner Bürger, insbesondere auf vertraglicher Grundlage in Schutz. ${ }^{217}$ Die Unterscheidung zwischen private law und public law ist in England seit jeher Anlass für heftige Diskussionen.218 $\mathrm{Zu}$ beantworten ist die Frage, ob das public law als „Sonderrecht des Staates“ anzusehen ist.

Nach englischer Rechtstradition und dem Verständnis des Verhältnisses Bürger-Staat steht ein „Sonderrecht des Staates“219 im Sinne eines public law in der Gefahr, eine Verschlechterung der Position des einzelnen Bürgers heraufzubeschwören. Man befürchtet, dass ein öffentliches Recht der Krone (auch mit Hilfe besonderer Gerichte) über die bestehenden Sonderpositionen hinaus zusätzliche Befugnisse verschafft und sie in eine dem Bürger übergeordnete Position bringt. 220

Diese Bedenken manifestieren sich vor allem, wenn besondere Gerichte für Klagen gegen die Krone geschaffen werden sollen. Seien nicht die gleichen Gerichte wie für Klagen von Bürgern gegeneinander zuständig, könne sich eine Verschiebung von Befugnissen des Gesetzgebers auf die Gerichte im „öffentlichen Bereich“ ergeben. Allgemein herrscht großes Misstrauen gegenüber der Befugnis besonderer Gerichte, das vom Parlament beschlossene public law durch ihre Rechtsprechung ,in unrichtiger Weise“ zu verändern. ${ }^{221}$ Spiegelbildlich zu diesem Misstrauen verhält sich das Vertrauen in die Effizienz parlamentarischer Kontrollen. ${ }^{222}$ Rechte, die in anderen Rechtsordnungen der

215 Civil Procedure Rules Rule 54.19.

216 Civil Procedure Rules Rule 54.1.

217 Arrowsmith, LQR 1990 (106), S. 290 f.

218 Finn, Public function - private action, in: Benn/Gaus, Public and private in social life, 1983, S. 93 ff; Samuel, MLR 1983 (46), S. 558 ff; Woolf, PL 1986, S. 220 ff; Taggart, "The peculiarities of the English": Resisting the public/private law distinction, in: Craig/Rawlings, Law and administration in Europe, 2003, S. 107 ff; aus rechtshistorischer Perspektive Allison, A continental distinction in the common law, 2000.

219 Dabei bleibt der Begriff des Staates nach deutschem Verständnis im englischen System unklar. Dort nimmt die Krone (the Crown) am ehesten diesen besonderen Status ein. Deren Sonderstellung zeigt sich an verschiedenen Stellen im Verfahren vor den common law Gerichten. Z.B. kann gem. Crown Proceedings Act 1947 sec. 25 die Verurteilung der Krone zur Zahlung einer Geldsumme nicht zwangsvollstreckt werden. Stattdessen wird lediglich ein certificate of the amount due ausgestellt, weil die Zahlungspflicht der Krone aufgrund dieser Bescheinigung per Gesetz angeordnet ist. Vgl. für weitere Beispiele Cane, Administrative law, 2004, S. 267 f; Loughlin, The state, the crown and the law, in: Sunkin/Payne, The nature of the crown, 1999, S. 33.

220 Dicey, Introduction to the study of the law of the Constitution, 1941 (1889), S. $183 \mathrm{ff}$; dazu Cane, Public law and private, in: Eekellaar, Oxford essays in jurisprudence, 1987, S. 61-64.

221 Yardley, Principles of administrative law, 1986, S. 1.

222 Mitchell, PL 1965, S. 100. 
Judikative zustehen, z.B. die Überprüfung von Gesetzen auf ihre Rechtmäßigkeit, werden im englischen System als entbehrlich angesehen, weil die Spielräume des Parlamentes nicht angetastet werden sollen. ${ }^{223}$ Dies geschieht mit dem Ziel, Einflüsse der Judikative zu beschränken. Neben Verfahren vor Gerichten führte man administrative tribunals ein, die dem Bürger (verwaltungsintern) Rechtsschutz gewähren sollen. ${ }^{224}$

Die Kriterien für die Abgrenzung public law-private law sind vor dem Hintergrund der Regelung rule 54.1 Civil Procedure Rules zu betrachten, die die Formulierung „exercise of a public function" enthält:

(1) Source of power approach

Zunächst prüfen die Gerichte, ob die Rechtsquelle für die Handlungsbefugnis dem common law entstammt oder als eine vom Parlament erlassene Rechtsnorm anzusehen ist (statutory power). Während jede Handlung, die auf statutory law basiert, diesem Ansatz folgend auch einen Rechtsstreit im öffentlichen Recht hervorruft, ist wegen der Verfügbarkeit von private law remedies bei jedem Vertrag mit dem Staat hingegen judicial review ausgeschlossen. Dieser rein formale Ansatz, der sogenannte source of power approach 225 , wurde deshalb von der Rechtsprechung 226 und der Rechtswissenschaft 227 weiter entwickelt, weil er für sich genommen einen zu großen, gerichtlich nicht kontrollierbaren Bereich von Verwaltungshandeln ausklammert, das vertragliche Handeln. Im Gegenzug schließt er zunehmend durch Gesetze geregelte, früher dem common law unterliegende Fragen ein (Gestaltung des Vertragsrechts mit Hilfe von statutes).

(2) Nature of the power approach

Nach dem sog. nature of the power approach 228 gehören Rechtsstreitigkeiten in den Bereich des public law, wenn die handelnde Stelle staatliche Gewalt ausübt (public power). Dabei besteht bei gewissen Institutionen Einigkeit (wie z.B. Ministerien), bei anderen ist die Abgrenzung nicht eindeutig (z.B. Gewerkschaften oder selbstverwaltete Institutionen, weil sie von der Organisationsstruktur privatrechtlich organisiert sind, aber indirekt staatliche Gewalt/state authority ausüben). ${ }^{229}$ Hinzu kommt, dass nach diesem Ansatz eine Rechtsstreitigkeit, deren Gegenstand aus einem Vertrag entstanden

223 Philips/Jackson, Constitutional and administrative law, 2001, S. 7; Pollard/Hughes, Constitutional and administrative law, 1990, S. 399. Aus diesem Grund gehört das Parlament aufgrund der doctrine of Parliamentary supremacy auch nicht dazu, wenn von der Krone die Rede ist. Vgl. Cane, Administrative law, 2004, S. 268.

224 Wiesner, Administrative Tribunals in Großbritannien, 1974.

225 O’Reilly v. Mackman [1983] 2 AC S. 237; kritisch Morris/Fredman, PL 1994, S. 69 ff.

226 Regina v. Panel on Take-overs and Mergers, Ex parte Datafin Plc. and Another [1987] QB S. 815; [1987] 2 WLR S. 699 (CA), diskutiert bei Hunt, Constitutionalism and the contractualisation of government in the United Kingdom, in: Taggart, The province of administrative law, 1997, S. $28 \mathrm{ff}$.

227 Beatson, LQR 1987, S. 47 ff; Craig, Public law and control over private power, in: Taggart, The province of administrative law, 1997, S. $198 \mathrm{f}$.

228 Regina v. Panel on Take-overs and Mergers, Ex parte Datafin Plc. and Another [1987] WLR 1987 S. 699 (CA); R. v Advertising Standards Authority Ex p. Insurance Services [1990] COD 42.

229 Beatson, LQR 1987, S. 50 f; Craig, Public law and control over private power, in: Taggart, The province of administrative law, 1997, S. $200 \mathrm{ff}$. 
ist, nie mit judicial review überprüft werden kann, weil es nicht nur eine handelnde Stelle gibt. Dies erscheint nicht angemessen. 230

(3) Functional approach

Ein anderer Ansatz geht von der Funktion der Unterscheidung zwischen public law und private law aus (functional approach). ${ }^{231}$ Sie führt die dogmatische Unterscheidung beziehungsweise Unterscheidbarkeit darauf zurück, dass die dem Staat obliegenden Aufgaben erfüllt werden müssen, egal, in welcher Rechtsform das passiert. ${ }^{232} \mathrm{Im}$ Grunde lehnt diese Ansicht die grundsätzliche Unterscheidbarkeit ab und fordert eine konkrete Einzelfallentscheidung mit dem Ziel, Theorien über die Zuordnung zu public law oder private law überflüssig zu machen. ${ }^{233}$

(4) Stellungnahme

Die Einordnung in die Kategorien public/private fällt offenbar auch der englischen Rechtswissenschaft schwer. Die Lösung des functional approach lässt pragmatisch die Suche nach einer allgemein passenden Theorie fallen und wählt keinen dogmatischen Zugang. Sie stellt damit sicher, dass die Besonderheiten jedes Falles gewürdigt werden können und nicht allein wegen theoretischer Überlegungen eine geeignete Zuordnung unterbleibt. Diese Lösung enthält deshalb einen Ansatz, der auch für das deutsche Recht erwogen werden sollte.

\section{Vertrag im öffentlichen Recht}

\section{Der verwaltungsrechtliche Vertrag im deutschen Recht}

\section{a) Hintergrund und historische Entwicklung bis zur gesetzlichen Regelung}

Im Jahre 1866 entwickelte Otto Mayer eine Lehre zum verwaltungsrechtlichen Vertrag als allgemeiner Handlungsform. ${ }^{234}$ Er verstand den ,verwaltungsrechtlichen Vertrag" 235 zwischen Staat und Bürger als zwei Verwaltungsakte, einen, der nach Zustimmung des Bürgers diesem eine Leistungspflicht auferlegt („Verwaltungsakt auf Unterwerfung ${ }^{“ 236}$ ) und einen, der die Entschädigung für die Leistung gewährt. Mayer konnte

230 Feldman/Birks, English public law, 2004, S. 90 f.

231 Harlow/Rawlings, Law and administration, 1997, S. 29 ff; Harlow, MLR 1980 (43), S. 241 ff; Oliver, PL 1997, S. 630 ff.

232 Zur Abgrenzung der Anwendung des Human Rights Acts mittels des Kriteriums public function vgl. Oliver, PL 2000, S. 476 ff.

233 So auch Freedland, Government by contract, in: Craig/Rawlings, Law and administration in Europe, 2003, S. 123 f; Cane, Administrative law, 2004, S. 13.

234 Mayer, Theorie des französischen Verwaltungsrechts, 1998 (1886); ders., AöR 1888 (3), S. 42 ff.

235 Anders beurteilt Otto Mayer die vertragliche Gestaltung des Verhältnisses zwischen zwei Trägern öffentlicher Gewalt. Hier erkennt er verwaltungsrechtliche Verträge an. Als ebenso möglich erachtet er, den Abschluss eines zivilrechtlichen Vertrages zwischen Staat und „Untertan, wenngleich als eng begrenzte Ausnahme." Vgl. Dewitz, Der Vertrag in der Lehre Otto Mayers, 2004, S. 16-29.

236 Dazu Schiedermair, Der Verwaltungsakt auf Unterwerfung, 1968. 
sich einen Vertrag nur zwischen zwei gleich gestellten Partnern vorstellen, so dass ein Vertrag zwischen Staat und Untertan nicht denkbar war.

Daran entzündete sich die Kritik Paul Labands. Er war der Auffassung, dass ein Vertrag nicht zwei gleichgestellte Kontrahenten voraussetze. Zwar hatte auch Laband die Vorstellung, dass der Staat durch keinen höheren Befehl gebunden und keiner Herrschaftsmacht unterworfen sei. Er drehte dieses Argument um und begründete damit seine positive Haltung zum Vertrag zwischen Staat und Bürger. Der Staat sei gerade deshalb fähig, sich nach eigenem Belieben aller Rechtsformen zu bedienen. Deshalb könne er auch einen Vertrag auf dem Gebiet des öffentlichen Rechts als Handlungsform wählen. 237

Im weiteren Verlauf der Entwicklung während der Weimarer Republik und der Zeit des Nationalsozialismus wird der verwaltungsrechtliche Vertrag in der Praxis immer bedeutsamer. ${ }^{238}$ Vereinbarungen zwischen zwei Trägern öffentlicher Gewalt sind als verwaltungsrechtliche Verträge anerkannt und Verträge zwischen Staat und Bürgern werden vermehrt geschlossen. Es werden gesetzliche Regelungen ausgearbeitet. ${ }^{239}$ Problematisch bleibt die Frage, ob eine ausdrückliche gesetzliche Ermächtigung für die Zulässigkeit des verwaltungsrechtlichen Vertrages als erforderlich anzusehen ist. Dies geht einher mit dem Problem, wie weit das Ermessen der Verwaltung bei der inhaltlichen Gestaltung des Vertrags reicht und wie weit mit dem Vertrag vom ,sachlich Richtigen" abgewichen werden darf. 240

Nach dem Zweiten Weltkrieg prägt die Vereinbarkeit mit dem Grundgesetz die dogmatische Beschäftigung mit dem verwaltungsrechtlichen Vertrag. Problematisch erschien, ob die Praxis, verwaltungsrechtliche Verträge abzuschließen, mit dem Grundsatz der Gesetzmäßigkeit der Verwaltung vereinbar sein würde. ${ }^{241}$

Mit der gesetzlichen Regelung des „öffentlich-rechtlichen Vertrages“ im Verwaltungsverfahrensgesetz in den Jahren 1976/1977242 tritt die Entwicklung des verwaltungsrechtlichen Vertrages in eine neue Phase. Es klärt sich unter anderem die Frage der Zulässigkeit des verwaltungsrechtlichen Vertrages mit der gesetzlichen Regelung der Handlungsform in $\S \S 54 \mathrm{ff} \mathrm{VwVfG}$ und der Anerkennung als verfahrensbeendigende

237 Laband, AöR 1887 (2), S. 149 ff.

238 Pakeerut, Die Entwicklung der Dogmatik des verwaltungsrechtlichen Vertrages, 2000, S. 46 ff.

239 So enthält der Württembergische Entwurf einer Verwaltungsrechtsordnung von 1931 Regelungen über die Zulässigkeit, Voraussetzungen für den Vertragsschluss und für die Nichtigkeit des Vertrages. Dazu Hofacker, Verwaltungsrechtsordnung für Württemberg, 1931, S. 28 f.

240 Dazu Pakeerut, Die Entwicklung der Dogmatik des verwaltungsrechtlichen Vertrages, 2000, S. 51 ff; im Einzelnen Kormann, System der rechtsgeschäftlichen Staatsakte, 1910, S. 28 ff; Apelt, Der verwaltungsrechtliche Vertrag, 1920, S. 41 ff; Burckhardt, Der Vertrag im Privatrecht und im öffentlichen Recht, 1924, S. 42.

241 Dazu ausführlich Pakeerut, Die Entwicklung der Dogmatik des verwaltungsrechtlichen Vertrages, 2000, S. 61 ff; beispielhaft genannt seien Imboden, Der verwaltungsrechtliche Vertrag, 1955, S. 66 ff; Kottke, Subordinationsrechtlicher Vertrag, 1966, S. 37 f; Bleckmann, VerwArch 1972, S. 416 ff.

242 Am 1.1.1977 in Kraft getreten, Gesetz v. 25.5.1976 (BGB1. I, S. 1253); dazu Spanner, DVB1 1964, S. 845 ff; Baring, DVB1 1965, S. 180 ff. 
Handlungsform neben dem Verwaltungsakt in $\S 9$ VwVfG. ${ }^{243}$ Die weitestgehend wörtliche Übernahme dieser Regelungen in die $\S \S 53$ ff SGB X ${ }^{244}$ führt diese Entwicklung fort.

\section{b) Begriff}

$\S 54 \mathrm{~S} .1 \mathrm{VwVfG}$ und $\S 53 \mathrm{~S} .1 \mathrm{SGB} X$ definieren den verwaltungsrechtlichen Vertrag nicht, sondern setzen ihn voraus. Sie sprechen von einem Vertrag, durch den ein Rechtsverhältnis auf dem Gebiet des öffentlichen Rechts begründet, geändert oder aufgehoben wird. Die Vertragsbegriffe des allgemeinen Verwaltungsrechts und des Sozialrechts sind insoweit identisch.

Aus dem Anwendungsbereich der Vorschriften ergibt sich gem. § 1 Abs. 1 VwVfG beziehungsweise $\S 1$ SGB X, dass es sich um Verträge einer Behörde beziehungsweise des dahinter stehenden Trägers öffentlicher Verwaltung 245 handeln muss. Staatsverträge oder Verwaltungsabkommen zwischen Bund und Ländern sind somit nicht erfasst. ${ }^{246}$ Im Weiteren wird deshalb der Begriff des verwaltungsrechtlichen Vertrags gewählt, der als Gegenbegriff zum privatrechtlichen Vertrag geeignet scheint.

In Literatur und Rechtsprechung werden subordinationsrechtliche und koordinationsrechtliche Verträge unterschieden. Koordinationsrechtlich sind die Verträge, die zwischen grundsätzlich gleichgeordneten Vertragspartnern, insbesondere zwischen rechtsfähigen Trägern öffentlicher Verwaltung geschlossen werden. Subordinationsrechtlich sind dagegen Verträge zwischen Parteien, die in einem Über-Unterordnungsverhältnis zueinander stehen, also typischerweise Verträge zwischen Verwaltung und Bürger. ${ }^{247}$

Diese Unterscheidung wird hier modifiziert und stattdessen von einem „Vertrag im Sinn des $\S 54$ S. 2 VwVfG“ gesprochen. ${ }^{248}$ Die Formulierung ,insbesondere“ in $\S 54 \mathrm{~S}$. 2 VwVfG, § 53 S. 2 SGB X bedeutet, dass der verwaltungsrechtliche Vertrag nicht nur in Fällen, in denen auch ein Verwaltungsakt erlassen werden könnte, abgeschlossen werden kann. ${ }^{249}$ Da der verwaltungsrechtliche Vertrag als Mittel der kooperativen Einbeziehung und der Mitwirkung des Bürgers in dem Prozess staatlicher Herrschaftsausübung und als gegenseitig funktional austauschbar anzusehen ist, ist diese Begrifflich-

243 Maurer, Der Verwaltungsvertrag, in: Hill, Verwaltungshandeln durch Verträge und Absprachen, 1990, S. 33; Krebs, VVDStRL, H. 52 (1993), S. 248 ff; Pitschas, Entwicklung der Handlungsformen im Verwaltungsrecht, in: Blümel/Pitschas, Reform des Verwaltungsverfahrensrechts, 1994, S. 230.

244 Neubekanntmachung des Sozialgesetzbuchs - Verwaltungsverfahren vom 18.8.1980 (BGB1. I, S. 1469).

245 Dazu Gurlit, Jura 2001, S. 663.

246 Dazu ausführlicher Schlette, Die Verwaltung als Vertragspartner, 2000, S. 18-20.

$247 \mathrm{Zu}$ diesen Begriffen Maurer, Allgemeines Verwaltungsrecht, 2009, S. 373 ff; vgl. speziell zum subordinationsrechtlichen Vertrag Gent, Der subordinationsrechtliche Vertrag (§ 53 SGB X) als geeignete Handlungsform zur Erbringung von Sozialleistungen, 1987.

248 So auch Schlette, Die Verwaltung als Vertragspartner, 2000, S. 383.

249 Gusy, DVB1 1983, S. 1225. 
keit passender. ${ }^{250}$ Hinzu kommt, dass der Abschluss jedes verwaltungsrechtlichen Vertrages als Erfüllung einer öffentlichen Aufgabe und damit in Ausübung öffentlicher Gewalt geschieht ${ }^{251}$, sodass in der Bezeichnung subordinationsrechtlicher Vertrag die Abgrenzung zu den koordinationsrechtlichen Verträgen verschwimmt.

\section{c) Abgrenzung zwischen öffentlichem Recht und Privatrecht}

Der Wortlaut der $\S 54$ S. 1 VwVfG, $\S 53$ S. 1 SGB X spricht von einem Rechtsverhältnis auf dem Gebiet des öffentlichen Rechts und setzt damit die Abgrenzbarkeit von öffentlichem Recht und Privatrecht voraus. Die genaue Abgrenzung von öffentlichem Recht und Privatrecht ist im Allgemeinen und im Besonderen bei der Anwendung der Vorschriften über den verwaltungsrechtlichen Vertrag umstritten. ${ }^{252}$ Die Arbeit beschränkt sich auf zentrale Abgrenzungsansätze 253 und folgt im Übrigen der Ansicht, dass die Abgrenzung nicht mit Hilfe einer einheitlichen Theorie, sondern aufgrund der jeweiligen Besonderheiten des Sachbereichs und damit unter Berücksichtigung der konkreten Sachlage erfolgen muss. ${ }^{254}$

\section{aa) Interessentheorie}

Die Interessentheorie geht auf eine Stelle in den Digesten zurück, in denen ius publicum und ius privatum unterschieden werden. ${ }^{255}$ Das Kriterium zur Abgrenzung ist das Interesse, dem der Rechtssatz, dessen Zuordnung geklärt werden soll, dient, also ob die Regelung im Interesse des einzelnen Bürgers oder des Gemeinwesens getroffen wurde. Dass diese Theorie später in Ansätzen in anderen Theorien aufgegriffen wurde ${ }^{256}$, aber nicht mehr in Reinform Anwendung findet, liegt daran, dass es Interessen gibt, die sowohl dem Bürger als auch dem Gemeinwesen zugeordnet werden können. Der Abgrenzungsansatz führt somit nicht zu eindeutigen Ergebnissen.

\section{bb) Subordinationstheorie}

Kriterium der Unterscheidung ist das Vorliegen eines Gleichordnungs- oder Unterordnungsverhältnisses zwischen den Beteiligten. Im Privatrecht stehen danach die Einzelnen grundsätzlich nebeneinander, während das öffentliche Recht nach dem zugrunde

250 Zur Frage der Beziehung des öffentlich-rechtlichen Vertrages zur Staatsform vgl. Waechter, JZ 2006, S. 166 ff.

251 Gusy, DVBl 1983, S. 1225.

252 Zur Entwicklung des Begriffs Stolleis, Öffentliches Recht und Privatrecht im Prozess der Entstehung des modernen Staates, in: Hoffmann-Riem/Schmidt-Aßmann, Öffentliches Recht und Privatrecht als wechselseitige Auffangordnungen, 1996, S. $41 \mathrm{ff}$.

253 Weitere Abgrenzungstheorien finden sich im Überblick bei Maurer, Allgemeines Verwaltungsrecht, 2009, S. 41 ff; Manssen, Privatrechtsgestaltung durch Hoheitsakt, 1994, S. 53-100; Stelkens, Verwaltungsprivatrecht, 2005, S. 330-344.

254 So auch Ossenbühl, NJW 2000, S. 2946.

255 Der berühmte Satz ist ein Zitat Ulpians in 1,1,1 22 der Digesten und lautet: ,publicum ius est quod ad statum rei Romanae spectat, privatum quod ad singulorum utilitatem."

256 Dazu im Einzelnen Schmidt, Die Unterscheidung von privatem und öffentlichem Recht, 1985, S. 86 ff. 
gelegten Staatsverständnis Verhältnisse zwischen verschiedenen Herrschaftssubjekten und deren Beziehungen zu den der Herrschaft Unterworfenen regelt. ${ }^{257}$

Die Formulierung ,alle Staatsgewalt geht vom Volke aus“, das Demokratieprinzip gem. Art. 20 Abs. 2 S. 1 GG, lässt Zweifel an der verfassungsrechtlichen Rechtfertigung einer grundsätzlichen Subordination des Bürgers gegenüber dem Staat aufkommen. ${ }^{258}$ In „klassischen Bereichen“ der Eingriffsverwaltung, z.B. im Polizeirecht, mag das Subordinationsverhältnis vorhanden sein, doch ergibt sich daraus nicht, dass der Staat aus diesem Grund die Befugnis habe, durch Verwaltungsakt verbindliche Regelungen aufzuerlegen. Das Recht, durch Verwaltungsakt zu handeln, ist erst Rechtsfolge der Anwendung öffentlichen Rechts. ${ }^{259}$ Auch das einfachgesetzliche öffentliche Recht vermag die Subordinationstheorie nicht zu erklären. Besteht ein subjektiv-öffentliches Recht, gibt es seinem Inhaber eine Rechtsposition, die es ihm ermöglicht, diese gerichtlich geltend zu machen (Art. 19 Abs. 4 GG), sodass sie mit der eines privatrechtlich Berechtigten vergleichbar ist. ${ }^{260}$ Angesichts des heutigen Staatsverständnisses reicht die Subordinationstheorie nicht mehr als Begründung für die Abgrenzung aus.

\section{cc) Subjektstheorie, Zuordnungstheorie (modifizierte Subjektstheorie)}

Nach der Subjektstheorie gehören zum öffentlichen Recht die Rechtssätze, die die Ordnung von Verhältnissen zum Gegenstand haben, an denen ein Träger öffentlicher Gewalt beteiligt ist. ${ }^{261}$ Dabei ist problematisch, dass der Staat nach deutscher Rechtstradition wie eine Privatperson handeln kann und dann das Privatrecht Anwendung findet. Die reine Beteiligung eines Trägers öffentlicher Gewalt ist als Abgrenzungsansatz deshalb nicht ausreichend. Die Zuordnungstheorie (modifizierte Subjektstheorie) berücksichtigt diesen Einwand und erkennt an, dass dem Staat auch das private Recht zur Verfügung steht. ${ }^{262}$ Die Griffigkeit dieses formalen Abgrenzungskriteriums darf nicht darüber hinwegtäuschen, dass sie keinen eigenständigen Erklärungswert besitzt und deshalb keine abschließende Lösung über die Abgrenzung von öffentlichem Recht und Privatrecht bietet.

\section{dd) Sonderrechtslehre}

Die Sonderrechtslehre greift ebenfalls den oben aufgeführten Einwand auf und definiert auf der Grundlage der Subjektstheorie das öffentliche Recht als Sonderrecht staatlicher Gewalt, das private Recht als das allgemeine Recht jedermanns. Das allgemeine Recht stehe auch dem Staat zu Verfügung, soweit nicht das öffentliche Recht als das Sonderrecht staatlicher Gewalt maßgeblich sei. In Abgrenzung zur (modifizierten) Sub-

257 Jellinek, Allgemeine Staatslehre, 1914/1959, S. 384.

258 Schmidt, Die Unterscheidung von öffentlichem Recht und Privatrecht, 1985, S. 96 f.

259 Schmidt, Die Unterscheidung von öffentlichem Recht und Privatrecht, 1985, S. 98 f.

260 Achterberg, Die Rechtsordnung als Rechtsverhältnisordnung, S. 40, 62, 75.

261 Mayer, Deutsches Verwaltungsrecht, 1914/2004, Bd. 1, S. 16.

262 Wolff, AöR 1950/51 (76), S. 205 ff. 
jektstheorie stellt die Sonderrechtslehre darauf ab, dass der Staat als solcher und damit die öffentliche Gewalt selbst beteiligt ist. 263

\section{ee) Kelsens Idee vom Einheitsrechtssystem}

Der rechtsvergleichende Ansatz der vorliegenden Arbeit rechtfertigt es, an dieser Stelle die Überlegungen Hans Kelsens darzustellen. Dieser folgte der Idee eines Einheitsrechtssystems, lehnte also die Trennung zwischen öffentlichem Recht und Privatrecht vollständig ab. ${ }^{264} \mathrm{Er}$ ordnete dem Staat das Moment der Überordnung nur in seiner Qualität als Wahrer der Rechtsordnung zu, nicht aber sofern er als Rechtssubjekt Träger von Rechten und Pflichten sein kann und damit der Rechtsordnung selbst unterworfen ist. Nach seinem Verständnis ist ein Vertrag zwischen Staat und Bürger nicht nach Zugehörigkeit zum öffentlichen Recht oder Privatrecht zu unterscheiden. Er ist vielmehr wirksam, wenn die Wirkungen des Vertrages nicht allein durch die Willenserklärung des Staates gesetzt werden, sondern gleichzeitig die Willenserklärung des Einzelnen erfordern. Somit lässt sich eine Ähnlichkeit zu der Auffassung im englischen Recht feststellen, die die Abgrenzung zwischen public law und private law für überflüssig hält und grundsätzlich Verträge zwischen Krone und Bürger an denselben Maßstäben wie Verträge zwischen zwei Privatpersonen misst. ${ }^{265}$ Das von Kelsen propagierte Einheitsrechtssystem hat sich nicht durchgesetzt. Dennoch konnte Kelsens Ansatz der deutschen Rechtswissenschaft dazu dienen, ihre Ablehnung gegenüber verwaltungsrechtlichen Verträgen zu untermauern. ${ }^{266}$

\section{d) Abgrenzung von verwaltungsrechtlichem und privatrechtlichem Vertrag}

Die eben dargestellte Abgrenzung zwischen öffentlichem Recht und Privatrecht hat nicht nur aus dogmatischen Gründen zu erfolgen ${ }^{267}$. Sie ist erforderlich, um die im Vorfeld bei der Vertragsgestaltung 268 anzuwendenden Verfahrens- und Formvorschriften auffinden und bei Streitigkeiten den richtigen Rechtsweg beschreiten zu können. ${ }^{269}$

Der Vertrag wird nach seinem Gegenstand unabhängig vom Willen der Vertragspartner dem öffentlichen Recht oder dem Privatrecht zugeordnet (Gegenstandstheo-

263 Bachof, Über öffentliches Recht, in: ders., FS Bundesverwaltungsgericht, 1978, S. 9 ff.

264 Kelsen, AöR 1913 (31), S. 53 ff, $190 \mathrm{ff}$.

265 Z.B. Freedland, Government by contract, in: Craig/Rawlings, Law and administration in Europe, 2003, S. 123 f; Cane, Administrative law, 2004, S. 13.

266 Dazu Maurer, Der Verwaltungsvertrag, in: Hill, Verwaltungshandeln durch Verträge und Absprachen, 1990, S. $21 \mathrm{f}$.

267 So auch Eichenhofer, SGb 2003, S. 365.

268 Grziwotz, Vertragsgestaltung im öffentlichen Recht, 2002, S. 25.

269 Krebs, VVDStRL, H. 52 (1993), S. 260 f; Renck, JuS 2000, S. 1001 ff; Bonk, in: Stelkens/Bonk/Sachs, Verwaltungsverfahrensgesetz, 2001, § 54 Rn. 177 ff; vgl. aus der privatrechtlichen Literatur zur Bedeutung der Abgrenzung nur Kramer, in: Münchener Kommentar, Bürgerliches Gesetzbuch, 2006, v § 145 Rn. 43; Hübner, Der öffentlichrechtliche Vertrag - ein Instrument zur Aushöhlung des Privatrechts?, in: Hofmann/Meyer-Cording/Wiedermann, FS Pleyer, 1986, S. $497 \mathrm{ff}$. 
rie). ${ }^{270}$ Entscheidend ist, ob sich die Vereinbarung auf öffentlich-rechtlich oder privatrechtlich geregelte Sachverhalte bezieht. ${ }^{271}$ Die Gegenstandstheorie wird immer wieder in Zweifel gezogen und kritisiert, ein anderer überzeugenderer, gleichermaßen für den Einzelfall flexibler Ansatz ist aber nicht erkennbar. ${ }^{272}$

\section{e) Abgrenzung zwischen verwaltungsrechtlichem Vertrag und Verwaltungsakt}

Die Abgrenzung zwischen verwaltungsrechtlichem Vertrag und Verwaltungsakt ist auf eine eindeutige Zuweisung ausgelegt, denn ein identischer Gegenstand kann nicht gleichzeitig Verwaltungsakt und verwaltungsrechtlicher Vertrag sein. ${ }^{273}$

Als Abgrenzungskriterium zwischen verwaltungsrechtlichem Vertrag und Verwaltungsakt dient beim verwaltungsrechtlichen Vertrag das Kriterium „Willenseinigung“, beim Verwaltungsakt „Verfügung, Entscheidung oder andere hoheitliche Maßnahme auf dem Gebiet des öffentlichen Rechts“. Dieser Voraussetzung entnimmt man die „einseitig verbindliche Regelung“, die ein Verwaltungsakt enthält. Dazu ist eine Bewertung der Gesamtumstände des Einzelfalles erforderlich ${ }^{274}$, die sich in der vorliegenden Arbeit nur in theoretischen Ausführungen erschöpfen kann.

Entscheidend ist die subjektive Willensrichtung der Beteiligten. ${ }^{275}$ Zur Feststellung der subjektiven Willensrichtung sind objektive Kriterien heranzuziehen, wie die Bezeichnung der Maßnahme, die äußere Form und mündliche oder schriftliche Äußerungen der Beteiligten während des Verwaltungsverfahrens. ${ }^{276}$ In unklaren Fällen gibt es keine Vermutung zugunsten eines Verwaltungsakts als Normalfall verwaltungsrechtlichen Handelns ${ }^{277}$, doch wird man in der Regel wegen des Fehlens einer wirksamen Willenserklärung keinen verwaltungsrechtlichen Vertrag annehmen können. ${ }^{278}$ Das bedeutet, Unklarheiten bezüglich der Handlungsform gehen stets zu Lasten der Verwaltung, da sie eine eindeutige Formenwahl treffen könnte. ${ }^{279}$

270 Pickel/Marschner, SGB X, Bd. 1, § 53 Rn. 14; Engelmann, in: Wulffen, SGB X, 2008, § 53 Rn. 7; Maurer, Allgemeines Verwaltungsrecht, 2009, S. 370 f; a.A. im Überblick bei Grziwotz, Vertragsgestaltung im öffentlichen Recht, 2002, S. 24.

271 BGHZ 97, 312=BVerwGE 74, 368 [370] in ständiger Rspr.; m.w.N. Bonk, in: Stelkens/Bonk/Sachs, Verwaltungsverfahrensrecht, 2001, § 54 Rn. 76; Krasney, in: Kasseler Kommentar, Bd. 2, SGB X, § 53 Rn. 3.

272 Kritik in jüngster Zeit von Stelkens, Verwaltungsprivatrecht, 2005, S. 713, aber auch Renck, JuS 2000, S. 1002.

273 Ziekow/Siegel, VerwArch 2003, S. 595 m.w.N. über die Rspr.

274 BVerwGE 60, 208 [210]; Kopp/Ramsauer, VwVfG, 2008, § 54 Rn. 21.

275 Bonk, in: Stelkens/Bonk/Sachs, VwVfG, 2001, § 54 Rn. 39; Henneke, in: Knack, VwVfG, 2000, § 54 Rn. 3.

276 Ule/Laubinger, Verwaltungsverfahrensrecht, 1995, § 68 I; Schlette, Die Verwaltung als Vertragspartner, 2000, S. $185 \mathrm{f}$.

277 Dazu im Einzelnen Schlette, Die Verwaltung als Vertragspartner, 2000, S. 198 ff; a.A. Kopp, VwVfG, 2008, § 54 Rn. 15, der im Zweifel von einem Verwaltungsakt ausgeht.

278 Schlette, Die Verwaltung als Vertragspartner, 2000, S. 187; Maurer, Allgemeines Verwaltungsrecht, 2009, S. $366 \mathrm{f}$.

279 So auch Spannowsky, Grenzen des Verwaltungshandelns durch Verträge und Absprachen, 1994, S. 78. 


\section{aa) Handlungsformermächtigung}

Aus $\S 54 \mathrm{VwVfG}, \S 53 \mathrm{SGB} \mathrm{X}$ ergibt sich, dass die Verwaltung grundsätzlich durch Vertrag handeln darf (Handlungsformermächtigung). Insoweit ist der Streit um die Zulässigkeit verwaltungsrechtlicher Verträge beigelegt. ${ }^{280}$ Diese allgemeine Ermächtigung der Verwaltung zu vertraglichem Handeln wird durch die normative Vorordnung des Abschlusses in Fachgesetzen konkretisiert und die Verwaltung auf diesen Ermessensspielraum aufmerksam gemacht. ${ }^{281}$

\section{bb) Gebundene Wahlfreiheit der Verwaltung}

Ein Wahlrecht der Verwaltung besteht im Hinblick auf die Handlungsform (Verwaltungsakt oder vertragliches Handeln) ${ }^{282}$, denn aus den Regelungen in $\S \S 9,54$ S. 2 VwVfG, $\S \S 8,53$ S. 1 SGB X kann keine Präferenz für eine Handlungsform abgeleitet werden. ${ }^{283}$ Bei der Ausübung des Wahlrechts handelt es sich um eine Ermessensentscheidung, §§ 54 S. 1 VwVfG, 53 S. 1 SGB X.

Fraglich ist aber, wie die Auswahlentscheidung der Verwaltung gestaltet sein muss, um rechtmäßig zu sein. Es gelten die allgemeinen Regeln für die Ermessensausübung entsprechend $\S \S 40$ VwVfG, 39 Abs. 1 SGB IV, im Besonderen die Ermessensfehlerlehre. ${ }^{284}$ Insbesondere muss die Verwaltung ihr Ermessen ausüben, also überhaupt eine Auswahlentscheidung treffen, sonst liegt ein Ermessensnichtgebrauch vor. Die Ermessenszwecke ergeben sich aus $\S 10 \mathrm{~S} .2 \mathrm{VwVfG}$, wo es heißt, dass das Verwaltungsverfahren einfach, zweckmäßig und zügig durchgeführt werden muss. ${ }^{285}$

Die umstrittene Frage, ob der einzelne Bürger das subjektiv-öffentliche Recht inne hat, eine ermessensfehlerfreie Entscheidung über die Handlungsform zu verlangen, hängt an der Beantwortung der Frage, welchen Interessen die Ermessensausübung der Verwaltung dient (Schutznormlehre). 286 Die Ermessensausübung dient neben dem öffentlichen Interesse an einem behördlichen Entscheidungsspielraum dem Interesse des Einzelnen als aktivem Verwaltungssubjekt an einem verwaltungsrechtlichen Vertrag statt Erlass eines Verwaltungsakts. ${ }^{287}$ Somit werden durch die Ermessensausübung

280 Vgl. erstes Kapitel B IV 1a) S. 82.

281 Dazu Klindt, NVwZ 2003, S. 308.

282 Vgl. erstes Kapitel B III 3a) aa) S. 76.

283 Schmidt-Aßmann, Das allgemeine Verwaltungsrecht als Ordnungsidee, 2004, S. 343 schreibt: „Einen Vorrang „Entscheiden vor Vereinbaren“ gibt es für die Verwaltung nicht.“ Dazu im Einzelnen Schlette, Die Verwaltung als Vertragspartner, 2000, S. $198 \mathrm{ff}$.

284 Allgemein Bonk, in: Bonk/Stelkens/Sachs, VwVfG, 2001, § 54 Rn. 14; Erichsen/Ehlers, Allgemeines Verwaltungsrecht, 2006, § 10 Rn. 60 ff; Ziekow/Siegel, VerwArch 2003, S. 603; Klindt, NVwZ 2003, S. 308.

285 Kopp/Ramsauer, VwVfG, 2008, § 54 Rn. 15.

286 Dazu Kopp/Ramsauer, VwVfG, 2008, Einf Rn. 71.

287 Schlette, Verwaltung als Vertragspartner, 2000, S. 203f; Punke, Verwaltungshandeln durch Vertrag, 1989, S. 94; Krebs, VVDStRL, H. 52 (1993), S. 248; a.A. Kopp/Ramsauer, VwVfG, 2008, § 35 Rn. 16. 
auch Interessen des einzelnen Bürgers geschützt. Er hat folglich einen Anspruch auf ermessensfehlerfreie Entscheidung über die zu verwendende Handlungsform.

\section{cc) Handlungsformverbote}

Existieren „entgegenstehende Vorschriften“, liegt ein Handlungsformverbot respektive ein Vertragsformverbot vor. Entgegenstehende Vorschriften sind nicht nur ausdrückliche gesetzliche Verbote, sondern auch solche, die sich aus der Systematik des Gesetzes oder dem Sinn und Zweck der Regelung ergeben, z.B. durch ausdrücklichen oder sonst bewussten Ausschluss der Anwendbarkeit der $\S \S 54 \mathrm{ff} \mathrm{VwVfG}$ zugunsten einer Regelung des Gegenstandes durch Verwaltungsakt. 288

Im Sozialverwaltungsrecht 289 besteht ein Handlungsformverbot für „reine Normvollzugsverträge“. $290 \S 53$ Abs. 2 SGB X enthält eine wesentliche Einschränkung der Zulässigkeit des verwaltungsrechtlichen Vertrags über Sozialleistungen. Dieser darf nur geschlossen werden, soweit die Erbringung der Leistung im Ermessen des Leistungsträgers steht. Das heißt, dass ein Handlungsverbot in Bezug auf die sozialen Leistungen errichtet ist, auf die der Berechtigte einen Anspruch hat.

\section{Public law contract}

a) Hintergrund und Kritik an der Idee eines public law contract

Bisher kennt das englische Recht keinen public law contract. Es kennt keinen Vertrag, der wegen der Beteiligung eines Verwaltungsträgers besonderen Regeln des public law unterliegen würde oder in einem eigenständigen Gesetz geregelt wäre. Bei der Diskussion um kooperative Handlungsformen der Verwaltung wird immer wieder vom Gesetzgeber gefordert, in dieser Richtung tätig zu werden. ${ }^{291}$ Bisher wird den Besonderheiten von Vertragsschlüssen der Verwaltung innerhalb des contract law und den Grundsätzen von judicial review Rechnung getragen. ${ }^{292}$

Es gibt aber bestimmte Arten von Vereinbarungen, bei denen dies nicht möglich ist, weil sie keine Verträge im Sinne des contract law sind. Bei den Steuerungsaspekten von Kooperation wurden bereits verwaltungsinterne Vereinbarungen angesprochen, die als public service agreements oder als framework documents bezeichnet werden und Beziehungen zwischen verschiedenen Verwaltungseinheiten regeln. Für diese Vereinbarun-

288 Bonk, in: Stelkens/Bonk/Sachs, VwVfG, 2001, § 54 Rn. 102-104; Maurer, Allgemeines Verwaltungsrecht, 2009, S. 385.

289 Gem. $§ 2$ Abs. 2 Nr. 4 VwVfG gelten die $\S \S 54$ ff VwVfG für Verfahren nach dem Sozialgesetzbuch nicht, sondern ausschließlich die $\S \S 53$ ff SGB X.

290 Bauer, Anpassungsflexibilität im öffentlich-rechtlichen Vertrag, in: Hoffmann-Riem/SchmidtAßmann, Innovation und Flexibilität des Verwaltungshandelns, 1994, S. 265.

291 So Harden, The contracting state, 1992, S. 75 ff; Craig, Administrative law, 2003, S. 126; Freedland, Government by contract re-examined, in: Craig/Rawlings, Law and administration in Europe, 2003, S. $133 \mathrm{ff}$.

292 Vgl. erstes Kapitel B III 3b) S. 77; McLean, OJLS 2004, S. 129 ff bezweifelt, dass den Besonderheiten damit ausreichend Rechnung getragen ist. 
gen wird die Geltung des contract law abgelehnt, weil der gesteuerten Verwaltungseinheit die capacity to contract fehlt. Sie sind rechtlich nicht eigenständig, sondern ebenso wie der andere Vertragspartner Teil der Krone. ${ }^{293}$

Ein anderer Anwendungsbereich sind die in der vorliegenden Arbeit als government contracts bezeichneten Verträge zur Erfüllung einer öffentlichen Aufgabe. Auch für diese werden eigenständige Lösungen verlangt, z.B. gesetzliche Vorgaben für Standardverträge. ${ }^{294}$

Die Kritik an einer rechtlichen Ausgestaltung eines public law contract knüpft insbesondere daran an, dass die Abgrenzung des Anwendungsbereichs dieser Regelungen schwierig wäre. Die verschiedenen Fälle, die vom Anwendungsbereich erfasst sein könnten, weisen zudem so unterschiedliche Facetten auf, dass eine gemeinsame vereinheitlichende Lösung für die Gegner einer solchen Lösung nicht vorstellbar erscheint. ${ }^{295}$

\section{b) Inhaltliche Ausgestaltung eines public contract law}

Die rechtliche Verankerung von Grundsätzen, die auf diese Besonderheiten eingestellt sind, wäre Ziel für die inhaltliche Ausgestaltung eines public contract law. Dieses müsste die virulenten Probleme, die durch die Nichtanwendung von contract law entstehen, beseitigen. Es handelt sich dabei bislang um nicht mehr als ,a project for public lawyers" ${ }^{296}$ Es geht vor allem um die Frage der Verantwortlichkeit für die Maßnahmen, welche die durch framework documents geschaffenen Stellen ergreifen (accountability). Dadurch dass es kein Verfahren im Falle eines Vertragsbruchs gibt, ist die Einhaltung des Vertrages nicht abgesichert. ${ }^{297}$ Hinzu kommt, dass der von den Maßnahmen betroffene Dritte, z.B. der Bürger, der Leistungen einer executive agency beansprucht, auf die vertragliche Ebene nicht Einfluss nehmen und ihre Erfüllung der Vereinbarungen des framework document nicht gerichtlich überprüfen lassen kann. ${ }^{298}$

\section{Vertragsähnliches Verwaltungshandeln}

\section{Begriff}

Die der Arbeit zugrunde liegende Definition von Kooperation ${ }^{299}$ beschränkt die zu beschreibenden Handlungsformen nicht auf die normierten Typen kooperativen Han-

293 Cane, Administrative law, 2004, S. 310 schreibt: "They are not ordinary contracts because such agencies remain part of the Crown, which, in law, is a single indivisible entity."

294 Turpin, Government procurement and contracts, 1989, S. 114; Craig, Administrative law, 2003, S. $126 \mathrm{f}$.

295 Vincent-Jones, MLR 2002 (65), S. 627.

296 Freedland, PL 1998, S. 307. Davies, Accountability: A public law analysis of government by contract, 2001, S. 185 ff entwirft dazu einen Lösungsansatz und stellt damit, wie sie selbst schreibt, "a challenging agenda for future research" auf.

297 Freedland, PL 1994, S. 102.

298 Collins, Government contracts, 2002, S. 305 f.

299 Vgl. erstes Kapitel A I 1 S. 48. 
delns und nimmt weder eine Eingrenzung auf einseitige Entscheidungen mit kooperativen Elementen noch auf Verträge vor, die privatrechtlichen oder öffentlich-rechtlichen Regelungen folgen. Dies hat seinen Grund darin, dass sich Handlungsinstrumente finden lassen, die auf einer Willenseinigung zwischen mindestens zwei Parteien über einen ausgehandelten Inhalt beruhen und den beschriebenen Formen des Vertrags nach bürgerlichem oder öffentlichem Recht nur ähnlich sind. Es handelt sich um verschiedene Formen informalen kooperativen Verwaltungshandelns, deren wesentliches, sofort erkennbares Merkmal die Tatsache ist, dass das Ergebnis des Zusammenwirkens zwischen der Verwaltung und einem Bürger nicht in rechtlich bekannten und etablierten Formen, insbesondere nicht in Form eines Vertrages, verbindlich ist. Begrifflichkeiten wie Agreement ${ }^{300}$, Arrangement ${ }^{301}$, Quasi-Vertrag ${ }^{302}$, oder im englischen Recht quasicontract $^{303}$, contract-like arrangement ${ }^{304}$, contract as symbol ${ }^{305}$ bezeichnen Vereinbarungen dieser Art.

Die vertragsähnliche Vorgehensweise der Verwaltung kann durch den Gesetzgeber angeordnet sein oder sich schlicht daraus ergeben, dass die Voraussetzungen vertraglichen oder einseitigen Vorgehens nicht erfüllt sind.

\section{Voraussetzungen}

Die Situation, in der die Vereinbarung geschlossen wird, ist nicht vergleichbar mit anderen „echten“ Vertragsschlüssen zwischen Staat und Bürger. Anders als bei Verträgen zur Regelung der Leistungserbringung auf einem freien Markt oder bei rein fiskalischen Geschäften, mit denen ein Bedarf der Verwaltung gedeckt wird, gestalten die Vereinbarungen die persönliche Beziehung zwischen dem Staat und dem einzelnen Bürger oder die Beziehung von Marktteilnehmern eines regulierten Marktes. ${ }^{306}$

Wesentliches Kriterium für vertragsähnliches Handeln ist, dass die Willenseinigung der Parteien keine Garantie gerichtlicher Durchsetzbarkeit begründen kann. Voraussetzung ist deshalb, dass es ein Kooperationsergebnis gibt, das in einem Aushandlungsprozess zwischen den Parteien entstanden ist. Das Ergebnis erlangt Bedeutung, weil sich die Parteien gebunden fühlen und dies z.B. durch Unterzeichnung eines Dokumentes auch nach außen Kund tun.

300 Bulling, DÖV 1989, S. 280 f, 287.

301 Bulling, DÖV 1989, S. 280.

302 Eberhard, Zivilrecht und Sozialrecht in der Beziehung von Kassenarzt und Kassenpatient, AcP 171 (1971), S. 301.

303 Collins, Regulating contracts, 2002, S. 315.

304 Freedland/King, Client contractualism between the employment service and jobseekers in the UK, in: Sol/Westerveld, Contractualism in employment services, 2005, S. 123.

305 Davies, Accountability: A public law analysis of government by contract, 2001, S. 56.

306 Vincent-Jones, MLR 2005 (68), S. 891. 


\section{Zulässigkeit vertragsähnlicher Vorgehensweise der Verwaltung}

Vertragsähnliches Handeln ist ausgeschlossen, wenn eine andere Handlungsform vorgeschrieben ist, z.B. bei der Zulassung zur Pflege der Abschluss eines Versorgungsvertrages gem. § 72 SGB XI. Daneben gibt es einige Fälle, in denen sich aus der rechtlichen Gestaltung der Beziehungen zwischen Staat und Leistungserbringer oder Bürger von einem vertragsähnlichen Handeln auszugehen ist. Auch die im zweiten Kapitel zu untersuchenden Instrumente der Arbeitsverwaltung, das $J S A$ und die Eingliederungsvereinbarungen sind daraufhin zu prüfen, ob sie als vertragsähnliche Handlungsformen einzuordnen sind.

\section{a) NHS-contract}

NHS-contracts ${ }^{307}$ sind Vereinbarungen innerhalb des National Health Service. Zwei Institutionen des National Health Service, der Anbieter einer Gesundheitsleistung (provider) und ein Käufer (purchaser), der gegenüber dem einzelnen Patienten zur Erbringung der Leistung verpflichtet ist, schließen diese Vereinbarung. ${ }^{308}$

Sie unterliegen per Gesetz ${ }^{309}$ nicht dem contract law und werden deshalb auch nicht vor den common law Gerichten verhandelt, sondern in einem administrativen Verfahren entschieden, das detailliert im Gesetz geregelt ist. 310

Es handelt sich nicht um Vereinbarungen auf einem freien Markt, denn innerhalb des National Health Service herrscht nur auf der Seite des Angebots Wettbewerb, nicht auf der Seite der Nachfrage. Die Nachfrage nach Leistungen des Gesundheitssystems steht in ihrer Größenordnung, und v.a. im Hinblick auf die einsetzbaren finanziellen Mittel, fest und kann deshalb nur bedingt Druck auf die Angebotsseite machen. Man spricht deshalb von einem quasi-market bzw. einem internal market. Das Gesundheitssystem im Vereinigten Königreich hofft, dass sich auf dem internal market die Vorteile der konventionellen Marktwirtschaft, v.a. Kostensenkungspotenziale durch Wettbewerb, mit den Vorteilen von bürokratischer Abwicklung von Vorgängen verbinden und auf diese Weise die jeweiligen Nachteile so weit wie möglich vermieden werden. ${ }^{311}$

307 National Health Service and Community Care Act 1990 sec. 4.

308 Hughes, MLR 1991 (54), S. 90 ff.

309 National Health Service \& Community Care Act 1990 sec. 4 subs. 3, dazu Hughes/McHale/Griffiths, Settling contract disputes in the National Health Service, in: Flynn/William, Contracting for health, 1997, S. 99 ff; Davies, Accountability: A public law analysis of government by contract, 2001, S. $28 \mathrm{ff}$.

310 Dazu Hughes/McHale/Griffiths, Settling contract disputes in the National Health Service, in: Flynn/William, Contracting for health, 1997, S. 99 ff; McHale /Hughes/Griffiths, Conceptualizing contractual disputes in the National Health Service Internal Market, in: Deakin/Michie, Contracts, cooperation and competition, 1997, S. $195 \mathrm{ff}$.

311 Freedland, PL 1999, S. 306. 
b) Disciplinary contract

Disciplinary contracts sind Vereinbarungen zwischen Einzelnem und Staat, bei denen die steuernde Wirkung von Kooperation im Vordergrund steht und die rechtliche Durchsetzbarkeit ebenfalls ausgeschlossen ist.

Es finden sich drei Anwendungsbeispiele: Vereinbarungen zwischen Schule, Eltern und einzelnem Schüler (home-school-agreements 312 ), Übereinkünfte zwischen Gefängnisinsasse und Gefängnisverwaltung und der youth offender contract zwischen jugendlichen Straftätern und Beratungsstellen für jugendliche Straftäter ${ }^{313}$.

Diesen Beispielen ist gemeinsam, dass es sich um bereits vor der Vereinbarung bestehende besondere „Gewaltverhältnisse“ (im untechnischen Sinn) handelt, bei denen der Einzelne dem Staat gegenüber eine typischerweise unterlegene oder anhängige Rolle einnimmt. Es soll zielgerichtet auf ein bestimmtes, in der Theorie oder im Gesetz ausgeformtes Ergebnis hin eine Verhaltensänderung beim Einzelnen erreicht werden. Den Weg zu dieser Verhaltensänderung will der Staat durch Einführung des Instruments „Vereinbarung“ steuern. ${ }^{314}$ Dies gelingt dadurch, dass der Einzelne bei der Festlegung des Vorgehens einbezogen wird und er durch Unterzeichnung dazu gebracht wird, dass er die Ergebnisse auch als eigene betrachtet. Auf diese Verträge ist common law nicht anwendbar, denn der abschließenden staatlichen Stelle fehlt grundsätzlich der Rechtsbindungswille bzw. die Fähigkeit, den Staat zu verpflichten.

\section{c) Leistungsabrede, $\S 12$ SGB XII}

$\S 12$ SGB XII regelt die Leistungsabrede, in der zwischen Sozialleistungsträger und Sozialhilfeempfänger das weitere Vorgehen des Sozialhilfebezugs vereinbart wird.

In der Gesetzesbegründung wird zum Zweck der Leistungsabsprache ausgeführt, dass damit eine kooperative Vorgehensweise der Sozialhilfeträger gesetzlich eingefordert wird. 315 Die erfolgreiche Überwindung der Notlage wie auch die Stärkung der Selbsthilfe zur aktiven Teilnahme am Leben in der Gemeinschaft ist in vielfältiger Weise von der aktiven Mitwirkung der Leistungsberechtigten abhängig. Der Einzelne wird (informell) zur Mitwirkung angehalten und erwartet, dass er sich aufgrund seiner Unterschrift unter das Dokument „verpflichtet fühlt“. Ziel des Abschlusses der Leistungsabrede ist neben der aktiven Beteiligung des Leistungsempfängers auch die stärkere Selbststeuerung des Sozialhilfeträgers. Dieser wird zur Vorbereitung der Leistungsabrede das Vorgehen mit dem Leistungsberechtigten planen, Entwicklungschancen suchen und dabei die Ansprüche des Leistungsberechtigten verwirklichen müssen (Chancenverbesserung).

Um die einfache und flexible Handhabung dieser Vorschrift zu sichern, stellte der Gesetzgeber klar, dass es sich bei der Abrede nicht um einen öffentlich-rechtlichen Ver-

312 School Standards and Framework Act $1998 \mathrm{sec} .110$.

313 Youth Justice and Criminal Evidence Act $1999 \mathrm{sec} .8$.

314 Freedland/King, Client contractualism between the employment service and jobseekers in the UK, in: Sol/Westerveld, Contractualism in employment services, 2005, S. $123 \mathrm{f}$.

315 BT-Drucksache 15/1514, S. 56. 
trag handelt. ${ }^{316}$ Inhaltlich steht die Umsetzung der in $\S 11$ SGB XII etablierten Beratungs- und Unterstützungspflicht des erfassten Personenkreises ${ }^{317}$ im Vordergrund, für die der Rechtsgrund aber das Gesetz bleibt, nicht die Vereinbarung. 318

\section{Inhaltliche Ausgestaltung und Wirkungen}

Vertragsähnliches Verwaltungshandeln hat die inhaltlichen Vorgaben zu erfüllen, die aus der Verwaltungsaufgabe folgen oder die gesetzlich vorgegeben sind. Auch die möglichen Wirkungen vertragsähnlichen Verwaltungshandelns können aus gesetzlichen Vorgaben resultieren. Feststeht, dass Wirkungen des vertragsähnlichen Verwaltungshandelns nicht Folge übereinstimmender Willenserklärungen sind, sondern aus den Regeln, die diese Vorgehensweise der Verwaltung vorsehen, abzuleiten sind. Der Abschluss einer solchen Vereinbarung kann z.B. Voraussetzung für den Erhalt staatlicher Unterstützung beziehungsweise für weiteres staatliches Tätig werden sein. In diesem Fall übernimmt der Einzelne infolge seiner Zustimmung verstärkt Verantwortung für die staatliche Unterstützung.

Die Nichteinhaltung der Vereinbarung („,breach of contract“) führt bei vertragsähnlichem Verwaltungshandeln nicht zur Anwendung von Privatrecht beziehungsweise contract law. Teilweise ist das ausdrücklich gesetzlich geregelt, z.B. im National Health Service \& Community Care Act 1990 sec. 4 subs. 3, teilweise ist dieses Ergebnis durch Auslegung des Gesetzes ${ }^{319}$ zu begründen. Ziel des Ausschlusses von Vertragsrecht/contract law ist u.a. die Vermeidung der bei der gerichtlichen Durchsetzung der Rechte entstehenden Kosten (costs of litigation). 320

Die Handlungsform wird praktiziert, ohne dass ihre konkrete Ausgestaltung und Abwicklung abschließend geklärt, geschweige denn geregelt wäre. Gerade die Auswirkungen, die die oben beschriebene Schwebestellung auf den Einzelnen hat, werden im dritten Kapitel am Beispiel von Vereinbarungen zwischen Arbeitsverwaltung und Einzelnem analysiert.

316 BT-Drucksache 15/1514, S. 56.

317 Nur ein kleiner Personenkreis ist erfasst, denn der Gesamtplan ( $\S 58,68$ Abs. 1 S. 2 SGB XII) geht für alle Personen vor, die Eingliederungshilfe erhalten. Es bleiben Personen, die Grundsicherung im Alter beziehen, § 44 Abs. 2 SGB XII i.V.m. § 12 SGB XII, und Personen, die noch nicht abschließend dem SGB XII oder SGB II zugeordnet sind. Dazu Baur, SozR akt 2006, S. 51 f.

318 Roscher, in: Münder, LPK-SGB XII, § 12 Rn. 3; Spellbrink, SozR akt 2006, S. 52 ff

319 Dazu Barker, MLR 1993 (56), S. 832 ff, insbesondere zu Fragen von restitution bei der Abwicklung von NHS contracts.

320 Collins, Regulating contracts, 2002, S. 316. 


\section{Systematisierungskriterien für kooperative Handlungsformen}

Die Systematisierung kooperativer Handlungsformen erfolgt anhand einer Reihe von Kriterien und verfolgt das Ziel, die Vielfalt bereits bestehender oder neuartiger Handlungsformen zu ordnen. Dabei sind nicht nur streng rechtliche Kategorien maßgeblich. Es soll vielmehr die rechtliche Analyse im zweiten Kapitel vorbereitet und ein Raster für die Auswertung des Rechtsvergleiches im dritten Kapitel erstellt werden. Die Kriterien werden bei der Auswertung so miteinander kombiniert, dass ein vollständiges Bild von Ausgestaltung und Wirkungen des Verwaltungshandelns entsteht.

\section{Beteiligungszeitpunkt}

Abgrenzbar sind verschiedene Handlungsformen anhand des Zeitpunkts, der betrachtet wird und in dem Kooperation stattfindet.

Es kann danach differenziert werden, ob die Kooperation lediglich im Vorfeld einer einseitigen Entscheidung stattfindet oder direkt bei der Entscheidung als Mitentscheidung. Es sind auch kooperative Handlungsformen denkbar, die erst bei Vollzug beziehungsweise Abwicklung einer einseitigen Entscheidung Anwendung finden.

Der Zeitpunkt der Beteiligung ist relevant für die Frage, auf welche Weise die Beteiligung Wirkungen entfalten kann und wie intensiv die Einwirkung des Einzelnen die Verwaltung beeinflussen kann.

\section{Beteiligte an der Kooperation: Leistungsempfänger - Leistungserbringer - Dritte}

Mit diesem Kriterium wird beschrieben, ob und in welchem (sozialleistungs-)rechtlichen Verhältnis die Verwaltung kooperativ vorgeht.

Kooperieren Leistungsempfänger und Leistungsträger, handelt es sich um eine Vereinbarung zwischen Staat und einzelnem Bürger, die die sozialrechtlichen Rechte und Pflichten von Staat und Bürger betrifft. Wird eine Vereinbarung zwischen Leistungsträger und Leistungserbringer geschlossen, werden dadurch Rechte des Einzelnen möglicherweise auch mit gestaltet. Es können aber auch Vereinbarungen mit Dritten sein, die weder den Einzelnen noch den Leistungserbringer betreffen.

Die Beteiligung am kooperativen Prozess ist für die Frage wichtig, wer vom Verwaltungshandeln in Rechten berührt wird und deshalb unter Umständen außerhalb des kooperativen Prozesses zu beteiligen ist. Mit bedacht werden muss auch eine Einbeziehung der jeweiligen Interessen bei den Verhandlungen. 
Die Kriterien einseitig - zweiseitig - mehrseitig geben Aufschluss darüber, wie viele Beteiligte mit der Verwaltung zusammenwirken und auch die getroffene Entscheidung mittragen.

Findet die kooperative Vorgehensweise in einer einseitigen Entscheidung der Verwaltung ihr Ende, handelt es sich um einseitiges Verwaltungshandeln. Dieses hat dann eine kooperative Komponente, wenn vor der einseitigen Entscheidung eine Absprache stattgefunden hat.

Bei zwei- und mehrseitiger Beteiligung verantwortet nicht nur die Verwaltung eine Entscheidung, sondern auch der oder mehrere Beteiligte. Es bleibt nicht nur bei der Mitwirkung an einer einseitigen Entscheidung, vielmehr liegt eine Willenseinigung vor, die legitimierende Wirkung entfalten kann.

\section{Förmlichkeit der Beteiligung}

Die Förmlichkeit von Kooperation ist ein Kriterium dafür, deren Außenwirkung zu verdeutlichen.

Sie ist nicht nur eine Verfahrensfrage. Jede Form, ob Schriftform oder eine strengere oder weniger strenge Form, erfüllt einen bestimmten Sinn und Zweck, an dem sich der Gesetzgeber orientiert. Wichtig ist die Beweisfunktion ${ }^{321}$, aber auch die Wirkung des schriftlichen unterzeichneten Dokuments für die Ernsthaftigkeit des Erklärten und der daran anknüpfenden Außenwirkung des Verwaltungshandelns. ${ }^{322}$

\section{Bindungswirkung des Kooperationsergebnisses}

Die Bindungswirkung eines Verwaltungshandelns für die Beteiligten hängt davon ab, ob sie sich an die Ergebnisse des kooperativen Prozesses halten müssen beziehungsweise ob sie sich verpflichtet haben, sich entsprechend zu verhalten. Dies ergibt sich jeweils aus der objektiven Rechtsordnung. ${ }^{323}$

Eine Bindungswirkung kann sich aus z.B. aus einer Selbstbindung der Verwaltung oder des Einzelnen ergeben, aber auch gesetzlich für den Einzelfall oder allgemein für eine Handlungsform angeordnet sein. Die Reichweite der Selbstbindung der Verwaltung ist nochmals zu unterteilen in Fälle der direkten Bindung an die Entscheidung oder eine indirekte, weniger konkrete Bindung im Rahmen einer nachfolgenden (dann meist einseitigen) Entscheidung, z.B. im Rahmen der Ermessensausübung.

321 Ziekow/Siegel, VerwArch 2004, S. 134 f.

322 Z.B. zur Schriftform des Verwaltungsaktes Stelkens, in: Stelkens/Bonk/Sachs, VwVfG, 2001, § 37 Rn. 33.

323 Krause, Rechtsformen des Verwaltungshandelns, 1974, S. 81. 
Die Durchsetzbarkeit des Kooperationsergebnisses kann durch Gerichte und Vollstreckungsorgane, aber auch durch vereinbarte Instanzen, z.B. Einigungsstellen sichergestellt sein. Sie kann für die Beteiligten an der Kooperation unterschiedlich ausgestaltet und auch für verschiedene Teile mehr oder weniger ausgeprägt sein.

So ist danach zu differenzieren, ob z.B. Ansprüche gegen die Verwaltung gerichtlich geltend gemacht werden können oder nicht. Daran schließt sich die Frage an, ob ein Verfahren zur Durchsetzung des Inhalts verfügbar ist.

Auf der anderen Seite stellt sich die Frage, ob für eine bestimmte Handlungsform der Verwaltung ein Verfahren vorgesehen ist, deren Inhalte gegenüber einem Einzelnen vor ein Gericht oder eine andere Instanz gebracht werden können. So ist es denkbar, dass genau diese Möglichkeit der Verwaltung bei Verwendung einer Handlungsform versperrt ist, während dies bei einer anderen Handlungsweise einfacher oder gänzlich unproblematisch ist. Daran schließt sich die Frage nach der Dichte der Kontrolle an. Stehen bei einer gerichtlichen Durchsetzung die gesamte Vereinbarung oder nur bestimmte Einzelfragen auf dem Prüfstand des unparteiischen Dritten?

Die Frage der Bindungswirkung betrifft einen Aspekt der gerichtlichen Durchsetzbarkeit, ist aber klar von dieser abzugrenzen. Eine materielle Verpflichtung wird im Rahmen der gerichtlichen Durchsetzbarkeit geprüft. Es ist aber denkbar, dass diese Pflicht bewusst nicht justiziabel ist und deshalb die Kriterien nicht deckungsgleich sind. 


\title{
Zweites Kapitel: Länderberichte
}

\author{
A. Englisches Recht
}

\section{Jobseekers Act 1995 und New Deals}

\section{Jobseekers Act 1995}

Im Vereinigten Königreich veränderte das Inkrafttreten des Jobseekers Act 1995 zum 7.10.1996 sowohl die sozialen Leistungen für den Fall der Arbeitslosigkeit als auch die Organisation der Arbeitsverwaltung grundlegend.

Die jobseeker's allowance ersetzte unemployment benefit und income support und vereinfachte so ein System aus zwei nebeneinander stehenden Leistungen durch eine Leistung mit einheitlichen Voraussetzungen. ${ }^{1}$

Unemployment benefit ${ }^{2}$ war eine Versicherungsleistung, auf die derjenige, der Beiträge (contributions) eingezahlt hat, einen Anspruch (entitlement) erwarb. Es entstanden dadurch keine verfassungsrechtlich geschützten (Eigentums-)Rechte, sodass deren Veränderung durch ein Parlamentsgesetz aufgrund der sovereignty of Parliament unproblematisch möglich war. ${ }^{3}$ Income support war eine je nach Bedürftigkeit des Arbeitslosen zu zahlende (means-tested) finanzielle Hilfe. ${ }^{4}$ Beide Leistungen schlossen einander nicht aus, konnten also gleichzeitig in Anspruch genommen werden. Sie folgten aber in ihren Voraussetzungen unterschiedlichen Prinzipien und Vorschriften. 5

Auf zentraler und regionaler Ebene waren mehrere Verwaltungsbehörden im Geschäftsbereich sowohl des Department for Education and Employment als auch des Department for Social Security für die Entscheidung über die oben aufgeführten Leistungen zuständig. Seit der Reform ist die Zuständigkeit für alle Unterstützungsleistungen in besonderen sozialen Lebenslagen auf zentraler Ebene beim neu gegründeten Department for Work and Pensions ${ }^{6}$ zusammengeführt. $^{7}$ Dieses besteht aus Fachabteilungen

1 HMSO, Jobseeker's allowance, Cm 2687, 1994, S. 14 ff; Finn, NE 1996, S. 60; Fulbrook, ILJ 1995 (24), S. 399.

2 Zur Entwicklung des unemployment benefit seit seiner Einführung durch den Unemployment Insurance Act 1920 vgl. Wikeley/Ogus, The law of social security, 2002, S. 496 ff; in deutscher Sprache Husmann, ZESAR 2009, S. 70 f.

3 Vgl. dazu die Ausführungen bei Hamisch, Der Schutz individueller Rentenrechte bei Rentenreformen, 2001, S. 305 ff, die auf die Leistungen der Arbeitslosenversicherung übertragbar sind.

4 Wikeley/Ogus, The law of social security, 2002, S. 336.

5 Wood u.a., Social security legislation 2006, Bd. 2, 2006, S. $31 \mathrm{ff}$.

6 Das DWP entstand am 8.6.2001. Der Minister ist Mitglied des Kabinetts. Es gibt noch zwei ministers of State und drei parlamentarische under-secretaries, die den Minister auf politischer Ebene unterstützen. Chef der Beamten ist der permanent secretary. Daneben gibt es advisory bodies, z.B. das Social Security Advisory Committee, die beratende Funktion haben. Sie geben ihre Meinung zu Vorhaben des Ministeriums ab (z.B. regulations) und erteilen Rat auf eigene Initiative hin (research 
entsprechend dem Lebensalter der Hilfe suchenden Menschen (Kindheit, erwerbsfähiges Alter, Ruhestand). Diese Struktur setzt sich in den untergeordneten Behörden fort (Child Support Agency, Jobcentre Plus, Pension Service). ${ }^{8}$

Auf regionaler Ebene sind die sog. Jobcentres Plus (JCP) für Personen im erwerbsfähigen Alter zuständig. Die gespaltene Zuständigkeit des früheren Employment Servi$c e^{9}$ für unemployment benefit und bestimmte Zuständigkeiten der Benefits Agency für income support betreffend Unterstützung für das Risiko Arbeitslosigkeit sind nun dort gebündelt. ${ }^{10}$ Damit wird der Forderung der Citizen's Charter genüge getan, wonach die Bereiche der Verwaltung, die direkt mit dem einzelnen Bürger zu tun haben, möglichst einen „Service aus einer Hand“ (one-stop-shop ${ }^{11}$ ) gewähren sollen. Die Verwaltungsstrukturen wurden der Transparenz und Einfachheit wegen gestrafft. ${ }^{12}$ Für die Umsetzung dieses Verwaltungsaufbaus und der Zusammenführung der beiden staatlichen Aufgaben von Employment Service und Benefits Agency hatte der Gesetzgeber ein Zeitfenster bis 2006 vorgesehen, wobei der Großteil der JCP seit 2002 eingerichtet ist. Bis dahin wurden die Aufgaben nach Absprache von den vorhandenen Behörden erledigt. ${ }^{13}$

\section{Jobseeker's allowance}

Im Jobseekers Act 1995 ist die staatliche Unterstützung, die ein Arbeitsloser im Vereinigten Königreich erhalten kann - die jobseeker's allowance - einheitlich geregelt. ${ }^{14}$ Einzelheiten der Durchführung sind in den Jobseeker's Allowance Regulations 199615

reports). Die Einholung ihres Rates ist in bestimmten Fällen zwingend (Verfahrensvoraussetzung). Dazu Wikeley/Ogus, The law of social security, 2002, S. 130-143.

7 Zur politischen Bedeutung der Reform der Verwaltungsstrukturen, insbesondere zur Gründung des DWP vgl. Carmel/Papadopoulos, The new governance of social security, in: Millar, Understanding social security, 2003, S. 40 f.

8 Wikeley/Ogus, The law of social security, 2002, S. 133.

9 Die Umstrukturierung des employment service erfolgte schrittweise seit 1979 und ging einher mit der allgemein für erforderlich gehaltenen Verschlankung des Staates durch Privatisierung und Contracting-out. Endpunkt war die Umstrukturierung des employment services in eine executive agency und damit seine Steuerung und Kontrolle über jährlich auszuhandelnde Vereinbarungen mit dem zuständigen Department for Education and Employment. Dazu Finn, The role of contracts and the private sector in delivering Britain's "employment first" welfare state, in: Sol/Westerveld, Contractualism in employment services, 2005, S. $104 \mathrm{ff}$.

10 Winkler, BArbB1 2004, S. 19.

11 HMSO, The Citizen's Charter, Cm 1599, 1991, S. 20; vgl. allgemein zur Einführung von one-stopshops im Rahmen der Reform der Verwaltung TSO, Modernising Government, Cm 4310, 1999, Kapitel 3.18.

12 HMSO, Jobseeker's allowance, Cm 2687, 1994, S. 14; Wikeley/Ogus, The law of social security, 2002, S. 143; Fletcher, PP 1997 (25), S. 173 ff.

13 Zum organisatorischen Umbau der Verwaltungsstrukturen Stafford, Service delivery and the user, in: Millar, Understanding Social Policy, 2003, S. 215; Winkler, ibv 2004, S. 3 f; Bertelsmann Stiftung, Exposé Arbeitsmarktpolitik, Juni 2002, S. 5 unter Bezugnahme auf www.DWP.gov.uk, this week news, 1.4.2002; Knuth/Finn, IAT-Report 2004-04, S. 1 ff.

14 Ein Überblick zur Rechtslage in deutscher Sprache findet sich bei Husmann, ZESAR 2009, S. 69 ff, $124 \mathrm{ff}$.

15 Im Weiteren SI 1996/207. 
geregelt. Übergangsvorschriften enthalten die Jobseeker's Allowance (Transitional Provisions) Regulations 1996. ${ }^{16}$ Das White Paper formuliert das Ziel der Änderung mit folgenden Worten: „The new jobseeker's allowance, as its title makes clear, will be a means of support while an unemployed person looks for work, not an income for a lifestyle divorced from work". ${ }^{17}$ Bereits zu Beginn der Reform wurde so seitens der Politik deutlich gemacht, dass in Zukunft die Versorgung mit passiven Leistungen zwar erforderlich sein, aber nur in Verbindung mit Wiedereingliederungsbemühungen stattfinden würde.

Die jobseeker's allowance umfasst zwei Arten von Hilfe, die einheitliche Grundvoraussetzungen (core conditions), aber auch unterschiedliche Voraussetzungen haben. Da ein Grundsatz bei den Reformen war, das vorhandene Geld aus dem System auf die Bedürftigsten zu verteilen und so die stets steigenden Ausgaben für soziale Leistungen zu begrenzen, sowie eine Vereinfachung der Regelungen zu erreichen, verwendete man als Grundgerüst für die Leistungen nach dem Jobseeker's Act 1995 die früheren Vorschriften für income support. ${ }^{18}$ Diese wurden als flexibler betrachtet und erschienen geeigneter, die angestrebten Ziele zu erreichen, als ein als Versicherung ausgestaltetes, starres System. 19

Der Jobseekers Act 1995 unterscheidet die contribution-based jobseeker's allowan$c e$, eine beitragsabhängige Unterstützung (Rest der Arbeitslosenversicherung), die für bis zu sechs Monaten gezahlt wird, und die income-based jobseeker's allowance, eine zeitlich unbeschränkte, bedürftigkeitsabhängige Unterstützung. Diese Unterscheidung wirkt sich grundsätzlich auf die Höhe der zu erwartenden Leistung nicht aus, denn es wird in beiden Varianten ein bestimmtes Sicherungsniveau garantiert. Die bedürftigkeitsabhängige Unterstützung setzt aber die Verwertung von Vermögen und Einkommen voraus, bevor staatliche Hilfe verlangt werden kann, während bei der beitragsabhängigen Form der jobseeker's allowance Vermögen und Einkommen grundsätzlich geschont werden. Damit wird derjenige besser gestellt, der sich in der National Insurance versichert. So entsteht ein einfachgesetzlicher Schutz durch Beiträge erworbener Rechtspositionen. Von einer verfassungsrechtlichen Garantie dieser Rechtsposition kann freilich nicht gesprochen werden. ${ }^{20}$

\section{a) Grundvoraussetzungen der jobseeker's allowance}

$\mathrm{Zu}$ den Grundvoraussetzungen der jobseeker's allowance gehören die sog. labour market conditions: Der Antragsteller muss zur Erwerbsarbeit bereit und in der Lage sein (available for employment ${ }^{21}$ ), aktiv Arbeit suchen (actively seeking employment ${ }^{22}$ ) und

16 SI 1996/2567.

17 HMSO, Jobseeker's allowance, Cm 2687, 1994, S. 10.

18 Einführend zur Handhabung der Einkommensgrenzen Wikeley, JSSL 2001 (8), S. 95 ff.

19 HMSO, Jobseeker's allowance, Cm 2687, 1994, S. 15.

20 Zur aktuellen Diskussion über den Schutz der Human Rights beispielhaft Herne Hill/Clapinska, Human Rights and the British Constitution, in: Jowell/Oliver, The changing Constitution, 2004, S. $62 \mathrm{ff}$.

21 Genaueres hierzu HMSO, Jobseeker's allowance, Cm 2687, 1994, S. 17 f. 
ein jobseeker's agreement ${ }^{23}$ mit einem Mitarbeiter des JCP abgeschlossen haben, das während des gesamten Leistungsbezugs wirksam sein muss. ${ }^{24}$

Available for work bedeutet, dass eine Person bereit und in der Lage 25 ist, unverzüglich jede bezahlte Arbeit (any employed earner's employment ${ }^{26}$ ) anzunehmen. ${ }^{27}$ Von diesem Grundsatz gibt es in Jobseekers Act 1995 sec. 6 subs. 2 abschließend aufgeführte Ausnahmen, wenn bestimmte anerkannte Umstände der Arbeitsaufnahme entgegenstehen (besondere, in der Person liegende Gründe, Ortsgebundenheit, Betreuungspflichten). Grundsätzlich besteht eine Verpflichtung zur Aufnahme einer Vollzeittätigkeit von mindestens 40 Stunden pro Woche. Kann nur eine Teilzeitstelle angeboten werden, besteht allerdings die Verpflichtung, sich mit dieser zu begnügen. ${ }^{28}$ Ausnahmen bestehen auch hier für Menschen, die Angehörige, insbesondere Kinder betreuen (caring responsibilities). ${ }^{29}$

Activeley seeking employment bedeutet, dass die Person die Schritte unternehmen muss, die von ihr erwartet werden dürfen, damit sie die besten Aussichten auf eine Arbeit (hier auch selbständige Tätigkeit ${ }^{30}$ ) wahrt. ${ }^{31}$ Die Arbeitslosigkeit muss unfreiwillig (unvoluntary unemployment) sein. Dies entspricht der bereits vor Inkrafttreten des Jobseekers Act 1995 geltenden Rechtslage. ${ }^{32}$

Daneben darf der Arbeitslose nicht bereits in größerem Umfang erwerbstätig sein (engaged in remunerative work), d.h. durchschnittlich 16 Stunden und mehr pro Woche gegen Entgelt oder zumindest dessen Erwartung arbeiten ${ }^{33}$. Ehrenamtliche Tätigkeiten und Trainingsmaßnahmen zur Eingliederung in den Arbeitsmarkt sind ausgenommen. ${ }^{34}$

22 Diese Voraussetzung wurde bereits durch den Social Security Act 1989 sec. 10 subs. 1 eingeführt, um den Charakter der Arbeitslosenunterstützung zu verändern und der aktiven Arbeitsuche Vorrang vor bloßer finanzieller Absicherung bei Eintritt des sozialen Risikos Arbeitslosigkeit einzuräumen. Dazu Wikeley, JLS 1989 (16), S. 291 ff.

23 Im weiteren $J S A$.

24 Jobseekers Act 1995 sec. 1 subs. 2 para. a-i.

25 Zur Abgrenzung zum Income Benefit bei Krankheit Bacon, LMT 2002, S. 195 ff.

26 Jobseekers Act 1995 sec. 6 subs. 9 i.V.m. Social Security Contributions and Benefits Act 1992 sec. 2, sec. 122 subs. 1. Damit ist nur abhängige Beschäftigung gemeint. Eine selbständige Beschäftigung kann zwar begonnen werden, die Arbeitsbereitschaft darf aber nicht auf die Aufnahme einer selbständigen Tätigkeit beschränkt werden.

27 Jobseekers Act 1995 sec. 6 subs. 1.

28 SI 1996/207 reg. 6 (1) bzw. (2).

29 Insgesamt zu dieser Voraussetzung der jobseeker's allowance Wood u.a., Social security legislation 2006, Bd. 2, 2006, S. 790.

30 Jobseekers Act 1995 sec. 7 subs. 8; dazu Wood u.a., Social security legislation 2006, Bd. 2, 2006, S. 63.

31 Jobseekers Act 1995 sec. 7 subs. 1.

32 Vgl. zweites Kapitel Fn. 22

33 Genaue Angaben, was als Arbeitszeit in diesem Sinn angerechnet wird, welche Tätigkeiten erfasst sind, z.B. Studienzeiten, finden sich bei Child Poverty Action Group, Welfare benefits and tax credits handbook, 2005, S. 751 ff. Ist eine Person mehr als 16 Stunden pro Woche beschäftigt, ist sie von der jobseeker's allowance ausgeschlossen. Dann ist an das Auffangsystem income support zu denken.

34 Jobseekers Act 1995 sec. 1 subs. 2 para. e-f; SI 1996/207 reg. 53. 
Auch eine Berufsausbildung (relevant education), z.B. ein Studium in Vollzeit ${ }^{35}$, führt dazu, dass die jobseeker's allowance nicht erteilt werden kann, es sei denn, dass die Maßnahme im Rahmen des New Deal for 18-24 year-olds durchgeführt wird. ${ }^{36}$ Ferner muss der Arbeitslose arbeitsfähig sein (capable of work) ${ }^{37}$ und darf die Altersgrenze für den Ruhestand (derzeit 60 Jahre für Frauen und 65 Jahre für Männer) noch nicht erreicht haben. Er muss in der sog. common travel area (Vereinigtes Königreich, Kanalinseln, Isle of Man) leben. Diese Voraussetzung wird in einem habitual residence test ${ }^{38}$ geprüft und stellt die Schnittstelle zu Personen dar, die dem Einwanderungsrecht unterstehen. 39

\section{b) Besondere Voraussetzungen der contribution-based jobseeker's allowance 40}

Zunächst ist zu prüfen, ob der Antragsteller in einem gesetzlich festgelegten Zeitraum vor Beantragung der jobseeker's allowance ausreichend Beiträge an die Arbeitslosenversicherung (National Insurance) eingezahlt oder sonst Ansprüche im Versicherungssystem erworben hat. Einzelheiten der Berechnung sind für die vorliegende Arbeit nicht relevant. 41

Die laufenden Einkünfte dürfen eine individuell zu bestimmende Summe nicht erreichen, die sich aus dem altersbezogenen Betrag der jobseeker's allowance zuzüglich eines bestimmten zulässigen Einkommens ergibt. Wird diese Grenze überschritten, wird die jobseeker's allowance gekürzt und nicht in voller Höhe ausgezahlt. ${ }^{42}$

Jobseeker's allowance und income support ${ }^{43}$ schließen einander grundsätzlich aus. ${ }^{44}$ Eine Person kann diese staatlichen Leistungen nicht nebeneinander beziehen (mutually

35 Seit Mitte der Siebziger Jahre werden Unterstützungen an Studierende nicht mehr als Leistungen der sozialen Sicherung angesehen, sondern aus dem education budget gezahlt. Rechtstechnisch wurde dies durch Einführung eines negativen Tatbestandsmerkmals (relevant education) erreicht, sodass Personen, die mehr als 12 Wochenstunden Unterricht haben (guided learning hours), keine jobseeker's allowance bekommen können.

36 Jobseekers Act 1995 sec. 1 subs. 2 para. g; SI 1996/207 reg. 54.

37 SI 1996/207 reg. 55: Personen, die erwartungsgemäß keinen Zugang zum Arbeitsmarkt finden werden, erhalten weiterhin Unterstützung in Form von income support oder incapacity benefit (Erwerbsminderungsrente). Dazu Wikeley/Ogus, The law of social security, 2002, S. 499; Wood u.a., Social security legislation 2006, Bd. 2, 2006, S. 869 ff.

38 Ähnlich wie im deutschen Recht steht dabei die Frage im Mittelpunkt, ob die gefestigte Absicht (settled intention) besteht, sich jedenfalls für eine gewisse Dauer in der common travel area aufzuhalten. Dazu Child Poverty Action Group, Welfare benefits and tax credits handbook, 2005, S. 702 ff.

39 Dabei ist die europäische RL 1408/71 zu beachten. Dazu Wikeley/Ogus, The law of social security, 2002, S. 339 f.

40 Jobseekers Act $1995 \mathrm{sec} .2$.

41 Ausführlich zu Einzelheiten der Berechnung Wood u.a., Social security legislation 2006, Bd. 2, 2006, S. 45 ff; Wikeley/Ogus, The law of social security, 2002, S. 501 ff; Matthewman/Ennals/Self, Tolley's social security and state benefits handbook, Rn. 3.4.

42 Dazu im Einzelnen SI 1996/207 reg. 56 i.V.m. reg. 99 (2), 101 (2), sched. 6; Child Poverty Action Group, Welfare benefits and tax credits handbook, 2005, S. 948.

$43 \mathrm{Zu}$ den Voraussetzungen von income support Child Poverty Action Group, Welfare benefits and tax credits handbook, 2005, S. $293 \mathrm{ff}$. 
exclusive benefits). Vorrang hat das Leistungsregime der jobseeker's allowance. ${ }^{45}$ Ist die contribution-based jobseeker's allowance niedriger als der Anspruch auf income support, wird diese Summe über die income-based jobseeker's allowance aufgestockt (top-up).

In bestimmten Fällen muss zwischen income support und jobseeker's allowance gewählt werden. Ist auf der einen Seite die jobseeker's allowance interessant, weil sog. contribution credits zur National Insurance abgeführt werden, die zwar nicht zu eigenen Ansprüchen führen, aber bei Erfüllung von Rahmenfristen Berücksichtigung finden, hat auf der anderen Seite das Regime des income support den Vorteil, dass es dort keine Anforderungen im Hinblick auf Arbeitsuche und Verfügbarkeit gibt.

\section{c) Besondere Voraussetzungen der income-based jobseeker's allowance}

Hat der Arbeitslose keinen Anspruch auf beitragsabhängige Leistungen, muss er die Voraussetzungen der einkommensabhängigen jobseeker's allowance erfüllen, um staatliche Unterstützung zu erhalten (income-based jobseeker's allowance 46 ).

Laufendes Einkommen und vorhandenes Vermögen dürfen jeweils eine bestimmte Höhe nicht erreichen. Die Grenze für das laufende Einkommen wird individuell bestimmt. Zunächst betrug die Vermögensgrenze pauschal 8.000 Pfund beziehungsweise 12.000 Pfund, wenn der Antragsteller oder sein Partner 60 Jahre oder älter war. Diese Beträge wurden im April 2006 erhöht auf bis zu 16.000 Pfund. ${ }^{47}$ Zudem darf der Antragsteller keine Rente beziehen (pension payments) und muss sich in der common travel area aufhalten (habitual residence test). ${ }^{48}$

Auch income-based jobseeker's allowance und income support schließen einander aus. ${ }^{49}$ Während contribution-based jobseeker's allowance und income support nicht von einer Person gleichzeitig bezogen werden können, aber innerhalb eines Haushalts lebende Personen unterschiedlichen Regimen (jobseeker's allowance bzw. income support) unterworfen sein können, wird im Fall einer Konkurrenz zwischen income-based jobseeker's allowance und income support der gesamte Haushalt nach demselben Leistungsrecht beurteilt. Bezieht ein Familienmitglied bereits Leistungen aus einer incomebased jobseeker's allowance, ist jedes weitere Familienmitglied mit einem eigenständigen Anspruch auf Grundlage einer income-based jobseeker's allowance ausgeschlossen und umgekehrt, wenn ein Familienmitglied berechtigt ist, income support zu beziehen. 50

44 Jobseekers Act 1995 sec. 2 subs. 1 (d); Social Security Contributions and Benefits Act 1992 sec. 124 subs. 1 para. f.

45 Dazu Child Poverty Action Group, Welfare benefits and tax credits handbook, 2005, S. 410, 837 f; Wood u.a., Social Security Legislation 2006, Bd. 2, 2006, S. 42 f.

46 Jobseekers Act $1995 \mathrm{sec}$. 3; SI 1996/207 reg. 82, 83.

47 Jobseekers Act 1995 sec. 13; SI 1996/207 reg. 107, 116 (1ZA); vgl. zu den Einzelheiten Matthewman/Ennals/Self, Tolley's social security and state benefits handbook, Rn. 3.13.

48 Vgl. zweites Kapitel A I 2a) S. $101 \mathrm{f}$.

49 Jobseekers Act 1995 sec. 3 subs. 1 para. b-c; Social Security Contributions and Benefits Act 1992 sec. 124 subs. 1 para. f.

50 Jobseekers Act 1995 sec. 3 subs. 1 para. d. 
d) Beantragung als joint-claim couple

Hat ein (verheiratetes oder nicht verheiratetes) Paar, bei dem ein oder beide Partner nach dem 28.10.1947 geboren sind, keine Kinder zu versorgen, hat es den Antrag auf eine jobseeker's allowance verpflichtend gemeinsam zu stellen (joint-claim couple ${ }^{51}$ ). 52

Die jobseeker's allowance wird schließlich erteilt, wenn beide Partner neben der gemeinsamen Antragstellung die labour market conditions erfüllen, also aktiv Arbeit suchen und erwerbsbereit sind. ${ }^{53}$ Auch das vorbereitende Interview (work-focused interview) muss mit beiden Partnern geführt ${ }^{54}$ und das $J S A$ anschließend von beiden unterzeichnet werden.

Vor dieser Änderung erhielt der Partner eines Arbeitslosen, der Unterstützung aufgrund einer income-based jobseeker's allowance bezog, eine zusätzliche Leistung für diesen Partner, ohne dass dieser die labour market conditions erfüllen musste. Es war zu beobachten, dass die Anzahl der Haushalte, in denen niemand arbeitete und beide Partner von staatlicher Unterstützung lebten, anstieg. Die Evaluierung der rechtlichen Änderung ergab, dass infolge der gemeinsamen Beantragung und Betreuung die Anzahl dieser Haushalte signifikant gesenkt werden konnte. 55

\section{e) Höhe der jobseeker's allowance}

Die jobseeker's allowance führt zu einem Anspruch auf eine wöchentlich ${ }^{56}$ auszuzahlende, in der Höhe nach Alter gestaffelte soziale Leistung. ${ }^{57}$ Daran ist die enge Verwandtschaft mit Leistungen nach den Prinzipien von income support zu erkennen. Die Leistung ist in der Höhe abgestimmt auf andere soziale Leistungen, um zu vermeiden, dass Arbeitsanreize im System sozialer Leistungen, insbesondere tax credits verloren gehen. 58

Sie beträgt bei der beitragsabhängigen Form der jobseeker's allowance seit April 2008 für die Altersgruppe zwischen 16 und 24 Jahren 47,95 Pfund, für die Altersgruppe

51 Abzugrenzen ist das joint-claim couple vom single-claim couple, bei dem es nur einen Antrag gibt und bei der Höhe der Leistung bestehende Unterhaltsverpflichtungen berücksichtigt werden.

52 Jobseekers Act $1995 \mathrm{sec}$. 3A. Die Regelung trat zum 19.3.2001 in Kraft und betraf Paare, in denen ein Partner oder beide am bzw. nach dem 18.3.1976 geboren sind. Sie wurde zunächst mit Wirkung vom 28.10.2002 auf Personen, die nach dem 28.10.1957 geboren sind, und ab dem 25.2.2008 auf den oben beschriebenen Personenkreis ausgedehnt.

53 Einzelheiten, insbesondere Ausnahmen, die im Detail geregelt sind, werden erläutert von Frobisher, WRB 2001 (160), S. 23 ff.

54 Employment Act 2002 sec. 49 subs. 1; SI 1996/207 reg. 23A.

55 Dazu Bewley/Dorsett/Thomas, Joint claims for JSA evaluation, DWP, Research Report Nr. 235, 2005, S. $25 \mathrm{ff}$.

56 Jobseekers Act $1995 \mathrm{sec} .1$ subs. 3.

57 Jobseekers Act $1995 \mathrm{sec} .1$ subs. 4.

58 HMSO, Jobseeker's allowance, Cm 2687, 1994, S. 21. Ein Überblick über die jobseeker's allowance ergänzende Leistungen findet sich bei Husmann, ZESAR 2009, S. 126 ff. 
ab 25 Jahren 60,50 Pfund. ${ }^{59}$ Diese Leistung wird 182 Tage lang bei jedem Fall von erneuter Arbeitslosigkeit gezahlt. 60

Die bedürftigkeitsabhängige Form der jobseeker's allowance wird wie income support an die Einkommens- und Vermögenssituation des Antragstellers angepasst. Die jeweilige Summe setzt sich aus drei Komponenten zusammen. Zunächst gibt es den Grundbetrag (personal allowance ${ }^{61}$ ), der in der Höhe den Leistungen der beitragsabhängigen jobseeker's allowance entspricht. Dieser wird aufgestockt durch Sonderzahlungen bei besonderem Bedarf (premiums ${ }^{62}$ ) und Zuschüssen zu Kosten der Unterkunft (housing costs $\left.{ }^{63}\right)^{64}$

In Fällen, in denen bei Vorliegen aller Voraussetzungen die Höhe der contributory jobseeker's allowance unter der Höhe der income-based jobseeker's allowance liegt, gibt es Regeln, die das zu zahlende top-up festlegen. 65

Der Antragsteller erhält in der Regel in den ersten drei Tagen der Arbeitslosigkeit, trotz Vorliegens aller Voraussetzungen der beitragsabhängigen oder einkommensabhängigen jobseeker's allowance, kein Geld (waiting days). Eine Ausnahme liegt dann vor, wenn der Arbeitslose bereits kurz zuvor arbeitslos war oder income support oder incapacity benefit erhält. ${ }^{66}$

Die gemeinsame Beantragung der jobseeker's allowance (joint-claim) hat auf die Höhe der Leistung keine Auswirkungen, es sei denn ein Partner erfüllt die labour market conditions nicht, oder es wurde eine Sanktion verhängt. ${ }^{67}$

\section{New Deal Programme}

Die jobseeker's allowance wurde nach dem Regierungswechsel von der Regierung Tom Majors hin zur Regierung Tony Blairs durch Programme ergänzt, die unter dem Namen New Deal ${ }^{68}$ speziellen Gruppen von Arbeitslosen besondere Förderung bei Einhaltung bestimmter Anforderungen mit dem Ziel der (Wieder-)Eingliederung in den Arbeitsmarkt zuteil werden lassen. ${ }^{69}$ Die Fördermaßnahmen wurden durch Privatisie-

59 Jobseekers Act 1995 sec. 4 subs. 1, 2; SI 2008/632. Ursprünglich wurden die Beträge nach drei Altersgruppen festgesetzt (unter 18 Jahre 35,65 Pfund, 18-24 Jahre 46,85 Pfund, ab 25 Jahre 59,15 Pfund, vgl. SI 1996/207 reg. 79; SI 2003/526 reg. 22).

60 Jobseekers Act $1995 \mathrm{sec} .5$.

61 SI 1996/207 reg. 83 (a), sched. 1 (1); SI 2003/526 sched. 10 reg. 1 (a)-(e).

62 SI 1996/207 reg. 83 (d,e), sched. 1 part III; SI 2003/526 sched. 11, 12.

63 SI 1996/207 reg. 83 (f), sched. 2.

64 Dazu detailliert Husmann, ZESAR 2009, S. 125 f.

65 Jobseekers Act $1995 \mathrm{sec} .4$ subs. 6.

66 Jobseekers Act 1995 sched. 1 (4); SI 1996/207 reg. 46.

67 Jobseekers Act 1995 sec. 4A; dazu Frobisher, WRB 2001 (160), S. 23 ff.

68 Einzelheiten und aktuelle Informationen zu den New Deal Programmen findet man unter http://www.jobcentreplus.gov.uk/JCP/Customers/New_Deal.

69 Zur Entwicklung und der Vorgeschichte der New Deals vgl. Finn, SPR 1998, S. 105-122, zur Beeinflussung durch die Workfare-Politik in den USA vgl. Daguerre, SPA 2004 (38), S. 41-56. 
rungsgewinne finanziert und belasteten somit nicht den laufenden Haushalt des Vereinigten Königreiches. ${ }^{70}$

\section{a) New Deal for Young People}

Im New Deal for Young People, der zum 6.4.1998 als erster New Deal als „Flaggschiff" ${ }^{\text {"71 }}$ der welfare-to-work Politik in Kraft trat, werden 18- bis 24-jährige Arbeitslose betreut, die bereits sechs Monate lang kontinuierlich die jobseeker's allowance erhalten haben. ${ }^{72}$

Das Programm hat drei Stufen. Die erste Stufe ( gateway $^{73}$ ) dauert bis zu vier Monate. Sie dient dazu, entweder direkt in nicht subventionierte Arbeit im ersten Arbeitsmarkt zu vermitteln, sofern dies aufgrund der Qualifikationen des jungen Arbeitslosen möglich ist, oder herauszufinden und zu beraten, welche kurzfristigen Maßnahmen der Person zu einer Stelle verhelfen könnten oder sie zur Teilnahme an der nächsten Stufe des New Deal zu motivieren. Der Schwerpunkt liegt auf der intensiven Beratung, Betreuung und Anleitung des Arbeitslosen durch seinen persönlichen Betreuer (personal adviser) in Gesprächen, aber auch durch eigene Aktivitäten des Betreuers wie beispielsweise Verhandlungen mit und Herstellung von Kontakten zu Arbeitgebern.

Dabei ist die Teilnahme am gateway-Programm nicht zwingend, kann aber in einer sog. jobseeker's direction, einer einseitigen Anordnung im Einzelfall verlangt werden. Bei Weigerung wird die Nichtbefolgung dieser Anordnung mit Entzug der jobseeker's allowance sanktioniert. ${ }^{74}$ Damit wird faktisch ein Zwang zur Teilnahme ausgeübt. ${ }^{75}$ Zudem ist seit April 2001 auch die Nichtteilnahme selbst durch die Nichterteilung der jobseeker's allowance für zwei Wochen sanktioniert. ${ }^{76}$

Nach Ablauf der ersten Stufe ist der Eintritt in die zweite Stufe (options), also die Teilnahme an einer der vier folgenden Maßnahmen zwingend. Bei Nichteinhaltung entfällt automatisch die jobseeker's allowance für zunächst zwei, bei Wiederholung innerhalb der letzten zwölf Monate für vier, bei weiterer Wiederholung innerhalb der letzten zwölf Monate für 26 Wochen. ${ }^{77}$ In dieser Zeit werden auch sog. hardship payments nicht unter den allgemeinen Voraussetzungen gezahlt, sondern nur im Ausnahmefall für bestimmte, in den Regelungen abschließend definierte Personengruppen (vulnerable groups), z.B. Schwangere. ${ }^{78}$ Es besteht entweder die Option, eine Ausbildung in Voll-

70 Wright, Activating the unemployed, in: Clasen, What future for social security?, 2001, S. $242 \mathrm{f}$.

71 Trickey/Walker, Steps to compulsion within labour market policies, in: Lodemel/Trickey, An offer you can't refuse, 2001, S. 199.

72 Die jüngste erhältliche Evaluierung zu dieser Personengruppe liefert Wilkinson, New Deal for young people: Evaluation of unemployment flows, 2003 und bescheinigt darin eine positive Entwicklung der Jugendarbeitslosigkeit, die er insbesondere auf den New Deal zurückführt.

73 SI 1996/207 reg. 75 subs. 1 (a) (i) (bb).

74 Jobseekers Act 1995 sec. 19 subs. 2, 5 (a); SI 1996/207 reg. 69 subs. 2 (a).

75 Child Poverty Action Group, WRB 1998 (142), S. 4.

76 Jobseekers act 1995 sec. 19 subs. 2, 5 (a); SI 1996/207 reg. 69 subs. 2 (a); SI 2001/1029 reg. 4.

77 Jobseekers act 1995 sec. 19 subs. 2, 5; SI 1996/207 reg. 69 subs. 2 (a, b, c).

78 SI 1996/207 reg. 140 para. 1; reg. 140A para. 1. 
zeit bei Bezug der jobseeker's allowance zu absolvieren 79 oder stets bei einem Tag pro Woche Aus- beziehungsweise Fortbildung eine subventionierte Stelle anzutreten, ehrenamtlich/gemeinnützig (voluntary sector option) tätig zu sein oder bei der Environmental Task Force mitzuarbeiten. ${ }^{80}$ Seit Oktober 2002 gibt es eine fünfte Option, die Vorbereitung einer Selbständigkeit innerhalb eines Zeitraums von bis zu 26 Wochen. ${ }^{81}$ Der Gesetzgeber hat somit klar gemacht, dass eine weitere Option, nämlich ,a life on benefit", nicht besteht. ${ }^{82}$

Die Optionen dauern in der Regel sechs Monate, die Aus- oder Fortbildung bis zu einem Jahr. Hat sich daran keine Festanstellung angeschlossen, tritt der Arbeitslose in die dritte Stufe (follow through) ein und erhält nochmals besondere Betreuung mit dem Ziel einer Beendigung des Leistungsbezugs. Diese Phase kann bis zu 26 Wochen dauern. Benötigt der Arbeitslose danach immer noch eine jobseeker's allowance, kann er nochmals die zweite Stufe durchlaufen, um mit Hilfe einer weiteren option seine Chancen auf Wiedereingliederung in den Arbeitsmarkt zu verbessern.

Der New Deal for Young People führt also zu Modifikationen der jobseeker's allowance. Die Nichtteilnahme an dem Programm zieht Sanktionen nach sich, die im bestehenden System der jobseeker's allowance in dieser Form keine Rechtsgrundlage haben. Das Programm stellt damit über die Sanktionierung indirekt Voraussetzungen für staatliche Unterstützung auf.

\section{b) New Deal for long-term unemployed people over 25}

Der New Deal for long-term unemployed people over 25 schließt an die oben beschriebene Zielgruppe an und erfasst Personen, die älter als 25 Jahre sind. Das Programm beginnt nach einer Arbeitslosigkeit von zwei Jahren. Es enthält für diese Personen keine erste Stufe (gateway) der Betreuung, Beratung und Förderung, die zunächst die genaue Orientierung des Arbeitslosen zum Ziel hätte, sondern beginnt direkt mit der Verpflichtung, an options teilzunehmen, also entweder eine subventionierte Arbeit anzunehmen oder eine Aus- oder Fortbildung zu machen. Unterstützt wird die Arbeitsuche im nicht subventionierten ersten Arbeitsmarkt durch regelmäßige Beratungsgespräche, die den Arbeitslosen bis zu sechs Monate begleiten. Wird eine Beschäftigung nicht angetreten oder schuldhaft wieder beendet, oder eine Ausbildung abgebrochen, entfällt die jobseeker's allowance für bis zu 26 Wochen. ${ }^{83}$

\section{c) Weitere New Deals}

Es gibt weitere New Deals, die andere Zielgruppen einbeziehen. So sind z.B. Alleinerziehende (New Deal for lone parents) oder Personen über 50 Jahre (New Deal 50

79 SI 1996/207 reg. 75 (1) (b) (ii).

80 SI 1996/207 reg. 75 (1) (a) (ii) (bb),(cc).

81 SI 1996/207 reg. 75 (1) (a) (ii) (aa); SI 2002/2314 reg. 4 (1) (b); dazu Child Poverty Action Group, WRB 1998 (146), S. 13.

82 Child Poverty Action Group, WRB 1998 (142), S. 7; Finn, SPR 1998, S. 115.

83 Dazu Child Poverty Action Group, WRB 1998 (146), S. 10-13. 
plus) Zielgruppen des New Deal. Diese Programme sind für den Einzelnen nicht verpflichtend und enthalten deshalb auch nicht die Möglichkeit, die Nichtteilnahme zu sanktionieren. Vielmehr steht die auf diese Personen abgestellte Betreuung und Beratung im Vordergrund. Die Bereitschaft zur Beendigung der Arbeitslosigkeit wird hier offenbar höher eingeschätzt, sodass eine erzwungene Teilnahme nicht erforderlich erscheint. ${ }^{84}$ Diese Programme entfalten keine Wirkungen für die jobseeker's allowance und das jobseeker's agreement.

\section{Jobseeker's agreement}

Das jobseeker's agreement (JSA) ist das Herzstück der Reform und soll unter anderem die Kontrolle sog. „, freiwilliger Arbeitslosigkeit“ ermöglichen. ${ }^{85}$

Vor der Einführung der jobseeker's allowance und des JSA war der sog. Back-toWork-Plan entsprechendes Instrument der Arbeitsvermittlung. ${ }^{86}$ Auf die Stärken dieses einseitig abgeschlossenen Instruments baut der Gesetzgeber des JSA auf. 87 Im Back-toWork-Plan wurden Maßnahmen zur Integration des Arbeitslosen in den Arbeitsmarkt festgelegt, wobei die Mitwirkung des Antragstellers darauf beschränkt war, den Inhalt des Plans mitzugestalten. Auf seine Zustimmung zur konkreten Ausgestaltung kam es gerade nicht an. Der Abschluss war freiwillig. ${ }^{88}$

Das JSA ersetzt den Back-to-Work-Plan. Ein Arbeitsloser erhält nur dann die jobseeker's allowance und infolgedessen soziale Leistungen, wenn er (neben den oben beschriebenen Voraussetzungen) ein $J S A$ unterzeichnet hat. ${ }^{89}$ Genaueres ist im Jobseekers Act $1995 \mathrm{sec} .9-10$ sowie in den SI 1996/207 reg. 31-40 und 66 geregelt.

\section{Definition}

Die Legaldefinition des JSA enthält Jobseekers Act 1995 sec. 9 subs. 1. Die Vorschrift besagt, dass ein $J S A$ vorliegt, wenn zwischen dem Antragsteller (claimant) und einem employment officer ${ }^{90}$ eine Vereinbarung geschlossen wird, die den zum Zeitpunkt des Abschlusses geltenden Anforderungen des Jobseekers Act 1995 entspricht.

Der employment officer ist Mitarbeiter der executive agency Jobcentre Plus (JCP) und vom $D W P$ zum Abschluss dieser Vereinbarungen ermächtigt. ${ }^{91}$

84 Meager, New Deal im Vereinigten Königreich, in: Weinert u.a., Beschäftigungsfähigkeit, 2001, S. 168.

85 Fulbrook, ILJ 1995 (24), S. 398.

86 Vgl. erstes Kapitel B II 2 S. 69.

87 HMSO, Jobseeker's allowance, Cm 2687, 1994, S. 11, 20.

88 Wood u.a., Social security legislation 2006, Bd. 2, 2006, S. 68; vgl. auch HMSO, Jobseeker's allowance, Cm 2687, 1994, S. 20.

89 Jobseekers Act $1995 \mathrm{sec} .1$ subs. 2 (2).

90 Jobseekers Act 1995 sec. 9 subs. 13.

91 Wood u.a., Social security legislation 2006, Bd. 2, 2006, S. 69. 
a) Gesetzlich geregelte Mindestanforderungen

Den geforderten Inhalt eines $J S A$ beschreibt SI 1996/207 reg. 31. Dort sind die zum Zeitpunkt des Antrags geltenden Anforderungen an die Vereinbarung im Sinn des Jobseekers Act $1995 \mathrm{sec} .9$ subs. 1 formuliert.

Die Vereinbarung muss den Namen des Antragstellers und das Datum der Vereinbarung enthalten. 92

Sie muss (shall) aufführen, wann und wie viele Stunden der Antragsteller pro Tag arbeiten kann (pattern of availability). Sie muss beschreiben, in welcher Hinsicht der Antragsteller in seiner Arbeitsfähigkeit beschränkt ist, insbesondere bezüglich des Ortes oder der Art der Arbeit. In der Vereinbarung müssen die gesuchte Tätigkeit und die Aktivitäten des Antragstellers hinsichtlich der Arbeitsuche und der Verbesserung der Chancen des Antragstellers auf dem Arbeitsmarkt festgelegt werden. 93

Ferner kann der genaue Anfangs- und Endtermin für Zeiten vereinbart werden, in denen der Arbeitsuchende, obwohl er nach den strengen Definitionen in Jobseekers Act $1995 \mathrm{sec}$. 6, 7 nicht arbeitsbereit ist oder nicht aktiv Arbeit sucht, trotzdem als arbeitsbereit/aktiv arbeitsuchend angesehen wird (permitted period). Das ist ein Zeitraum von bis zu 13 Wochen, in dem ihm nur Stellen in seinem bisherigen Beruf und/oder zu seinem bisherigen Gehalt angeboten werden und er selbst auch nur nach dieser Art von Stellen suchen muss. ${ }^{94}$ Die Einschränkungen bei der Bereitschaft zur Erwerbstätigkeit beziehungsweise bei einer aktiven Arbeitsuche sind mit der Definition von available for work und actively seeking employment nicht vereinbar. Da aber für eine bestimmte Zeit ein solcher Schutz für den Antragsteller sinnvoll sein kann, um einen möglichst langen Verbleib in dem neu gefundenen Arbeitsverhältnis unabhängig von staatlicher Unterstützung zu erreichen, bleibt die Möglichkeit, jenen Freiraum durch das JSA zu nutzen und für den Einzelfall passend auszufüllen.

Ferner enthält das $J S A$ eine Erklärung, mit der die Kenntnisnahme des Antragstellers vom Inhalt der Vereinbarung und den darin enthaltenen Bedingungen bestätigt wird. ${ }^{95}$

Es muss eine Belehrung darüber enthalten sein, welche Rechte der Antragsteller hat, wenn zwischen employment officer und Antragsteller keine Einigung über den Inhalt des JSA zustande kommt (statement on the claimants rights). ${ }^{96}$ Dazu mehr im Rahmen des Rechtsschutzes. ${ }^{97}$

92 SI 1996/207 reg. 31 (a) und (h).

93 SI 1996/207 reg. 31 (b-e).

94 Jobseekers Act 1995 sec. 6 subs. 4-7, sec. 7 subs. 4-5, 7; SI 1996/207 reg. 16, 20, 31 (f).

95 HMSO, Jobseeker's allowance, Cm 2687, 1994, S. 21.

96 SI 1996/207 reg. 31 (g); Child Poverty Action Group, Welfare benefits and tax credits handbook, 2005, S. 370.

97 Vgl. zweites Kapitel A II 9 S. 131 ff. 
b) Prüfungsrecht bzw. Prüfungspflicht des employment officer

Der employment officer muss den ausgehandelten Inhalt der Vereinbarung prüfen. Er darf erst dann die Vereinbarung abschließen, wenn er der Auffassung ist, dass die vereinbarten Maßnahmen die Voraussetzungen „bereit zur Erwerbsarbeit“ und „aktive Arbeitsuche" erfüllen.

Bestehen Zweifel bei ihm oder verlangt es der Antragsteller, kann die Frage, ob die gesetzlichen Anforderungen an das JSA erfüllt sind, einem decision maker ${ }^{98}$ übertragen werden. Dieser muss innerhalb von 14 Tagen entscheiden. ${ }^{99}$ Die Entscheidung des $d e-$ cision maker 100 ist bei einem SSAT anfechtbar. Es wird erwartet, dass sich wegen dieser Kontrollmöglichkeit eine einheitliche Begrifflichkeit für die beiden zentralen labour market conditions herausbildet. 101

\section{Formelle Gesichtspunkte}

Das JSA muss schriftlich niedergelegt und von den Parteien unterschrieben sein. ${ }^{102}$ Der Antragsteller muss eine Kopie der Vereinbarung erhalten. ${ }^{103}$ Der englische Gesetzestext formuliert ,shall be in writing and be signed“, was einem „Müssen“ entspricht, also einer Pflicht der Verwaltung, auf ein unterzeichnetes Dokument hinzuwirken. ${ }^{104}$

\section{Rechtliche Einordnung}

a) Rechtliche Handlungsfähigkeit auf Seiten des employment officer

Parteien der Vereinbarung sind der Antragsteller und der sog. employment officer, ein Mitarbeiter des $J C P$.

Die rechtliche Handlungsberechtigung eines Verwaltungsbeamten hängt davon ab, in welcher Behörde er arbeitet. Man unterscheidet die Beamten, die direkt im Ministerium (department) als central civil service politische Entscheidungen des Ministers fachlich vorbereiten ${ }^{105}$, von denen, die im dem Ministerium nachgeordneten Bereich in executive agencies 106 tätig sind und ausschließlich dem einzelnen Bürger gegenüber auftreten.

98 Vgl. zweites Kapitel A II 9 S. $131 \mathrm{f}$.

99 Jobseekers Act 1995 sec. 9 subs. 5.

100 Vgl. zweites Kapitel A II 9 S. $131 \mathrm{f}$.

101 Wood u.a., Social security legislation 2006, Bd. 2, 2006, S. 69.

102 Jobseekers Act 1995 sec. 9 subs. 3.

103 Jobseekers Act 1995 sec. 9 subs. 4.

104 Foulkes, Administrative law, 1995, S.178 f; Wade/Forsyth, Administrative law, 2004, S. 265; Harding, Public duties and public law, 1989, S. 14.

105 Drewry, PL 1988, S. 508; Harden, The contracting state, 1992, S. 23 f.

106 Sie wurden next step agencies genannt nach dem Bericht der Efficiency Unit, HMSO, Improving management in government: the next steps, 1988. 
Executive agencies entstanden im Zuge der Verwaltungsreformen im Vereinigten Königreich (New Public Management ${ }^{107}$ ) durch Ausgliederung bestimmter Bereiche der Ministerialverwaltung. Zunächst hatte man das Ziel, dadurch die Verantwortlichkeiten der Minister (ministerial responsibility ${ }^{108}$ ) zu verringern und diese zu entlasten. ${ }^{109}$ Man ging davon aus, dass eine rechtliche Verantwortung (accountability) als Verantwortlichkeit gegenüber dem Parlament bestehe, die sich darauf beziehe, bei allgemeinen Fragen und Missständen dem Parlament gegenüber Auskunft geben zu müssen, nicht aber über Entscheidungen im Einzelfall. ${ }^{110}$ Es ist inzwischen im Verhaltenscodex der Minister (Ministerial Code) niedergelegt, dass jeder Minister auch für die einzelnen Entscheidungen der executive agencies rechtliche Verantwortung trägt, sodass es letztlich wie vor Einführung der executive agencies bei einer vollumfänglichen Verantwortung des Ministers gegenüber dem Parlament geblieben ist. ${ }^{111}$ Deshalb folgte nicht allein aus der Veränderung der Organisationsstruktur eine Beschränkung der Verantwortlichkeit des Fachministers.

Die executive agencies werden in (Rahmen-)Vereinbarungen (framework documents ${ }^{112}$ ) mit dem Ministerium zu bestimmten Tätigkeiten in eigener Verantwortung mit einem ihnen zugewiesenen Budget unter Leitung eines chief executive verpflichtet. ${ }^{113}$ In diesen Rahmenvereinbarungen werden auch die Handlungsmöglichkeiten der Mitarbeiter dieser Behörde festgelegt. Executive agencies sind deshalb grundsätzlich rechtlich nicht selbständig. Sie haben keine legal personality, die nur entsteht, wenn die executive agency Trägerin von Rechten und Pflichten und unabhängig von der ihr übergeordneten Stelle ist. ${ }^{114}$ Aufgrund der Ausgestaltung der Rahmenvereinbarungen nimmt das Ministerium derzeit weit reichend Einfluss auf die executive agency, denn die gesamten Abläufe, die Finanzierung und auch die Möglichkeit, welche Vereinbarungen überhaupt geschlossen werden können, werden vom Ministerium bestimmt. ${ }^{115}$ In Folge dessen handeln die Mitarbeiter der executive agency in der Regel im Namen des für sie zuständigen Ministeriums. ${ }^{116}$

107 Vgl. erstes Kapitel A I 2a) S. 49.

108 Dazu allgemein Cane, Administrative law, 2004, S. 355 ff; Woodhouse, Ministerial responsibility, in: Bogdanor, The British Constitution in the twentieth century, 2004, S. $281 \mathrm{ff}$.

109 Baldwin, MLR 1988 (51), S. 625 f; Drewry/Giddings, The origins of the next steps programme, in: Giddings, Parliamentary accountability, 1995, S. 14.

110 Philips/Jackson, Constitutional and administrative law, 2001, S. 378 f.

111 Pyper, Ministerial responsibility and next step agencies, in: Giddings, Parliamentary accountability, 1995, S. 20; vgl. auch HMSO, Civil service management reform: the next steps, Cm 524, 1988, S. 9; $H M S O$, The financing and accountability of next steps agencies, Cm 914, 1989, S. 16.

$112 \mathrm{Zu}$ deren (rechtlicher) Einordnung vgl. Harden, The contracting state, 1992, S.26-28, 37-51; Vincent-Jones, The new public contracting, 2006, S. $154 \mathrm{f}$.

113 Harden, The contracting state, 1992, S. 22-26; Oliver/Drewry, Public service reforms, 1996, S. 35, 41.

114 Harden, The contracting state, 1992, S. 38.

115 Cane, Administrative law, 2004, S. 315.

116 So auch Craig, Administrative law, 2003, S. $149 \mathrm{ff}$. 
Der employment officer verpflichtet also nicht sich selbst, sondern handelt für das zuständige Ministerium. ${ }^{117}$ Somit kommt es auf die fehlende rechtliche Selbständigkeit des JCP nicht an. ${ }^{118}$ Das DWP selbst ist rechtsfähig. Es leitet diese Fähigkeit von der Fähigkeit der Krone ab, sich in gleichem Umfang wie jeder „Untertan“ durch Verträge binden zu können. ${ }^{119}$ Das bedeutet für das $J S A$, dass dieses nur in Übereinstimmung mit den Rahmenverträgen geschlossen werden kann. ${ }^{120}$

Enthielte also der Rahmenvertrag, der die executive agency JCP begründet, die Ermächtigung zu rechtlich bindenden Verträgen, stünde die fehlende Rechtsfähigkeit des $J C P$ einer Einordnung als government contract nicht entgegen. Doch fehlt in den Rahmenvereinbarungen mit den $J C P$ bewusst diese Ermächtigung, sodass keine wirksamen Verträge auf der Grundlage des common law geschlossen werden können. ${ }^{121}$

\section{b) Vereinbarung von Pflichten}

Während bei government contracts bereits die Pflicht zur Erfüllung einer Aufgabe vertraglich übertragen wird und schließlich vom Vertragspartner zu erfüllen ist, ist dies im $J S A$ nicht der Fall.

Im $J S A$ werden zwar auch die Aufgaben der Verwaltung berührt, es geht aber nur um die Abwicklung der Aufgabenerfüllung. Die Aufgaben selbst bleiben bei der Behörde, nämlich sowohl die Gewährung finanzieller Unterstützung als auch die Vermittlung des Arbeitslosen. Im JSA werden „lediglich“ eine Bestandsaufnahme und eine Konkretisierung der Rechte und Pflichten der Parteien der Vereinbarung vorgenommen. Insbesondere werden die labour market conditions umschrieben und auf den konkreten Einzelfall bezogen. ${ }^{122}$

Es besteht insoweit ein wesentlicher Unterschied zur Ausgangssituation von government contracts.

\section{c) Freiwilligkeit - Vertragsfreiheit}

Der gesetzliche Hintergrund des $J S A$ als solcher beziehungsweise die Anordnung im Gesetz, ein JSA abzuschließen (statutory background), steht der Annahme einer freiwillig eingegangenen Vereinbarung freilich nicht entgegen. Es gibt verschiedenste Verträge, deren Abschluss angeordnet und deren Inhalt gesetzlich umschrieben ist. ${ }^{123}$ Solange

117 Vgl. zweites Kapitel A II 1 S. 109.

118 Anders beim australischen Newstart Activity Agreement. Dort spricht der Wortlaut des Social Security Act 1991 (Cth) sec. 604 von einer Vereinbarung mit dem „Commonwealth Employment Center", das selbst keine Rechtspersönlichkeit besitzt. Dazu Seddon, Newstart activity agreements: Are they contracts?; Brennan, Newstart activity agreements: Are they contracts?, beide in: Creyke/Sassella, Targeting accountability and review, 1998, S. $82 \mathrm{ff}$ bzw. $87 \mathrm{ff}$.

119 Vgl. erstes Kapitel B III 3b) S. 77.

120 Vincent-Jones, The new public contracting, 2006, S. $237 \mathrm{f}$.

121 Vincent-Jones, The new public contracting, 2006, S. $237 \mathrm{f}$.

122 HMSO, Jobseeker's allowance, Cm 2687, 1994, S. 20 f.

123 Seddon, Government contracts, 1999, S. 83. 
die Abschlussentscheidung selbst als freiwillig bezeichnet werden kann, kann man von einem freiwillig eingegangenen Vertrag sprechen.

Freiwillig bedeutet nämlich, dass die Vereinbarung in Ausübung des Prinzips der Vertragsfreiheit geschlossen wird. ${ }^{124}$ Interessant ist, inwiefern beide Elemente der Vertragsfreiheit, die Abschluss- und die Gestaltungsfreiheit, in der zu untersuchenden Situation beim Abschluss eines $J S A$ vorhanden sind.

\section{aa) Abschlussfreiheit des Antragstellers}

Das Element der Abschlussfreiheit ist erfüllt, wenn die Entscheidung, ob man sich vertraglich bindet oder nicht, freiwillig getroffen werden kann.

Kontrahierungszwang schränkt zwar die Abschlussfreiheit einer Vertragspartei ein. Auf der anderen Seite dient der Kontrahierungszwang dem Schutz der schwächeren Partei und bewahrt diese davor, ein bestimmtes wichtiges Gut wie z.B. Strom oder Wasser nicht zu erhalten. Ein Kontrahierungszwang, der sich zu Lasten der schwächeren Partei auswirkt, ist mit dem Prinzip der Vertragsfreiheit allerdings nicht vereinbar. ${ }^{125}$

Beim Abschluss des JSA ist Freiwilligkeit in diesem Sinn nicht gegeben. Die finanzielle Unterstützung des Arbeitslosen und die Vereinbarung sind miteinander in der Weise verbunden, dass ohne Vereinbarung keine Leistung gewährt wird. Angesichts der Notsituation, die dem Bedürftigen ohne staatliche Unterstützung droht, kann von einer freiwilligen Unterzeichnung keine Rede sein. ${ }^{126}$ Bei den oft sehr kurzen Gesprächen steht auf der Seite des Antragstellers die staatliche Unterstützung als ,the real issue“ im Mittelpunkt, weil sie ihm das finanzielle Auskommen absichert. ${ }^{127}$ Es handelt sich um einen Fall eines faktischen Kontrahierungszwanges, der die schwächere Partei, den Arbeitslosen, belastet. Die Ausübung dieses Zwanges ist mit dem dargestellten Begriff von Vertragsfreiheit nicht vereinbar.

Ferner gibt es nicht die Möglichkeit, den „Anbieter“ der Sozialleistung zu wählen, da „finanzielle Unterstützung im Fall der Arbeitslosigkeit“ nur von einer staatlichen Stelle, also vom $J C P$ angeboten wird. ${ }^{128}$ Es gibt somit im Hinblick auf den Anbieter der Leistung keine Wahlfreiheit. Die Verwaltung darf auf der Grundlage des Jobseekers Act 1995 außer in den dort umschriebenen Härtefällen ohne JSA keine Leistungen gewähren. Insgesamt führt dies zu einer abhängigen Position des Antragstellers, die der Annahme einer Abschlussfreiheit entgegensteht.

124 Vgl. erstes Kapitel B III 2 S. $71 \mathrm{ff}$.

125 Vgl. erstes Kapitel B III 2 S. 71 ff.

126 So Wikeley/Ogus, The law of social security, 2002, S. 351 unter Hinweis auf die Parlamentsdebatte; Wood u.a., Social security legislation 2006, Bd. 2, 2006, S. 69.

127 So auch Handler, Social citizenship and workfare in the United States and Western Europe, 2004, S. 150.

128 Fulbrook, ILJ 1995 (24), S. 400; vgl. dazu allgemein zu diesen Veränderungen in der sozialen Sicherung Carmel/Papadopoulos, The new governance of social security in Britain, in: Millar, Understanding social security, 2003, S. 39. 


\section{bb) Gestaltungsfreiheit der Verwaltung}

Aus verschiedenen Gründen ist die Gestaltungsfreiheit des employment officer bei der Aushandlung des Inhalts des $J S A$ ebenfalls eingeschränkt.

Einschränkungen ergeben sich aus der Tatsache, dass gesetzlich festgelegt ist, wie die vereinbarten Maßnahmen inhaltlich auszusehen haben. So statuiert Jobseekers Act $1995 \mathrm{sec} .9$ subs. 5, dass der employment officer das $J S A$ solange nicht abschließen darf, bis er überzeugt ist, dass im JSA die Voraussetzungen available for employment und activeley seeking employment erfüllt sind, beziehungsweise das dafür erforderliche Verhalten des Antragstellers beschrieben ist. ${ }^{129}$ Auch bei einer Änderung des Inhalts des $J S A$ sind diese Kriterien unbedingt zu erfüllen. ${ }^{130}$

Andere Abweichungen, wie z.B. die Vereinbarung von permitted periods, ist ausdrücklich geregelt, was so verstanden werden muss, dass die Möglichkeiten der Arbeitsverwaltung, kreative Ideen zur Eingliederung des Arbeitsuchenden in den Arbeitsmarkt in das $J S A$ aufzunehmen, ausgeschlossen sind.

\section{d) (Gesetzlicher) Ausschluss der Anwendung von contract law}

Das JSA ist durch den Jobseekers Act 1995 geregelt. Es handelt sich also um statutory law. Entsprechend der gesetzlichen Regelung entfaltet das JSA nur Wirkungen, soweit diese mit der Beantragung einer jobseeker's allowance zusammen hängen. ${ }^{131}$ Dies wird so verstanden, dass die Anwendung von contract law auf das JSA durch gesetzliche Regelung ausgeschlossen ist. ${ }^{132}$

\section{e) Vertragsähnliches Verwaltungshandeln}

Das JSA ist kein government contract, auf den modifiziert das law of contract Anwendung finden könnte. Vielmehr handelt es sich um vertragsähnliches Verwaltungshandeln. Das JSA ist ein quasi-contract: Die Situation, in der die Vereinbarung geschlossen wird, ist nicht vergleichbar mit anderen Vertragschlüssen zwischen Staat und Einzelnem. Es handelt sich um eine Vereinbarung zwischen dem Staat und dem einzelnen Bürger, bei der die Ausgestaltung der Rechtsbeziehung, insbesondere die Voraussetzungen der jobseeker's allowance durch das Gesetz bereits konkretisiert sind, und kein ausreichend weiter (Ver-)Handlungsspielraum mehr bleibt. Es fehlt der Vereinbarung zudem die Garantie gerichtlicher Durchsetzbarkeit, weil diese gerade vom Gesetzgeber ausgeschlossen ist. ${ }^{133}$

129 Wikeley/Ogus, The law of social security, 2002, S. 353.

130 Jobseekers Act 1995 sec. 10 subs. 4.

131 Jobseekers Act 1995 sec. 9 subs. 2.

132 Wood u.a., Social security legislation 2006, Bd. 2, 2006, S. 68; Wikeley/Ogus, The law of social security, 2002, S. 351.

133 Jobseekers Act 1995 sec. 9 subs. 2. 


\section{Funktion des JSA}

Ganz allgemein gesprochen soll das $J S A$ das Nebeneinander von Rechten und Pflichten verdeutlichen, das beim Bezug von finanzieller Unterstützung im Fall von Arbeitslosigkeit besteht. Der Antragsteller hat nicht nur ein Recht auf diese Unterstützung, sondern auch Pflichten, die im JSA möglicherweise neu begründet, zumindest aber konkretisiert werden. ${ }^{134}$ Damit steht das JSA im Zusammenhang mit der Umsetzung der Forderungen der Citizen's Charter, mit der Weiterentwicklung der social citizenship sowie mit einem veränderten Verständnis des social contracts.

\section{a) Citizen's Charter und Customer's Charter des JCP}

Das JSA ist als Anwendungsfall der Standards, die die Citizen's Charter ${ }^{135}$ setzt, anzusehen.

Im Mittelpunkt der Citizen's Charter steht neben der Verbesserung des Verhältnisses Staat - Bürger die Effizienz der Verwaltung, die in dieser Arbeit bereits als ein Aspekt kooperativer Verwaltung beschrieben worden ist. ${ }^{136}$ Es gilt aber zu vermeiden, dass die Motivation der Citizen Charter auf diese beiden Aspekte reduziert wird. ${ }^{137}$ Bezug zum $J S A$ hat vielmehr der Katalog von (Verfahrens-),,Rechten“ des Einzelnen, der insbesondere durch die Forderung nach gerichtlicher Kontrolle der Verwaltung gewichtig erscheint. ${ }^{138}$

Der Einzelne soll sich durch Mitwirkung am Verwaltungsverfahren besser mit diesem auseinander setzen, den Informationsfluss hin zur Verwaltung befördern und letztendlich dadurch auch beitragen, die Verwaltung insgesamt voran zu bringen. Wichtig erscheint, dass in den Vorgaben der Charter soweit möglich vermieden wird, rechtliche Verpflichtungen für den Staat in den Vordergrund zu stellen. ${ }^{139}$

Wie in der Citizen's Charter vorgesehen, werden deren recht allgemein gehaltene Vorgaben durch die Customer's Charter des $J C P^{140}$ ergänzt und konkretisiert. In dieser

134 Bryson, ILJ 1995 (24), S. 204 ff.

135 HMSO, The Citizen's Charter, Raising the standard, Cm 1599, 1991; dazu allgemein zur Einführung und Implementierung Doern, PP 1993 (21), S. 17-29.

136 Vgl. erstes Kapitel A II 1 S. 54 f; dazu Woodhouse, In pursuit of good administration, 1997, S. 49 ff; Lewis, PQ 1993 (64), S. 316 ff; im Einzelnen zur Effizienzsteigerung durch Wettbewerb bei $O s$ borne/Gaebler, Reinventing government, 1992, S. $71 \mathrm{ff}$.

137 Barron/Scott, MLR 1992 (55), S. 537 f.

138 Dazu Page, The Citizen's Charter and administrative justice, in: Harris/Partington, Administrative justice in the 21 st century, 1999, S. $85 \mathrm{ff}$.

139 Lewis, PQ 1993, S. 320; Drewry, PL 2002, S. 10. Im Einzelnen Kingdom, Citizen or state consumer? in: Chandler, The Citizen's Charter, 1996, S. 7 ff.

140 Als service standards einsehbar unter http://www.jobcentreplus.gov.uk/JCP/stellent/groups/jcp/ documents/websitecontent/dev_011795.pdf. Insofern bestätigt sich die Aussage von Drewry, PL 2002, S. 9 f nicht, dass die Citizen's Charter in Vergessenheit geraten ist, denn diese Standards gelten weiterhin. Durch die weitgehende Übernahme ihrer Grundsätze, insbesondere die Betonung der Dienstleistung Verwaltung, wie aus der Formulierung ,service first", dem Nachfolgeprogramm der Labour-Regierung, hervorgeht (vgl. TSO, Modernising Government, Cm 4310, 1999) kann eben- 
werden - nochmals konkreter - Standards für den Umgang mit den Arbeitslosen festgelegt, z.B. höflicher Umgang miteinander, prompte Antwort der Verwaltung auf Anfragen und Terminwünsche der Bürger innerhalb festgelegter Fristen. Während der der Vereinbarung vorausgehenden Gespräche und Verhandlungen zwischen Behörde und Arbeitslosem werden Situationen entstehen, in denen der Einzelne von den in der Citizen's Charter oder Customer's Charter des JCP festgelegten Standards betroffen ist, weil er selbst, aber auch das $J C P$ diese einzuhalten haben.

\section{b) Social citizenship}

Das Konzept social citizenship entwickelte und verteidigte der Soziologe $T$. $H$. Marshall und betrachtete es als „krönenden Abschluss“ 141 bei der Entstehung der Stellung des Bürgers im Staat (citizenship). Heute wird, und darin liegt die Bedeutung des $J S A$, das Konzept der social citizenship im englischen Arbeitsförderungsrecht nicht (mehr) verfolgt. ${ }^{142}$ Aktivierung steht nach der hier vertretenen Definition dem Konzept einer social citizenship entgegen.

T.H. Marshall beschrieb drei Phasen der Entwicklung der Stellung des Bürgers im Staat ${ }^{143}$ : civil citizenship beinhaltete die Rechte, die der Bürger brauchte, um individuelle Freiheit zu genießen, also z.B. persönliche Freiheit, das Recht auf Eigentum und gewisse Rechte gegenüber der Justiz. Political citizenship als zweite Phase stand für politische Beteiligungsrechte, insbesondere das aktive und passive Wahlrecht. Als dritte und letzte Phase der Entwicklung betrachtete Marshall die social citizenship, was bedeutet, dass der Einzelne nicht nur ein Recht auf angemessene wirtschaftliche Sicherheit, sondern auch ein weiterreichendes Recht auf Beteiligung an sozialen Standards hat, die in der Gesellschaft verwirklicht sind. Marshall entwickelte diese Ideen Ende des Zweiten Weltkriegs in einer Zeit, in der man eher hoffnungsvoll in die Zukunft blickte und ein großes Interesse daran hatte, Ungleichheiten der „Klassengesellschaft“ zu beseitigen. ${ }^{144}$ Eine mögliche Umsetzung dieses Konzepts ist die Einführung eines basic income/Grundeinkommens, welches jedem Bürger gewährt wird. ${ }^{145}$

Versteht man social citizenship als Recht auf konkrete Leistungen, wird dieses durch aktivierende Maßnahmen und die Verknüpfung von Rechten und Pflichten nicht mehr gewährleistet. Zwar gab Marshall zu, dass einem Bürger im Staat nicht nur Rechte zukommen, sondern dass ihn auch Pflichten treffen, doch verstand er letztere als jeder-

falls als Beweis dienen, dass über die Erfordernisse, die die Citizen's Charter ansprach, weiterhin Einigkeit besteht.

141 Fraser/Gordon, Civil citizenship against social citizenship?, in: Steenbergen, The condition of citizenship, 1994, S. 92.

142 King, In the name of liberalism, 1999, S. 246.

143 Marshall, Citizenship and social class, in: Marshall, Sociology at the crossroads and other essays, 1963, S. 74.

144 Fraser/Gordon, Civil citizenship against social citizenship?, in: Steenbergen, The condition of citizenship, 1994, S. 92.

145 Handler, Social citizenship and workfare in the United States and Western Europe, 2004, S. 272 ff; kritisch zur Verbindung der Idee eines basic income mit der Idee vom welfare contractualism: White, BJPS 2000 (30), S. 528. 
mann treffende allgemeine Pflichten, wie die Pflicht, Steuern zu zahlen oder Beiträge zur Sozialversicherung (national insurance) abzuführen. Er betrachtete aber die Einhaltung der Pflichten nicht als Voraussetzung für die Entstehung der Rechte gegenüber dem Staat. 146

Versteht man social citizenship dagegen nur als Möglichkeit des Zugangs zu sozialen Rechten, ist aktivierende Arbeitsmarktpolitik auch eine Ausprägung des Konzepts der social citizenship. ${ }^{147}$ Denn aktivierende Arbeitsmarktpolitik sieht zwar nicht vor, Rechte des Einzelnen auf soziale Leistungen ohne die Betonung einer aus der Berechtigung resultierenden Verpflichtung zu einem die Arbeitslosigkeit möglichst beendigenden oder zumindest verkürzenden Verhalten des Einzelnen zu stärken. ${ }^{148}$ Doch führt Aktivierung dazu, dass der Arbeitslose, der sich aktivieren lässt, die vorgesehenen Leistungen erhält. Es besteht also weiterhin die Möglichkeit, staatliche Unterstützung zu erhalten.

Diese weite Interpretation, die bereits den Zugang zu sozialen Rechten ausreichen lässt, bleibt indes fragwürdig. Die Stärkung der Pflichten im Rahmen aktivierender Maßnahmen ist nicht nur eine „kosmetische Sache“. Sie führt zu Änderungen in der Rechtsstellung des Einzelnen und damit zu einer Änderung seiner sozialen Stellung im Fall der Arbeitslosigkeit. ${ }^{149}$ Die Entwicklung der quasi-contracts als Instrument zur Definition und eventuell auch Neubegründung von Pflichten des Arbeitslosen geschieht unter Berücksichtigung der Gesetzesmaterialien gerade nicht, um Rechte des Einzelnen zu erweitern, sondern zu kontrollieren und Grundlage für Disziplinierungsmaßnahmen zu schaffen. ${ }^{150}$ Die Stellung des Einzelnen und die Verantwortung für den Eintritt der Arbeitslosigkeit werden damit nicht im Sinn der social citizenship ausgebaut. Auf die mögliche Verbesserung der Rechtsstellung bei „gefälligem“ Verhalten kommt es im Verständnis der social citizenship nicht an. Das Ergebnis, dass durch die Verankerung aktivierender Maßnahmen, insbesondere mit Hilfe des JSA, die Idee einer social citizenship aus dem englischen Arbeitsförderungsrecht verdrängt wird, war demnach politisches Ziel und ist auch erreicht worden.

\section{c) Social contract}

In der Idee vom social contract spielen die eben dargestellten Ansätze zusammen. Sie greift die Abkehr vom Konzept einer social citizenship auf und verfolgt die eben beschriebenen Ziele weiter. Die Konstruktion eines Vertrages zwischen Staat und Einzelnem dient der Betonung von Pflichten des Bürgers gegenüber dem Staat. ${ }^{151}$ Die

146 Marshall, Citizenship and social class, in: Marshall, Sociology at the crossroads and other essays, 1963, S. 122 f.

147 Dazu genauer White, BJPS 2000 (30), S. 508-512.

148 Vgl. Einführung A I S. 28 ff.

149 King, In the name of liberalism, 1999, S. 232 f.

150 Collins, Regulating contracts, 2002, S. 20.

151 So allgemein auch Harden, The contracting state, 1992, S. 33, der schreibt: “ The new element is not „,consumer sovereignity“, however, or greater rights for individuals. Rather it is the fact that the 
Pflichten werden besonders hervorgehoben, um die Anspruchshaltung des früheren Leistungsberechtigten (entstanden vor dem Hintergrund einer social citizenship) abzubauen und zu erreichen, dass jeder einzelne Bürger das Nebeneinander von Rechten und Pflichten stärker als zuvor wahrnimmt. ${ }^{152}$ Damit wird der Schritt vom bloßen Bezieher einer Leistung hin zum Kunden mit Rechten und Pflichten vollzogen. ${ }^{153}$

Der Aspekt, dass durch eine Vereinbarung zwischen Bürger und Staat auch Pflichten des Staates begründet oder zumindest betont werden könnten, tritt dagegen zurück. In den Vereinbarungen werden in erster Linie die Pflichten des Einzelnen dargestellt und konkretisiert. Die Pflichten des Staates werden außen vor gelassen. Darin liegt auch der Grund dafür, dass die rechtliche Durchsetzbarkeit der Pflichten des Staates durch den Bürger nicht erwünscht ist und gesetzgeberisch bei der Einführung des $J S A$ auch nicht umgesetzt wurde.

d) Principal of conditionality: empowerment, self-responsibility, control, sanctions

Mit dem sog. principal of conditionality 154 wird der status quo beschrieben. Seit der Einführung des $J S A$ werden die Pflichten des Einzelnen neben seinem Anspruch auf soziale Leistungen betont. Damit wird das Prinzip der social citizenship zugrundegelegt und gleichzeitig dessen Defizit, die fehlende Anbindung sozialer Rechte an Pflichten gegenüber dem Staat, behoben. ${ }^{155}$

Das $J S A$ hat also Bedeutung für die Konkretisierung, welche Maßnahmen der Antragsteller ergreifen muss, um (zurück) in Beschäftigung zu finden (empowerment). Während der Verhandlungen über das $J S A$ werden die persönlichen Defizite des einzelnen Arbeitslosen festgestellt, um Fördermaßnahmen auf ihn ausrichten zu können. Denn die fehlenden Fähigkeiten des Arbeitslosen, seine Arbeitslosigkeit zu beenden, werden als wichtigstes zu behebendes Problem angesehen. ${ }^{156}$

Die Verantwortung des Einzelnen für sein eigenes Leben beziehungsweise Auskommen (self-responsibility) ist Teil der Pflichten, die jedem Bürger zukommen. Marshall bezeichnete sie sehr allgemein als "general obligation to live the life of a good citizen"157, was Teil der Kritik an seinem Ansatz war. ${ }^{158}$ Die Unbestimmtheit dieser Pflichten ist für die Klarheit der Stellung des Bürgers im Staat nicht förderlich. Das JSA wirkt an dieser Stelle und ist Anwendungsbeispiel für einen "new contract between the

parties to the contract have separate interests. [...] Contract thus appears as a way of dissolving the old dilemma of independence versus control by using the former to promote accountability.".

152 Mead, Citizenship and social policy: T.H. Marshall and poverty, in: Paul, The welfare state, 1997, S. $221 \mathrm{f}$.

153 Mather, Government by contract, in: Vibert, Britain's constitutional future, 1991, S. 89.

154 Dwyer, CSP 1998 (57), S. 493-517.

155 King, In the name of liberalism, 1999, S. 232 f, 249, 256.

156 House of Commons Sessions 1995-6, Employment Committee Second Report, The right to workfare (London HMSO 13. Februar 1996) p. XXIV; zit. nach King, In the name of liberalism, 1999, S. 248.

157 Marshall, Citizenship and social class, in: Marshall, Sociology at the crossroads and other essays, 1963, S. 122 f.

158 Vgl. eine Übersicht über die Kritiker bei Dwyer, Understanding social citizenship, 2004, S. 45. 
citizens and the state based on rights and responsibilities", wie es die Regierung formulierte. 159

Ferner können über die niedergeschriebenen Maßnahmen diese Schritte besser überwacht werden (control). Die zuständige Stelle kann regelmäßig und effektiv feststellen, ob der Arbeitslose sich dem vereinbarten Ziel entsprechend verhält. ${ }^{160}$ Dazu wird die Vereinbarung als Grundlage der Gespräche zwischen Antragsteller und Sachbearbeiter des $J C P$ herangezogen. ${ }^{161}$ Bei Nichteinhaltung ist die Vereinbarung nicht nur eine Form bürokratischer Kontrolle, sondern auch „hartes Instrument zur Disziplinierung und Bestrafung"162, denn die Nichteinhaltung von Verpflichtungen führt zu Kürzungen oder Wegfall der Leistungen (sanctions). ${ }^{163}$

Das $J S A$ soll abschreckend (deterrent) wirken. Zum einen führt das Aushandeln der einzelnen Schritte auf dem Weg in Beschäftigung zu einer Kontrolle durch die Behörden. Es wird vermutet, dass sich eine bestimmte Gruppe arbeitsloser Menschen aus dem Leistungsbezug zurückzieht, weil sie sich dieser Kontrolle nicht aussetzen will. ${ }^{164}$

Zum anderen verspricht sich der Gesetzgeber von der Verankerung der Pflichten in einer Vereinbarung und deren Sanktionierung, dass die Androhung der Leistungskürzung bereits die Bereitschaft des Arbeitslosen erhöht, möglichst schnell den Leistungsbezug zu beenden. 165

Ob sich diese Annahmen in der Praxis bestätigen werden, bleibt abzuwarten. Die bislang verfügbaren Evaluierungen haben gezeigt, dass das JSA als solches in dieser Hinsicht keine große Bedeutung erlangt hat. Zwar hat es keine negativen Auswirkungen, doch bleiben die allein auf die Vereinbarung zurückgehenden Erfolge sehr begrenzt. Es wird vor allem das vereinbart, was ohnehin nahe liegt oder getan worden wäre, beziehungsweise Informationen werden gegeben, die auch ohne eine Niederlegung eines $J S A$ hätten gegeben werden müssen. ${ }^{166}$

Bei einzelnen Betroffenen ist anzunehmen, dass die Vereinbarung überflüssig ist, weil der Wille, wieder in Arbeit zu kommen, ohnehin vorhanden ist. So knüpft die Einführung des principal of conditionality an Vorurteile gegen Empfänger sozialer Leistungen an, die keinesfalls in jedem Fall gerechtfertigt sind und insbesondere den ,redlichen Arbeitslosen“" in Verruf bringen. ${ }^{167}$

159 HMSO, New ambitions for our country: A new contract for welfare, Cm 3805, 1998.

160 Wood u.a., Social security legislation 2006, Bd. 2, 2006, S. 80.

161 Child Poverty Action Group, WRB 1996 (134), S. 10.

162 Collins, Regulating contracts, 2002, S. 20.

163 Vgl. zweites Kapitel A II 6b) S. 122 f.

164 King, In the name of liberalism, 1999, S. 248; Wood u.a., Social security legislation 2006, Bd. 2, 2006, S. 79 hält diese Annahme aufgrund fehlender Zahlen nur für einen Wunsch des Gesetzgebers.

165 King, In the name of liberalism, 1999, S. 256.

166 Wood u.a., Social security legislation 2006, Bd. 2, 2006, S. 68.

167 Dazu Dwyer, CSP 1998 (57), S. 514. 
a) Voraussetzung für den Leistungsbezug

\section{aa) Anspruchsvoraussetzung}

Der Abschluss eines JSA und dessen wirksames Fortbestehen während des Leistungsbezugs ist Voraussetzung für den Bezug finanzieller Unterstützung aus der jobseeker's allowance. ${ }^{168}$ Es wird keine soziale Leistung ausgezahlt, solange und sobald kein formgerechtes $J S A$ (mehr) vorliegt. Dies gilt insbesondere für Zeiten, in denen wegen Meinungsverschiedenheiten zwischen Antragsteller und Mitarbeiter des JCP das vorgeschlagene $J S A$ von dritter Stelle überprüft wird. ${ }^{169}$ Davon gibt es Ausnahmen, die im Folgenden beschrieben werden.

\section{bb) Fiktion eines JSA}

Eine Ausnahme ist die Fiktion eines JSA. Der Antragsteller wird so behandelt, als hätte er ein $J S A$ abgeschlossen. ${ }^{170}$ Die Fiktion ist für Situationen vorgesehen, in denen sich das Fehlen der Vereinbarung nicht zu Lasten des Antragstellers auswirken soll, insbesondere weil die tatsächlichen Entwicklungen die verwaltungsmäßigen Abläufe überholen.

Hat eine Person einen Antrag auf Erteilung einer jobseeker's allowance gestellt, und werden die Ergebnisse des Gespräches mit dem employment officer in einem JSA festgehalten, wird für den Leistungsbeginn auf den Zeitpunkt der Antragstellung abgestellt. Die Sozialleistung kann rückwirkend ab diesem Zeitpunkt gewährt werden, nicht erst ab Unterzeichnung des $J S A .{ }^{171}$

Erledigt sich der Antrag, z.B. durch Arbeitsantritt, bevor die Möglichkeit bestand, ein $J S A$ abzuschließen, wird der Antragsteller ebenfalls so behandelt, als hätte ein $J S A$ bereits zum Zeitpunkt der Antragstellung vorgelegen. ${ }^{172}$

Daneben gibt es eine Auffangvorschrift, die eine Fiktion des JSA vorsieht. Gerät der normale Ablauf im JCP aus Gründen, die nicht im Verantwortungsbereich des Antragstellers entstanden sind, ins Stocken und ergeben sich Verzögerungen, bleibt das Fehlen des $J S A$ folgenlos. ${ }^{173}$ Das ist z.B. der Fall, wenn eine Flut von Anträgen auf Erteilung einer jobseeker's allowance eingeht, weil ein Hauptarbeitgeber einer Region Massenentlassungen vornehmen muss, sodass eine rasche Abarbeitung aller Anträge aus Kapazitätsgründen vorübergehend nicht möglich ist. ${ }^{174}$

168 Jobseekers Act 1995 sec. 1 subs. 2 (b).

169 Dazu zweites Kapitel A II 9c) S. 132 ff.

170 Jobseekers Act 1995 sec. 19 subs. 10; SI 1996/207 reg. 34.

171 SI 1996/207 reg. 34 (a).

172 SI 1996/207 reg. 34 (b)

173 SI 1996/207 reg. 34 (d).

174 Wood u.a., Social security legislation 2006, Bd. 2, 2006, S. 68. 
Neben der Fiktion des Abschlusses eines JSA gibt es Regelungen, nach denen das $J S A$ zurückdatiert werden kann (back-dating). Es ist eine entsprechende Entscheidung erforderlich. Der Antragsteller wird bei der Erteilung der jobseeker's allowance so behandelt, als hätte das erst später abgeschlossene JSA zum Zeitpunkt der Antragstellung bereits vorgelegen.

Dies ist entweder der Fall, weil das JSA nach dem Antrag unterzeichnet wird, ohne dass Meinungsverschiedenheiten über den Inhalt entstanden sind, z.B. wenn wegen Überlastung des employment officer kein früherer Gesprächstermin gefunden werden konnte. 175

Ferner kann ein decision maker 176 die Rückdatierung anordnen, wenn Meinungsverschiedenheiten über den Inhalt des $J S A$ entstanden sind. ${ }^{177}$ Er kann dies nach Berücksichtigung aller relevanten Umstände tun, wenn sich der Antragsteller zu Recht gegen das vorgeschlagene $J S A$ gewehrt hat, oder andere Zweifel über den Inhalt der Vereinbarung zu klären waren. ${ }^{178}$

\section{b) Verhängung von Sanktionen gegenüber dem Antragsteller}

Da ohne JSA eine Voraussetzung der jobseeker's allowance fehlt, sodass keine finanzielle Unterstützung ausgezahlt wird, besteht für weitere Konsequenzen einer Weigerung des Antragstellers, ein JSA abzuschließen, kein Bedarf. Das JSA ist Voraussetzung für den Leistungsbezug. Deshalb wird dem Antragsteller von Seiten von Interessenvertretern der Arbeitslosen empfohlen, auf jeden Fall ein JSA abzuschließen und bei Unzufriedenheit über die enthaltenen Verpflichtungen eine Abänderung anzustreben. Wichtig ist, dass bei der Verwaltung keine Zweifel an der Bereitschaft des Antragstellers darüber entstehen, dass er (zumindest bis zu einer endgültigen Entscheidung) das JSA einhalten und die labour market conditions erfüllen wird. Ansonsten besteht die Gefahr von Sanktionen. ${ }^{179}$

Sanktionen können allerdings nicht allein aufgrund der Nichteinhaltung des $J S A$ verhängt werden. Dafür fehlt ein entsprechender Mechanismus im Gesetz. Sanktionen werden vielmehr angeknüpft an das Verhalten des Arbeitsuchenden. Es muss noch ein Zwischenschritt zwischen Nichteinhaltung der Vereinbarungen aus dem JSA und der Sanktion erfolgen. ${ }^{180}$

Dieser Zwischenschritt ist z.B. eine sog. jobseeker's direction, eine einseitig angeordnete Weisung des employment officers. ${ }^{181}$ Erfüllt ein Arbeitsloser die ihm gegenüber verhängten Weisungen des employment officers ohne rechtfertigenden Grund nicht, er-

175 Jobseekers Act 1995 sec. 9 subs. 11; SI 1996/207 reg. 35.

176 Vgl. zweites Kapitel A II 9 S. 131 f.

177 Jobseekers Act 1995 sec. 9 subs. 6, 7 (c).

178 SI 1996/207 reg. 32.

179 Child Poverty Action Group, Welfare benefits and tax credits handbook, 2005, S. 371.

180 Jobseekers Act 1995 sec. 19 subs. 5.

181 Dazu Child Poverty Action Group, Welfare benefits and tax credits handbook, 2005, S. 423. 
hält er für bis zu 26 Wochen keine jobseeker's allowance und damit keine soziale Leistung. ${ }^{182}$ Gleiches gilt, wenn der Arbeitsuchende wegen Fehlverhaltens seinen Platz in einer Trainingsmaßnahme verliert. ${ }^{183} \mathrm{Im} J S A$ ist die Teilnahme an der Trainingsmaßnahme verbindlich vereinbart, nicht aber die Sanktion bei Verlust des Platzes. Die jobseeker's allowance und damit der Leistungsbezug enden also erst, wenn die Trainingsmaßnahme nicht mehr weitergeführt wird.

Aus gen angeführten Beispielen ergibt sich, dass Sanktionen entweder gesetzlich angeordnet sind oder im Einzelfall in einem eigenständigen Rechtsakt verhängt werden, dass sie aber nicht unmittelbar auf der Grundlage des $J S A$ eintreten.

\section{c) Auslegung (unbestimmter) Rechtsbegriffe und Ermessensausübung (discretion)}

Im Rahmen der Entscheidung über die Erteilung einer jobseeker's allowance sind verschiedene Voraussetzungen zu prüfen. Zentral sind die sog. labour market conditions. ${ }^{184}$ Bei der Auslegung 185 dieser Begriffe ist der Inhalt des JSA zu beachten. Ein decision maker ${ }^{186}$ kann sich über die dem Abschluss eines JSA zugrundeliegende Willenseinigung nicht ohne weiteres hinwegsetzen. Wird beispielsweise im JSA ausdrücklich behandelt, wie der Arbeitslose wieder Arbeit finden soll, kann der Begriff actively seeking employment nur so verstanden werden, wie ihn das JSA versteht. Das gilt auch dann, wenn das bedeutet, dass überhaupt keine Arbeitsuche stattfindet, wenn diese im Einzelfall nicht sinnvoll wäre. Es ist bislang nicht endgültig geklärt, ob das $J S A$ abschließend ist und kein darüber hinausgehender Aspekt mehr berücksichtigt werden darf. 187

Ähnliches gilt bei Entscheidungen im Zusammenhang mit der jobseeker's allowance, die im Ermessen der Verwaltung stehen. Dort können für den Nachweis von Verschulden des Antragstellers die Formulierungen des JSA herangezogen werden. Es besteht insoweit die Vermutung, dass sich der Antragsteller über die Bedeutung des $J S A$ im Klaren war und ihm nun sein dem $J S A$ entgegengesetztes Verhalten bewusst ist. Das gilt auch für die Entscheidung über das Vorliegen eines Grundes (good cause), der der Durchsetzung einer jobseeker's direction entgegenstehen kann. ${ }^{188}$ Bei der Prüfung, ob diese erlassen werden darf, sind alle Umstände des Einzelfalles zu berücksichtigen. 189 Auch hier liefert das im JSA Vereinbarte die relevanten Anhaltspunkte für eine nachfolgende Entscheidung. 190

182 Jobseekers Act $1995 \mathrm{sec} .19$ subs. 2, 5 (a), 10.

183 Jobseekers Act 1995 sec. 19 subs. 5 (c).

184 Dazu zweites Kapitel A I 2 S. 100 ff.

185 Kirby, SLR 2003 (24), S. 95 ff.

186 Vgl. zweites Kapitel A II 9 S. $131 \mathrm{f}$.

187 So ausdrücklich CJSA/2162/2001 S. 11; dazu Wood u.a., Social security legislation 2006, Bd. 2, 2006, S. 69.

188 Jobseekers Act 1995 sec. 19 subs. 5 (a).

189 Jobseekers Act $1995 \mathrm{sec} .9$ subs. 7 (b).

190 Wood u.a., Social security legislation 2006, Bd. 2, 2006, S. 68 f. 
d) Ausschluss weiterer Wirkungen

Ein JSA hat nur Wirkungen für den Zweck der Beantragung einer jobseeker's allowance. ${ }^{191}$ Weitergehende rechtliche Konsequenzen ergeben sich nicht, insbesondere kann nicht auf privatrechtlichem Wege gegen die Nichteinhaltung der Vereinbarung vorgegangen werden. Das wurde auch in der bislang einzigen explizit zum JSA ergangenen Entscheidung des Social Security Commissioners für die jobseeker's allowance bestätigt. 192

\section{Störungen bei der Abwicklung des vertragsähnlichen Verhältnisses}

\section{a) Veränderung der tatsächlichen Verhältnisse}

Das JSA kann durch Vereinbarung zwischen dem Antragsteller und dem employment officer jederzeit abgeändert werden. ${ }^{193}$ Sinn und Zweck dieser Abänderungsmöglichkeit ist es, eine flexible Anpassung der Vereinbarung an die Veränderungen auf dem Arbeitsmarkt oder an die Situation des Antragstellers zu ermöglichen. ${ }^{194}$ Die Abänderungsvereinbarung muss in Schriftform verfasst und unterschrieben sein. ${ }^{195}$ Auch von der Abänderungsvereinbarung muss der Antragsteller eine Kopie erhalten. ${ }^{196}$ Es findet wiederum eine Inhaltskontrolle durch den employment officer statt, in der überprüft wird, ob die Vereinbarung nach der Abänderung noch den (Kern-)Voraussetzungen der jobseeker's allowance entspricht, nämlich ob der Antragsteller, verhält er sich entsprechend der Vereinbarung, als zur Erwerbsarbeit bereit und als aktiv arbeitsuchend angesehen werden kann. ${ }^{197}$

Hinsichtlich der Abänderungsvereinbarung kann der Mitarbeiter des JCP eine Entscheidung des decision maker/Secretary of State herbeiführen, wenn Zweifel an der Zulässigkeit der Abänderung bestehen. ${ }^{198}$ Verlangt dies der Antragsteller ausdrücklich, muss eine Entscheidung des decision maker/Secretary of State folgen. Während der Zeit, in der der decision maker über die Anfrage entscheidet, bleibt das frühere JSA in Kraft und muss auch eingehalten werden. 199

Erfüllen sowohl der Vorschlag des employment officer als auch der Vorschlag des Antragstellers die Voraussetzungen der labour market conditions (available for work, actively seeking work), muss der decision maker ${ }^{200}$ dem Vorschlag des Antragstellers

191 Jobseekers Act 1995 sec. 9 subs. 2.

192 CJSA/2162/2001 S. 9; so auch Wikeley/Ogus, The law of social security, 2002, S. 351; Wood u.a., Social security legislation 2006, Bd. 2, 2006, S. 68.

193 Jobseekers Act 1995 sec. 10 subs. 1.

194 Vincent-Jones, The new public contracting, 2006, S. $238 \mathrm{f}$.

195 Jobseekers Act 1995 sec. 10 subs. 2; SI 1996/207 reg. 37.

196 Jobseekers Act 1995 sec. 10 subs. 3.

197 Jobseekers Act 1995 sec. 10 subs. 4.

198 Jobseekers Act 1995 sec. 10 subs. 5.

199 Child Poverty Action Group, Welfare benefits and tax credits handbook, 2005, S. 372.

200 Vgl. zweites Kapitel A II 9 S. 131 f. 
Vorrang einräumen. ${ }^{201}$ Der decision maker kann den Abschluss einer Vereinbarung bestimmten Inhalts anweisen. ${ }^{202}$ Der Antragsteller hat dann 21 Tage Zeit, diesen Vorschlag anzunehmen. Tut er dies nicht, wird auch das frühere $J S A$ außer Kraft gesetzt. ${ }^{203}$ Folge ist, dass die jobseeker's allowance wegfällt und der Antragsteller (außer bei Vorliegen eines Härtefalls) auch während der (gerichtlichen) Überprüfung kein Geld erhält.

\section{b) Nichteinhaltung der Vereinbarungen aus dem JSA durch das JCP}

Der hier zu behandelnde Störungsfall tritt ein, wenn ein abgeschlossenes JSA, das weder beendet noch aus einem anderen Grund keinen Bestand mehr hat, inhaltlich durch die Behörde nicht erfüllt wird. Das kann z.B. der Fall sein, weil sie anders als der Antragsteller die versprochenen Maßnahmen nicht mehr für sinnvoll hält ${ }^{204}$, oder weil es ihr aus bestimmten Gründen nicht möglich ist, das Versprochene einzuhalten.

Die Nichteinhaltung einer vertraglich übernommenen Pflicht stellt im contract law einen Vertragsbruch dar (breach of contract). Es stellt sich nun die Frage, welche Rechtsfolgen die Nichteinhaltung eines quasi-contracts hat. Dieses Problem ist im Jobseekers Act 1995 und den zugehörigen Regulations nicht geregelt.

Im common law gilt, dass der Staat nur unter bestimmten Voraussetzungen zur Erfüllung von Pflichten gegenüber dem Einzelnen verpflichtet ist. Liegt ein solcher Fall nicht vor, kann der Antragsteller auch keine gerichtliche Durchsetzung der Pflichten bewirken. Denn er hat nur einen rechtlichen Anspruch auf eine öffentliche Leistung, wenn die Gerichte aus der gesetzlichen Formulierung die Entstehung eines private right erkennen und daraus eine Pflicht zur Erfüllung dieser Pflicht ableiten. ${ }^{205}$

\section{aa) Doctrine of executive necessity}

Die Anwendung der im contract law entwickelten doctrine of executive necessity 206 hätte zur Folge, dass die Verwaltung unter bestimmten Voraussetzungen die Vereinbarung brechen dürfte. Die Verwaltung kann nicht zur Einhaltung eines Vertrages gezwungen werden, wenn dagegen schwerwiegende Gründe sprechen. Die Gerichte gehen davon aus, dass hinter der Nichteinhaltung des Vertrages eine politische Entscheidung stehen muss, nicht die bloße Unzufriedenheit mit dem Vertragspartner oder dem Vertragsinhalt. ${ }^{207}$ Die Anwendung der doctrine of executive necessity darf auch im common law nicht dazu führen, dass die Folgen der Nichteinhaltung einer Pflicht aufgrund

201 Jobseekers Act 1995 sec. 10 subs. 7 (a); SI 1996/207 reg. 39; sowie para. 21951 Decision makers guide (DMG), einsehbar unter http://www.dwp.gov.uk/publications/dwp/dmg/index.asp.

202 Jobseekers Act 1995 sec. 10 subs. 6 (b).

203 Jobseekers Act 1995 sec. 10 subs. 6 (c); SI 1996/207 reg. 38.

204 Der Widerspruch zum Antragsteller ist erforderlich, weil sonst unproblematisch eine Abänderungsvereinbarung geschlossen werden könnte. Vgl. zweites Kapitel A II 7a) S. 124.

205 Harden, The contracting state, 1992, S. 39.

206 Vgl. erstes Kapitel B III 3b) bb) (2) S. 78.

207 Seddon, Government contracts, 1999, S. 169 ff. 
eines die gesamte Verwaltung betreffenden Grundes von einem Einzelnen getragen werden müssen. 208

Unabhängig von der Antwort auf die Frage der Anwendbarkeit dieser Lehre auf vertragsähnliches Verwaltungshandeln, dürfte die Möglichkeit der Verwaltung, diese besondere Situation für das Wohlergehen des Staates anzunehmen, sehr selten sein. $\mathrm{Ihr}$ Eintritt ist aber durchaus möglich, insbesondere angesichts beschränkter Budgets für aktive Arbeitsmarktpolitik.

Folge der Anwendung der Regel wäre, dass die im $J S A$ vereinbarten Leistungen entfielen und damit gerade der Einzelne belastet wäre. Da es sich beim $J S A$ allein um eine Leistungsvoraussetzung für die jobseeker's allowance handelt und sich keine weiteren Folgen aus dem JSA ergeben dürfen, bleibt für die doctrine of executive necessity kein Anwendungsbereich. Die Verwaltung ist bereits durch den gesetzlichen Ausschluss weiterer Wirkungen davor geschützt, ohne vorhandene Ressourcen auf einer vertragsähnlichen Grundlage Leistungen erbringen zu müssen.

\section{bb) Rule against fettering future executive action}

Ferner ist zu prüfen, ob sich die Verwaltung, obwohl common law grundsätzlich keine Anwendung findet, auf die rule against fettering future executive action ${ }^{209}$ berufen kann.

Die Regel besagt, dass die Verwaltung nicht durch Verträge bei der Ausübung ihrer zukünftigen Verwaltungstätigkeit eingeschränkt werden darf. Die Rechtsfolgen ihrer Anwendung sind nicht abschließend geklärt. Verträge werden als (teilweise) unwirksam betrachtet oder als wirksam, aber nicht durchsetzbar. Sie werden auch als schlechthin wirksam und durchsetzbar angesehen und hätten dann die Nachteile, die für den Vertragspartner aus der Nichteinhaltung durch die Verwaltung resultieren. Damit verbunden ist die Diskussion, ob mit einer Rückabwicklung wegen unrechtmäßiger Bereicherung (unjust enrichment) zu rechnen ist. ${ }^{210}$

Für Entscheidungen der Arbeitsverwaltung gilt, dass die Festlegungen des $J S A$ bei der Subsumtion unter Gesetzesbegriffe heranzuziehen sind. ${ }^{211}$ Die jobseeker's allowance wird insofern bei Vorliegen der Voraussetzungen ohne weitere Ermessensausübung erteilt. Für die Heranziehung der rule against fettering future executive action besteht deshalb kein Bedarf.

c) Nichteinhaltung der Vereinbarungen aus dem JSA durch den Antragsteller

In Folge der Einordnung als vertragsähnliches Verwaltungshandeln entsteht auch auf Seiten des Arbeitslosen keine rechtliche Bindung. Der Störungsfall der Nichteinhaltung der Vereinbarungen aus dem $J S A$ durch den Arbeitslosen ist deshalb bereits geklärt: Das $J S A$ ist für ihn nicht nach common law Grundsätzen bindend. Die Nichteinhaltung der

208 Mitchell, The contracts of public authorities, 1954, S. 77 f, der damit begründet, dass es eine Pflicht zur Kompensation gibt.

209 Vgl. erstes Kapitel B III 3b) bb) (3) S. 79.

210 Seddon, Government contracts, 1999, S. $177 \mathrm{ff}$.

211 CJSA/2162/2001 S. 11. 
Vereinbarung hat ohne weitere Zwischenschritte, v.a. ohne die Erteilung einer jobseeker's direction ${ }^{212}$, keine Konsequenzen.

Die Tatsache, dass die Nichteinhaltung des JSA durch den Antragsteller nicht automatisch sanktioniert wird, ist zugleich Folge der Einordnung des JSA als social contract, der statt einer direkten Wirkung vor allem indirekt Einfluss auf das Verhalten der Vertragsparteien hat.

\section{d) Restitution}

Erlangt eine Partei aufgrund besonderer Umstände auf Kosten der anderen Vertragspartei unrechtmäßig einen Vorteil, kommt im common law eine Klage auf restitution in Betracht. Bei erfolgreichem Verfahren wird das Erlangte ausgeglichen.

Für das $J S A$ wird diese Situation kaum diskutiert. ${ }^{213}$ Die umfangreich diskutierten Probleme des sog. NHS contracts $^{214}$ sind aber vergleichbar. ${ }^{215}$ NHS contracts sind Vereinbarungen zwischen zwei Institutionen des National Health Service, einem Anbieter einer Gesundheitsleistung (provider) und dessen Käufer (purchaser), der gegenüber dem einzelnen Patienten zur Erbringung der Leistung verpflichtet ist. Diese Vereinbarungen unterliegen, wie oben bereits erwähnt ${ }^{216}$, per Gesetz nicht dem contract law und werden deshalb auch nicht vor den common law Gerichten verhandelt, sondern in einem administrativen Verfahren entschieden, das detailliert im Gesetz geregelt ist.

Die Zulassung einer Klage auf Ausgleich unrechtmäßig zustande gekommener Vermögensverschiebungen würde dazu führen, dass zwar der NHS contract nicht vor die common law Gerichte gebracht werden kann, dass aber diese bei Störungsfällen über die Klage auf restitution entscheiden müssten.

In der Diskussion zu den NHS contracts wurden Kriterien für die Prüfung entwickelt, die hier angeführt und auf das $J S A$ angewendet werden. So wird Sinn und Zweck des Ausschlusses von zivilrechtlichen Ansprüchen auf Grundlage des JSA deutlich.

\section{aa) Grundsätzliche Zulässigkeit einer Klage auf restitution}

Die Klage auf restitution ${ }^{217}$ setzt voraus, dass kein wirksamer oder zumindest kein durchsetzbarer Vertrag (incomplete bzw. legally unenforceable contract) vorliegt, der Grundlage für Ansprüche sein könnte. Ferner muss der Kläger beweisen, dass eine „Bereicherung“ (enrichment) auf Seiten der beklagten Partei vorliegt, die auch einen Vermögenswert (gain) haben muss. Zudem muss der Kläger einen besonderen Klagegrund (restitutionary cause of action) geltend machen können, der begründet, warum es un-

212 Vgl. zweites Kapitel A II 6b) S. 122 f.

213 Zur Anwendbarkeit eines claim on restitution auf public functionaries vgl. Cane, Administrative law, 2004, S. 316-318.

214 Dazu einführend Harden, The contracting state, 1992, S. 42 f.

215 Grundsätzlich dazu Hughes, MLR 1991 (54), S. 88 ff; Barker, MLR 1993 (56), S. 832 ff; Allen, MLR 1995 (58), S. 321 ff; Bennet/Ferlie, Pub Admin 1996 (74), S. 49 ff.

216 Vgl. zweites Kapitel A II 4d) S. 115.

217 Allgemein dazu Birks, An introduction to the law of restitution, 1989, S. 9-16; 294-299 mit den Nachweisen zum case law. 
gerecht wäre (unjust), wenn der Beklagte die „Bereicherung“ behalten dürfte. Denn die Rückabwicklung eines bereits durchgeführten Vertrages ist im common law grundsätzlich nicht möglich, es sei denn, die bereicherte Partei kannte die Unwirksamkeit des Vertrages und zöge ohne Rückabwicklung daraus Vorteile. Zuletzt steht dem Erfolg einer Klage entgegen, dass der Gesetzgeber die „Bereicherung“ gerade in Kauf genommen hat (policy bar ${ }^{218}$ ), sodass eine Rückabwicklung gegen den Willen des Gesetzgebers verstoßen würde. Hierbei handelt es sich um den ausführlich zu besprechenden Ausschluss einer Rückabwicklung aufgrund einer gesetzgeberischen Entscheidung.

Diese Voraussetzungen sind auf ihre Anwendbarkeit auf das $J S A$ hin zu überprüfen, weil es sich beim $J S A$ um keinen wirksamen oder durchsetzbaren Vertrag handelt, sondern um einen quasi-contract, der rechtlich nicht durchsetzbar ist.

Es gibt verschiedene Situationen, in denen eine Vermögensverschiebung mit Vermögenswert angenommen werden kann. Z.B. ergibt sich eine Vermögensverschiebung, wenn sich der Antragsteller auf eine Trainingsmaßnahme, die der employment service versprochen hat, vorbereitet, indem er auf eigene Rechnung einen vorbereitenden Kurs belegt. Er verbessert damit seine Vermittlungschancen, was der Behörde diese Qualifizierungsmaßnahme erspart, und trägt dafür selbst die Kosten. Lehnt die Behörde später entgegen des Vereinbarten ab, die Trainingmaßnahme anzubieten, hat sie diese Verbesserung in den Vermittlungsaussichten des Antragstellers erlangt. Diese haben einen Vermögenswert, nämlich die Kosten der Maßnahme, die der Arbeitslose selbst bezahlt hat.

Es ist nun zu fragen, ob dem Antragsteller diese Kosten erstattet werden. Dies richtet sich letztendlich danach, ob ein common law Gericht die Klage aufgrund eines policy bar für ausgeschlossen erachten wird oder nicht.

\section{bb) Ausschluss der Klage aufgrund einer Entscheidung des Gesetzgebers (policy bar)}

Beim Ausschluss der Klage wurden im Bezug auf die NHS contracts verschiedene Prinzipien ausgemacht, die zu prüfen sind. 219

Es kommt nicht darauf an, weshalb der Vertrag keine Grundlage für Ansprüche sein kann. $\mathrm{Ob}$ er wegen einer gesetzlichen Bestimmung unwirksam oder nur nicht durchsetzbar ist, ist unerheblich. Vielmehr ist zu prüfen, ob der Zweck des Gesetzes, welches die Unwirksamkeit/Nichtdurchsetzbarkeit anordnet, unterlaufen wird, wenn eine Klage Erfolg hätte. Dabei ist zu beachten, dass ein Unterlaufen des Gesetzeszweckes vorliegen kann, obwohl das Gesetz keinen ausdrücklichen Ausschluss einer Klage auf restitution enthält. 220

Welche Überlegungen sind also dem Ausschluss der Anwendung des contract law vorausgegangen? Der Ausschluss der Anwendung von contract law wird damit begründet, dass es im Jobseekers Act 1995 sec. 9 subs. 2 heißt, dass das $J S A$ nur Wirkung für die Beantragung einer jobseeker's allowance entfaltet. Diese Formulierung enthält kei-

218 Vgl. zweites Kapitel A II 7d) bb) S. 127.

219 Barker, MLR 1993 (56), S. 837 ff.

220 Dazu m.w.N. Barker, MLR 1993 (56), S. 839. 
nen ausdrücklichen Ausschluss, was der Annahme eines policy bar aber nicht entgegensteht.

Die Gründe, die über diese gesetzliche Vorschrift hinaus für die Annahme eines policy bar sprechen, sind zahlreich. Im Ergebnis würde die Zulassung einer Klage auf restitution den Zweck der ausschließlichen Wirkung des JSA für die jobseeker's allowance unterlaufen.

Zum einen, und das dürfte der wichtigste Grund für den Ausschluss der Klage sein, handelt es sich bei den zu erwartenden Streitigkeiten um solche, die der Gesetzgeber besser im administrativen Verfahren aufgehoben glaubt. ${ }^{221}$ An der Entscheidung über die jobseeker's allowance ist auf Verwaltungsebene nur der employment service beziehungsweise das DWP beteiligt. Common law Gerichte würden über Fragen entscheiden, für die sich gerade das besondere Verfahren vor administrative tribunals entwickelt hat. Die Annahme der Klage unterliefe die Entscheidung des Gesetzgebers, die er durch die ausdrückliche Regelung des Verfahrens für die Auslegung des Jobseekers Act 1995 zum Ausdruck gebracht hat.

Hier - wie bei den NHS contracts - besteht die Gefahr, dass der Rechtsweg (common law Gericht oder tribunal) von der Formulierung der Klage und somit vom Vorbringen der Streitparteien abhinge. Macht der Kläger allein die Nichteinhaltung des JSA geltend, müsste er einen appeal formulieren (d.h. Zuständigkeit eines Social Security Appeal Tribunal). Stellt er auf eine Bereicherung der Behörde ab, käme die Klage auf restitution in Betracht (d.h. Zuständigkeit eines common law Gerichtes). Ob der Antragsteller die Klage auf restitution überhaupt erheben kann, ist wiederum eine Frage des Zeitpunkts, in dem der Konflikt mit der Behörde auftritt, und folglich in gewisser Weise dem Zufall überlassen. Dadurch wird der Anschein eines willkürlichen Verfahrens erweckt und damit das Vertrauen in eine geordnete Rechtsprechung erschüttert. ${ }^{22}$

Die zwangsläufige Aufspaltung der Zuständigkeiten bei Zulassung dieser Klageart brächte zudem unerwünschte Probleme für Rechtssicherheit und Klarheit der Verfahren mit sich.

Unabhängig von der Verfahrensaufspaltung ist bei Unzulässigkeit einer Klage auf restitution sichergestellt, dass die Wirkungen des $J S A$ tatsächlich beschränkt sind. Damit wird der besonderen Situation bei der Entstehung des JSA Tribut gezollt und zum Schutze des Antragstellers eine zu große Bedeutung des $J S A$ für verschiedenste andere Zwecke verhindert. Denn der Ausschluss von zivilrechtlichen Klagen gegen ihn auf Grundlage des $J S A$ bedeutet auch für den Antragsteller eine Beschränkung seiner Haftung. Die Zwangslage, in der er das JSA unterzeichnet, wirkt sich damit nur auf die jobseeker's allowance aus. Eine weitergehende Haftung ist ausgeschlossen.

221 Vgl. die Diskussion über public law und private law im ersten Kapitel B III 3b) dd) S. 55 ff.

222 Barker, MLR 1993 (56), S. 841. 
a) Erledigung des Antrags auf jobseeker's allowance

Das JSA entfaltet keine Wirkungen mehr, wenn sich der Antrag des Antragstellers erledigt hat. 223

Die Ausnahmen zu diesem Grundsatz sind abschließend aufgeführt. ${ }^{224}$ Es handelt sich dabei in SI 1996/207 reg. 36 (a) um die Situation, dass bereits innerhalb von 14 Tagen ein weiterer Antrag auf Erteilung einer jobseeker's allowance gestellt ist, so dass die erneute Abfassung eines $J S A$ keine neuen Erkenntnisse erwarten lässt.

SI 1996/207 reg. 36 (b) enthält die Regelung, dass bei einer zeitweisen Aufhebung (period of suspension) der jobseeker's allowance durch den Secretary of State das einmal abgeschlossene JSA für die Dauer der außer Kraft Setzung der jobseeker's allowance aus Vereinfachungsgründen wirksam bleiben soll.

SI 1996/207 reg. 36 (c) regelt, dass das JSA auch im Falle von Leistungen aus der früheren Sozialversicherung (national insurance credits) relevant bleiben und dass deshalb die Erledigung des Antrags nicht zur Beendigung des $J S A$ führen soll (Übergangsrecht).

b) Einvernehmliche Abänderung des JSA

Zudem führt die Abänderung des $J S A$ gem. Jobseekers Act $1995 \mathrm{sec} .10$ zu einer Beendigung der Ausgangsvereinbarung. Hierbei handelt es sich um eine einvernehmliche Abänderung, die unter denselben Voraussetzungen wie eine erstmalige Vereinbarung zustande kommen muss. 225

\section{c) Angeordnete Abänderung}

Daneben wird das JSA durch die Entscheidung des Secretary of State beendet, wenn dieser ein neues $J S A$ anderen Inhalts vorschlägt. Dieses soll dann zwischen employment officer und Antragsteller abgeschlossen werden. Der Secretary of State wird von den Parteien angerufen und regt einen anderen Inhalt an, weil es zwischen den Parteien Meinungsverschiedenheiten über den zu vereinbarenden Inhalt gab. Es handelt sich um eine Abänderung des $J S A$ in Folge verwaltungsinterner Kontrollwege. 226

d) Keine einseitige Beendigung

Weitere Möglichkeiten gibt es nicht, das JSA zu beenden, insbesondere kann das JSA nicht auf andere Weise einseitig beendet werden. Die Social Security Commissioners führten aus, dass, wäre eine einseitige Beendigung möglich, das $J S A$ keine substantielle Bedeutung (substantive effect) haben könne. Dies sei vom Gesetzgeber nicht ge-

223 Jobseekers Act 1995 sec. 9 subs. 12.

224 SI 1996/207 reg. 36.

225 Vgl. zweites Kapitel A II 7a) S. $124 \mathrm{f}$.

226 Vgl. zweites Kapitel A II 9c) S. 132 ff. 
wünscht. Ferner würde das Verfahren zur Beendigung nach den Vorgaben des Gesetzgebers durch Anerkennung weitergehender Beendigungsmöglichkeiten unterlaufen. ${ }^{227}$

\section{Rechtsschutz}

\section{a) Belehrung}

Das JSA muss gem. SI 1996/207 reg. 31(g) eine Belehrung über die (Verfahrens-) Rechte des Antragstellers (statement on the claimant's right) enthalten. Diese klärt den Antragsteller darüber auf, dass er eine vorgeschlagene Vereinbarung an den Secretary of State weiterreichen und hinsichtlich jeder Festlegung oder Anweisung derselben Stelle deren Überprüfung verlangen kann. Ferner hat er die Möglichkeit, vor ein appeal tribunal zu ziehen, um eine Festlegung oder Anweisung des Secretary of State überprüfen zu lassen.

Fehlt die Belehrung, was angesichts der üblichen Verwendung von Standardformularen für das $J S A$ selten sein dürfte, kann der Rechtsbehelf verspätet (sog. late appeal) eingelegt werden. Die Unkenntnis einer Frist reicht als Rechtfertigung dafür aus, dass man die Frist nicht eingehalten hat. Bei nachgewiesener Kenntnis gibt es keine gesetzliche Verlängerung der Frist oder eine Wiedereinsetzung. ${ }^{228}$

b) Ausschluss einer private law procedure

Contract law findet auf das JSA selbst keine Anwendung. Auch ohne die Anwendbarkeit von contract law ist es aber möglich, dass im Wege einer private law procedu$r e^{229}$ Rechtsschutz gewährt wird. Für public law contracts gilt grundsätzlich die private law procedure, es sei denn die gesamte Situation ist vom public law geprägt. Besteht eine Mischsituation, in der Elemente des private law und des public law zu finden sind, ist zu prüfen, ob das public law ein Verfahren zum judicial review vorsieht und kein Missbrauch des Verfahrens droht. 230

Für Streitigkeiten über das JSA selbst ist ein judicial review nicht vorgesehen. Das Vertragsmodell wie aus dem contract law bekannt, ist zwar Hintergrund für die im JSA enthaltenen Rechte und Pflichten. Es wird jedoch durch die Ausgestaltung im Jobseekers Act 1995 stark modifiziert. Der Gesetzgeber hat, wie noch darzustellen ist, den Rechtsschutz im Jobseekers Act 1995 ausführlich und abschließend geregelt.

Insbesondere besteht Einigkeit darüber, dass die Formulierung im Jobseekers Act 1995 sec. 9 subs. 2 so zu verstehen ist, dass alle privatrechtlichen Ansprüche und damit auch eine private law procedure ausgeschlossen sind.231

\section{CJSA/2162/2001 S. 7.}

228 Child Poverty Action Group, Welfare benefits and tax credits handbook, 2005, S. 1263.

229 Einführend dazu Walker/Ward, Walker\&Walker's English legal system, 1994, S. 277 ff; Slapper/Kelly, Sourcebook on the English legal system, 2001, S. $197 \mathrm{ff}$.

230 Brownsword, Contract law: Themes for the twenty-first century, 2000, S. 197-208.

231 Vgl. zweites Kapitel A II 6d) S. 124. 
c) Verwaltungsinterne Kontrolle: decision making, revision, supersession

\section{aa) Decision making}

Der employment officer kann einen decision maker anrufen, der bei Meinungsverschiedenheiten zwischen ihm und dem Antragsteller entscheidet. ${ }^{232}$ Es handelt sich dabei um eine verwaltungsinterne Kontrolle. Den decision makers wurde zwar zunächst eine Existenz in einer ,twilight world " 233 bescheinigt, weil sie einerseits wie die Judikative Entscheidungen der Verwaltung überprüften, andererseits aber dieselben Aufgaben haben wie die Verwaltung selbst, nämlich Entscheidungen über soziale Leistungen $\mathrm{zu}$ treffen. Inzwischen ist geklärt, dass decision making keine Ausübung judikativer Gewalt ist, sondern als verwaltungsinterne Kontrolle anzusehen ist. ${ }^{234}$

Die Meinungsverschiedenheiten zwischen employment officer und Antragsteller bestehen in erster Linie darüber, ob der Antragsteller der jobseeker's allowance arbeitsfähig (available for work) ist und/oder aktiv Arbeit sucht (actively seeking employment) beziehungsweise ob erwartet werden kann, dass ein JSA diesen Inhalts vom Antragsteller unterzeichnet wird. ${ }^{235}$ Verlangt der Antragsteller diese Überprüfung, muss der employment officer den decision maker anrufen. ${ }^{236}$

Beim decision maker handelt es sich um einen civil servant, einen Mitarbeiter des employment service, der innerhalb der handelnden Behörde im Auftrag und Namen des zuständigen Ministeriums (DWP) als decision maker auftreten darf. ${ }^{237} \mathrm{Er}$ ist an das Gesetz (hier den Jobseekers Act 1995 und dessen Ausführungsbestimmungen) in seiner Auslegung durch die Gerichte (case law) und die Social Security Comissioners gebunden. Er entscheidet somit über die Streitfrage grundsätzlich unabhängig von internen Vorgaben des Ministeriums. ${ }^{238}$

Im Regelfall soll er innerhalb von 14 Tagen nach Eingang der Anfrage entscheiden, wobei diese Pflicht gerichtlich nicht durchsetzbar ist. ${ }^{239}$ Der decision maker hat die

232 Jobseekers Act $1995 \mathrm{sec} .9$ subs. 6.

233 Cane, Administrative law, 2004, S. 389.

234 Bis zur Entscheidung R. v. Deputy Industrial Injuries Commissioner ex p. Moore [1965] 1 QB, S. 456 [486] und bis zur Bestätigung durch Jones v. Department of Employment [1988] 2 WLR S. 493 (CA) herrschte Streit darüber.

235 Es wird erwartet, dass sich wegen dieser Kontrollmöglichkeit eine einheitliche Begrifflichkeit für die beiden zentralen labour market conditions herausbilden wird. Dazu Wood u.a., Social security legislation 2006, Bd. 2, 2006, S. 69.

236 Jobseekers Act 1995 sec. 9 subs. 6.

237 Früher rief man einen adjudication officer an, der ein Mitarbeiter des zuständigen Ministeriums war, aber unabhängig entscheiden sollte, vgl. Social Security Act 1975 Teil III. Diese unklare Zugehörigkeit wurde durch den Social Security Act 1998 sec. 1 aufgehoben. Da die Aufgaben des decision maker denen des früheren adjudication officers entsprechen, gilt das bereits entwickelte case law weiter. Dazu Wikeley/Ogus, The law of social security, 2002, S. $182 \mathrm{f}$.

238 HMSO, Social Security Appeal Tribunals, A guide to procedure, 1988, S. 2; Baldwin/Wikeley/Young, Judging social security, 1992, S. $29 \mathrm{f}$.

239 Social Security Act 1975 sec. 99; R. v Secretary of State for Social Services, ex p. Child Poverty Action Group [1990] 2 QB, S. 540 [545 f]. 
Begründung seiner Entscheidung innerhalb eines Monats zu liefern 240 und kann z.B. anweisen, dass der employment officer ein $J S A$ bestimmten Inhalts abschließen muss. Der decision maker soll selbst Vorschläge machen, unter welchen Voraussetzungen der employment officer die Vereinbarung abschließen darf. Ferner ordnet er das sog. backdating 241 an. Das (abgeschlossene/unterzeichnete) JSA muss so behandelt werden, als sei es bereits zum Zeitpunkt des Antrags abgeschlossen gewesen. Die Entscheidung des decision maker ist für die Verwaltung wegen einer entsprechenden gesetzlichen Anordnung nicht bindend. 242

\section{bb) Revision}

Die sog. revision ist einer der Wege, eine Entscheidung nochmals verwaltungsintern überprüfen zu lassen. Dabei muss ein anderer decision maker die Entscheidung auf ihre Richtigkeit hin untersuchen. ${ }^{243}$ Diese Art des Rechtsschutzes ist entweder nicht fristgebunden, dann aber vom Vorliegen bestimmter Gründe abhängig (any time revision), oder fristgebunden innerhalb eines Monats ohne Vorliegen eines Grundes möglich (any ground revision). ${ }^{244}$

Das Vortragen folgender Gründe eröffnet ein Verfahren zur Überprüfung des JSA im Rahmen einer any time revision:

Macht ein Mitarbeiter der Verwaltung bei der Entscheidung einen Fehler (sog. official error), kann die Entscheidung überprüft werden. ${ }^{245}$ Dasselbe gilt für Fehler die Tatsachengrundlage der Entscheidung betreffend oder deren Unkenntnis (mistake/ignorance of facts), sodass der Antragsteller unrechtmäßig gezahlte Leistungen erhalten hat. ${ }^{246}$ Die Überprüfung findet also auch zu Ungunsten des Leistungsempfängers statt. Die Entscheidung kann ferner überprüft werden, wenn der Antragsteller Anspruch auf andere Leistungen hätte, die den Bezug der beantragten Leistung ausschließen oder vermindern. ${ }^{247}$ Daneben kann stets während eines Verfahrens vor einem SSAT, aber vor einer Sachentscheidung die angegriffene Entscheidung geändert werden. ${ }^{248}$

Ein speziell für die jobseeker's allowance geltender Grund ist eine Entscheidung, in der die Gewährung der jobseeker's allowance wegen Vorliegens einer Sanktion abgelehnt wurde. 249

Ergebnis einer erfolgreichen revision ist entweder eine neue Entscheidung, die die angefochtene Entscheidung entweder trotz oder ohne Vorliegen eines Grundes für die

240 SI 1999/991, reg. 28 (1) (b).

241 Jobseekers Act 1995 sec. 9 subs. 7.

242 Die Entscheidung war früher bindend für die Verwaltung gem. Jobseekers Act 1995 sec. 9 subs. 9. Die Rechtslage wurde geändert durch Social Security Act 1998 sched. 7 sec. 136 subs. 4 .

243 Child Poverty Action Group, Welfare benefits and tax credits handbook, 2005, S. 1197.

244 SI 1999/991 reg. 3 (1) (b), 4.

245 SI 1999/991 reg. 3 (5) (a).

246 SI 1999/991 reg. 3 (5) (b).

247 SI 1999/991 reg. 3 (7).

248 SI 1999/991 reg. 3 (4).

249 SI 1999/991 reg. 3 (6); Jobseekers act $1995 \mathrm{sec} .19$. 
revision bestätigt, oder eine Entscheidung, die den Grund bestätigt und eine Änderung der Ausgangsentscheidung verlangt. 250

\section{cc) Supersession}

Supersession ist ein weiterer verwaltungsinterner Weg, eine Entscheidung abzuändern. Sie kann in Abgrenzung zur revision verfolgt werden, wenn die revision zwar grundsätzlich zulässig ist, aber zusätzliche Umstände hinzutreten, die bei einer revision die Abänderung nicht begründen können. ${ }^{251}$ Die Möglichkeit einer supersession schließt also eine Lücke der verwaltungsinternen Überprüfbarkeit einer Entscheidung. Sie wird (allerdings nicht zwingend schriftlich) eingelegt bei der Stelle, die die anzufechtende Entscheidung gefällt hat. Dann entscheidet wiederum ein sog. decision maker, also ein Mitarbeiter des DWP über den Erfolg des Antrags.

Hauptanwendungsfall der supersession ist die wesentliche Veränderung der Verhältnisse (relevant change of circumstances) beziehungsweise deren Bevorstehen. ${ }^{252} \mathrm{Sie}$ kann auch verfolgt werden, wenn rechtliche oder tatsächliche Fehler vorliegen oder eine andere soziale Leistung in Aussicht steht, die die Gewährung des Beantragten ausschließt oder einschränkt. ${ }^{253}$ Der Sonderfall, der bei der revision einschlägig ist, ist ebenso für eine supersession geregelt: Wird die Gewährung der jobseeker's allowance wegen Vorliegens einer Sanktion abgelehnt, kann diese Entscheidung im Wege der supersession überprüft werden. ${ }^{254}$

Während des Entscheidungsverfahrens kann der decision maker Beweise einholen und deren Beibringung durch den Antragsteller verlangen, z.B. ein medizinisches Gutachten. ${ }^{255}$

Ergebnis einer erfolgreichen supersession ist eine neue Entscheidung, die entweder die vorhergehende Entscheidung bestätigt oder deren Abänderung anregt. ${ }^{256}$

\section{dd) Zusammenfassung}

Diese drei Verfahren verwaltungsinterner Kontrolle sollen bereits im Vorfeld externer Kontrolle die Aufdeckung von Fehlern bei der Anwendung der bestehenden Vorschriften ermöglichen. Besonders ist, dass die Entscheidung des decicion maker seit Social Security Act 1975 sec. 104 weitgehend frei von Abhängigkeiten innerhalb der entscheidenden Behörde sein soll. Die Strukturen stehen seitdem einer politischen Beeinflussung stärker entgegen. Es gibt für die politische Ebene keinen offiziellen Weg mehr, auf die verwaltungsinterne Kontrolle einzuwirken. Die Gefahr, dass eine „Entscheidung von oben" in Umgehung des einzelnen decision maker abgeändert wird, ist

250 Child Poverty Action Group, Welfare benefits and tax credits handbook, 2005, S. 1197 f.

251 SI 1999/991 reg. 6 (3).

252 SI 1999/991 reg. 6 (2) (a).

253 SI 1999/991 reg. 6 (2).

254 SI 1999/991 reg. 6 (2) (f).

255 Child Poverty Action Group, Welfare benefits and tax credits handbook, 2005, S. 1197.

256 Child Poverty Action Group, Welfare benefits and tax credits handbook, 2005, S. 1204. 
deshalb verringert, wenn auch nicht auszuschließen ist, dass inoffizielle Möglichkeiten genutzt werden. 257

\section{d) Appeal}

\section{aa) Social Security Appeal Tribunal (SSAT)}

(1) Entstehung und deren Gründe

Social Security Appeal Tribunals wurden 1984 als besondere Entscheidungsgremien für Streitigkeiten über soziale Leistungen (social security benefits) gegründet. ${ }^{258}$ Das ausführliche Rechtsschutzsystem von common law Gerichten erschien für die Masse der entstehenden Fälle betreffend soziale Leistungen, insbesondere auch nach Einführung der jobseeker's allowance, nicht geeignet. ${ }^{259}$ Gerade die Personengruppen, die Sozialleistungen benötigen, lassen sich auf informelle Institutionen eher ein als auf die (altehrwürdigen) common law Gerichte. Es entstehen zudem Kosteneinsparungen, die sich in erster Linie durch die niedrigere Bezahlung der Mitglieder der SSATs ergeben. Auch die erforderliche fachliche Spezialisierung, die zunehmend notwendig ist, ist ein Grund für die Schaffung der SSATs. 260

Seit ihrer Gründung wurden sie vor allem durch den Social Security Act 1998 weiterentwickelt und zu wichtigen Säulen des Rechtsschutzes bei social security benefits ausgebaut. $^{261}$ Ihre Zuständigkeit ergibt sich stets durch ausdrückliche gesetzliche Eröffnung des Rechtsweges zu einem bestimmten SSAT.

\section{(2) Zusammensetzung des Entscheidungsgremiums}

Das Entscheidungsgremium in den SSATs setzte sich bis zum Social Security Act 1998 aus drei Personen zusammen, die nicht zwingend juristische Kenntnisse haben mussten. Seit der Rechtsänderung muss jedenfalls der Vorsitzende lawyer (Jurist) sein, die anderen beiden Mitglieder verfügen über in der Praxis erworbene Expertise im jeweiligen Aufgabengebiet der Sozialverwaltung. 262

257 Vgl. zur Rechtslage zu Zeiten der adjudication officers die ausführliche Studie von Baldwin/Wikeley/Young, Judging social security, 1992, S. 66.

258 Health and Social Services and Social Security Adjudications Act 1983; zur Vorgeschichte Wikeley, AdminLR 1994 (46), S. 183 ff; Wikeley, MLR 2000 (63), S. 476 ff.

259 So schreibt Street, Justice in the welfare state, 1985, S. 3: „For these cases we don't want a RollsRoyce system of justice."

260 Wade/Bradley, Constitutional and administrative law, 1985, S. 704 f; Harlow/Rawlings, Law and administration, 1997, S. 480 f; Cane, Administrative law, 2004, S. 392 ff.

261 Grundlage war der Entwurf HMSO, Improving decision making and appeals in social security, Cm 3228, 1996. Dieser wurde vor allem wegen der Verschlechterung der Verfahrensposition des Einzelnen kritisiert. Dazu Adler, PQ 1997 (68), S. 388 ff; Sainsbury, The reform of social security adjudication, in: Harris/Partington, Administrative justice in the 21st century, 1999, S. 462, Wikeley, JSSL 1999 (6), S. 155 ff. Neueste Reformbestrebungen kommentiert Adler, JSSL 2002 (9), S. 177 ff.

262 Zur Entwicklung Wikeley, AdminLR 1994 (46), S. 197 ff. 
Über die jobseeker's allowance entscheidet grundsätzlich nur eine Person, die dann auch Jurist (lawyer) sein muss. 263

\section{(3) Gegenstand des Verfahrens}

Gegenstand des Verfahrens sind Entscheidungen des decision maker, sowohl erstmals ergangene als auch bereits im Wege der revision oder supersession ergangene Entscheidungen. Ein SSAT kann über Anträge des decision maker entscheiden, Entscheidungen aufheben und die Zahlung von Leistungen in Eilfällen anordnen (urgent needs payments). ${ }^{264}$

Betreffend die jobseeker's allowance kann die Frage Gegenstand eines Verfahrens werden, ob das jobseeker's agreement zweckmäßig (reasonable) ist. Auch Fragen betreffend die Verhängung einer Sanktion oder die Befolgung einer jobseeker's direction, insbesondere das Vorliegen einer Rechtfertigung (good cause) für die Weigerung, ein $J S A$ abzuschließen, können Gegenstand einer Klage vor einem SSAT sein. ${ }^{265}$

\section{(4) Formfragen}

Die Klageschrift ist innerhalb eines Monats ab Erhalt des Schreibens der Behörde einzureichen. ${ }^{266}$ Die Frist beginnt am Tag nach Erhalt des Schreibens und endet einen Kalendermonat nach dem Tag, der dem Tag des Erhalts entspricht. ${ }^{267}$ Zur Einhaltung dieser Frist ist es deshalb nicht zwingend erforderlich, die Entscheidung des decision maker abzuwarten. Daneben gibt es besondere Fälle, in denen die Monatsfrist verlängert werden oder außerhalb der Frist binnen Jahresfrist ab Ablauf der Monatsfrist Klage erhoben werden kann. ${ }^{268}$ Neben Gründen für die Verspätung ist die Klage von einer Zulassung durch die angerufene Stelle abhängig. ${ }^{269}$

(5) Verfahren

Das Verfahren vor den SSATs verläuft nach anderen Prinzipien als das Verfahren vor common law Gerichten. Das SSAT handelt zwar genauso auf der Grundlage von Recht und Gesetz, vermeidet Parteilichkeit und bewahrt die Unabhängigkeit von der Sozialverwaltung ${ }^{270}$. Es gibt aber keine sog. adversarial procedure, also keinen Parteiprozess, in dem das Gericht nur das Vorgetragene als Grundlage der Entscheidung heranziehen darf. Im Gegenteil gilt, dass das SSAT alles, was zugunsten und zuungunsten des An-

263 SI 1999/991 reg. 36; kritisch zu den damit verbundenen Konsequenzen für den Rechtsuchenden Wikeley, JSSL 1999 (6), S. 165.

264 HMSO, Social Security Appeal Tribunals, A guide to procedure, 1988, S. 7.

265 Child Poverty Action Group, Welfare benefits and tax credits handbook, 2005, S. 1220.

266 SI 1999/991 reg. 31 (1).

267 Child Poverty Action Group, Welfare benefits and tax credits handbook, 2005, S. 1223.

268 SI 1999/991 reg. 32 (1).

269 SI 1999/991 reg. 32 (3), dazu im Einzelnen Child Poverty Action Group, Welfare benefits and tax credits handbook, 2005, S. 1224, $1261 \mathrm{ff}$.

270 Der Vorwurf der Parteilichkeit besteht. Die Mitglieder der tribunals arbeiten häufig in den Behörden, deren Entscheidungen sie überprüfen. Ferner werden die tribunals von diesen Behörden finanziell unterstützt. Dazu Cane, Administrative law, 2004, S. 390. 
tragstellers spricht und im Verfahren auch nur nebenbei auftaucht, in Erfahrung bringen muss (inquisitorial approach ${ }^{271}$ ). Es hat Tatsachenfeststellungen zu treffen, die Umstände des Falles zu untersuchen und auf der Grundlage des festgestellten Sachverhalts das geltende Recht anzuwenden. ${ }^{272}$ Dabei ist es nicht an das formelle Beweisrecht (law of evidence) gebunden. ${ }^{273}$

In der Praxis tritt angesichts der Masse der Fälle die Ermittlung des Sachverhalts in den Hintergrund ${ }^{274}$, besonders dann, wenn der Antragsteller selbst nicht bei der mündlichen Erörterung des Antrags (hearing) anwesend ist. ${ }^{275}$ Seit einer Änderung der Verfahrensregeln kann bei der Antragstellung bereits auf ein hearing verzichtet werden. Dies wird als Aufforderung verstanden, dies möglichst auch zu tun. ${ }^{276}$ Ebenso legt Social Security Act 1998 sec. 10 subs. 8 fest, dass bei der Entscheidung im Regelfall die Tatsachenlage zugrundezulegen ist, wie sie im Zeitpunkt der Entscheidung der Verwaltung bestand, was mit einem inquisitorial approach nur schwer vereinbar ist. Aufgrund dieser Rechtsänderungen wird eine Entwicklung des Verfahrens weg von einem inquisitorial approach hin zu einer mehr von Aktenlage und schriftlichem Vortrag ohne weitergehende Ermittlungspflichten des Gerichtes bestimmten Vorgehensweise in den SSATS beschrieben. Es entsteht der Eindruck, dass sich das Verfahren vor einem SSAT immer mehr dem Verfahren vor common law Gerichten annähert. ${ }^{277}$

Die Entscheidung ist schriftlich unter Angabe der Gründe, die sich aus der Darstellung des zugrunde gelegten Sachverhalts und einer rechtlichen Begründung zusammensetzen, festzuhalten. ${ }^{278}$ Diese Pflicht ist insbesondere deshalb wichtig, weil nur so die Konsistenz der „Rechtsprechung“ gewahrt werden kann, indem die Begründungen der Entscheidungen nachvollziehbar und die Vergleichbarkeit zweier Fälle überprüfbar gemacht wird. 279

(6) Entscheidung und Anfechtbarkeit

Die Entscheidung des SSAT ist nur noch in bestimmten Fällen anfechtbar. Bei Schreib- oder Tippfehlern sind diese von Mitarbeitern des SSAT zu korrigieren. Treten neue Tatsachen zu Tage, kommt eine supersession in Betracht. Über diese entscheidet

271 Logie/Watchman, CJQ 1989 (8), S. 109 ff; a.A. Genn, MLR 1993 (56), S. 395, die diesen Begriff nicht für richtig hält, weil es sich ihrer Ansicht nach um formlose (informal) Verfahren handelt.

272 Zu den Verfahrensregeln SI 1999/991 reg. 49 ff.

273 Dazu Mesher, Social security law, in: Kiralfy, The burden of proof, 1987, S. 211-227.

274 Cane, Administrative law, 2004, S. 389; Wikeley/Ogus, The law of social security, 2002, S. 197.

275 Baldwin/Wikeley/Young, Judging social security, 1992, S. $104 \mathrm{ff}$ nach der Untersuchung vor verschiedenen Social Security Appeal Tribunals verhandelter Fälle.

276 Dazu im Einzelnen Matthewman/Ennals/Self, Tolley's social security and state benefits handbook, Rn. 23A.9.

277 Dazu Wikeley, MLR 2000 (63), S. 494 ff.

278 SI 1999/991 reg. $53 \mathrm{f}$.

279 Cane, Administrative law, 2004, S. 389. 
ein Social Security Commissioner. ${ }^{280}$ Liegen Rechtsfehler vor, kommt ebenfalls eine Überprüfung durch eine Social Security Commission in Betracht. ${ }^{281}$

\section{bb) Social Security Commission}

In bestimmten Fällen können der Antragsteller oder das Ministerium wegen einer Entscheidung des SSAT die sog. Social Security Commissioners anrufen. Diese Instanz ist nur dann vorgesehen, wenn ein error of law im Raum steht.

Die Commissioners gehören einem juristischen Beruf (sollicitors, barristers) an, haben mindestens zehn Jahre Berufserfahrung und arbeiten nach ihrer Ernennung durch die Krone hauptamtlich grundsätzlich als (Einzel-)Richter, bei schwierigeren Fragen in einem dreiköpfigen Gremium. ${ }^{282}$

Die Social Security Commission entscheidet meist nach Aktenlage ohne mündliche Verhandlung selbst inhaltlich über den Streit, erlässt also eine neue Entscheidung, wenn alle Tatsachen feststehen und die Entscheidung rechtlich falsch war. ${ }^{283}$ Normalerweise wird bei Erfolg des Rechtsbehelfs der Fall an ein anderes tribunal zurückgeschickt und mit Vorgaben, wie die Entscheidung zu treffen ist, versehen. ${ }^{284}$

\section{e) Judicial Review}

Judicial review ist allgemein im Gegensatz zur stets per Gesetz (statute) angeordneten administrativen Kontrolle von Entscheidungen eine gerichtliche Überprüfung von Entscheidungen der Verwaltung auf ihre Übereinstimmung mit Regeln und Prinzipien des administrative law (lawfulness) hin. ${ }^{285}$ Es geht stets um die Prüfung, ob die Verwaltung ihre vom Parlament übertragenen Befugnisse überschritten hat.

\section{aa) Gegenstand des Verfahrens}

Aufgrund der Regelung in Jobseekers Act 1995 sec. 9 subs. 2 hat ein JSA nur Wirkungen für die Beantragung einer jobseeker's allowance, nicht für andere Zwecke. Das heißt, dass sich keine anderen rechtlichen Konsequenzen ergeben können. Vor common law Gerichten kann gegen die Nichteinhaltung der Vereinbarung nicht vorgegangen werden. 286

Gegen Entscheidungen des SSATs kann im Wege des judicial review vorgegangen werden, wenn bestimmte Voraussetzungen erfüllt sind. Ist beispielsweise ein Verfahren vor einem SSAT ausgeschlossen oder wurde die Zulassung zu einem SSAT verweigert,

280 SI 1999/991 reg. 57 (5).

281 Dazu Child Poverty Action Group, Welfare benefits and tax credits handbook, 2005, S. 1241.

282 Social Security Administration Act 1992, ch. 5 § 52; dazu im Einzelnen Yardley, Principles of administrative law, 1986, S. 190 f; Wikeley, AdminLR 1994 (46), S. 206 f.

283 Social Security Act 1998 sec. 14 subs. 7.

284 Social Security Act 1998 sec. 14 subs. 8 (b).

285 Vgl. Civil Procedure Rules rule 54. Kritisch dazu Oliver, PL 2002, S. 91 ff, weil sie durch das Sonderverfahren das bestehende System des common law gestört sieht.

286 Wood u.a., Social security legislation 2006, Bd. 2, 2006, S. 68. 
kommt eine Überprüfung durch judicial review in Betracht. ${ }^{287}$ Es gibt für Fragen betreffend das $J S A$, z.B. Nichteinhaltung eines $J S A$, keine speziell geregelten Möglichkeiten, vor common law Gerichten zu klagen.

\section{bb) Zuständiges Gericht und Verfahren}

Das Verfahren findet vor besonderen Kammern der common law Gerichte statt und folgt besonderen Regeln. ${ }^{288}$ Es ist wichtig, dass für die Klage ein passendes Verfahren zur Verfügung steht. Aufgrund der Rechtsweggarantie, die seit Einführung des Human Rights Act 1998 sec. 7 bekannt ist, ist es umso mehr Aufgabe der Gerichte, ein solches Verfahren bereitzustellen. Es gilt seitdem der Grundsatz, dass bei eigener Betroffenheit (victim of the unlawful act) gegen unrechtmäßige Akte der öffentlichen Gewalt (public authority) vorgegangen werden kann. Die Gerichte legen diesen Grundsatz weit aus und machen, soweit möglich, alle Akte öffentlicher Gewalt einer gerichtlichen Kontrolle zugänglich. ${ }^{289}$

Im Vereinigten Königreich ist darüber hinaus kein Verfassungsrechtsweg in vergleichbarer Weise wie im deutschen System vorhanden. Es gibt weder ein entsprechendes Verfassungsgericht noch ein besonderes Verfahren oder Klagearten. ${ }^{290}$ Verfassungsrecht ist gleichwohl von Verwaltung, SSATs und Gerichten ohne höchstrichterlichem Entscheidungsmonopol bei einem bestimmten Gericht anzuwenden. ${ }^{291}$

\section{f) Kosten}

Das Verfahren vor den SSATs ist kostenfrei. Es gibt ferner kein System zur Finanzierung der Vertretung vor einem SSAT. Das ist damit begründbar, dass es Organisationen wie das Citizen's Advice Bureaux gibt, die die Vertretung kostenlos anbieten. Es handelt sich dabei allerdings nicht um einen Rechtsbeistand im engen Sinn. ${ }^{292}$

Judicial review vor dem Court of Appeal, dem High Court und dem Court of Session ist kostenfrei. Wünscht man anwaltlichen Beistand, muss dieser selbst bezahlt werden, es sei denn, man erfüllt die Voraussetzungen für free legal aid. ${ }^{293}$

287 Child Poverty Action Group, Welfare benefits and tax credits handbook, 2005, S. 1253.

288 Dazu Walker/Ward, Walker\&Walker's English legal system, London, 1994, S. 127 ff, $131 \mathrm{ff}$.

289 Schwarze, European administrative law, 2006, S. $140 \mathrm{f}$.

290 Blumenwitz, Einführung in das anglo-amerikanische Recht, 2003, S. 164.

291 Einführend zur Wirkung des Verfassungsrechts im Vereinigten Königreich Marshall/Yardley, ZaöRV 1962, S. $540 \mathrm{ff}$.

292 Matthewman/Ennals/Self, Tolley's social security and state benefits handbook, Rn. 24.3, 24.26C; vgl. Genn, MLR 1993 (56), S. 393-411, die die Frage bejaht, dass die Vertretung des Antragstellers Auswirkungen auf den Ausgang des Verfahrens hat und damit gegen den Grundsatz eines möglichst formlosen Verfahrens vor tribunals argumentiert.

293 Child Poverty Action Group, Welfare benefits and tax credits handbook, 2005, S. 1253; allgemein dazu Matthewman/Ennals/Self, Tolley's social security and state benefits handbook, Rn. 24. 


\section{B. Deutsches Recht}

\section{Arbeitsförderungsrecht}

\section{SGB III und Job-AQTIV-Gesetz}

\section{a) Arbeitsförderungsgesetz und SGB III}

Das Recht der Arbeitsförderung 294 wurde durch das Arbeitsförderungsreformgesetz (AFRG) ${ }^{295}$ mit Wirkung zum 1.1.1998 in das Sozialgesetzbuch als Drittes Buch Sozialgesetzbuch (SGB III) ${ }^{296}$ eingegliedert und dabei grundlegend geändert.

Seitdem ist, angeregt von den beschäftigungspolitischen Leitlinien der EU, der präventive Charakter der Arbeitsförderung betont, und Förderinstrumente sind zeitlich vorverlagert. Anders als das AFG knüpft das SGB III Leistungen der Arbeitsförderung, z.B. Arbeitsbeschaffungsmaßnahmen, nicht mehr an lang andauernde Arbeitslosigkeit an 297 , sondern hat die schnellstmögliche Wiedereingliederung in den Arbeitsmarkt zum Ziel. Der Gesetzgeber erweiterte die Angebote für Berufsberatung und die Förderung beruflicher Weiterbildung und gestaltete sie im Hinblick auf ein früheres staatliches Tätig werden zur Vermeidung von lang andauernder Arbeitslosigkeit um. ${ }^{298}$ Beabsichtigt war ferner die Vereinfachung des Arbeitsförderungsrechts, was wegen des stetigen Wandels in einer modernen Gesellschaft als schwieriges Unterfangen erscheint und deshalb angesichts des detaillierten Katalogs an Eingliederungsleistungen auch nicht als erreicht betrachtet werden kann. Die Übersichtlichkeit des Eingliederungsinstrumentariums des Arbeitsförderungsrechts wurde oftmals der für Arbeitgeber, Arbeitnehmer und Maßnahmeträger erforderlichen Planungssicherheit geopfert, insbesondere durch das komplizierte Nebeneinander verschiedener Übergangsregelungen. Das Instrumentarium wurde auf bestimmte Zielgruppen ausgerichtet und deren Förderbedarf in den verschiedenen Instrumenten genauestens rechtlich umschrieben. ${ }^{299}$ Obwohl im Koalitionsvertrag zwischen CDU/CSU und SPD $^{300}$ eine Neuordnung der arbeitsmarktpolitischen Instrumente für die laufende Legislaturperiode bis 2009 angekündigt, und diese Ankündigung von Seiten der Bundesregierung offiziell wiederholt worden ist ${ }^{301}$, blieben die

294 Geregelt im Arbeitsförderungsgesetz (AFG) vom 25.6.1969 (BGB1. I, S. 582).

295 BT-Drucksache 13/4941.

296 Gesetz v. 24.3.1997 (BGBl. I, S. 594)

297 In jüngster Zeit sind Gegentendenzen erkennbar, z.B. Qualifizierungszuschuss für jüngere Arbeitnehmer gem. § 4210 SGB III; Eingliederungszuschuss für Ältere gem. § 421f SGB III.

298 Körner, NZA 2002, S. 242.

299 Bolay/Eisenreich/Isele, Die neue Arbeitsförderung, 2005, S. 16.

300 Koalitionsvertrag zwischen CDU/CSU und SPD „Gemeinsam für Deutschland. Mit Mut und Menschlichkeit" vom 11.11.2005, S. 32 ff.

301 Rede des Bundesministers für Arbeit und Soziales Olaf Scholz zum Haushaltsgesetz 2008 vor dem Deutschen Bundestag am 29.11.2007 in Berlin, Bulletin Nr. 134-1 vom 29.11.2007. 
gesetzlichen Änderungen zur „Neuausrichtung der arbeitsmarktpolitischen Instrumente“ hinter diesen Ankündigungen zurück. ${ }^{302}$

\section{b) Job-AQTIV-Gesetz}

Im Job-AQTIV-Gesetz ${ }^{303}$, dem Gesetz zur Reform der arbeitsmarktpolitischen Instrumente, durch das das SGB III mit Wirkung zum 1.1.2002304 geändert wurde, verstärkte der Gesetzgeber nochmals den präventiven Ansatz der Arbeitsförderung, um damit dem Vorwurf mangelnder Effizienz und Ressourcenvergeudung in der Bundesanstalt für Arbeit ${ }^{305}$ zu begegnen. Erklärte Ziele des Gesetzesentwurfs sind die Ermittlung des individuellen Hilfebedarfs, die Optimierung des Vermittlungsprozesses, der Einsatz arbeitsmarktpolitischer Instrumente ohne Einhaltung von Mindestfristen von Arbeitslosigkeit und eine verbesserte Verzahnung des Einsatzes arbeitsmarktpolitischer Instrumente mit dem Instrumentarium anderer Politikbereiche. Es ging dem Gesetzgeber um die Bekämpfung der vorhandenen und um die Verhinderung der Entstehung neuer, insbesondere lang andauernder Arbeitslosigkeit. ${ }^{306}$ Dieses Ziel verfolgte auch die Große Koalition zwischen CDU, CSU und SPD weiter und hob im Koalitionsvertrag ausdrücklich hervor, dass zentrale Aufgabe der Regierungspolitik der Abbau der Arbeitslosigkeit sei. 307

Im Gesetz werden diese Aufgabenstellungen in der Neufassung der allgemeinen Vorschriften unter anderem in $\S 1$ SGB III (Ziele der Arbeitsförderung), § 4 SGB III (Vorrang der Vermittlung) und $\S 5$ SGB III (Vorrang der aktiven Arbeitsförderung) deutlich. Interessant ist, dass der Gesetzgeber in $§ 1$ Abs. $1 \mathrm{~S}$. 4 SGB III alle Leistungen der Arbeitsförderung in ein Abhängigkeitsverhältnis zur Sozial- und Wirtschaftspolitik, insbesondere aber auch zur Finanzpolitik der Bundesregierung stellt. Das bedeutet für die Bundesagentur für Arbeit eine vorher in dieser Form nicht vorhandene Bindung an politische Ziele der Bundesregierung. ${ }^{308}$

Zur Erreichung der Ziele der Arbeitsförderung werden folgende Schritte vorgeschlagen:

Das Verhältnis zwischen Agentur für Arbeit und einzelnem Arbeitslosen soll sich verbessern. Die Vermittlungstätigkeit der Agenturen für Arbeit hat unbedingten Vorrang vor Leistungen zum Ersatz des Arbeitsentgelts bei Arbeitslosigkeit, § 4 SGB III. Die Tätigkeit der Bundesagentur für Arbeit darf sich nicht nur darauf beschränken, Arbeitsuchende und Arbeitgeber zusammenzubringen, sondern muss die aktive Suche of-

302 Gesetz zur Neuausrichtung der arbeitsmarktpolitischen Instrumente v. 21.12.2008 (BGB1. I, S. 2917). Dazu Voelzke, jurisPR-SozR 5/2009 Anm. 4.

303 „AQTIV“ steht für Aktivieren, Qualifizieren, Trainieren, Investieren und Vermitteln.

304 Gesetz v. 10.12.2001 (BGBl. I, S. 3443).

305 Seit dem Dritten Gesetz für moderne Dienstleistungen v. 23.12.2003 (BGBl. I, S. 2848) Bundesagentur für Arbeit; dazu Schütze/Oschmiansky, ZSR 2006, S. 5 ff.

306 BT-Drucksache 14/6944, S. 1.

307 Koalitionsvertrag zwischen CDU/CSU und SPD „Gemeinsam für Deutschland. Mit Mut und Menschlichkeit“"vom 11.11.2005, S. 15; dazu Feckler, in: Ambs, GK-SGB III, § 1 Rn. 1a.

308 Feckler, in: Ambs, GK-SGB III, § 1 Rn. 3. 
fener Stellen umfassen. ${ }^{309}$ Spätestens bei der Arbeitslosmeldung muss ein Bewerberprofil, nötigenfalls mit Hilfe eines Assessment-Centers (§ 35 Abs. 3 SGB III) erstellt werden. In diesem sollen berufliche und persönliche Merkmale (Kenntnisse, Qualifikation, Berufserfahrung, Aktualität der Qualifikation und Kenntnisse sowie Weiterbildungsfähigkeit und -bereitschaft), aber auch die Gegebenheiten des Arbeitsmarktes, auf den sich die Vermittlungsbemühungen des Arbeitslosen erstrecken, erfasst werden. Dieses Bewerberprofil ist zusammen mit der individuellen Vermittlungsstrategie in einer Eingliederungsvereinbarung festzuhalten. 310

Daneben steht die Förderung von Infrastrukturmaßnahmen, innerhalb derer Arbeitslose in Beschäftigung gebracht werden können (§ 279a SGB III). 311

Besonderes Augenmerk erhalten bestimmte Beschäftigtengruppen, um deren Chancen auf dem Arbeitsmarkt zu erhöhen (z.B. Jugendliche, Behinderte, ältere Arbeitnehmer). Dabei soll die gewährte Unterstützung nicht auf Dauer angelegt sein, sondern momentane Ungleichgewichte ausgleichen. ${ }^{312}$

c) Überblick über die Leistungen nach dem SGB III

\section{aa) Versicherungspflichtverhältnis}

Seit der Eingliederung des Arbeitsförderungsrechts in das Sozialgesetzbuch spricht man nicht mehr von einer Beitragspflicht ${ }^{313}$, auch wenn sie rechtlich betrachtet weiterhin besteht, sondern von einer Versicherungspflicht der in der Arbeitslosenversicherung versicherten Personen, um eine einheitliche Terminologie in der Sozialversicherung zu erreichen. ${ }^{314}$ Es wird gem. § 24 SGB III ein Versicherungspflichtverhältnis begründet, das Oberbegriff für alle versicherungsrechtlich relevanten Lebenssachverhalte ist. 315

Das Versicherungspflichtverhältnis beginnt gem. § 24 Abs. 2, 4 SGB III grundsätzlich mit dem Tag des Eintritts in ein Beschäftigungsverhältnis und endet mit dem Tag des Ausscheidens.

Versicherungspflichtig sind in erster Linie Personen, die gegen Arbeitsentgelt oder zu ihrer Berufsausbildung beschäftigt sind, $\S 25$ Abs. 1 S. 1 SGB III. 316

309 Hielscher, WSI-Mitteilungen 2006, S. $119 \mathrm{ff}$.

310 BT-Drucksache, 14/6944, S. 28 f.

311 Kaltenstein, in: Hauck/Noftz, SGB III, K § 279a Rn. 6 ff; Schlegel/Eicher, in: Hennig, SGB III, § 279a Rn. 22 ff.

312 Feckler, in: Ambs, GK-SGB III, § 1 Rn. 16.

313 Zur verfassungsrechtlichen Rechtfertigung der Beitragspflicht vgl. Spellbrink, JZ 2004, S. 538 ff, der sie im Ergebnis infolge der Veränderung des SGB III nicht mehr für gegeben hält.

314 Dazu Schulin/Igl/Welti, Sozialrecht, 2007, S. 347.

315 BT-Drucksache 13/4941, S. 157.

316 Die Besonderheiten, die für Auszubildende nach dem Berufsbildungsgesetz und Wehrpflichtige beziehungsweise Zivildienstleistende gelten, sind $\S 24$ Abs. 1 S. 2, § 24 Abs. 2 SGB III zu entnehmen. Sonstige Versicherungspflichtige sind in $\S 26$ SGB III aufgeführt, z.B. Jugendliche in Einrichtungen der beruflichen Rehabilitation nach $\S 35$ SGB IX. 
Was unter Leistungen der aktiven Arbeitsförderung zu verstehen ist, definiert erstmals $\S 3$ Abs. 4 SGB III. Leistungen der aktiven Arbeitsförderung sind alle in $\S 3$ Abs. 1 SGB III aufgeführten Leistungen mit Ausnahme der Leistungen, auf die der Versicherte einen Rechtsanspruch hat, weil sie aufgrund der Versicherungspflicht des Arbeitnehmers als Entgeltersatzleistungen unmittelbar bei Eintritt der (Teil-)Arbeitslosigkeit zu zahlen sind. So sind das Arbeitslosengeld bei Arbeitslosigkeit, das Teilarbeitslosengeld und das Insolvenzgeld zwar Leistungen der Arbeitsförderung, nicht aber der aktiven Arbeitsförderung.

Im Folgenden werden einige Beispiele für Leistungen der aktiven Arbeitsförderung aufgeführt, die direkt oder indirekt auf Nachfrage- oder Angebotsseite auf den Arbeitsmarkt einwirken: Berufsberatung, Ausbildungs- und Arbeitsvermittlung und diese unterstützende Leistungen, Maßnahmen der Eignungsfeststellung, Trainingsmaßnahmen zur Verbesserung der Eingliederungsaussichten, Mobilitätshilfen zur Aufnahme einer Beschäftigung, Gründungszuschuss zur Aufnahme einer selbständigen Tätigkeit und die Übernahme von Weiterbildungskosten während der Teilnahme an einer beruflichen Weiterbildung.

Diese Leistungen der aktiven Arbeitsförderung sind grundsätzlich Ermessensleistungen. In $\S 3$ Abs. 5 SGB III sind einige Ausnahmen aufgeführt, bei denen es sich um Versicherungsleistungen handelt, die der Absicherung des Lebensunterhalts dienen. Diese sind Pflichtleistungen, denn Ermessen soll nur in Bezug auf Leistungen bestehen, die ihrer Art nach Veränderungen oder Absicherungen auf dem Arbeitsmarkt herbeiführen können und sollen. ${ }^{317}$ Pflichtleistungen sind die Beauftragung von Dritten mit der Vermittlung nach sechs Monaten, Überbrückungsgeld, Berufsausbildungshilfe, besondere Leistungen zur Teilhabe am Arbeitsleben ${ }^{318}$, Arbeitslosengeld bei beruflicher Weiterbildung, Kurzarbeitergeld, Wintergeld und Leistungen zur Förderung der Teilnahme an Transfermaßnahmen.

Gem. § 17 SGB III können bereits von Arbeitslosigkeit bedrohte Personen Maßnahmen der aktiven Arbeitsförderung erhalten, um die Gefahr, tatsächlich arbeitslos zu werden, bereits vor dem Eintritt zu bannen. Denn es ist schwieriger aus einer Arbeitslosigkeit herauszukommen, als aus einer ungekündigten Stelle heraus eine neue zu finden. 319

\section{cc) Entgeltersatzleistungen, $\S \S 116 f f$ SGB III}

Die für diese Arbeit relevante Entgeltersatzleistung ist das Arbeitslosengeld bei Arbeitslosigkeit gem. $\S 116$ Nr. 1 SGB III. Entgeltersatzleistungen, durch die andere versicherte Risiken als das der Arbeitslosigkeit abgedeckt werden, wie z.B. das Insolvenz-

317 Hünecke, in: Gagel, SGB III, § 3 Rn. 2; Feckler, in: Ambs, GK-SGB III, § 3 Rn. 19.

318 Seit der Gesetzesänderung (BT-Drucksache 13/6845, S. 35) haben Behinderte einen Rechtsanspruch auf die besonderen Leistungen zur Teilhabe am Arbeitsleben, wenn die allgemeinen Leistungen die berufliche Eingliederung behinderter Menschen nicht sichern können. Dazu Feckler, in: Ambs, GKSGB III, § 3 Rn. 20.

319 Zur Definition der Voraussetzungen Timme, in: Hauck/Noftz, SGB III, § 17 Rn. 4 ff. 
geld gem. $\S 183$ ff SGB III oder das Kurzarbeitergeld gem. $\S 169$ ff SGB III werden im Folgenden nicht behandelt, weil sie keine Bezüge zur Eingliederungsvereinbarung aufweisen.

Der Anspruch auf Arbeitslosengeld ergibt sich aus § 118 Abs. 1 SGB III und besteht bei Arbeitslosigkeit und beruflicher Weiterbildung. Derjenige Arbeitnehmer, der arbeitslos ist, sich beim Arbeitsamt arbeitslos gemeldet und die Anwartschaftszeit erfüllt hat, erhält Arbeitslosengeld bei Arbeitslosigkeit, § 118 Abs. 1 SGB III. Dieser Anspruch ist ein vermögenswertes Rechtsgut und fällt deshalb in den Schutzbereich des Art. 14 Abs. 1 GG. ${ }^{320}$

Arbeitslos ist gem. § 119 Abs. 1 SGB III, wer nicht in einem Beschäftigungsverhältnis steht (Beschäftigungslosigkeit), sich bemüht, seine Beschäftigungslosigkeit zu beenden (Eigenbemühungen) und den Vermittlungsbemühungen der Arbeitsagentur zur Verfügung steht (Verfügbarkeit). Beschäftigungslos ist derjenige, der gar nicht oder weniger als 15 Stunden pro Woche arbeitet, § 118 Abs. 2 SGB III. Die Verfügbarkeit setzt voraus, dass der Arbeitslose arbeitsfähig, das heißt die objektiven Kriterien des Leistungsvermögens erfüllt, und arbeitsbereit ist, das heißt, dass er subjektiv arbeiten will, § 119 Abs. 2 SGB III.

Der Arbeitslose ist bei der Arbeitsuche nur verpflichtet, zumutbare Arbeit zu suchen und anzunehmen, § 119 Abs. 5 Nr. 1, 121 SGB III. Darauf, ob und wie sich die Einschränkung auf zumutbare Arbeit in der Praxis auswirkt, hat vor allem die Rechtsprechung Einfluss, indem sie diese in Einzelfallentscheidungen auslegt. Der Gesetzgeber hat in den letzten Gesetzesänderungen die Rechtslage deutlich verschärft. Es gibt weder sog. Berufsschutz noch den Schutz einmal erworbener beruflicher Qualifikationen, $\S$ 121 Abs. 5 SGB III. Diese Voraussetzung hat im Übrigen nicht nur Bedeutung als Voraussetzung des Anspruchs auf Arbeitslosengeld, sondern darüber hinaus auch bei der Prüfung der Voraussetzungen einer Sanktion auf der Grundlage des $\S 144$ Abs. 1 S. 2 Nr. 2 SGB III und für die Zulässigkeit der Festlegungen über die Eigenbemühungen in einer Eingliederungsvereinbarung. Denn vereinbart und sanktioniert werden kann nur Zumutbares i.S.d. § 121 SGB III. ${ }^{321}$

Die Höhe des Arbeitslosengeldes ist in $\S 129$ SGB III geregelt und abhängig vom Familienstatus, der Lohnsteuerklasse und dem wöchentlichen früheren Arbeitsentgelt des Arbeitslosen, sodass er bei Vorhandensein mindestens eines Kindes entweder 67 Prozent oder bei Fehlen von Kindern 60 Prozent des pauschalierten Nettoarbeitsentgeltes $^{322}$ für grundsätzlich 12 Monate 323 erhält.

320 BVerfGE 72, 9 [ 18 ff]; 74, 203 [213 ff]; dazu Lauer, in: Wissing u.a., SGB III, 2005; § 121 Rn. 9; allgemein Papier, in: Maunz/Dürig, Grundgesetz, Art. 14 Rn 152 f.

321 Dazu Marschner, in: Ambs, GK-SGB III, § 121 Rn. 2 ff.

322 Vgl. Einzelheiten zu den entsprechenden Berechnungen des Bemessungsentgeltes gem. § 132 SGB III und des Leistungsentgeltes gem. § 133 SGB III bei Köhler, Arbeitsförderungsrecht, in: Plagemann, Münchener Anwaltshandbuch Sozialrecht, 2009, S. 279-283.

323 Alters- und beitragszeitabhängig kann Arbeitslosengeld gem. § 434r SGB III bis maximal 24 Monate gewährt werden, vgl. Siebtes Gesetz zur Änderung des Dritten Buches Sozialgesetzbuch und anderer Gesetze v. 8.4.2008 (BGBl. I, S. 681). 


\section{a) Definition}

In $\S 6$ Abs. 1 S. $3, \S 35$ Abs. 4 S. 1 SGB III 324 ist festgelegt, dass die Agentur für Arbeit mit dem Arbeitslosen eine Eingliederungsvereinbarung trifft.

Die Agentur für Arbeit ist die unterste Verwaltungseinheit der Bundesagentur für Arbeit, einer rechtsfähigen bundesunmittelbaren Körperschaft des öffentlichen Rechts mit Selbstverwaltung 325 , und stellt deren örtliche Verwaltungsebene dar. ${ }^{326}$ Für die Agentur für Arbeit handelt ein zuständiger Sachbearbeiter, der die Agentur für Arbeit berechtigt und verpflichtet, für die er arbeitet.

Verhandlungspartner der Agentur für Arbeit ist der Arbeitslose. Arbeitslos im Sinn des $\S 35$ Abs. 4 SGB III ist gem. $§ 16$ SGB III wie beim Arbeitslosengeld gem. $§ 119$ Abs. 1 SGB III, wer vorübergehend nicht in einem Beschäftigungsverhältnis steht, eine versicherungspflichtige Beschäftigung sucht, dabei den Vermittlungsbemühungen der Agentur für Arbeit zur Verfügung steht und sich bei der Agentur für Arbeit arbeitslos gemeldet hat.

\section{b) Abgrenzung zum Eingliederungsvertrag, $\S \S 229$ ff SGB III a. F.}

Die Eingliederungsvereinbarung ersetzt den Eingliederungsvertrag nach $\S \S 229 \mathrm{ff}$ SGB III a. F. 327 In der Praxis blieb dieses Instrumente unbedeutend ${ }^{328}$ und ist durch das Job-AQTIV-Gesetz zum 31.12.2001 wieder abgeschafft worden. ${ }^{329}$ Der Eingliederungsvertrag ging auf eine Modellinitiative des Bundes Katholischer Unternehmer zurück. Dieser betrachtete die Risiken, die eine herkömmliche arbeitsvertragliche Bindung bedeuteten, als Hemmschwelle bei der Einstellung von Langzeitarbeitslosen. ${ }^{330}$ Die Arbeitgeber erwarteten bei dieser Gruppe von Arbeitslosen erhebliche krankheitsbedingte Fehlzeiten. Der Gesetzgeber reagierte mit der Einführung des Eingliederungsvertrages auch auf die vorgetragene Erfahrung, dass Langzeitarbeitslose oftmals erhebliche Defizite bei den fachlichen Kenntnissen, verringerte Leistungsbereitschaft sowie größere Schwierigkeiten hätten, sich in regelmäßige betriebliche Abläufe einzugliedern und konstante und effektive Leistungen zu erbringen. 331

$324 \S \S 6,35$ Abs. 4 SGB III sind durch das Gesetz zur Neuausrichtung der arbeitsmarktpolitischen Instrumente v. 21.12.2008 (BGBl. I, S. 2917), in Kraft seit 1.1.2009 aufgehoben worden. Seither ist die Eingliederungsvereinbarung in ähnlicher Form in $\S 37$ Abs. 2 und 3 SGB III geregelt. Die Arbeit behält die alte Zitierung bei, weil die frühere Rechtslage analysiert wird.

$325 \S 367$ Abs. 1 SGB III.

$326 \S 367$ Abs. 2 SGB III.

327 Die $\S \S 229$ ff SGB III a.F. traten zum 1.1.1998 in Kraft, Arbeitsförderungsreformgesetz v. 24.3.1997 (BGBl. I, S. 594).

328 Körner, NZA 2002, S. 242.

329 Gesetz v. 10.12.2001 (BGBl. I, S. 3443).

330 Dazu Stindt, Innovative Arbeitsförderung, in: Engelen-Kefer u.a., Die Reform der Arbeitsförderung, 1997, S. 52 ff.

331 BT-Drucksache 13/4941, S. 194. Dazu im Einzelnen Bepler, in: Gagel, SGB III, 2001, § 229 Rn. 3 11; allgemein zum Eingliederungsvertrag Hanau, DB 1997, S. 1278 ff; Rolfs, NZA 1998, S. 19 ff. 
Der Eingliederungsvertrag wurde zwischen Arbeitgeber und Arbeitnehmer abgeschlossen. Es wurde ein „privatrechtliches Probebeschäftigungsverhältnis“ ${ }^{332}$ begründet. Der Arbeitgeber konnte einen Langzeitarbeitslosen für einen Zeitraum von mindestens zwei Wochen bis längsten falls sechs Monate einstellen. Währenddessen übernahm das Arbeitsamt die Kosten für Ausfallzeiten, z.B. wegen Krankheit. Ziel war dabei die Übernahme des Beschäftigten in ein unbefristetes, jedenfalls in ein befristetes ordentliches Arbeitsverhältnis.

Die rechtliche Einordnung des Eingliederungsvertrags blieb bis zu seiner Abschaffung umstritten. Die Gewerkschaften forderten die Entstehung eines herkömmlichen Arbeitsverhältnisses. Sie hielten den Eingliederungsvertrag für einen „Probelauf für ein Arbeitsleben ohne oder nur mit stark reduziertem Arbeitsrecht" und sahen dieses modifizierte Beschäftigungsverhältnis als geeignet an, das Arbeitsrecht als Errungenschaften der Gewerkschaften zum Schutz des Arbeitsnehmers zu unterlaufen. ${ }^{333}$ Unter Berücksichtigung des gesetzgeberischen Interesses an einer Entlastung der Arbeitgeber sprechen die besseren Argumente dafür, dass durch den Vertragsschluss lediglich ein vom Arbeitsamt subventioniertes Beschäftigungsverhältnis im Sinn des § 7 SGB IV begründet wird und gerade kein Arbeitsverhältnis mit allen daraus entstehenden Rechten und Pflichten. Die rechtliche Einordnung bleibt im Übrigen wegen der Regelung in $\S 231$ Abs. 2 SGB III unbedeutend ${ }^{334}$, wonach Arbeitsrecht ohnehin Anwendung findet, soweit keine abweichenden Regelungen im SGB III getroffen wurden. ${ }^{335}$ Es handelt sich also um einen privatrechtlichen Vertrag, der erst nach Zustimmung durch das Arbeitsamt die Förderung durch das Arbeitsamt und weitere Modifikationen erfährt. ${ }^{336}$

Anders als beim Eingliederungsvertrag nach $\S \S 229$ ff SGB III a.F. wird die Eingliederungsvereinbarung nach $\S 35$ Abs. 4 SGB III zwischen Staat und Bürger abgeschlossen (kooperatives Instrument). Der Eingliederungsvertrag aber ist eine besondere Form des privatrechtlichen Vertrages, jedenfalls wird er zwischen zwei Privaten, dem Arbeitgeber und dem Arbeitnehmer, abgeschlossen. Die Arbeitsverwaltung beteiligt sich nicht an der konkreten Vertragsgestaltung ${ }^{337}$, sondern fördert nur den Abschluss des Vertrages durch verbesserte (Rahmen-)Bedingungen für den Arbeitgeber. Sie begleitet die Integration des Arbeitslosen durch weitere arbeitsförderungsrechtliche Instrumente, wie z.B. Trainingsmaßnahmen, berufliche Weiterbildung und Eingliederungszuschüsse. ${ }^{338}$ Sie ist selbst nicht am Vertragsverhältnis beteiligt. Die besonderen Probleme kooperativer Verwaltung im Verhältnis Staat - Bürger stellen sich demzufolge nicht.

332 Bepler, in: Gagel, SGB III, 2001, § 229 Rn. 7.

333 Dazu Gerntke/Ulber, AiB 1997, S. 512 f.

334 Bader, AuR 1997, S. 381 ff.

335 Niesel, SGB III, 1998 (1. Auflage), § 231 Rn. 3; Bepler, in: Gagel, SGB III, 2001, § 231 Rn. 5 ff.

336 Zur Diskussion um die rechtliche Einordnung zusammenfassend Bepler, in: Gagel, SGB III, 2001, § 231 Rn. 39-42; Heinz, in: Wissing u.a., SGB III, 1999 (wegen Rechtsänderung alte Auflage), § 231 Rn. 7-17.

337 Sie stellt nur einen Mustervertrag zur Verfügung, abgedruckt bei Bepler, in: Gagel, SGB III, 2001, $\S 231$ Anlage 1.

338 Strobel, Der Eingliederungsvertrag zur Integration von Arbeitslosen, in: Charbonnier, Beschäftigungsförderung durch neues Arbeits- und Sozialrecht, 1998, S. 146 f. 
a) Niederlegung der ,individuellen Vermittlungsstrategie“

Die Regelungen in $\S 6$ Abs. 1 S. 3, § 6 Abs. 2 S. 2 , § 35 Abs. 4 SGB III sind denkbar kurz. Der Gesetzgeber beschränkt sich auch in der Gesetzesbegründung auf die Formulierung, dass in der Vereinbarung die sog. ,individuelle Vermittlungsstrategie“ niedergelegt werden soll. 339

In der Vereinbarung werden die Vermittlungsbemühungen der Agentur für Arbeit und die Eigenbemühungen des Arbeitslosen festgelegt. Zudem wird aufgenommen, welche künftigen Leistungen der aktiven Arbeitsförderung, sofern die Voraussetzungen dafür vorliegen, ergriffen, und in welcher zeitlichen Abfolge diese erbracht werden. Gegenüber Arbeitslosen, die einen Eingliederungsgutschein nach $§ 223$ SGB III erhalten, soll die Ausgabe des Eingliederungsgutscheins in der Eingliederungsvereinbarung mit einem Arbeitsangebot oder einer Vereinbarung über die notwendigen Eigenbemühungen zur Einlösung des Eingliederungsgutscheins verbunden werden, § 35 Abs. 4 S. 2 SGB III.

Um bei späteren Entscheidungen den Aushandlungsprozess besser nachvollziehen zu können, ist die Aufnahme der wesentlichen Gründe, die für die Vereinbarung der Maßnahmen ausschlaggebend waren, in den Text der Vereinbarung sinnvoll. 340

\section{b) Vermittlungsbemühungen der Agentur für Arbeit}

Was „Vermittlungsbemühungen“ sind, ist in $\S 35$ Abs. 1 S. 1, 2 SGB III definiert. Vermittlung ist die Tätigkeit der Agentur für Arbeit, die darauf gerichtet ist, Ausbildungsuchende mit Arbeitgebern zur Begründung eines Ausbildungsvertrages und Arbeitsuchende mit Arbeitgebern zur Begründung eines Beschäftigungsverhältnisses zusammenzubringen. Beschäftigungsverhältnis ist der Oberbegriff für Beschäftigung als Arbeitnehmer oder Heimarbeiter, unabhängig davon, ob in eine versicherungspflichtige oder z.B. nur in eine geringfügige Beschäftigung vermittelt wird. Begrifflich ausgeschlossen sind damit Beschäftigungen als Beamter oder Selbständiger, wobei dies nicht als ein Verbot für die Agenturen für Arbeit zu verstehen ist, in diese Formen von Beschäftigung zu vermitteln. ${ }^{341}$

Bei ihrer Vermittlungstätigkeit hat die Agentur für Arbeit die Neigung, Eignung und Leistungsfähigkeit des Arbeitsuchenden und die Anforderungen der angebotenen Stellen zu berücksichtigen, $\S 35$ Abs. 2 S. 2 SGB III (Prinzip der Individualisierung). Die genauen Anforderungen für die Arbeitsverwaltung sind nicht geregelt und hängen damit in gewissem Maße am Engagement der Mitarbeiter in den Agenturen für Arbeit. Derjenige, der sich bei der Bundesagentur für Arbeit meldet, hat ein subjektiv-öffentliches Recht auf deren Tätig werden (Anspruch auf sachgerechte Vermittlung). Die Bundesagentur für Arbeit hat aber bei Leistungen der aktiven Arbeitsförderung, die gem. § 3

339 BT-Drucksache 14/6944, S. 28.

340 Luthe, SGb 2002, S. 80.

341 Peters-Lange, in: Gagel, SGB III, § 35 Rn. 11. 
Abs. 5 SGB III keine Pflichtleistungen sind, bei der Auswahl der konkreten Eingliederungsleistungen einen Spielraum, der es ihr erlaubt, in pflichtgemäßer Ermessensausübung zwischen verschiedenen Möglichkeiten auszuwählen. ${ }^{342}$

\section{c) Eigenbemühungen des Arbeitslosen}

Entsprechend der Legaldefinition in $\S 119$ Abs. 1 Nr. 2 i.V.m. § 119 Abs. 4 SGB III sind Eigenbemühungen des Arbeitslosen die Bemühungen des Arbeitnehmers, seine Beschäftigungslosigkeit zu beenden. Er hat dazu alle Möglichkeiten zu seiner beruflichen Eingliederung zu nutzen. Hierzu gehören nach dem Gesetzestext die Wahrnehmung der Verpflichtungen aus der Eingliederungsvereinbarung, die Mitwirkung bei der Vermittlung durch Dritte und die Inanspruchnahme der Selbstinformationseinrichtungen der Agentur für Arbeit.

Ist das Vorliegen von Eigenbemühungen als Leistungsvoraussetzung und damit als echte Rechtspflicht oder deren Fehlen als bloßes versicherungswidriges Verhalten (Obliegenheit) anzusehen?

In $\S 119$ Abs. 1 Nr. 3 SGB III werden Eigenbemühungen als Leistungsvoraussetzung aufgeführt. Bei der Regelung des Ruhens des Arbeitslosengeldes wegen einer Sperrzeit in $\S 144$ Abs. 1 S. 2 Nr. 3 SGB III ist die Unterlassung der von der Agentur für Arbeit geforderten Eigenbemühungen als versicherungswidriges Verhalten sanktioniert.

Es ist zu unterscheiden zwischen Eigenbemühungen, die bloß (teilweise) fehlen und einem Verhalten des Arbeitsuchenden, das seine völlige Passivität zeigt und das vollständige Ablehnen von Eigeninitiative zum Ausdruck bringt. ${ }^{343}$ Die Formulierung in $\S$ 144 Abs. 1 S. 2 Nr. 3 SGB III ist so zu verstehen, dass sanktioniert wird, wenn Nachweishandlungen, z.B. die Vorlage von Bewerbungsschreiben, fehlen, also der Nachweis von Eigenbemühungen nicht gelungen ist. Fehlt jegliches Tätigwerden des Arbeitsuchenden, entsteht der Anspruch auf Arbeitslosengeld erst gar nicht, weil eine Leistungsvoraussetzung fehlt.

In der Eingliederungsvereinbarung werden die von der Agentur für Arbeit geforderten Handlungen des Arbeitsuchenden fixiert. Das Gespräch über den Abschluss einer Eingliederungsvereinbarung dürfte bereits das $\mathrm{Ma} ß$ an Eigenbemühungen erfüllen, das für die Leistungsvoraussetzung in $\S 119$ Abs. 1 Nr. 3 SGB III erforderlich ist.

Aussagen über den Inhalt einer Eingliederungsvereinbarung können nicht ohne Kenntnis des Begriffs „Eigenbemühungen“ getroffen werden. Vorzugehen ist deshalb so, dass zuerst abstrakt der Begriff Eigenbemühungen definiert und sodann die konkreten Maßnahmen für einen einzelnen Arbeitslosen in der Eingliederungsvereinbarung fixiert werden müssen.

342 Dazu Peters-Lange, in: Gagel, SGB III, § 35 Rn. 18.

343 So entschied das BSG, BSGE 95, 176 [183], dass bei einer fehlenden Konkretisierung der Obliegenheit zu Eigenbemühungen diese nur verneint werden können, wenn der Arbeitslose über die Einschaltung der Dienste der Agentur für Arbeit hinaus selbst nichts unternimmt. So bereits Voelzke, NZS 2005, S. 282; Steinmeyer, in: Gagel, SGB III, § 119 Rn. 118. 
Welche Leistungen der aktiven Arbeitsförderung auf der Grundlage des SGB III erbracht werden können, regelt $\S 3$ Abs. 4 SGB III. ${ }^{344}$

Diese Leistungen können nur insoweit in der Vereinbarung festgelegt werden, als deren Voraussetzungen vorliegen, $\S 35$ Abs. 4 S. 1 SGB III. So können z.B. Maßnahmen der beruflichen Weiterbildung nur vereinbart werden, wenn der Arbeitslose die allgemeinen Förderbedingungen gem. § 77 SGB III erfüllt. ${ }^{345}$ Das stellt eine Einschränkung des Vereinbarungsspielraums der Parteien einer Eingliederungsvereinbarung dar, der bei der Verhandlung über deren möglichen Inhalt berücksichtigt werden muss.

Es muss vor Abschluss der Vereinbarung bereits eine Vorabprüfung dieser Voraussetzungen stattgefunden haben, sonst besteht die Gefahr, dass die Vereinbarung ins Leere geht. Inwieweit dann die Vereinbarung noch bindend sein kann, insbesondere weil Leistungen der Agentur für Arbeit und Gegenleistung des Arbeitslosen nicht mehr ausgewogen sind, beziehungsweise der Arbeitslose unter diesen Bedingungen sein Einverständnis nicht erteilt hätte, ist im weiteren Verlauf der Arbeit noch zu klären.

Problematisch ist ferner, wie es sich auf den Verhandlungsspielraum auswirkt, dass der Arbeitsverwaltung nicht für alle Leistungen der aktiven Arbeitsförderung ein Ermessensspielraum eingeräumt wurde. Im Ermessen der Verwaltung stehen nur die Berufsberatung, Arbeitsvermittlung und diese unterstützenden Leistungen, Maßnahmen der Eignungsfeststellung, Trainingsmaßnahmen zur Verbesserung der Eingliederungsaussichten und die Übernahme von Weiterbildungskosten während der Teilnahme an einer beruflichen Weiterbildung. Die anderen Maßnahmen der aktiven Arbeitsförderung sind Entgeltersatzleistungen und damit Versicherungsleistungen, die durch den Beitrag „erkauft" wurden und somit möglicherweise nicht verhandelbar sind.

\section{e) Geltungszeitraum der Vereinbarung}

Aus $\S 35$ Abs. 4 S. 5 SGB III geht hervor, dass die Eingliederungsvereinbarung ohne erneute Überprüfung längsten falls für sechs Monate, bei arbeitslosen und ausbildungsuchenden Jugendlichen sowie in den Fällen des Satzes 2 (Eingliederungsgutschein) längsten falls für drei Monate abgeschlossen werden darf. Die Eingliederungsvereinbarung ist für einen konkreten Zeitraum abzuschließen. ${ }^{346}$ Innerhalb dieser Fristen soll jedenfalls im Regelfall ein Treffen zwischen Arbeitslosem und Mitarbeiter in der Agentur für Arbeit stattfinden, um zu überprüfen, ob sich die Annahmen aus der Eingliederungsvereinbarung bewahrheiten oder nicht. ${ }^{347}$ Dies gilt insbesondere für die Gefahr der Entstehung einer Langzeitarbeitslosigkeit. Möglichst frühzeitig soll dieses Risiko, auch vom Arbeitslosen, erkannt und entsprechende Maßnahmen der Arbeitsverwaltung ergriffen werden.

344 Vgl. zweites Kapitel B I 1c) S. 142 ff.

345 Steinmeyer, info also 2002, S. 6.

346 So auch Steinmeyer, info also 2002, S. 6; Rademacher, in: Ambs, GK-SGB III, § 35 Rn. 99.

347 BT-Drucksache 14/6944, S. 31. 
Die Eingliederungsvereinbarung nach SGB III ist schriftlich abzuschließen, § 35 Abs. 4 S. 3 SGB III. Es muss eine Unterschrift unter das Dokument gesetzt werden. Dem Arbeitslosen ist eine Ausfertigung der Vereinbarung auszuhändigen, § 35 Abs. 4 S. 3 SGB III.

Schriftlichen Erklärungen werden von den Erklärenden selbst größere Bedeutung zugemessen als mündlichen Absprachen (Warnfunktion ${ }^{348}$ ). Die Betonung der Ernsthaftigkeit der schriftlichen Erklärung entspricht auch dem Interesse des Gesetzgebers. ${ }^{349}$ Dieser legt Wert auf persönlichen Kontakt zwischen Fallmanager und Arbeitslosem und verlangt ein Aushandeln der Eingliederungsvereinbarung.

Der Ausschluss einer telefonischen Arbeitslosmeldung gem. § $37 \mathrm{~b} \mathrm{SGB} \mathrm{III}{ }^{350}$ zeugt davon, dass der Versicherungspflichtige verpflichtet ist, sich unverzüglich nach Kenntnis des Beendigungszeitpunkts persönlich bei der Agentur für Arbeit als arbeitsuchend zu melden. Das deutsche Arbeitsförderungsrecht erachtet den persönlichen Kontakt zwischen Arbeitslosem und Mitarbeiter der Agentur für Arbeit als besonders wichtig.

Dieser Gedanke setzt sich bei der Eingliederungsvereinbarung fort. Wird lediglich eine Strategie nach Aktenlage schriftlich und ohne persönlichen Kontakt zugeleitet, ist dies ebenso wenig zulässig, wie die schlichte Übergabe eines Formulars, das mit Eingliederungsvereinbarung überschrieben, aber weder durchgesprochen wird noch Spielraum für den Einzelfall lässt. Für den Arbeitslosen hat die Schriftform allerdings den Vorteil, dass er, verhält er sich entsprechend der Vereinbarung, mit diesem Dokument den geforderten Nachweis über die von der Agentur für Arbeit geforderten Eigenbemühungen gem. § 119 Abs. 5 SGB III erbringen kann (Beweis- oder Kontrollfunktion $\left.^{351}\right) .{ }^{352}$ Sie sollte deshalb auch von den Arbeitslosen nicht als bloße Formalität begriffen, sondern ernst genommen werden.

\section{Rechtliche Einordnung}

Den Worten Krauses folgend, das „Recht [dürfe] die Handlungen der Verwaltung nicht als vorgegebene quasi-natürliche Vorgänge hinnehmen und sie erst dann Handlungsformen zuordnen, nachdem sie vorgenommen wurden; es [habe] sie als Tätigkeit mit spezifischer Funktion in den Griff zu nehmen und durch Formen und Verfahren zu disziplinieren“ 353 wird nun die rechtliche Einordnung der Eingliederungsvereinbarung nach dem SGB III vorgenommen.

348 Weihrauch, VerwArch 1991, S. 558, der Faber, Verwaltungsrecht, 1995, S. 277 insoweit widerspricht. Faber hält lediglich die Beweisfunktion der Schriftform im Verwaltungsrecht für vom historischen Gesetzgeber gewollt, nicht aber die Warnfunktion.

349 BT-Drucksache 14/6944, S. 31.

350 Otto, NZS 2005, S. $288 \mathrm{ff}$.

351 Dazu Kopp/Ramsauer, VwVfG, 2008, § 57 Rn. 1; Henneke, in: Knack, VwVfG, 2000, § 57 Rn. 3.

352 Steinmeyer, info also 2002, S. 6.

353 Krause, Rechtsformen des Verwaltungshandelns, 1974, S. 16. 
Im deutschen öffentlichen Recht gibt es bestimmte gesetzlich geregelte Handlungsformen, wobei es sich dabei nicht um einen abschließenden Katalog handelt. Die Verwaltung genießt eine sog. Formenwahlfreiheit. 354

\section{a) Verwaltungsakt, $\S 31 \mathrm{SGB} X$}

Der Verwaltungsakt ist zunächst eine typisch einseitige, nicht-kooperative Handlungsform der Verwaltung. Doch kann, wie im ersten Kapitel dieser Arbeit dargestellt, der Verwaltungsakt kooperative Elemente aufweisen. ${ }^{355}$ In welcher Art und Weise Kooperation stattfinden müsste, um die Eingliederungsvereinbarung als Verwaltungsakt einordnen zu können ${ }^{356}$, wird im Folgenden untersucht.

Verwaltungsakt ist jede Verfügung, Entscheidung oder andere hoheitliche Maßnahme, die eine Behörde zur Regelung eines Einzelfalles auf dem Gebiet des öffentlichen Rechts trifft und die auf unmittelbare Rechtswirkung nach außen gerichtet ist, $\S 31 \mathrm{~S} .1$ SGB X.

\section{aa) Hoheitliche Maßnahme}

Hängt die Ausarbeitung der Eingliederungsvereinbarung überwiegend vom Einverständnis des Arbeitslosen ab (dann weist die Maßnahme eine vertragliche Prägung auf) oder ist sie nur von seiner Mitwirkung an einseitigen Festlegungen der Agentur für Arbeit geprägt (dann liegt ein Verwaltungsakt vor)?

Die Definition der einseitigen Maßnahme wird zu dem vom Gesetzgeber des SGB III geforderten und in der Gesetzesbegründung beschriebenen Verhalten der Arbeitsverwaltung in Beziehung gesetzt. Es kann keine Subsumtion stattfinden, weil dazu das Verhalten der Parteien im Einzelfall betrachtet werden müsste.

Die hoheitliche Maßnahme 357 ist gekennzeichnet durch deren Einseitigkeit. 358 „Einseitig“ bezieht sich auf das Verfahren, nicht auf die getroffene Regelung als Ergebnis der Maßnahme. ${ }^{359}$ Das gilt auch für den mitwirkungsbedürftigen Verwaltungsakt ${ }^{360}$, bei dem die Maßnahme zwar beantragt beziehungsweise ihr zugestimmt, letztendlich aber einseitig von der Behörde festgelegt und verantwortet wird.

Einseitigkeit ist als Gegensatz zur vertraglichen Regelung zu verstehen. ${ }^{361}$ So wird die Maßnahme beim verwaltungsrechtlichen Vertrag ausgehandelt. Aushandeln bedeutet, dass sich die Parteien über den Inhalt der Vereinbarung austauschen bzw. darüber sprechen. Dazu ist es notwendig, dass es einen Spielraum für den Inhalt gibt, dass also nicht bereits der gesamte Inhalt aufgrund gesetzlicher oder anderer Bindungen feststeht.

\footnotetext{
354 Vgl. erstes Kapitel B III 3a) aa) S. 76.

355 Vgl. erstes Kapitel B I 1 S. 65 ff.

356 Vgl. z.B. Steinmeyer, info also 2002, S. 4 f.

357 Stelkens, in: Stelkens/Bonk/Sachs, VwVfG, 2001, § 35 Rn. 41 f zum Begriff der „Maßnahme“.

358 Engelmann, in: Wulffen, SGB X, 2008, § 31 Rn. 10.

359 Krause, Rechtsformen des Verwaltungshandelns, 1974, S. 126.

360 Vgl. erstes Kapitel B I 1b) aa) S. 66.

361 Vgl. erstes Kapitel B IV 1f) S. 89 f.
} 
Im Privatrecht geht man davon aus, dass während des Aushandelns die wechselseitige Beeinflussung sichergestellt wird und wegen der abschließenden Einigung im Vertrag grundsätzlich einseitige Ergebnisse vermieden werden. ${ }^{362}$ Es ist deshalb nicht erforderlich, dass ein gegenseitiges Nachgeben ${ }^{363}$ wie beim Vergleich stattfindet. Es ist auch nicht abträglich, dass sich eine Partei mit ihren Forderungen ganz durchsetzt, während die andere nur Minimalforderungen oder gar keine stellt. Dies kann zwar dazu führen, dass von einem Verhandlungsungleichgewicht der Parteien auszugehen ist, schränkt aber nicht bereits den Verhandlungsbegriff ein. ${ }^{364}$

Ein Aushandeln kommt bei der Eingliederungsvereinbarung folglich in Betracht, wenn im Einzelfall ein echtes Gespräch zwischen Arbeitsverwaltung und Arbeitslosem stattfindet. Der Inhalt ist zwar nur eingeschränkt von Seiten der Verwaltung verhandelbar, aber es gibt Spielräume, insbesondere bei der Auswahl verschiedener Eingliederungsleistungen, aber auch bei den Eigenbemühungen des Arbeitslosen. ${ }^{365}$ Die Bindung der Verwaltung, gesetzmäßig zu handeln, schließt nicht von vornherein ein Aushandeln aus, sonst wäre ein verwaltungsrechtlicher Vertrag undenkbar. Auf Seiten des Arbeitslosen besteht ohnehin ein Verhandlungsspielraum, sowohl im Hinblick auf das Maß und die Ausgestaltung seiner Eigenbemühungen als auch im Hinblick auf die Maßnahmen aktiver Arbeitsförderung, die für ihn interessant erscheinen. ${ }^{366}$ Die Abhängigkeit von finanzieller Unterstützung in Form von Arbeitslosengeld wirkt sich nur bedingt aus. Das Arbeitslosengeld selbst kann niemals Verhandlungsmasse sein, da der Arbeitslose darauf einen Anspruch hat, den er auch einklagen kann. ${ }^{367}$ Sanktionsmöglichkeiten der Verwaltung im Hinblick auf die Erzwingung einer Vereinbarung sind nicht vorhanden. ${ }^{368}$ Insofern steht einem Aushandeln im obigen Sinn nichts entgegen.

Eine vertragliche Regelung als Gegenbegriff zur hoheitlichen Maßnahme setzt ferner voraus, dass die Parteien mit Vertragswillen handeln. Nur wenn sie einen Vertrag abschließen wollen, kann auch ein Vertrag zustande kommen. Entscheidend ist die subjektive Willensrichtung der Beteiligten. Ein Vertrag liegt vor, wenn die Rechtsfolgen eines verwaltungsrechtlichen Vertrags gewollt, ein Verwaltungsakt, wenn die Rechtsfolgen der $\S 335 \mathrm{ff}$ VwVfG, $\S \S 31 \mathrm{ff}$ SGB X beabsichtigt sind. ${ }^{369}$ Zur Feststellung der subjektiven Willensrichtung sind objektive Kriterien heranzuziehen, wie die Bezeichnung der Maßnahme, die Form und mündliche oder schriftliche Äußerungen der Beteiligten wäh-

362 Brox/Walker, Allgemeiner Teil des Bürgerlichen Rechts, 2008, Rn. 70 f.

363 Vgl. dazu Kopp/Schenke, VwGO, 2007, § 106 Rn. 3, der unter Hinweis auf HansOLG MDR 1977, S. 502 schreibt: ,jeder muss nachgeben“.

364 Schweiger, NZS 2002, S. 411 vermengt an dieser Stelle die Frage des Verhandlungsgleichgewichts mit dem Begriff des Aushandelns.

365 Vgl. zweites Kapitel B I 3 S. 147 ff.

366 A.A. Schweiger, NZS 2002, S. 412, der die Verhandlungsstärke des einzelnen Arbeitslosen als zu gering ansieht, als dass ein Verhandeln möglich wäre. Er negiert auch den kooperativen Charakter der Eingliederungsvereinbarung und spricht von einseitigem informellem Verwaltungshandeln.

367 Beim verwaltungsrechtlichen Vertrag regelt dies $\S 53$ Abs. 2 SGB X.

368 Vgl. zweites Kapitel B I 8b) S. 177 f; im Ergebnis so auch Schweiger, NZS 2002, S. 412.

369 Ule/Laubinger, Verwaltungsverfahrensrecht, 1995, § 67 I; Bonk, in Stelkens/Bonk/Sachs, VwVfG, 2001, § 54 Rn. 39; Henneke, in: Knack, VwVfG, 2000, § 54 Rn. 3. 
rend des Verwaltungsverfahrens. ${ }^{370}$ In unklaren Fällen gibt es keine Vermutung zugunsten eines Verwaltungsakts als Normalfall verwaltungsrechtlichen Handelns ${ }^{371}$, doch wird man in der Regel wegen des Fehlens einer wirksamen Willenserklärung keinen verwaltungsrechtlichen Vertrag annehmen können. Es ist zudem nicht Voraussetzung, dass die Partner des Vertrages „gleich“ im Sinne von gleich stark, gleich mächtig oder gleich unabhängig sind. 372

Bezogen auf die Anwendung dieser Definition im Arbeitsförderungsrecht steht das mögliche Verhandlungsungleichgewicht auf Seiten des Arbeitslosen der Annahme einer ausgehandelten Vereinbarung, z.B. in Form eines verwaltungsrechtlichen Vertrages nicht entgegen. Das Verhandlungsgewicht des Einzelnen ist zwar von außen betrachtet geringer als das der Verwaltung, weil diese einseitig Maßnahmen festsetzen und die Nichteinhaltung der Regelungen sanktionieren kann. Doch steht der Arbeitsverwaltung das Recht, einseitig tätig zu werden, nur zu, wenn eine Einigung über die Eingliederungsstrategie nicht gelingt. Die Drohung mit dem Leistungsentzug ist deshalb nicht bereits von vornherein möglich. Die Behörde soll nach dem Willen des Gesetzgebers gerade nicht einseitig tätig werden. Dies folgt aus der Anmerkung in der Gesetzesbegründung, dass erst bei fehlender Einigung eine einseitige Aufgabe zum Tätig werden bei der Agentur für Arbeit übrig bleibt. ${ }^{373}$ Um rechtmäßig zu handeln, muss die Agentur für Arbeit also zunächst ausschließen, dass eine Vereinbarung der individuellen Vermittlungsstrategie nicht möglich ist. Scheitert diese Einigung, bleibt der Verwaltung die Möglichkeit, hoheitlich tätig zu werden. ${ }^{374}$

Dass an Verhandlung zwischen Arbeitsverwaltung und Arbeitslosem nicht zu denken sei, weil der Arbeitslose die im Ermessen der Verwaltung stehenden Maßnahmen der aktiven Arbeitsförderung nicht ohne weiteres einklagen könne ${ }^{375}$, kann nicht überzeugen. Die Ermessensverwaltung ist gerade das ,genuine Gebiet“ des verwaltungsrechtlichen Vertrages, wie sich aus $\S 53$ Abs. 2 SGB X ableiten lässt, der das Verhandeln über Rechtsansprüche ausschließt. Die Konkretisierung dieser allgemein gehaltenen gesetzlichen Regelungen erfolgt über die Aushandlung der Eingliederungsvereinbarung. Insofern ist die fehlende Einklagbarkeit kein Druckmittel für die Verwaltung. Auch diese kann nicht ohne weiteres fehlende Eigenbemühungen sanktionieren, ohne sie für den Einzelnen verständlich konkretisiert zu haben. ${ }^{376}$

370 Schlette, Die Verwaltung als Vertragspartner, 2000, S. 185 f.

371 Schmidt - Aßmann, Das allgemeine Verwaltungsrecht als Ordnungsidee, 2004, S. 343; Schlette, Die Verwaltung als Vertragspartner, 2000, S. 187; a.A. Kopp, VwVfG, § 54 Rn. 15; Kottke, Subordinationsrechtlicher Vertrag, 1966, S. 21; Kirchhof, in: Isensee/Kirchhof, Handbuch des Staatsrechts, 2005, Bd. 3, § 59 Rn. 122 ff, 149 ff.

372 Vgl. erstes Kapitel B III 2c) S. 73; a.A. Schweiger, NZS 2002, S. 412.

373 BT-Drucksache 14/6944, S. 31.

374 Vgl. erstes Kapitel A IV 2 S. 62 zur Handlungsfähigkeit der Verwaltung bei kooperativem Vorgehen.

375 Schweiger, NZS 2002, S. 412.

376 Bieback, Umbau der Arbeitsförderung nach den Vorschlägen der Hartz-Kommission, in: SDSRV 52 (2004), S. $73 \mathrm{f}$. 
Ferner wird eingewandt, dass ein Aushandeln grundsätzlich in der Situation der Arbeitslosigkeit undenkbar sei, weil der Arbeitslose seine Interessen gegenüber der Verwaltung niemals vertreten könne. Es mag einzelne Arbeitslose geben, die zu einer in irgendeiner Weise förderlichen Vertretung eigener Interessen nicht in der Lage sind, doch muss in diesem Fall an deren Erwerbsfähigkeit insgesamt gezweifelt werden oder an deren Fähigkeit, sich überhaupt rechtsgeschäftlich zu binden. Das sind Ausnahmefälle. Es ist von einer grundsätzlich möglichen Verhandlung der Eingliederungsstrategie zwischen Arbeitsverwaltung und Arbeitslosem auszugehen.

Bei der Eingliederungsvereinbarung nach dem SGB III fehlt in der Regel die hoheitliche Maßnahme, weil die Ausarbeitung der Vereinbarung überwiegend vom Einverständnis des Arbeitslosen abhängt. Kommt aber trotz redlichem Bemühen der Parteien keine Einigung zwischen Arbeitslosem und Arbeitsverwaltung zustande, bleibt der Behörde die Möglichkeit, hoheitlich vorzugehen. Es handelt sich dann aber nicht um eine Eingliederungsvereinbarung, sondern um einen Eingliederungsplan. ${ }^{377}$

\section{bb) Regelung}

Eine Regelung setzt voraus, dass eine verbindliche Rechtsfolge gesetzt wird und so ein Lebenssachverhalt einseitig verbindlich gestaltet, d.h. Rechte des Betroffenen unmittelbar begründet, geändert, aufgehoben, mit bindender Wirkung festgestellt oder verneint werden. ${ }^{378}$

Die Intention des Gesetzgebers und der Sinn und Zweck der Regelung in $\S 35$ Abs. 4 SGB III schließen eine einseitige Regelung im Sinn des § 31 SGB X aus. Der Gesetzgeber hat ausdrücklich formuliert, dass die Eingliederungsvereinbarung kein neues Rechtsverhältnis begründet. 379 Zudem wurde eine Eingliederungs"vereinbarung" 380 eingeführt, was darauf hinweist, dass deren Inhalt nicht allein von der Behörde festgelegt, sondern von beiden Parteien der Vereinbarung getragen werden soll. ${ }^{381}$

Zwar ist die Mitwirkung des Einzelnen kein entscheidendes Kriterium für eine vertragliche Regelung, da es auch den mitwirkungsbedürftigen Verwaltungsakt gibt, doch geht das für die Eingliederungsvereinbarung vorgesehene Kooperieren weit über das beim mitwirkungsbedürftigen Verwaltungsakt Übliche hinaus. ${ }^{382}$ Es lässt sich für die Eingliederungsvereinbarung nicht leugnen, dass die Agentur für Arbeit ihren Inhalt, der gesetzlich nur vage umschrieben ist, nicht allein bestimmen kann. Es ist die Information durch den einzelnen Arbeitslosen erforderlich, sodass ein Mindestmaß an Kommunika-

377 Vgl. im Folgenden zweites Kapitel B I 5b) S. 155.

378 BVerwGE 36, 192 [194]; 77, 268 [271]; Henneke, in: Knack, VwVfG, 2000, § 35 Rn. 32 f; Stelkens, in: Stelkens/Bonk/Sachs, VwVfG, 2001, § 35 Rn. 77 ff.

379 BT-Drucksache 14/6944, S. 31, bestätigend Buchheit, BArbB1 2002, S 5 f.

380 BVerwGE 25, 72 [78 f] zur Wertung als VA trotz Bezeichnung „Vereinbarung.

381 So auch Bieback, Umbau der Arbeitsförderung nach den Vorschlägen der Hartz-Kommission, SDSRV 52 (2004), S. 72 f; Peters-Lange, in: Gagel, SGB III, § 35 Rn. 29; Rademacher, in: Ambs, GK-SGB III, § 35 Rn. 105, der überdies deutlich eine Veränderung der Rechtsverhältnisse durch die Eingliederungsvereinbarung ablehnt.

382 Bieback, Umbau der Arbeitsförderung nach den Vorschlägen der Hartz-Kommission, SDSRV 52 (2004), S. 73. 
tion vorliegen muss. Das Ergebnis der Kommunikation, die Vermittlungsstrategie, muss zudem auch vom Arbeitslosen mitgetragen werden. Dies wäre bei einer einseitigen Regelung nicht möglich.

\section{cc) Zwischenergebnis}

Beim Abschluss einer Eingliederungsvereinbarung fehlt sowohl eine hoheitliche Maßnahme als auch eine Regelung, um die Eingliederungsvereinbarung rechtlich als Verwaltungsakt einordnen zu können.

\section{b) „Eingliederungsplan“}

Der Plan ist eine weder im Verwaltungsverfahrensgesetz noch im SGB X ausdrücklich geregelte Handlungsform. Es gibt ihn speziell im Sozialrecht z.B. als Hilfeplan gem. § 36 Abs. 2 SGB VIII oder als Gesamtplan auf der Grundlage des § 58 SGB XII. Über die Rechtsnatur des Plans besteht - wie oben dargestellt - Uneinigkeit. Es lassen sich aus der Diskussion Eigenschaften des Plans als kooperativer Handlungsform der Verwaltung herausfiltern. ${ }^{383}$

Ein Plan mit kooperativen Elementen setzt voraus, dass das Verfahren seiner Ausarbeitung unter Mitwirkung des Betroffenen erfolgt, ohne dass die Mitwirkung Wirksamkeitsvoraussetzung wäre. Das Ergebnis der Planung wird im Plan einseitig von der Verwaltung niedergelegt, sodass der Betroffene darauf keinen Einfluss mehr hat.

In Abgrenzung zum Verwaltungsakt enthält ein Plan keine eigenständige Verwaltungsentscheidung mit unmittelbarer Außenwirkung. Er hat vorbereitende und begründende Bedeutung für den sich anschließenden Leistungsbescheid. Er wird mit der Einbeziehung Teil des Leistungsbescheids als maßgeblichem Verwaltungsakt, sofern ein solcher erlassen wird. Ansonsten hat er keine rechtliche Relevanz für die Verwaltung oder den Bürger. 384

Bezogen auf die Eingliederungsvereinbarung nach SGB III kommt ein sog. „Eingliederungsplan“385 in Betracht, wenn keine Einigung zwischen Arbeitslosem und Agentur für Arbeit zustande kommt. Diesen Fall sieht die Gesetzesbegründung ausdrücklich vor. Sie weist darauf hin, dass es bei fehlender Einigung bei der einseitigen Vermittlungsund Beratungsaufgabe der Agentur für Arbeit und einer Entscheidung über Leistungen der aktiven Arbeitsförderung bleibt. ${ }^{386}$ Dieser Eingliederungsplan ist nicht als Verwaltungsakt einzuordnen, weil er keine Regelung enthält. Die Regelung enthält erst die auf der Grundlage der Planung ergangene Entscheidung über die konkrete Maßnahme. ${ }^{387}$

383 Vgl. erstes Kapitel B II 1 S. 68 f.

384 Dazu Luthe, SGb 2002, S. 79.

385 Buchheit, BArbB1 2002, S. 5.

386 BT-Drucksache 14/6944, S. 31.

387 Köhler, Arbeitsförderung, in: Plagemann, Münchener Anwaltshandbuch Sozialrecht, 2009, S. 402. 
Daraus ergibt sich im Umkehrschluss, dass die Arbeitsverwaltung ansonsten nicht einseitig handelt, um zu vermeiden, dass sie sich rechtswidrig verhält ${ }^{388}$, mithin also nur bei fehlgeschlagener Einigung nach Ausarbeitung eines Eingliederungsplans einen Verwaltungsakt erlässt. ${ }^{389}$

\section{c) Verwaltungsrechtlicher Vertrag, $\S \S 53$ ff SGB X}

\section{aa) Allgemeine Voraussetzungen}

Der verwaltungsrechtliche Vertrag setzt gem. § 53 Abs. 1 SGB X voraus, dass ein Rechtsverhältnis auf dem Gebiet des öffentlichen Rechts durch Vertrag begründet, geändert oder aufgehoben wird, soweit Rechtsvorschriften nicht entgegenstehen.

\section{bb) Abgrenzung zum privatrechtlichen Vertrag}

Zunächst ist eine Abgrenzung zum Privatrecht vorzunehmen, die über den Gegenstand der vertraglichen Regelung erfolgt. Maßgeblich ist im Zweifel der Schwerpunkt der vertraglichen Regelungen. Für die Einordnung kann auch der mit der Vereinbarung verfolgte Zweck herangezogen werden. Ist eine eindeutige Zuordnung nicht möglich, ist keine Aufspaltung in verschiedene Vertragsbestandteile und deren unterschiedliche $\mathrm{Zu}$ ordnung geboten. ${ }^{390}$

Ein verwaltungsrechtlicher Vertrag ist anzunehmen, wenn der Vertrag Rechte/Pflichten öffentlich-rechtlicher Art begründen, inhaltlich verändern oder aufheben soll. Eine öffentlich-rechtliche Pflicht beziehungsweise ein öffentliches Recht wird begründet, wenn auf einer Seite zwingend auch ein Träger öffentlicher Gewalt als solcher berechtigt oder verpflichtet ist. 391

Wenn die Eingliederungsvereinbarung überhaupt Rechte/Pflichten begründet oder inhaltlich ändert, was erst noch zu prüfen ist, handelt es sich um solche aus dem SGB III, da künftige Leistungen der aktiven Arbeitsförderung vereinbart werden. Diese Pflichten treffen in jedem Fall die Agenturen für Arbeit, also einen Träger öffentlicher Verwaltung. Da es nicht möglich ist, dass andere Rechte/Pflichten vereinbart werden als im SGB III vorgesehene ${ }^{392}$, wird jedenfalls der Schwerpunkt des Vereinbarten stets im öffentlichen Recht liegen.

388 So ist nach Ansicht Steinmeyers, info also 2002, S. 5 das einseitige Ausarbeiten eines Eingliederungsplans nur bei dem Arbeitslosen nicht rechtswidrig, der einer Vereinbarung überhaupt nicht zugänglich ist oder überzogene Forderungen stellt.

389 A.A. Luthe, SGb 2002, S. 79, der die Eingliederungsvereinbarung stets als, ,individualisierten Gesamtplan“ bezeichnet, der eine später folgende Entscheidung über eine Maßnahme der aktiven Arbeitsförderung vorbereitet. In diese Richtung geht auch die Einordnung bei Peters-Lange, in: Gagel, SGB III, § 35 Rn. 27, die die Ähnlichkeit mit dem Reha-Plan ohne weitere Begründung und eindeutige Position nennt.

390 Vgl. erstes Kapitel B IV 1e) S. 88.

391 Ule/Laubinger, Verwaltungsverfahrensrecht, 1995, § 67 I 3.

392 Vgl. zweites Kapitel B I 3 S. 147 ff. 
cc) Ausschluss des verwaltungsrechtlichen Vertrages, $\S 53$ Abs. 2 SGB $X$

Der Vertragsschluss ist nur bei Leistungen zulässig, deren Erbringung im Ermessen der Arbeitsverwaltung liegt, § 53 Abs. 2 SGB X. Es besteht also ein Handlungsformverbot für den verwaltungsrechtlichen Vertrag über Leistungen, die der Gesetzgeber als Ansprüche ausgestaltet hat.

Die Reduzierung des Anwendungsbereichs des verwaltungsrechtlichen Vertrages ist Ausdruck des Misstrauens des Gesetzgebers, der sozialstaatliche Ansprüche nicht einem Aushandlungsprozess zwischen der Arbeitsverwaltung und dem einzelnen Arbeitslosen überlassen wollte beziehungsweise will. 393

Führt man die allgemeinen Ausführungen über die Ermessensspielräume der Arbeitsverwaltung im Arbeitsförderungsrecht gem. § 3 Abs. 4, 5 SGB III mit der Zulässigkeit des verwaltungsrechtlichen Vertrages gem. $§ 53$ Abs. 2 SGB X zusammen, so ergibt sich daraus, dass insbesondere das Arbeitslosengeld bei Arbeitslosigkeit, das Teilarbeitslosengeld und das Insolvenzgeld sowie die Leistungen der aktiven Arbeitsförderung, die der Entgeltsicherung dienen, z.B. das Arbeitslosengeld bei beruflicher Weiterbildung oder das Überbrückungsgeld, nicht verhandelbar sind. Das heißt, diesbezüglich ist der Abschluss eines verwaltungsrechtlichen Vertrages ausgeschlossen.

Verhandelbar sind beispielsweise die Berufsberatung, Arbeitsvermittlung und diese unterstützende Leistungen, Trainingsmaßnahmen oder die Übernahme von Weiterbildungskosten während der Teilnahme an einer beruflichen Weiterbildung. 394

\section{dd) Vertragsschluss}

Der verwaltungsrechtliche Vertrag kommt zustande, wenn eine Einigung der Parteien erzielt werden kann. Dass dies grundsätzlich möglich ist, wurde bei der Ablehnung der Einordnung als Verwaltungsakt bereits geprüft und festgestellt, dass ein Aushandeln trotz Verhandlungsungleichgewichts der Vertragsparteien nicht ausgeschlossen ist. 395

Thematisiert werden muss an dieser Stelle ein Abschlusszwang, der die Vertragsfreiheit der Beteiligten weitgehend einschränkt und der als Eingriff in Art. 2 Abs. $1 \mathrm{GG}$ zu rechtfertigen wäre. Ein Abschlusszwang kann direkt angeordnet sein oder sich indirekt aus den Umständen ergeben.

Im SGB III gibt es keinen Abschlusszwang direkter Art. Der Abschluss einer Eingliederungsvereinbarung ist nicht zwingend vorgeschrieben.

Ein Abschlusszwang könnte sich zwar auf Seiten der Behörde wegen der Formulierung einer Soll-Vorschrift in $\S 35$ Abs. 4 SGB III ergeben, da Soll-Vorschriften für die Verwaltung im Regelfall eine Verpflichtung zum Tätig werden beinhalten. ${ }^{396}$ Die Verwaltung darf von dieser Pflicht aber dann abweichen und eine eigene Entscheidung treffen, wenn eine atypische Konstellation vorliegt. Das Vorliegen eines Sonderfalles kann

393 Wolff/Bachof/Stober, Verwaltungsrecht, Bd. 2, 2000, S. 206 f.

394 Vgl. zweites Kapitel B I 3 S. 147 ff; B I 1c) S. 142 f.

395 Vgl. zweites Kapitel B I 5a) S. 151 ff.

396 Vgl. BVerwGE 12, 284 [285]; 42, 26 [28 f]; 90, 88 [93]. 
voll gerichtlich überprüft werden. ${ }^{397}$ Besonders im Sozialrecht sind die Fälle, in denen ein Sonderfall von den Gerichten anerkannt wurde, sehr selten. ${ }^{398}$ Dieser Entscheidungsspielraum der Behörde genügt aber, um einen Abschlusszwang auf Seiten der Behörde abzulehnen. Die Arbeitsverwaltung muss vielmehr eine Entscheidung darüber fällen, ob ein Sonderfall gegeben ist. Dadurch ist ausgeschlossen, dass die Behörde sich zum Abschluss gezwungen fühlt, ohne eine Prüfung vorzunehmen.

Auch auf Seiten des Arbeitslosen besteht kein Abschlusszwang. Die Eingliederungsvereinbarung ist keine Leistungsvoraussetzung für Entgeltersatzleistungen. ${ }^{399} \mathrm{Da}$ es keine Möglichkeit gibt, den Abschluss einer Eingliederungsvereinbarung im SGB III zu erzwingen oder die Erklärung des Antragstellers zu ersetzen, ist der Vertragsschluss für den Antragsteller nicht mit einem Abschlusszwang belegt. 400

\section{ee) Begründung, Änderung oder Aufhebung eines Rechtsverhältnisses}

Durch den Abschluss einer Eingliederungsvereinbarung müsste ein Rechtsverhältnis begründet, inhaltlich geändert oder aufgehoben werden.

\section{(1) Rechtsverhältnis}

Unter einem Rechtsverhältnis versteht man jede sich aus einem konkreten Sachverhalt ergebende rechtliche Beziehung eines Rechtssubjekts zu einem anderen oder einer Sache. ${ }^{401}$ Das Rechtsverhältnis muss sich auf einen konkreten Einzelfall beziehen, sodass Vereinbarungen über eine Vielzahl von Fällen nicht als verwaltungsrechtliche Verträge anzusehen sind. 402 Bei der Eingliederungsvereinbarung ist das zu betrachtende Rechtsverhältnis das Verhältnis zwischen Bundesagentur für Arbeit und einzelnem Arbeitslosen, mithin also die rechtliche Beziehung zwischen zwei Rechtssubjekten. Sie ist in § 24 SGB III als Versicherungspflichtverhältnis näher konkretisiert.

\section{(2) Aufhebung eines Rechtsverhältnisses}

Dass dieses Rechtsverhältnis durch die Eingliederungsvereinbarung aufgehoben werden könnte, liegt nicht im Interesse der Vorschrift über die Eingliederungsvereinbarung. Die Aufhebung setzt voraus, dass durch den Vertrag ein Rechtsverhältnis beseitigt wird. ${ }^{403}$ Durch die Eingliederungsvereinbarung wird das Verhältnis zwischen Agentur für Arbeit und Arbeitslosem gerade gestaltet, also keinesfalls aufgehoben. ${ }^{404}$

397 BVerwGE 90, 275 [278 ff].

398 So die Auswertung von Brinktrine, Verwaltungsermessen in Deutschland und England, 1998, S. 42.

399 Vgl. zweites Kapitel B I 7a) S. 166 f.

400 So auch Peters-Lange, in: Gagel, SGB III, § 35 Rn. 98; Rademacher, in: Ambs, GK-SGB III, § 35 Rn. 98; Rademacker, in: Hauck/Noftz, SGB III, K § 35 Rn. 53.

401 BVerwGE 14, 235 [236]; 89, 327 [329]; 100, 262 [264].

402 Bonk, in: Stelkens/Bonk/Sachs, VwVfG, 2001, § 54 Rn. 84.

403 Bonk, in: Stelkens/Bonk/Sachs, VwVfG, 2001, § 54 Rn. 82.

404 Es ist zudem nicht möglich, dieses Versicherungspflichtverhältnis, welches durch Gesetz entsteht, durch einfachen Parteiwillen aufzuheben. 
(3) Begründung eines Rechtsverhältnisses

Durch die Eingliederungsvereinbarung wird kein Rechtsverhältnis begründet.

Die Begründung eines Rechtsverhältnisses, z.B. durch Abschluss eines Verpflichtungsvertrages, liegt vor, wenn sich ein Teil des Vertrages zu einer noch zu erbringenden Leistung verpflichtet. Der Verpflichtungsvertrag schafft ein Schuldverhältnis und begründet damit Rechte und Pflichten der Vertragspartner zueinander. Eine solche Verpflichtung kann auf Seiten der Behörde in dem Erlass eines Verwaltungsaktes oder einem sonstigen Verwaltungshandeln bestehen, auf Seiten des Bürgers kommt jede im Zivilrecht oder im öffentlichen Recht mögliche Leistung in Betracht. 405

Wie oben beschrieben, hat der Gesetzgeber in der Gesetzesbegründung die Entstehung eines neuen Rechtsverhältnisses durch die Eingliederungsvereinbarung abgelehnt. ${ }^{406}$ Das für eine vertragliche Regelung relevante Rechtsverhältnis ist damit das Versicherungspflichtverhältnis, das gem. § 24 Abs. 1 SGB III mit dem Tag des Eintritts in das Beschäftigungsverhältnis entsteht. Es kann also durch die Eingliederungsvereinbarung nicht begründet werden, weil es bereits automatisch durch das Gesetz unabhängig von einer Willenserklärung der Beteiligten entsteht.

(4) Inhaltliche Änderung eines Rechtsverhältnisses

Es kommt zuletzt die inhaltliche Änderung des Versicherungspflichtverhältnisses in Betracht. Auch diese ist abzulehnen.

Sie liegt vor, wenn das Rechtsverhältnis durch den verwaltungsrechtlichen Vertrag inhaltlich umgestaltet wird. ${ }^{407}$ Dabei steht es den Beteiligten frei, gesetzlich bestehende Schuldverhältnisse zu ändern, sofern nicht gesetzliche Vorschriften entgegenstehen. ${ }^{408}$

Das Gebot der Gesetzesbindung der Verwaltung, das im Sozialrecht in § 31 SGB I geregelt ist, steht einer inhaltlichen Änderung des Versicherungspflichtverhältnisses durch die Verwaltung entgegen. ${ }^{409}$ Es folgt daraus, dass durch die Eingliederungsvereinbarung keine Rechte und Pflichten, insbesondere nicht Sanktionsmöglichkeiten gegenüber dem Bürger geändert werden dürfen. 410

Es folgt daraus nicht, dass sich die Verwaltung in pflichtgemäßer Ausübung ihres Ermessens in einem verwaltungsrechtlichen Vertrag gegenüber dem einzelnen Arbeitslosen in Bezug auf eine Eingliederungsleistung bindet oder eine Zusicherung im Sinn des $\S 34$ SGB X abgibt. Da aber die „Gegenleistungen“ des Bürgers, seine Eigenbemü-

405 Pickel/Marschner, SGB X, Bd. 1, § 53 Rn. 28 ff.

406 BT-Drucksache 14/6944, S. 30 f.

407 Bonk, in: Stelkens/Bonk/Sachs, VwVfG, 2001, § 54 Rn. 82.

408 BVerwGE 84, 257 [262].

409 Dazu Hauck, in: Hauck/Noftz, SGB I, K § 31 Rn. 4, 9.

410 So auch Rademacher, in: Ambs, GK-SGB III, § 35 Rn. 105; Peters-Lange, in: Gagel, SGB III, § 35 Rn. 27; Rixen, in: Eicher/Spellbrink, SGB II, § 15 Rn. 2 SGB II; a.A. Rademacker, in: Hauck/Noftz, SGB III, K § 35 Rn. 51, der aber zugibt, dass diese Einordnung nicht dem Willen des Gesetzgebers entspricht; Körner, NZS 2002, S. 242; Kretschmer, DÖV 2006, S. 895, die die Eingliederungsvereinbarung als verwaltungsrechtlichen Vertrag einordnen und dabei ohne weitere Begründung $\S 31$ SGB I hinzu zitieren. 
hungen, nicht in derselben Weise verbindlich, sprich einklagbar sind, dürfte das Interesse der Verwaltung dementsprechend gering sein. Es ist deshalb davon auszugehen, dass auch die Verwaltung die Eingliederungsvereinbarung nur dazu nutzt, ihre Eingliederungsbemühungen entsprechend auf den Einzelnen auszurichten, ohne aber Rechtsansprüche des Arbeitslosen auf vertraglicher Ebene entstehen zu lassen. ${ }^{411}$

$\mathrm{Zu}$ klären ist an dieser Stelle, wie die Formulierung ,nach der Eingliederungsvereinbarung obliegende Pflichten" aus $\S 38$ Abs. 2 SGB III einzuordnen ist. Ist das ein Hinweis darauf, dass in der Eingliederungsvereinbarung Pflichten inhaltlich geändert werden?

Die Vorschrift gibt nicht direkt Auskunft darüber. Das Versicherungspflichtverhältnis wird nicht deshalb durch die Eingliederungsvereinbarung inhaltlich geändert, weil sich Rechtsfolgen aus der Nichterfüllung der Pflichten aus einer abgeschlossenen Eingliederungsvereinbarung ergeben. ${ }^{412} \S 38$ Abs. 2 SGB III gibt vielmehr Aufschluss darüber, ob Inhalte der Eingliederungsvereinbarung tatsächlich echte Rechtspflichten sind oder nur Obliegenheiten.

Eine Obliegenheit im Privatrecht ist eine „Pflicht geringerer Intensität“".413 Sie zeichnet sich dadurch aus, dass dem „Berechtigten“ regelmäßig weder ein Erfüllungsanspruch noch eine Klage- und Vollstreckungsmöglichkeit noch ein Verletzungsschadensersatzanspruch zustehen. Der Belastete hat für den Fall der Nichtbeachtung andere Rechtsnachteile zu erwarten, vor allem den Verlust einer günstigen Rechtsposition. Man kann deshalb sagen, dass der Belastete, wenn er der Obliegenheit nachkommt, in eigenem Interesse handelt, ohne dass ihm bei Nichteinhaltung der Obliegenheit der Vorwurf rechtwidrigen Verhaltens gemacht werden könnte. ${ }^{414}$

Im Sozialversicherungsrecht spricht man von Obliegenheiten des Versicherten ebenfalls bei unvollkommenen Verpflichtungen, die dem Versicherungsnehmer zur Wahrung seiner Ansprüche auferlegt sind. ${ }^{415}$ Wesentlich für das Vorliegen einer Obliegenheit ist, dass diese das Verhalten des Versicherungsnehmers steuern sollen, ohne dass eine Möglichkeit bestünde, das Verhalten ohne Mitwirkung oder gegen den Willen des Versicherungsnehmers zu bewirken. ${ }^{416}$ Die Auferlegung von Obliegenheiten ist ein typisches versicherungstechnisches Mittel, das den Zweck hat, den Versicherer und die Versichertengemeinschaft vor vermeidbaren Belastungen und ungerechtfertigten Ansprüchen zu schützen. ${ }^{417}$ Das kann so weit gehen, dass die Obliegenheiten so gestaltet werden, dass das Verhalten zur Voraussetzung für die Leistung (Leistungsfreiheit als

411 Rademacher, in: Ambs, GK-SGB III, § 35 Rn. 108.

412 So aber Rademacker, in: Hauck/Noftz, SGB III, K § 35 Rn. 50.

413 Schmidt, Die Obliegenheiten, 1953, S. 104 f.

414 Teichmann, in: Soergel, Bürgerliches Gesetzbuch, 1999, vor § 241 Rn. 8; Grothe, in: Münchener Kommentar, Bürgerliches Gesetzbuch, 2006, § 194 Rn. 6.

415 Voelzke, Die Herbeiführung des Versicherungsfalles im Sozialversicherungsrecht, 2004, S. 84, der bei der Begriffsbildung auf Quellen aus dem Privatrecht (Medicus, Schuldrecht I, 2002, § 59 Rn. 675; Larenz, Allgemeiner Teil des Bürgerlichen Rechts, 1997, § 12 II 4) zurückgreift.

416 BSG, Urteil v. 26.5.1983, SozR 1200 § 66 Nr. 10.

417 BGH, VersR 1977, S. 274. 
gegebenenfalls eintretender Rechtsnachteil) wird oder zumindest zu einer Leistungsbeschränkung führt. 418

Die Eingliederungsvereinbarung enthält nur Obliegenheiten. Die Rechtspflichten der Parteien sind gesetzlich geregelt. Die Nichteinhaltung der Eingliederungsvereinbarung nach SGB III führt zu Rechtsverlusten, die sich aus den gesetzlich geregelten Sanktionsmöglichkeiten ergeben. $§ 38$ Abs. 2 SGB III sieht die Einstellung der Vermittlung vor, § 144 SGB III den Eintritt einer Sperrzeit. Von einer Erfüllung der Pflichten ist nicht die Rede. Spricht deshalb $\S 38$ Abs. 2 SGB III von „Pflichten“, sind damit lediglich Obliegenheiten gemeint.

Festzuhalten ist zudem, dass auch ohne eine Eingliederungsvereinbarung das Fehlen von Eigenbemühungen sanktioniert werden kann, § 144 Abs. 1 S. 2 Nr. 3 SGB III, so dass davon auszugehen ist, dass auch ohne Konkretisierung durch eine Eingliederungsvereinbarung abstrakt klar ist, was der einzelne Arbeitslose zu tun hat, um nicht mit einer Sperrzeit belegt zu werden. ${ }^{419}$

\section{(5) Zusammenfassung}

Durch die Eingliederungsvereinbarung werden Rechte und Pflichten weder begründet noch inhaltlich geändert. Die Voraussetzungen des $\S 53$ Abs. 1 SGB X liegen somit nicht vor.

\section{ff) Zwischenergebnis}

Leistungen, auf die der Arbeitslose einen gesetzlich ausgestalteten Anspruch hat, können von vornherein nicht im Wege des verwaltungsrechtlichen Vertrags aufgrund des bestehenden Handlungsverbots gem. § 53 Abs. 2 SGB X verhandelt werden.

In Bezug auf die Ermessensleistungen der Arbeitsförderung ist der verwaltungsrechtliche Vertrag ebenfalls nicht einschlägig.

\section{d) Vertragsähnliches Verwaltungshandeln}

Die Eingliederungsvereinbarung ist genauso wie das $J S A$ ein Beispiel für vertragsähnliches Verwaltungshandeln. ${ }^{420}$ Keine der bislang beschriebenen Handlungsformen rechtfertigt eine Einordnung der Eingliederungsvereinbarung im SGB III nach der genauen Überprüfung der jeweiligen Voraussetzungen. Es ist aber auch keine andere Handlungsform gesetzlich vorgeschrieben, sodass vertragsähnliches Handeln zulässig ist.

Die wesentlichen (kooperativen) Elemente der Eingliederungsvereinbarung im SGB III wurden bereits im Rahmen der Prüfung, ob die Einordnung als gesetzlich geregelte Handlungsform möglich ist, angesprochen. Sie lassen sich wie folgt zusammenfassen:

418 Voelzke, Die Herbeiführung des Versicherungsfalles im Sozialversicherungsrecht, 2004, S. 85.

419 Bestätigend Peters-Lange, in: Gagel, SGB III, § 35 Rn. 27.

420 Mutschler, in: Wissing u.a., SGB III, 2005, § 35 Rn. 42 spricht von einer „quasivertraglichen Situation" und stellt die Eingliederungsvereinbarung in den Zusammenhang zu Zielvereinbarungen. 
Die Eingliederungsvereinbarung ist grundsätzlich auszuhandeln, d.h. es muss im Einzelfall ein Kontakt, in der Regel ein Gespräch zwischen Arbeitsverwaltung und Arbeitslosem stattfinden. Die Intention des Gesetzgebers und der Sinn und Zweck der Regelung in $§ 35$ Abs. 4 SGB III schließen eine einseitige Regelung im Sinn des § 31 SGB $\mathrm{X}$ aus und verlangen, dass die Veränderungen im Lebenssachverhalt nicht allein von der Behörde ausgehen, sondern von beiden Parteien der Vereinbarung getragen werden sollen. Verhandelt wird sowohl über die Auswahl der Eingliederungsleistungen als auch über die Eigenbemühungen des Arbeitslosen. In beiden Fragen lässt das Gesetz Spielräume, die die Parteien der Eingliederungsvereinbarung ausfüllen. Die Agentur für Arbeit bestimmt folglich den Inhalt der Eingliederungsvereinbarung, der gesetzlich nur vage umschrieben ist, nicht allein. Es sind die Informationen des einzelnen Arbeitslosen erforderlich, der das Ergebnis der Kommunikation, die Vermittlungsstrategie, auch mittragen soll.

Die Eingliederungsvereinbarung kommt zustande, wenn eine Einigung der Parteien über ein Kooperationsergebnis erzielt worden ist. Die Vertragsfreiheit wird durch die gesetzlichen Regelungen nicht berührt, insbesondere besteht kein Abschlusszwang auf Seiten des Arbeitslosen.

Die Wirkungen der Eingliederungsvereinbarung konnten ebenfalls bereits herausgearbeitet werden. Die Nichteinhaltung der Eingliederungsvereinbarung nach SGB III führt zu Rechtsverlusten, die sich aus den gesetzlich geregelten Sanktionsmöglichkeiten in $\S 38$ Abs. 2 SGB II und $\S 144$ SGB III ergeben. Die Eingliederungsvereinbarung enthält folglich nur Obliegenheiten. Die echten Rechtspflichten der Parteien sind im Recht der Arbeitsförderung gesetzlich geregelt.

In allgemeinen Worten gesprochen enthält die Vereinbarung verbindliche Aussagen, die von beiden Seiten verantwortet werden. Sie wurde ausgehandelt, aber das Vereinbarte kann rechtlich nicht eingefordert werden (Obliegenheit).

\section{Funktion der Eingliederungsvereinbarung}

Im Folgenden wird die Funktion der Eingliederungsvereinbarung im System des SGB III genauer beschrieben. In Abgrenzung zu den Wirkungen der Eingliederungsvereinbarung steht bei der Darstellung der Funktionen im Mittelpunkt, in welchem Kontext die Vereinbarung steht. Konkrete rechtliche Konsequenzen der Vereinbarung werden unter dem Gesichtspunkt „Wirkungen der Eingliederungsvereinbarung“ dargestellt. ${ }^{421}$

\section{a) Grundsatz vom Fördern und Fordern}

Die Eingliederungsvereinbarung nach SGB III wurde eingeführt, um den Grundsatz des Förderns und Forderns in rechtliche Formen zu gießen 422 und dem Arbeitslosen vor Augen zu führen, dass das ,soziale Recht nur bei Erfüllung von Pflichten zu haben

421 Vgl. zweites Kapitel B I 7 S. 166 ff.

422 BT-Drucksache 14/6944, S. 28. 
ist". 423 Wie im ersten Kapitel beschrieben, wird ausgehandelten oder zumindest mit dem Betroffenen abgestimmten Maßnahmen der Arbeitsverwaltung eine höhere Akzep$\operatorname{tanz}$ bei den Leistungsberechtigten zugeschrieben. ${ }^{424}$ Diese hat ihren Grund sowohl in der genaueren Abstimmung auf den Einzelfall als auch in dem hoheitlichen Maßnahmen allgemein entgegengebrachten Missmut. 425

\section{b) Neubegründung von Leistungspflichten}

Aufgrund des Versicherungscharakters besteht nicht die Möglichkeit, in der Eingliederungsvereinbarung Leistungspflichten, sowohl für den Arbeitslosen als auch für die Arbeitsagentur, zu begründen. Begründet ein zivilrechtlicher oder öffentlich-rechtlicher Vertrag ein vorher nicht bestehendes Rechtsverhältnis mit Rechten und Pflichten, so tut dies die Eingliederungsvereinbarung nach dem Willen des Gesetzgebers nicht. 426

Es bleibt der Verwaltung allerdings unbenommen, dem Arbeitslosen gegenüber verbindliche Zusagen zu machen, mit denen sie im gesetzlich umschriebenen Handlungsspielraum verbleibt, wenn sie dabei die Ziele des Arbeitsförderungsrechts einhält. ${ }^{427}$ Der Abschluss eines verwaltungsrechtlichen Vertrages über eine bestimmte Eingliederungsleistung ist deshalb möglich, nicht aber als Eingliederungsvereinbarung, sondern an Stelle eines Verwaltungsakts.

Die Entwicklung eines individuellen Eingliederungsinstrumentes ist in diesem Zusammenhang auf $\S 10$ SGB III (freie Förderung) beschränkt und kann ebenso nicht im Rahmen einer Eingliederungsvereinbarung erfolgen. Dort ist vorgesehen, dass die freie Förderung den Zielen und Grundsätzen der gesetzlichen Instrumente entsprechen muss und diese nicht aufstocken darf (Aufstockungsverbot). Die Arbeitsagenturen befinden sich also auch im Rahmen des $\S 10$ SGB III im engen Korsett des gesetzlich geregelten Eingliederungsinstrumentariums. Aufgrund der Bindung der Bundesagentur für Arbeit an die Finanzpolitik der Bundesregierung und angesichts der Tatsachen, dass eine „freiere" Eingliederungsleistung auf der Grundlage des § 10 SGB III gesetzlich möglich ist, ist für darüber hinaus gehende innovative Leistungen kein Geld im Eingliederungsbudget der Bundesagentur für Arbeit vorgesehen, sodass eine entsprechende vertragliche Vereinbarung nicht in Betracht kommt.

\section{c) Mitwirkungspflicht, $\S 38$ SGB III}

In $\S 38$ Abs. 1, 1a, 2 SGB III sind die Mitwirkungspflichten des Arbeitslosen ausdrücklich geregelt. Deshalb greifen die allgemeinen Mitwirkungspflichten gem. $\S 60 \mathrm{ff}$

423 Eichenhofer, SGb 2004, S. 207.

424 Vgl. erstes Kapitel A II S. 54 ff.

425 Bestätigend Ebsen, Der Arbeitslose als Sozialbürger und Klient, in: Wulffen, FS 50 Jahre Bundessozialgericht, 2004, S. 738.

426 Vgl. dazu zweites Kapitel B I 5 S. 150 ff.

427 Geiger, info also 2002, S. 200 f. 
SGB I 428 nur noch in Bezug auf Geldleistungen ein, für den Bereich der Vermittlung und Eingliederung lediglich ergänzend. ${ }^{429}$

Die Bedeutung der Eingliederungsvereinbarung liegt darin, dass damit das Rechteund Pflichtengeflecht zwischen Agentur für Arbeit und Arbeitslosem im Hinblick auf die Vermittlung konkretisiert werden kann. Die Pflichten aus der Eingliederungsvereinbarung können sich sowohl auf die Eigenbemühungen des Arbeitslosen als auch auf die vereinbarten Vermittlungs- und sonstigen Leistungen der aktiven Arbeitsförderung der Agentur für Arbeit beziehen. ${ }^{430}$ In $\S 38$ Abs. 2 2. Alt. SGB III ist vorgesehen, dass die Agentur für Arbeit die Vermittlung einstellen kann, solange der Arbeitsuchende die ihm nach der Eingliederungsvereinbarung obliegenden Pflichten nicht erfüllt. ${ }^{431}$ Dadurch dass der Gesetzgeber die Verhängung einer Sanktion bei Nichteinhaltung von „Pflichten" durch den Arbeitslosen ins Ermessen der Arbeitsagentur gestellt hat, erhöht er deren rechtliche Relevanz. So wird die Bedeutung der Vereinbarung insgesamt neben der allgemeinen Mitverantwortung des Arbeitsuchenden betont. 432

Es deutet sich hier bereits an, dass die Nichteinhaltung von Pflichten durch die Verwaltung vom Gesetzgeber vernachlässigt wird. Denn beschrieben werden allein Konsequenzen, die die Rechtsstellung des Arbeitslosen verschlechtern.

\section{d) Auslegung unbestimmter Rechtsbegriffe}

Die Auslegung unbestimmter Rechtsbegriffe ${ }^{433}$, die von der Ermessensausübung strikt zu trennen ist, erfolgt nach den allgemeinen Auslegungsregeln. Die Gerichte können die Auslegung unbestimmter Rechtsbegriffe grundsätzlich uneingeschränkt nachprüfen und die Begriffe eigenständig auslegen. ${ }^{434}$ Nach Ansicht der Verfassungsrechtsprechung ist sie dazu verpflichtet, um das subjektive Recht auf gerichtliche Kontrolle gem. Art. 19 Abs. 4 GG zu wahren. ${ }^{435}$

428 Dazu Rüfner, VSSR 1977, S. 347 ff; Gronwald, SGb 1989, S. 17 ff; Grupp, VerwArch 1989, S. 44 ff; Steck, BKK 1992, S. 349 ff.

429 Rademacher, in: Ambs, GK-SGB III, § 38 Rn. 2.

430 Rademacher, in: Ambs, GK-SGB III, § 38 Rn. 22.

431 Vgl. zweites Kapitel B I 7c) bb) S. 174 f.

432 Sienknecht, in: Spellbrink/Eicher, Kasseler Handbuch des Arbeitsförderungsrechts, 2003, S. 1247 Rn. 69.

433 Unbestimmte Rechtsbegriffe sind Begriffe im Gesetzestext, die von ihrer inhaltlichen Bedeutung her nicht eindeutig beziehungsweise inhaltlich nicht klar bestimmt sind (dazu Maurer, Allgemeines Verwaltungsrecht, 2009, S. 143 f). Bezogen auf das Arbeitsförderungsrecht stellt z.B. der Begriff „wichtiger Grund“ gem. § 144 Abs. 1 SGB III einen unbestimmten Rechtsbegriff dar.

434 Eine a.A. in der Literatur spricht vom „Beurteilungsspielraum der Verwaltung“, was bedeutet, dass der Gesetzgeber die Verwaltung durch Verwendung eines unbestimmten Rechtsbegriffs zu eigenverantwortlichen und gerichtlich nur bedingt überprüfbaren Entscheidungen ermächtigt hat. Vgl. dazu Bachof, JZ 1955, S. 97 ff.

435 Z.B. BVerfGE 84, 50, vgl. dazu ausführlich Schulze-Fielitz, JZ 1993, S. 772 ff, der die Entwicklung der Rechtsprechung zum Beurteilungsspielraum der Verwaltung bei unbestimmten Rechtsbegriffen darstellt und zu dem Ergebnis kommt, dass der Spielraum, der zunächst ausufernd zugestanden wurde, nur noch im Ausnahmefall vor dem Grundgesetz Bestand hat. 
Unbestimmte Rechtsbegriffe sind abstrakt auszulegen und anschließend im konkreten Einzelfall anzuwenden. Deshalb ist es bezogen auf die Eingliederungsvereinbarung wesentlich, dass diese die erforderlichen Informationen für die konkrete Anwendung des unbestimmten Rechtsbegriffs enthält. So können die fachlichen und ökonomischen Auswahlkriterien des Gesetzgebers und die individuelle Fallgeschichte, wie sie in der Eingliederungsvereinbarung zum Ausdruck kommt, beachtet werden. Deshalb ist es auch zweckmäßig, die wesentlichen Gründe, auf die sich die vereinbarten Maßnahmen stützen, mit in den Text der Eingliederungsvereinbarung aufzunehmen. ${ }^{436}$ Die Nachvollziehbarkeit z.B. der Ablehnung eines wichtigen Grundes und infolgedessen der Eintritt einer Sperrzeit gem. § 144 SGB III wird erhöht, wenn die Umstände und die Gründe für die Aushandlung bestimmter Eigenbemühungen aus einem schriftlichen Dokument hervorgehen.

\section{e) Eigenbemühungen, § 119 Abs. 4 Nr. 1 SGB III}

Die Eigenbemühungen sind ein Aspekt der Definition der Arbeitslosen gem. § 119 Abs. 1 SGB III.

Im Rahmen der Eigenbemühungen hat der Arbeitslose alle Möglichkeiten zur beruflichen Eingliederung zu nutzen. Hierzu gehört gem. § 119 Abs. 4 Nr. 1 SGB III die Wahrnehmung der Verpflichtungen aus der Eingliederungsvereinbarung. Auch hier hat der Gesetzgeber ausdrücklich die rechtliche Relevanz der in der Eingliederungsvereinbarung vereinbarten Pflichten hervorgehoben. Somit kann der Leistungsanspruch auf Arbeitslosengeld ruhen, wenn der Arbeitslose nicht die Erfüllung der vereinbarten Pflichten nachweist. 437

\section{f) Qualitätssicherung und Qualitätsprüfung}

Aus dem Sinn und Zweck der Eingliederungsvereinbarung, die Vermittlungsstrategie nachprüfbar zu dokumentieren, folgt, dass sich die Bundesagentur für Arbeit und die örtlichen Agenturen für Arbeit damit einem Qualitätssicherungsprozess unterziehen. ${ }^{438}$ Es wird schriftlich fixiert, welche Schwierigkeiten und welche konkreten Chancen die örtliche Arbeitsagentur bei der Eingliederung des Arbeitslosen in den Arbeitsmarkt sieht. Später kann überprüft werden, ob sie mit dieser Prognose richtig lag. Daraus können Rückschlüsse auf die Prognosekompetenz und damit auf die Qualität der Vermittlungstätigkeit der Agenturen für Arbeit gezogen werden.

Ferner wird festgehalten, was die Arbeitsverwaltung zur Beendigung der Arbeitslosigkeit beitragen wollte. Damit wird überprüfbar, ob die richtigen Maßnahmen, die den Arbeitslosen wieder in Arbeit bringen, vereinbart wurden. Es geht also sowohl um eine

\footnotetext{
436 Luthe, SGb 2002, S. 80.
}

437 Vgl. zweites Kapitel B I 7c) S. $171 \mathrm{ff}$.

438 BT-Drucksache 14/6944, S. 31; so bestätigend Peters-Lange, in: Gagel, SGB III, § 35 Rn. 28; Rademacher, in: Ambs, GK-SGB III, § 35 Rn. 109. 
Möglichkeit für die Behörde, in regelmäßigen Abständen ihre Arbeitsweise zu überprüfen, als auch darum, eine Grundlage für Kontrollen im Einzelfall zu haben. 439

Daraus ergibt sich für den Einzelnen allerdings kein Anspruch. Es ist vielmehr Beispiel dafür, dass die Eingliederungsvereinbarung im Zusammenhang mit kooperativer Verwaltung steht und auch mit deren verwaltungsinterner Komponente zu tun hat.

\section{Wirkungen der Eingliederungsvereinbarung}

a) Voraussetzung für Leistungen der Agenturen für Arbeit

Bei den Leistungsvoraussetzungen ist zwischen Vermittlungstätigkeit der Agenturen für Arbeit und Entgeltersatzleistungen zu unterscheiden.

\section{aa) Vermittlungstätigkeit}

Anders als im Jobseekers Act 1995 geregelt hat der deutsche Gesetzgeber nicht vorgesehen, dass jeder Arbeitslose eine Eingliederungsvereinbarung abschließen muss, um von Vermittlungsbemühungen der Agentur für Arbeit profitieren zu können.

Es gibt zwar eine Pflicht zum Abschluss einer Eingliederungsvereinbarung, die sowohl für die Agentur für Arbeit als auch den Arbeitslosen gilt. Dies ergibt sich aus dem Wortlaut des $\S 6$ Abs. 1 S. 3 SGB III (,halten fest"). Danach ist es zwingend vorgeschrieben, dass die Agentur für Arbeit und der Arbeitslose auf den Abschluss einer Eingliederungsvereinbarung hinwirken.

Die Nichteinhaltung dieser Pflicht ist jedoch nicht sanktioniert. Es bleibt vielmehr bei fehlender Einigung bei der einseitigen Beratungs- und Vermittlungsaufgabe der Agentur für Arbeit, sowie einer anschließenden Entscheidung über Maßnahmen der aktiven Arbeitsförderung. ${ }^{440}$ Es gibt zudem Fälle, in denen der Abschluss einer Eingliederungsvereinbarung nicht sinnvoll erscheint, z.B. wenn bereits eine Anschlussstelle gefunden ist und Leistungen der Arbeitslosenversicherung nur zur Überbrückung in Anspruch genommen werden. ${ }^{441}$

Daraus ist unter Zugrundelegung des Sinns und Zwecks der Regelung zu folgern, dass eine Vereinbarung nur im Regelfall geschlossen werden muss. ${ }^{442}$

\section{bb) Entgeltersatzleistungen, insbesondere Arbeitslosengeld}

Das Vorliegen einer Eingliederungsvereinbarung ist nicht Voraussetzung für Entgeltersatzleistungen nach $\S \S 116$ ff SGB III. Dies ergibt sich für das Arbeitslosengeld bei Arbeitslosigkeit und Weiterbildung aus den Anspruchsvoraussetzungen in $\S \S 117$, 118, 124a SGB III. Die Leistungsansprüche der Arbeitslosenversicherung finden also

439 Spellbrink, SGb 2004, S. 80.

440 BT-Drucksache 14/6944, S. 31.

441 Steinmeyer, info also 2002, S. 5.

442 Eine entsprechende Vereinbarung mit einem Ausbildungsuchenden ist nach $\S 6$ Abs. 2 SGB III bis spätestens zum 30.9. eines Kalenderjahres nur dann verpflichtend zu schließen, wenn dieser zu Beginn des Ausbildungsjahres (September/Oktober) noch nicht vermittelt ist. 
auch nach deren Modernisierung ihren Rechtsgrund nicht in einer vertraglichen Vereinbarung, sondern im Gesetz. ${ }^{443}$

cc) Zwischenergebnis

Der Abschluss einer Eingliederungsvereinbarung kann nicht als Leistungsvoraussetzung für Leistungen nach dem SGB III angesehen werden. Es bleibt bei fehlender Einigung stets die Aufgabe der Agentur für Arbeit, einseitig tätig zu werden.

\section{b) Ermessensausübung}

\section{aa) Verwaltungsermessen: Begriff und Abgrenzung}

Verwaltungsermessen ist ein normativ (in Gesetzen, Rechtsverordnungen und Satzungen) eröffneter Spielraum für Entscheidungen der Verwaltung. ${ }^{444}$ Dieser Begriff entspricht der überwiegend verwendeten Terminologie und findet für das Sozialverwaltungsrecht seine normative Grundlage in $\S 39$ Abs. 1 SGB I. Eine Ermessensleistung im Sinn des $\S 39$ Abs. 1 SGB I liegt vor, wenn der Leistungsträger ermächtigt ist, bei der Entscheidung über Sozialleistungen nach seinem Ermessen zu handeln.

Gesetzlich unbestimmt ist deshalb die Rechtsfolge, nicht ein Tatbestands- oder Rechtsfolgebegriff. ${ }^{445}$ Die Gerichte sprechen von „volitivem Handlungs- oder Verhaltensermessen“. Damit ist gemeint, dass der Ermessensbereich erst beginnt, wenn die Tatbestandsvoraussetzungen einer Vorschrift erfüllt sind. ${ }^{446}$

Die Ermessensermächtigung kann sich auf die Frage nach dem Tätigwerden (sog. Entschließungsermessen) oder/und auf die Frage nach der Auswahl aus verschiedenen denkbaren Möglichkeiten oder Mitteln (sog. Auswahlermessen) beziehen. ${ }^{447}$

Im Rahmen einer Ermessensentscheidung hat der Leistungsträger das Ermessen entsprechend dem Zweck der Ermächtigung auszuüben und die gesetzlichen Grenzen des Ermessens einzuhalten. Somit handelt der Leistungsträger erst dann pflichtgemäß, wenn keine Ermessensüberschreitung vorliegt, die Verwaltungsentscheidung dem Zweck der Ermächtigung zur Ermessenentscheidung entspricht und nicht gegen die Selbstbindung

443 Eichenhofer, SGb 2004, S. 205.

444 Dieser Begriff des Verwaltungsermessens wird hergeleitet und dargestellt von Brinktrine, Verwaltungsermessen in Deutschland und England, 1998, S. 23-26. Dort findet sich eine zusammenfassende Übersicht über die einschlägigen Fundstellen in der Literatur, die diesem Ermessensbegriff folgen. Beispielhaft genannt seien Maurer, Allgemeines Verwaltungsrecht, 2009, S. 135 f; Tettinger, Rechtsanwendung und Kontrolle, 1980, S. 105; Ossenbühl, Rechtsquellen und Rechtsbindungen der Verwaltung, in: Erichsen/Ehlers, Allgemeines Verwaltungsrecht, 2006, § 10 Rn. 10.

445 In Abgrenzung zur hier verwendeten Terminologie ist der unbestimmte Rechtsbegriff keine Frage der Ermessensausübung. Vgl. dazu Maurer, Allgemeines Verwaltungsrecht, 2009, S. 143 f; Brinktrine, Verwaltungsermessen in Deutschland und England, 1998, S. 14.

446 Vgl. nur BVerwGE 61, 15 [20]; 72, 38 [53]. Sog. „kognitives (Beurteilungs-)Ermessen“ liegt der Anwendung unbestimmter Rechtsbegriffe zugrunde (so Wolff/Bachof/Stober, Verwaltungsrecht, Bd. 1, 1999, S. 455). Dazu im Einzelnen Brinktrine, Verwaltungsermessen in Deutschland und England, 1998, S. 31.

447 Dazu allgemein Brinktrine, Verwaltungsermessen in Deutschland und England, 1998, S. 31. 
der Verwaltung und gegen das Gebot der Gleichbehandlung aller gleichgelagerten Fälle verstößt. 448

\section{bb) Ermächtigung}

Im Recht der Arbeitsförderung besteht ein Ermessensspielraum der Agenturen für Arbeit grundsätzlich nur im Rahmen der aktiven Arbeitsförderung, § 3 Abs. 5 SGB III. Auf Leistungen, die aufgrund der Versicherteneigenschaft des Arbeitnehmers als Entgeltersatzleistungen unmittelbar bei Eintritt der (Teil-)Arbeitslosigkeit zu zahlen sind, also Arbeitslosengeld bei Arbeitslosigkeit, Teilarbeitslosengeld und Insolvenzgeld, hat der Versicherte einen Rechtsanspruch, § 3 Abs. 4 SGB III. Kein Ermessenspielraum besteht für bestimmte Leistungen der aktiven Arbeitsförderung, die das Gesetz ausnimmt (Beauftragung von Dritten mit der Vermittlung nach sechs Monaten, Überbrückungsgeld, Berufsausbildungshilfe, besondere Leistungen zur Teilhabe am Arbeitsleben, Arbeitslosengeld bei beruflicher Weiterbildung, Kurzarbeitergeld, Wintergeld, und Leistungen zur Förderung der Teilnahme an Transfermaßnahmen). ${ }^{449}$

\section{cc) Zweck der Ermächtigung}

Bei der Ausübung des Ermessens ist der Zweck der jeweiligen Ermächtigungsnorm festzustellen. $\mathrm{Zu}$ beachten sind die Ermächtigungsvorschrift selbst, aber auch gesetzliche Leitlinien, die die Intention eines Regelungswerkes beschreiben. ${ }^{450}$ Vorliegend findet sich die Ermächtigung für die Arbeitsverwaltung in § 3 SGB III i.V.m. den verschiedenen Rechtsgrundlagen für Eingliederungsleistungen im SGB III.

Der Ermächtigungsspielraum besteht, um es zu ermöglichen, dass die Auswahl der möglichen Hilfen auf den Einzelfall abgestellt werden kann. Eine kasuistische Auflistung der Voraussetzungen der jeweiligen Eingliederungsinstrumente würde den Rahmen sprengen. Die unterschiedliche Vorbildung und die Ausgangssituation des einzelnen Arbeitslosen bei der Arbeitsuche machen eine individuelle Entscheidung erforderlich.

Diese allgemeine Ermächtigung ist im Licht weiterer Vorschriften zu sehen.

Eine der relevanten Vorschriften ist $\S 5$ SGB III. Der Vorrang der aktiven Arbeitsförderung vor passiven Leistungen zum Ersatz des Arbeitsentgelts gem. § 5 SGB III bedeutet, dass für die Auswahl der arbeitsmarktpolitischen Leistungen der Agenturen für Arbeit ein Informations- und Beratungsbedarf besteht. Um dieses Prinzip entsprechend seinem Ziel auszufüllen, besteht das Auswahlermessen der Arbeitsverwaltung. Bezogen auf die Eingliederungsvereinbarung im Zusammenspiel mit dem dem Abschluss der Eingliederungsvereinbarung vorausgehenden Instrument individueller Chanceneinschätzung (Profiling) auf der Grundlage des § 35 Abs. 3 SGB III wird während deren Ausarbeitung die Grundlage für die richtige Auswahl der Leistungen vorbereitet. 451

448 Feckler, in: Ambs, GK-SGB III, § 3 Rn. 20.

449 Vgl. zweites Kapitel B I 1c) S. 142 f.

450 Brinktrine, Verwaltungsermessen in Deutschland und England, 1998, S. 94 ff.

451 Feckler, in: Ambs, GK-SGB III, § 5 Rn. 2a. 
Der Ermessensspielraum ist bei der Vermittlung gem. § 35 SGB III auf ein Auswahlermessen beschränkt („hat Vermittlung anzubieten“), während ansonsten auch das $\mathrm{Ob}$ des Tätigwerdens bei Ermessensleistungen im Ermessen der Arbeitsagentur liegt. ${ }^{452}$

Gesetzliche Grenzen der Ermessensausübung ergeben sich im Arbeitsförderungsrecht aus $\S 7$ SGB III, in dem geregelt ist, wie die Ermessensleistungen der aktiven Arbeitsförderung auszuwählen sind. Zentral sind dabei die Grundsätze der Wirtschaftlichkeit und Sparsamkeit, die dem $\S 7$ Bundeshaushaltsordnung (BHO) entnommen sind.

Der Grundsatz der Wirtschaftlichkeit ist eingehalten, wenn sich Verhalten und Entscheidung der Verwaltung orientiert an bestimmten Vorgaben als planvolles Handeln darstellen. Es bedeutet nicht einfach die Auswahl des billigsten Angebots, sondern die Auswahl desjenigen, welches unter Berücksichtigung des Aufwands die besten Erfolgsaussichten im Hinblick auf die Wiedereingliederung des einzelnen Arbeitslosen in den Arbeitsmarkt verspricht (Zweck-Mittel-Relation), wobei grundsätzlich alle Berechtigten in gleicher Lage in gleicher Weise zu fördern sind. ${ }^{453}$ Leitziel ist dabei $\S 1$ SGB III und der Gedanke der Effektivität und Effizienz der Maßnahme. Die Haushaltslage der Bundesagentur für Arbeit ist ebenfalls zu berücksichtigen. Das bedeutet unter anderem, dass die zugewiesenen Mittel so zu bewirtschaften sind, dass eine Bewilligung und Erbringung der einzelnen Leistungen im gesamten Haushaltsjahr gewährleistet sind. ${ }^{454}$

Der Grundsatz der Sparsamkeit ergänzt den Grundsatz der Wirtschaftlichkeit von Maßnahmen und bedeutet, dass der Mitteleinsatz nicht schlichtweg möglichst gering zu halten ist, sondern dass nur die zur Erfüllung der Aufgaben notwendigen Ausgaben getätigt werden. Im Zusammenspiel mit dem Grundsatz der Wirtschaftlichkeit bedeutet das, dass auch die Erfolgsaussichten der Maßnahme zu berücksichtigen sind und eine längerfristige Betrachtung der Wiedereingliederungschancen vorgenommen werden muss. 455

\section{ee) Grenzen aus der Eingliederungsvereinbarung}

Die Ermessensentscheidung ist eine Entscheidung über den Einzelfall. ${ }^{456}$ Es ist deshalb möglich, dass sich eine Bindung der Verwaltung aus dem konkreten Verhältnis zwischen Arbeitsverwaltung und Einzelnem ergibt.

In der Theorie der Ermessenslehre wird diese Konstellation als ermessensreduzierender Umstand bei der Ermessensreduzierung auf Null aufgeführt. ${ }^{457}$ Sie betrifft Fälle, in

452 Dazu Timme, in: Hauck/Noftz, SGB III, K § 3 Rn. 15; Niesel, SGB III, 2005, § 7 Rn. 3 ff.

453 Feckler, in: Ambs, GK-SGB III, § 7 Rn. 4.

454 Interpretation des $\S 71$ a Abs. 4 SGB IV, vgl. Feckler, in: Ambs, GK-SGB III, § 3 Rn. 22, § 7 Rn. 4.

455 Feckler, in: Ambs, GK-SGB III, § 7 Rn. 6-8.

456 Vgl. zweites Kapitel B I 7b) aa) S. 167.

457 Bei der Einordnung der Selbstbindung gibt es unterschiedliche Ansätze. Teilweise wird sie als Unterfall der Ermessensreduzierung angesehen (so z.B. Di Fabio, VerwArch 1995, S. 223 ff), teilweise als eigenständige Fallgruppe neben der Ermessensreduzierung auf Null (Ossenbühl, in Erichsen/Ehlers, Allgemeines Verwaltungsrecht, 2006, § 10 Rn. 20). Vgl. dazu Brinktrine, Verwaltungsermessen in Deutschland und England, 1998, S. 157 f. 
denen sich die Behörde nicht durch zeitlich andauernde Verwaltungspraxis ${ }^{458}$, sondern durch eine rechtmäßige Zusicherung oder einen wirksamen Vertrag zu einem bestimmen Verhalten verpflichtet hat. ${ }^{459}$ Die Eingliederungsvereinbarung ist lediglich als vertragsähnliches Handeln einzuordnen, sie ist in ihren Inhalten aber trotzdem verbindliche. ${ }^{460}$ Es können sich also ermessenskonkretisierende Aspekte aus der Eingliederungsvereinbarung ergeben, über die sich die Verwaltung nicht ohne erheblichen Begründungsaufwand hinwegsetzen kann. ${ }^{461}$

Die Eingliederungsvereinbarung wirkt als „Katalysator für Einzelfallgesichtspunkte“"462. Die gesetzlichen Tatbestände im Arbeitsförderungsrecht enthalten kaum detaillierte Anweisungen für die Ermessensausübung, sodass erst die Konkretisierung der Voraussetzungen und Maßnahmen der Förderung unter Einbeziehung des Betroffenen erforderlich ist. Dabei kommt der Eingliederungsvereinbarung die wichtige Aufgabe zu, die große Anzahl von Möglichkeiten auf die zu begrenzen, die für den konkreten Einzelfall sinnvoll sind. Sie führt also zu einer Vorstrukturierung der Entscheidung, die der Vermeidung von Ermessens- und Abwägungsfehlern dient.

Die Bindung der Verwaltung an ihre Verpflichtung erscheint ausgeschlossen, wenn die einzige verbleibende Möglichkeit, das Ermessen auszuüben auf rechtliche Hindernisse stößt, z.B. in Form einer Unmöglichkeit oder der Unzumutbarkeit. 463 Interessant ist, wie sich diese Konstellation auf Verpflichtungen aus der Eingliederungsvereinbarung auswirkt. In diesem Fall wird die Pflicht zur Abänderung der Vereinbarung relevant. Dann entfällt nicht nur die unmögliche/unzumutbare Verpflichtung der Behörde, sondern auch die Gegenleistungspflicht des Arbeitlosen, die dieser bei der Vereinbarung über die Abänderung geltend machen kann, bevor er einer Abänderung zustimmt.

\section{ff) Gerichtliche Überprüfbarkeit des Ermessens}

Die Sozialgerichtsbarkeit kann die Ermessensentscheidungen der Arbeitsverwaltung im Rahmen ihrer Kompetenzen grundsätzlich gerichtlich überprüfen und Ermessensfehler feststellen. Die Vereinbarung vermag hier nicht bereits die gerichtliche Kontrolldichte zu verringern. Es handelt sich gerade nicht um einen verwaltungsrechtlichen Vertrag, dessen Inhalt teilweise einer gerichtlichen Überprüfung entzogen ist. ${ }^{464}$ Da die Einglie-

458 Fallgruppe Gleichbehandlungsgebot, Art. 3 Abs. 1 GG als ermessensreduzierender Umstand bei der Ermessensreduzierung auf Null: Die Pflicht zur Gleichbehandlung mit der Folge einer Verengung des Ermessensspielraums auf eine einzige Entscheidung entsteht bei einer Selbstbindung der Verwaltung durch eigenes Entscheidungsverhalten. Dazu Brinktrine, Verwaltungsermessen in Deutschland und England, 1998, S. 157; Gubelt, in: Münch/Kunig, GG, Bd. 1, Art. 3 Rn. 39 ff.

459 Faber, Verwaltungsrecht, 1995, S. 300.

460 Dazu zweites Kapitel B I 5d) S. $161 \mathrm{f}$.

461 So auch Bieback, Umbau der Arbeitsförderung nach den Vorschlägen der Hartz-Kommission, SDSRV 52 (2004), S. 73 f; Rademacher, in: Ambs, GK-SGB III, § 35 Rn. 108.

462 Luthe, SGb 2002, S. 79.

463 Brinktrine, Verwaltungsermessen in Deutschland und England, 1998, S. 159.

464 Vgl. erstes Kapitel B IV 1 S. 82. 
derungsvereinbarung den Ermessensspielraum der Behörde konkretisiert, sind Abweichungen von der Vereinbarung besonders gründlich darzustellen und zu begründen. ${ }^{465}$

\section{c) Verhängung von Sanktionen}

Sanktionen im Arbeitsförderungsrecht sind Maßnahmen der Agenturen für Arbeit, die dazu führen, dass Entgeltleistungen zum Ersatz des Arbeitsentgelts oder andere Maßnahmen der aktiven Arbeitsförderung (auf bestimmte Zeit) nicht mehr gewährt werden.

\section{aa) Sperrzeit, § 144 SGB III}

Die Sperrzeit gem. § 144 SGB III gewährt der Versichertengemeinschaft Schutz vor Risikofällen, deren Eintritt der Versicherte selbst zu vertreten hat oder an deren Behebung er in unbegründeter Weise nicht mithilft ${ }^{466}$ und hat deshalb keinen ,strafrechtlichen Charakter“467. Sie tritt kraft Gesetzes ein und läuft kalendermäßig ab, unabhängig davon, ob sich Wirkungen für den Anspruch auf Arbeitslosengeld ergeben. ${ }^{468}$

Unmittelbare Rechtsfolge einer Sperrzeit ist, dass währenddessen der Anspruch auf Arbeitslosengeld ruht, $\S 144$ Abs. 1 erster Halbs. SGB III. Der Anspruch auf Arbeitslosengeld besteht zwar (sog. Stammrecht), aber das Arbeitslosengeld gelangt für die Dauer der Sperrzeit nicht zur Auszahlung. ${ }^{469}$ Das bedeutet, das Arbeitslosengeld muss nach Ablauf der Sperrzeit nicht erneut beantragt werden. ${ }^{470}$ Ferner vermindert sich die Anspruchsdauer gem. $\S 128$ SGB III bei bestimmten Sperrzeiten. Der Anspruch auf Arbeitslosengeld erlischt aber nur im Ausnahmefall für die Dauer der Sperrzeit gem. § 147 SGB III, wenn eine bestimmte Anzahl von Sperrzeiten über den Arbeitslosen verhängt worden sind (sog. wiederholte Sperrzeit, § 147 Abs. 1 Nr. 2 SGB III). ${ }^{471}$ Der Zeitraum, für den eine Sperrzeit besteht, wird unter Umständen nicht an das Ende der Bezugsdauer für das Arbeitslosengeld angehängt. Die finanzielle Leistung entfällt dann definitiv für diesen Zeitraum. ${ }^{472}$

\section{Geiger, info also 2002, S. 201.}

466 Vgl. nur BSG, SozR 3-4100 § 119 Nr. 3; zusammenfassend BSG, SGb 2004, S, 639 ff; allgemein zum Zweck der Sperrzeit Estelmann, VSSR 1997, S. 323-326.

467 Marschner, in: Ambs, GK-SGB III, § 144 Rn. 6; Winkler, in: Gagel, SGB III, § 144 Rn. 27 führt dazu aus, dass $§ 144$ SGB III zwar nach allgemeiner Auffassung keine Strafvorschrift ist, dass dies aber von Arbeitnehmern und Arbeitslosen meist anders gesehen wird. Die Länge der Sperrzeit ergebe - verglichen mit den Kriminalstrafen bei Kleinkriminalität - immerhin 84 Tagessätze.

468 Marschner, in: Ambs, GK-SGB III, § 144 Rn. 15.

469 Verfassungsrechtliche Aspekte der Sperrzeit sind nachzulesen bei Hünecke, in: Gagel, SGB III, § 1 Rn. 18 ff; Winkler, in: Gagel, SGB III, § 144 Rn. 19 ff; Valgolio, in: Hauck/Noftz, SGB III, K § 144 Rn. 14-21.

470 BSGE 32, 253 [256]; 33, 1 [7]; Marschner, in: Ambs, GK-SGB III, § 144 Rn. 235.

471 Voelzke, NZS 2005, S. $282 \mathrm{f}$ spricht in diesem Zusammenhang von einer „Verdichtung der Obliegenheiten des Arbeitsförderungsrechts, weil auch Sperrzeiten, die vor der Entstehung des Anspruchs auf Arbeitslosengeld entstanden sind, zum Erlöschen des Anspruchs beitragen.

472 Mittelbare Auswirkungen hat die Verhängung einer Sperrzeit auf die Rentenversicherung, weil die Sperrzeit mangels Beitragszahlung weder eine Beitragszeit im Sinne des $§ 54$ GB VI darstellt noch als Anrechnungszeit gem. § 58 SGB VI angesehen wird. Um eine Lücke im Versicherungsverlauf 
Sperrzeiten knüpfen direkt an das Verhalten des Arbeitslosen an, an sog. versicherungswidriges Verhalten. ${ }^{473}$

Die Weigerung, eine Eingliederungsvereinbarung abzuschließen, oder die Nichteinhaltung vereinbarter Pflichten erfüllt keinen in § 144 SGB III aufgeführten Tatbestand. ${ }^{474}$ Der Abschluss einer Eingliederungsvereinbarung kann nicht faktisch mittels Verhängung einer Sperrzeit und infolgedessen eintretender finanzieller Konsequenzen erzwungen werden. Es findet sich keine entsprechende Regelung im SGB III, insbesondere kann nicht in direktem Durchgriff wegen mangelnder Kooperationsbereitschaft oder Ablehnung einer Einigung zum Abschluss einer Eingliederungsvereinbarung eine Sperrzeit für das Arbeitslosengeld verhängt werden. Dies hat der Gesetzgeber in der Gesetzesbegründung deutlich gemacht. ${ }^{475}$

\section{(2) Sperrzeit bei unzureichenden Eigenbemühungen, § 144 Abs. 1 S. 2 Nr. 3 SGB III}

Die Nichterfüllung einer abgeschlossenen Eingliederungsvereinbarung hat hingegen rechtliche Folgen: Der Anspruch des Arbeitslosen auf Arbeitslosengeld ruht wegen versicherungswidrigen Verhaltens für zwei Wochen, wenn der Arbeitslose trotz Belehrung über die Rechtsfolgen ohne wichtigen Grund die von der Agentur für Arbeit geforderten Eigenbemühungen nicht nachweist, § 144 Abs. 1 S. 2 Nr. 3 i.V.m. § 144 Abs. 5 SGB III. Somit wird die Einhaltung der Eingliederungsvereinbarung als versicherungsrechtliche Obliegenheit qualifiziert. 476

Der Begriff der Eigenbemühungen entstammt § 119 Abs. 1 Nr. 2 i.V.m. § 119 Abs. 4 SGB III, der die Voraussetzungen des Anspruchs auf Arbeitslosengeld regelt. In $\S 119$ Abs. 4 Nr. 1 SGB III ist die Wahrnehmung von Verpflichtungen aus der Eingliederungsvereinbarung ausdrücklich als Element der Definition der „Eigenbemühungen“ genannt. Der fehlende Nachweis kann also zum Ruhen des Anspruchs führen.

Die Arbeitsverwaltung muss den Arbeitslosen zu konkreten Eigenbemühungen aufgefordert haben unter Hinweis darauf, dass nur bei Erbringen von Eigenbemühungen auch von Arbeitslosigkeit auszugehen ist. Für die Erfüllung muss die Arbeitsverwaltung eine Frist setzen und über die Sanktionsmöglichkeit belehren. Diese Aufforderung ist kein Verwaltungsakt, Sie muss trotzdem umfassend die vom Arbeitslosen geforderten Aktivitäten enthalten. Das Sperrzeitereignis tritt schließlich ein, wenn ein fristgerechter

zu vermeiden, ist die Möglichkeit einer freiwilligen Beitragsleistung zu prüfen. In der Kranken- und Pflegeversicherung treten keine wesentlichen Nachteile ein. Dazu Benkel, in: Wissing u.a., SGB III, 2005, § 144, Rn. 122 ff; Marschner, in: Ambs, GK-SGB III, § 144 Rn. 10 ff.

473 Deshalb wird teilweise davon gesprochen, dass es nur noch den Sperrzeittatbestand des ,versicherungswidrigen Verhaltens" gibt, der in die einzelnen Hauptgruppen aufgefächert wird (so Henke, in: Eicher/Schlegel, SGB III, § 144 Rn. 82). Dazu überzeugend a.A. Marschner, in: Ambs, GK-SGB III, § 144 Rn. 16, der darin nur die Zweckrichtung und den inneren Grund der Sperrzeitnorm sieht.

474 So auch Schweiger, NZS 2002, S. 412 f.

475 Vgl. BT-Drucksache 14/6944, S. 31.

476 Vgl. zweites Kapitel B I 5c) ee) S. 158 ff; Voelzke, NZS 2005, S. 282, der dazu allgemein ohne Bezug auf die Eingliederungsvereinbarung Stellung nimmt. 
Nachweis über das Erbringen der geforderten Eigenbemühungen nicht erbracht wird, z.B. die Dokumentation der Zahl der getätigten Bewerbungen durch Vorlage von Fotokopien. 477

Problematisch ist, wie konkret die Eigenbemühungen von der Agentur für Arbeit gefordert werden müssen. ${ }^{478}$ Hier kann die Eingliederungsvereinbarung als besondere Form dienen, dem Arbeitslosen die bestehenden gesetzlichen Verpflichtungen vor Augen zu führen ${ }^{479}$, aber auch den Einwand eröffnen, dass bestimmte Eigenbemühungen nicht zu erbringen sind. Hier ist allerdings zu beachten, dass die Eingliederungsvereinbarung von der Rechtsprechung im Einzelfall nicht als abschließend betrachtet wurde. Es soll demnach möglich sein, dass die Arbeitsverwaltung über das in der Eingliederungsvereinbarung geforderte Maß hinaus Eigenbemühungen verlangen kann. ${ }^{480}$ Dies ist sicher richtig für den nicht seltenen Fall, dass Eingliederungsvereinbarungen halbherzig abgeschlossen und nicht weiterentwickelt und angepasst werden. Ansonsten sollte die Anerkennung der abschließenden Darstellung der Obliegenheiten des Arbeitslosen in der Eingliederungsvereinbarung Einzug in die Verwaltungspraxis halten.

Mit Hilfe der Eingliederungsvereinbarung kann die Agentur für Arbeit auch ihrer Pflicht, über die Rechtsfolge eines Untätigbleibens, der Sperrzeit wegen unzureichender Eigenbemühungen, Rechtsfolgen zu belehren, gerecht werden, § 144 Abs. 1 S. 2 Nr. 3 SGB III, wenn sie diese Belehrung in die Eingliederungsvereinbarung aufnimmt. 481

\section{(3) Verhältnis von Sperrzeit und § 2 Abs. 5 SGB III}

Seit der Formulierung in $\S 2$ Abs. 5 SGB III, wonach die Arbeitnehmer zur Vermeidung oder zur Beendigung von Arbeitslosigkeit insbesondere ein zumutbares Beschäftigungsverhältnis fortzusetzen, eigenverantwortlich nach Beschäftigung zu suchen, bei bestehendem Beschäftigungsverhältnis frühzeitig vor dessen Beendigung eine zumutbare Beschäftigung aufzunehmen und an einer beruflichen Eingliederungsmaßnahme teilzunehmen haben, ist unklar, ob darin über die in $\S 144$ SGB III zu findenden Sanktionstatbestände hinaus bei Verstößen Sanktionen verhängt werden dürfen. Diese Pflichten sollen Inhalt der Eingliederungsvereinbarung sein.

Entsprechend der Gesetzesbegründung fehlt den Regelungen über Sperrzeiten ein erzieherisches Element. ${ }^{482}$ Es geht vielmehr um den Schutz der Versichertengemeinschaft davor, dass der Anspruchsberechtigte das Risiko seiner Arbeitslosigkeit manipuliert.

Die mit dem Dritten Gesetz für moderne Dienstleistungen am Arbeitsmarkt in $\S 2$ Abs. 5 SGB III statuierten Pflichten haben nur Appellfunktion. Der Gesetzgeber weist zu Beginn des SGB III bereits auf die Selbstverantwortung des Versicherten hin und darauf, dass das Anspruchsdenken der Versicherten nicht mit den Rechtsfolgen bei der

477 BSG, Urteil v. 20.10.2005, B 7a AL 18/05 R. Dazu auch Marschner, in: Ambs, GK-SGB III, § 144 Rn. 118 ff; Valgolio, in: Hauck/Noftz, SGB III, K § 144 Rn. 194 ff.

478 Marschner, in: Ambs, GK-SGB III, § 144 Rn. 123 f.

479 So auch ausdrücklich BSGE 95, 176 [183].

480 LSG München, Urteil vom 18.6.2004, L8 AL 145/03.

481 Valgolio, in: Hauck/Noftz, SGB III, K § 144 Rn. 200.

482 Bereits im Regierungsentwurf zum AFG (BT-Drucksache V/2291, S. 83). 
Verletzung dieser Pflichten (Ruhen/Erlöschen des Anspruchs auf Arbeitslosengeld z.B. gem. $§ 144$ Abs. 1 S. 2 Nr. 4 SGB III) übereinstimmt. ${ }^{483}$. Die Regelung erweitert somit nicht die Sperrzeitvorschriften, sondern hebt die Pflichten des Arbeitslosen hervor, ohne damit gleichzeitig eine Grundlage für eine neben $§ 144$ SGB III stehende Sanktion zu schaffen. Sie beeinflusst mithin nicht unmittelbar und verbindlich die Rechte und v.a. auch die Pflichten des Versicherten. ${ }^{484}$

\section{bb) Einstellung der Vermittlung, $\S 38$ Abs. 2 SGB III}

Gem. § 38 Abs. 2 SGB III kann die Agentur für Arbeit die Vermittlung einstellen, solange der Arbeitsuchende nicht ausreichend mitwirkt oder die ihm nach der Eingliederungsvereinbarung obliegenden Pflichten nicht erfüllt. Somit regelt $\S 38$ SGB III nicht nur die Mitwirkung des Arbeitsuchenden, wie die Überschrift vermuten ließe, sondern er enthält die Ermächtigung für die Agentur für Arbeit, das Fehlen unterstützenden Verhaltens des Arbeitslosen zu sanktionieren. Abs. 2 entspricht in seiner Funktion der allgemeinen Vorschrift in § 66 SGB I. 485 Die Eingliederungsvereinbarung enthält somit Obliegenheiten im Rechtssinn, bei deren Nichteinhaltung ein Rechtsverlust des „Verpflichteten“ droht. ${ }^{486}$ Die Vorschrift enthält hingegen keine Pflicht zum Abschluss einer Eingliederungsvereinbarung, denn der Wortlaut (,die ihm nach der Eingliederungsvereinbarung obliegenden Pflichten") setzt eine bereits abgeschlossene Vereinbarung voraus. ${ }^{487}$

Die Einstellung der Vermittlung folgt nicht automatisch aus dem Gesetz bei Vorliegen der Voraussetzungen, sie liegt im pflichtgemäßen Ermessen der Agentur für Arbeit, sodass diese über die tatsächliche Einstellung im Einzelfall zu entscheiden hat. ${ }^{488}$

Rechtsfolge der Entscheidung, die Vermittlungstätigkeit ganz oder teilweise einzustellen, ist, dass der Anspruch auf Vermittlungsleistungen gem. § 35 Abs. 1 SGB III zwar bestehen bleibt, dass er aber, obwohl die Voraussetzungen dafür vorliegen, nicht realisiert werden kann. Fallen die Voraussetzungen für eine Einstellung weg, z.B. weil sich der Arbeitslose wieder entsprechend der Eingliederungsvereinbarung verhält, lebt der Anspruch auf Vermittlungstätigkeit automatisch wieder auf. Insofern sind sich die Einstellung der Vermittlung nach $\S 38$ Abs. 2 SGB III und das Ruhen von Ansprüchen, $\S 142$ ff SGB III, zum Beispiel wegen einer Sperrzeit gem. § 144 SGB III, ähnlich. ${ }^{489}$

483 Gesetz vom 23.12.2003 (BGBl. I, S. 2848).

484 BSG, Urteil v. 27.3.2003 - B 7 AL 4102 R; ebenso Henke, in: Eicher/Schlegel, SGB III, § 144 Rn. 78 ff; Timme, in: Hauck/Noftz, SGB III, K § 2 Rn. 57. a.A. Eichenhofer, SGb 2000, S. 289, der von einer Sperrzeit wegen mangelnder Selbstverantwortung spricht.

485 Kruse, in: Gagel, SGB III, § 38 Rn. 6; Brand, in: Niesel, SGB III, 2005, § 38 Rn. 5.

486 Köhler, Arbeitsförderung, in: Plagemann, Münchener Anwaltshandbuch Sozialrecht, 2009, S. 402.

487 So im Ergebnis auch Rademacher, in: Ambs, GK-SGB III, § 38 Rn. 28.

488 Brand, in: Niesel, SGB III, 2005, § 38 Rn. 5.

489 Rademacher, in: Ambs, GK-SGB III, § 38 Rn. 30 f. 
d) Darlegungs- und Beweislastverteilung

\section{aa) Untersuchungsgrundsatz im Verwaltungsverfahren}

Die Darlegungs- und Beweislastverteilung im Verwaltungsverfahren entspricht grundsätzlich den allgemeinen verwaltungsverfahrensrechtlichen Regeln. ${ }^{490}$ Es gilt der Untersuchungsgrundsatz gem. § 20 SGB X, wonach die Behörde den Sachverhalt von Amts wegen ermitteln muss. Im Verwaltungsverfahren folgen in der Regel aus einer Eingliederungsvereinbarung keine automatischen Veränderungen bei der Darlegungsund Beweislast.

\section{bb) Umkehr der Darlegungs- und Beweislast bei einer Sperrzeit}

Bei der Verhängung einer Sperrzeit gem. § 144 Abs. 1 S. 3 SGB III ist eine Umkehr der Darlegungs- und Beweislast angeordnet, sodass der Abschluss einer Eingliederungsvereinbarung in diesen Fällen Wirkungen für die Darlegungs- und Beweislast entfalten kann. Der Anspruch auf Arbeitslosengeld ruht für die Dauer einer Sperrzeit, wenn der Arbeitslose sich ohne wichtigen Grund versicherungswidrig verhalten hat. Der Arbeitslose muss die Tatsachen, die für die Beurteilung eines wichtigen Grundes maßgebend sind, darlegen und beweisen. Der wichtige Grund hat die Funktion, die berechtigten Interessen des Arbeitslosen mit den Interessen der Versichertengemeinschaft an der Vermeidung der Arbeitslosigkeit abzuwägen. ${ }^{491}$

Es ist bislang umstritten, ob daraus folgt, dass der Arbeitslose die objektive Beweislast trägt, wenn Ungewissheit über den wichtigen Grund herrscht ${ }^{492}$, oder ob es bei dem Grundsatz bleibt, dass in der klassischen Eingriffsverwaltung Ungewissheiten über eingriffsbegründende Tatsachen zu Lasten der Behörde gehen. Das bedeutete, dass bei Zweifeln die Behörde vom Vorliegen eines wichtigen Grundes für das Verhalten des Arbeitslosen ausgehen muss. ${ }^{493}$ Die Formulierung der Vorschrift und die Beibehaltung der Formulierung bei der jüngsten Änderung des SGB III sprechen dafür, dass der Gesetzgeber dem Arbeitslosen das Darlegungs- und Beweisrisiko für diejenigen Tatsachen aufbürden will, die aus seiner Sphäre oder seinem Verantwortungsbereich stammen. ${ }^{494}$ Damit hat der Arbeitslose die Sperrzeit hinzunehmen, wenn ihm der Nachweis eines wichtigen Grundes nicht gelingt. ${ }^{495}$

Die Eingliederungsvereinbarung ist als schriftliches Dokument geeignet, so sie entsprechende Ausführungen enthält, Hilfestellungen bei der Erfüllung der Darlegungs-

490 Pestalozza, Der Untersuchungsgrundsatz, in: Schmitt Glaeser, Verwaltungsverfahren, FS zum 50jährigen Bestehen des Richard Boorberg Verlags, 1977, S. 185 ff.

491 Voelzke, NZS 2005, S. 285 f.

492 Marschner, in: Ambs, GK-SGB III, § 144 Rn. 67.

493 Bieback, KJ 2003, S. 31.

494 Vgl. Gesetz v. 23.12.2002 (BGBl. I, S. 4607); Gesetz v. 30.7.2004 (BGBl. I, S. 2014).

495 Marschner, in: Ambs, GK-SGB III, § 144 Rn. 167. 
und Beweislast zu geben, indem z.B. darin festgehaltene Einschränkungen des Arbeitslosen nachgewiesen werden können. 496

\section{cc) Beweisfunktion im gerichtlichen Verfahren}

Für das gerichtliche Verfahren gibt es ebenso wie im Verwaltungsverfahren keine ausdrücklichen Regelungen über die Auswirkungen einer Eingliederungsvereinbarung auf das Beweisrecht. Es sollte hier von einer Beweisfunktion der Eingliederungsvereinbarung gesprochen werden. Derjenige, der sich bei seinem Vortrag auf von der Vereinbarung abweichende Absprachen beruft, muss deren Vorliegen beweisen. Dies gilt dann sowohl für den Arbeitslosen als auch für die Behörde. Auf diese Weise wird die Verbindlichkeit der Vereinbarung ernst genommen und nicht nur auf ein pro forma Dokument beschränkt. 497

\section{Störungen bei der Abwicklung des vertragsähnlichen Verhältnisses}

a) Veränderung der tatsächlichen Verhältnisse

Die Eingliederungsvereinbarung ist gem. § 35 Abs. 4 S. 4 SGB III sich ändernden Verhältnissen anzupassen. Ihr Inhalt muss stets daraufhin überprüft werden, ob die Annahmen in der Vereinbarung noch mit den tatsächlichen Verhältnissen und deren Entwicklung übereinstimmen.

Dies ist für die Frage, ob eine Langzeitarbeitslosigkeit i.S.d. § 18 SGB III, also eine Arbeitslosigkeit von einem Jahr und länger droht, gesetzlich vorgeschrieben. Diese Annahme in der Eingliederungsvereinbarung muss spätestens nach sechsmonatiger Arbeitslosigkeit, bei arbeitslosen und ausbildungsuchenden Jugendlichen sowie in den Fällen des Satzes 2 (Eingliederungsgutschein) bereits nach drei Monaten überprüft werden, $\S 35$ Abs. 4 S. 5 SGB III.

Ferner kommt eine Abänderung der Vereinbarung in Betracht, wenn Voraussetzungen einer vereinbarten künftigen Leistung der aktiven Arbeitsförderung nicht mehr erfüllbar, aber gem. $§ 35$ Abs. 4 S. 1 SGB III in der Eingliederungsvereinbarung aufgeführt sind.

Die Abänderung kann nur einvernehmlich erfolgen. Dies ergibt sich zwar nicht direkt aus der Vorschrift des $\S 35$ Abs. 4 SGB III, aber aus dem Regelungszusammenhang. Wäre eine einseitige Abänderung möglich, ginge die Bindungswirkung der ursprünglichen Vereinbarung verloren. Sie muss genauso wie die Eingliederungsvereinbarung selbst vereinbart werden.

496 Boecken, VSSR 2003, S. 45 ff wehrt sich mit verfassungsrechtlichen Argumenten gegen die Verwendung der Eingliederungsvereinbarung in diesem Kontext und verweist auf Degenhart, ZfA 1999, S. 531 ff, der die Vermutungsregelung in $\S 7$ Abs. 3 SGB IV für verfassungsrechtlich problematisch hält.

497 Ebenso Köhler, Arbeitsförderungsrecht, in: Plagemann, Münchener Anwaltshandbuch Sozialrecht, 2009, S. $401 \mathrm{f}$. 
b) Nichteinhaltung von vereinbarten Pflichten durch den Arbeitslosen

\section{aa) Kein Erfüllungsanspruch}

Aus dem Gesetz ergibt sich gegenüber dem Arbeitslosen kein Anspruch der Agentur für Arbeit auf Einhaltung der in der Vereinbarung niedergelegten Pflichten. 498 Da die Eingliederungsvereinbarung nicht als verwaltungsrechtlicher Vertrag einzuordnen ist, gibt es aus diesem Grund auch keinen vertraglichen Erfüllungsanspruch der Verwaltung. Inhalt der Eingliederungsvereinbarung sind bloße Obliegenheiten. ${ }^{499}$

\section{bb) Schadensersatz}

Sekundärrechte sind mangels eines Vertrages ausgeschlossen.

Anders als in $\S 15$ Abs. 3 SGB II 500 ist im SGB III nicht geregelt, dass Schadensersatz zu zahlen ist, wenn der Arbeitslose eine vereinbarte Bildungsmaßnahme abbricht. $\mathrm{Da}$ es sich beim Schadensersatz um eine weitere, über den Rahmen des Gesetzes hinausgehende Sanktion handelt, wäre dies ohnehin ausgeschlossen, § 31 SGB I.501 Vielmehr ist der Sanktionstatbestand $\S 144$ Abs. 1 S. 2 Nr. 5 SGB III erfüllt, wenn eine Eingliederungsmaßnahme abgebrochen wird, sodass der Arbeitslose gleichwohl zum Durchhalten, wenn auch mittels (Negativ-)Anreize, angehalten wird.

\section{cc) Vertragsstrafe}

Die Vereinbarung einer Vertragsstrafe ${ }^{502}$ scheitert ebenfalls am Fehlen eines Vertrages. Die Vertragsstrafe ist ein Instrument, das die Einhaltung eines Vertrages durch einen finanziellen Anreiz beziehungsweise einen Negativanreiz abzusichern versucht, um zu vermeiden, dass ungewisse, schwer nachweisbare Folgeschäden nicht ersetzt werden. ${ }^{503}$ Doch auch die Vereinbarung einer Vertragsstrafe dürfte über den Vorbehalt des Gesetzes in $\S 31$ SGB I hinausgehen. Denn es handelt sich dabei um eine Sanktion, die keine gesetzliche Grundlage hat, und damit um eine negative Folge einer Eingliederungsvereinbarung, die der Gesetzgeber nicht wollte. 504

\section{c) Nichteinhaltung von vereinbarten Pflichten durch die Agentur für Arbeit}

Da die Eingliederungsvereinbarung keine Vereinbarung im Sinne eines verwaltungsrechtlichen Vertrags ist, kann diese keine Rechte und Pflichten der Verwaltung begründen.

498 Kossens, AuA 2002, S. 17; Bieback, Umbau der Arbeitsförderung nach den Vorschlägen der HartzKommission, SDSRV 52 (2004), S. 74; a.A. ohne Begründung Mutschler, in: Wissing u.a., SGB III, 2005, § 35 Rn. 46, der von einem „klagbaren Anspruch aus der Eingliederungsvereinbarung“, aber gleichzeitig von einer quasi-vertraglichen Situation spricht.

499 Geiger, info also 2002, S. 201.

500 Vgl. zweites Kapitel B II 8d) aa) S. 234.

501 Vgl. dazu zweites Kapitel B I 5c) S. 156 ff.

502 Allgemein zur Definition im Privatrecht Gottwald, in: Münchener Kommentar, 2007, v § 339 Rn. 1.

503 Winkler, in: Gagel, SGB III, § 144 Rn. 28.

504 Vgl. auch § 32 SGB I über das Verbot nachteiliger Folgen einer privatrechtlichen Vereinbarung. 
Hält sich die Behörde nicht an Vereinbartes, kann die Vereinbarung als eine vorweggenommene Ermessenausübung beziehungsweise eine den Ermessensspielraum konkretisierende Bindung gesehen werden, sodass sich die Behörde bei Versagung der Leistung widersprüchlich verhält. Sie muss in jedem Fall Gründe für ihr Abweichen von der Vereinbarung der Eingliederungsstrategie vorweisen. ${ }^{505}$ Folge wäre eine rechtswidrige Entscheidung, gegen die der Arbeitslose über Widerspruch und Klage auf dem Sozialgerichtsweg vorgehen kann. ${ }^{506}$ Auf das Instrument der Dienstaufsichtsbeschwerde macht die Gesetzesbegründung aufmerksam. ${ }^{507}$

\section{Beendigung der Eingliederungsvereinbarung}

\section{a) Erledigung infolge der Beendigung der Arbeitslosigkeit}

Endet die Arbeitslosigkeit, erledigt sich die Eingliederungsvereinbarung. Für künftige Leistungen aktiver Arbeitsförderung liegen die Voraussetzungen nicht mehr vor. Vermittlungsbemühungen der Arbeitsagentur würden ins Leere laufen und Eigenbemühungen des Arbeitslosen machen keinen Sinn mehr.

Die Eingliederungsvereinbarung hat nur Bestand, solange die Vermittlungssituation zwischen Arbeitsagentur und Arbeitslosem gegeben ist, mithin solange die Arbeitslosigkeit besteht. Suchte man ein rechtliches Instrument, könnte man daran denken, dass die Willenserklärung der Verwaltung auflösend bedingt abgegeben wurde. Die Bedingung tritt ein, wenn der Arbeitslose nicht mehr in den Arbeitsmarkt eingliedert werden muss.

\section{b) Zeitablauf}

Die Vereinbarung wird auf eine bestimmte Zeit hin abgeschlossen. Das ergibt sich daraus, dass eine Pflicht besteht, eine Fortschreibung der Vereinbarung zu veranlassen, wenn die Arbeits-/Ausbildungsplatzsuche in dem ursprünglich vereinbarten Geltungszeitraum nicht von Erfolg gekrönt ist, § 35 Abs. 4 S. 4 2. Halbs. SGB III. 508 Damit endet die Eingliederungsvereinbarung mit deren Zeitablauf.

\section{c) Abänderung}

Die in $\S 35$ Abs. 4 S. 5, 6 SGB III vorgeschriebene Anpassung an sich ändernde Verhältnisse und die Überprüfung der Eingliederungsvereinbarung insbesondere auf das Risiko einer drohenden Langzeitarbeitslosigkeit hin kann zu einer Beendigung der Eingliederungsvereinbarung führen, wenn die ursprüngliche Vereinbarung durch eine Än-

505 Rademacher, in: Ambs, GK-SGB III, § 35 Rn. 106.

$506 \mathrm{Im}$ Ergebnis so Bieback, Umbau der Arbeitsförderung nach den Vorschlägen der HartzKommission, SDSRV 52 (2004), S. 74; Geiger, info also 2002, S. 201.

507 BT-Drucksache 14/6944 S. 31.

508 So auch Knoblauch/Hübner, NDV 2005, S. 281. 
derungsvereinbarung vollständig abgelöst wird. Im Übrigen endet nur der Teil, der geändert worden ist.

\section{d) Kündigung}

Eine ausdrückliche gesetzlich vorgesehene Kündigungsmöglichkeit als einseitige Beendigungsmöglichkeit besteht für die Eingliederungsvereinbarung nicht.

Die Vorschriften über den verwaltungsrechtlichen Vertrag sind nicht anzuwenden, mithin nicht $\S 59$ SGB X.509 Die Möglichkeiten einvernehmlicher Abänderung der Vereinbarung sind ausreichend und mit dem Sinn und Zweck einer vereinbarten Strategie zur Beendigung der Arbeitslosigkeit besser zu vereinbaren, als eine einseitige Kündigungsmöglichkeit. Die Gesprächssituation zwischen Arbeitsverwaltung und Arbeitslosem bleibt auf diese Weise aufrechterhalten.

\section{Rechtsschutz}

\section{a) Außergerichtlicher Rechtsschutz}

Beim außergerichtlichen Rechtsschutz ist zu unterscheiden, ob eine Eingliederungsvereinbarung ausgehandelt werden soll oder ob eine solche bereits abgeschlossen wurde.

\section{aa) Beratung und Entscheidung des Vorgesetzten}

Im Verhandlungsprozess kann es zu unterschiedlichen Ansichten über die vorzusehenden Maßnahmen kommen. Bei Differenzen kann der Arbeitslose eine Beratung und eine Entscheidung des Vorgesetzten des zuständigen Sachbearbeiters der Agentur für Arbeit verlangen. Hierbei kann er zu seiner Unterstützung einen Berater seines Vertrauens hinzuziehen. Kann auch bei diesem Einigungsversuch kein Einvernehmen erzielt werden, und kommt deshalb keine Eingliederungsvereinbarung zustande, bleibt es dabei, dass die Agentur für Arbeit Vermittlungsvorschläge unterbreitet und über Maßnahmen der aktiven Arbeitsförderung entscheidet. ${ }^{510}$

\section{bb) Dienstaufsichtsbeschwerde}

Eine Dienstaufsichtsbeschwerde kommt in Betracht, wenn der einzelne Mitarbeiter der Agentur für Arbeit sich nicht an die Vereinbarung hält. Eine weitere außergerichtliche Überprüfungsmöglichkeit bezüglich der Eingliederungsvereinbarung steht dem Arbeitslosen nicht zur Verfügung. Ob dieser Weg im Normalfall erfolgversprechend ist, darf mit Recht in Zweifel gezogen werden. ${ }^{511}$ Er kann aber ein zusätzliches Druckmittel sein, wenn der Fallmanager sich persönlich unkorrekt verhält. In allen anderen Fällen

509 A.A. Knoblauch/Hübner, NDV 2005, S. 281, allerdings aufgrund der Tatsache, dass sie vom Abschluss eines verwaltungsrechtlichen Vertrags ausgehen.

510 BT-Drucksache 14/6944, S. 31.

511 Kruse, in: LPK-SGB III, 2008, § 35 Rn. 36. 
wird die Dienstaufsichtsbeschwerde nur zu einer weiteren Verschlechterung des Verhältnisses zwischen Arbeitslosem und Arbeitsagentur führen.

b) Widerspruch gegen einseitige Entscheidung

Gegen die Eingliederungsvereinbarung selbst gibt es keinen Rechtsbehelf. Widerspruch gem. § $78 \mathrm{SGG}$ ist insbesondere nicht statthaft, weil die Eingliederungsvereinbarung kein Verwaltungsakt im Sinn des $\S 31$ SGB X ist. Nur gegen Verwaltungsakte, gegen die die Anfechtungsklage statthaft ist ${ }^{512}$, ist Widerspruch einzulegen, $\S 78$ Abs. 1 SGG.

c) Gerichtlicher Rechtsschutz

Dem Arbeitslosen steht der gerichtliche Rechtsschutz offen, wenn sein Begehren nach allgemeinen Verfahrensregelungen des SGG gerichtlich überprüfbar ist. Im Folgenden werden nur die besonderen, die Eingliederungsvereinbarung betreffenden Fragen besprochen, ohne im Detail auf den Rechtsschutz gegen Akte der Arbeitsverwaltung einzugehen.

aa) Rechtsweg

Der Rechtsweg zu den Sozialgerichten ist gem. § 51 Abs. 1 SGG eröffnet, denn es handelt sich um eine Angelegenheit der Arbeitsförderung.

\section{bb) Klägerisches Begehren}

Die Arbeitsverwaltung kann nicht direkt auf Einhaltung der Vereinbarung klagen, da die Eingliederungsvereinbarung nicht als verwaltungsrechtlicher Vertrag einzuordnen ist. Vertragsähnliches Handeln kann nicht gerichtlich durchgesetzt werden. Der Arbeitsverwaltung steht nur das oben beschriebene Sanktionsinstrumentarium zur Durchsetzung vereinbarter Pflichten zur Verfügung.

Der Arbeitslose als Kläger kann sich nicht gegen die Eingliederungsvereinbarung selbst wehren. Bei Widerstand gegen den von der Behörde vorgeschlagenen Inhalt für die Eingliederungsvereinbarung bleiben ihm außergerichtliche Rechtsschutzmöglichkeiten. Die Gerichte kann er nicht bemühen. Klägerisches Begehren auf Seiten des Arbeitslosen kann es aber sein, dass Sanktionen überprüft oder Leistungen gewährt werden.

\section{cc) Klage gegen den Sanktionsbescheid}

Hat die Agentur für Arbeit eine Sanktion auf der Grundlage des $\S 144$ Abs. 1 S. 2 Nr. 3 SGB III verhängt, weil der Arbeitslose in der Eingliederungsvereinbarung vereinbarte Eigenbemühungen nicht ausreichend nachgewiesen hat, kann dieser Bescheid angefochten werden.

512 Vgl. zweites Kapitel B I 10c) S. 180 f. 
Die Aufhebung der Leistungsbewilligung gem. $\S \S 45,48$ SGB X wegen Eintritts einer Sperrzeit ist ein Verwaltungsakt im Sinn des $\S 31$ SGB X. ${ }^{513}$ Dieser Bescheid wird bei Vorliegen der formellen und materiellen Voraussetzungen mittels einer Anfechtungsklage gem. § 54 Abs. 1 S. 1 1. Alt. SGG, die häufig mit einem Leistungsantrag verbunden wird, § 54 Abs. 4 SGG, aus der Welt geschafft, sodass der ursprüngliche Bescheid wieder auflebt. 514

\section{dd) Klage gegen die Einstellung der Vermittlung}

Der Bescheid, in dem dem Arbeitslosen die Entscheidung mitgeteilt wird, dass die Vermittlung wegen Nichterfüllung von Pflichten aus der Eingliederungsvereinbarung eingestellt wird ( $§ 38$ Abs. 2 SGB II), stellt einen Verwaltungsakt im Sinn des $\S 31$ SGB X dar. ${ }^{515}$ Aufgrund der darin enthaltenen Regelung im Einzelfall mit Außenwirkung stellt die Arbeitsverwaltung ihre Vermittlungsbemühungen ein.

Dieser Bescheid kann mit einer Anfechtungsklage beseitigt werden, § 54 Abs. $1 \mathrm{~S} .1$ SGG.

\section{d) Kosten}

In Bezug auf die Kosten des Rechtsschutzes gibt es keine Besonderheiten:

Im Vorverfahren hat der Widerspruchsführer gem. § 63 Abs. 1 SGB X einen Kostenerstattungsanspruch, soweit er erfolgreich Widerspruch eingelegt hat. Die Gebühren und Auslagen eines Rechtsanwalts sind im Vorverfahren erstattungsfähig, wenn die Zuziehung notwendig war, $\S 63$ Abs. 2 SGB X.

Für die Gerichtskosten gilt der Grundsatz der Kostenfreiheit. Das Verfahren vor den Gerichten der Sozialgerichtsbarkeit ist für Versicherte und Leistungsempfänger kostenfrei, soweit sie in dieser jeweiligen Eigenschaft als Kläger oder Beklagter beteiligt sind, $\S 183$ S. 1 SGG. Es gibt gem. § 73a SGG i.V.m. §§ 114 ff ZPO die Möglichkeit, in allen Instanzen der Sozialgerichtsbarkeit Prozesskostenhilfe zu beantragen.

513 Die Feststellung über den Eintritt einer Sperrzeit in einem mit dem Bewilligungsbescheid einheitlichen Bescheid enthält einen „,deklaratorischen Verfügungssatz“, der als Verwaltungsakt im Sinn des $\S 31$ SGB X anzusehen ist. Mit diesem wird die getroffene Regelung in Bezug auf die Leistungsbewilligung begründet. Dazu BSG, Urteil v. 3.6.2004, B 11 AL 71/03 R; BSGE 95, 80 [82]; Valgolio, in: Hauck/Noftz, SGB III, K § 144 Rn. 311 f; Henke, in: Eicher/Schlegel, SGB III, § 144 Rn. 580; anders noch Winkler, in Gagel, SGB III, § 144 Rn. 15 unter Verweis auf BSG, Urteil v. 21.3.1998, B 7 AL 44/01 R.

514 Zum Verfahren Winkler, in: Gagel, SGB III, § 144 Rn. 243.

515 So auch Rademacker, in: Hauck/Noftz, SGB III, K § 38 Rn. 13; Rademacher, in: Ambs, GK-SGB III, § 38 Rn. 29. 


\section{SGB II und Viertes Gesetz für moderne Dienstleistungen am Arbeitsmarkt}

\section{a) Zusammenführung von Arbeitslosenhilfe und Sozialhilfe: „Hartz IV“}

Im Vierten Gesetz für moderne Dienstleistungen am Arbeitsmarkt vom 24.12.2003516 („Hartz IV“517), das im Wesentlichen zum 1.1.2005 in Kraft getreten ist, wird im Kern in einem Zweiten Buch Sozialgesetzbuch (SGB II) die „Grundsicherung für Arbeitsuchende“ geregelt. Unter dem Motto „Fördern und Fordern“, das auch das erste Kapitel des SGB II überschreibt und immer wieder der Bildung von Grundsätzen zugrunde liegt, werden die bisherige Arbeitslosenhilfe und die Sozialhilfe für erwerbsfähige Personen zusammengeführt. 518 Damit wird ein im Abschlussbericht der HartzKommission als intransparent und verwaltungsmäßig aufwändig bezeichnetes System vereinfacht ${ }^{519}$, das über alle politischen Parteien hinweg als überholungsbedürftig angesehen wurde. 520

Das frühere System war dreigegliedert: Arbeitslosengeld als reine Versicherungsleistung stand neben Arbeitslosenhilfe und Sozialhilfe als jeweils steuerfinanzierte Leistungen, wobei Arbeitslosengeld bzw. Arbeitslosenhilfe zur Sicherstellung des Existenzminimums gleichzeitig beantragt werden konnten. So konnte die fehlende Familienkomponente der Arbeitslosenhilfe aufgefangen und mit einer anderen, reinen Fürsorgeleistung ergänzt werden. 521

Seit dem 1.1.2005 werden die sog. erwerbsfähigen Hilfebedürftigen im Rahmen der Regelungen des SGB II betreut und erhalten Arbeitslosengeld II, nicht erwerbsfähige Personen in Bedarfsgemeinschaft mit einem Antragsteller nach SGB II bekommen Sozialgeld. Alle anderen nicht erwerbsfähigen Personen ${ }^{522}$ werden nach den Vorschriften des SGB XII betreut. ${ }^{523} \mathrm{Im}$ Unterschied zur Sozialhilfe sind die Empfänger von Ar-

516 Gesetz v. 24.12.2003 (BGB1. I, S. 2954).

517 Sehr polemisch Zuck, NJW 2005, S. 649 ff, der „Hartz IV“ wegen des Aufgreifens des Namens Peter Hartz' als Nonsensbegriff bezeichnet.

518 Zur Diskussion über die Zusammenlegung, deren Auswirkungen und mögliche Schwierigkeiten vgl. Boecken, SGb 2002, S. 357 ff; Adamy, SozSich 2003, S. 285 ff; Kruse, ZIAS 2003, S. 301 ff; Spindler, SozSich 2003, S. 338 ff; Deutscher Verein für öffentliche und private Fürsorge, NDV 2003, S. 496 ff; Bauer, DÖV 2004, S. 1017 ff; Korenke, SGb 2004, S. 525 ff; zum Menschenbild Becker, Sozialmodell und Menschenbild in der „Hartz-IV” Gesetzgebung, in: Behrends, Gesetzgebung, Menschenbild und Sozialmodell im Familien- und Sozialrecht, 2008, S. 39 ff.

519 Hartz, Moderne Dienstleistungen am Arbeitsmarkt, 2002, S. 126.

520 Agenda 2010, Koalitionsvereinbarung SPD/Die Grünen, Programmatik der CDU/CSU; dazu Berlit, info also 2003, S. 195.

521 Dazu und ausführlich zu den Funktionen der Arbeitslosenhilfe Ebsen, Die Arbeitslosenhilfe zwischen Sozialversicherung und Fürsorge, in: Ebsen, Stand und Entwicklung des Arbeitsförderungsrechts, 1997, S. $162 \mathrm{f}$.

$522 \mathrm{Zu}$ Abgrenzungsproblemen von SGB II und SGB XII Rothkegel, ZfSH/SGB 2004, S. 396-409; Blüggel, SGb 2005, S. 377 ff.

523 Neu geregelt im Gesetz zur Einordnung des Sozialhilferechts in das Sozialgesetzbuch v. 27.12.2003 (BGBl. I, S. 3022). 
beitslosengeld II pflichtversichert in der Krankenversicherung ( 5 Abs. 1 Nr. 2a SGB V), der Pflegeversicherung ( $\$ 20$ Abs. 1 S. 2 Nr. 2a SGB XI) und der Rentenversicherung 524 ( 33 S. 1 Nr. 3a SGB VI). Da es sich beim Arbeitslosengeld II lediglich um eine Grundsicherung handelt, während in der Arbeitslosenhilfe Prozentsätze vormals erzielten Einkommens gewährt wurden, führte die Zusammenführung von Arbeitslosenhilfe und Sozialhilfe vor allem zu einer Verschlechterung für die Menschen, die vor der Arbeitslosigkeit gut verdient haben. 525

\section{b) Kommunales Optionsgesetz}

Probleme bei der organisatorischen Umsetzung und der damit eng verwobenen Abwicklung der finanziellen Folgen der Zusammenlegung von Arbeitslosen- und Sozialhilfe bereitete die Festlegung der Trägerschaft für die Leistungen nach SGB II.

Während bislang die Trägerschaft für die Sozialhilfe mitsamt der finanziellen Belastung bei den Kommunen lag, hatte diese die Bundesagentur für Arbeit im Rahmen der Arbeitslosenhilfe inne. Die Bundesagentur für Arbeit erbrachte die Leistungen aus Steuermitteln des Bundes, da es sich bei der Arbeitslosenhilfe um eine Fürsorge-, nicht um eine Versicherungsleistung handelte. Die Kommunen führten auf örtlicher Ebene Maßnahmen zur Wiedereingliederung von Sozialhilfeempfängern in den ersten Arbeitsmarkt durch. Diese Erfahrungen im Rahmen der Hilfe zur Arbeit auf der Grundlage der $\S \S 18 \mathrm{ff}$ BSHG sollten auch nach der Zusammenführung genutzt werden. ${ }^{526} \mathrm{Oft}$ fand bereits zu dieser Zeit eine Zusammenarbeit mit den örtlichen Agenturen für Arbeit statt, teilweise auch gefördert im Rahmen von Projekten wie z.B. MoZArT.527

In der Diskussion waren verschiedene Organisationsformen: eine alleinige Trägerschaft der Kommunen (bei einer (Teil-) Finanzierung durch den Bund), eine Zusammenarbeit von Kommunen und Bundesagentur für Arbeit unter der Federführung der Bundesagentur für Arbeit, aber auch eine alleinige Leistungsträgerschaft der Bundesagentur für Arbeit.

Am Ende stand das Gesetz zur optionalen Trägerschaft von Kommunen nach dem Zweiten Buch Sozialgesetzbuch (Kommunales Optionsgesetz) ${ }^{528}$, ein im Vermittlungsausschuss gefundener Kompromiss, der sich in $\S 6$ Abs. 1, 2 SGB II ausdrückt: Die Trägerschaft für die Regelleistung gem. $\S \S 20,28$ SGB II und die Eingliederungsleistungen gem. § 16 SGB II mit Ausnahme der flankierenden Eingliederungsleistungen gem. $§ 16$ Abs. 2 S. 2 Nr. 1-4 SGB II ${ }^{529}$ erhält die Bundesagentur für Arbeit. Ihre

524 Dazu Chojetzki/Klönne, DRV 2004, S. 513-531.

525 Simulationsrechnungen bei Blos/Rudolph, IAB-Kurzbericht 2005, S. 1 ff.

526 Berlit, Hilfe zur Arbeit im Kontext aktueller sozialpolitischer Entwicklungen, in: Fuchs/SchulzeBöing, Hilfe zur Arbeit und kommunale Beschäftigungspolitik, 1999, S. 15 ff.

527 Modellprojekt zur Verbesserung der Zusammenarbeit von Arbeitsämtern und Trägern der Sozialhilfe, Gesetz v. 29.11.2000 (BGBl. I, S. 1590), vgl. Reher, BArbBl 2002, S. 8 f; Genz/Schwendy, TuP 2004, S. 8.

528 Gesetz v. 30.7.2004 (BGB1. I, S. 2014).

529 Zur Umgestaltung durch das Gesetz zur Neuausrichtung der arbeitsmarktpolitischen Instrumente vgl. Fn. 536. 
Kompetenz insbesondere im Beratungs- und Vermittlungsbereich wird damit nicht in Frage gestellt. Die Trägerschaft der nach Landesrecht zuständigen Träger ${ }^{530}$ (kommunale Träger, § 6 Abs. 3 SGB II) erstreckt sich auf die Leistungen für Unterkunft und Heizung nach $\S 22$ SGB II, die einmaligen Leistungen nach § 23 SGB II sowie auf die sog. flankierenden Eingliederungsleistungen nach $\S 16$ Abs. 2 S. 2 Nr. 1-4 SGB II (Kinderbetreuung, Schuldnerberatung, psychosoziale Betreuung, Suchtberatung).

Zur gemeinsamen Wahrnehmung dieser Aufgaben sollten gem. § 44b SGB II Arbeitsgemeinschaften zwischen den Trägern der Grundsicherung für Arbeitsuchende errichtet werden, deren Rechtsform und rechtliche Handlungsmöglichkeiten zunächst ungeklärt und sofort mit dem Inkrafttreten der Vorschriften Gegenstand einer Verfassungsbeschwerde von elf Kreisen und Landkreisen waren. Diese brachten u.a. vor, dass es sich bei den Arbeitsgemeinschaften um verfassungswidrige Mischverwaltung zwischen einer Bundesbehörde und kommunalen Behörden handelte, die die Kreise und Landkreise in ihrem Recht auf eigenverantwortliche Aufgabenwahrnehmung verletzte (Art. 28 Abs. 2 i.V.m. Art. 83 GG). ${ }^{531}$ Mit Urteil vom 20.12.2007 hat das Bundesverfassungsgericht $\S 44 \mathrm{~b}$ SGB II für verfassungswidrig erklärt und für eine Neuorganisation des Vollzugs des SGB II eine Übergangsfrist bis 31.12.2010 gewährt. ${ }^{532}$ Seither werden zwischen Bund, Ländern, Kommunalen Spitzenverbänden und Bundesagentur für Arbeit Verhandlungen darüber geführt, welche Veränderungen erforderlich sind und wie dem Urteil Rechnung getragen werden kann, um einen möglichst bürgernahen, aber verfassungskonformen Vollzug sicherzustellen. Eine Einigung auf politischer Ebene steht bislang aus. 533

Sofern kommunale Träger Aufgaben nach $\S 6$ Abs. 1 Nr. 1 SGB II, also Aufgaben, die grundsätzlich die Agenturen für Arbeit erfüllen, nach Zulassung durch die Bundesagentur für Arbeit entsprechend der Experimentierklausel in § 6a SGB II wahrnehmen, tun sie dies nach Errichtung besonderer Einrichtungen gem. § 6a Abs. 6 SGB II in alleiniger kommunaler Verantwortung an Stelle der Bundesagentur für Arbeit und erhalten die entstehenden Kosten erstattet (sog. Optionskommunen). Die Zahl der insgesamt in Deutschland zugelassenen Träger beträgt höchstens 69, § 6a Abs. 3 S. 1 SGB II. Die

530 In Bayern sind die Landkreise und kreisfreien Gemeinden die nach Landesrecht zuständigen Träger der Grundsicherung für Arbeitsuchende, Art 2 Abs. 1 AGSG.

531 Henneke, DÖV 2005, S. 177 ff; ders., DÖV 2006, S. 726 ff; vgl. auch Buscher, WiW 2004, S. 284; Adamy, SozSich 2004, S. 124 ff; ders., SozSich 2004, S. 332 ff; Strobel, NVwZ 2004, S. 1195 ff; Quaas, SGb 2004, S. 723 ff; Faber, NZS 2005, S. 76; Ruge, DVBl 2005, 403 ff; Bieback, RsDE 2006, S. 24 ff; Dyllick/Lörincz/Neubauer, ZfSH/SGB 2007, S. 397 ff; Wahrendorf, SozR akt 2009, S. $2 \mathrm{f}$.

532 BVerfG, Urteil v. 20.12.2007, 2 BvR 2433/04; dazu Anm. Klaus, in: jurisPR-SozR 5/2008 Anm. 2.

533 Zusammenfassend Henneke, Der Landkreis 2008, 59 ff; Korioth, DVBl 2008, S. 812 ff; Becker, Sozialmodell und Menschenbild in der „Hartz-IV” Gesetzgebung, in: Behrends, Gesetzgebung, Menschenbild und Sozialmodell im Familien- und Sozialrecht, 2008, S. 51-54. Auf einer Informationsplattform des IAB (http://infosys.iab.de/infoplattform/thema.asp) werden aktuelle Entwicklungen dargestellt. 
Zulassung hängt von der Zustimmung der zuständigen obersten Landesbehörde ab, § 6a Abs. 4 S. 1 SGB II und ist derzeit bis 31.12 .2010 befristet. ${ }^{534}$

\section{c) Überblick über die Leistungen nach dem SGB II}

\section{aa) Leistungen zur Eingliederung in Arbeit, $\S \S 14$ ff SGB II}

Die zuständigen Träger der Grundsicherung für Arbeitsuchende werden in erster Linie mit dem Ziel tätig, die erwerbsfähigen Hilfebedürftigen wieder in den Arbeitsmarkt einzugliedern. Der in $\S 14$ SGB II geregelte Grundsatz des Förderns ist der wichtigste Grundsatz, der bei der Entscheidung beziehungsweise Verhandlung über Leistungen zur Eingliederung beachtet werden muss. 535

Das zeitliche Element dieser Hilfe wird in dem seit 01.08.2006 geltenden sog. Sofortangebot gem. $\S 15$ a SGB II ${ }^{536}$ stark betont. Personen, die in den letzten beiden Jahren weder Geldleistungen zur Sicherung des Lebensunterhaltes auf der Grundlage des SGB II noch des SGB III bezogen haben, sollen bei Beantragung von Leistungen der Grundsicherung für Arbeitsuchende unverzüglich Leistungen zur Eingliederung in Arbeit angeboten werden.

Grundsätzlich stehen den Agenturen für Arbeit die Instrumente des Arbeitsförderungsrechts zur Verfügung, was aus der Verweisung auf die entsprechenden Vorschriften des SGB III in $\S 16$ Abs. 1 SGB II folgt.

$\S 16$ Abs. 2 S. 1 SGB II enthält eine Generalklausel, die es der Agentur für Arbeit ermöglicht, weitere Leistungen zur Eingliederung zu erbringen. § 16 Abs. 2 S. 2 SGB II konkretisiert diese weiteren Leistungen mit Hilfe eines nicht abschließenden Katalogs von Leistungen (,insbesondere“), die es dem besonderen Bedarf der erwerbsfähigen Hilfebedürftigen entsprechend ermöglichen soll, jenen verstärkt Unterstützung zuteil werden zu lassen. Beispielhaft genannt seien z.B. die Schuldnerberatung, psychosoziale Betreuung oder das Einstiegsgeld nach $\S 29$ SGB II. 537

Für die „weiteren Leistungen“ gem. $\S 16$ Abs. 2 S. 1 sowie das Einstiegsgeld und Leistungen nach dem Altersteilzeitgesetz gem. $§ 16$ Abs. 2 S. 2 Nr. 5 und 6 SGB II sind die Agenturen für Arbeit, für die flankierenden Eingliederungsleistungen nach $\S 16$ Abs. 2 S. 2 Nr. 1-4 SGB II die kommunalen Träger der Grundsicherung für Arbeitsuchende zuständig, $\S 6$ Abs. 1 S. 1 Nr. 2 SGB II. 538

534 Dazu Henneke, Das Optionsmodell bei der Grundsicherung für Arbeitssuchende, in: Henneke, Künftige Funktionen und Aufgaben der Kreise, 2004, S. 95 ff.

535 Kapitel 3 Abschnitt 1 (Leistungen zur Eingliederung in Arbeit) ist durch das Gesetz zur Neuausrichtung der arbeitsmarktpolitischen Instrumente v. 21.12.2008 (BGBl. I, S. 2917) weitreichend umgestaltet worden. Die Arbeit behält die frühere Zitierung bei. Die Änderungen betreffen v.a. die Regelungen zu den im SGB II-Bereich zulässigen Eingliederungsleistungen, nicht aber die Eingliederungsvereinbarung als solche.

536 Eingeführt mit Wirkung zum 1.8.2006 durch Gesetz vom 20.7.2006 (BGBl. I, S. 1706).

537 Zur Abgrenzung zum Katalog des $§ 16$ Abs. 1 SGB II Schumacher, in: Oestreicher, SGB XII/SGB II, § 16 Rn. 26 ff; Hafenrichter, in: Hohm, GK-SGB II, § 16 Rn. 235 ff.

538 Vgl. zweites Kapitel B II 1b) S. 183 f. 
Finden die erwerbsfähigen Hilfebedürftigen keine Arbeit, sollen Arbeitsgelegenheiten geschaffen werden, § 16 Abs. 3 SGB II.

\section{bb) Leistungen zur Sicherung des Lebensunterhaltes, $\S \S 19$ ff SGB II}

Die Leistungen zur Sicherung des Lebensunterhaltes sind in den $\S \S 19$ ff SGB II geregelt. An den erwerbsfähigen Hilfebedürftigen wird Arbeitslosengeld II gezahlt. Nicht erwerbsfähige Mitglieder der Bedarfsgemeinschaft im Sinn des $§ 7$ Abs. 3 SGB II erhalten unter bestimmten Voraussetzungen Sozialgeld gem. § 28 SGB II. 539

Das Arbeitslosengeld II besteht gem. § 19 SGB II aus Leistungen zur Sicherung des Lebensunterhaltes 540 einschließlich der angemessenen Kosten für Unterkunft und Heizung und einem befristeten Zuschlag gem. § 24 SGB II, der den Übergang vom Bezug von Arbeitslosengeld als Leistung der Arbeitslosenversicherung zum Arbeitslosengeld II abfedern soll. Die Regelleistung gem. § 20 Abs. 2, 4 SGB II ist eine Pauschale ${ }^{541}$, die den gesamten Lebensunterhalt abdeckt. ${ }^{542}$

Hilfe zum Lebensunterhalt nach dem dritten Kapitel des SGB XII ist grundsätzlich gem. § 5 Abs. 2 SGB II ausgeschlossen ${ }^{543}$, und zwar nicht erst bei Leistungsbezug nach SGB II, sondern bereits dann, wenn dem Grunde nach ein Anspruch auf Leistungen der Grundsicherung für Arbeitsuchende besteht, wenn also insbesondere ein erwerbsfähiger Hilfebedürftiger Leistungsempfänger wäre. ${ }^{544}$

Die Regelleistung betrug zunächst in den alten Bundesländern einschließlich Berlin (Ost) 345 Euro, in den neuen Bundesländern 331 Euro. ${ }^{545}$ Mit dem Ersten Gesetz zur

539 Dabei handelt es sich trotz der Vertretungsregelung in $\S 38$ SGB II um eigenständige Ansprüche jedes Mitglieds der Bedarfsgemeinschaft, vgl. BSG, Urteil vom 7.11.2006-B 7b AS8/06R -, SozR 4$4200 \S 22$ Nr. 1, Rz. 12.

540 Zur Zusammensetzung der Regelleistung vgl. Schwabe, ZfF 2008, S. 145 ff. 204.

541 Zur verfassungsrechtlichen Rechtfertigung der Pauschalierung O'Sullivan, SGb 2005, S. 370; Hebeler, SGb 2008, S. 8 ff.

542 BT-Drucksache 15/1516, S. 56; Hüttenbrink, Sozialhilfe und Arbeitslosengeld II, 2004, S. 37 f zählt Ernährung, Kleidung, Körperpflege, Hausrat, Bedarfe des täglichen Lebens sowie in vertretbarem Umfang auch Beziehungen zur Umwelt und eine Teilnahme am kulturellen Leben auf.

543 Zur Bemessung des Regelsatzes nach dem SGB XII Frommann, NDV 2004, S. 246 ff.

$544 \mathrm{Zu}$ diesem Ausschluss aus verfassungsrechtlicher Sicht Krahmer, ZfF 2004, S. 180 f.

545 Diskutiert wird das Verfahren der Festlegung der Regelleistung in $\S 20$ SGB II und die Frage, ob dieser Betrag bedarfsdeckend ist für die Sicherung des verfassungsrechtlich gewährleisteten „,soziokulturellen Existenzminimums“ (BVerfGE 82, 60 [80]). Vgl. Krahmer, ZfF 2004, S. 179-181; Luthe/Dittmar, SGb 2004, S. 272 ff; Bieback, NZS 2005, S. 337-339; Däubler, NZS 2005, S. 225 ff; Könemann, Der verfassungsunmittelbare Anspruch auf das Existenzminimum, 2005, S. $181 \mathrm{ff} ; O$ ckenga, ZfSH/SGB 2006, S. 143 ff; Rothkegel, SGb 2006, S. 76; Hebeler, SGb 2008, S. 8 ff; zusammenfassend Becker, Sozialmodell und Menschenbild in der „Hartz-IV” Gesetzgebung, in: Behrends, Gesetzgebung, Menschenbild und Sozialmodell im Familien- und Sozialrecht, 2008, S. 6163. Zur Frage, ob höhere Regelleistungen im Klageweg zu erwarten sind, vgl. Sartorius, info also 2005, S. 56-58. Die Verfassungsmäßigkeit bestätigend u.a. BayLSG, Urteil v. 18.5.2006, L 11 AS 111/05; BSG, Urteil v. 23.11.2006, B 11b AS 1/06 R; BSG, Beschluss v. 16.5.2007, B 11b AS 71/06 B; BSG, Urteil v. 22.4.2008, B 1 KR 10/07 R; BSG, Beschluss v. 16.12.2008, B 4 AS 69/08 B; BVerfG, Beschluss v. 7.11.2007 1 BvR 1840/07. Unentschieden ist die Frage der Höhe des Sozialgeldes für Kinder und Jugendliche, vgl. BSG, Vorlagebeschluss zum BVerfG v. 27.1.2009, B 14/11b AS 9/07 R. 
Änderung des Zweiten Sozialgesetzbuchs wurde diese Unterscheidung zugunsten einer bundeseinheitlichen Zahlung eines Arbeitslosengeldes II in Höhe von 345 Euro als Regelleistung aufgegeben. ${ }^{546}$ Diese Leistung wird jeweils zum ersten Juli eines Jahres um den Vomhundertsatz angepasst, um den sich der aktuelle Rentenwert in der gesetzlichen Rentenversicherung verändert. ${ }^{547} \mathrm{Ab}$ 1. Juli 2009 beträgt die Leistung 359 Euro. 548

Daneben gibt es Leistungen für Mehrbedarfe beim Lebensunterhalt gem. § 21 SGB II, die entsprechend den Berechnungen des Gesetzgebers nicht durch die Regelleistung abgedeckt sind, z.B. erhalten werdende Mütter nach der 12. Schwangerschaftswoche einen Mehrbedarf von 17 vom Hundert der Regelleistung.

Die Leistungen für Unterkunft und Heizung sind in § 22 SGB II geregelt. Sie werden in Höhe der tatsächlichen Aufwendungen erbracht, soweit diese angemessen sind. 549

Gem. § 23 SGB II kann ein Darlehen gewährt werden, mit dem unabweisbare Bedarfe, die nicht mit dem Regelsatz oder vorhandenem Vermögen gedeckt werden konnten, finanziert werden. Es ist grundsätzlich mit der in der Regelleistung enthaltenen Sparrate zurückzuzahlen. ${ }^{550}$

\section{Definition der Eingliederungsvereinbarung und Abgrenzung}

\section{a) Begriff}

$\S 15$ Abs. 1 S. 1 SGB II enthält eine Legaldefinition der Eingliederungsvereinbarung. Die Agentur für Arbeit soll im Einvernehmen mit dem kommunalen Träger mit jedem erwerbsfähigen Hilfebedürftigen die für seine Eingliederung erforderlichen Leistungen vereinbaren.

546 Erstes Gesetz zur Änderung des Zweiten Sozialgesetzbuchs v. 22.12.2005 (BGB1.I, S. 3675), in Kraft seit 1.1.2006.

547 Dazu kritisch Becker, Sozialmodell und Menschenbild in der „Hartz-IV” Gesetzgebung, in: Behrends, Gesetzgebung, Menschenbild und Sozialmodell im Familien- und Sozialrecht, 2008, S. 63. $\mathrm{Zu}$ einem Alternativvorschlag für einen spezifischen Preisindex zur Anpassung der Regelleistung vgl. Martens, SozSich 2008, S. 68 ff.

548 Verkündet am 17.6.2009 (BGBl. I, S. 1342).

549 Der Begriff der „Angemessenheit der Unterkunftskosten“ ist als unbestimmter Rechtsbegriff uneingeschränkt gerichtlich überprüfbar und beschäftigt bereits intensiv die Gerichte und wegen der Bundesbeteiligung an den Leistungen für Unterkunft und Heizung gem. § 46 SGB II den Bundesrechnungshof. Die „Angemessenheit" wird bestimmt vom anspruchslimitierenden Bedarfsdeckungsprinzip und ist u.a. in Bezug zu setzen zum örtlichen Wohnungsmarkt. Dazu allgemein Rothkegel, in: Rothkegel, Sozialhilferecht, 2005, S. 264 ff; Kofner, WuM 2007, S. 310f; überblicksmäßig Groth/Siebel-Huffmann, NZS 2007, S. 69 ff unter Berücksichtigung der jüngsten höchstrichterlichen Rechtsprechung des Bundessozialgerichtes, Urteil v. 7.11.2006, SozR 4-4200 § 22 Nr. 1; aktuell Wahrendorf, SozR akt 2009, S. 3 f.

550 Vgl. dazu kritisch Krahmer, ZfF 2004, S. 180 ff; Däubler, NJW 2005, S. 1545 ff; Ockenga, ZFSH/SGB 2006, S. 146 f; Armborst, info also 2006, S. 58 ff; Becker, Sozialmodell und Menschenbild in der „Hartz-IV” Gesetzgebung, in: Behrends, Gesetzgebung, Menschenbild und Sozialmodell im Familien- und Sozialrecht, 2008, S. 65. 
Die Eingliederungsvereinbarung nach SGB II findet im arbeitsförderungsrechtlichen $\S 35$ Abs. 4 SGB III ihr Vorbild und hat nicht nur denselben Namen. ${ }^{551}$ Sie weist aber auch grundsätzliche Unterschiede zur Vereinbarung nach SGB III auf und ist deshalb gesondert darzustellen.

b) Parteien der Vereinbarung und Einvernehmen des kommunalen Trägers

Parteien der Vereinbarung sind die Agentur für Arbeit und der sog. erwerbsfähige Hilfebedürftige.

\section{aa) Fallmanager als Bevollmächtigter der Agentur für Arbeit}

Die Agentur für Arbeit ist Partei der Vereinbarung auf Seiten der (Arbeits-)Verwaltung. Sie handelt durch ihre Mitarbeiter, die insoweit ihre Bevollmächtigten sind. 552

Hat die Kommune gem. §6a SGB II optiert, handelt es sich um einen Mitarbeiter des kommunalen Trägers, der gem. § 6b Abs. 1 S. 2 SGB II - soweit seine Zulassung reicht - die Rechte und Pflichten des Fallmanagers der Agentur für Arbeit hat. Durch die Vereinbarung wird dann die Kommune, die an Stelle der Bundesagentur für Arbeit als deren Organ handelt, berechtigt und verpflichtet. ${ }^{553}$ Handelt eine Arbeitsgemeinschaft gem. § 44b SGB II, nimmt sie die Aufgaben der Agentur für Arbeit wahr, § 44b Abs. 3 S. 1 SGB II. ${ }^{554}$ Zur Vereinfachung wird in der Arbeit trotzdem vom „Mitarbeiter der Agentur für Arbeit" gesprochen.

Dieser Mitarbeiter der Agentur für Arbeit ist in der Regel der sog. „Fallmanager“555, ein Begriff, der im Gesetzestext nicht auftaucht, der aber beschreibt, was in $\S 4$ Abs. 1 Nr. 1, § 14 S. 2 SGB II gemeint ist, wenn es heißt, dass die Agentur für Arbeit für jeden erwerbsfähigen Hilfebedürftigen einen persönlichen Ansprechpartner benennen soll. Diese Benennungspflicht dient der möglichst schnellen und erfolgreichen Förderung in jedem Einzelfall, weil der Fallmanager die Bedürfnisse des jeweiligen Hilfebedürftigen aufgrund des durch ihn durchgeführten Profilings besser kennenlernen kann. Der vom Gesetzgeber anvisierte Betreuungsschlüssel ${ }^{556}$ bei zu betreuenden Hilfebedürftigen zwischen 15 und 25 Jahren von 1:75 war im Bundesdurchschnitt im Jahr 2005 fast erreicht (1:77), inzwischen hat er sich wieder verschlechtert und liegt bei 1:87. Unwesentlich anders hat sich die Situation im Erwachsenenbereich entwickelt, wo ein Schlüssel von 1:150 geplant war, der derzeit bei 1:174 liegt (Ausgangslage Anfang 2005 von 1:214). Erreicht ist lediglich der anvisierte Betreuungsschlüssel bei der Leistungsgewährung in den Bedarfsgemeinschaften. Dort wollte man ein Verhältnis von 1:140 herstellen. Im Jahr 2007 hatte ein Betreuer „nur“ 125 Bedarfsgemeinschaften zu versor-

551 Löschau/Marschner, Hartz IV, 2004, Rn. 414; Brühl/Hofmann, Sozialgesetzbuch Zweites Buch, 2004, S. 102.

552 Stark, in: Estelmann, SGB II, § 15 Rn. 39.

553 Stark, in: Estelmann, SGB II, § 15 Rn. 39; Schumacher, in: Oestreicher, SGB XII/SGB II, § 15 Rn. $16 \mathrm{f}$.

554 Vgl. zweites Kapitel B II 1b) S. 183 f.

555 Löschau, in: Estelmann, SGB II, § 14 Rn. 4, 15 ff; Müller, in: Hauck/Noftz, SGB II, K § 14 Rn. 8.

556 BT-Drucksache 15/1516, S. 2. 
gen. ${ }^{557}$ Mit Hilfe der Festlegung von Betreuungsschlüsseln soll nach dem Willen des Gesetzgebers ein effektiveres Erbringen der Eingliederungsleistungen nach dem SGB II unterstützt werden. 558

\section{bb) Erwerbsfähiger Hilfebedürftiger}

Auf der anderen Seite der Vereinbarung steht der erwerbsfähige Hilfebedürftige. 559 Wer erwerbsfähiger Hilfebedürftiger ist, ist eigens für das SGB II definiert. $§ 7$ Abs. 1 S. 1 Nr. 1-4 SGB II legen fest, dass der Arbeitsuchende im Alter zwischen 15 und 65 Jahren, erwerbsfähig und hilfebedürftig sein muss. Voraussetzung ist ferner ein gewöhnlicher Aufenthalt 560 in der Bundesrepublik. 561

Ausgeschlossen ist in der Regel, wer für voraussichtlich weniger als 6 Monate stationär untergebracht ist (ein gerichtlich angeordneter Freiheitsentzug ist gleichgestellt) oder Renten wegen Alters oder ähnliche Leistungen öffentlich-rechtlicher Art bezieht, § 7 Abs. 4 SGB II. Auch ausgeschlossen sind grundsätzlich Personen, die sich in Schuloder Hochschulausbildung befinden, § 7 Abs. 5, 6 SGB II ${ }^{562}$ und die sich ohne Zustimmung ihres persönlichen Ansprechpartners außerhalb des in der Erreichbarkeitsanordnung definierten Bereichs aufhalten, $\S 7$ Abs. 4a SGB II. ${ }^{563}$

In $\S 8$ Abs. 1 SGB II ist der Begriff der Erwerbsfähigkeit definiert. Erwerbsfähig ist demnach, wer nicht wegen Krankheit oder Behinderung auf absehbare Zeit außerstande ist, unter den üblichen Bedingungen des Arbeitsmarktes mindestens drei Stunden täglich erwerbstätig zu sein. Dieses Kriterium ist angelehnt an die Definition einer vollen Erwerbsminderung gem. $\S 43$ Abs. 2 S. 2 SGB VI, $\S 41$ Abs. 1 Nr. 2 SGB XII. 564

557 Bundesagentur für Arbeit, Jahresbericht 2007, SGB II, 2007, S. 69. In diesem Bericht wird angemerkt, dass die im Jahr 2005 festgelegten Kundendefinitionen derzeit von Bundesministerium für Arbeit und Soziales und der Bundesagentur für Arbeit überarbeitet werden.

558 Löschau, in: Estelmann, SGB II, § 14 Rn. 15 ff.

559 Es gelten die allgemeinen Voraussetzungen der Verfahrensfähigkeit gem. § 11 SGB X, sodass die Handlungen von minderjährigen Hilfebedürftigen nur bei entsprechend wirksamer Vertretung wirksam sind. Die Handlungsfähigkeit zur Beantragung und Entgegennahme von Sozialleistungen, die gem. § 36 SGB I mit Vollendung des fünfzehnten Lebensjahres gegeben ist, kann wegen des Wortlauts der Vorschrift nicht auf den Abschluss von Verträgen ausgedehnt werden. Dazu Schumacher, in: Oestreicher, SGB XII/SGB II, § 15 Rn. 17.

560 Seit 1.8.2006 legt $\S 36$ S. 3 SGB II fest, dass bei nicht feststellbarem gewöhnlichen Aufenthalt auf den tatsächlichen Aufenthaltsort abzustellen ist. Vgl. auch BSG, Urteil v. 16.12.2008, B 4 AS 1/08 R. Zur Frage der Definition des gewöhnlichen Wohnsitzes bei drittstaatsangehörigen Ausländern (vgl. BT-Drucksache 15/1516, S. 52) Sieveking, ZAR 2004, S. 283 ff; Geiger, Info AuslR 2004, S. 360 f; Strick, NJW 2005, S. 2182 ff; Fuchs, NZS 2007, S. 1 ff.

561 Ausländerrechtliche Fragen, insbesondere zum Leistungsausschluss gem. § 7 Abs. 1 S. 2 SGB II werden nicht behandelt. Dazu im Detail Schumacher, in: Oestreicher, SGB XII/SGB II, § 7 Rn. 11 ff.

562 Dazu Schumacher, in: Oestreicher, SGB XII/SGB II, § 7 Rn. 31 ff.

563 Eingeführt mit Wirkung zum 1.8.2006 durch Gesetz vom 20.7.2006 (BGBl. I, S. 1706). Dazu Winkler, info also 2007, S. 3 ff.

564 Dazu Rixen, info also 2006, S. 153. Problematisch sind verfahrensrechtliche Regelungen zur Feststellung der Erwerbsfähigkeit auf der Grundlage der § 44a SGB II und § 45 SGB XII und damit zusammenhängende gesetzlich angeordnete Bindungswirkungen an Entscheidungen der zuständigen Stellen. Dazu Schoch, NDV 2006, S. 512 ff, 543 ff. 
Hilfebedürftig ist gem. § 9 Abs. 1 SGB II, wer seinen Lebensunterhalt, seine Eingliederung in Arbeit und den Lebensunterhalt der mit ihm in einer Bedarfsgemeinschaft lebenden Personen nicht oder nicht ausreichend aus eigenen Kräften und Mitteln, vor allem aber nicht durch Aufnahme einer zumutbaren ${ }^{565}$ Arbeit und dem zu berücksichtigenden Einkommen oder Vermögen sichern kann und die erforderliche Hilfe nicht von anderen, insbesondere Angehörigen oder von Trägern anderer Sozialleistungen erhält.

Auf Seiten des Leistungsberechtigten erfolgt keine Anknüpfung an abhängige Beschäftigung in einem Angestelltenverhältnis, sodass auch (gescheiterte oder ,in den Startlöchern befindliche“) Selbständige Berechtigte sind. Andererseits gibt es keine $\S 17$ SGB III entsprechende Vorschrift, die bei drohender Hilfebedürftigkeit präventiv Hilfeleistungen vorsehen würde. 566

\section{cc) Einvernehmen des kommunalen Trägers, § 15 Abs. 1 S. 1 SGB II}

Gem. § 15 Abs. 1 S. 1 SGB II soll die Eingliederungsvereinbarung im Einvernehmen mit dem kommunalen Träger abgeschlossen werden. Erst mit dem Kommunalen Optionsgesetz ${ }^{567}$ wurde das Mitwirkungsrecht der kommunalen Träger in den Wortlaut des $\S 15$ Abs. 1 S. 1 SGB II eingefügt. Die rechtliche Betroffenheit entstand durch die Regelungen über die Zuständigkeit für die flankierenden Eingliederungsleistungen gem. § 16 Abs. 2 S. 2 Nr. 1-4 SGB II. ${ }^{568}$ Dadurch sollen die Rechte des vom Inhalt der Eingliederungsvereinbarung betroffenen kommunalen Trägers gesichert werden. ${ }^{569} \mathrm{Im}$ Übrigen tragen die kommunalen Träger die Kosten der Unterkunft und Heizung und haben deshalb ein großes finanzielles Interesse an einer realistischen Eingliederungsstrategie, die den Einzelnen wieder unabhängig von Leistungen der Grundsicherung für Arbeitsuchende macht. 570

Für die Praxis der getrennten Aufgabenwahrnehmung von Bundesagentur für Arbeit und kommunalem Träger wird, um eine einfache Handhabung der Mitwirkung zu erzielen, vorgeschlagen, die Eingliederungsvereinbarung während eines gemeinsamen Termins zwischen Arbeitsuchendem, Fallmanager und dem Vertreter der Kommune abzuschließen und schriftlich niederzulegen. ${ }^{571} \mathrm{Ob}$ dies z.B. aufgrund möglicher terminlicher Engpässe tatsächlich eine einfachere Handhabung erlaubt, muss hier dahinstehen.

565 Zum Begriff der Zumutbarkeit Kothe, SozSich 2005, S. 146 ff.

566 Zu den Folgen der Einbeziehung Selbständiger Knoblauch/Hübner, NDV 2006, S. 375 ff.

567 Gesetz v. 30.7 .2004 (BGB1. I, S. 2014).

568 Vgl. den Wortlaut der entsprechenden BT-Drucksache 15/2816, S. 12: „, Da die kommunalen Träger neben der Zuständigkeit für die Kosten der Unterkunft und Heizung auch für die Eingliederungsleistungen nach $\S 16$ Abs. 2 S. 2 Nr. 1-4 SGB II zuständig sind, ist es sinnvoll, dass die von der Agentur für Arbeit abzuschließende Eingliederungsvereinbarung im Einvernehmen mit dem kommunalen Träger erfolgt.“

569 Rixen, in: Eicher/Spellbrink, SGB II, § 15 Rn. 5 spricht deshalb von einem „kostenbegrenzenden Vetorecht" der kommunalen Träger.

570 Vgl. zweites Kapitel B II 8b) S. 231 ff.

571 Stark, in: Estelmann, SGB II, § 15 Rn. 112 sieht dies als Möglichkeit an, durch die Anwesenheit einer dritten Person dem Vorwurf zu begegnen, es finde keine ausreichende (rechtsstaatliche) Kontrolle der Verhandlungssituation beim Abschluss der Eingliederungsvereinbarung statt. Dieses Ar- 
In einer Arbeitsgemeinschaft im Sinn des $\S 44 b$ SGB II entfällt das gesondert für jeden Einzelfall einzuholende Einvernehmen, wenn der kommunale Träger diese Aufgabe auf die Arbeitsgemeinschaft übertragen hat, $\S 44 \mathrm{~b}$ Abs. 3 S. 2 SGB II. ${ }^{572}$ In einer Optionskommune gem. § 6a Abs. 1 S. 1 SGB II ist ein eigenständiges Vetorecht ebenfalls nicht erforderlich. Die Kommune handelt an Stelle der Agenturen für Arbeit und kann dabei die eigenen Interessen wahren. 573

\section{c) Bedarfsgemeinschaft, $§ 7$ Abs. 3 SGB II}

Besonders hervorzuheben ist, dass gem. § 15 Abs. 2 SGB II zwischen dem erwerbsfähigen Hilfebedürftigen und der Agentur für Arbeit vereinbart werden kann, welche (Eingliederungs-)Leistungen eine Person erhalten soll, die mit dem Hilfebedürftigen in einer Bedarfsgemeinschaft lebt. ${ }^{574}$ In $\S 15$ Abs. 2 S. 2 SGB II ist geregelt ${ }^{575}$, dass diese Personen zu beteiligen sind. Wie die Beteiligung aussehen soll, lässt der Gesetzgeber offen.

Die Inhalte werden mit dem erwerbsfähigen Hilfebedürftigen vereinbart, die anderen Personen werden nicht automatisch Vertragspartner der Eingliederungsvereinbarung. Dass der Hilfebedürftige seine Bedarfsgemeinschaft bei der Beantragung und Entgegennahme der Leistungen auf der Grundlage des $\S 38$ S. 1 SGB II vertritt, kann wegen des Wortlauts „bei Entgegennahme und Beantragung von Leistungen“ nicht angenommen werden, denn es handelte sich um eine Vertretung bei einem Vertragschluss, in dem die Eingliederungsstrategie vereinbart wird. Deshalb geht die Bundesagentur für Arbeit davon aus, dass für jeden erwerbsfähigen Hilfebedürftigen gesondert eine Eingliederungsvereinbarung abgeschlossen werden sollte. ${ }^{576}$ Die bloße Beteiligung an dem Gespräch dürfte nicht ausreichend sein. ${ }^{577}$ Erforderlich ist vielmehr ein echtes Mitwirkungsrecht auf der Grundlage des $\S 57$ Abs. 1 SGB X. 578

gument läuft aber in einer Arbeitsgemeinschaft leer, da ein Mitarbeiter der Arbeitsgemeinschaft beide Leistungsträger in Personalunion vertritt.

572 So auch Berlit, in: Münder/Armborst, LPK-SGB II, 2005, § 15 Rn. 10.

573 Berlit, SozR akt 2006, S. 43.

574 Zur Bedarfsgemeinschaft gehören gem. § 7 Abs. 3 SGB II vereinfacht formuliert der Hilfebedürftige, seine Eltern, sofern der Hilfebedürftige noch minderjährig ist und sie in einem Haushalt leben, sein Ehegatte oder Lebenspartner, der Partner einer eheähnlichen Lebensgemeinschaft und die minderjährigen, dem Haushalt angehörenden eigenen Kinder oder Kinder des Ehegatten oder des Lebenspartners, es sei denn sie bestreiten ihren Lebensunterhalt aus eigenem Einkommen oder Vermögen, z.B. Kindesunterhalt. Kritisch äußerte sich Spellbrink, NZS 2007, S. 121 ff zur Bedarfsgemeinschaft und bezeichnete sie aufgrund vor allem verfahrensrechtlicher Lücken als „Fehlkonstruktion“. Ähnlich Becker, Sozialmodell und Menschenbild in der „Hartz-IV” Gesetzgebung, in: Behrends, Gesetzgebung, Menschenbild und Sozialmodell im Familien- und Sozialrecht, 2008, S. 50; Labrenz, ZfF 2008, S. 217 ff.

575 Diese Regelung fand erst mit Verabschiedung des Kommunalen Optionsgesetzes (BT-Drucksache, $15 / 2816$ S. 12) Eingang in $\S 15$ SGB II. Es soll sich dabei um eine „Klarstellung“ handeln.

576 So für die Bundesagentur für Arbeit Kocialkowski, SozR akt 2006, S. 39.

577 Dazu Löschau/Marschner, Hartz IV, 2004, Rn. 417; Stark, in: Estelmann, SGB II, § 15 Rn. 197.

578 So Berlit, SozR akt 2006 S. 43. 
d) Abgrenzung zur Vereinbarung gem. § 17 Abs. 2 BSHG und zur Leistungsabrede gem. § 12 SGB XII

\section{aa) Bundessozialhilfegesetz (BSHG)}

Während der Geltung des BSHG gab es für den Sozialhilfeträger und den Hilfebedürftigen die Möglichkeit, eine Vereinbarung gem. § 17 Abs. 2 BSHG abzuschließen. Regelungskontext war die Hilfe zum Lebensunterhalt und der Anspruch des Hilfebedürftigen auf Beratung und Unterstützung durch den Sozialhilfeträger, § 17 Abs. 1 BSHG (sog. Auswegberatung 579 ).

Diese Vereinbarung sollte die Verbindlichkeit der jeweiligen Absprachen unterstreichen, entfaltete aber normalerweise keine rechtliche Bindungswirkung. Ihre Bedeutung wurde deshalb, insbesondere wenn keine finanzielle Verpflichtung für den Hilfebedürftigen vorgesehen war, als gering eingestuft und in den Bereich informeller Vereinbarungen verwiesen. Es war anerkannt, dass diese Vereinbarung lediglich pädagogische/therapeutische Wirkung im Hilfeprozess entfalten konnte. Es bestand aber stets die Möglichkeit, eine solche Vereinbarung als verwaltungsrechtlichen Vertrag gem. $\S \S 53$ ff SGB X auszugestalten und ihr damit eine größere Wirkungskraft zu geben. ${ }^{580}$ Ein Verbot der vertraglichen Handlungsform ist weder dem Gesetz noch der Gesetzesbegründung zu entnehmen. ${ }^{581}$ Trotzdem waren die Fälle solcher Verträge rar. ${ }^{582}$

Wichtig ist festzuhalten, dass es an anderer Stelle des BSHG keine weitere Bezugnahme auf diese Vereinbarungen gab, insbesondere keine Abschlussverpflichtung für den Hilfebedürftigen oder eine Sanktion bei fehlender Mitwirkung, sodass die Ablehnung eines solchen Vertrages nicht als fehlende Mitwirkung gem. § 66 SGB I verstanden werden konnte. ${ }^{583}$ War die Vereinbarung als verwaltungsrechtlicher Vertrag geschlossen worden, bestanden aber die Möglichkeiten, die das öffentliche Vertragsrecht vorsieht, um die vereinbarten Rechte und Pflichten durchzusetzen.

Die besonderen Mitwirkungspflichten, die im Rahmen der Hilfe zur Arbeit gem. § 18 BSHG bestanden und bei Nichterfüllung über $\S 25$ BSHG zum Wegfall des Anspruchs auf Hilfe zum Lebensunterhalt führten, basierten nicht auf einer Vereinbarung, sondern auf dem Gesetz. 584 Diese Konstruktion kann deshalb nicht als Vorläufer der Eingliede-

579 Spindler, NDV 2002, S. 361.

580 Widersprüchlich ist die Formulierung im Gesetzesentwurf BT-Drucksache 13/2440, S. 20: „Außerhalb von Rechtsansprüchen wird eine größere Verlässlichkeit zwischen dem Träger der Sozialhilfe und dem Hilfeempfänger hergestellt.“

581 Bieback, GMH 2003, S. 26 votiert sogar dafür, diese Vereinbarungen stets als verwaltungsrechtliche Verträge anzusehen.

582 Im Einzelnen dazu Spellbrink, SozR akt 2006, S. 53.

583 Wenzel, in: Fichtner, Bundessozialhilfegesetz, 2003, § 17 Rn. 9.

584 Spindler, Aktivierende Ansätze in der Sozialhilfe, in: Dahme u.a., Soziale Arbeit für den aktivierenden Staat, 2003, S. 231. 
rungsvereinbarung betrachtet werden 585 , auch wenn inhaltlich genau diese Pflichten heute Gegenstand der Eingliederungsvereinbarung sind. ${ }^{586}$

bb) Zwölftes Buch Sozialgesetzbuch (SGB XII)

Nach Eingliederung des Sozialhilferechts in das Zwölfte Buch Sozialgesetzbuch findet sich eine Vereinbarung in $\S 12$ SGB XII, die Leistungsabrede. Der Gesetzgeber hat gleichzeitig ausdrücklich ausgeschlossen, den weiteren Sozialhilfebezug im Wege eines verwaltungsrechtlichen Vertrages zu vereinbaren. Deshalb ist die Leistungsabrede zwar das Nachfolgeinstrument zu $\S 17$ BSHG, kann aber nicht mehr zu einem gerichtlich durchsetzbaren Vertrag über die in § 11 SGB XII etablierten Beratungs- und Unterstützungspflichten führen. ${ }^{587}$ Die Leistungs“abrede" ist vielmehr als vertragsähnliches Verwaltungshandeln zu bezeichnen. 588

\section{Inhalt der Eingliederungsvereinbarung}

a) Niederlegung ,verbindlicher Aussagen zum Fördern und Fordern“

Gem. $\S 15$ Abs. 1 S. 1 SGB II vereinbart die Agentur für Arbeit im Einvernehmen mit dem kommunalen Träger mit dem erwerbsfähigen Hilfebedürftigen die für seine Eingliederung erforderlichen Leistungen. In der Eingliederungsvereinbarung wird damit, so die Gesetzesbegründung, das Sozialrechtsverhältnis zwischen dem Erwerbsfähigen und der Agentur für Arbeit konkretisiert. Sie enthält verbindliche Aussagen zum Fördern und Fordern. 589

Diese allgemeinen Sätze werden in $\S 15$ Abs. 1 S. 2 Nr. 1-2 SGB II konkretisiert. Die Eingliederungsvereinbarung soll insbesondere bestimmen, welche Leistungen der erwerbsfähige Hilfebedürftige zur Eingliederung in Arbeit erhält, welche Bemühungen zur Eingliederung in Arbeit er in welcher Häufigkeit mindestens unternehmen muss und in welcher Form er diese nachzuweisen hat. Damit wird in einer nicht abschließenden Aufzählung (,insbesondere“) beschrieben, was jedenfalls Gegenstand der Eingliederungsvereinbarung sein soll. 590

b) Leistungen zur Eingliederung, $\S 15$ Abs. 1 S. 2 Nr. 1 , $\S 16$ ff SGB II

Gegenstand der Eingliederungsvereinbarung können dem Wortlaut des § 15 SGB II folgend nur Leistungen zur Eingliederung gem. $\S \S 14$ ff SGB II sein, nicht Leistungen zur Sicherung des Lebensunterhaltes, $§ 19$ ff SGB II.

585 So aber Lehmann-Franßen, NZS 2005, S. 520.

586 Fuchs, Hilfe zur Arbeit als Integrationsprozess, in: Fuchs/Schulze-Böing, Hilfe zur Arbeit und kommunale Beschäftigungspolitik, 1999, S. 87 ff.

587 Roscher, in: Münder, LPK-SGB XII, § 12 Rn. 3; Spellbrink, SozR akt 2006, S. 52 ff

588 Vgl. dazu im Einzelnen erstes Kapitel B V 3c) S. 69.

589 BT-Drucksache 15/1516, S. 54.

590 Luthe/Dittmar, Das neue Fürsorgerecht in der Praxis, 2005, S. 281. 
Die Formulierung in $\S 15$ Abs. 1 S. 1 SGB II ,erforderliche Leistungen zur Eingliederung" bedeutet sowohl eine wesentliche Inhaltsbeschränkung als auch eine Inhaltskontrolle. Der Gesetzgeber ordnet eine Prognoseentscheidung der Arbeitsverwaltung an und bindet sie dadurch in ihren Handlungsmöglichkeiten. Der Begriff ,erforderlich“ hat zwei Facetten: Zum einen darf die Agentur für Arbeit nichts „Unmögliches, Übertriebenes oder sonst Ungeeignetes“ vom Hilfebedürftigen erwarten. Zum anderen legt der Begriff „erforderlich“ für den Arbeitsuchenden fest, dass dieser die erforderlichen Bemühungen erbringen muss und jedenfalls nicht weniger Aufwand bei der Arbeitsuche betreiben darf. 591

aa) § 16 Abs. 1 SGB II

Der Inhalt der Eingliederungsvereinbarung nach SGB II ist - bezogen auf die erforderlichen Leistungen zur Eingliederung - von einer weitgehenden Bezugnahme auf das Arbeitsförderungsrecht geprägt. ${ }^{592} \mathrm{Gem} . \S 16$ Abs. 1 SGB II kann die Agentur für Arbeit den Großteil der Instrumente der aktiven Arbeitsförderung nach SGB III anwenden. 593

Im Unterschied zu den Regelungen im SGB III handelt es sich bei den Eingliederungsleistungen nach $\S 16$ Abs. 1 S. 1 SGB II ausschließlich um Ermessensleistungen, auch wenn es sich im SGB III um Pflichtleistungen handelt. ${ }^{594}$ Das Ermessen bezieht sich nicht nur auf die Auswahl des geeigneten Instrumentes, sondern auch auf das Entscheidungsermessen und damit die Frage, ob überhaupt eine Leistung zur (Wieder-)Eingliederung erbracht wird. 595

Bei der Ausfüllung des eben beschriebenen Ermessensspielraums sind die Leistungsgrundsätze des $\S 3$ SGB II zu beachten.

Hervorzuheben ist, dass gegenüber erwerbsfähigen Hilfebedürftigen, die das 25. Lebensjahr noch nicht erreicht haben, die Verpflichtung besteht, diese unverzüglich nach Antragstellung auf Leistungen nach dem SGB II in eine Arbeit, eine Ausbildung oder eine Arbeitsgelegenheit zu vermitteln, $\S 3$ Abs. 2 S. 1, $§ 16$ Abs. 1 S. 1 SGB II i.V.m. $\S \S 35$ ff SGB III. Die anderen Eingliederungsleistungen, wie z.B. Teilnahme an Trainingsmaßnahme gem. § 48 SGB III i.V.m. § 16 Abs. 1 S. 1 SGB II, sind auch bei Hilfebedürftigen unter 25 Jahren Ermessensleistungen. 596

Zusätzlich zu den Leistungsvoraussetzungen des SGB II sieht $§ 16$ Abs. 1 S. 3 SGB II vor, dass die jeweiligen Voraussetzungen der Leistungen nach SGB III erfüllt sind, soweit das SGB II nicht abweichende Voraussetzungen enthält. Es handelt sich deshalb bei den Verweisungen in $\S 16$ Abs. 1 SGB II um Rechtsgrundverweisungen, soweit das

591 Stark, in: Estelmann, SGB II, § 15 Rn. 61.

592 Spellbrink, JZ 2004, S. 542 kritisiert diese Tatsache und begründet damit eine Entwertung der Beiträge zur Arbeitslosenversicherung.

593 Eine ausführliche Darstellung der zur Verfügung stehenden Leistungen enthält die Übersicht bei Voelzke, in: Hauck/Noftz, SGB II, K § 16 Rn. 11.

594 Stark, in: Estelmann, SGB II, § 16 Rn. 68.

595 Voelzke, in: Hauck/Noftz, SGB II, K § 16 Rn. 12; Luthe, in: Hauck/Noftz, SGB II, K 33 Rn. 34.

596 Zur Problematik der Schnittstellen zur Jugendhilfe Kunkel, ZfSH/SGB 2006, S. 76 ff. 
SGB II nicht selbst Voraussetzungen regelt. ${ }^{597}$ Auch die Erfüllung dieser Voraussetzungen des SGB III ist bei der Vereinbarung einer Leistung im Wege einer Eingliederungsvereinbarung nach SGB II zu berücksichtigen, beziehungsweise vor deren Vereinbarung durch die Agentur für Arbeit zu prüfen. Dies dürfte die inhaltliche Gestaltung der konkreten Eingliederungsvereinbarung wesentlich verkomplizieren. ${ }^{598}$

\section{bb) § 16 Abs. $2 S G B I I$}

Mittels einer Generalklausel in $\S 16$ Abs. 2 S. 1 SGB II und „Hauptbeispielen“599 in $\S 16$ Abs. 2 S. 2 SGB II wird der Katalog der Eingliederungsleistungen mit dem Ziel erweitert, eine möglichst flexible Gestaltung der Eingliederung zu erreichen. Daraus ergeben sich im Zusammenspiel mit dem SGB III verschiedenste Möglichkeiten, die Eingliederung erwerbsfähiger Hilfebedürftiger auch über das bekannte Eingliederungsinstrumentarium des SGB III hinaus zu unterstützen.

Wichtig für das Verständnis der Generalklausel ist das sog. Aufstockungsverbot in $\S$ 16 Abs. 2 S. 1 zweiter Halbsatz SGB II, wonach die Leistungen nach § 16 Abs. 1 SGB II nicht durch sonstige Leistungen aufgestockt werden dürfen. 600

Ausdrücklich genannt sind in $\S 16$ Abs. 2 S. 2 SGB II die Betreuung minderjähriger oder behinderter Kinder oder die häusliche Pflege von Angehörigen, die Schuldnerberatung, die psychosoziale Betreuung, die Suchtberatung, das Einstiegsgeld nach § 29 SGB II 601 und Leistungen zur Beschäftigungsförderung nach § 16a SGB II. ${ }^{602}$ Zur Frage der Zuständigkeit über die Erbringung der jeweiligen Leistung kann auf die Ausführungen zum Kommunalen Optionsgesetz verwiesen werden. ${ }^{603}$

Inwieweit die kommunalen Träger der Grundsicherung für Arbeitsuchende die sog. flankierenden Eingliederungsleistungen ausführen, wird Gegenstand der Evaluierungen der Instrumente des SGB II sein.

cc) § 16 Abs. $3 S G B I I$

Für erwerbsfähige Hilfebedürftige, die keine Arbeit finden können, sollen Arbeitsgelegenheiten geschaffen werden. Vor Abschluss der Eingliederungsvereinbarung ist festzustellen und darüber zu verhandeln, welche Tätigkeit die Eingliederungschance des Hilfebedürftigen verbessern kann.

597 Zunächst war dies strittig. Von einer Rechtsgrundverweisung ging Stark, in: Estelmann, SGB II, § 16 Rn. 38 aus, von einer Rechtsfolgenverweisung Spellbrink, JZ 2004, S. 542; Lehmann-Franßen, NZS 2005, S. 522.

598 Voelzke, in: Hauck/Noftz, SGB II, K § 16 Rn. 19.

599 BT-Drucksache 15/1516, S. 54.

600 Dazu Schumacher, in: Oestreicher, SGB XII/SGB II, § 16 Rn. 51 ff.

601 Vgl. wegen der Unbestimmtheit der Voraussetzungen und der Ungebundenheit des Fallmanagers die Kritik bei Spellbrink, NZS 2005, S. 231.

602 Definitionen der entsprechenden Leistungen sowie weiterführende Hinweise z.B. bei Voelzke, in: Hauck/Noftz, SGB II, K $§ 16$ Rn. 35-48; Stark, in: Estelmann, SGB II, § 16 Rn. 46 ff; Schumacher, in: Oestreicher, SGB XII/SGB II, § 16a n.F..

603 Vgl. zweites Kapitel B II 1b) S. 183 f. 
Die Arbeitsgelegenheiten, v.a. in der Variante der Mehraufwandsentschädigung gem. $\S 16$ Abs. 3 S. 2 SGB II (,Zusatz-Jobs“), sind in der Praxis, aber auch in der wissenschaftlichen Diskussion umstritten. Der Begriff der ,im öffentlichen Interesse liegenden, zusätzlichen Arbeit" ist zwar theoretisch, nicht aber in der Praxis geklärt und verleitet zu rechtswidriger Anwendung. ${ }^{604}$ Dient nämlich die konkrete angebotene Arbeitsgelegenheit nicht der Verbesserung der Eingliederungschancen des Hilfebedürftigen, und führt die Tätigkeit lediglich zur Einsparung regulärer, zur Erfüllung öffentlicher Aufgaben gehörender Arbeiten, ist die Heranziehung des Hilfebedürftigen auch damit nicht zu rechtfertigen, dass der Einsatz der Arbeitskraft der Allgemeinheit auf Grund des „Solidaritäts- beziehungsweise Gegenseitigkeitsprinzips geschuldet ist“ ${ }^{\text {“ }} 605$ $\mathrm{Zu}$ einem ernüchternden Ergebnis kam der Bericht des Bundesrechnungshofes, der 25 Prozent der Maßnahmen als schlicht rechtswidrig, weitere 50 Prozent der Maßnahmen als rechtlich unklar betrachtete. 606

\section{c) Bemühungen des erwerbsfähigen Hilfebedürftigen, § 15 Abs. 1 S. 2 Nr. 2 SGB II}

Die in $\S 15$ Abs. 1 S. 2 Nr. 2 SGB II geforderten Bemühungen des erwerbsfähigen Hilfebedürftigen sind auf den Grundsatz des Forderns in $\S 2$ SGB II zu beziehen. Dort heißt es, dass der erwerbsfähige Hilfebedürftige und die mit ihm in einer Bedarfsgemeinschaft lebenden Personen alle Möglichkeiten zur Beendigung oder Verringerung ihrer Hilfebedürftigkeit ausschöpfen müssen.

Das bedeutet in erster Linie, dass der erwerbsfähige Hilfebedürftige nicht abwarten darf, bis ihm die Agentur für Arbeit eine Arbeitsstelle vermittelt. ${ }^{607}$ Die Erwirtschaftung seines Lebensunterhaltes bleibt trotz Unterstützung des Staates in seiner persönlichen Verantwortung. Ein entsprechendes Verhalten ist Voraussetzung dafür, dass er diese Unterstützung weiter beziehen kann.

Wichtig ist für den Einzelfall zu klären, welche Eigenbemühungen erwartet werden können. Das Gesetz bleibt zwangsläufig ungenau. Aus § 31 SGB II können bestimmte Verhaltensweisen abgeleitet werden, deren Fehlen zu Sanktionen führt. Relevant sind auch die Gepflogenheiten der jeweiligen Berufssparte, die den Arbeitsuchenden aufnehmen soll, sowie das Einstellungsverhalten der Arbeitgeber. ${ }^{608}$

604 Dazu Stahlmann, ZfSH/SGB 2006, S. 131 ff; Spindler, info also 2006, S. 162 ff; Krahmer, SGb 2006, S. 581 ff. Inzwischen hat das BAG, Urteil v. 8.11.2006, 5 AZB 36/06, bestätigt, dass für Streitigkeiten über Zusatz-Jobs die Sozialgerichte zuständig sind. Vgl. auch Gagel, Anm. zu BAG, Urteil v. 26.9.2007, 5 AZR 857/06, jurisPR-ArbR 7/2008 Anm. 3.

605 BT-Drucksache 15/6951, S. 2, vgl. zu dieser Diskussion Rothkegel, in: Rothkegel, Sozialhilferecht, 2005, S. 299-301; Bieback, NZS 2005, S. 341-343; Zwanziger, AuR 2005, S. 8-15; Becker/Sichert, SpuRt 2005, S. 187 ff; Bieritz-Harder, ZfSH/SGB 2005, S. 259 ff; Krahmer/Spindler, NDV 2005, S. 17 ff; Rixen, SozSich 2005, S. 152 ff; Rixen/Pananis, NJW 2005, S. 2177 ff. Die Bundesagentur für Arbeit hat die Neuregelung der Arbeitsgelegenheiten gefordert.

606 Bundesrechnungshof, Bericht über die Durchführung der Grundsicherung für Arbeitsuchende, 2006, S. $15 \mathrm{ff}$.

607 Müller, in: Hauck/Noftz, SGB II, K § 15 Rn. 33.

608 Müller, in: Hauck/Noftz, SGB II, K $§ 15$ Rn. 35. 
So gehört z.B. zu den Eigenbemühungen das Schreiben und der Nachweis einer bestimmten Anzahl von Bewerbungen, der Besuch von Fortbildungen, eine gewisse zumutbare räumliche Mobilität, Annahme von Arbeiten und die Duldung ärztlicher Untersuchungen und Behandlungen. Auch die Vereinbarung einer bestimmten Anzahl von Terminen mit dem Fallmanager ist mögliche Verhandlungsmasse bei der Aushandlung der Eingliederungsvereinbarung. ${ }^{609}$ Diese Aufzählung ist keinesfalls abschließend, schließlich werden darüber Verhandlungen zwischen den Parteien geführt. 610

Neben den in $\S 15$ Abs. 1 SGB II vorgeschriebenen Mindestinhalten beschränkt die Kreativität der Parteien der Eingliederungsvereinbarung lediglich der Grundsatz, dass das Vereinbarte nicht gegen bestehende Vorschriften verstoßen darf.611 Dazu gehört insbesondere die Regelung über die Zumutbarkeit von Arbeit, § 10 SGB II. 612 § 10 Abs. 1 SGB II enthält einen abschließenden Katalog von Tatbeständen, die der Zumutbarkeit einer Arbeitsaufnahme entgegenstehen. ${ }^{613}$ Das bedeutet, dass die Aufnahme einer unzumutbaren Arbeit nicht vereinbart werden darf. ${ }^{614}$

\section{d) Schadensersatzpflicht, $\S 15$ Abs. 3 SGB II}

Bei Vereinbarung einer Bildungsmaßnahme muss bereits in der Eingliederungsvereinbarung festgelegt werden, dass und in welchem Umfang der Hilfebedürftige schadensersatzpflichtig ist, wenn er die Maßnahme aus einem von ihm zu vertretenden Grund nicht zu Ende führt, $§ 15$ Abs. 3 SGB II. 615

\section{e) Geltungszeitraum der Vereinbarung}

Die Eingliederungsvereinbarung nach SGB II soll für sechs Monate abgeschlossen werden, $\S 15$ Abs. 1 S. 3 SGB II. ${ }^{616}$ Danach soll eine neue Eingliederungsvereinbarung abgeschlossen werden, bei der die bisher gewonnenen Erfahrungen zu berücksichtigen sind, $\S 15$ Abs. 1 S. 4, 5 SGB II.

Sinn und Zweck der Befristung der Vereinbarungen ist es sicherzustellen, dass eine intensive Betreuung stattfindet und zeitnah überlegt wird, ob die vereinbarten Maßnahmen immer noch zur beruflichen Eingliederung des Hilfebedürftigen geeignet erscheinen. ${ }^{617}$

609 Müller, in: Hauck/Noftz, SGB II, K § 15 Rn. 35.

610 Stark, in: Estelmann, SGB II, § 15 Rn. 63.

611 Stark, in: Estelmann, SGB II, § 15 Rn. 73.

612 Die Zumutbarkeitsregelung wurde im Zuge der Reformen wesentlich ausgedehnt, sodass im Grundsatz jede Arbeit zumutbar ist. Es gibt z.B. keinen qualitativen Berufsschutz, auch eine gewisse Entfernung vom Beschäftigungsort kann verlangt werden. Dazu Valgolio, in: Hauck/Noftz, SGB II, K § 10 Rn. $2 \mathrm{ff}$.

613 Rixen, SGb 2005, S. 509 ff zur Auslegung des Auffangtatbestandes in $\S 10$ Abs. 1 Nr. 5 SGB II.

614 Dazu zweites Kapitel B II 7c) cc) S. 218 f.

615 Vgl. zweites Kapitel B II 8d) aa) S. 233 f.

616 Für die Zeit bis 31.12.2006 regelt die Übergangsvorschrift in $\S 65$ Abs. 6 SGB II, dass $\S 15$ Abs. 1 S. 2 SGB II mit der Maßgabe gilt, dass Eingliederungsvereinbarungen bis dahin für bis zu zwölf Monate geschlossen werden sollen.

617 BT-Drucksache 15/1516, S. 54. 
Explizite Regelungen zur Form der Eingliederungsvereinbarung enthält das SGB II nicht. Aus der Vorbildfunktion der Eingliederungsvereinbarung nach SGB III618 ist abzuleiten, dass die Schriftform einzuhalten ist. ${ }^{619}$ So fordert die Gesetzesbegründung, dass die Eingliederungsvereinbarung mit dem Fallmanager gemeinsam erarbeitet und unterzeichnet, mithin also schriftlich abgeschlossen werden soll. ${ }^{620}$

Ist die Eingliederungsvereinbarung als verwaltungsrechtlicher Vertrag gem. $§ 53 \mathrm{ff}$ SGB X einzuordnen, was im Anschluss überprüft wird, muss die Schriftform ohnehin wegen $\S 56$ SGB X eingehalten werden. Am Ende der Urkunde muss eigenhändig durch Namensunterschrift oder notariell beglaubigtes Handzeichen unterzeichnet werden. Mündlich abgeschlossene Verträge oder Verträge, die die Schriftform nicht erfüllen, sind gem. $\S 58$ Abs. 1, 61 S. 2 SGB X i.V.m. § 125 BGB nichtig. ${ }^{621}$ Die Schriftform hat im öffentlichen Recht vor allem Warn- und Kontroll- beziehungsweise Beweisfunktion. ${ }^{622}$ Sie bezieht sich auf alle Vertragserklärungen aller Vertragsteile, sodass sich Leistung und Gegenleistung eindeutig und zweifelsfrei aus den Vertragsurkunden selbst ergeben müssen. 623

\section{Rechtliche Einordnung}

Bei der rechtlichen Einordnung der Eingliederungsvereinbarung nach dem SGB II stellen sich zwar durchaus ähnliche Fragen wie bei der Eingliederungsvereinbarung nach dem SGB III. Wegen der in bestimmten Bereichen bereits im Grundsatz unterschiedlichen Ausgestaltung des SGB II ist aber eine eigenständige Prüfung erforderlich. ${ }^{624}$

\section{a) Verwaltungsakt, $\S 31$ SGB $X$}

\section{aa) Hoheitliche Maßnahme}

Die hoheitliche Maßnahme ist ein Kennzeichen für Verwaltungshandeln durch Verwaltungsakt, § 31 SGB X. Es setzt voraus, dass das zwischen Arbeitsverwaltung und Arbeitsuchendem Besprochene einseitig von der Arbeitsverwaltung festgelegt und ver-

618 Löschau/Marschner, Hartz IV, 2004, Rn. 414; Brühl/Hofmann, Sozialgesetzbuch Zweites Buch, 2004, S. 102.

619 Vgl. zweites Kapitel B I 4 S. 150.

620 BT-Drucksache 15/1516, S. 46.

621 An dieser unflexiblen Nichtigkeitsfolge knüpft die Kritik am geltenden verwaltungsrechtlichen Vertrag an. Vgl. z.B. Maurer, Der Verwaltungsvertrag, in: Hill, Verwaltungshandeln durch Verträge und Absprachen, 1990, S. 25; Schmitz, DVB1 2005, S. 19.

622 Dazu Ziekow/Siegel, VerwArch 2004, S. 134 f.

623 BVerwGE 84, 236 [244].

624 Insofern ist die vermischte Prüfung bei Knoblauch/Hübner, NDV 2005, S. $277 \mathrm{ff}$ zu verschwommen. 
antwortet wird. Dazu ist erforderlich, dass eine vertragliche Prägung dieses Prozesses ausgeschlossen werden kann. 625

Die Willensrichtung der Parteien ist festzustellen. Es ist klar, dass es nicht um die Überprüfung eines konkreten Verhältnisses zwischen einem bestimmten Arbeitsuchenden und einem Fallmanager gehen kann, sondern nur um die theoretische Abwägung des Für und Wider einer Vereinbarung vor dem Hintergrund des vom Gesetzgeber Geregelten und Gewollten.

Die Betrachtung objektiver Kriterien führt zu keinem eindeutigen Ergebnis für die subjektive Willensrichtung der Parteien:

Die Form - Schriftform - wird wegen ihrer Vorteile, insbesondere der Beweisfunktion, sowohl bei vertraglicher Vereinbarung als auch bei Tätigwerden durch Verwaltungsakt verwendet. Die Bezeichnung Eingliederungsvereinbarung, mit der das Dokument überschrieben wird, deutet auf einen verwaltungsrechtlichen Vertrag hin. Ein Verwaltungsakt wird eher mit dem Begriff „Bescheid“ überschrieben.

Das Verfahren kann hingegen für einen Verwaltungsakt sprechen. Denn, obwohl hier kein konkretes Verfahren betrachtet werden kann, wird es bereits im Gesetz dominiert von dem Fallmanager, der ungleich mehr Prüfungspflichten hat als ein gewöhnlicher Vertragspartner. ${ }^{626}$ Hierbei liegt deshalb eine einseitige Festlegung des Inhalts nahe, weil die entscheidenden Grenzen des Möglichen vom Fallmanager bestimmt werden.

Neben objektiven Kriterien ist auch das Interesse der Parteien an einem Vertragschluss ausschlaggebend.

Für den Arbeitsuchenden bringt der Verwaltungsakt zwar eine klare Struktur mit sich, die sich für ihn besonders wegen der eindeutigen gerichtlichen Überprüfbarkeit positiv auswirkt. Ferner kann es für einen Arbeitsuchenden, der sich mit dem „Konsum“ passiver Leistungen zufrieden gibt ${ }^{627}$, positiv sein, dass ihm die Arbeitsverwaltung einseitig entgegenkommt und ihm dem Aufwand abnimmt, sich zu überlegen wie seine Integration in den Arbeitsmarkt gestaltetet werden könnte. Angesichts der Tatsache, dass die allgemeinen Mitwirkungspflichten der $\S \S 60 \mathrm{ff}$ SGB I auch im SGB II Geltung haben und dem Arbeitslosen bereits ein hohes Maß an Mitwirkung abverlangt werden kann, ist dieses Argument zu vernachlässigen. Es geht schlicht nicht mehr, Leistungen der Grundsicherung für Arbeitsuchende nur „einzustecken“, ohne dafür etwas zu tun. An diesem Prozess freiwillig mitzuwirken, kann deshalb nur im Interesse des Hilfebedürftigen liegen.

Die Mitwirkung beim verwaltungsrechtlichen Vertrag dagegen bietet gegenüber passivem Konsum folgende Vorteile: Der Hilfebedürftige kann die Erfassung seiner Bedürfnisse bei der Eingliederung steuern und beeinflussen. Damit kann auch die Richtung seiner (weiteren) Berufstätigkeit in eine zum Arbeitsuchenden passende Richtung gelenkt werden. Er kann falsche Annahmen der Arbeitsverwaltung frühzeitig erkennen und sie richtig stellen. Interessant ist für ihn auch, dass eine (vertragliche) Bindung der

625 Vgl. zweites Kapitel B I 5 S. 150 ff.

626 Vgl. zweites Kapitel B I 3 S. 147 ff.

627 Vgl. Oschmiansky/Kull/Schmid, Faule Arbeitslose? Politische Konjunkturen einer Debatte, Discussion Paper FSI 01 - 206; Oschmiansky, APuZ 2003, B 6-7. 
Verwaltung eintritt. Denn auch die Verwaltung übernimmt beim verwaltungsrechtlichen Vertrag Verpflichtungen, die sie nach Vertragschluss erfüllen muss. Zwar besteht weiterhin die Möglichkeit, den Vertrag abzuändern, sollte dies insbesondere aus Sicht der Verwaltung nötig sein. Doch legt sich die Verwaltung in jedem Fall auf unterstützende Maßnahmen fest, die die Ausübung ihres Entschließungs- und Auswahlermessens in Bezug auf eingliedernde Maßnahmen bestimmt.

Die Verwaltung hat ebenfalls mehr Interesse an einem verwaltungsrechtlichen Vertrag als an einem Verwaltungsakt. ${ }^{628}$ Die höhere gerichtliche Kontrolldichte bei Verwaltungsakten im Vergleich zum verwaltungsrechtlichen Vertrag ${ }^{629}$ spricht für ein größeres Interesse an letzterem. Die der Verwaltung verbleibenden Spielräume sind bei eingeschränkter gerichtlicher Kontrolle größer und können für eine bessere Abstimmung der Leistungen auf den Einzelfall genutzt werden. Die psychologische Wirkung einer Einbeziehung des Arbeitsuchenden erhöht ebenfalls das Interesse der Verwaltung an einer vertraglichen Regelung. ${ }^{630}$ Die Abgabe von Verantwortung an den Einzelnen, insbesondere für die konkrete Auswahl der Maßnahmen zur Eingliederung entlastet die Verwaltung und ermöglicht ihr, mehr Energien auf die vermittelnde Tätigkeit zu verwenden, Kontakt zu Arbeitgebern aufzunehmen und den Arbeitsmarkt auf der Suche nach offenen Stellen beziehungsweise Marktlücken für eine Selbständigkeit zu erkunden.

\section{bb) „Ersatzvornahme“ durch die Verwaltung, § 15 Abs. 1 S. 6 SGB II}

$\S 15$ Abs. 1 S. 6 SGB II enthält ein unabhängig von den Interessen der Parteien bestehendes Argument dafür, dass die Eingliederungsvereinbarung im Regelfall als vertragliche und nicht als einseitige Regelung anzusehen ist. Kommt eine Einigung zwischen Agentur für Arbeit und erwerbsfähigem Hilfebedürftigen nicht zustande, sollen die Regelungen 631 nach $\S 15$ Abs. 1 S. 2 SGB II durch Verwaltungsakt erfolgen. ${ }^{632}$

Diese ausdrückliche gesetzliche Regelung unterstreicht den Willen des Gesetzgebers, dass zunächst eine Einigung zwischen Arbeitsverwaltung und Einzelnem versucht werden soll. Erst dann steht der Arbeitsverwaltung, um ihre Handlungsfähigkeit nicht zu gefährden und die Eingliederung des Arbeitslosen nicht zu verzögern, der Weg über den Verwaltungsakt offen. Das bedeutet für die Auslegung der Willensrichtung der Arbeitsverwaltung einen gesetzlichen Auftrag zu verhandeln und auf den Abschluss einer Ver-

628 Da die Fallzahlen beim Arbeitslosengeld II ca. 3,5 Mio. ausmachen, kann man den Begriff Massenverfahren zweifellos verwenden. Für diese wird, im Allgemeinen gesprochen, die Entscheidung mittels Verwaltungsaktes ,als in der Regel effizienter“ betrachtet. So Diering, in: Diering/Timme/Waschull, LPK-SGB X, vor $\S \S 53-61$, Rn. 3. Dem widerspricht Kretschmer, DÖV 2006, S. 893, wenn er schreibt, dass sich auch in der Massenverwaltung, v.a. im Sozialrecht, der Vertrag einen sicheren Platz erkämpft hat.

629 Vgl. erstes Kapitel B IV 1 S. 82 ff.

630 Vgl. erstes Kapitel A II S. 54 ff; so auch Luthe/Timm, SGb 2005, S. 262 ohne weitere Begründung.

631 Spellbrink, SozR akt 2006, S. 54 weist darauf hin, dass die Formulierung „Regelung“ in $\S 15$ Abs. 1 S. 6 SGB II ein Argument gegen die Annahme eines verwaltungsrechtlichen Vertrages liefere.

632 Stark, in: Estelmann, SGB II, § 15 Rn. 128 ff spricht von einer „Ersatzvornahme“ durch die Verwaltung bzw. einem „Eingliederungsbescheid““. 
einbarung hinzuwirken. ${ }^{633}$ Darin ist ein Handlungsformverbot hinsichtlich eines Verwaltungsakts zu sehen, das solange besteht, bis das Scheitern der Verhandlungen feststeht. 634

\section{cc) Zwischenergebnis}

Im Ergebnis scheitert die Einordnung als Verwaltungsakt daran, dass die Einseitigkeit der Maßnahme fehlt, wenn die Maßnahmen zur Eingliederung sowie die Bemühungen des erwerbsfähigen Hilfebedürftigen in einer Eingliederungsvereinbarung von Hilfebedürftigem und Arbeitsverwaltung gemeinsam ausgearbeitet und vereinbart werden.

b) „Eingliederungsplan“, „Hilfeplan“

Aufgrund des aufzählenden Charakters der Eingliederungsvereinbarung und der Formulierung „Niederlegung verbindlicher Aussagen“ könnte die Eingliederungsvereinbarung nach SGB II als Plan bezeichnet werden. Die Einordnung aufgrund dieser formalen Argumentation hält einer Überprüfung anhand der maßgeblichen Kriterien 635 für planendes Verwaltungshandeln jedoch nicht stand. Die Eingliederungsvereinbarung nach SGB II ist keine Vorstufe einer sich anschließenden Verwaltungsentscheidung, sondern eigenständig. Sie hat zwar zusätzlich Auswirkungen bei späteren Entscheidungen, stellt aber für sich genommen ein eigenständiges Tätigwerden der Verwaltung dar.

Im Übrigen ergibt sich aus der Anordnung in $\S 15$ Abs. 1 S. 6 SGB II, dass bei fehlender Einigung ein Verwaltungsakt erlassen werden soll. Der Plan als eigenständige Handlungsform ist durch die gesetzliche Entscheidung zugunsten einer anderen Handlungsform (Verwaltungsakt für den Fall einer fehlenden Einigung) ausgeschlossen.

\section{c) Verwaltungsrechtlicher Vertrag, $\S \S 53$ ff SGB X}

Der verwaltungsrechtliche Vertrag setzt gem. § 53 Abs. 1 SGB X voraus, dass ein Rechtsverhältnis auf dem Gebiet des öffentlichen Rechts durch Vertrag begründet, geändert oder aufgehoben wird, soweit Rechtsvorschriften nicht entgegenstehen. Ein verwaltungsrechtlicher Vertrag über Sozialleistungen kann nur geschlossen werden, soweit die Erbringung der Leistungen im Ermessen des Leistungsträgers steht, § 53 Abs. 2 SGB X.

\section{aa) Abgrenzung zum privatrechtlichen Vertrag}

Die Einordnung als privatrechtlicher Vertrag scheitert. Die möglichen vertraglichen Inhalte sind schwerpunktmäßig dem öffentlichen Recht zuzuordnen.

Die Pflichten und Rechte der Agentur für Arbeit, die aus der Vereinbarung folgen können, ergeben sich ausschließlich aus dem SGB II oder haben zumindest ihre Grundlage im SGB II. Diese Vorschriften sind unproblematisch dem Sozialrecht als Teil des

633 Hannes, SozSich 2007, S. 68.

634 So auch Luthe/Timm, SGb 2005, S. 262; Berlit, in: Münder/Armborst, LPK-SGB II, 2005, § 15 Rn. 8 .

635 Vgl. erstes Kapitel B II 1 S. 68 f. 
öffentlichen Rechts zuzuordnen. Eine Anwendung privatrechtlicher Vorschriften scheidet deshalb aus. Auch die Schadensersatzpflicht gem. § 15 Abs. 3 SGB II, die in die Eingliederungsvereinbarung aufzunehmen ist, hat ihren Grund in einer Regelung des SGB II und gehört damit dem öffentlichen Recht an.

\section{bb) Ausschluss des verwaltungsrechtlichen Vertrages, § 53 Abs. 2 SGB X}

Der Abschluss eines verwaltungsrechtlichen Vertrages ist gem. §53 Abs. 2 SGB X nur zulässig, soweit die Erbringung der Leistung im Ermessen der Behörde steht. Es ist darüber hinaus zu prüfen, ob $\S 15$ SGB II eine abweichende Regelung im Sinn des $\S 37$ SGB I enthält, die insoweit eine Modifizierung des SGB X zuließe.

Wie bereits dargestellt 636 , wird über die Erbringung der Leistungen zur Eingliederung gem. § 16 SGB II grundsätzlich in einer Ermessensentscheidung entschieden, während der Hilfebedürftige auf die Leistungen zur Sicherung des Lebensunterhaltes stets einen Anspruch hat. Damit steht fest, dass Leistungen zur Sicherung des Lebensunterhaltes und Eingliederungsleistungen, auf die der Hilfebedürftige einen gesetzlichen Anspruch hat, nicht im Wege eines verwaltungsrechtlichen Vertrages vereinbart werden dürfen.

$\S 15$ SGB II enthält auch keine abweichende Regelung im Sinn des $\S 37$ SGB I, sodass es beim Grundsatz des $\S 53$ Abs. 2 SGB X bleibt: $\S 15$ SGB II steht im Abschnitt 1 des dritten Kapitels des SGB II und soll den Eingliederungsprozess gestalten, nicht aber die Leistungen zur Sicherung des Lebensunterhaltes. Der mögliche Inhalt der Eingliederungsvereinbarung ist zwar nicht abschließend aufgelistet, doch prägen die Beispiele die mögliche Richtung der inhaltlichen Ausgestaltung. Der Abschluss einer Eingliederungsvereinbarung über Leistungen zur Sicherung des Lebensunterhaltes ist ausgeschlossen. 637

\section{cc) Vertragschluss und Kontrahierungszwang}

Wie der privatrechtliche Vertrag setzt der verwaltungsrechtliche Vertrag zwei übereinstimmende Willenserklärungen voraus, die als Angebot und Annahme in Bezug zueinander abgegeben werden, § 61 SGB X i.V.m. § 145 ff BGB. Es ist stets eine Frage des Einzelfalles, ob ein Vertragschluss vorliegt.

In der vorliegenden Arbeit wird nun untersucht, ob die rechtliche Ausgestaltung der Eingliederungsvereinbarung im SGB II bereits in der Theorie, d.h. ohne die Betrachtung der konkreten Umstände einer Eingliederungsvereinbarung, einem Vertragschluss entgegensteht. In erster Linie ist der Wortlaut im Gesetz zu betrachten, der grundsätzlich Ausdruck der gesetzgeberischen Intention ist.

Während des Gesetzgebungsverfahrens 638 wurde die Formulierung „,vereinbart" statt „festgelegt“ in allen drei Absätzen des $\S 15$ SGB II in Abänderung des Entwurfes verwendet, um mit dieser Terminologie zu verdeutlichen, dass der Inhalt der Eingliede-

636 Vgl. zweites Kapitel B II 3b) S. 194 ff.

637 So auch Berlit, in: Münder/Armborst, LPK-SGB II, 2005, § 15 Rn. 8.

638 Beschlussempfehlung des Ausschusses für Wirtschaft und Arbeit, in: BT-Drucksache 15/1728, S. 2 f, $15 \mathrm{ff}, 168 \mathrm{ff}$. 
rungsvereinbarung nicht einseitig durch die Agenturen für Arbeit verfasst, sondern „partnerschaftlich“ mit dem erwerbsfähigen Hilfebedürftigen zu Stande kommen, also besprochen und gemeinsam niedergelegt werden soll. 639 Der erwerbsfähige Hilfebedürftige und die Agentur für Arbeit in der Person des Fallmanagers sind beim Abschluss der Eingliederungsvereinbarung nach dem Willen des Gesetzgebers grundsätzlich gleichberechtigte Partner. 640 Die heutige Formulierung beziehungsweise die präzisen Veränderungen in der früheren Formulierung des SGB II-Entwurfs sprechen für eine vertragliche Vereinbarung des Inhalts der Eingliederungsvereinbarung. 641

\section{(1) Die Willenserklärung des Bürgers}

Die Einwilligung des Bürgers in den Vertragsabschluss ist Wirksamkeitsvoraussetzung des verwaltungsrechtlichen Vertrages, nicht nur Rechtmäßigkeitsvoraussetzung. Sie ist eine Willenserklärung ${ }^{642}$, also eine Willensäußerung, die auf eine Rechtsfolge gerichtet ist. ${ }^{643}$ Durch das Erfordernis einer Willenserklärung des Bürgers wird das Verwaltungsverfahren von diesem gleichberechtigt mit abgeschlossen. ${ }^{644}$ Dadurch erhält der vertragschließende Bürger die Mitverantwortung für das Verhandlungsergebnis. 645

Dem Ermessen der Behörde, durch Vertrag zu handeln (Formenwahlfreiheit), korrespondiert ein entsprechendes „Entschließungsermessen“ des Bürgers, zu unterzeichnen (Abschlussfreiheit). Damit hat es der Bürger in der Hand, ob ein Vertrag zustande kommt oder ein Verwaltungsakt erlassen werden muss/kann.

Insoweit ist der Vertragschluss grundsätzlich Freiheitsgebrauch im Sinn des Art. 2 Abs. 1 GG. ${ }^{646}$ Vertragsfreiheit wie im Zivilrecht bis zur Grenze der Gesetzes- und Sit-

639 BT-Drucksache 15/1728, S. 15, 177.

640 Ausschussbericht, in: BT-Drucksache 15/1749, S. 32; dazu Müller, in: Hauck/Noftz, SGB II, K $\S 15$ Rn. 2.

641 Ebenso Luthe/Timm, SGb 2005, S. 261 f; Hannes, SozSich 2007, S. 68; a.A. Spellbrink, SozR akt 2006, S. 54, der diese Änderungen ohne weitere Begründung als „kosmetisch“ bezeichnet.

642 Krause, VerwArch 1970, S. 302 spricht insoweit vom „eigenen Rechtsinstitut der Willenserklärung des öffentlichen Rechts“.

643 Erichsen/Ehlers, Allgemeines Verwaltungsrecht, 2006, § 27 Rn. 1; Wolff/Bachof/Stober, Verwaltungsrecht, Bd. 1, 1999, S. 551; ausführlich zu möglichen Auslegungsgrundsätzen sowie Bindung, Widerruf und Anfechtung Kluth, NVwZ 1990, S. 609 ff; Krause, VerwArch 1970, S. 322-326.

644 Maurer, Allgemeines Verwaltungsrecht, 2009, S. 378.

645 In diesem Sinn formuliert Efstratiou, Die Bestandskraft des öffentlich-rechtlichen Vertrags, 1988, auf S. $127 \mathrm{ff}$ „Die Verantwortung des Bürgers besteht darin, dass die inhaltliche Gestaltung der Entscheidung auf ihn zurückgeht, dass er seine Rechte und Interessen im Verwaltungsverfahren vorgetragen und geltend gemacht hat und dass die Entscheidung erst durch seine formell und ausdrücklich erklärte Zustimmung in vertraglicher Form ergehen konnte. Somit wird die Mitwirkung des Bürgers institutionalisiert. Statt bloßer Mitwirkung durch die Verfahrenseinleitung mittels Antrags wird ihm ein Teil der Verantwortung für die Verwaltungsentscheidung übertragen. Der Bürger hat das Recht und die Last, im Rahmen der Gesetze und des geltenden Rechts maßgeblichen Einfluss auf die inhaltliche Gestaltung der Verwaltungsentscheidung selbst zu nehmen.“

646 Göldner, JZ 1976, S. 355; Efstratiou, Die Bestandskraft des öffentlich-rechtlichen Vertrags, 1988, S. 272; Punke, Verwaltungshandeln durch Vertrag, 1989, S.171; Krebs, VVDStRL, H. 52 (1993), S. 265; so auch Schlette, Die Verwaltung als Vertragspartner, 2000, S. 66, der kritisiert, dass allzu oft 
tenwidrigkeit besteht wegen der besonderen rechtlichen Bindungen der Verwaltung aber nicht. Diese öffentlich-rechtliche Bindung wirkt sich aber auf die Freiheit des einzelnen Bürgers aus. ${ }^{647}$ Stern wies noch vor Erlass des VwVfG in diesem Zusammenhang darauf hin, dass diese Bindungen die vertragliche Gestaltungsfreiheit in ihrem ,institutionellen Sinngehalt" nicht verletzen dürfen. ${ }^{648}$ Bestehen Zweifel an der Freiwilligkeit, d.h. an der Tatsache, dass die Erklärung des Bürgers frei von Willensmängeln und nicht unter der erdrückenden Verhandlungsmacht der Verwaltung abgegeben wurde, ist über die Lösung dieses Problems im Rahmen der Fehlerfolgen des Vertragsschlusses nachzudenken. ${ }^{649}$

(2) Die Willenserklärung und die Abschlusspflicht der Arbeitsverwaltung

Die für den Vertragsschluss erforderliche Willenserklärung der Verwaltung unterliegt der Gesetzesbindung650: Die Verwaltung bleibt trotz der Miteinbeziehung des Bürgers allein voll verantwortlich für die Übereinstimmung des Vertragsinhalts mit dem Gesetz. Auf Vertragsfreiheit im Sinn des Art. 2 Abs. 1 GG kann sich die Verwaltung nicht berufen. ${ }^{651}$ Deshalb ist der verwaltungsrechtliche Vertrag zwar keinesfalls unmöglich ${ }^{652}$, denn es kommt durch übereinstimmende Willenserklärung von Verwaltung und Bürger ein Vertrag zustande. Dieser Vorgang stellt sich für die Verwaltung nur nicht als Ausübung von Freiheitsrechten, sondern als Aufgabenerfüllung dar. ${ }^{653}$

Die Verwaltung „soll“ die erforderlichen Leistungen zur Eingliederung ,vereinbaren“, § 15 Abs. 1 SGB II. Damit wurde eine Abschlusspflicht begründet, die nicht mit einem Kontrahierungszwang verwechselt werden darf. Der Kontrahierungszwang ist stets ein Eingriff in den Schutzbereich des Art. 2 Abs. 1 GG und muss gerechtfertigt sein. Auf Art. 2 Abs. 1 GG könnte sich die Verwaltung als Teil des Staates aber ohnehin nicht berufen. ${ }^{654}$ Zudem enthalten Soll-Vorschriften für die Verwaltung im Regelfall die Verpflichtung zum Tätigwerden. ${ }^{655}$ Eine abweichende Vorgehensweise ist nur bei atypischen Einzelfällen möglich. Das ist der Fall, wenn gut vertretbare und überwiegende Gründe für ein Absehen von der Anwendung der Norm sprechen, wobei diese Grün-

diese Komponente hinter der Frage der Beeinträchtigung der Grundrechte des Bürgers zurückstehen müsse.

647 Dazu Bonk, in: Stelkens/Bonk/Sachs, VwVfG, 2001, § 54 Rn. 90; Schlette, Die Verwaltung als Vertragspartner, 2000, S. 68.

648 Stern, AöR 84 (1959), S. 285.

649 Bonk, in Stelkens/Bonk/Sachs, VwVfG, § 54 VwVfG, Rn. 32; Ziekow/Siegel, VerwArch 2003, S. 596; als Ausgangspunkt der viel zitierte Aufsatz von Schilling, VerwArch 1996, S. 191 ff, in dem er für ,unfreiwillige“ Verträge mit der öffentlichen Hand einen sich direkt aus der Verfassung ergebenden Anspruch auf Aufhebung des Vertrages vorschlägt.

650 Kluth, NVwZ 1990, S. 610; Schmidt-Aßmann, Das allgemeine Verwaltungsrecht als Ordnungsidee, 2004, S. 342.

651 Vgl. erstes Kapitel B III 2 S. $71 \mathrm{ff}$.

652 So noch die Gegner des verwaltungsrechtlichen Vertrages.

653 Ziekow/Siegel, VerwArch 2004, S. 298.

654 Vgl. erstes Kapitel B III 2b) S. 72.

655 Vgl. in st. Rspr. BVerwGE 12, 284 [285]; 42, 26 [28 f]; 90, 88 [93]. 
de nicht von der Behörde selbst zu vertreten sein dürfen. ${ }^{656}$ Eine atypische Konstellation liegt ferner vor, wenn der Sachverhalt zwar vom abstrakten Rahmen des Gesetzes umfasst ist, aber auf die Zweckbestimmung des Gesetzes nicht abzielt. Das Vorliegen eines Sonderfalles kann voll gerichtlich überprüft werden. ${ }^{657}$ Besonders im Sozialrecht sind die Entscheidungen, in denen ein Sonderfall von den Gerichten anerkannt wurde, sehr selten. 658

Ein atypischer Fall kann nicht darin gesehen werden, dass der Abschluss einer Eingliederungsvereinbarung in bestimmten Fällen nicht aussichtsreich erscheint, z.B. weil die Vermittlung in Arbeit in einer strukturschwachen Gegend bei Vorliegen einer relevanten örtlichen Bindung geradezu unmöglich ist. Es ist davon auszugehen, dass der Gesetzgeber auch in diesen Fällen eine Pflicht der Behörde zum Abschluss einer Eingliederungsvereinbarung sieht. ${ }^{659} \mathrm{Er}$ sieht den Aushandlungsprozess beziehungsweise die einseitige Anordnung als zentrale Grundlage der Eingliederungsbemühungen an. 660

Andererseits kann der Abschluss einer Eingliederungsvereinbarung als bloße Förmelei angesehen und damit ein atypischer Fall bejaht werden, wenn ein Anschlussarbeitsverhältnis bereits gefunden ist und bei Arbeitslosmeldung beziehungsweise bei Beantragung von Arbeitslosengeld II feststeht, dass die Arbeitslosigkeit nicht andauern wird (feste Einstellungszusage innerhalb von acht Wochen). ${ }^{661}$ Hier spricht eine KostenNutzen-Überlegung dafür, keine weiteren Ressourcen in den Arbeitslosen zu investieren, da dessen Eingliederung bereits so gut wie vollzogen ist. 662

Vorübergehend kann die Arbeitsverwaltung von einer Eingliederungsvereinbarung absehen, wenn Erwerbstätigkeit nicht zumutbar ist. Es handelt sich dabei v.a. um Fälle des $\S 10$ Abs. 1 SGB II (z.B. Erziehung eines Kindes bis zur Vollendung des dritten Lebensjahres, $\S 10$ Abs. 1 S. 1 Nr. 3 SGB II). ${ }^{663}$

Ein anderer atypischer Fall dürfte gegeben sein, wenn der kommunale Träger sein Einvernehmen verweigert, sodass eine Vereinbarung nicht wirksam werden und ein Verwaltungsakt erlassen werden muss. ${ }^{664}$ Für den Fall eines Dissens über den Vertragsinhalt greift $\S 15$ Abs. 1 S. 6 SGB II direkt ein und ordnet den Erlass eines Verwal-

656 Stark, in: Estelmann, SGB II, § 15 Rn. 94 unter Verweis auf eben genannte Rspr.

657 BVerwGE 90, 275 [280].

658 So die Auswertung bei Brinktrine, Verwaltungsermessen in Deutschland und England, 1998, S. 42.

659 Dazu Stark, in: Estelmann, SGB II, § 15 Rn. 93 ff.

660 BT-Drucksache 15/1516, S. 46.

661 Stark, in: Estelmann, SGB II, § 15 Rn. 100 unter Bezugnahme auf Steinmeyer, info also 2002, S. 5, der diese Aussage über die Pflicht zum Abschluss einer Eingliederungsvereinbarung nach SGB III machte; Kocialkowski, SozR akt 2006, S. 38; Schumacher, in: Oestreicher, SGB XII/SGB II, § 15 Rn. 29.

662 An dieser Stelle sei darauf hingewiesen, dass die Fälle, in denen ein Anschlussarbeitsverhältnis bereits gefunden ist, selten sein werden. Schließlich geht dem Bezug von Arbeitslosengeld II meist ein Zeitraum von ca. einem Jahr Bezug von Arbeitslosengeld nach SGB III voraus, in der der Arbeitsuchende gerade keine neue Arbeitstelle gefunden hat.

663 Kocialkowski, SozR akt 2006, S. 38; Schumacher, in: Oestreicher, SGB XII/SGB II, § 15 Rn. 28.

664 Vgl. zweites Kapitel B II 2b) cc) S. 190; B II 8b) S. 231 ff. 
tungsaktes an, sodass nicht der Umweg über die Begründung eines atypischen Falles gemacht werden muss. 665

Die Soll-Vorschrift räumt der Arbeitsverwaltung folglich einen Entscheidungsspielraum ein. Dieser lässt es nicht zu, von einem Abschlusszwang der Behörde zu sprechen. Es handelt sich lediglich um eine Abschlusspflicht, die der Verwaltung vom Gesetzgeber unproblematisch auferlegt werden kann.

\section{(3) Kontrahierungszwang infolge § 2 S. 2 SGB II}

Der erwerbsfähige Hilfebedürftige muss gem. § 2 S. 2 SGB II aktiv an allen Maßnahmen zu seiner Eingliederung mitwirken, insbesondere eine Eingliederungsvereinbarung abschließen. Die Soll-Vorschrift in $\S 15$ Abs. 1 S. 1 SGB II richtet sich lediglich an die Verwaltung. Diese Abschlusspflicht führt jedenfalls dann zu einem zu rechtfertigenden Eingriff in den Schutzbereich des Art. 2 Abs. 1 GG, wenn der Vertragschluss auch gegen den Willen des Hilfebedürftigen herbeigeführt werden könnte. Denn es gibt kein ,ausdrückliches Mitwirkungsverweigerungsrecht bei qualitativ schlechter Beratung und Hilfeleistung“, eben so wenig wie einen geschriebenen Unzumutbarkeitsgrund „fachlich schlechte Betreuung“، 666

Die in $\S 2$ Abs. 1 SGB II enthaltene Pflicht zum Abschluss einer Eingliederungsvereinbarung ist nicht durchsetzbar, denn eine Ersetzung der Erklärung des erwerbsfähigen Hilfebedürftigen ist nicht geregelt. Es bleibt beim Grundsatz des $\S 888$ Abs. 1 ZPO, wonach nicht vertretbare Handlungen nur mit den Zwangsmitteln Zwangsgeld und Zwangshaft erzwungen werden können, nicht aber die Handlung selbst. Die Sanktion in $\S 31$ Abs. 1 S. 1 Nr. 1a SGB II sieht „lediglich” die Absenkung des Arbeitslosengeldes II vor, nicht aber die Ersetzung einer verbindlichen Willenserklärung. Folglich handelt es sich bei der Abschlusspflicht in § 2 S. 2 SGB II um eine bloße Obliegenheit 667.668 Diese zwingt den Arbeitsuchenden zwar dazu, den Sanktionsbescheid abzuwarten und gegen diesen gerichtlich vorzugehen, was aufgrund der materiellen Richtigkeitsgewähr des Vereinbarten 669 und Beweislastvorschriften 670 Schwierigkeiten bereiten kann, doch bleibt es dabei, dass die Erteilung der Erklärung nicht ersetzt werden kann. Eine Abschlusspflicht, die durchgesetzt werden könnte, besteht somit nicht. Ein verfassungswidriger Eingriff in Art. 2 Abs. 1 GG liegt nicht vor. 671

665 A.A. Müller, in: Hauck/Noftz, SGB II, K § 15 Rn. 10, 14, 21.

666 Stark, in: Estelmann, SGB II, § 15 Rn. 103.

667 Zum Begriff Obliegenheit im Sozialrecht vgl. zweites Kapitel B I 5c) ee) (4) S. 159 ff.

668 O'Sullivan, SGb 2005, S. 372; Blüggel, SozSich 2005, S. 14; Berlit, in: Münder/Armborst, LPKSGB II, § 2 Rn. 25 f; Spellbrink, in: Eicher/Spellbrink, SGB II, § 2 Rn. 5 spricht von der Norm als ,allgemeine Interpretationsfolie“.

669 Luthe/Timm, SGb 2005, S. 263.

670 Vgl. zweites Kapitel B II 7e) S. 225.

671 Spellbrink, in: Eicher/Spellbrink, SGB II, § 2 Rn. 9; a.A. Berlit, in: Münder/Armborst, LPK-SGB II, 2005, § 15 Rn. 14. 
(4) Faktischer Kontrahierungszwang infolge $\S 2$ S. 2 i.V.m. $§ 31$ Abs. 1 S. 2 Nr. 1a SGB II

Die Abschluss"obliegenheit" ist zwar nicht durchsetzbar, aber der Gesetzgeber hat ein Druckmittel zu deren Erfüllung eingeführt: Mit der Regelung in § 2 S. 2 SGB II („muss abschließen“) im Zusammenspiel mit der Sanktionsvorschrift in § 31 Abs. 1 S. 2 Nr. 1a SGB II besteht ein faktischer Kontrahierungszwang auf Seiten des Arbeitsuchenden, der ohne verfassungsrechtliche Rechtfertigung zu einer Verletzung der Vertragsfreiheit gem. Art. 2 Abs. 1 GG führt und damit einem wirksamen Vertragschluss entgegensteht. 672

Die freiwillige Abgabe der Willenserklärung steht in Frage und damit ein Eingriff in Art. 2 Abs. 1 GG, weil angesichts der drohenden Kürzung des Arbeitslosengeldes II ein beträchtlicher finanzieller Druck für den Hilfebedürftigen entsteht. 673 Der Gesetzgeber wollte gerade diesen Zusammenhang herstellen und erzieherisch auf den Hilfebedürftigen einwirken. ${ }^{674}$ Die Verknüpfung von Abschlusspflicht und Kürzung des Arbeitslosengeldes II führt zu einem Eingriff in die Vertragsfreiheit.

Die vom Gesetzgeber angestrebten Ziele sind aber verfassungsrechtlich legitim und der Eingriff kann gerechtfertigt werden.

Die Einsicht, dass sich kooperatives Vorgehen auf die Wiedereingliederung eines Hilfebedürftigen positiv auswirkt, und das Ziel, die Leistungen passgenauer und situationsangemessener auf den Einzelnen zu erbringen ${ }^{675}$, aber auch allgemeiner das Ziel, Massenarbeitslosigkeit zu bekämpfen durch effiziente (Wieder-)Eingliederung in den Arbeitsmarkt ${ }^{676}$, sind beachtlich und haben Verfassungsrang. ${ }^{677}$

Die Vereinbarung ist dazu ein geeignetes Instrument, denn deren Aushandlung bietet die Möglichkeit, den Inhalt der Vereinbarung und damit die Leistungen zu diskutieren und gemeinsam festzulegen. Es ist auch erforderlich, eine Sanktion zu verhängen, weil es kein gleich wirksames, weniger eingreifendes Instrument gibt. Die Alternative - eine nicht oder weniger sanktionierte Vereinbarung - droht nicht beachtet zu werden. Das reicht aus, die zweite Stufe des Verhältnismäßigkeitsgrundsatzes zu bejahen. Hinzukommt, dass es sich um steuerfinanzierte Leistungen handelt, die der Einzelne zur Wiedereingliederung erhält. Die optimale Nutzung von Steuermitteln rechtfertigt die erzwungene Kooperationsbereitschaft. Auch das Übermaßverbot ist gewahrt. Der ,wichtige Grund" in $\S 31$ Abs. 1 S. 2 SGB II stellt sicher, dass legitime Gründe für eine Verweigerung des Vertragsschlusses geltend gemacht und berücksichtigt werden kön-

672 Däubler, info also 2005, S. $51 \mathrm{ff}$ zur Frage der verfassungskonformen Auslegung. Krahmer, NDV 2006, S. 507 ff begründet mit dem Nebeneinander von Freiwilligkeit und Zwang die Aussage, dass die Konstruktion der Eingliederungsvereinbarung im SGB II rundum widersprüchlich sei.

673 Vgl. zweites Kapitel B II 7c) S. 218 ff.

674 Vgl. Überschrift des Unterabschnitts 2 des SGB II „Anreize und Sanktionen“, in dem § 31 SGB II steht.

675 Vgl. erstes Kapitel A II S. 54 ff.

676 BR-Drucksache 558/03 S. 1, 126.

677 BVerfGE 21, 245 [251]. 
nen. ${ }^{678}$ Die Auslegung dieses unbestimmten Rechtsbegriffes ist der Ansatzpunkt für eine verfassungskonforme Interpretation der Sanktion der Verweigerung der Eingliederungsvereinbarung gem. $\S 31$ Abs. 1 S. 2 Nr. 1a SGB II. ${ }^{679}$ Wird diese Interpretation im Einzelfall beachtet, ist der Eingriff in Art. 2 Abs. 1 GG gerechtfertigt.

\section{(5) Faktischer Kontrahierungszwang aufgrund der Monopolstellung}

Ein faktischer Kontrahierungszwang aufgrund der Monopolstellung der Arbeitsverwaltung ergibt sich daraus, dass der Arbeitsuchende, will er an staatliche Unterstützung kommen, keinen anderen potentiellen Vertragspartner für die Eingliederungsvereinbarung als die Agentur für Arbeit hat. ${ }^{680}$ Diese eingeschränkte Gestaltungsfreiheit ist eine typische Situation, in der im Privatrecht aufgrund der existenziell bedrohlichen Situation und der Monopolstellung ein Kontrahierungszwang für den Monopolisten angenommen wird. Doch auch im Privatrecht führt der Kontrahierungszwang zu Lasten des Monopolisten nicht zum Ausschluss eines Vertrages, sondern dazu, dass zugunsten der schwächeren Partei schützende Vorschriften eingreifen. 681

Ein faktischer Kontrahierungszwang auf Seiten des Arbeitsuchenden steht der Annahme eines verwaltungsrechtlichen Vertrages auch nicht entgegen. Denn es ist nicht so, dass die Vertragsfreiheit weder im Privat-, noch im Sozial- oder Verwaltungsrecht jeden Kontrahierungszwang verbieten würde. Gerade Fälle des faktischen Zwanges sind zahlreich und lassen sich rechtfertigen. Aus $\S 53$ SGB X ergibt sich nicht, dass im öffentlichen Recht vollständige Vertragsfreiheit herrschen würde. In gleicher Weise, wie es im Privatrecht Einschränkungen der Vertragsfreiheit sowohl hinsichtlich der Gestaltungsfreiheit als auch hinsichtlich der Abschlussfreiheit gibt, gelten auch im öffentlichen Recht Grenzen der Vertragsfreiheit. 682 Für die Behörde gilt z.B. das Übermaßverbot. Leistungen, die aufgrund der Monopolstellung erbracht werden, und Gegenleistungen müssen in einem angemessenen Verhältnis zueinander stehen.

Der Gesetzgeber hat im Gesetzestext sein Interesse daran festgeschrieben, dass der erwerbsfähige Hilfebedürftige sich voll und ganz auf die aktive Arbeitsuche einlässt. Deshalb stellt er das Element „Aushandlung einer Einigung“ in den Mittelpunkt. Der Arbeitsuchende hat, geht man davon aus, dass es nicht um den Einzelfall des Arbeitsunwilligen geht, selbst ein Interesse daran, beste Vermittlungsarbeit der Agentur für Arbeit abzurufen und so die Unterstützung zu erlangen, die er benötigt. Diese Interessenlage verringert die Bedrohung durch die Eingliederungsvereinbarung 683 auch in Be-

678 Rixen, in: Eicher/Spellbrink, SGB II, § 15 Rn. 15 f; a.A. Berlit, in: Münder/Armborst, LPK-SGB II, 2005, § 31 Rn. 12.

679 So auch Lang, NZS 2006, S. 184; Rixen, in: Eicher/Spellbrink, SGB II, § 15 Rn. 16; vom Ansatz ähnlich Schön, SGb 2006, S. 296.

680 Luthe/Timm, SGb 2005, S. 262.

681 Vgl. erstes Kapitel B III 2 S. 71 ff.

682 Henneke, in: Knack, VwVfG, 2000, § 54 Rn. 25 f.

683 Berlit, info also 2003, S. 205 bezeichnet wegen dieser in seinen Augen schwachen Verhandlungsposition die Eingliederungsvereinbarung als „Vereinbarung im Schatten der Macht“. In einer jüngeren Veröffentlichung (SozR akt 2006, S. 50) äußert er sich allerdings aufgeschlossener über das Instrument der Eingliederungsvereinbarung und schreibt: „Bei problembewusster, sachgerechter Anwen- 
zug auf den erwerbsfähigen Hilfebedürftigen. Begründet nämlich die Eingliederungsvereinbarung nach SGB II anders als im SGB III auch Pflichten der Behörde auf Erfüllung des Vereinbarten, verbessert sich dadurch die Position des Einzelnen im Vergleich zum einseitigen Verwaltungshandeln. Die Monopolstellung der Arbeitsverwaltung steht wegen der überwiegend für den Einzelnen begünstigenden Folge der Vereinbarung einem wirksamen Vertragsschluss nicht entgegen.

\section{(6) Faktischer Kontrahierungszwang aufgrund $\S 15$ Abs. 1 S. 6 SGB II}

Ein weiterer Ansatzpunkt für einen Kontrahierungszwang liegt vor, wenn die Weigerung, eine Eingliederungsvereinbarung abzuschließen, bei der einseitigen Entscheidung über Eingliederungsleistungen gem. $\S 15$ Abs. 1 S. 6 SGB II berücksichtigt werden muss. Dann entstünde zusätzlicher Druck auf den Hilfebedürftigen, weil sich seine Position möglicherweise verschlechtert. Ist dies das berühmte „Tüpfelchen auf dem i“, das dazu führt, dass von Seiten des Arbeitsuchenden keine freiwillige Erklärung abgegeben wird? Angesichts der Tatsache, dass die Weigerung, eine angebotene Eingliederungsvereinbarung abzuschließen, bereits Konsequenzen für das Arbeitslosengeld II hat, weitere Sanktionen vom Gesetzgeber aber nicht angeordnet sind, ist die Einbeziehung der Weigerung in die Abwägung bei der einseitigen Entscheidung nicht zulässig. Zuviel an Sanktion zerstört die Motivation des Arbeitsuchenden. Sie trägt ferner der Behandlung des Arbeitsuchenden als Kunden und Klienten nicht Rechnung, die auf ein Miteinander abzielt.

Zudem ist aus Sicht des Hilfebedürftigen das „Drohpotenzial“ eines Verwaltungsaktes nicht besonders hoch. Die Eingliederungsmaßnahmen werden dann einseitig angeordnet, die Ermessensentscheidungen sind aber auf ihre Rechtmäßigkeit hin vollumfänglich gerichtlich nachprüfbar. Obwohl es für den Arbeitsuchenden eine größere Einflussnahme bedeutet, wenn eine Eingliederungsvereinbarung geschlossen wird ${ }^{684}$, hält sich der sich aus einem Verwaltungsakt ergebende Nachteil des Hilfebedürftigen trotzdem in Grenzen. Ein Eingriff in seine Entscheidungsfreiheit lässt sich damit jedenfalls nicht begründen.

\section{(7) Kontrahierungszwang im Rahmen des $\S 31$ Abs. 5 SGB II}

Problematisch ist die Situation bei $\S 31$ Abs. 5 SGB II. Dieser ordnet einen noch stärkeren faktischen Zwang an, eine Eingliederungsvereinbarung abzuschließen. Denn bei erwerbsfähigen Hilfebedürftigen, die das 15. Lebensjahr, jedoch nicht das 25. Lebensjahr vollendet haben, treten gewichtigere Sanktionen ein. ${ }^{685}$ Auf der anderen Seite besteht bei diesem Personenkreis die Verpflichtung der Arbeitsverwaltung, unverzüglich eine Arbeit, Ausbildung oder Arbeitsgelegenheit anzubieten. Aus diesem Grund erscheint die Verpflichtung, die Ergebnisse des Profiling schriftlich festzuhalten, um

dung kann die Eingliederungsvereinbarung ein sinnvolles und hilfreiches Mittel auf dem Weg sein, bei schwieriger Arbeitsmarktlage dem Ziel einer Eingliederung in Arbeit näher zu kommen.“

684 Vgl. dazu zweites Kapitel B II 5a) aa) S. 199 ff.

685 Vgl. zweites Kapitel B II 7c) dd) S. 224. 
den Eingliederungserfolg einer vermittelten Arbeit nicht zu gefährden, sinnvoll und ausgewogen. 686

\section{dd) Begründung, Änderung oder Aufhebung von Rechten und Pflichten}

Werden durch die Eingliederungsvereinbarung nach SGB II tatsächlich Rechte und Pflichten begründet, geändert oder aufgehoben?

Die Aufhebung setzt voraus, dass durch den Vertrag ein Rechtsverhältnis beseitigt wird. ${ }^{687}$ Eine Aufhebung scheidet aber aus, denn das Rechtsverhältnis zwischen Arbeitsuchendem und Agentur für Arbeit, das entstand, als sich der erwerbsfähige Hilfebedürftige z.B. durch einen Antrag auf Arbeitslosengeld II an die Agentur für Arbeit wandte, besteht weiter fort. Es wird ausgefüllt durch die Erbringung von Anspruchsleistungen wie das Arbeitslosengeld II oder anderen Leistungen zur Sicherung des Lebensunterhalts und von Leistungen zur Eingliederung, die durch die Eingliederungsvereinbarung vereinbart oder durch einen Verwaltungsakt angeordnet wurden.

Die Begründung eines Rechtsverhältnisses, mithin der Abschluss eines Verpflichtungsvertrages liegt vor, wenn sich ein Teil des Vertrages zu einer noch zu erbringenden Leistung verpflichtet. Der Verpflichtungsvertrag schafft ein Schuldverhältnis und begründet damit Rechte und Pflichten der Vertragspartner zueinander. Eine solche Verpflichtung kann auf Seiten der Behörde in dem Erlass eines Verwaltungsaktes oder einem sonstigen Verwaltungshandeln bestehen, auf Seiten des Bürgers kommt jede im Zivilrecht oder im öffentlichen Recht mögliche Leistung in Betracht. ${ }^{688}$ Die Eingliederungsvereinbarung soll verbindliche Aussagen zum Fördern und Fordern des Erwerbsfähigen enthalten und sie soll das Sozialrechtsverhältnis zwischen Erwerbsfähigem und der Agentur für Arbeit konkretisieren. ${ }^{689}$ Anders als in der Gesetzesbegründung zu $§ 35$ Abs. 4 SGB III heißt es nicht, dass kein neues Rechtsverhältnis begründet wird. Daraus kann man schließen, dass die Vereinbarung der Eingliederungsstrategie dazu führt, dass die Pflichten des SGB II, die sehr allgemein formuliert sind und angesichts der drohenden Sanktionen keine große Rechtssicherheit bieten, auf die im Einzelfall anwendbaren konzentriert und damit vertraglich begründet werden.

Der Vorgang kann auch als inhaltliche Änderung betrachtet werden. ${ }^{690}$ Dies setzt voraus, dass das Rechtsverhältnis durch den verwaltungsrechtlichen Vertrag inhaltlich umgestaltet wird. ${ }^{691}$ Sieht man die Pflichten des SGB II trotz der weiten Ermessensspielräume der Verwaltung als ausreichend konkret an, werden sie durch die Vereinbarung lediglich weiter konkretisiert und inhaltlich verbindlich gemacht. Dies gilt insbe-

686 Münder, in: Münder/Armborst, LPK-SGB II, 2005, Einl. Rn. 18; a.A. Vor, in: Estelmann, SGB II, § 3 Rn. 31 spricht von sozialer Verelendung infolge dieser bloß finanziellen Sanktionen, ohne auf der anderen Seite Hilfe anzubieten. Er bezieht aber nicht in die Tatsache mit ein, dass $\S 3$ Abs. 2 S. 1 SGB II die stärkere Pflicht zur Förderung des Jugendlichen enthält, indem er unverzüglich in eine Arbeit, Ausbildung beziehungsweise Arbeitsgelegenheit vermittelt werden muss.

687 Bonk, in: Stelkens/Bonk/Sachs, VwVfG, 2001, § 54 Rn. 82.

688 Pickel/Marschner, SGB X, Bd. 1, § 53 Rn. 28 ff.

689 BT-Drucksache 15/1516, S. 54.

690 Berlit, SozR akt 2006, S. 42.

691 Bonk, in: Stelkens/Bonk/Sachs, VwVfG, 2001, § 54 Rn. 82. 
sondere für die Mitwirkungspflichten des Arbeitslosen, deren Grundlage nach Abschluss der Eingliederungsvereinbarung diese selbst wird. ${ }^{692}$

ee) Einordnung als Vertrag i.S.d. § 53 Abs. 1 S. 2 SGB X

Die Eingliederungsvereinbarung ist als Vertrag i.S.d. § 53 Abs. 1 S. 2 SGB X einzuordnen. ${ }^{693}$ Er wird zwischen Agentur für Arbeit und Einzelnem geschlossen und ist vorrangig gegenüber einer Regelung durch Verwaltungsakt, die gem. § 15 Abs. 1 S. 6 SGB II nur bei Fehlschlagen einer Einigung erlaubt ist. Mit der Bezugnahme auf eine Regelung durch Verwaltungsakt in $\S 53$ Abs. 2 SGB X wird lediglich klargestellt, dass es um das Verhältnis eines Einzelnen mit der Verwaltung geht, die klassischerweise mittels Verwaltungsakt gestaltet wird. Ein solcher Fall liegt hier vor. ${ }^{694}$

Es handelt sich nicht um einen Austauschvertrag i.S.d. § 55 SGB X, für den der Ausschluss des $\S 53$ Abs. 2 SGB X, also der Ausschluss des verwaltungsrechtlichen Vertrages für Anspruchsleistungen, nicht gelten würde, § 55 Abs. 3 SGB III. ${ }^{695}$ Ein Austauschvertrag setzt voraus, dass Leistung und Gegenleistung im synallagmatischen Verhältnis zueinander stehen und sich die Gegenleistung des Vertragspartners der Behörde aus der Vereinbarung ergibt und nicht bereits aus dem Gesetz. ${ }^{696}$ Die Eingliederungsleistungen werden nicht wegen der Eingliederungsbemühungen des Hilfebedürftigen erbracht und die Gegenleistung des Bürgers ist schon als Zweck der Verwaltungsleistung in den $\S 15$ Abs. 1 Nr. 2, 31 Abs. 1 Nr. 1 SGB II sowie als Mitwirkungspflicht gem. $\S \S 60$ ff SGB I gesetzlich vorgesehen. ${ }^{697}$ Damit wird sie nicht mehr vertraglich vereinbart und ist keine echte Gegenleistung i.S.d. § 55 SGB X.

Es liegt auch kein „hinkender Austauschvertrag“ 698 vor. Dieser ist eine besondere Form des Austauschvertrags im Sinn des $\S 55$ SGB X und setzt wie dieser voraus, dass sich die Gegenleistung aus dem Vertrag ergibt, was gerade nicht angenommen wird. ${ }^{699}$

692 Spellbrink, SozR akt 2006, S. 53 ff knüpft daran seine These vom normenkonkretisierenden Verwaltungshandeln sui generis und behauptet, dass der Vertrag zwischen Hilfebedürftigem und Arbeitsverwaltung Fiktion sei. Ihn treiben v.a. Rechtsschutzaspekte. Diese können auch anders befriedigend gelöst werden, z.B. durch Zulassung einer Feststellungsklage zur Überprüfung der Nichtigkeit der Eingliederungsvereinbarung. Vgl. zweites Kapitel B II 10c) dd) S. 240.

693 Vgl. erstes Kapitel B IV 1b) S. 84. Dort wurde geklärt, dass der Begriff „,subordinationsrechtlicher Vertrag“ die Stellung des Bürgers in der Vertragsbeziehung als Über - Unterordnungsverhältnis und deshalb unangemessen umschreibt. Er wird aus diesem Grund in vorliegender Arbeit nicht verwendet, sondern die Formulierung „Vertrag i.S.d. $§ 53$ Abs. 1 S. 2 SGB X“.

694 Vgl. zweites Kapitel B II 2b) S. 188 f.

695 Luthe/Timm, SGb 2005, S. 261; Berlit, SozR akt 2006, S. 42; a.A. Knoblauch/Hübner, NDV 2005, S. 278; Becker, Sozialmodell und Menschenbild in der „Hartz-IV” Gesetzgebung, in: Behrends, Gesetzgebung, Menschenbild und Sozialmodell im Familien- und Sozialrecht, 2008, S. 47 wegen fehlender Kontrolle, ob es sich um gesetzliche Pflichten handelt.

696 Klückmann, SGb 1979, S. 413; Becker, in: Hauck/Noftz, SGB X, K § 55 Rn. 16 ff.

697 Blüggel, SozSich 2005, S. 12 ff.

698 Engelmann, in: Wulffen, SGB X, 2008, § 55 Rn. 11.

699 So aber Berlit, in: Münder/Armborst, LPK-SGB II, 2005, § 15 Rn. 9; ders., SozR akt 2006, S. 42. 
d) Zwischenergebnis

Die Eingliederungsvereinbarung nach SGB II ist als verwaltungsrechtlicher Vertrag, insbesondere als Vertrag i.S.d. § 53 Abs. 1 S. 2 SGB X einzuordnen.

\section{Funktion der Eingliederungsvereinbarung}

Die Eingliederungsvereinbarung gem. § 15 SGB II enthält verbindliche Aussagen zum Fördern und Fordern des erwerbsfähigen Hilfebedürftigen. Gerade diese Verbindlichkeit ist als das wirklich Neue der Eingliederungsvereinbarung nach § 15 SGB II zu bezeichnen. ${ }^{700}$

$\S 15$ SGB II ist die Grundsatznorm, die die wesentlichen Merkmale und das Zustandekommen der Eingliederungsvereinbarung regelt. Er konkretisiert den vorausgehenden $\S 14$ SGB II, der den Grundsatz des Förderns enthält. 701

\section{a) Zusammenführung von Arbeitslosenhilfe und Sozialhilfe}

Mit der Einführung des Zweiten Buches Sozialgesetzbuch wurden die Arbeitslosenhilfe und die Sozialhilfe für erwerbsfähige Personen zusammengeführt. Es entstand eine eigenständige Leistung, die bestimmte Aufgaben der beiden früheren Leistungen erfüllen muss. Diese Gesetzesänderung prägt die Funktion der Eingliederungsvereinbarung nach SGB II. Die Eingliederungsleistungen werden weitgehend unter Rückgriff auf das im SGB III geregelte Recht der aktiven Arbeitsförderung erbracht. Die Leistungen zur Sicherung des Lebensunterhalts, insbesondere das Arbeitslosengeld II, sind dem Sozialhilferecht des früheren BSHG (jetzt SGB XII) nachgebildet. ${ }^{702}$

Die Eingliederungsvereinbarung spielt in beiden Bereichen eine Rolle. Sie betrifft zunächst nur die Leistungen zur Eingliederung, wie der Wortlaut (,die für die Eingliederung erforderlichen Leistungen vereinbaren") und die Stellung im Gesetz (Kapitel 3, Abschnitt 1, Leistungen zur Eingliederung) beweisen. Daneben hat die Eingliederungsvereinbarung infolge der Sanktion in $\S 31$ Abs. 1 Nr. 1a und 1b SGB II auch Bedeutung für die Höhe des Arbeitslosengeldes II, weil das Gesetz eine Absenkung beziehungsweise den Wegfall des Arbeitslosengeldes II vorsieht, wenn der erwerbsfähige Hilfebedürftige sich weigert, eine Eingliederungsvereinbarung abzuschließen oder vereinbarte Pflichten einzuhalten. 703

Aufgrund der Tatsache, dass im SGB II beide Aufgaben - Eingliederung und Sicherung des Lebensunterhaltes - erfüllt werden sollen, ist bei der Auslegung der Regelungen, die die Eingliederungsvereinbarung betreffen, zu berücksichtigen, ob es sich um einen Aspekt der Arbeitsförderung oder der Sozialhilfe handelt, der von der Vereinbarung berührt wird. Ferner ist im Rahmen der Auslegung und Anwendung des SGB II zu

700 So auch Luthe/Timm, SGb 2005, S. 261.

701 Stark, in: Estelmann, SGB II, § 15 Rn. 7.

702 Löschau/Marschner, Hartz IV, 2004, Rn. 406.

703 Dazu zweites Kapitel B II 7c) S. 218 ff. 
berücksichtigen, dass es kein unterhalb des SGB II rangierendes Auffangnetz mehr gibt, da die Sozialhilfe aufgrund $\S 5$ Abs. 2 SGB II ausgeschlossen ist. ${ }^{704}$

\section{b) Erwerbsfähigkeit und Hilfebedürftigkeit, §§ 8, 9 SGB II}

In der Eingliederungsvereinbarung können nicht die Begriffe Erwerbsfähigkeit und Hilfebedürftigkeit, $\S \S 8$ und 9 SGB II, näher beschrieben werden. Es ist nicht zulässig, im Profiling gewonnene Ergebnisse zur Prüfung dieser Leistungsvoraussetzungen in der Eingliederungsvereinbarung verbindlich zu machen.

Aus der Systematik des Gesetzes ergibt sich, dass zwischen dem erwerbsfähigen Hilfebedürftigen und der Agentur für Arbeit eine Eingliederungsvereinbarung abgeschlossen werden soll. Damit steht fest, dass, bevor über die Vereinbarung nachgedacht werden kann, die Agentur für Arbeit klären muss, ob die Definitionen der $\S \S 8,9$ SGB II erfüllt sind, § 44a SGB II. ${ }^{705}$ Damit scheidet es aus, dass erst in der Vereinbarung geklärt wird, ob Erwerbsfähigkeit und/oder Hilfebedürftigkeit vorliegen.

\section{c) Unbestimmte Rechtsbegriffe}

Bedeutung erlangt die Eingliederungsvereinbarung nach SGB II ebenso wie das arbeitsförderungsrechtliche Instrument der Eingliederungsvereinbarung nach SGB III bei der Auslegung unbestimmter Rechtsbegriffe. So ist z.B. der wichtige Grund im Sinn des $\S 31$ Abs. 1 S. 2 SGB II im Lichte der Eingliederungsvereinbarung zu verstehen, auch wenn es nicht um darin festgelegte Pflichten, sondern um andere Sanktionstatbestände geht. Die Vereinbarung enthält Informationen, die bei der Auslegung berücksichtigt werden müssen. Damit zusammen hängen Darlegungs- und Beweislasten vor Gericht, die noch dargestellt werden. ${ }^{706}$

\section{d) Qualitätssicherung und Qualitätsprüfung}

Die Eingliederungsvereinbarung nach SGB II soll für sechs Monate abgeschlossen werden, $\S 15$ Abs. 1 S. 3 SGB II. Danach soll eine neue Eingliederungsvereinbarung abgeschlossen werden, bei der die bisher gewonnen Erfahrungen zu berücksichtigen sind, § 15 Abs. 1 S. 4, 5 SGB II. Sinn und Zweck dieser Befristung der Vereinbarungen ist es sicherzustellen, dass eine intensive Betreuung stattfindet und zeitnah überlegt wird, ob die vereinbarten Maßnahmen immer noch der beruflichen Eingliederung des Hilfebedürftigen zu dienen geeignet erscheinen. ${ }^{707}$ Dementsprechend stellt die Eingliederungsvereinbarung auch für den Rechtskreis des SGB II ein Instrument der Qualitäts-

704 Münder, in: Münder/Armborst, LPK-SGB II, 2005, Einl. Rn. 25.

705 Nicht weiter vertieft wird an dieser Stelle die Zuständigkeit einer gemeinsamen Einigungsstelle gem. § 45 i.V.m. § 44a SGB II bei Streitigkeiten über die Erwerbsfähigkeit oder Hilfebedürftigkeit, die im Geflecht der Zuständigkeiten zwischen Agentur für Arbeit, kommunalen Trägern und den bei voller Erwerbsminderung zuständigen Leistungsträgern entstehen können. Dazu Mrozynski, ZfSH/SGB 2004, S. 201; Blüggel, SGb 2005, S. 377 ff; Schoch, NDV 2006, S. 512 ff.

706 Vgl. zweites Kapitel B II 7e) S. 225.

707 BT-Drucksache 15/1516, S. 54. 
sicherung für die Agenturen für Arbeit dar. ${ }^{708}$ Die Bundesagentur für Arbeit kann mit Hilfe der abgeschlossenen Eingliederungsvereinbarungen Informationen über Leistungen zur Eingliederung und deren Entwicklung erhalten und dadurch die Effektivität der unteren örtlichen Ebene kontrollieren. 709

\section{Wirkungen der Eingliederungsvereinbarung}

a) Voraussetzung für Leistungen der Grundsicherung für Arbeitsuchende a) Leistungen zur Eingliederung in Arbeit, $\$ \S 14$ ff SGB II

Für Leistungen zur Eingliederung in Arbeit gem. § 16 SGB II ist der Abschluss einer Eingliederungsvereinbarung nicht Voraussetzung.

Gem. § 15 Abs. 1 S. 1 SGB II „soll“ die Agentur für Arbeit mit dem erwerbsfähigen Hilfebedürftigen eine Eingliederungsvereinbarung abschließen und darin die Leistungen zur Eingliederung vereinbaren. Ein „soll“ ist nach allgemeinen Grundsätzen ein „Muss“, von dem die Verwaltung nur in begründeten atypischen Fällen abweichen darf. ${ }^{710}$ Das würde bedeuten, dass Eingliederungsleistungen de facto ohne eine Eingliederungsvereinbarung nicht gewährt werden können.

Dem steht jedoch $\S 15$ Abs. 1 S. 6 SGB II entgegen, der vorsieht, dass das, was normalerweise Inhalt der Eingliederungsvereinbarung wäre, durch Verwaltungsakt geregelt werden soll, wenn eine Einigung nicht zustande kommt. Diese Entscheidung hat die Verwaltung einseitig-hoheitlich nach pflichtgemäßem Ermessen zu treffen. Daraus lässt sich ableiten, dass ein erwerbsfähiger Hilfebedürftiger trotzdem Leistungen zur Eingliederung in Arbeit erhalten kann, auch wenn keine Eingliederungsvereinbarung zustande kommt.

Es ist ferner aus dem Wortlaut des $\S 16$ SGB II nicht ablesbar, dass irgendeine Form der Anordnung mit dem Inhalt des $§ 15$ SGB II für die Gewährung von Leistungen zur Eingliederung Voraussetzung ist. Es ist also denkbar, dass die Arbeitsverwaltung Leistungen zur Eingliederung gewährt, ohne gleichzeitig die Eigenbemühungen des Arbeitsuchenden konkretisiert zu haben. Dieses Vorgehen entspräche zwar nicht der Intention des Gesetzgebers, der die Eingliederungsvereinbarung als Instrument der Eingliederung ansieht, wäre aber mit den geltenden Vorschriften vereinbar. ${ }^{711}$

Es verbleibt somit der Arbeitsverwaltung die Möglichkeit, der Erbringung von Leistungen zur Eingliederung Vorrang vor der verfahrensmäßigen Einbeziehung und Regelung der Pflichten des Arbeitsuchenden einzuräumen.

708 Brühl/Hofmann, Sozialgesetzbuch Zweites Buch, 2004, S. 102.

709 Problematisch sind dabei datenschutzrechtliche Aspekte. Dazu Müller-Thele, NJW 2005, S. 1541 ff; Stahlmann, info also 2006, S. $10 \mathrm{ff}$.

710 BVerwGE 49, 16 [23]; 64, 318 [323]; 90, 88 [93]; an Stelle vieler Maurer, Allgemeines Verwaltungsrecht, 2009, S. 136 f; in diesem Zusammenhang auch Stark, in: Estelmann, SGB II, § 15 Rn. 59.

711 So auch Stark, in: Estelmann, SGB II, § 15 Rn. 69. 
Der Abschluss einer Eingliederungsvereinbarung ist nicht Leistungsvoraussetzung für den Bezug von Leistungen zur Sicherung des Lebensunterhaltes. Die Argumentation wird beispielhaft an den Voraussetzungen des Arbeitslosengeldes II gem. §§ $19 \mathrm{ff} \mathrm{SGB}$ II vollzogen, ist aber auf die anderen Leistungen zur Sicherung des Lebensunterhaltes übertragbar.

Gem. § 19 SGB II erhalten erwerbsfähige Hilfebedürftige als Arbeitslosengeld II grundsätzlich Leistungen zur Sicherung des Lebensunterhaltes einschließlich der angemessenen Kosten für Unterkunft und Heizung. Daraus ergibt sich, dass Voraussetzung lediglich die Berechtigung nach § 7 SGB II ist, nämlich insbesondere die Erwerbsfähigkeit, die Hilfebedürftigkeit und der gewöhnliche Aufenthalt in der Bundesrepublik Deutschland. Auf die Prüfung der Erwerbsfähigkeit gem. § 8 SGB II und der Hilfebedürftigkeit gem. $\S 9$ SGB II hat die Eingliederungsvereinbarung keinen Einfluss ${ }^{712}$, genauso wenig auf das Vorliegen des gewöhnlichen Aufenthaltes.

\section{cc) Zwischenergebnis}

Die Eingliederungsvereinbarung ist nicht Voraussetzung für die Leistungen nach SGB II. Es geht aber aus den Vorschriften der klare Wille des Gesetzgebers hervor, dass die Eingliederungsvereinbarung als Instrument genutzt werden muss, auch wenn deren Aushandlung und Abschluss im Einzelfall ein aufwändigeres Verfahren erfordert als eine einseitige Regelung durch Verwaltungsakt.

\section{b) Ermessensausübung}

Im Folgenden werden die Auswirkungen einer abgeschlossenen Eingliederungsvereinbarung auf die Ermessensausübung dargestellt. Es ergeben sich verschiedene Unterschiede zur Eingliederungsvereinbarung nach $\S 35$ Abs. 4 SGB III, deren Wirkungen besonders im Rahmen der Ermessensausübung deutlich werden.

\section{aa) Ermächtigung}

Ein Ermessensspielraum besteht grundsätzlich bei allen Eingliederungsleistungen ${ }^{713}$, $\S 16$ Abs. 1-4 SGB II. Danach „kann“ die Agentur für Arbeit bestimmte Leistungen zur Eingliederung gewähren. Zur Verfügung steht im Wesentlichen das Eingliederungsinstrumentarium des Arbeitsförderungsrechts aufgrund der Verweisung auf bestimmte Vorschriften des SGB III; daneben gibt es eigenständige, dem SGB II vorbehaltene Eingliederungsleistungen. Diesen Ermessensspielraum deutet auch $\S 3$ Abs. 1 S. 1 SGB II aus, in dem die Leistungsgrundsätze festgelegt sind.

Der Ermessensspielraum erstreckt sich sowohl auf das Entschließungs- als auch auf das Auswahlermessen. ${ }^{714}$ Das Entschließungsermessen ist insofern beschränkt, als sich

712 Vgl. zweites Kapitel B II 6b) S. 213.

713 Eine Ausnahme bilden Eingliederungsleistungen auf der Grundlage des $§ 16$ Abs. 1 S. 3 SGB II, auf die bei Vorliegen der Voraussetzungen ein Anspruch besteht.

714 So auch Lehmann-Franßen, NZS 2005, S. 522. 
aus $\S 3$ Abs. 3 SGB II ein Vorrang der Leistungen zur Eingliederung gegenüber Leistungen zur Sicherung des Lebensunterhaltes ergibt. ${ }^{715}$

Leistungen zur Sicherung des Lebensunterhaltes gem. $\S 19$ ff SGB II sind dagegen als Ansprüche ausgestaltet (,erhalten“), sodass ein Ermessensspielraum der Agentur für Arbeit für diese Leistungen nicht besteht.

Das gilt auch für die Vermittlung von erwerbsfähigen Hilfebedürftigen, die das 25. Lebensjahr noch nicht erreicht haben. Sie müssen unverzüglich nach Antragstellung auf Leistungen nach dem SGB II in eine Arbeit, eine Ausbildung oder eine Arbeitsgelegenheit vermittelt werden, $\S 3$ Abs. 2 S. 1, 16 Abs. 1 S. 1 SGB II i.V.m. $\S 33$ ff SGB III. Damit ist ein Anspruch auf diese Leistung zur Eingliederung begründet 716 und das besondere Interesse des Gesetzgebers an dieser Personengruppe ${ }^{717}$ und der Wille, diese in entschlossener Weise zu fördern, in Gesetzesform gefasst. 718

Die anderen Leistungen zur Eingliederung sind auch bei Hilfebedürftigen unter 25 Jahren Ermessensleistungen.

\section{bb) Zweck der Ermächtigung}

$\S 1$ SGB II beschreibt den Zweck der Einräumung von Ermessen für die Aufgaben und Ziele der Grundsicherung für Arbeitsuchende: Die Grundsicherung für Arbeitsuchende soll die Eigenverantwortung von erwerbsfähigen Hilfebedürftigen stärken und dazu beitragen, dass sie ihren Lebensunterhalt unabhängig von der Grundsicherung für Arbeitsuchende aus eigenen Mitteln und Kräften bestreiten. Sie soll erwerbsfähige Hilfebedürftige bei der Aufnahme oder Beibehaltung einer Erwerbstätigkeit unterstützen und den Lebensunterhalt sichern, soweit sie ihn nicht auf andere Weise bestreiten können. Das bedeutet, dass bei der Entscheidung über Leistungen zur Eingliederung beide Komponenten, das Fördern und das Fordern in ein angemessenes Maß gebracht werden müssen, wobei die Verantwortung des Einzelnen, selbst für den eigenen Lebensunterhalt zu sorgen, betont wird. Darin liegt sicherlich eine Abkehr von Grundsätzen, wie sie das Recht der Arbeitslosenhilfe kannte, denn dort wurde die Beziehung zu einer einmal ausgeübten Beschäftigung als ausreichend erachtet, auf der Grundlage des damaligen Einkommens eine soziale Leistung auszuzahlen. Das betraf auch wiedereingliedernde Maßnahmen. ${ }^{719}$ Das Ermessen eröffnet Spielräume für die flexible Auswahl des richtigen Instrumentes für den Einzelfall.

715 Vor, in: Estelmann, SGB II, § 3 Rn. 16.

716 Problematisch und umstritten ist allerdings die Einklagbarkeit dieses Anspruches, weil in den verschiedenen die Leistungen zur Eingliederung regelnden Vorschriften, insbesondere $\S 3$ Abs. 1 SGB II („Kann-Vorschrift“), § 3 Abs. 2 SGB II (,Ist-Bestimmung“) und § 16 Abs. 3 SGB II („SollBestimmung") jeweils unterschiedliche Maßstäbe zu finden sind. Die Diskussion zusammenfassend bedeutet dies, dass die Altersgruppe unter 25 Jahren bei der Vermittlung bevorzugt behandelt werden muss, ohne dass sie die Schaffung einer Arbeit, Ausbildung oder Arbeitsgelegenheit einklagen könnte. Dazu Luthe, in: Hauck/Noftz, SGB II, K § 3 Rn. 65 f.

717 BT-Drucksache 15/1516, S. 51.

718 Luthe, in: Hauck/Noftz, SGB II, K § 3 Rn. 64; Vor, in: Estelmann, SGB II, § 3 Rn. 1.

719 Dazu Ebsen, Die Arbeitslosenhilfe zwischen Sozialversicherung und Fürsorge, in: Ebsen, Stand und Entwicklung des Arbeitsförderungsrechts, 1997, S. 162 ff, 167. 
Im Recht der Grundsicherung für Arbeitsuchende ist $\S 3$ SGB II, der Leistungsgrundsätze für die Erbringungen von Leistungen zur Eingliederung in Arbeit enthält, als Grenze für die Ermessensausübung anzusehen.

Gem. § 3 Abs. 1 S. 1 SGB II kann eine Leistung erbracht werden, wenn sie zur Vermeidung oder Beseitigung, Verkürzung oder Verminderung der Hilfebedürftigkeit für die Eingliederung erforderlich ist. In $\S 3$ Abs. 1 S. 2 SGB II sind als konkrete Kriterien für die Ermessensausübung die Eignung, die individuelle Lebenssituation, insbesondere die familiäre Situation, die voraussichtliche Dauer der Hilfebedürftigkeit und die Dauerhaftigkeit der Eingliederung des erwerbsfähigen Hilfebedürftigen genannt.

Ferner sind gem. § 3 Abs. 1 S. 3 SGB II die Grundsätze der Wirtschaftlichkeit und Sparsamkeit einzuhalten. ${ }^{720}$ Interessant bleibt, da die Rechtsprechung hierzu sich oftmals noch auf das Arbeitsförderungsrecht bezieht, ob es grundsätzlich nicht zu beanstanden ist, wenn die Verwaltung die Haushaltslage in die Ermessensentscheidung einbezieht, solange sie die Ablehnung der Maßnahme nicht allein auf die Erschöpfung der Haushaltsmittel stützt. ${ }^{721}$

\section{dd) Grenzen aus der Eingliederungsvereinbarung}

Die verbindliche Eingliederungsvereinbarung ist als öffentlich-rechtlicher Vertrag zu qualifizieren. ${ }^{722}$ Die Verwaltung hat das Ermessen gem. $§ 39$ Abs. 1 SGB I pflichtgemäß auszuüben. Enthält die Eingliederungsvereinbarung eine konkret bestimmte Leistung, ergibt sich daraus für den Hilfebedürftigen ein Vertragserfüllungsanspruch. ${ }^{723}$ Rechtsgrundlage für die Leistung ist insoweit dann der Vertrag. Eine Ermessensausübung findet nicht mehr statt.

Es gibt aber auch Eingliederungsvereinbarungen, die keine in der Weise konkretisierten Leistungen vorsehen, dass bereits ein Erfüllungsanspruch entstünde. In diesem Fall ist der Ermessensspielraum der Behörde bezogen auf das Vereinbarte auszuüben und auf die Regelungen in der Eingliederungsvereinbarung abzustimmen. Es können daher die Voraussetzungen einer Ermessensreduzierung auf Null eintreten. Enthält die Eingliederungsvereinbarung nämlich so detaillierte Aussagen über das Fördern und Fordern und die zu erbringenden Eingliederungsleistungen, ohne ein Maßnahme zu benennen, ist es gleichwohl möglich, dass nur noch eine Entscheidung Trägers der Grundsicherung für Arbeitsuchende ermessensfehlerfrei ergehen kann. Das bedeutet, dass bei der letztendlich ergehenden Anordnung der Maßnahme die Behörde kein Auswahlermessen mehr hat, sondern nur noch das Vereinbarte in einer einseitigen Anordnung ausspricht.

720 Vgl. zu den Definitionen zweites Kapitel B I 7b) dd) S. 169.

721 BSG, Urteil v. 25.10.1990, 7 RAr 14/90; BSG, Urteil v. 27.6.1996, 11 RAr 97/95; dazu Voelzke, in: Hauck/Noftz, SGB II, K § 16 Rn. 14.

722 Vgl. zweites Kapitel B II 5c) S. $201 \mathrm{ff}$.

723 Berlit, in: Münder/Armborst, LPK-SGB II, 2005, § 15 Rn. 12, der auch eine vertragliche Zusicherung gem. § 34 SGB X anspricht. 
Zu klären ist das Verhältnis der Grundsätze der Ermessensfehlerlehre zur Nichtigkeitskontrolle gem. § 58 SGB X. Problematisch ist dabei, dass ein Vertrag inhaltlich nicht mit derselben Kontrolldichte überprüft werden kann wie ein Verwaltungsakt, und es deshalb zu unterschiedlichen Ergebnisses kommen kann, wenn eine Ermessensausübung überprüft oder eine Nichtigkeitskontrolle gem. § 58 SGB X durchgeführt wird. 724

Ermessensentscheidungen sind von den Gerichten unter den Gesichtspunkten der Ermessensfehlerlehre überprüfbar. Abweichend vom Grundsatz in $\S 40$ Abs. 2 SGB I entsteht der Anspruch auf fehlerfreie Ermessensausübung gem. § 39 Abs. 2 SGB I zum Zeitpunkt des Abschlusses der Eingliederungsvereinbarung und nicht bei Bekanntgabe der Entscheidung. ${ }^{725}$ Das bedeutet, dass die Behörde bereits beim Abschluss der Eingliederungsvereinbarung die für die Ausübung ihres Ermessens relevanten Gesichtspunkte sammeln, überdenken und berücksichtigen muss.

Daraus folgt, dass Nichtigkeitskontrolle und Ermessensentscheidung an denselben Zeitpunkt anknüpfen. Ob trotzdem eine unterschiedliche Bewertung eintritt, hängt davon ab, wie Rechtsverstöße im Rahmen der Ermessensausübung bei der Nichtigkeitskontrolle gem. $§ 58$ SGB X behandelt werden. ${ }^{726}$

\section{c) Verhängung von Sanktionen, § 31 SGB II}

$\S 31$ SGB II ist überschrieben mit den Worten „Absenkung und Wegfall des Arbeitslosengeldes II“. Dem Grundsatz des Förderns und Forderns entsprechend soll der erwerbsfähige Hilfebedürftige verpflichtet werden, konkrete Schritte zur Behebung seiner Hilfebedürftigkeit zu unternehmen. Die Verknüpfung einer finanziellen, für den erwerbsfähigen Hilfebedürftigen negativen Folge mit einem Verhalten, das sich dadurch auszeichnet, dass der erwerbsfähige Hilfebedürftige nicht alle Möglichkeiten zur Beendigung oder Verringerung seiner Hilfebedürftigkeit ausschöpft, konkretisiert den Grundsatz des Forderns, der in § 2 SGB II verankert ist. ${ }^{727}$ Ähnlich wie die Sperrzeiten versteht der Gesetzgeber das Instrument positiv und nicht als Bestrafungsinstrument. ${ }^{728}$ Es liegt in der Intention des Gesetzgebers, zum geforderten Verhalten zu motivieren. $\mathrm{Ob}$ jener Motivationsversuch erfolgreich sein kann, bleibt abzuwarten, wird aber vehement bestritten und der Vorschrift stattdessen ein ordnungsrechtlicher Charakter zugewie-

724 Luthe, in: Hauck/Noftz, SGB II, K § 3 Rn. 8.

725 Luthe, in: Hauck/Noftz, SGB II, K § 3 Rn. 11.

726 Vgl. zweites Kapitel B II 8a) cc) S. 228 ff.

727 BT-Drucksache 15/1516, S. 60; Berlit, ZFSH/SGB 2005, S. 707 ff; 2006, S. 11 ff spricht vom „,neuen Sanktionensystem“.

728 So heißt es in der Gesetzesbegründung BT-Drucksache 15/1516, S. 47, dass die Aufnahme einer Erwerbstätigkeit nicht nur über Anreize gefördert, sondern auch mit Hilfe von Sanktionen gefordert wird, während der Zugang zu Beratung und Betreuung stets erhalten bleibt. Dazu Valgolio, in: Hauck/Noftz, SGB II, K § 31 Rn. 6. 
sen. ${ }^{729}$ Zur Formulierung in $\S 2$ SGB II wird angemerkt, dass die die Verwaltung treffenden Pflichten kaum erwähnt und jedenfalls nicht in ähnlicher Weise betont werden, wie die der Hilfebedürftigen. ${ }^{730}$ Dies setzt sich in der Sanktionsvorschrift des $\S 31$ SGB II fort, der „Fehlverhalten“ der Verwaltung nicht gesondert sanktioniert.

§ 31 SGB II zählt in den Absätzen 1-5 verschiedene Tatbestände auf, bei deren Erfüllung das Arbeitslosengeld II aufgrund der gesetzgeberischen Entscheidung gesenkt wird und der Zuschlag nach § 24 SGB II wegfällt. Dabei hat der Gesetzgeber bewusst verschiedene Prozentsätze der Absenkung eingeführt und die wiederholte Pflichtverletzung stärker als die einfache sanktioniert, um so seine Bewertung des Schweregrads der Pflichtverletzung deutlich zu machen. ${ }^{731}$ Seit 1.1.2007 hat der Gesetzgeber den Sanktionstatbestand in $\S 31$ SGB II wesentlich verschärft. Ab der zweiten Wiederholung innerhalb eines Jahres wird das Arbeitslosengeld II um 100 vom Hundert gemindert. Das heißt, es werden auch keine Kosten für Unterkunft und Heizung mehr übernommen, § 31 Abs. 1 S. 4 SGB II. Bei nachträglicher Einhaltung entfällt die Sanktion nicht vollständig, sondern kann lediglich auf 60 Prozent begrenzt werden, § 31 Abs. 1 S. 5 SGB II. Um der staatlichen Pflicht, das Existenzminimum zu gewährleisten ${ }^{732}$, gerecht zu werden, hat der Gesetzgeber bei einer Absenkung von mehr als 30 vom Hundert vorgesehen, dass die Erbringung von ergänzenden Sachleistungen oder geldwerten Leistungen im Ermessen des zuständigen Trägers steht und z.B. in Form von Lebensmittelgutscheinen erfüllt werden kann, $\S 31$ Abs. 3 S. 6 SGB II. 733 Diese Sachleistung ist ausreichend, um das Existenzminimum zu garantieren, das durch das Sozialstaatsprinzip in Verbindung mit dem Schutz der Menschenwürde (Art. 20 Abs. 1 i.V.m. Art. 1 Abs. 1 GG) verfassungsrechtlich verankert ist. 734

In $\S 31$ Abs. 6 SGB II werden Beginn und Dauer der Absenkung und des Wegfalls von Arbeitslosengeld II geregelt. So ist die Sanktion auf einen Zeitraum von drei Monaten beschränkt, beginnend mit dem Kalendermonat, der auf den Monat folgt, in dem der Feststellungsbescheid über die Absenkung wirksam, also grundsätzlich bekannt gege-

729 Dauber, in: Mergler/Zink, SGB II, § 31 Rn. 3, der wegen der Formulierung „Sanktionen“ auf den ordnungsrechtlichen Charakter hinweist.

730 Berlit, in: Münder/Armborst, LPK-SGB II, 2005, § 2 Rn. 4.

731 BT-Drucksache 15/1516, S. 60.

732 Vgl. zweites Kapitel B II 1c) bb) S. 186; allgemein zum Existenzminimum Neumann, NVwZ 1995, S. 426 ff; Sartorius, Das Existenzminimum im Recht, 2000; Könemann, Der verfassungsunmittelbare Anspruch auf das Existenzminimum, 2005; Soria, JZ 2005, S. 644 ff; Wallerath, JZ 2008, S. 157 ff.

733 Um die mit dem Hilfebedürftigen in einer Bedarfsgemeinschaft lebenden minderjährigen Kinder nicht mit der Absenkung allzu stark zu belasten, ist das Ermessen zur Erbringung von ergänzenden Sachleistungen durch eine „Soll-Vorschrift“ stärker gebunden, § 31 Abs. 3 S. 7 SGB II. Vgl. BTDrucksache 15/1516, S. 61.

734 Deshalb wird ein Verstoß überwiegend abgelehnt. Vgl. Berlit, in: Münder/Armborst, LPK-SGB II, 2005, § 31 Rn. 12; O'Sullivan, SGb 2005, S. 370 f; Luthe, SGb 2006, S. 637 ff; a.A. Krahmer, ZfF 2004, S. 180 ff; Mrozynski, ZFSH/SGB 2004, S. 219; zusammenfassend Becker, Sozialmodell und Menschenbild in der „Hartz-IV” Gesetzgebung, in: Behrends, Gesetzgebung, Menschenbild und Sozialmodell im Familien- und Sozialrecht, 2008, S. 64. Allgemein zum argumentativen Umgang mit der Würde des Menschen Hassemer, EuGRZ 2005, S. 300 ff. 
ben wird. Daneben wird klargestellt, dass während der Absenkung beziehungsweise des Wegfalls kein Anspruch auf ergänzende Hilfe zum Lebensunterhalt nach den Vorschriften des SGB XII besteht, § 31 Abs. 6 S. 3 SGB II.

\section{aa) Ablehnung einer angebotenen Vereinbarung, § 31 Abs. 1 Nr. Ia SGB II}

Gem. § 31 Abs. 1 S. 1 Nr. 1a SGB II wird das Arbeitslosengeld II in einer ersten Stufe um 30 Prozent der maßgeblichen Regelleistung unter Wegfall des Zuschlags nach $\S$ 24 SGB II abgesenkt, wenn sich der erwerbsfähige Hilfebedürftige trotz vorheriger Belehrung über die Rechtsfolgen ohne wichtigen Grund weigert, eine ihm angebotene Eingliederungsvereinbarung abzuschließen.

\section{(1) Angebot einer Eingliederungsvereinbarung}

Was unter einer angebotenen Eingliederungsvereinbarung im Sinn des $\S 31$ Abs. $1 \mathrm{~S}$. 1 Nr. 1a SGB II zu verstehen ist, ist noch ungeklärt. Die Formulierung darf wohl nicht so verstanden werden, dass der Inhalt einseitig von der Arbeitsverwaltung ausgearbeitet und dann dem Arbeitslosen zur Unterschrift vorgelegt wird. Das würde der gesamten Konstruktion der Eingliederungsvereinbarung als kooperatives Instrument zuwiderlaufen. Andererseits wird deutlich, dass eine Sanktion nur dann möglich ist, wenn die Verwaltung ihrerseits tätig geworden ist. Es ist also nicht zulässig, eine Sanktion zu verhängen, wenn auch von Seiten der Verwaltung nichts getan wurde, um zu einem Verhandlungsergebnis zu kommen. „Angeboten“ heißt deshalb in diesem Fall, dass ein Versuch von Seiten der Verwaltung gemacht wurde, für den konkreten Einzelfall eine einvernehmliche Lösung „,im Gesamtpaket“, das dem Arbeitslosen vorgelegt wurde, zu finden und zu vereinbaren.

\section{(2) Abschlussverweigerung}

Eine Sanktion setzt ferner voraus, dass der erwerbsfähige Hilfebedürftige sich weigert, eine ihm angebotene Eingliederungsvereinbarung abzuschließen. Die Weigerung des erwerbsfähigen Hilfebedürftigen braucht nicht ausdrücklich erklärt zu werden. Es genügt ein Verhalten, das diese Absicht ausdrückt. ${ }^{735}$ Es ist aufgrund der Formulierung „verweigern“ aber nicht ausreichend, dass in Folge subjektiv vorwerfbaren Verhaltens der Vertrag nicht zustande kommt, sondern das Verhalten des Hilfebedürftigen muss seinen Willen ausdrücken, in voller Absicht eine Eingliederungsvereinbarung nicht unterzeichnen zu wollen. ${ }^{736}$

Es wird deshalb empfohlen, vor der Unterzeichnung einer Vereinbarung diese zunächst schriftlich zu fixieren und dann um Zeit zum Überlegen zu bitten, um zu vermeiden, dass man sich voreilig verbindlich $\mathrm{zu}$ etwas verpflichtet, was erst wieder mit Rechtsmitteln - soweit überhaupt möglich - korrigiert werden könnte. ${ }^{737}$ Ein solches Verhalten darf nicht als Weigerung im Sinn des $\S 31$ Abs. 1 Nr. 1a SGB II verstanden

735 Valgolio, in: Hauck/Noftz, SGB II, K § 31 Rn. 10.

736 Rixen, in: Eicher/Spellbrink, SGB II, § 31 Rn. 9.

737 Geiger, Grundsicherung für Arbeitsuchende, in: Plagemann, Münchener Anwaltshandbuch Sozialrecht, 2009, S. 483 unter Berufung auf LSG NRW v. 7.2.2008 - L 7 B 201/07. 
werden. ${ }^{738}$ Es ist vielmehr dem Hilfebedürftigen ein Prüfungsrecht zuzugestehen, das es ihm ermöglicht, die Vereinbarung zu überdenken.

Keine für eine Sanktion relevante Weigerung des erwerbsfähigen Hilfebedürftigen liegt ferner vor, wenn der Hilfebedürftige über die Vereinbarung verhandelt, mit einzelnen Punkten der angebotenen Vereinbarung nicht einverstanden ist, und darüber keine Einigung mit der Arbeitsverwaltung erzielt werden kann. Die Sanktion darf in diesem Fall nicht verhängt werden, weil $\S 15$ Abs. 1 S. 6 SGB II eingreift und damit die Interessen der Agentur für Arbeit durch die Möglichkeit einer einseitigen Regelung im Wege des Verwaltungsakts ausreichend gewahrt sind. ${ }^{739}$ Eine für eine Sanktion relevante Weigerung setzt auch voraus, dass die Behörde sicherstellen kann, dass der Inhalt des Vorgeschlagenen z.B. trotz einer Sprachbarriere tatsächlich verstanden worden ist. ${ }^{740}$

\section{(3) Wichtiger Grund für die Abschlussverweigerung}

Die Prüfung eines wichtigen Grundes gem. $\S 31$ Abs. 1 S. 2 SGB II ${ }^{741}$, der die Abschlussverweigerung rechtfertigen könnte, ist eine zusätzliche Zumutbarkeitskontrol1e. ${ }^{742}$ Der wichtige Grund bezieht sich auf die Weigerung, die vorgeschlagenen Pflichten zu vereinbaren. ${ }^{743}$ Sind die Vorbehalte gegen die Vereinbarung begründbar, woran aufgrund der erforderlichen verfassungskonformen Auslegung des § 31 SGB II keine allzu hohen Anforderungen gestellt werden dürfen ${ }^{744}$, liegt grundsätzlich ein wichtiger Grund gem. $\S 31$ Abs. 1 S. 2 SGB II vor. ${ }^{745}$ Im Rahmen dieses Prüfungspunktes muss zunächst von der Verwaltung und anschließend vom Gericht geprüft werden, ob der Vertragsinhalt mit der Rechtslage vereinbar ist. Es findet also vor Abschluss der Eingliederungsvereinbarung keine Beschränkung auf die Prüfung von Nichtigkeitsgründen statt. ${ }^{746} \S 31$ Abs. 1 S. 2 SGB II stellt somit sicher, dass im Einzelfall bestehende Gründe, die den Abschluss der Eingliederungsvereinbarung aufgrund verfassungsrechtlicher Bedenken verbieten, Beachtung finden und auch als Tatbestandsvoraussetzung gerichtlich überprüft werden können. ${ }^{747}$

738 Rixen, in: Eicher/Spellbrink, SGB II, § 31 Rn. 12.

739 So auch Valgolio, in: Hauck/Noftz, SGB II, K § 31 Rn. 11.

740 Valgolio, in: Hauck/Noftz, SGB II, K § 31 Rn. 12.

741 Davon zu unterscheiden sind Probleme der Beweislastumkehr. Vgl. zweites Kapitel B II 7e) S. 225 ff.

742 Rixen, in: Eicher/Spellbrink, SGB II, § 31 Rn. 34.

743 Andere definieren den wichtigen Grund im Hinblick auf die bestehenden gesetzlichen Pflichten des erwerbsfähigen Hilfebedürftigen und lehnen es ab, dass über diese hinausgehende Pflichten vereinbart werden dürfen. So Valgolio, in: Hauck/Noftz, SGB II, K § 31 Rn. 12.

744 Vgl. zweites Kapitel B II 5c) cc) (4) S. 207 f; so auch Lang, NZS 2006, S. 184; Rixen, in: Eicher/Spellbrink, SGB II, § 15 Rn. 16; kritisch zu dieser Vorgehensweise Müller, in: Hauck/Noftz, SGB II, K § 15 Rn. 20.

745 Brühl/Hofmann, Sozialgesetzbuch Zweites Buch, 2004, S. 104.

746 So auch Berlit, in: Münder/Armborst, LPK-SGB II, 2005, § 31 Rn. 59; Berlit, SozR akt 2006, S. 46.

747 Zur Einbettung dieser Prüfung in das System gerichtlichen Rechtsschutzes und der Zulässigkeit einer Feststellungsklage vgl. zweites Kapitel B II 10c) dd) S. 240. 
(4) Rechtsfolgenbelehrung

Sanktionen auf der Grundlage des $\S 31$ Abs. 1 SGB II sind vor ihrem Eintritt anzukündigen. Zwar ist die vorherige Belehrung nicht im Gesetzestext zu finden, lässt sich aber aus der Gesetzesbegründung herauslesen. ${ }^{748}$ Die Belehrung kann über den Zeitpunkt ihrer Erteilung hinaus ihre Warnfunktion nur erfüllen, wenn sie konkret, verständlich und vollständig ist, und dem Hilfebedürftigen die Folgen seines Verhaltens unmissverständlich vor Augen geführt werden. Dazu muss die Belehrung ausnahmslos im Vorhinein erfolgen. Hinweise in Warnblättern genügen für die Erfüllung dieser Belehrungspflichten nicht. Es ist auch unerheblich, ob es sich um einen informierten Hilfebedürftigen handelt, da die Belehrung in jedem Fall im unmittelbaren Zusammenhang mit der Sanktionierung stattfinden muss. ${ }^{749}$ Eine schriftliche Belehrung ist empfehlenswert. 750

Eine fehlende Belehrung führt dazu, dass eine Voraussetzung für eine Sanktion nicht vorliegt und damit das Arbeitslosengeld II nicht abgesenkt werden kann. ${ }^{751}$

\section{bb) Nichteinhaltung vereinbarter Pflichten, § $31 \mathrm{Abs} .1 \mathrm{Nr}$. $1 \mathrm{~b}$ SGB II}

Um die rechtliche Verbindlichkeit der abgeschlossenen Vereinbarung und darin enthaltener Pflichten klarzumachen, ist deren Nichteinhaltung sanktioniert. Das von beiden Seiten vereinbarte und damit zu erwartende Verhalten ist Gegenstand des Sozialrechtsverhältnisses geworden. ${ }^{752}$

Gem. § 31 Abs. 1 S. 1 Nr. 1b SGB II wird das Arbeitslosengeld II unter Wegfall des Zuschlags nach $\S 24$ SGB II in einer ersten Stufe um 30 Prozent der Regelleistung abgesenkt, wenn der erwerbsfähige Hilfebedürftige sich trotz vorheriger Belehrung über die Rechtsfolgen ohne wichtigen Grund weigert, in der Eingliederungsvereinbarung festgelegte Pflichten zu erfüllen, insbesondere in ausreichendem Umfang Eigenbemühungen nachzuweisen.

\section{(1) Anwendungsbereich der Sanktionsvorschrift}

Eine erweiternde Auslegung dergestalt, dass die Sanktionsvorschrift auch auf die Regelung der Eigenbemühungen durch Verwaltungsakt gem. § 15 Abs. 1 S. 6 SGB II angewendet werden könnte, ist nicht möglich.753 Der Wortlaut ist insoweit eindeutig. Darüber hinaus ist der Sinn und Zweck der Vorschrift entsprechend der Gesetzbegrün-

748 BT-Drucksache 15/1516, S. 60.

749 Rixen, in: Eicher/Spellbrink, SGB II, § 31 Rn. 44.

750 Valgolio, in: Hauck/Noftz, SGB II, K § 31 Rn. 36, 78; Dauber, in: Mergler/Zink, SGB II, § 31 Rn. 16.

751 Dauber, in: Mergler/Zink, SGB II, § 31 Rn. 16; Rixen, in: Eicher/Spellbrink, SGB II, § 31 Rn. 44.

752 Valgolio, in: Hauck/Noftz, SGB II, K § 31 Rn. 13.

753 Stark, in: Hauck/Noftz, SGB II, K § 15 Rn. 86 unter Hinweis auf den Deutschen Juristinnenbund, der in der Nichtanwendbarkeit eine Besserstellung der nichtverhandlungsbereiten Arbeitslosen gesehen hat; Valgolio, in: Hauck/Noftz, SGB II, K § 31 Rn. 19; a.A. Schumacher, in: Oestreicher, SGB XII/SGB II, § 31 Rn. 30 f, der den Normzweck auf die Gleichstellung der durch Verwaltungsakt angeordneten Eingliederungsbemühungen ausdehnt. 
dung, dass das Vereinbarte verbindlich ist, weil es auf dem Willen beider Parteien beruht. Deshalb kann die Nichteinhaltung einer einseitigen Verpflichtung nicht mit demselben Maß gemessen werden. Für die Durchsetzung von Verwaltungsakten steht im Übrigen das Instrumentarium des allgemeinen Verwaltungsrechtes zur Verfügung, z.B. die Titelfunktion des Verwaltungsaktes bei der Verwaltungsvollstreckung, sodass eine zusätzliche Sanktion nicht erforderlich ist.

\section{(2) Verletzung der Nachweispflicht}

Die in der Eingliederungsvereinbarung vereinbarten Pflichten müssen eindeutig formuliert sein. Es muss dem Antragsteller der Unterschied zwischen der Verletzung allgemeiner Mitwirkungspflichten mit der Folge des § 66 SGB I und der Verletzung der vereinbarten Pflichten nach $\S 31$ SGB II klar sein. ${ }^{754}$ Die Vereinbarung der Pflichten ändert deren Qualität und hat deshalb schärfere Sanktionen zur Folge.

Auch die Nachweispflicht muss ausreichend konkret sein, und der Arbeitsuchende muss ausdrücklich auf die Folgen der Verletzung dieser Pflicht hingewiesen worden sein, bevor die Pflichtverletzung begangen worden ist. Die Betonung der Nachweispflicht in Bezug auf die Eigenbemühungen und deren ausdrückliche Hervorhebung in $\S$ 15 Abs. 1 S. 2 Nr. 2 SGB II erscheint übertrieben. 755

\section{(3) Wichtiger Grund}

Wann ein wichtiger Grundes i.S.d. § 31 Abs. 1 S. 2 SGB II vorliegt, ist auch für diesen Sanktionstatbestand umstritten. Die in der Eingliederungsvereinbarung vereinbarten Pflichten müssen bei der Auslegung des wichtigen Grundes eine wichtige Rolle spielen. ${ }^{756}$ Diese Pflichten sind aufgrund einer Willenseinigung Teil eines verwaltungsrechtlichen Vertrages geworden und enthalten damit eine speziellere Regelung als das Gesetz. Dies gilt auch für den Fall, dass gesetzliche Pflichten zum Nachteil des Antragstellers modifiziert worden sind. ${ }^{757}$ Jedes andere Verständnis würde die möglichen Wirkungen des Vertragsschlusses zu stark einschränken. ${ }^{758}$

Problematisch ist, ob es bereits zur Annahme eines wichtigen Grundes ausreicht, dass eine Eingliederungsvereinbarung rechtswidrig, aber nicht gem. § 58 SGB X nichtig ist. Da das (Sozial-)Verwaltungsrecht keine rechtswidrigen Verträge kennt, sondern nur nichtige, und deshalb eine gegen Gesetze verstoßende Eingliederungsvereinbarung wirksam ist, muss sie eingehalten werden. Eine Ausnahme ist nur dann gegeben, wenn über die Rechtswidrigkeit hinaus ein Nichtigkeitsgrund gem. § 58 SGB X gegeben ist. 759

754 Valgolio, in: Hauck/Noftz, SGB II, K § 31 Rn. 17.

755 Valgolio, in: Hauck/Noftz, SGB II, K § 31 Rn. 16.

756 So auch Luthe/Timm, SGb 2005, S. 263; dazu zweites Kapitel B II 6c) S. 213.

757 Dazu zweites Kapitel B II 5c) dd) S. 210.

758 Dazu erstes Kapitel B III 2c) S. 73.

759 Vgl. dazu zweites Kapitel B II 8a) S. 226 ff. 
Ferner besteht die Möglichkeit, dass das Verhalten des erwerbsfähigen Hilfebedürftigen den Sanktionstatbestand nach $\S 31$ Abs. 1 Nr. 1c SGB II erfüllt, der die Absenkung des Arbeitslosen wegen der Weigerung, eine zumutbare Arbeit aufzunehmen, anordnet. Diese Sanktion knüpft an die Nichterfüllung vereinbarter Pflichten an, nicht an die Weigerung, Pflichten zu vereinbaren.

Ein Bezug zur Eingliederungsvereinbarung ergibt sich dann, wenn in ihr die Kriterien für eine zumutbare Arbeit beschrieben sind. Hier besteht Konfliktpotenzial in Bezug auf die Regelung zumutbarer Arbeit in $§ 10$ SGB II. Eine Einschränkung, aber auch eine Ausdehnung des Zumutbarkeitsbegriffs durch vertragliche Vereinbarung widerspricht dem Grundsatz der Gesetzmäßigkeit der Verwaltung beziehungsweise dem Vorrang des Gesetzes. ${ }^{760}$

Bei der Definition des wichtigen Grundes gem. $\S 31$ Abs. 1 S. 2 SGB II ist zu berücksichtigen, dass vereinbarten Pflichten größeres Gewicht zukommt, und dass die Vereinbarung gesetzlich unbestimmte Pflichten modifizieren kann. Die Eingliederungsvereinbarung ist für die Auslegung der zumutbaren Arbeit heranzuziehen und dabei dem Vereinbarten eine große Bedeutung zuzumessen.

\section{dd) Sonderregelung für Jugendliche, § 31 Abs. 5 SGB II}

Der Wille, Jugendliche im Rahmen der Leistungen zur Eingliederung besonders zu fördern, wie er sich z.B. aus dem Anspruch auf unverzügliche Vermittlung gem. § 3 Abs. 2 SGB II ableiten lässt, findet sein Pendant in einer besonders strengen Sanktionierung 761 , die mit dem sog. SGB II-Fortentwicklungsgesetz ${ }^{762}$ noch weiter verschärft worden ist. 763

$\S 31$ Abs. 5 SGB II sieht vor, dass erwerbsfähige Hilfebedürftige, die das 25. Lebensjahr noch nicht vollendet haben, nur die Kosten für Unterkunft und Heizung gem. § 22 SGB II erhalten, die ihrerseits direkt an den Vermieter oder einen anderen Empfangsberechtigten ausgezahlt werden sollen, wenn die Voraussetzung für eine Senkung des Arbeitslosengeldes II nach den Abs. 1-4 SGB II erfüllt sind, z.B. also eine angebotene Eingliederungsvereinbarung nicht abgeschlossen oder eine in der Eingliederungsvereinbarung festgelegte Pflicht nicht erfüllt wird, § 31 Abs. 5 S. 1 SGB II. Bei wiederholter Pflichtverletzung i.S.d. § 31 Abs. 3 S. 4 SGB II wird das Arbeitslosengeld II um 100 Vom Hundert gesenkt, doch bei Jugendlichen können bei nachträglicher Bereitschaft auch die Kosten der Unterkunft und Heizung insgesamt, nicht nur gemindert auf 60 Prozent erbracht werden, $\S 31$ Abs. 5 S. 3 SGB II. Der Lebensunterhalt kann während dieser Sanktion durch Gewährung von ergänzenden Sachleistungen oder geldwerten Leistungen sichergestellt werden, $\S 31$ Abs. 5 S. 6, Abs. 3 S. 6 SGB II. Eine vorher-

760 Vgl. dazu zweites Kapitel B II 8a) aa) S. 226 f.

761 BT-Drucksache 15/1516, S. 61.

762 Gesetz zur Fortentwicklung des Zweiten Buches Sozialgesetzbuch vom 20.7.2006 (BGB1. I, S. 1706).

763 Dazu Fahlbusch/Müller/Rixen, NDV 2006, S. 411 ff; Wunder/Diehm, SozSich 2006, S. 195 ff. 
gehende Belehrung über die Rechtsfolgen nach $\S 31$ Abs. 5 S. 3 SGB II a.F. ist nicht mehr vorgesehen.

\section{d) Einstellung der Vermittlung}

Es besteht aufgrund der Verweisung in $\S 16$ Abs. 1 S. 3, 1 SGB II i.V.m. § 38 Abs. 2 SGB III die Möglichkeit, die Vermittlungstätigkeit einzustellen und somit neben finanziellem Druck dem Hilfebedürftigen auch die Seite der Förderung zu verschließen, wirkt er nicht beim Abschluss einer Eingliederungsvereinbarung mit. 764

Es dürfte aber als Mitwirkung anzusehen sein, wenn der Hilfebedürftige über den Inhalt der Vereinbarung verhandelt und mit begründbaren Vorbehalten den Inhalt der vorgeschlagenen Vereinbarung nicht unterzeichnen will. In diesem Fall ist eine Einstellung der Vermittlung unzulässig. ${ }^{765}$

\section{e) Darlegungs- und Beweislastverteilung im Verwaltungsverfahren}

Im Verfahren über Entscheidungen nach dem SGB II erfolgt keine allgemeine Beweislastumkehr im Rahmen einseitiger Entscheidungen der Arbeitsverwaltung, denen eine Eingliederungsvereinbarung vorausgeht. Aufgrund des Untersuchungsgrundsatzes (Amtsermittlung) trägt grundsätzlich die Verwaltung die objektive Beweislast, § 20 SGB X.766

Eine Ausnahme hat der Gesetzgeber des SGB II vorgesehen, wenn Sanktionen verhängt werden. Weigert sich der erwerbsfähige Hilfesuchende trotz Belehrung über die Rechtsfolgen, eine ihm angebotene Eingliederungsvereinbarung abzuschließen oder eine in der Eingliederungsvereinbarung festgelegte Pflicht, insbesondere vereinbarte Eigenbemühungen, zu erfüllen, wird ohne Ermessensspielraum der Agentur für Arbeit das Arbeitslosengeld II abgesenkt. In diesen Fällen sieht § 31 Abs. 1 S. 2 SGB II vor, dass eine Sanktion nicht verhängt werden darf, wenn der erwerbsfähige Hilfebedürftige einen wichtigen Grund für sein Verhalten nachweist. Damit scheint dem Hilfebedürftigen die objektive Beweislast dafür aufgebürdet zu sein, in jedem Fall einen wichtigen Grund nachzuweisen. Bei Nichterweislichkeit des wichtigen Grundes (non-liquet) darf die Sanktion verhängt werden.

Es ist strittig, ob diese Regelung verfassungskonform ist. Die Verfassungswidrigkeit wird damit begründet, dass beim Handeln durch Vereinbarungen die Verwaltung nicht mehr in die Rechte des Bürgers eingreifen darf, als dies bei einseitigem Handeln möglich wäre. Dort hat die Behörde bei belastenden Maßnahmen den Nachweis zu führen, dass der zugrunde gelegte Sachverhalt wie angenommen vorliegt. ${ }^{767}$ Die Beweislastumkehr kann sich deshalb nur auf Tatsachen beziehen, die in die Sphäre oder den Ver-

764 Dem widerspricht die Aussage in der Gesetzesbegründung, dass dem erwerbsfähigen Hilfebedürftigen stets der Zugang zu Beratung und Betreuung erhalten bleibt, während eine Sanktion eingreift. Vgl. BT-Drucksache 15/1516, S. 47.

765 Brühl/Hofmann, Sozialgesetzbuch Zweites Buch, 2004, S. 104.

766 Dazu Spannowsky, Grenzen des Verwaltungshandelns durch Verträge und Absprachen, 1994, S. 78.

767 Dazu Krahmer, ZfF 2004, S. 178 f. 
antwortungsbereich des Hilfebedürftigen fallen. 768 Denn diese lassen sich vom Hilfebedürftigen selbst leicht nachweisen, sodass keine übermäßige Belastung zu befürchten ist. Eine Tatsache fällt in die Sphäre des Hilfebedürftigen, wenn die Behörde deren Relevanz im Zeitpunkt der Entscheidung nach den behördlich verfügbaren und datenschutzrechtlich erschließbaren Informationen nicht erkennen kann, insbesondere weil der Hilfebedürftige das Bekanntwerden der Tatsache unter Ausschluss der Behörde zu steuern vermag.

Die Darlegungs- und Beweislast im Rahmen des $\S 31$ Abs. 1 S. 2 SGB II ist damit genauso auszulegen wie bei der Verhängung einer Sperrzeitnorm gem. § 144 Abs. $1 \mathrm{~S}$. 2 SGB III ${ }^{769}$. Dies entspricht im Ergebnis der amtlichen Begründung zu $\S 31$ Abs. $1 \mathrm{~S}$. 2 SGB II. 770

\section{Störungsfälle}

a) Nichtigkeit des verwaltungsrechtlichen Vertrages, § 58 SGB X

\section{aa) Grundsatz der Gesetzmäßigkeit der Verwaltung}

Die materiellen Anforderungen an den verwaltungsrechtlichen Vertrag sind bestimmt vom Grundsatz der Gesetzmäßigkeit der Verwaltung, der für den verwaltungsrechtlichen Vertrag gilt und durch Einführung der $\S \S 54 \mathrm{ff}$ VwVfG, $53 \mathrm{ff}$ SGB X konkretisiert wurde. ${ }^{771}$ Die stetige Weiterentwicklung des Grundsatzes der Gesetzmäßigkeit der Verwaltung führt dazu, dass der Spielraum für die Verwaltung enger wird.

Der Grundsatz der Gesetzmäßigkeit der Verwaltung hat zwei Aspekte, den Vorbehalt des Gesetzes und den Vorrang des Gesetzes. ${ }^{772}$

Unter dem Vorbehalt des Gesetzes versteht man, dass die Verwaltung bei Grundrechtseingriffen nur tätig werden darf, wenn sie dazu durch Gesetz ermächtigt worden ist. ${ }^{773}$ Der Grundsatz ergibt sich aus dem Demokratieprinzip und der Rechtsgebot sowie den Grundrechten. Das bedeutet mehr, als dass die Verwaltung ohne gesetzliche Ermächtigung in ihren Handlungsmöglichkeiten nur eingeschränkt ist. ${ }^{774}$ Bezogen auf den verwaltungsrechtlichen Vertrag gilt, dass dem Vorbehalt des Gesetzes mit der gesetzli-

768 So auch Dauber, in: Mergler/Zink, SGB II, § 31 Rn. 21; Däubler, info also 2005, S. 53 f; Rixen, in: Eicher/Spellbrink, SGB II, § 31 Rn. 41 ff.

769 Vgl. zweites Kapitel B I 7d) bb) S. 175.

770 BT-Drucksache 15/1728, S. 186; BT-Drucksache 15/1749, S. 33; ausführlich Rixen, in: Eicher/Spellbrink, SGB II, § 31 Rn. 40.

771 Bonk, in: Stelkens/Bonk/Sachs, VwVfG, 2001, § 54 Rn. 90.

772 Dazu Ossenbühl, Vorrang und Vorbehalt des Gesetzes, in: Isensee/Kirchhof, Handbuch des Staatsrechts, Bd. 3, 2004, § 62; Maurer, Allgemeines Verwaltungsrecht, 2009, S. 115-133.

773 Ossenbühl, Vorrang und Vorbehalt des Gesetzes, in: Isensee/Kirchhof, Handbuch des Staatsrechts, Bd. 3, 2005, § 62 Rn. 7.

774 Maurer, Allgemeines Verwaltungsrecht, 2009, S. 116. 
chen Ausgestaltung in den Verwaltungsverfahrensgesetzen genüge getan ist. ${ }^{775}$ Der Vertragsschluss wird als Freiheitsgebrauch verstanden, der insoweit nicht vorbehaltsrelevant ist. 776

Hinter dem Vorrang des Gesetzes wiederum verbirgt sich, dass der Gesetz gewordene Wille des Gesetzgebers bei Verwaltungsentscheidungen unbedingt zu beachten ist, was sich aus Art. 20 Abs. 3 GG ergibt. 777 Die Verwaltung hat auf der einen Seite das geltende formelle Recht anzuwenden (Anwendungsgebot) als auch gesetzeswidrige Maßnahmen zu unterlassen (Abweichungsverbot). ${ }^{778}$ Auch im öffentlichen Recht hat die Verwaltung aufgrund dieser Bindung keine Gestaltungsfreiheit im zivilrechtlichen Sinn. 779

\section{bb) Grundsätze des Verwaltungsverfahrensrechts und Rezeption des Privatrechts}

Von Bedeutung ist darüber hinaus die Bindung der Verwaltung an die allgemeinen Grundsätze des Verwaltungs(verfahrens)rechts, die sich aus der Verweisung in $\S 9$ i.V.m. $\S 62$ S. 1 VwVfG, $\S 8$ i.V.m. § 61 S. 1 SGB X ergibt. Die Rezeption des Privatrechts über $\S 62 \mathrm{~S} .2 \mathrm{VwVfG}, \S 61 \mathrm{~S} .2 \mathrm{SGB} X$ führt ebenfalls zu Vorgaben für die materielle Wirksamkeit eines verwaltungsrechtlichen Vertrages. Eine wichtige Rolle spielt die Kontrolle der Allgemeinen Geschäftsbedingungen, die über die Eingliederung des AGBG in die $\S \S 305 \mathrm{ff}$ BGB unstreitig auch im Verwaltungsrecht notwendig wird. 780

Die Nichtigkeit des verwaltungsrechtlichen Vertrages ist in $\S 58$ SGB X geregelt. Aus der Formulierung der Vorschrift lässt sich ableiten, dass bei allen anderen Rechtsmängeln der verwaltungsrechtliche Vertrag wirksam ist. Das SGB X kennt zwar ebenso wie das allgemeine Verwaltungsverfahrensrecht den wirksamen und den nichtigen Vertrag, nicht aber einen aufhebbaren rechtswidrigen. ${ }^{781}$ Der nichtige Vertrag kann nicht Rechtsgrundlage für Ansprüche sein, der wirksame mit Rechtsfehlern behaftete Vertrag schon. ${ }^{782}$ Dieser mit Rechtsfehlern behaftete Vertrag ist aufgrund der vom Gesetz zugewiesenen uneingeschränkten Wirksamkeit ${ }^{783}$ auch nicht inhaltlich gerichtlich überprüfbar, sodass im Vergleich zum Verwaltungsakt, der gerade auf seine Rechtmäßigkeit

775 Bonk, in: Stelkens/Bonk/Sachs, VwVfG, 2001, § 54 Rn. 90; Kunig, DVB1 1992, S. 1197 f; Bauer, Anpassungsflexibilität im öffentlich-rechtlichen Vertrag, in: Hoffmann-Riem/Schmidt-Aßmann, Innovation und Flexibilität des Verwaltungshandelns, 1994, S. 268.

776 So grundlegend bereits vor der Regelung im VwVfG Göldner, JZ 1976, S. 355; zur heutigen Rechtslage Schmidt-Aßmann/Krebs, Rechtsfragen städtebaulicher Verträge, 1992, S. 182 ff; Scherzberg, Jus 1992, S. 211 f; Krebs, VVDStRL, H. 52 (1993), S. 265 f.

777 Für viele Maurer, Allgemeines Verwaltungsrecht, 2009, S. 115.

778 Schlette, Die Verwaltung als Vertragspartner, 2000, S. 81; Bauer, Anpassungsflexibilität im öffentlich-rechtlichen Vertrag, in: Hoffmann-Riem/Schmidt-Aßmann, Innovation und Flexibilität des Verwaltungshandelns, 1994, S. 269.

779 Vgl. erstes Kapitel B III 2 S. 71 ff.

780 Geis, NVwZ 2002, S. 386.

781 Krasney, in: Kasseler Kommentar, Bd. 2, SGB X, § 58 Rn. 2; Bonk, in: Stelkens/Bonk/Sachs, VwVfG, 2001, § 59 Rn. 2, 11.

782 Ziekow/Siegel, VerwArch 2004, S. 290; Bonk, in: Stelkens/Bonk/Sachs, VwVfG, 2001, § 59 Rn. 12.

783 Ausnahme ist die schwebende Unwirksamkeit bei fehlender Beteiligung von Dritten und Behörden gem. $§ 57$ SGB X. 
hin überprüft wird, eine Rechtsschutzverkürzung durch die Verwendung des verwaltungsrechtlichen Vertrages eintritt. Diese unflexible Fehlerfolgenregelung wird kritisiert und als Hauptgrund für die insgesamt zögerliche Verwendung des verwaltungsrechtlichen Vertrages betrachtet. 784

cc) Schwerwiegender Verstoß gegen ein Verbotsgesetz, § 58 Abs. 1 SGB X i.V.m. § 134 $B G B$

Die Nichtigkeit einer Eingliederungsvereinbarung nach $\S 58$ Abs. 1 SGB X i.V.m. § 134 BGB setzt voraus, dass ein Verbotsgesetz den Vertragschluss als solchen oder mit diesem Inhalt verbietet. Der Begriff des Verbotsgesetzes im Privatrecht ist von dem des öffentlichen Rechts zu unterscheiden. Handelt es sich bei § 134 BGB im Privatrecht um einen Fall negativer Abschlusskontrolle, durch die bestimmte Regelungsgehalte den Vertragsparteien entzogen werden, dient $§ 134$ BGB im öffentlichen Recht dazu, dem Verhalten der Verwaltung Grenzen zu setzen. Das Verbotsgesetz muss sich deshalb nur an die Verwaltung richten. ${ }^{785}$ Dabei ist das gesetzliche Verbot nicht schlicht eine Ausprägung des Grundsatzes der Gesetzmäßigkeit der Verwaltung. Der Rechtsverstoß der Behörde muss vielmehr ein gewisses Gewicht haben („qualifizierter Rechtsverstoß“786), was sich nur im Einzelfall ermitteln lässt. ${ }^{787}$

Im Hinblick auf die Nichtigkeit einer Eingliederungsvereinbarung enthalten $\S 15$ SGB II und § 3 SGB II Anhaltspunkte für einen Verstoß gegen ein Verbotsgesetz.

So ist es ein Verstoß gegen $\S 15$ SGB II, wenn vom Hilfebedürftigen Eingliederungsbemühungen verlangt werden, die für seine Eingliederung nicht erforderlich sind, z.B. solche, die keine wiedereingliedernde Wirkung haben. Das gilt insbesondere für Arbeitsgelegenheiten nach $\S 16$ Abs. 3 SGB II oder das Verlangen unzähliger Bewerbungen bei einer aussichtslosen Arbeitsmarktlage. 788

Ferner stellt es einen Verstoß gegen das Gebot, eine Eingliederungsvereinbarung auszuhandeln, dar, wenn deren Inhalt standardmäßig vorbereitet und nur zur Unterschrift vorlegt wird, also ohne tatsächliche Abstimmung oder auch nur ein Gespräch über mögliche Inhalte zustande kommt. Hier sind auch die Grundsätze des § 3 SGB II verletzt, der als Leistungsgrundsatz die Berücksichtigung der Eignung und der individuellen Lebenssituation vorsieht. 789

784 Ziekow/Siegel, VerwArch 2004, S. 290.

785 Ziekow/Siegel, VerwArch 2004, S. 284; Bonk, in: Stelkens/Bonk/Sachs, VwVfG, 2001, § 59 Rn. 56 m.w.N.; a.A. Krasney, in: Kasseler Kommentar, Bd. 2, SGB X, § 58 Rn. 5, der davon ausgeht, dass sich das Verbotsgesetz an beide Vertragsparteien richten muss, weil er keine Differenzierung zwischen der Anwendung des § 134 BGB im Privatrecht und im öffentlichen Recht vornimmt.

786 BVerwGE 89, 7 [10]; 98, 58 [63].

787 Vgl. zur Anwendbarkeit des § 134 BGB auf verwaltungsrechtliche Verträge Höfling/Krings, Jus 2000, S. 631 f; Maurer, Allgemeines Verwaltungsrecht, 2009, S. 395 ff; Ziekow/Siegel, VerwArch 2004, S. 283 ff; Bonk, in: Stelkens/Bonk/Sachs, VwVfG, 2001, § 59 Rn. 49 ff; Krasney, in: Kasseler Kommentar, Bd. 2, SGB X, § 58 Rn. 5; Engelmann, in: Wulffen, SGB X, 2008, § 58 Rn. 6.

788 Berlit, SozR akt 2006, S. 48 betrachtet dies als Verstoß gegen $\S 58$ Abs. 2 Nr. 4 SGB X, weil er von der Anwendbarkeit des $\S 55$ SGB X auf die Eingliederungsvereinbarung ausgeht.

789 So auch Berlit, SozR akt 2006, S. 48. 
Aufgrund des faktischen Abschlusszwanges, der wegen der Sanktion in § 31 SGB II entsteht, und der drohenden Absenkung der Leistungen zur Sicherung des Lebensunterhaltes besteht die erhöhte Gefahr, dass die Willenserklärung des Hilfebedürftigen zum Abschluss einer Eingliederungsvereinbarung von diesem unter Druck doch erteilt wird, während er in anderen Lebenslagen ohne existenzielle Ängste anders gehandelt hätte. Mit der allgemein angenommenen inhaltlichen Richtigkeitsgewähr von Verträgen lässt sich deshalb eine allzu enge Auslegung des qualifizierten Rechtsverstoßes nicht rechtfertigen. Die gerichtliche Kontrolldichte ist an die Umstände des Vertragsschlusses anzupassen und deshalb im Fall der Eingliederungsvereinbarung auf Verstöße gegen die Ziele kooperativer Verwaltung, insbesondere manifestiert in den $\S \S 3,15$ SGB II, auszudehnen.

\section{dd) Sittenwidrigkeit, $\S 58$ Abs. 1 SGB Xi.V.m. $\S 138$ BGB}

Das im Rahmen der rechtlichen Einordnung allgemein diskutierte Problem des ungleichen Verhandlungsgleichgewichts erlangt bei der Prüfung der Nichtigkeit der Eingliederungsvereinbarung Bedeutung. In Betracht kommt eine Sittenwidrigkeit gem. $\S$ 138 BGB. 790

Ein sittenwidriger verwaltungsrechtlicher Vertrag liegt vor, wenn der Sozialleistungsträger den Vertrag unter Ausnutzung seiner Überlegenheit insbesondere an personellen und sachlichen Ressourcen abschließt und dabei die Willensfreiheit des Vertragspartners unzumutbar einschränkt (Missbrauch des Verhandlungsgewichts ${ }^{791}$ ). ${ }^{792}$ Das Ergebnis der Verhandlungen muss ein Vertrag sein, der in wesentlichen Teilen nach der Einschätzung aller gerecht Denkenden schlechterdings unerträglich ist („Stempel der Verwerflichkeit"). Hierfür reicht in der Regel der deutliche Machtmissbrauch einer hoheitlichen Überlegenheit unter Ausnutzung einer Zwangs- oder Notsituation aus. ${ }^{793}$

Ein Beispiel könnte der Fall eines rein willkürlich handelnden Fallmanagers sein, der beim Vertragsschluss sich nicht an den Grundsatz der Gesetzesbindung der Verwaltung hält und den Bürger mit der vorgeschlagenen Vereinbarung unter Androhung der möglichen Sanktionen nur schikanieren will. Ein solcher Fall wird in der Regel nicht gegeben sein. Es kann deshalb keine Rede davon sein, dass die Eingliederungsvereinbarung bereits aufgrund des Abschlusszwanges und der Sanktionierungsmöglichkeiten als stets sittenwidrig anzusehen ist. Eine Sittenwidrigkeit im Einzelfall ist allerdings durchaus denkbar.

790 Knoblauch/Hübner, NDV 2005, S. 279; Berlit, in: Münder/Armborst, LPK-SGB II, 2005, § 15 Rn. 9.

791 Ziekow/Siegel, VerwArch 2004, S. 283; Höfling, JuS 2000, S. 632.

$792 \mathrm{Zu}$ prüfen ist in diesem Zusammenhang auch, ob die Voraussetzungen einer Anfechtung wegen Täuschung oder rechtswidriger Drohung gem. § 123 BGB gegeben sind. Dazu Höfling/Krings, JuS 2000, S. 632.

793 Bonk, in: Stelkens/Bonk/Sachs, VwVfG, 2001, § 59 Rn. 59; BVerfG, Urteil v. 19.10.1993, 1 BvR 567/89, 1 BvR 1044/89, ZIP 1993, S. 1775; BVerwGE 42, 331 [342 f]. 
Gem. § 58 Abs. 2 Nr. 1 SGB X i.V.m. § 40 SGB X ist ein verwaltungsrechtlicher Vertrag nichtig, wenn der Vertrag unter schweren Inhalts- und Formfehlern leidet.794 Nach der vor der Geltung des VwVfG entwickelten Evidenztheorie führt nur ein besonders schwerer Form- oder Inhaltsfehler, der mit der Rechtsordnung unter keinen Umständen vereinbar ist und überdies für einen urteilsfähigen Bürger offensichtlich sein muss, zur Nichtigkeit eines Verwaltungsaktes. Die Generalklausel für die Nichtigkeit von Verwaltungsakten in $\S 44$ Abs. $1 \mathrm{VwVfG}$ ist auf die Nichtigkeit des verwaltungsrechtlichen Vertrages zu übertragen. Abgestellt wird auf das Gewicht und die Bedeutung des Fehlers, nicht auf Fehlerarten oder gar auf ein mehr oder weniger schuldhaftes Verhalten des Behördenvertreters. Der Verstoß gegen eine wichtige Rechtsbestimmung allein, selbst gegen eine Verfassungsbestimmung wie Art. 20 Abs. 3 GG oder gegen die Grundrechte, führt nicht zur Nichtigkeit. ${ }^{795}$ Nichtig sein dürften dagegen (bewusst) ohne Rücksicht auf die gesetzlichen Anforderungen getroffene Willkürmaßnahmen, und zwar sowohl solche zum Nachteil des Adressaten als auch ihn begünstigende Regelungen. 796

Je nach Ausgestaltung des Falles ist also auch $\S 58$ Abs. 2 Nr. 1 SGB X ein Prüfungspunkt, an dem das willkürliche Handeln eines Fallmanagers die Nichtigkeit der Eingliederungsvereinbarung herbeiführen kann. Um den Nichtigkeitsgrund „Sittenwidrigkeit“ vom Vorliegen eines schweren Inhalts- oder Formmangels gem. § 40 SGB X abgrenzen zu können, kann darauf abgestellt werden, ob das willkürliche Verhalten der Schikane des Hilfebedürftigen „dient“, also die bewusste Beeinflussung des freien Willens des Hilfebedürftigen, oder ob ein anderer Fehler des Fallmanagers (z.B. willkürliche Eingliederungsleistung) die Eingliederungsvereinbarung nichtig macht.

\section{ff) Nichtigkeit gem. $\S 58$ Abs. 2 Nr. 2 SGB X}

$\S 58$ Abs. 2 Nr. 2 SGB X erfasst die Situation eines kollusiven Zusammenwirkens der Vertragsparteien zur Herbeiführung eines rechtswidrigen Erfolges. Er setzt voraus, dass die Rechtswidrigkeit eines Verwaltungsaktes mit entsprechendem Inhalt beiden Parteien bekannt war, nicht nur die tatsächlichen Umstände, die zur Rechtswidrigkeit führen würden. Gerade Ermessens- und Abwägungsfehler sind dem Bürger aber nur selten bekannt, sodass der Anwendungsbereich der Vorschrift faktisch eingeschränkt ist. 797

794 Bonk, in: Stelkens/Bonk/Sachs, VwVfG, 2001, § 59 Rn. 19; Henneke, in: Knack, VwVfG, 2000, § 59 Rn. 18; Kopp/Ramsauer, VwVfG, 2008, § 59 Rn. 23 f.

795 So BVerwGE 19, 284 [287]; 23, 237 [238]; 27, 295 [299]; vgl. Sachs, in: Stelkens/Bonk/Sachs, VwVfG, 2001, § 44 Rn. 100 ff m.w.N..

796 Im konkreten Fall für grobe Fehler bei der Schätzung der Besteuerungsgrundlagen verneinend BFH, Urteil v. 1.10.1992, IV R 34/90, NVwZ 1993, S. $1231 \mathrm{f}$.

797 Bonk, in: Stelkens/Bonk/Sachs, VwVfG, 2001, § 59 Rn. 30 ff; Henneke, in: Knack, VwVfG, 2000, § 59 Rn. 19; Krasney, in: Kasseler Kommentar, Bd. 2, SGB X, § 58 Rn. 9. 
Es müsste ein Zusammenwirken zwischen Fallmanager und Hilfebedürftigem stattfinden, das dazu führt, dass z.B. Leistungen zur Eingliederung trotz Fehlens der Voraussetzungen wider besseres Wissen der Vertragsparteien vereinbart werden.

\section{gg) Nichtigkeit gem. $\$ \S 58$ Abs. 2 Nr. 3, 4 SGB X}

Die Nichtigkeitsgründe der $\S \S 58$ Abs. 2 Nr. 3, 4 SGB X sind auf die Eingliederungsvereinbarung nicht anwendbar. Es handelt sich bei der Eingliederungsvereinbarung weder um einen Vergleichsvertrag im Sinn des § 54 SGB X noch um einen Austauschvertrag im Sinn des $\S 55$ SGB X. 798

\section{hh) Zwischenergebnis}

Die Regelung der Nichtigkeit in $\S 58$ SGB X geht im Spannungsfeld zwischen der Vertragsverbindlichkeit und dem Prinzip der Gesetzmäßigkeit der Verwaltung einen „Mittelweg“799, weist dem verwaltungsrechtlichen Vertrag aber eine höhere Bestandskraft zu als dem Verwaltungsakt. ${ }^{800}$ Das heißt auch, dass die gerichtliche Kontrolldichte aufgrund $\S 58$ SGB X in Bezug auf die Eingliederungsvereinbarung geringer ist als bei einer Festsetzung durch Verwaltungsakt gem. § 15 Abs. 1 S. 6 SGB II. Aufgrund der sanktionierten Abschlussverpflichtung ist die Gefahr des Missbrauchs des unbestritten unausgewogenen Verhandlungsgewichtes zwischen Staat und Einzelnen Anlass genug, die gerichtliche Kontrolldichte dieser Tatsache anzupassen. Dazu ist das geltende Recht des verwaltungsrechtlichen Vertrags bei entsprechender Auslegung der Vorschriften durchaus in der Lage. Zudem wird eine verfahrensrechtliche Absicherung der Rechte des Bürgers als erforderlich angesehen. 801

b) Nichterteilung des Einvernehmens des kommunalen Trägers, § 15 Abs. 1 S. 1 SGB II

Welche Folgen die Nichterteilung des Einvernehmens des kommunalen Trägers hat, hängt davon $a b$, ob das Zustimmungserfordernis Wirksamkeitsvoraussetzung ist oder bloßes internes Mitwirkungsrecht, § 57 SGB X.

Die in $\S 57$ SGB X enthaltenen Zustimmungserfordernisse bringen zum Ausdruck, dass Verträge zu Lasten Dritter auch im Verwaltungsrecht grundsätzlich unzulässig sind. Diese Wertung basiert auf der Schranke der Vertragsfreiheit in Art. 2 Abs. 1 GG, den Rechten anderer. ${ }^{802}$ Es gibt Zustimmungserfordernisse eines Dritten, in dessen

798 Vgl. zweites Kapitel B II 5c) ee) S. 211; a.A. Berlit, SozR akt 2006, S. 47 f, der die entsprechende Anwendbarkeit des $\S 55$ auf den hinkenden Austauschvertrag voraussetzend zu $\S 58$ Abs. 2 Nr. 4 SGB X Ausführungen macht.

799 Bonk, in: Stelkens/Bonk/Sachs, VwVfG, 2001, § 59 Rn. 7.

800 Krasney, in: Kasseler Kommentar, Bd. 2, SGB X, § 58 Rn. 3; Freischmidt, in: Hauck/Noftz, SGB X, K $\S 58$ Rn. 4.

801 Zur Zulässigkeit einer Klage auf Feststellung der Nichtigkeit der Eingliederungsvereinbarung vgl. zweites Kapitel B II 10c) dd) S. 223 f.

802 BVerwG, DVB1 1993, S. 434; Kopp/Ramsauer, VwVfG, 2008, § 58 Rn. 1; Bonk, in: Stelkens/Bonk/Sachs, VwVfG, 2001, § 58 Rn. 2. Kritik an diesem Ansatz übt Ziekow/Siegel, VerwArch 2004, S. 140, der darauf hinweist, dass $\S 58$ VwVfG auf der subjektiven Richtigkeitsgewähr durch 
Rechte durch den verwaltungsrechtlichen Vertrag eingegriffen wird, und Zustimmungserfordernisse, die eine andere Behörde betreffen, §58 VwVfG, § 57 SGB X. Die Abgrenzung zwischen dem Dritten gem. § 58 Abs. 1 VwVfG, § 57 Abs. 1 SGB X und der Behörde in $\S 58$ Abs. 2 VwVfG, $\S 57$ Abs. 2 SGB X erfolgt danach, welche Rechtsposition durch die Zustimmung bzw. Mitwirkung geschützt werden soll: In Abs. 1 werden subjektiv-öffentliche Rechte Dritter geschützt, mit Abs. 2 wird die öffentliche Kompetenzordnung gewahrt werden. ${ }^{803}$

Auf die Eingliederungsvereinbarung nach SGB II findet insgesamt $§ 57$ Abs. 2 SGB $\mathrm{X}$ Anwendung. Abzulehnen ist wegen des über die flankierenden Leistungen hinausgehenden Interesses des kommunalen Trägers die Beschränkung des Mitwirkungsrechts auf die Inhalte der Eingliederungsvereinbarung, die die flankierenden Leistungen betreffen. ${ }^{804}$ Es wird eine eigenständige Rechtsposition des kommunalen Trägers geschützt werden. 805

Ein ohne diese Zustimmung beziehungsweise Mitwirkung geschlossener Vertrag ist schwebend unwirksam, bindet aber die Parteien bis zur endgültigen Verweigerung oder Erklärung der Zustimmung beziehungsweise Vornahme der Mitwirkungshandlung. Die später erteilte Zustimmung oder vorgenommene Mitwirkung wirkt ex tunc auf den Zeitpunkt des Vertragsschlusses zurück. ${ }^{806}$ Die Vorschrift ist auch anwendbar, wenn das Einvernehmen direkt für den Fall des Abschlusses eines verwaltungsrechtlichen Vertrages vorgesehen ist. ${ }^{807}$

Die Eingliederungsvereinbarung ist also solange schwebend unwirksam, bis der kommunale Träger sein Einvernehmen in der vorgeschriebenen Form erteilt hat. 808 Wird es nie erteilt, kommt der Vertrag nicht zustande. Dann kann die Agentur für Arbeit nicht auf der Grundlage des $\S 31$ Abs. 1 S. 1 Nr. 1a SGB II sanktionieren, da nicht die Weigerung des Hilfebedürftigen zum Nichtabschluss der Eingliederungsvereinbarung geführt hat, sondern das Fehlen einer Mitwirkungshandlung. Vielmehr ist der angestrebte und ausgehandelte Inhalt der Vereinbarung mittels eines Verwaltungsaktes umzusetzen und so die Verbindlichkeit für den Einzelnen herzustellen. 809

privatautonomen Interessenausgleich basiert, nicht aber auf dem die Verwaltung bindenden Ansatz einer objektiven Richtigkeitsgewähr.

803 Dazu Bonk, in: Stelkens/Bonk/Sachs, VwVfG, 2001, § 58 Rn. 3; Ziekow/Siegel, VerwArch 2004, S. $137 \mathrm{f}$.

804 So aber Berlit, SozR akt 2006, S. 43; wohl auch Schumacher, in: Oestreicher, SGB XII/SGB II, § 15 Rn. 17.

805 Müller, in: Hauck/Noftz, SGB II, K § 15 Rn. 26; Rixen, in: Eicher/Spellbrink, SGB II, § 15 Rn. 5; a.A. Berlit, in: Münder/Armborst, LPK-SGB II, 2005, $§ 15$ Rn. 10, der ohne Begründung eine interne Mitwirkungshandlung annimmt. Dazu im Einzelnen zweites Kapitel B II 2b) cc) S. 190 ff.

806 Ziekow/Siegel, VerwArch 2004, S. 137; Bonk, in: Stelkens/Bonk/Sachs, VwVfG, 2001, § 58 Rn. 19; Kopp/Ramsauer, VwVfG, 2008, § 58 Rn. 19.

807 Zur Eingliederungsvereinbarung Becker, in: Hauck/Noftz, SGB X, K § 57 Rn. 42.

808 Diese Rechtsunsicherheit bei allen verwaltungsrechtlichen Verträgen mit einem Mitwirkungsrecht einer anderen Behörde kritisiert Schmidt-Aßmann, Das allgemeine Verwaltungsrecht als Ordnungsidee, 2004, S. 347 f und schlägt stattdessen eine Einspruchsmöglichkeit mit Frist vor, um der Zeit der schwebenden Unwirksamkeit ein klares Ende zu bereiten.

Rixen, in: Eicher/Spellbrink, SGB II, § 15 Rn. 5. 
Es besteht die Möglichkeit, das Einvernehmen des kommunalen Trägers im Wege der Aufsicht oder gerichtlich ersetzen zu lassen. 810

c) Veränderung der tatsächlichen Verhältnisse

Die Eingliederungsvereinbarung nach SGB II durfte für eine Übergangszeit bis 31.12.2006 für zwölf Monate abgeschlossen werden (§ 65 Abs. 6 SGB II). Danach soll die Vereinbarung gem. $\S 15$ Abs. 1 S. 3 SGB II grundsätzlich sechs Monate gelten. Nach dem Ende der Laufzeit soll eine neue Vereinbarung getroffen werden, in die die Erkenntnisse der bisherigen Umsetzung einfließen müssen (§ 15 Abs. 1 S. 4, 5 SGB II).

Es ist umstritten, ob unabhängig von den gesetzlich vorgegebenen Laufzeiten die Vereinbarung nach SGB II jederzeit geändert werden kann. ${ }^{811}$ Diese einfache Anpassung würde ermöglichen, dem Sinn und Zweck der Vereinbarung, der möglichst passgenauen Förderung des einzelnen Hilfebedürftigen, zu entsprechen. Doch steht die Bindungswirkung des verwaltungsrechtlichen Vertrages diesem formlosen Weg der Vertragsanpassung in einem kooperativen Verhältnis entgegen. Denn es gilt $\S 59$ Abs. $1 \mathrm{~S}$. 1 SGB X Rechnung zu tragen. ${ }^{812}$

Haben sich die Verhältnisse, die für die Festsetzung des Vertragsinhalts maßgebend gewesen sind, seit Abschluss des Vertrages so wesentlich geändert, dass einer Vertragspartei das Festhalten an der ursprünglichen vertraglichen Regelung nicht zuzumuten ist, so kann diese Vertragspartei eine Anpassung des Vertragsinhalts an die geänderten Verhältnisse verlangen, § 59 Abs. 1 S. 1 SGB X. Es ist bislang noch nicht entschieden, wann dieser Fall gegeben ist. Es ist aber davon auszugehen, dass im Sinne einer Verbindlichkeit der Vereinbarung diese Möglichkeit eher eng auszulegen ist. So wird z.B. die Änderung von Förderrichtlinien innerhalb der Agentur für Arbeit nicht als ausreichende Änderung der Verhältnisse betrachtet, die die Unzumutbarkeit für die Verwaltung begründen könnte. ${ }^{813}$

d) Nichteinhaltung der vereinbarten Pflichten durch den erwerbsfähigen Hilfebedürftigen

In der Eingliederungsvereinbarung können Pflichten nach dem SGB II verbindlich vereinbart werden. Für den Fall der Nichteinhaltung dieser Pflichten sieht das SGB II bestimmte Sanktionen vor. Diese sind in $\S 31$ SGB II abschließend geregelt. 814

Gem. § 60 Abs. 1 SGB X können beide Vertragspartner die sofortige Vollstreckbarkeit vereinbaren. Der Vertrag hat dann Titelfunktion und ist der einseitigen Auferlegung von Pflichten des erwerbsfähigen Hilfebedürftigen ebenbürtig. ${ }^{815}$ Dies ist vor allem für

810 Dazu Engelmann, in: Wulffen, SGB X, 2008, § 57 Rn. 10.

811 So Löschau/Marschner, Hartz IV, 2004, Rn. 416.

812 Vgl. dazu zweites Kapitel B II 9c) S. 237.

813 Knoblauch/Hübner, NDV 2005, S. 281.

814 Vgl. zweites Kapitel B II 7c) S. 218 ff.

815 Maurer, Der Verwaltungsvertrag, in: Hill, Verwaltungshandeln durch Verträge und Absprachen, 1990, S. 23. 
die Arbeitsverwaltung interessant, die das in der Eingliederungsvereinbarung Vereinbarte nicht mittels Verwaltungsakt festsetzen darf, sobald der Hilfebedürftige über den Inhalt verhandelt. Die sofortige Vollstreckbarkeit ist aber nur dann hilfreich, wenn die Zwangsvollstreckung nach zivilprozessualen Grundsätzen zulässig ist. Das ist jedenfalls für nicht vertretbare Handlungen nicht der Fall, $\S 888$ Abs. 1 ZPO. Diese können nur mittels Zwangsgeld und Zwangshaft durchgesetzt werden. Die Vereinbarung einer sofortigen Vollstreckbarkeit der Eingliederungsvereinbarung wird deshalb regelmäßig nicht erfolgen. Zwar würde die Verhängung von Zwangsgeld zu staatlichen Einnahmen führen, wenn der Hilfebedürftige das Zwangsgeld begleichen kann, doch wird dadurch das Ziel, vereinbarungskonformes Verhalten des Hilfebedürftigen zu bewirken, nicht erreicht werden. Es wird lediglich der Druck auf den Hilfebedürftigen erhöht. 816

\section{aa) Schadensersatz bei Abbruch einer Bildungsmaßnahme, § 15 Abs. 3 SGB II}

Das Gesetz ermächtigt die Parteien der Vereinbarung ausdrücklich nur im Einzelfall, selbst Sanktionen zu vereinbaren. § 15 Abs. 3 SGB II regelt einen solchen Fall. Rechtsgrundlage ist dann die Eingliederungsvereinbarung.

Wenn der erwerbsfähige Hilfebedürftige eine vereinbarte Bildungsmaßnahme ${ }^{817}$ aus einem von ihm zu vertretenden Grund abbricht, sind bereits in der Vereinbarung eine Schadensersatzpflicht des erwerbsfähigen Hilfebedürftigen und deren Umfang vorzusehen. 818 Damit soll über die drohende Absenkung des Arbeitslosengeldes II gem. § 31 Abs. 1 Nr. 2 SGB II hinaus ein finanzieller Anreiz geschaffen werden, die begonnene Maßnahme zu Ende zu führen. 819

In Abgrenzung zu bekannten Fällen, in denen z.B. ein Beamter oder Soldat nach dem Durchlaufen einer Ausbildung auf Staatskosten, ohne die vereinbarte Zeit im Staatsdienst geblieben zu sein, ausscheidet und dann die Ausbildungskosten erstatten muss, ist hier gerade nicht die Kostenerstattung nach der Ausbildung geregelt. Die Sanktion ist vielmehr direkt an den Abbruch geknüpft. Die frühere Regelung in $\S 46$ Abs. 3 S. 2 AFG in der bis zum 31.12.1997 geltenden Fassung wurde nicht übernommen. ${ }^{820}$ Eine andere entsprechende Regelung findet sich nicht im SGB II. ${ }^{821}$

Das unterstreicht, dass es dem Gesetzgeber nicht um einen Beitrag zu den bei der Weiterbildungsmaßnahme entstehenden Kosten geht, sondern darum, durch einen finanziellen Anreiz die Beendigung der Maßnahme zu fördern. Dem Abschluss der Ausbildung wird Priorität eingeräumt mit dem Ziel der Verbesserung der Eingliederungschancen.

816 Berlit, in: Münder/Armborst, LPK-SGB II, 2005, § 15 Rn. 11; ders., SozR akt 2006, S. 43.

817 Zum Begriff Fuchsloch, in: Gagel, SGB II, § 15 Rn. 87 ff.

818 Spellbrink, SozR akt 2006 S. 55 f weist darauf hin, dass $\S 15$ Abs. 3 SGB II keine Rechtsgrundlage für einen nochmaligen Verwaltungsakt zur Feststellung der Schadensersatzpflicht enthält.

819 BT-Drucksache 15/1516, S. 54.

820 Sie lautete: „Die Leistungen sind zurückzuzahlen, wenn der Antragsteller innerhalb von vier Jahren nach Abschluss der Maßnahme ohne wichtigen Grund nicht mindestens drei Jahre lang eine die Beitragspflicht begründende Beschäftigung ausgeübt hat."

821 Dazu Stark, in: Estelmann, SGB II, § 15 Rn. $81 \mathrm{ff}$. 
$\mathrm{Da}$ es sich bei der Eingliederungsvereinbarung um einen verwaltungsrechtlichen Vertrag handelt, sind die Vorschriften über eine Vertragsstrafe grundsätzlich entsprechend anwendbar gem. § 61 S. 2 SGB X i.V.m. §§ 339 ff BGB. Voraussetzung ist, dass die Anwendung bürgerlichen Rechts nicht gegen Grundgedanken des öffentlichen Rechts verstößt. ${ }^{822}$ Die Vertragsstrafe dient auch beim verwaltungsrechtlichen Vertrag (nur) dazu, den Schuldner - gleichgültig ob Verwaltung oder Bürger - zur Erbringung der geschuldeten Leistung anzuhalten. Die Vereinbarung einer Vertragsstrafe muss sich also sachlich rechtfertigen lassen. Das bedeutet, die Nichterfüllung des Vertrages muss besondere Vorteile für den Bürger oder besondere Nachteile für die Behörde entstehen lassen, die mit Hilfe einer Vertragsstrafe ausgeglichen werden sollen. Sie enthält einen Zuschlag zur geschuldeten Hauptleistung und setzt Verschulden voraus. Sie ist insbesondere keine Gegenleistung für den Erlass einer Maßnahme, sondern dient der ordnungsgemäßen Erfüllung des Vertrages. Ein Verstoß gegen das Koppelungsverbot ist deshalb grundsätzlich bei der Vereinbarung einer Vertragsstrafe in einem verwaltungsrechtlichen Vertrag nicht zu befürchten. 823

Wird eine Regelung über die Schadensersatzpflicht nach Abbruch einer Weiterbildungsmaßnahme nach $\S 15$ Abs. 3 SGB II in die Eingliederungsvereinbarung aufgenommen, kann dies nicht als eine Form von vereinbarter Vertragsstrafe betrachtet werden. Ein Schaden ist nicht Voraussetzung einer Vertragsstrafe, weil die Vertragsstrafe nicht nur dem Ausgleich von Vermögensinteressen dient, sondern vor allem auf die Sicherung immaterieller Interessen abzielt. ${ }^{824} \S 15$ Abs. 3 SGB II spricht aber von Schadensersatz und setzt somit einen Schaden voraus. Dieser Schaden kann nie höher als die verauslagten Kosten für die Maßnahme sein. Bei $\S 15$ Abs. 3 SGB II handelt es sich somit nicht um eine Vertragsstrafe. 825 Die Vorschrift steht aber andererseits der Vereinbarung einer Vertragsstrafe nicht entgegen.

Die Vertragstrafe zu Lasten des Hilfebedürftigen wird durch die abschließende Sanktionsvorschrift des $\S 31$ SGB II überlagert. Damit ist ausgeschlossen, dass zusätzlich zu den gesetzlich vorgesehenen Sanktionen eine Vertragsstrafe wegen Nichterfüllung der Eingliederungsvereinbarung zu Lasten des Hilfebedürftigen vereinbart werden kann. Die Sanktionierung auf der Grundlage des § 31 SGB II geht gem. § 37 SGB I der Verweisungsnorm des $\S 61$ SGB X vor. Die Sanktionierung des Hilfebedürftigen tritt bei Verweigerung des Abschlusses, aber eben auch bei Nichterfüllung der Eingliederungsvereinbarung ein. Eine weitere zusätzliche „Strafe“ des Hilfebedürftigen kann somit nicht vereinbart werden. 826

822 Schilling, Die Vertragsstrafe in Verträgen mit der öffentlichen Hand, VerwArch 1993, S. 226; Kessler/Kortmann, DVB1 1977, S. 690 ff.

823 Maurer, Der Verwaltungsvertrag, in: Hill, Verwaltungshandeln durch Verträge und Absprachen, 1990, S. 27; Bonk, in: Stelkens/Bonk/Sachs, VwVfG, 2001, § 62 Rn. 37.

824 Gottwald, in: Münchener Kommentar, 2007, § 340 Rn. 8.

825 So auch Stark, in: Estelmann, SGB II, § 15 Rn. 55.

826 So auch Berlit, in: Münder/Armborst, LPK-SGB II, 2005, § 15 Rn. 11. 
Dieselbe Begründung steht der Entstehung eines Schadensersatzanspruchs gem. § 61 S. 2 SGB X i.V.m. § 280 BGB entgegen. Die Sanktionsvorschrift in § 31 SGB II regelt abschließend die Folgen der Nichteinhaltung der Eingliederungsvereinbarung. Zudem handelt es sich bei der Eingliederungsvereinbarung nicht um einen synallagmatischen Vertrag, sodass die entsprechende Anwendung von privatrechtlichen Institutionen, die das Synallagma voraussetzen, ebenfalls ausscheiden und einem Absenkungsbescheid nicht entgegengehalten werden können, z.B. Nachbesserungs-, Zurückbehaltungs-, oder sonstige Leistungsverweigerungsrechte. ${ }^{827}$

\section{e) Nichteinhaltung der vereinbarten Pflichten durch die Agentur für Arbeit}

\section{aa) Erfüllungsanspruch}

Eine Regelung, die das Nichteinhalten der Eingliederungsvereinbarung durch die Verwaltung parallel zu $\S 31$ SGB II ahnden würde, gibt es nicht. Es bleibt deshalb für die Verwaltung bei den Folgen, die das Vertragsrecht auf verwaltungsrechtlicher Grundlage kennt. Vom Vorliegen eines verwaltungsrechtlichen Vertrages ausgehend entfaltet jeder echte Vertrag Bindungswirkung gegenüber dem Hilfebedürftigen und führt zur gerichtlichen Durchsetzbarkeit des Vereinbarten. ${ }^{828}$ Es entsteht somit ein vertraglicher Erfüllungsanspruch, wenn die vereinbarten Leistungen zur Eingliederung nach Grund, Umfang oder Zeitpunkt konkret festgelegt sind.

Fehlt hingegen diese Bestimmtheit, sind die getroffenen Abreden als vertragliche $\mathrm{Zu}-$ sicherung zu verstehen, die näher zu bestimmenden Leistungen auf der Grundlage eines Verwaltungsaktes zu erbringen. ${ }^{829}$ Gem. $§ 34$ Abs. 3 SGB X ist die Verwaltung bei einer Änderung der Verhältnisse, wie sie in $\S 59$ SGB X für den verwaltungsrechtlichen Vertrag geregelt ist, nicht mehr an die Zusicherung gebunden. ${ }^{830}$

bb) Vertraglicher Schadensersatz und Aufwendungsersatz, $\S 61$ S. 1 SGB X i.V.m. $\S 280$ bzw. $284 B G B$

Daneben stehen vertragliche Ansprüche auf Schadensersatz und Aufwendungsersatz. ${ }^{831}$ Voraussetzung ist das Vorliegen haftungsbegründender Kausalität sowie ein bezifferbarer Schaden. Die Bezifferbarkeit setzt voraus, dass in der Eingliederungsvereinbarung Anhaltspunkte für die Entstehung eines Schadens aufgeführt sind. ${ }^{832}$ Die bloße fehlgeschlagene Vermittlung bei hinreichend konkreten Vermittlungsbemühun-

827 Berlit, SozR akt 2006, S. 49.

828 Müller, in: Hauck/Noftz, SGB II, K $§ 15$ Rn. 42; so auch ohne weitere Begründung Stark, in: Estelmann, SGB II, § 15 Rn. 78.

829 Berlit, in: Münder/Armborst, LPK-SGB II, 2005, § 15 Rn. 12.

830 Vgl. zweites Kapitel B II 8c) S. 233; zweites Kapitel B II 9c) S. 237.

831 Knoblauch/Hübner, NDV 2005, S. 281.

832 Berlit, SozR akt 2006, S. 49. 
gen der Arbeitsagentur als solche wird sicher nicht ausreichen, um Ansprüche des Einzelnen begründen zu können.

\section{cc) Amtshaftungsansprüche}

Amtshaftungsansprüche kommen stets in Betracht ${ }^{833}$, sind aber wegen ihrer strengen Voraussetzungen, insbesondere einer schuldhaft verletzten Amtspflicht gegenüber dem Geschädigten nur selten erfolgreicher als vertragliche Anspruchsgrundlagen. Ein Anwendungsgebiet ist sicherlich der schuldhaft schlecht betreute Arbeitslose, der nicht nur Opfer der Überlastung des Fallmanagers ist, sondern einem willkürlich handelnden Mitarbeiter der Agentur für Arbeit gegenübersteht. Hier kommen tatsächlich Schadensersatzansprüche aus Amtshaftung in Betracht, die aber ohne eine konkrete Situation nicht genauer beurteilt werden können.

\section{Beendigung der Eingliederungsvereinbarung}

\section{a) Erledigung}

Auch die Eingliederungsvereinbarung nach $\S 15$ SGB II erledigt sich durch Beendigung des Zustands der Arbeitslosigkeit, denn dann ist die Voraussetzung ,erwerbsfähiger Hilfebedürftiger" nicht mehr gegeben, und die Vereinbarung wird gegenstandslos.

\section{b) Zeitablauf}

Die Eingliederungsvereinbarung wird im Regelfall für sechs Monate abgeschlossen, $\S 15$ Abs. 1 S. 3 SGB II. Besteht die Arbeitslosigkeit fort, soll eine Anschlussvereinbarung geschlossen werden, $\S 15$ Abs. 1 S. 4, 5 SGB II. Nach diesem Zeitraum und wenn keine Anschlussvereinbarung geschlossen wird, endet die ursprüngliche Vereinbarung wegen Fristablaufs. Ist keine Befristung vereinbart, endet die Vereinbarung theoretisch nie, außer im Fall einer Erledigung. 834

\section{c) Änderung der Verhältnisse, §59 Abs. 1 S. 1 SGB X}

Die ursprüngliche Vereinbarung wird durch die Anpassung des Vertragsinhaltes gem. $§ 59$ Abs. 1 S. 1 SGB X abgeändert. Sie besteht also fort. ${ }^{835}$ Im Fall der Eingliederungsvereinbarung nach SGB II führt deshalb eine Änderung der Verhältnisse nicht zu einem Beendigungstatbestand.

\section{d) Kündigung des verwaltungsrechtlichen Vertrags, § 59 SGB X}

In $\S 59$ Abs. 1 S. 1 ist für den Fall ein Kündigungsrecht vorgesehen, dass die Vertragsanpassung aufgrund veränderter Verhältnisse nicht möglich oder einer Partei nicht zumutbar ist. Da die Grenze zwischen einer hinzunehmenden Vertragsstörung und einer

833 So auch Berlit, SozR akt 2006, S. 49.

834 Knoblauch/Hübner, NDV 2005, S. 281.

835 Bonk, in: Stelkens/Bonk/Sachs, VwVfG, 2001, § 60 Rn. 22 ff. 
Störung, die zur Kündigung berechtigt, liegt und von den Umständen im Einzelfall abhängt ${ }^{836}$, ist an dieser Stelle keine abschließende Stellungnahme möglich. Bislang hat die Rechtsprechung noch nicht Position beziehen müssen. Es ist aber von einer engen Auslegung auszugehen, da die Bindung an einen Vertrag nicht ohne ein gewisses Gewicht der Gründe beseitigt können werden soll.

Als ultima ratio hat die Verwaltung ein Kündigungsrecht, um schwere Nachteile für das Gemeinwohl zu verhüten oder zu beseitigen. Der Begriff „schwerer Nachteil“ für das Gemeinwohl ist eng auszulegen. Es müssen besondere, erhebliche, überragende Interessen der Allgemeinheit bedroht sein. Bestünde der Vertrag fort, würden dem Staat auf allen seinen Ebenen unzumutbare Lasten auferlegt. Die in $\S 58$ SGB X beschriebenen Nichtigkeitsgründe des verwaltungsrechtlichen Vertrages dürfen durch das Kündigungsrecht mithin nicht ausgehöhlt werden. ${ }^{837}$ Aufgrund des engen Anwendungsbereichs der Vorschrift wird sie bei den Größenordnungen von Leistungen, die in einer einzelnen Eingliederungsvereinbarung vereinbart werden können, keine Anwendung finden. Sie wird hier eher der Vollständigkeit halber genannt, um die Regelungsdichte des verwaltungsrechtlichen Vertrages im deutschen Recht aufzuzeigen.

\section{Rechtsschutz}

\section{a) Außergerichtlicher Rechtsschutz}

\section{aa) Dienstaufsichtsbeschwerde}

Die Dienstaufsichtsbeschwerde ist auch für den Fall eines willkürlich handelnden Fallmanagers ein Druckmittel, aber sicher kein objektives Überprüfungsinstrument. Problematisch und für den Bürger schwer feststellbar ist die Zuständigkeit für die Dienstaufsicht in den Arbeitsgemeinschaften. Diese richtet sich danach, ob der Arbeitsgemeinschaft die Dienstherreneigenschaft zukommt. Dies ist derzeit unterschiedlich von Bundesland zu Bundesland. Teilweise wurden Arbeitsgemeinschaften in öffentlichrechtlichen Formen gegründet und haben deshalb Dienstherreneigenschaft. Dann ist die Dienstaufsichtbeschwerde dort einzulegen. ${ }^{838}$ In den anderen Arbeitsgemeinschaften muss sich der Bürger an die Körperschaft halten, bei der der betreffende Mitarbeiter beschäftigt ist. Von außen ist dies nicht erkennbar und deshalb ein Handlungsfeld für den Gesetzgeber, sobald grundsätzlich über die Neuorganisation des Vollzugs des SGB II entschieden ist.

\section{bb) Ombudsrat}

Für die Zeit vom 1.1.2005 bis 30.6.2006 setzte der damalige Bundesminister für Wirtschaft und Arbeit Wolfgang Clement in Abstimmung mit dem damaligen Bundeskanzler Gerhard Schröder den Ombudsrat „Grundsicherung für Arbeitsuchende“ ein.

836 Dazu allgemein Bonk, in: Stelkens/Bonk/Sachs, VwVfG, 2001, § 60 Rn. 17.

837 Vgl. Bonk, in: Stelkens/Bonk/Sachs, VwVfG, 2001, § 60 Rn. 26 ff.

838 Z.B. VG Arnsberg, Urteil v. 22.3.2007, 20 K 2029/06.PVL. 
Außerhalb des regulären Widerspruchs- und Klageverfahrens konnten dort Einzelfälle, Fälle von besonderer Bedeutung oder häufig wiederkehrende Fragestellungen nach dessen eigenem Ermessen aufgegriffen werden. Einwände der künftigen Empfänger von Arbeitslosengeld II waren nach wie vor an die zuständige Behörde zu richten, konnten dort aber unbürokratisch aufgegriffen und auf das politische Tableau gebracht werden. So sollten Schwachstellen der Regelungen des SGB II schneller aufgedeckt und dem zuständigen Bundesminister zur Weiterentwicklung des Gesetzes und seiner Anwendung zugeleitet werden. Die schriftlichen Eingaben wurden in einer eigens eingerichteten Geschäftsstelle bearbeitet, telefonische Anfragen von der Infostelle beantwortet. Während der Übergangszeit gab es so eine zusätzliche Auskunftsstelle für Fragen und Probleme der Grundsicherung für Arbeitsuchende. 839

\section{b) Widerspruchsverfahren}

In $\S 39$ SGB II ist vorgesehen, dass der Widerspruch gegen einen Verwaltungsakt, der über Leistungen der Grundsicherung für Arbeitsuchende entscheidet, keine aufschiebende Wirkung hat. Einen solchen stellt der Sanktionsbescheid gem. $\S 31$ Abs. 6 SGB II dar, sodass es in diesem Fall neben der Einlegung des Widerspruchs erforderlich ist, die Wiederherstellung der aufschiebenden Wirkung zu beantragen, § 86a Abs. $2 \mathrm{Nr}$. 4 SGG. Auch für andere Bescheide über Eingliederungsleistungen gelten diese Ausführungen.

Für die Eingliederungsvereinbarung als solche gibt es keine spezielle Regelung. Das Widerspruchsverfahren ist damit bei Zweifeln über die Rechtmäßigkeit der Eingliederungsvereinbarung nicht einzuleiten, weil es sich nicht um einen Verwaltungsakt im Sinn des $\S 31$ SGB X handelt.

\section{c) Gerichtlicher Rechtsschutz}

Beim gerichtlichen Rechtsschutz sind drei Konstellationen zu unterscheiden: Es stehen Klagen mit dem Ziel der Erfüllung der Eingliederungsvereinbarung neben Klagen, in denen eine einseitige Sanktion angefochten wird und Klagen auf Feststellung der Rechtswidrigkeit der Eingliederungsvereinbarung.

aa) Rechtsweg, § 51 SGG

Der Rechtsweg zu den Sozialgerichten ist für die drei Fallgestaltungen gem. § 51 Abs. 1 Nr. 4a SGG eröffnet, denn es handelt sich bei den Streitigkeiten stets um solche über die Grundsicherung für Arbeitsuchende, die im SGB II geregelt ist.

\section{bb) Rechtsschutz bei Umsetzung der Vereinbarung}

Die Vertragserfüllung ist im Wege der Leistungsklage gem. § 54 SGG zu erwirken, es sei denn, der Vertrag enthält eine Unterwerfungsklausel, die als Vollstreckungsklau-

839 Ombudsrat - Grundsicherung für Arbeitsuchende, Schlussbericht vom 23.6.2006, S. 5 f; dazu Rexin, SozSich 2006, S. 243 ff. 
sel dienen kann. ${ }^{840}$ Dann kann das Zwangsvollstreckungsverfahren eingeleitet werden. Problematisch kann bei der Leistungsklage wegen Schadensersatz auf der Grundlage des $\S 15$ Abs. 3 SGB II sein, dass in der Eingliederungsvereinbarung die wesentlichen Faktoren für die Entstehung des Schadensersatzanspruchs enthalten sein müssen.

\section{cc) Rechtschutz gegen Sanktionen und Einstellung der Vermittlung}

\section{(1) Klägerisches Begehren}

Das klägerische Begehren des erwerbsfähigen Hilfebedürftigen ist bei einer Senkung des Arbeitslosengeldes II gem. § 31 SGB II die Auszahlung der ungesenkten Summe. Bei der Einstellung der Vermittlungstätigkeit ist es die Aufhebung des Bescheids, in dem die Arbeitsverwaltung diese Entscheidung bekannt gibt.

\section{(2) Klageart}

Hat die Arbeitsverwaltung einen Verwaltungsakt erlassen und richtet sich das klägerische Begehren auf die Beseitigung dieses Verwaltungsaktes, ist grundsätzlich die Anfechtungsklage statthafte Klageart, § 54 Abs. 1 S. 1 SGG. Das gilt auch für den Bescheid, in dem die Einstellung der Vermittlung mitgeteilt wird.

Auch bei einem Sanktionsbescheid im Sinn des $\S 31$ Abs. 6 SGB II ist statthafte Klageart grundsätzlich die Anfechtungsklage. Die Senkung des Arbeitslosengeldes II gem. § 31 SGB II geschieht zwar durch Gesetz. ${ }^{841}$ Es handelt sich deshalb bei dem Sanktionsbescheid um einen feststellenden Verwaltungsakt, der besagt, was de lege lata bereits gilt. Dieser Bescheid stellt die Rechtslage verbindlich fest, enthält mithin also eine Regelung im Sinn des § 31 SGB X. Denn solange der Bescheid in der Welt ist, ist er Rechtsgrundlage für die Kürzung der Leistung. Der erwerbsfähige Hilfebedürftige wird also durch diesen Bescheid in Kenntnis gesetzt, dass er eine abgesenkte Summe Arbeitslosengeld II ausbezahlt bekommt. Wird dieser Bescheid aufgehoben, lebt der bisher wirksame Leistungsbescheid wieder auf.

Anders ist der Fall, wenn noch kein Leistungsbescheid erlassen worden ist. Dann genügt nicht die Aufhebung des Sanktionsbescheides, mithin also nicht die Anfechtungsklage, weil er damit nicht sein Ziel, die Auszahlung des Arbeitslosengeldes II in ungesenkter Höhe, erreichen kann. Statthaft ist vielmehr für diesen Fall die Verpflichtungsklage auf Erlass eines Bescheides über die Auszahlung eines Arbeitslosengeldes II in ungekürzter Höhe.

\section{dd) Feststellung der Nichtigkeit der Eingliederungsvereinbarung}

In der Eingliederungsvereinbarung wird ein grundsätzlich feststellungsfähiges Rechtsverhältnis im Sinn des $\S 55 \mathrm{Nr}$. 1 SGG begründet oder jedenfalls inhaltlich geändert. Ein Vorverfahren ist bei der Feststellungsklage nicht durchzuführen, da dieses nur

840 BSGE 35, 47 [50 f].

841 Vgl. zweites Kapitel B II 7c) S. 218 ff. 
bei Verwaltungsakten, nicht aber bei verwaltungsrechtlichen Verträgen vorgesehen ist, $\S 78$ SGG i.V.m. § 39 Nr. 1 SGB II.

Problematisch ist, ob ein berechtigtes Interesse des Hilfebedürftigen an der Feststellung vorliegt, dass das Rechtsverhältnis, das die Eingliederungsvereinbarung begründet hat, nicht gegeben ist. Dem steht entgegen, dass der Hilfebedürftige den Sanktionsbescheid abwarten und gegen diesen vorgehen könnte. Verweist man den Hilfebedürftigen auf eine Inzidenzprüfung im Rahmen des Rechtsschutzes gegen diesen Bescheid, weist man ihm das Risiko einer Fehleinschätzung der Rechtmäßigkeit der Eingliederungsvereinbarung zu. Denn er müsste zunächst die Absenkung hinnehmen und schließlich bei einem non-liquet die Last der Nichterweislichkeit eines wichtigen Grundes i.S.d. § 31 Abs. 1 S. 2 SGB II tragen. Die Wahl der Rechtsform Vertrag hätte rechtsschutzverkürzende Wirkung, wenn die Eingliederungsvereinbarung selbst nicht im Wege einer Feststellungsklage gesondert überprüfbar ist. Damit wäre Art. 19 Abs. 4 GG berührt. Dies begründet unabhängig von der Frage nach dem gerichtlichen Prüfungsmaßstab für die Nichtigkeit der Eingliederungsvereinbarung die Zulässigkeit der Feststellungsklage zur Überprüfung der Nichtigkeit der Eingliederungsvereinbarung. ${ }^{842}$

d) Kosten

Es gilt der Grundsatz der Gebührenfreiheit im Sozialrecht. Das Verfahren vor den Gerichten der Sozialgerichtsbarkeit ist für Leistungsempfänger der Grundsicherung für Arbeitsuchende kostenfrei, soweit sie in dieser jeweiligen Eigenschaft als Kläger oder Beklagter beteiligt sind, § 183 S. 1 SGG.

842 So auch Berlit, SozR akt 2006, S. 49. 


\section{Drittes Kapitel: Ergebnisse und Auswertung des Rechtsvergleichs}

\section{A. Zusammenfassung der Ergebnisse}

\section{Jobseeker's agreement}

Das jobseeker's agreement wird schriftlich zwischen dem Leistungsempfänger, dem Arbeitslosen, und der Arbeitsverwaltung als Leistungsträger abgeschlossen. Die Kooperation findet grundsätzlich zwischen zwei Personen (Arbeitsloser und employment officer) statt, es sei denn, es wird ein Antrag als joint claim couple gestellt. Dann hat das $J S A$ drei Parteien. Die Parteien kooperieren vor der eigentlichen Entscheidung über die jobseeker's allowance, da das Vorliegen eines wirksamen agreement Leistungsvoraussetzung ist. ${ }^{1}$

Eine Änderung des $J S A$ ist möglich, aber nur in derselben Form wie das ursprüngliche $J S A$, denn das Vereinbarte hat verbindlichen Charakter. Das $J S A$ ist aber nicht verbindlich in dem Sinn, dass aus der Vereinbarung Ansprüche der Parteien resultieren. ${ }^{2}$ Es handelt sich um vertragsähnliches Verwaltungshandeln. ${ }^{3}$

Eine durch ein Social Security Administrative Tribunal sichergestellte eigenständige Durchsetzbarkeit des Vereinbarten kennt das englische Recht nicht. Es wird nur im Wege der Feststellung, ob die Leistungsvoraussetzungen vorlagen, das Vorhandensein eines wirksamen $J S A$ festgestellt. Das $J S A$ ist somit voll überprüfbar, sowohl im Hinblick auf den Abschluss als auch im Hinblick auf den Inhalt. Spielräume, die allein durch Verhandlung zwischen den Parteien ausgefüllt werden könnten und der rechtlichen Kontrolle entzogen wären, gibt es nicht. ${ }^{4}$

Die Möglichkeit, im Wege von judicial review gesondert gegen das jobseeker's agreement vorzugehen, besteht nicht. ${ }^{5}$

\section{Eingliederungsvereinbarung nach dem SGB III}

Bei der Eingliederungsvereinbarung nach dem SGB III handelt es sich um Kooperation zwischen einem einzelnen Arbeitslosen und einem Mitarbeiter der Arbeitsagentur, also auch um Kooperation zwischen Leistungsempfänger und Leistungsträger. Sie ist stets zweiseitig und bezieht keine weitere Person ein, insbesondere weil am Versicherungsverhältnis nur der Versicherte und die Bundesagentur für Arbeit beteiligt sind.

1 Vgl. zweites Kapitel A II 1, 2, 3 S. 109 ff.

2 Vgl. zweites Kapitel A II 7 S. 124 ff.

3 Vgl. erstes Kapitel B V S. 91 ff; zweites Kapitel A II 4 S. 111 ff.

4 Vgl. zweites Kapitel A II 9d) S. 135 ff.

5 Vgl. zweites Kapitel A II 9e) S. 138 f. 
Zeitlich betrachtet findet die Verhandlung vor einer einseitigen Entscheidung über Eingliederungsleistungen statt. 6

Das Kooperationsergebnis, welches in der schriftlichen ${ }^{7}$ Eingliederungsvereinbarung festgehalten und als sichtbares Zeichen für die Verbindlichkeit von den Parteien unterzeichnet wird, ist sowohl für den Arbeitslosen als auch für die Arbeitsverwaltung bindend. Es handelt sich um vertragsähnliches Verwaltungshandeln. ${ }^{8}$ Beide Parteien geben eine Erklärung mit Bindungswillen ab, die sich nach der Erteilung des Einverständnisses der anderen Partei nach der Abgabe nicht mehr einseitig verändern lässt. Die Bindung der Arbeitsverwaltung wirkt bei der Ermessensausübung vor einer einseitigen Entscheidung über Eingliederungsleistungen fort. ${ }^{9}$

Die Eingliederungsvereinbarung ist gerichtlich nicht durchsetzbar. Es fehlt bei vertragsähnlichem Handeln die beim privatrechtlich oder öffentlich-rechtlichen Vertrag bestehende Gewissheit, dass staatliche Stellen die Einhaltung des Vereinbarten sicherstellen. Die Eingliederungsvereinbarung ist eine Handlungsform, die als solche nicht im Verwaltungsprozess überprüft werden kann. ${ }^{10}$

Indirekt erfolgt eine Durchsetzung möglicher Kooperationsergebnisse über die Androhung von Sperrzeiten bereits im Vorfeld des Abschlusses der Eingliederungsvereinbarung. Doch sanktioniert der im SGB III enthaltene Sperrzeittatbestand nicht die Nichteinhaltung der Eingliederungsvereinbarung unmittelbar, sondern den fehlenden Nachweis ungenügender Eigenbemühungen, die zudem nicht zwangsläufig abschlieBend in der Eingliederungsvereinbarung aufgeführt sind. ${ }^{11}$

Die gerichtliche Überprüfbarkeit des Vereinbarten bleibt der Kontrolle der Ermessensentscheidung über Eingliederungsleistungen vorbehalten. Sie erfolgt damit nur im Wege der Ermessensfehlerlehre und schließt eine Zweckmäßigkeitskontrolle des Vereinbarten aus. ${ }^{12}$ Hinzukommt, dass der Arbeitslose die Sperrzeit abwarten muss, bis er die Eingliederungsvereinbarung überprüfen lassen kann. Das bringt die Hinnahme einer möglicherweise rechtswidrigen Sanktion mit sich und damit zunächst einen Nachteil für den Arbeitslosen. Letztendlich aber ist die Entscheidung über die Eingliederungsleistung vollumfänglich überprüfbar, sodass sich kein bleibender Nachteil für den Arbeitslosen einstellen kann. ${ }^{13}$

6 Vgl. zweites Kapitel B I 2, 3 S. 145 ff.

7 Vgl. zweites Kapitel B I 4 S. 150.

8 Vgl. erstes Kapitel B V S. 91 ff; zweites Kapitel B I 5 S. 150 ff.

9 Vgl. zweites Kapitel B I 7c) S. $171 \mathrm{ff}$.

10 Vgl. zweites Kapitel B I 10c) S. $180 \mathrm{ff}$.

11 Vgl. zweites Kapitel B I 7d) S. 175 ff.

12 Vgl. zweites Kapitel B I 7c) S. 171.

13 Vgl. zweites Kapitel B I 10c) S. 180 f. 
Bei der Eingliederungsvereinbarung nach dem SGB II handelt es sich grundsätzlich um eine Kooperation zwischen erwerbsfähigem Hilfebedürftigem und Arbeitsverwaltung, also ebenfalls um Kooperation zwischen Leistungsempfänger und Leistungsträger. ${ }^{14}$ Die schriftliche Eingliederungsvereinbarung des SGB II ${ }^{15}$ kann zwei oder mehr Parteien haben. Die Bedarfsgemeinschaft kann vom Inhalt der Eingliederungsvereinbarung betroffen sein. Sobald der Hilfebedürftige die Eingliederungsvereinbarung in Ausübung einer Vertretungsbefugnis abschließt, und sobald ein Mitglied der Bedarfsgemeinschaft selbst am Kooperationsprozess teilnimmt, indem es mit unterzeichnet, kommt eine weitere Willenserklärung dazu. Dann handelt es sich um eine mehrseitige Vereinbarung. ${ }^{16}$

Die Eingliederungsvereinbarung wird als verwaltungsrechtlicher Vertrag geschlossen und ist allein deshalb bereits als verbindliche kooperative Handlungsform einzuordnen. ${ }^{17}$ Die Beteiligung erfolgt als Mitentscheidung, denn das Kooperationsergebnis wird von den Vertragspartnern verantwortet. 18

Das Kooperationsergebnis ist grundsätzlich gerichtlich durchsetzbar, bei entsprechender Gestaltung der Vereinbarung sofort vollstreckbar. ${ }^{19}$

Die rechtliche Kontrolle ist vor Abschluss des Vertrages uneingeschränkt möglich, denn bei der Überprüfung einer Sanktion wegen Ablehnung der Eingliederungsvereinbarung hat das Gericht insbesondere zu prüfen, ob für die Abschlussverweigerung ein wichtiger Grund vorgelegen hat. Im Rahmen der Prüfung dieses wichtigen Grundes wird festgestellt, ob der Vertragsinhalt mit der Rechtslage vereinbar ist. ${ }^{20}$

Nach Abschluss der Vereinbarung ist die Überprüfbarkeit auf die im SGB X vorgesehene Nichtigkeitskontrolle beschränkt. Diese ist weit auszulegen, um den besonderen Folgen der Eingliederungsvereinbarung gerecht zu werden. ${ }^{21}$ Wird eine Sanktion wegen Nichteinhaltung einer vereinbarten Pflicht überprüft, ist auch das Gericht auf diesen Prüfungsmaßstab beschränkt. ${ }^{22}$ Eine Überprüfungsmöglichkeit (Feststellung der Nichtigkeit) besteht auch bereits vor Verhängung der Sanktion. ${ }^{23}$

Wird ein Anspruch auf eine konkrete Leistung, die in der Eingliederungsvereinbarung vereinbart wurde, geltend gemacht, ist die Eingliederungsvereinbarung direkt Gegenstand einer gerichtlichen Entscheidung. ${ }^{24}$

14 Vgl. zweites Kapitel B II 2a) 187.

15 Vgl. zweites Kapitel B II 4 S. 198.

16 Vgl. zweites Kapitel B II 2b), 2c) S. 188 ff.

17 Vgl. erstes Kapitel B IV S. 82 ff; zweites Kapitel B II 8d) S. 233 ff.

18 Vgl. zweites Kapitel B II 5 S. 198 ff.

19 Vgl. zweites Kapitel B II 8d) S. 233 ff.

20 Vgl. zweites Kapitel B II 7c) aa) S. 218 ff; zweites Kapitel B II 10c) cc) S. 240.

21 Vgl. zweites Kapitel B II 8a) S. 226 ff.

22 Vgl. zweites Kapitel B II 7c) bb) S. 222 ff.

23 Vgl. zweites Kapitel B II 10c) dd) S. 240.

24 Vgl. zweites Kapitel B II 10c) bb) S. 239. 


\section{B. Vergleich und Bewertung}

\section{Kooperation - Ziele erreicht?}

\section{Steigerung der Effizienz in der Verwaltungsarbeit}

Die Steigerung der Effizienz in der Verwaltungsarbeit ist das oberste Ziel kooperativen Verwaltungshandelns. Im ersten Kapitel ist die Begründung für dieses Ziel dargestellt: Großer Aufwand und das Bedürfnis nach Flexibilität, aber auch sachliche Zwänge machen in bestimmten Aufgabenfeldern der Verwaltung ein Abgehen von einseitigem Verwaltungshandeln erforderlich. So können z.B. Standards für die Leistungserbringung mittels Verträgen im Einvernehmen festgelegt werden. Vereinbarungen füllen zielgerichtet und wirkungsvoll vorhandene Spielräume aus. Kooperation ist allgemein gesprochen ein „dritter Weg“ 25 zwischen hoheitlich-hierarchischer Erledigung öffentlicher Aufgaben und gesellschaftlicher Selbststeuerung ohne staatliche Beteiligung. ${ }^{26}$

Effiziente Verwaltungsarbeit im Verhältnis zum Einzelnen erfordert, dass die Aufgaben, die die Verwaltung weiterhin gegenüber dem einzelnen Bürger erbringen soll, flexibel und transparent erfüllt werden. Dadurch wird in den Augen der Betroffenen nicht nur Verständnis für die Rechtslage geschaffen oder der Grund für die Verhängung von Sanktionen deutlich gemacht, kurz: die Akzeptanz für das Verwaltungshandeln gesteigert, sondern es wird auch schnelle und passgenaue Vermittlung in den Arbeitsmarkt ermöglicht. Unter diesen Gesichtspunkten kann Kooperation effiziente Verwaltungsarbeit gerade im Verhältnis zum einzelnen Bürger sicherstellen. ${ }^{27}$

Bei den untersuchten Vereinbarungen wird dieses Ziel nicht erreicht. Der Gesetzgeber ordnet in beiden Rechtsordnungen die Kooperation gesetzlich an. ${ }^{28}$ Grund dafür war die Programmatik aktivierender Arbeitsmarktpolitik/activating labour market policy und die Überzeugung des Gesetzgebers, dass Vereinbarungen zwischen Bürger und Staat im Sozialrecht Verbesserungen bringen werden. ${ }^{29}$ Die Vereinbarungen sind im englischen wie im deutschen Recht so ausgestaltet, dass sie ganz auf den Einzelfall auszurichten sind.

In England haben Evaluierungen ergeben, dass sich seit der Einführung der jobseeker's allowance die Vorstellung verbreitet hat, dass die Rechtsbeziehungen zwischen Staat und Arbeitslosem einen vertraglichen Charakter haben. Ein Grundanliegen der aktivierenden Arbeitsmarktpolitik ist also erfüllt. Diese Veränderung lässt sich aber nicht auf das JSA zurückführen, denn dieses wurde zwar abgeschlossen und grundsätzlich eingehalten, aber nicht als für die Wiedereingliederung in den Arbeitsmarkt dienlich betrachtet. Es hat deshalb zwar eine Effizienzsteigerung in der Arbeitsverwaltung

25 Hill, DVB1 1993, S. 976; Benz, Kooperative Verwaltung, 1994, S. 15, 59.

26 Vgl. erstes Kapitel A II 1 S. 54 f.

27 Vgl. erstes Kapitel A II 1 S. 54 f.

28 Vgl. drittes Kapitel A I - III S. 243-245.

29 Dazu Einführung A I S. 28 ff. 
stattgefunden, ein Zusammenhang zur kooperativen Handlungsform JSA lässt sich aber nicht herstellen. ${ }^{30}$

Für Deutschland ist die Evaluierung nicht so weit fortgeschritten, dass belastbare Ergebnisse vorlägen. Vor allem für das SGB III fehlen jegliche veröffentlichte Daten. Es lässt sich aber aus den anlässlich der Neuausrichtung der arbeitsmarktpolitischen Instrumente vorgenommenen Änderungen zur Eingliederungsvereinbarung im SGB III ${ }^{31}$ ableiten, dass der Gesetzgeber an diesem Instrument festhalten will. Er sieht die Eingliederungsvereinbarung offenbar insbesondere als geeignet an, bei der Wiedereingliederung älterer Arbeitsloser positiv zu wirken. § 35 Abs. 4 SGB III a.F. wurde deshalb insoweit ergänzt, als die Ausstellung des Eingliederungsgutscheins entweder mit einem konkreten Arbeitsangebot oder mit einer Vereinbarung über die notwendigen Eigenbemühungen verbunden werden sollte. ${ }^{32}$ Diese Änderung wurde in die neue Regelung zur Eingliederungsvereinbarung, § 37 SGB III, übernommen.

Für den Anwendungsbereich des SGB II sind nach Untersuchung der Jahre 2005 und des ersten Quartals 2006 durch den Bundesrechnungshof nicht etwa Effizienzsteigerungen, sondern vielmehr Vollzugsdefizite aufgedeckt worden. Der Bundesrechnungshof stellte fest, dass die Träger der Grundsicherung für Arbeitsuchende den Grundsatz des „Förderns" nicht ausreichend umgesetzt haben, da nicht alle Möglichkeiten ausgeschöpft worden sind, erwerbsfähige Hilfebedürftige zu integrieren. Eingliederungsvereinbarungen sind durchschnittlich erst nach vier Monaten abgeschlossen, und in der Hälfte der Fälle sind überhaupt keine Vereinbarung getroffen worden. ${ }^{33}$ Diese Defizite hat das Bundesarbeitsministerium mit Anlaufschwierigkeiten gerechtfertigt. ${ }^{34}$ Als Reaktion auf die Kritik durch den Bundesrechnungshof hat die Bundesagentur für Arbeit eine Arbeitshilfe und eine Geschäftsanweisung (immer noch Stand: 14.9.2006) herausgegeben, die den als SGB II-Träger tätigen Arbeitsagenturen Vorgaben für den Abschluss der Eingliederungsvereinbarung machen. ${ }^{35}$ Es ist davon auszugehen, dass die Vollzugsdefizite aufgrund flächendeckender Anwendung der Arbeitshilfe inzwischen deutlich verringert sind. Ob sich letztendlich eine messbare auf die Eingliederungsvereinbarung zurückzuführende Steigerung der Verwaltungseffizienz herausstellen wird, bleibt genauso wie weitere Evaluierungen der Eingliederungserfolge abzuwarten.

30 Vgl. Rayner u.a., Evaluating the jobseeker's allowance, 2005, S. 2.

31 Gesetz v. 21.12.2008 (BGBl., S. 2917).

32 BT-Drs. 16/7460, S. 9.

33 BT-Drs. 16/3200, S. 16 f, 111 f, 116.

34 BT-Drs. 16/3200, S. 16.

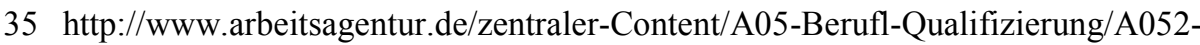
Arbeitnehmer/Publikation/pdf/Arbeitshilfe-Eingliederungsvereinbarung-P15.pdf; http://www.arbeitsagentur.de/zentraler-Content/A07-Geldleistung/A071Arbeitslosigkeit/Publikation/pdf/GA-Anforderung-Eingliederungsvereinbarung.pdf. 
Für das Sozialrecht spielt die Kommunikation zwischen Sozialverwaltung und Leistungsberechtigtem eine wichtige Rolle. Wird der Einzelne nur so weit wie unbedingt nötig einbezogen, entsteht oftmals ein Informationsdefizit bei der Verwaltung in Bezug auf die tatsächlichen Fähigkeiten, Fertigkeiten und Bedürfnisse des Einzelnen. Kooperatives Vorgehen ist ein möglicher Weg, eine gewisse Offenheit des Leistungsempfängers gegenüber der Verwaltung zu erreichen und während des Leistungsbezugs aufrechtzuerhalten. ${ }^{36}$

Die Ausgestaltung der Instrumente in den untersuchten Rechtsordnungen unterscheidet sich darin, dass die Informationsgewinnung im Bereich des $J S A$ ohne rechtliche Kategorien auskommt, während das deutsche Recht die Information der Verwaltung neben der Eingliederungsvereinbarung mit Mitwirkungspflichten sicherstellt.

Die Customer's Charter ist die Grundlage für die Einbeziehung des Einzelnen in die Verwaltungsarbeit. Dort wird die Mitwirkung des Bürgers bewusst nicht als rechtliche Verpflichtung eingeordnet und spielt deshalb - anders als im SGB II - bei der Entscheidung über die jobseeker's allowance und beim Abschluss des JSA keine Rolle. Die Gewinnung von Informationen über den Einzelnen ist zwar ein zentrales Anliegen, um auch hier die Eingliederungsdefizite des Arbeitslosen möglichst zielgerichtet beseitigen zu können. Im englischen Recht sind dafür aber keine eigenständigen rechtlichen Kategorien zu finden. Vielmehr findet man Standards für den Umgang miteinander, deren Einhaltung verpflichtend ist, aber keine rechtlichen Konsequenzen entfalten. ${ }^{37}$

Im deutschen Recht finden sich gesetzlich geregelte allgemeine Mitwirkungspflichten in $\S \S 60 \mathrm{ff}$ SGB I, die in erster Linie der Information der Verwaltung dienen. ${ }^{38}$ Diese können im SGB III nicht bilateral mit einer Eingliederungsvereinbarung modifiziert werden, insbesondere weil das Versicherungsverhältnis in Bezug auf die Eingliederungsleistungen abschließend gesetzlich geregelt ist. ${ }^{39}$ Die Verweigerung der Sozialleistung wegen fehlender Mitwirkung gem. § 66 SGB I bleibt im SGB III rechtlich möglich, aber nur soweit die Entgeltersatzleistung betroffen ist. In Bezug auf die Mitwirkungspflichten bei der Vermittlung ist $\S 38$ Abs. 2 SGB III die speziellere Regelung. ${ }^{40}$ Obwohl die Eingliederungsvereinbarung im SGB III selbst keine Verbindung zwischen Eingliederungsleistung und Mitwirkung herstellt oder fehlende Mitwirkung sanktionierbar macht, ist seit der Einführung einer speziellen Mitwirkungspflicht in $\S 38$ SGB III die Informationsgewinnung mit einer rechtlichen Pflicht abgesichert.

In der Eingliederungsvereinbarung nach dem SGB II werden die allgemeinen Mitwirkungspflichten der $\S \S 60$ ff SGB I konkretisiert und oftmals gleichzeitig verschärft. Die Informationsgewinnung ist nicht nur mit Hilfe allgemeiner Mitwirkungspflichten sichergestellt, sondern die Nichterfüllung einer Pflicht zur Information hat direkten Ein-

36 Vgl. erstes Kapitel A II 2 S. 55 f

37 Vgl. zweites Kapitel A II 5 S. 116 ff.

38 Hauck, in: Hauck/Noftz, SGB I, K $\S 60$ Rn. 1 m.w.N..

39 Vgl. zweites Kapitel B I 6c) S. 163.

40 Vgl. zweites Kapitel B I 7d) S. 175 f. 
fluss auf die Leistungen zum Lebensunterhalt. ${ }^{41}$ Die von kooperativem Vorgehen erwartete Offenheit auf Seiten des Arbeitslosen zur freiwilligen Information ist zwar nicht ausgeschlossen, aber wegen der klaren rechtlichen Absicherung und der Möglichkeit einer Sanktionierung jedenfalls zweifelhaft.

\section{Legitimierende Wirkung}

Im kooperativen Prozess wird nicht nur der Adressat z.B. bei der Entscheidungsfindung beteiligt, und damit die Akzeptanz der Entscheidung erhöht, sondern es wird eine Rechtfertigung für das Verwaltungshandeln erarbeitet und transparent gemacht. Das Ergebnis eines kooperativen Prozesses entfaltet auf diese Weise legitimierende Wirkung. Verträge können sogar vollständig an die Stelle einer Norm als Rechtsgrundlage treten, Vereinbarungen können die Rechtsgrundlage für das Verwaltungshandeln ergänzen und konkretisieren. 42

Im englischen Recht ist das $J S A$ Leistungsvoraussetzung für die jobseeker's allowance und gehört damit zum gesetzlichen Tatbestand. Dies ist nach deutschem Recht weder im SGB III noch im SGB II der Fall.

Die Vereinbarungen im englischen Recht entfalten darüber hinaus legitimierende Wirkung, weil Abweichungen von gesetzlichen Vorschriften durch Vereinbarung zulässig sind: Im JSA können sog. permitted periods festgelegt werden. Das sind Zeiträume, in denen der Arbeitslose entgegen der strengen Definition des Jobseekers Act $1995 \mathrm{sec}$. 6, 7 nicht arbeitsbereit ist oder nicht aktiv Arbeit sucht, aber trotzdem als arbeitsbereit/aktiv arbeitsuchend angesehen wird. In einem Zeitraum von bis zu 13 Wochen werden ihm nur Stellen in seinem bisherigen Beruf und/oder zu seinem bisherigen Gehalt angeboten, und er selbst muss auch nur nach dieser Art von Stellen suchen. Für eine bestimmte Zeit wird dieser Schutz für den Antragsteller als sinnvoll betrachtet, um einen möglichst langen Verbleib in dem neu gefundenen Arbeitsverhältnis unabhängig von staatlicher Unterstützung zu erreichen. Damit wird zugelassen, dass ein gesetzlicher Tatbestand der jobseeker's allowance durch das JSA für den Einzelfall passend ausgestaltet wird. ${ }^{43}$

Die Eingliederungsvereinbarungen nach deutschem Recht befinden sich in „engerem Korsett" rechtlicher Gegebenheiten. Leistungsvoraussetzungen dürfen nicht aufgrund einer bilateralen Vereinbarung modifiziert werden. Dies gilt insbesondere für den Begriff der Zumutbarkeit, den sowohl das SGB III als auch das SGB II kennt, und der am ehesten die Funktion der permitted periods erfüllt. Was einem Arbeitslosen zumutbar ist, steht nach deutschem Recht abstrakt betrachtet fest. Für eine Aushandlung ist hier kein Raum. In der Eingliederungsvereinbarung kann lediglich das, was allgemein, wenn auch in Form eines unbestimmten Rechtsbegriffs feststeht und damit auch für den konkreten Arbeitslosen gilt, diesem verständlich formuliert vor Augen geführt werden. ${ }^{44}$

41 Vgl. zweites Kapitel B II 7d) S. 226; zweites Kapitel B II 7c) bb) S. 222 f.

42 Vgl. erstes Kapitel A II 3 S. 56.

43 Vgl. zweites Kapitel A II 2a) S. 110.

44 Vgl. zum SGB III zweites Kapitel B I 3c) S. 148; zum SGB II zweites Kapitel B II 3c) S. 196. 
Allgemein gesprochen ist es also im englischen Recht mittels Vereinbarung möglich, unbestimmte Rechtsbegriffe für den Einzelfall zu konkretisieren, aber auch in gleichartigen Fällen unterschiedlich auszulegen ${ }^{45}$, während im deutschen Recht lediglich abstrakt bestehende Regelungen so formuliert werden können, dass sie für den Einzelnen verständlicher sind. ${ }^{46}$

\section{Psychologisch-pädagogischer Effekt}

Die Verwaltung steht bei einer kooperativen Zusammenarbeit auf einer Ebene mit dem Einzelnen und hofft so, Einfluss auf den Einzelnen ausüben zu können. Stets verfolgt sie dabei das Ziel, den Grund für die Sozialleistung schrittweise zusammen mit dem Arbeitslosen zu beseitigen. Dazu genügt es oft nicht, wenn die Arbeitsverwaltung nur die rechtlichen Möglichkeiten in rechtmäßiger Weise ausschöpft, sondern der Leistungsempfänger muss selbst seinen Beitrag leisten, aber auch motiviert werden, den Leistungsbezug hinter sich zu lassen. ${ }^{47}$

Die Vereinbarung zwischen Arbeitsverwaltung und Einzelnem spielt in dieser Hinsicht nach englischem Recht nur eine untergeordnete Rolle. Die Prozesshaftigkeit des Zusammenwirkens ist wegen der Tatsache, dass das $J S A$ Leistungsvoraussetzung ist, auf ein Mindestmaß beschränkt. Von einer Abschlussfreiheit kann keine Rede sein. Der Einfluss, den die Verwaltung während des Verhandelns ausüben kann, soll sicherstellen, dass sich der Arbeitslose seiner Pflicht, baldmöglichst wieder in Arbeit zu kommen, bewusst ist. 48

Das SGB III lässt der Arbeitsverwaltung in dieser Hinsicht viel Handlungsspielraum. Eine Verknüpfung von Abschluss der Eingliederungsvereinbarung und Leistungsbezug ist im SGB III nicht zu finden. ${ }^{49}$ Aufgrund fehlender veröffentlichter Untersuchungen zum Einsatz der Eingliederungsvereinbarung im SGB III und dem Fehlen von Arbeitshilfen und Anweisungen für den Einsatz der Eingliederungsvereinbarung im Arbeitsförderungsrecht lässt sich an dieser Stelle nur vermuten, dass die Arbeitsverwaltung diesen Spielraum derzeit nicht ausfüllt und die positiven Wirkungen der Eingliederungsvereinbarung nicht zum Tragen kommen, weil die Eingliederungsvereinbarung nicht flächendeckend eingesetzt wird. ${ }^{50}$ Ein weiterer Versuch zu erklären, warum der pädagogische Effekt der Eingliederungsvereinbarung im SGB III weniger eingesetzt wird, ist, dass die Sanktionierung des Einzelnen nicht im Vordergrund steht und wohl nach geltender Rechtslage auch gesetzgeberisch nicht gewollt ist. Eine Verhaltensänderung beim Arbeitslosen zu erwirken, ist nicht unbedingt Aufgabe der Arbeitsverwaltung und der Ar-

45 Vgl. zweites Kapitel A II 6c) S. 123.

46 Vgl. zweites Kapitel B I 6d) S. 164; zweites Kapitel B II 6c) S. 213.

47 Vgl. erstes Kapteil A II 4 S. 31.

48 Vgl. zweites Kapitel A II 4c) S. 113.

49 Vgl. zweites Kapitel B I 7 S. 166 ff.

50 Dass dies auch der Gesetzgeber so sieht, bestätigen die Änderungen durch das Gesetz zur Neuausrichtung der arbeitsmarktpolitischen Instrumente v. 21.12.2008 (BGB1. I, S. 2917), insbesondere die detaillierte Regelung der Eingliederungsvereinbarung in $\S 37$ SGB III n.F.. 
beitslosenversicherung. Eine über die bestehenden gesetzlichen Pflichten hinausgehende Motivation des Arbeitslosen, sich schnell wieder um Arbeit zu bemühen, scheint nicht angestrebt zu sein. Durch die Beitragszahlung erkauft sich der Arbeitslose gewisse Spielräume, in die die Arbeitsverwaltung auch auf kooperativem Wege nicht eingreift. Die Einflussnahme mit Hilfe von Zumutbarkeitskriterien und der dort verankerte Schutz der Entscheidungsfreiheit des Arbeitslosen sind im Rahmen der Arbeitslosenversicherung ausreichend. 51

Ganz anders ist dies bei der Eingliederungsvereinbarung des SGB II. Dort ist die Wiedereingliederung in den Arbeitsmarkt unbedingtes Ziel jeder Maßnahme. Auch die Eingliederungsvereinbarung hat deshalb das Ziel, den einzelnen Arbeitslosen in dieser Richtung zu beeinflussen und ihn auch dazu zu motivieren. Da die Leistungen nach dem SGB II in den meisten Fällen erst dann relevant werden, wenn bereits eine Arbeitslosigkeit von gewisser Dauer vorliegt, liegt es für den Gesetzgeber nahe, den Druck auf den Einzelnen zu erhöhen und von diesem, wenn nötig, eine Verhaltensänderung zu verlangen. Damit wird zwar bereits in der Theorie die Verantwortung für das Risiko, arbeitslos zu werden und zu bleiben, in gewisser Weise auf den Einzelnen verlagert. Es ist die „bereitwillige“ Suche nach jedem Weg heraus aus der Arbeitslosigkeit, der dem Einzelnen mit Hilfe der Eingliederungsvereinbarung gezeigt werden soll. 52

Der psychologisch-pädagogische Effekt kann dann beeinträchtigt sein, wenn sich die Arbeitsverwaltung darauf beschränkt, statt zu motivieren, auf die Möglichkeit einer Sanktionierung zu verweisen und den Zusammenhang zu den Leistungen zur Sicherung des Lebensunterhaltes systematisch als Drohkulisse zu verwenden.53 Ob der dadurch entstehende Druck ausschließlich die Personen erfasst, bei denen eine Verhaltensänderung erforderlich ist, ist zumindest fraglich. Eine Überbetonung dieses Zusammenhangs im Beratungsgespräch oder während des Aushandelns der Vereinbarung erscheint jedenfalls kontraproduktiv. Ähnlich wie bei rein pädagogisch-psychologisch wirkenden Vereinbarungen - erforscht für den Bereich sozialer Arbeit ${ }^{54}$ - sollte im SGB II die freiwillige, eventuell über positive Anreize bewirkte Verhaltensänderung in den Vordergrund gerückt werden. Die positiven Effekte kooperativen Vorgehens drohen sonst unterzugehen.

51 Vgl. zweites Kapitel B I 6 S. 162 ff.

52 Vgl. zweites Kapitel B II 3 S. 193 ff.

53 Vgl. zweites Kapitel B II 7c) S. 218 ff.

54 Vgl. Maluccio/Marlow, Social Work 1974, S. 35; Corden, BJSW 1980, S. 143 ff; Nelken, CLP 1987, S. 207 ff; Rojek/Collins, BJSW 1987, S. 200; Walsh u.a., Contracts for public services, in: Campbell/Vincent-Jones, Contracts and economic organisation: socio-legal initiatives, 1996, S. 212 ff; Freedland/King, Client contractualism between the employment service and jobseekers in the United Kingdom, in: Sol/Westerveld, Contractualism in employment services, 2005, S. 124 f. 


\section{1. Übervorteilung und Verhandlungsungleichgewicht}

Die Gefahr der Übervorteilung des Bürgers besteht in beiden Rechtsordnungen, denn die Vereinbarungen stehen jeweils in mehr oder weniger unmittelbarem Zusammenhang mit einer sozialen Leistung, die den Lebensunterhalt des Leistungsempfängers sicherstellen, jedenfalls aber einen Entgeltersatz gewähren soll. Im ersten Kapitel sind zur Entstehung des Verhandlungsungleichgewichtes folgende grundlegende Ausführungen gemacht worden: Es handelt sich stets um sozial angespannte Lebenssituationen, in denen auf Seiten des Bürgers eine gewisse Einschränkung der Entscheidungsstärke feststellbar sein kann, während die Verwaltung aufgrund fehlender persönlicher Betroffenheit unbeeinflusst agiert. Daraus kann ein Missverhältnis zwischen der Verhandlungsstärke der Sozialverwaltung und der Verhandlungsfähigkeit des einzelnen Bürgers entstehen. Es ist deshalb ein äußerst wirkungsvolles Druckmittel, eine Sozialleistung von der „Mitwirkung“ des Bürgers abhängig zu machen. 55

Insgesamt betrachtet ist das häufig zu beobachtende „Grundmisstrauen“ gegenüber der Verwaltung zwar nicht angebracht. Auch bei Vorliegen eines gewissen Machtgefälles zwischen Verwaltung und Bürger ist die Gefahr für den Einzelnen, von behördlicher Seite übervorteilt zu werden, gering. Interessant ist, dass diese Gefahr in beiden Rechtsordnungen erkannt, aber der Mechanismus, wie diese Gefahr ausgeschlossen oder möglichst gering gehalten wird, unterschiedlich ist:

Im englischen Recht ist das $J S A$ rechtlich weder ein Vertrag nach common law ${ }^{56}$ noch wird der Bürger über Umwege durch die Zulassung eines entsprechenden Verfahrens (z.B. restitution) an das Vereinbarte gebunden. ${ }^{57}$ Das $J S A$ entfaltet nur Wirkungen für den konkreten sozialrechtlichen Anspruch auf Erteilung einer jobseeker's allowan$c e .58$ Jede darüber hinausgehende Wirkung wird konsequent ausgeschlossen. Das Vereinbarte ist im administrativen Verfahren überprüfbar, sodass kein Raum entsteht, in dem die Verwaltung das Machtgefälle ohne anschließende Kontrolle ausnützen könnte. Ferner haben die kontrollierenden Stellen die Kompetenz, das Verwaltungshandeln abzuändern, sodass auch faktisch alle Mittel vorhanden sind, Machtmissbrauch nicht nur aufzudecken, sondern auch eventuelle negative Auswirkungen für den Einzelnen zu beenden. 59

Auf ähnliche Weise wirkt die Eingliederungsvereinbarung im SGB III. Das gesetzlich aufgrund der Sozialversicherungspflicht entstehende Versicherungsverhältnis entfaltet schützende Wirkung für den Bürger. Die Position des Bürgers ist dadurch gestärkt, dass er als Versicherter durch Beiträge erworbene Leistungen nicht ohne weiteres

55 Vgl. erstes Kapitel A III 1 S. $58 \mathrm{f}$.

56 Vgl. zweites Kapitel A II 4 S. 111 ff.

57 Vgl. zweites Kapitel A II 7d) S. 127 ff.

58 Vgl. zweites Kapitel A II 6d) S. 124.

59 Vgl. zweites Kapitel A II 9d) S. 135 ff. 
verlieren kann. Diese Sicherheit kann er als Gewicht bei den Verhandlungen einbringen. Die Gefahr der Übervorteilung des Bürgers infolge (allerdings weniger stark ausgeprägten) Verhandlungsungleichgewichts wird zudem dadurch minimiert, dass das Vereinbarte für die Eingliederungsleistungen im Rahmen der Ermessensausübung an denselben Maßstäben gemessen wird, wie wenn keine Vereinbarung vorläge. Es geht um die Feststellung von Ermessensfehlern. ${ }^{60}$ Werden zudem bestimmte Ermessensleistungen zusätzlich als verwaltungsrechtlicher Vertrag vereinbart, ist die Position des Arbeitslosen darüber hinaus gestärkt, weil er vertragliche Ansprüche gegen die Verwaltung erwirbt. 61

Im SGB II besteht wegen der im Gesetz vorgesehenen Sanktionen ein großes Machtgefälle zwischen Arbeitslosem und Arbeitsverwaltung. Der Einzelne bringt nur den Informationsvorsprung über sich selbst mit und kann die eigenen Stärken und Schwächen als Gewicht bei der Aushandlung der Vereinbarung in die Waagschale werfen. Er hat aber keine Stellung als Versicherter. Das Verhandeln kann sich nur auf mögliche Eingliederungsleistungen, nicht auf Leistungen zur Sicherung des Lebensunterhaltes beziehen, denn nur diese sind Gegenstand der Eingliederungsvereinbarung. Die Vereinbarung legt fest, welche Leistungen der Arbeitslose zur Eingliederung erhält, und was er selbst tun muss, um wieder in Arbeit zu kommen. Es wird also der Gegenstand, der dem Verhandeln zugänglich ist, auf Leistungen beschränkt, die bei der Wiedereingliederung des Leistungsempfängers in den Arbeitsmarkt helfen sollen und insoweit uneingeschränkt positiv sind. Die Leistungen zum Lebensunterhalt sind dagegen nicht verhandelbar.

Nach der Untersuchung der verschiedenen Mechanismen steht fest, dass die Gefahren einer Übervorteilung und eines Verhandlungsungleichgewichts zwischen den Parteien nicht von vornherein dem Abschluss von Vereinbarungen zwischen Staat und Bürger entgegenstehen. Anders als im Zivilrecht, das insoweit Generalklauseln oder gesetzliche Regelungen zum Schutz der benachteiligten Partei kennt ${ }^{62}$, kennt das Sozialverwaltungsrecht weitere Mechanismen, die negative Folgen für den Bürger vermeiden können: Beschränkung der Wirkungen eines Vertrages auf eine bestimmte Situation, (vollständige) gerichtliche Überprüfbarkeit einer vertraglichen Einigung, Bindungen der stärkeren Partei, etc..

\section{Ausschluss aus dem System}

Dem befürchteten Ausschluss bestimmter Personen aus dem Leistungssystem durch kooperative Vorgehensweise kann wirkungsvoll durch die rechtliche Ausgestaltung der Vereinbarungen in der jeweiligen Rechtsordnung und den Vollzugsbedingungen allgemein vorgebeugt werden.

60 Vgl. zweites Kapitel B I 7b) S. 167 ff.

61 Vgl. erstes Kapitel B IV 1 S. 82 ff.

62 Vgl. erstes Kapitel B III 2c) S. 73 ff. 
Im englischen Recht ist die Vereinbarung Leistungsvoraussetzung. Fehlende Verhandlungsfähigkeiten auf Seiten des Bürgers führen deshalb in der Tat zu einem Leistungsausschluss, wenn infolgedessen kein JSA abgeschlossen wird. Durch den günstigen Betreuungsschlüssel, der im Vereinigten Königreich bereits seit längerem eingeführt und durch Aufstockung des Personals auch erreicht worden ist, ist davon auszugehen, dass im Gespräch mit dem Einzelnen dieses Defizit ausgeglichen und deshalb durch eine dichtere Betreuung des Einzelfalles, v.a. im Rahmen der New Deals, aufgefangen wird. ${ }^{63}$

Im SGB III ist die Vereinbarung keine Leistungsvoraussetzung, weder für Entgeltersatzleistungen noch für Eingliederungsleistungen. Gerade die durch Beitragszahlung erworbenen Ansprüche sind durch eine fehlende Eingliederungsvereinbarung nicht gefährdet. Insoweit ist ein Ausschluss bei funktionierender Betreuung nicht denkbar.

Im SGB II ist die Eingliederungsvereinbarung ebenso keine Leistungsvoraussetzung. Der Konnex zu den Leistungen zur Sicherung des Lebensunterhaltes ist aber ungleich stärker als im SGB III. Aktivierung bedeutet, dass der Einzelne sich jedenfalls mittels einer Eingliederungsvereinbarung in den Eingliederungsprozess einbringt. Dazu ist ein Verhandeln auf gleicher Augenhöhe, und nicht nur eine Rhetorik vom „Fördern und Fordern" erforderlich. Diese setzt einen Beratungs-, Hilfe- und Aushandlungsprozess voraus, dessen Erfolg gerade für den Bereich des SGB II daran zu messen ist, ob geeignete Betreuung durch die Träger der Grundsicherung für Arbeitsuchende finanziell möglich ist. Die auch für das SGB II in der Gesetzesbegründung geforderten Betreuungsschlüssel sind derzeit zwar im Bereich der Leistungsgewährung erreicht, nicht aber bei der Eingliederungsbetreuung, weder bei Jugendlichen noch bei erwachsenen Hilfebedürftigen. ${ }^{64}$ Ohne ausreichendes Betreuungsangebot besteht weiterhin bei der Gestaltung im SGB II das Risiko, dass sich der Personenkreis, der Unterstützung bei der (Wieder-)Eingliederung in den Arbeitsmarkt am nötigsten hat, nicht in den Verhandlungen vor Abschluss der Eingliederungsvereinbarung entsprechend seinem Hilfebedarf äußern kann. Dies ist durch weiteren Ausbau. Jedenfalls durch Aufrechterhaltung der erreichten Betreuungsdichte im Bereich der Grundsicherung für Arbeitsuchende sicherzustellen.

\section{Verrechtlichung}

Eine Gefahr für das Gelingen von Kooperation ist eine Verrechtlichung in dem Sinn, dass die zulässigen Verfahren und Instrumente des Rechts den positiven Effekten kooperativen Vorgehens im Weg stehen oder ihnen zu wenig Raum lassen. Sinkt aus diesem Grund die Motivation beider Seiten der Vereinbarung, dieses Instrument zu nutzen, oder werden die positiven Effekte durch den Aufwand, den die Maßgaben der Rechts-

63 Vgl. zweites Kapitel A I 3 S. 106 ff.

64 Bundesagentur für Arbeit, Jahresbericht 2007, SGB II, 2007, S. 69, vgl. dazu zweites Kapitel B II 2b) aa) S. $188 \mathrm{f}$. 
ordnung vorgeben, aufgewogen und deshalb Lösungen vom Einzelfall weg verallgemeinert und standardisiert, ist die Erfolglosigkeit von Kooperation vorprogrammiert. ${ }^{65}$

Die Unterscheidung von öffentlichem Recht und Privatrecht ${ }^{66}$ sollte dabei nicht überbetont werden. Angesichts der zahlreichen Abgrenzungstheorien, deren Schwächen hinlänglich bekannt sind, darf man vermuten, dass die dogmatische Unterscheidung überholt und vor allem deren Aussagekraft überschätzt wird. Vertragsfreiheit und die bindende Qualität von vertraglichen Verpflichtungen begründen zusammen das Konzept der individuellen Autonomie, sowohl im Verhältnis der Bürger zueinander als auch im Verhältnis zum Staat. Auf diese Erkenntnisse baut kooperative Verwaltung auf. Das öffentliche Recht und das Privatrecht sind deshalb nicht voneinander abzugrenzen, sondern füreinander fruchtbar zu machen.

Im Vereinigten Königreich sind in Bezug auf die Verrechtlichung kooperativer Verwaltung zwei gegenläufige Entwicklungen erkennbar: Einerseits werden rechtliche Formen vermieden, da das $J S A$ ausdrücklich nicht als ,echter Vertrag“ angesehen werden kann. Andererseits ist das JSA Leistungsvoraussetzung und damit insgesamt als rechtliche Kategorie zu behandeln. Die rechtlichen Voraussetzungen sind folglich auf ein Mindestmaß beschränkt (Willenseinigung und Unterzeichnung einer Vereinbarung), das Kooperationsergebnis wird für das weitere Verwaltungshandeln vollständig nutzbar gemacht. Die Anwendbarkeit von contract law auf die Vereinbarungen zwischen Arbeitslosem und Arbeitsverwaltung hätte zur Folge gehabt, dass ausschließlich die common law Gerichtsbarkeit zur Entscheidung berufen gewesen wäre, was die englische Rechtsordnung als Gefahr für die Wahrung öffentlicher Interessen, nicht unbedingt als Gefahr für kooperative Prozesse betrachtet hätte. ${ }^{67}$ Die Forderung nach einem public law contract für Fälle, in denen die Anwendung des contract law wie beim JSA an der fehlenden Rechtspersönlichkeit einer Partei scheitert, hat bislang kaum Unterstützer gefunden. ${ }^{68}$ Als Modell wird der NHS contract angesehen, bei dem Vertragschluss, Preisfestlegung und Streitschlichtung eigens festgelegt sind. ${ }^{69}$ Denn die Einführung einer entsprechenden rechtlich verankerten Handlungsform ist bei der derzeitigen Gestaltung des Rechts der jobseeker's allowance nicht erforderlich und könnte vielmehr die bei der Betreuung Arbeitsloser vorhandene Flexibilität der Verwaltung bremsen. Ein möglicher Vorteil einer geregelten Handlungsform und der Einhaltung eines bestimmten Verfahrens, das willkürliche Verwaltung ausschließen soll, wird beim JSA auf andere Weise durch die gerichtliche Kontrolle vor Social Security Administrative Tribunals erreicht. Das JSA ist folglich insgesamt betrachtet so ausgestaltet, dass die Vorteile kooperativen Vorgehens trotz der Tatsache, dass das JSA Leistungsvoraussetzung ist, erhalten bleiben.

Im deutschen Recht sind Tendenzen zu einer Verrechtlichung im oben beschriebenen Sinn im SGB III ebenfalls nicht zu beobachten. Dort bleibt die Eingliederungsvereinba-

65 Vgl. erstes Kapitel A III 3 S. 60.

66 Vgl. erstes Kapitel B III 3b) dd) S. 80 ff; erstes Kapitel B IV 1c) S. 85 ff.

67 Vgl. zweites Kapitel A II 9d) aa) S. 135 ff.

68 Vgl. erstes Kapitel B IV 2 S. 90 ff

69 Vgl. erstes Kapitel B V 3a) S. 93. 
rung weitgehend außerhalb des rechtlich Greifbaren und stellt vor allem eine Strategie dar, wie die Wiedereingliederung des Arbeitslosen im besten Fall ablaufen kann. Der Einfluss, den die Eingliederungsvereinbarung auf die Ermessensausübung hat, stellt keine Gefahr für den Mehrwert kooperativer Handlungsweise dar, sondern beugt schlicht willkürlicher Verwaltungspraxis vor.

Die Eingliederungsvereinbarung im SGB II ist ein verwaltungsrechtlicher Vertrag und hat deshalb den Vorschriften des SGB X zu folgen. Die Ausgestaltung des verwaltungsrechtlichen Vertrages im SGB X lässt bestimmte Aspekte des Vertragsverhältnisses in der Verantwortung der Vertragsparteien. Insoweit findet grundsätzlich nur eine gerichtliche Nichtigkeitskontrolle statt. ${ }^{70}$ Die inhaltliche Gestaltungsmacht vor allem für die Verwaltung, aber auch für den Bürger ist dadurch eingeschränkt, dass der Gesetzgeber Regelungen getroffen hat, die den Inhalt der Eingliederungsvereinbarung festlegen. So müssen z.B. bei Eingliederungsleistungen, die in einer Eingliederungsvereinbarung vereinbart wurden, sämtliche gesetzlichen Voraussetzungen erfüllt sein. Vorgeschrieben sind zudem die Sanktionierung von Fehlverhalten des Bürgers und die weitere Abwicklung des Vereinbarten bei Geltung des öffentlich-rechtlichen Vertragsrechts. Für die Entstehung eines Mehrwertes kooperativen Vorgehens ist nicht entscheidend, dass es sich um rechtlich nicht greifbares Vorgehen handelt, entscheidend ist, dass der Aushandlungsprozess - wie im SGB II möglich - außerhalb der geregelten Handlungsform verwaltungsrechtlicher Vertrag stattfinden kann. Insoweit sind keine rechtlichen Vorgaben zu beachten. Die Gefahr, die durch Verrechtlichung im oben definierten Sinn droht, ist deshalb auch im SGB II gebannt.

\section{Haftung und Rechtsschutz}

\section{a) Haftung}

Die Verantwortung für das Verwaltungshandeln bleibt unklar, wenn die Haftung nicht eindeutig bestimmbar ist. Dann sind die öffentlichen Interessen und die Interessen der Bürger am Verwaltungshandeln möglicherweise nicht gewahrt. Haftung ist jedenfalls im Privatrecht für vertragliche Beziehungen geklärt. Es stellt sich deshalb die Frage, ob die für das $J S A$ und die Eingliederungsvereinbarungen vorgesehenen Lösungen diese Gefahr eines Haftungsdefizits im Interesse von Staat und Bürger auflösen können.

Feststeht, dass für das $J S A$ und die Eingliederungsvereinbarung nach dem SGB III keine vertraglichen Haftungszuweisungsvorschriften Anwendung finden, denn es handelt sich nicht um echte Verträge. Im SGB II sind eventuelle Ansprüche des Einzelnen als Folgeansprüche aus einem verwaltungsrechtlichen Vertrag nach analog anzuwendenden privatrechtlichen Regelungen abzuwickeln. Insoweit besteht kein Haftungsdefizit.

Im englischen Recht entsteht wegen der gesetzlich bestimmten Wirkungen des JSA kein Haftungsdefizit im Sinn eines Verantwortungsvakuums, obwohl es sich um eine vertragsähnliche Beziehung handelt. Das Privatrecht und die entsprechenden gerichtli-

70 Vgl. zweites Kapitel B II 8a) S. 226 ff. 
chen Verfahren zur Durchsetzung vereinbarter Pflichten wurden in England bezogen auf das $J S A$ bewusst ausgeschlossen. Die Verantwortung der Verwaltung für ihr Tun beruht deshalb nicht auf Vertragsrecht, sondern auf allgemeinen Prinzipien und wird insbesondere daran gemessen, ob das Vorgehen für sich betrachtet rechtmäßig (lawful) ist. Die politische Verantwortung hat das DWP auch ohne eine durchgehende rechtliche Haftungskette zu übernehmen. ${ }^{71}$ Die Konzentration auf die Wiedereingliederung des Arbeitslosen in den Arbeitsmarkt hat vielmehr Vorrang und behindert den Eingliederungsprozess nicht durch rechtliche Grenzen. Ein Interessenkonflikt zwischen Verwaltung und Bürger ist ebenso wenig wie ein Konflikt mit öffentlichen Interessen zu erwarten. Insoweit liegt eine andere Situation vor wie bei den Verträgen zwischen Verwaltung und privaten Leistungserbringern, wo möglicherweise die Interessen der Leistungsempfänger nicht ausreichend berücksichtigt werden. ${ }^{72}$

In Deutschland ist das bürgerlich-rechtliche Vertragsrecht ebenfalls nicht unmittelbar anwendbar, deshalb bleiben Haftungsfragen bei der Eingliederungsvereinbarung nach SGB III offen. Die Verbindlichkeit der Vereinbarung findet keine entsprechende Fortsetzung bei der Umsetzung des Vereinbarten. Die Ermessensfehlerlehre ordnet zwar Verantwortlichkeiten zu und schützt so den Einzelnen durch die Garantie einer willkürfreien Entscheidung, aber sie stellt nicht sicher, dass Nachteile, die der Einzelne hat, weil die Verwaltung in der Eingliederungsvereinbarung Vereinbartes nicht einhält, ausgeglichen werden. Bei einer vertragsähnlichen Vorgehensweise wie im SGB III, bei der das Recht keine weiteren Lösungsmöglichkeiten anbietet, entsteht ein Verantwortungsvakuum, das als Haftungsdefizit bezeichnet werden muss.

\section{b) Defizite beim Rechtsschutz}

Der privatrechtliche Rechtsweg ist in beiden Rechtsordnungen ausgeschlossen. Haben die Vereinbarungen weniger Durchsetzungskraft und weist die Konstruktion in der jeweiligen Rechtsordnung deshalb Defizite auf?

Der Rechtsschutz des Einzelnen ist in England durch die SSATs sichergestellt. Ein Defizit ist insoweit nicht festzustellen, es hat aber Veränderungen in den SSATs gegeben, die den Rechtsschutz suchenden Bürger belasten. In den Anfängen ihrer Gründung entschieden die SSATs in möglichst informellen Verfahren ohne ausdrücklich festgelegte Verfahrensgrundsätze über die jobseeker's allowance. Formelle Fragen nahmen keinen Raum ein und konnten der materiellen Überprüfung der Verfahrensgegenstände deshalb auch nicht im Wege stehen. Man spricht in England von einer judicialisation of appeal system. Damit ist gemeint, dass eine Verrechtlichung des Verfahrens vor SSATs stattfindet. So ging man vom Laienprinzip ab. Heute entscheiden Juristen als Richter an SSATs statt früher einer Kombination von Richtern und Laien. Dadurch ist zwar eine größere Unabhängigkeit von der Verwaltung gegeben, da diese früher in der Regel die SSATS mit „Laien“ besetzten. Folge war auch eine Entmachtung der Gewerkschaften verbunden mit der Hoffnung, dass dadurch die Entscheidungen weniger ideologisch

71 Vgl. zweites Kapitel A II 4a) S. 111.

72 Vgl. dazu erstes Kapitel B IV 2 S. 65 ff. 
belastet sein würden. Die Entscheidung eines Einzelrichters statt eines Gremiums von drei Personen soll auch die Konzentration auf rechtliche Kategorien sicherstellen, beschneidet aber den Einzelnen auf die Fachkompetenz und Beurteilungsfähigkeiten einer Person. Negative Auswirkungen für die Stellung des Antragstellers hat ferner die Einführung bestimmter Verfahren, die eine schnelle Entscheidung verhindern und das Verfahren verlängern, z.B. prehearing procedures und das Erfordernis eines hearings vor einer Entscheidung des SSATs. ${ }^{73}$

Im deutschen Recht kann weder die Arbeitsverwaltung noch der einzelne Arbeitslose gerichtlichen Rechtsschutz auf Einhaltung der Eingliederungsvereinbarung nach SGB III für sich in Anspruch nehmen. Erst die Feststellung, dass eine Sperrzeit eingetreten ist, ist ein Verwaltungsakt, der mit Widerspruch und Klage angefochten werden kann. Im Rahmen dieses Verfahrens werden die Inhalte der Eingliederungsvereinbarung für die gerichtliche Entscheidung relevant. ${ }^{74}$

Die Eingliederungsvereinbarung nach SGB II selbst kann sowohl Gegenstand einer außergerichtlichen, aber auch einer sozialgerichtlichen Auseinandersetzung sein. Der Rechtsschutz geht deshalb verglichen mit den anderen Vereinbarungen am weitesten. Es können darüber hinaus auch Sanktionen und die Einstellung der Vermittlung gerichtlich überprüft werden. Ein Defizit in Bezug auf effektiven Rechtsschutz ist deshalb nicht festzustellen.

Diskutiert wird die beschränkte gerichtliche Kontrolldichte ${ }^{75}$ bei der Überprüfung eines verwaltungsrechtlichen Vertrages, weil sie Freiräume für die Verwaltung, aber auch - was oft übersehen wird - für den Bürger bedeutet. Die beschränkte gerichtliche Kontrolldichte stellt sicher, dass sich ein zwischen „Schutzauftrag“ und „Bewirkungsauftrag" ausgewogenes Vertragsrecht entwickelt. ${ }^{76}$ Der Schutzauftrag des öffentlichen Vertragsrechts wäre mithin überbewertet, verlangte man, dass Verträge zwischen Staat und Bürger vergleichbar mit dem Verwaltungsakt vollumfänglich gerichtlicher Prüfung zugänglich sein müssen. Das deutsche Recht wäre dann zu unflexibel. Da sich die Verwaltung vermehrt kooperativer Handlungsformen bedient, sollte diese Freiheit von einem entsprechend angepassten Verständnis der Nichtigkeitsgründe begleitet werden.

\section{Kosten - Nutzen - Vergleich}

Halten sich die Kosten der Aushandlung der Vereinbarung die Waage mit den Einsparungen, die sich aus den Ergebnissen der Vereinbarung ergeben? Zur Beantwortung dieser Frage muss diese Arbeit weitgehend theoretische Ausführungen machen und greift, soweit vorhanden, auf empirische Erhebungen zurück. Feststeht, dass sich der Aufwand kooperativer Verwaltung für die untersuchten Instrumente in Grenzen hält, selbst wenn die Verwaltung den Auftrag zu kooperieren ernst nimmt:

73 Vgl. zweites Kapitel A II 9d) aa) S. 135 ff.

74 Vgl. zweites Kapitel $\square$ I 10c) S. 180 ff.

75 Vgl. zweites Kapitel B II 8a) S. 226 ff.

76 Schlette, Verwaltung als Vertragspartner, 2000, S. 378; so auch Spannowsky, Verträge und Absprachen, 1994, S. 34 ff, 295 ff. 
In England werden standardisierte Verfahren und Muster verwendet, die zwar durchgesprochen, aber nicht in jedem Detail ausgehandelt werden. Aus der derzeitigen Praxis ergibt sich nur geringer Nutzen, denn die Evaluierung ergab, dass der Inhalt des JSA für die Wiedereingliederungsstrategie nur selten als dienlich angesehen wurde. Es wird zudem nicht erwartet, dass sich der Nutzen im derzeitigen System der jobseeker's allowance steigern lässt. ${ }^{77}$ Der geringe Aufwand wird im Vereinigten Königreich wohl weiter betrieben werden.

In der deutschen Rechtsordnung ist der formelle Aufwand der Ausarbeitung einer Eingliederungsvereinbarung ähnlich groß wie in England. Es gibt Arbeitshilfen der Bundesagentur für Arbeit für das SGB II, die mehr und mehr verwendet werden. ${ }^{78}$

Ein gewisser Aufwand im Vollzug des SGB II und SGB III ergibt sich v.a. daraus, dass die Eingliederungsleistungen, insbesondere für SGB II-Leistungen überwiegend an Prognosen der Arbeitsverwaltung anknüpfen. Das hat Einfluss auf den Aufwand, eine Eingliederungsvereinbarung auszuhandeln. So ist es bereits bei Abschluss der Eingliederungsvereinbarung ein wichtiger Schritt festzustellen, welche Eingliederungshindernisse vorliegen und in die Eingliederungsvereinbarung aufgenommen werden. Denn dadurch wird nach derzeit bestehender Rechtslage das später eröffnete Eingliederungsinstrumentarium festgelegt. Der rechtliche Rahmen des Eingliederungsinstrumentariums ist dabei sehr eng und die Auswahl des „richtigen“ Instrumentes komplex. Es kann zu Konflikten zwischen Eingliederungsziel und dem Vereinbarten kommen, denn alle Überlegungen zu den Voraussetzungen von Eingliederungsleistungen müssen bei $\mathrm{Ab}-$ schluss der Eingliederungsvereinbarung bereits angestellt sein, um nicht Widersprüche zur späteren Ermessensausübung hervorzurufen. Eine Überforderung des Instrumentes ist deshalb zu befürchten. Die Gefahr von Fehlern ist groß, und deshalb ist ständiger Änderungsbedarf in Bezug auf die Eingliederungsvereinbarung vorprogrammiert. So wird der rechtmäßige Gebrauch der Eingliederungsleistungen nicht gefördert, sondern die positive Wirkung von kooperativem Vorgehen im Einzelfall geschwächt. Hinzukommt, dass der Abschluss der Vereinbarungen in der Überwachung seiner Umsetzung in jeden Eingliederungsschritt zusätzlichen Verwaltungsaufwand bedeutet.

Die Ergebnisse der gesetzlich verankerten Evaluierungen für das SGB II liegen noch nicht vor. ${ }^{79}$ Für die Eingliederungsvereinbarung nach SGB II ist zu erwarten, dass Aufwand und Nutzen sich jedenfalls die Waage halten werden, und das Instrument verstärkt Anwendung finden wird. Im Zuge der im Koalitionsvertrag zwischen CDU/CSU und $\mathrm{SPD}^{80}$ angekündigten Neuordnung der arbeitsmarktpolitischen Instrumente ${ }^{81}$ wird

77 Vincent, Jobseeker's allowance evaluation 1998; McKay u.a., Unemployment and jobseeking after the introduction of jobseeker's allowance, 1999; Rayner u.a., Evaluating the jobseeker's allowance, 2000.

78 Bundesagentur für Arbeit, Jahresbericht 2006, SGB II, 2006, S. 43.

79 Zur vom Institut für Arbeitsmarkt und Berufsforschung erwarteten Forschungsaufgabe Beckmann u.a., IAB-Forschungsbericht 2004, S. 1 ff; Brinkmann u.a., IAB-Forschungsbericht 2005, S. 1 ff. Erste veröffentlichte Ergebnisse wurden für Oktober 2007 erwartet, liegen aber noch nicht vor.

80 Koalitionsvertrag zwischen CDU/CSU und SPD „Gemeinsam für Deutschland. Mit Mut und Menschlichkeit" vom 11.11.2005, S. 32 ff. 
sich zeigen, ob der Gesetzgeber das Verhältnis von Aufwand und Nutzen der Eingliederungsvereinbarung im SGB III ebenfalls positiv einschätzt oder gesetzgeberisch nachsteuert.

\section{Kooperation an ihren Grenzen}

\section{Verfassungsrechtlicher Rahmen}

a) Eigentum

Kooperatives Vorgehen findet dort eine Grenze, wo die Vereinbarung den verfassungsrechtlich garantierten Schutz des Eigentums berührt.

Im Vereinigten Königreich sind Beiträge in das Sozialversicherungssystem nicht ausdrücklich geschützt. ${ }^{82}$ Seit dem Jobseekers Act 1995 handelt es sich bei der jobseeker's allowance um eine einheitliche Leistung, die das Risiko der Arbeitslosigkeit absichert. Die Umgestaltung des Systems war vor dem Hintergrund der supremacy of Parliament kein Problem. ${ }^{83}$ Deshalb hat auch das JSA in Bezug auf die Garantie des Eigentums keine verfassungsrechtliche Grenze, denn die Parteien agieren im einfachgesetzlichen Kontext.

Anders in der deutschen Arbeitslosenversicherung. Die Eigentumsgarantie in Bezug auf die Beiträge zur Arbeitslosenversicherung steht der Zusammenlegung einer Versicherungsleistung und einer Fürsorgeleistung, wie z.B. der Sozialhilfe, entgegen. Daraus ergibt sich auch die unterschiedliche Bedeutung der Eingliederungsvereinbarung im SGB III und SGB II, insbesondere in Bezug auf deren rechtliche Einordnung. Auf eine Modifizierung von Versicherungsleistungen muss sich ein Arbeitsloser auf der Grundlage des SGB III nicht einlassen. Er genießt insoweit verfassungsrechtlichen Schutz. ${ }^{84}$ Der Empfänger der Grundsicherung für Arbeitsuchende kann sich dagegen nur auf $\S 53$ Abs. 2 SGB X berufen, der regelt, dass Leistungen, auf die ein Anspruch besteht, nicht durch öffentlich-rechtlichen Vertrag verändert werden dürfen. ${ }^{85}$

\section{b) Handlungsfreiheit}

Handlungsfreiheit ist das Stichwort, unter dem geprüft wird, ob die Regelungen über die Eingliederungsvereinbarung und das JSA den Schutz der Vertragsfreiheit garantieren. In den Vergleichsrechtsordnungen sind verschiedene Eingriffstiefen feststellbar, sodass die Rechtfertigung auch in unterschiedlicher Weise zu begründen ist.

81 Diese Ankündigung wurde in der Rede des Bundesministers für Arbeit und Soziales Olaf Scholz zum Haushaltsgesetz 2008 vor dem Deutschen Bundestag am 29.11.2007 in Berlin, Bulletin Nr. 134-1 vom 29.11.2007, wiederholt.

82 Vgl. zweites Kapitel A I 2 S. 100.

83 Vgl. zweites Kapitel A I 1 S. 99.

84 Vgl. zweites Kapitel B I 1c) cc) S. 143.

85 Vgl. zweites Kapitel B II 5c) cc) S. 202. 
Im englischen Recht lässt die Situation, in der das $J S A$ geschlossen wird, dem Antragsteller kaum Handlungsspielraum. Es gibt auf der Stufe der Prüfung der jobseeker's allowance keinen Punkt, an dem rechtfertigende Gründe des Antragstellers für die Verweigerung des Abschlusses eines $J S A$ geprüft werden müssten. Erst bei einer Fiktion des $J S A$ beziehungsweise der Rückdatierung durch einen decision maker (backdating) wird rechtfertigenden Gründen auf Seiten des Antragstellers Rechnung getragen. ${ }^{86}$ Es ist deshalb systemkonform, dass das $J S A$ kein Vertrag ist und keine anderen Wirkungen hat, als Voraussetzung einer jobseeker's allowance zu sein. Es wäre sonst eine Rechtfertigung dieses Eingriffs in die Vertragsfreiheit erforderlich.

Während bei Abschluss einer Eingliederungsvereinbarung nach SGB III kein Abschlusszwang und damit kein Eingriff in die Handlungsfreiheit des Einzelnen festzustellen ist, ist dessen Rechtfertigung im Rahmen der verfassungskonformen Auslegung der Sanktionsvorschrift in $\S 31$ SGB II damit zu begründen, dass die vom Gesetzgeber verfolgten Ziele verfassungsrechtlich legitim sind. Der wichtige Grund in § 31 Abs. 2 SGB II stellt sicher, dass rechtfertigende Gründe für eine Verweigerung des Vertragsschlusses geltend gemacht und berücksichtigt werden können. ${ }^{87}$

\section{c) Existenzminimum}

Die verfassungsrechtliche Garantie des Existenzminimums ist eine Position, der in den Vergleichsrechtsordnungen unterschiedliches Gewicht zukommt.

Während es diese Garantie im Vereinigten Königreich nicht als rechtliche Kategorie gibt und die verfassungsrechtliche Garantie des Existenzminimums im SGB III nur am Rande eine Rolle spielt, steht diese im Zentrum der Diskussion um die Eingliederungsvereinbarung nach SGB II.

Grund dafür ist, dass anders als bei den allgemeinen Mitwirkungspflichten gesetzlich die Kürzung der Leistungen zur Sicherung des Lebensunterhaltes direkt an das Engagement des Einzelnen geknüpft ist, seine berufliche Eingliederung aktiv mit zu gestalten. Dabei ist die Eingliederungsvereinbarung nach SGB II nur ein Element neben anderen Sanktionstatbeständen, das diesen Zusammenhang vermittelt. Die verfassungsrechtliche Garantie des Existenzminimums ist gleichwohl eine Grenze, die nicht durch Konsens zwischen den Parteien unterschritten werden kann. Das ist auch der Grund dafür, dass eine Weiterentwicklung des verwaltungsrechtlichen Vertrages oder der Vorschriften über die Eingliederungsvereinbarung erfolgen müssen, die sicherstellen, dass (rechtswidrige) Vereinbarungen keine Sanktionen nach sich ziehen können. ${ }^{88}$

Auf einen (konkludent in der Eingliederungsvereinbarung nach SGB II erklärten) Grundrechtsverzicht kommt es in diesem Zusammenhang nicht an. Eine einvernehmliche Modifizierung der Leistungen zur Sicherung des Lebensunterhaltes ist bereits einfachgesetzlich aufgrund $\S 53$ Abs. 2 SGB X ausgeschlossen und wird deshalb nicht Inhalt einer Eingliederungsvereinbarung sein.

86 Vgl. zweites Kapitel A II 6a) S. 121.

87 Vgl. z.B. zweites Kapitel B II 7c) aa) (3) S. 221.

88 Vgl. zweites Kapitel B II 8a) cc) S. 228. 
d) Rechtsweggarantie

Wird jemand durch die öffentliche Verwaltung in seinen Rechten verletzt, so steht ihm der Rechtsweg offen (Art. 19 Abs. 4 GG und ähnlich Art. 6 EMRK). Ist im Fall des $J S A$ und der Eingliederungsvereinbarungen ein Rechtsweg für jeden Akt der Verwaltung garantiert oder ist die Vereinbarung kein Akt, gegen den ein Rechtsweg offen stehen muss?

Das englische Recht betrachtet die Zurverfügungstellung von Social Security Administrative Tribunals als Rechtsweg. ${ }^{89}$ Da das $J S A$ als Leistungsvoraussetzung voll überprüfbar ist, ist der Garantie genügt.

Für die Eingliederungsvereinbarung nach SGB III ist kein Rechtsweg zu einem Gericht vorgesehen. Die Eingliederungsvereinbarung als solche führt auch zu keiner Rechtsverletzung. Eine solche kann sich erst ergeben, wenn die anschließende Ermessensentscheidung über eine Eingliederungsleistung rechtswidrig ist. Diese wiederum ist gerichtlich überprüfbar. Eine Gefahr für die Einhaltung der Rechtsweggarantie besteht deshalb nicht.

Eine Gefahr für die Einhaltung der Rechtsweggarantie ist auch nicht festzustellen bei der Eingliederungsvereinbarung nach SGB II, denn diese kann selbst Gegenstand einer sozialgerichtlichen Klage auf Feststellung der Nichtigkeit der Eingliederungsvereinbarung sein. ${ }^{90}$ Es ist gerade nicht erst auf einen sich anschließenden Verwaltungsakt über eine Eingliederungsleistung zu warten. Im Gegenteil, die Eingliederungsvereinbarung kann gerade die Rechtsgrundlage für eine Eingliederungsleistung darstellen, wenn der Inhalt der Vereinbarung entsprechend gestaltet ist. Es wird dem Verwaltungsakt im Vergleich zum kooperativen Verwaltungshandeln nicht selten eine besondere „rechtsstaatliche Dignität" beigemessen, weil durch einen spezifisch ausgebauten Rechtsschutz im Verwaltungs- und Gerichtsverfahren die rechtsstaatlich problematischen Seiten des Verwaltungsaktes (einseitige Zugriffsmöglichkeit, restriktive Fehlerfolgenregelung) in den Hintergrund gestellt werden. ${ }^{91}$ Der Vertrag ist von seinem Ausgangspunkt einer Gleichberechtigung der Vertragsparteien besser geeignet, der Achtung des einzelnen Bürgers entgegenzukommen. Zugegebenermaßen ist die gerichtliche Kontrolldichte beim verwaltungsrechtlichen Vertrag geringer, aber wegen der Mitgestaltungsmöglichkeiten des Bürgers dort auch nicht im selben Umfang wie bei den Verwaltungsakten erforderlich. Die Gefahr für die Verletzung der Rechtsweggarantie ist deshalb auch im Fall der Eingliederungsvereinbarung nach SGB II gebannt.

89 Vgl. zweites Kapitel A II 9d) aa) S. 135 ff; im Einzelnen Matthewman/Ennals/Self, Tolley's social security and state benefits handbook, Rn. 23A.14.

90 Vgl. zweites Kapitel B II 10c) dd) S. 240.

91 Dazu Schmidt-Aßmann, Das allgemeine Verwaltungsrecht als Ordnungsidee, 2004, S. 337. 
a) Fairer Umgang mit dem Bürger

Die Konkretisierung der Vorgaben der Citizen's Charter in der Customer's Charter des JCP stellt sicher, dass der Umgang mit dem Bürger jedenfalls in der Theorie gewissen Standards entspricht. Die konkrete Umsetzung im jeweiligen Zwei-PersonenVerhältnis kann in dieser Arbeit nicht überprüft werden. Das $J S A$ ist jedenfalls als Anwendungsfall der Customer's Charter anzusehen.

In der deutschen Rechtsordnung findet sich in dieser konkreten Form kein Pendant. Die Neuausrichtung der Bundesagentur für Arbeit und die Umsetzung der Grundsicherung für Arbeitsuchende deuten aber darauf hin, dass auch hier verstärkt eine Kundenorientierung stattfinden soll ${ }^{92}$, die im Ergebnis durchaus mit den Forderungen der Customer's Charter vergleichbar sind.

Im deutschen Recht wird der Verfahrensaspekt des fairen Umgangs vielmehr in einen materiell-rechtlichen Aspekt umgewandelt. Wird dem Bürger eine Eingliederungsvereinbarung vorgelegt, ohne dass Verhandlungsspielräume aufgezeigt oder eingeräumt werden, führt dies im SGB II dazu, dass die Verweigerung der Unterzeichnung einer solchen angebotenen Eingliederungsvereinbarung nicht sanktioniert werden kann, weil dieses Verhalten der Arbeitsverwaltung einen wichtigen Grund im Sinn des § 31 Abs. 1 S. 2 SGB II darstellt. 93

\section{b) Untersuchungsgrundsatz}

Die Verpflichtung der Verwaltung zur Aufklärung des Sachverhaltes (inquisitorial approach) ist in der Arbeitsverwaltung des Vereinigten Königreiches nicht ausgeprägt. Es besteht zwar vor Abschluss eines JSA eine Prüfungspflicht des employment officers im Hinblick darauf, ob der Inhalt der Vereinbarung rechtlichen Anforderungen entspricht. Diese Entscheidung ist anfechtbar und begrenzt die Möglichkeiten, die Arbeitsverwaltung in einer Vereinbarung zu binden. Die Pflicht betrifft aber ausschließlich die Vereinbarkeit des Vereinbarungsinhaltes mit materiellem Recht, nicht die Richtigkeit der Angaben des Arbeitsuchenden.94

Im deutschen Recht wird die Verpflichtung der Arbeitsverwaltung, den Sachverhalt selbständig zu ermitteln (Untersuchungsgrundsatz, § 20 SGB X), durch die Mitwirkungspflichten eingegrenzt. Diese sind für das Arbeitsförderungsrecht in $\S 38$ SGB III, für das Recht der Grundsicherung für Arbeitsuchende als allgemeine Mitwirkungspflicht in $\S \S 60$ ff SGB I bzw. in $\S 2$ SGB II geregelt. ${ }^{95}$ Soweit diese Informationspflichten des Arbeitsuchenden beinhalten, trifft die Verwaltung darüber hinaus keine Untersuchungspflicht mehr. Wird der Bürger diesen Pflichten nicht gerecht, kann die

92 Vgl. zweites Kapitel B I 1b) S. 141; zweites Kapitel B I 6f) S. 165; zweites Kapitel B II 6d) S. 213.

93 Vgl. zweites Kapitel B II 7c) aa) S. 220 ff.

94 Vgl. zweites Kapitel A II 2b) S. 111.

95 Vgl. zweites Kapitel B I 6c) S. 163; zweites Kapitel B II 5a) aa) S. 199; zweites Kapitel B II 5c) cc) (3) S. 206. 
Behörde aber die vorgesehenen Sanktionen verhängen. Daraus folgt, dass die richtige Information der Arbeitsverwaltung in den Verantwortungsbereich des Einzelnen verlagert worden ist. Eine Grenze für kooperatives Vorgehen stellt deshalb der Untersuchungsgrundsatz weder im SGB III noch im SGB II dar.

\section{c) Ermessensausübung}

Bei der Erteilung der jobseeker's allowance findet keine Ermessensausübung statt. Liegen die Voraussetzungen des Jobseekers Act 1995 vor, ist sie zu erteilen. Bei Entscheidungen über Leistungen aus dem New Deal Programm (options), werden die Inhalte des JSA berücksichtigt, ohne dass eine rechtliche Bindung durch das JSA eintreten würde. Das gilt ebenso für Entscheidungen über Sanktionen wegen Nichteinhaltung der Vereinbarungen aus dem $J S A$, insbesondere bei der Prüfung, ob ein Verschulden des Arbeitsuchenden gegeben ist. Regeln über die Ermessensausübung wirken sich deshalb nicht als Grenze für die Inhalte eines $J S A$ aus. Der Inhalt des $J S A$ fließt nur als Anhaltspunkt in eine spätere Entscheidung ein, die aber bei entsprechender Begründung dem Wortlaut des $J S A$ widersprechen kann. ${ }^{96}$

Im deutschen Recht werden Ermessensentscheidungen im Wege der sog. Ermessensfehlerlehre überprüft. Im SGB III fließen die verbindlichen Inhalte der Eingliederungsvereinbarung direkt in eine Entscheidung über eine Eingliederungsleistung ein. Neben der Eingliederungsvereinbarung sind aber auch andere Aspekte zu beachten, sodass Korrekturen des Vereinbarten mit entsprechender Begründung möglich sind. ${ }^{97}$ Im SGB II sind die von der Verwaltung versprochenen Eingliederungsleistungen aufgrund des Vertragscharakters einklagbar. Das Ermessen im Sinn des $\S 16$ Abs. 1 SGB II ist in diesem Fall auf Null reduziert bzw. Rechtsgrundlage für die Eingliederungsleistung ist die Eingliederungsvereinbarung selbst. 98 Das kooperative Vorgehen verdrängt insoweit also eine Ermessensentscheidung. Auch im deutschen Recht wirken sich die Regeln über die Ermessensausübung nicht als Grenze für Kooperation aus.

\section{Bindung inter partes}

Vereinbarungen binden stets nur die Vertragspartner. Diese Grenze kooperativer Verwaltung wird in den Rechtsordnungen in unterschiedlicher Weise relevant.

Im englischen Recht wird das $J S A$ grundsätzlich als zweiseitige Vereinbarung zwischen Arbeitslosem und Arbeitsverwaltung geschlossen. Ist der Arbeitslose unterhaltspflichtig, z.B. gegenüber Kindern, oder hat er einen mit ihm zusammenlebenden Partner, werden diese Personen nie am JSA beteiligt. Eine Ausnahme ist nur für ein sog. joint claim couple vorgesehen. In diesem Fall stellen zwei Arbeitslose, die ohne Kinder zusammenleben, einen gemeinsamen Antrag und unterzeichnen gemeinsam das JSA.

96 Vgl. zweites Kapitel A II 6c) S. 123.

97 Vgl. zweites Kapitel B I 7b) ee) S. 169.

98 Vgl. zweites Kapitel B II 7b) dd) S. 215. 
Nur dann sind beide Antragsteller gebunden an die Inhalte eines $J S A .{ }^{99}$ Scheitert eine jobseeker's allowance am Fehlen eines JSA, gibt es die Möglichkeit von hardship payments, die vor allem für den Fall vorgesehen sind, dass Kinder an der Nichterteilung der jobseeker's allowance zu leiden hätten. ${ }^{100}$

Im deutschen Recht sieht die Eingliederungsvereinbarung nach SGB III nur einen zweiseitigen Abschluss vor. Am Versicherungsverhältnis, welches den Rahmen für die Eingliederungsvereinbarung bildet, sind keine weiteren Personen beteiligt. Einer Betroffenheit anderer Personen wird nicht Rechnung getragen, weil auch die Leistungen auf die versicherte Person beschränkt sind. Die Bindung inter partes ist deshalb unproblematisch.

Das SGB II erfasst in Bezug auf die Leistungen zur Sicherung des Lebensunterhaltes die Bedarfsgemeinschaft als gesetzlich in $\S 7$ Abs. 3 SGB II definierte soziale Einheit. Es ist vorgesehen, dass Personen, die mit dem erwerbsfähigen Hilfebedürftigen in einer Bedarfsgemeinschaft leben, von diesem sowohl bei der Antragstellung als auch bei der Entgegennahme der Fürsorgeleistungen vertreten werden, § 38 S. 1 SGB II. Die Eingliederungsvereinbarung bindet wegen des Grundsatzes der Bindung inter partes zunächst nur Arbeitslosen und Arbeitsverwaltung. Um die Bedarfsgemeinschaft - wie auch im Leistungsrecht - erfassen zu können, sieht § 15 Abs. 2 S. 2 SGB II vor, dass die Mitglieder der Bedarfsgemeinschaft an der Vereinbarung zu beteiligen sind. Dabei kann nicht auf $\S 38$ S. 1 SGB II zurückgegriffen werden. Gefordert wird eine Einbeziehung mit einer eigenen Willenserklärung, also ein echtes Mitwirkungsrecht gem. 57 Abs. 1 SGB X.101

\section{Kooperation und Aktivierung als Rahmen eines Politiktransfers}

Die Reformen der Arbeitsmarktpolitik im Vereinigten Königreich und in Deutschland - schlagwortartig beschrieben mit den Begriffen activating labour market policy bzw. aktivierende Arbeitsmarktpolitik - verfolgten ähnliche Ziele. Oberstes Ziel war der Kampf gegen (Langzeit-)Arbeitslosigkeit. Das Leitbild des aktivierenden Sozialstaates bediente sich zum Teil der Instrumente kooperativen Verwaltungshandelns. Die kooperative Vorgehensweise der Verwaltung stand im Dienst der Aktivierung. Kooperation hat aber auch eigenständige Ziele, die im ersten Kapitel ausführlich dargestellt worden sind. Das Erreichen dieser Ziele ist in den untersuchten Rechtsordnungen analysiert worden. Interessant ist es nun, einen Makrovergleich anzuschließen und damit den Vergleich der Einzelvorschriften zu einem Ganzen zusammen zu fügen.

Die wesentlichen Unterschiede in den Rechtsordnungen treten zu Tage, wenn man die rechtstechnische Umsetzung der Idee von einer Vereinbarung zwischen Arbeitslosem und Arbeitsverwaltung betrachtet. Die Unterschiede bei der Konstruktion als eigen-

99 Vgl. zweites Kapitel A I 2d) S. 105.

100 Vgl. zweites Kapitel A I 3a) S. 107.

101 Vgl. zweites Kapitel B II 2c) S. 191. 
ständiges Instrument in Deutschland und als Leistungsvoraussetzung in England machen folgendes deutlich:

Im Vereinigten Königreich wird eine konsequentere Durchsetzung von Kooperationsergebnissen verfolgt, während auf der anderen Seite die Kooperation selbst weniger frei und nur in engen Grenzen stattfinden kann. Diese Gestaltung passt in das englische System, das die Stellung des Einzelnen kaum betont und dafür klare Vorgaben für die Verwaltung aufweist. Aktivierung durch das $J S A$ ist wesentlich weniger auf den Einzelnen und dessen Hilfebedarf ausgerichtet. Stattdessen geben die New Deals der Förderung des Einzelfalles Priorität ${ }^{102}$ und stellen so eine Verknüpfung zur Gewährung der jobseeker's allowance her.

In Deutschland ist die Eingliederungsvereinbarung ein Instrument unter vielen aktivierenden Ansätzen, sowohl im Arbeitsförderungsrecht als auch in der Grundsicherung für Arbeitsuchende103. Ihre tatsächliche Anwendung unterbleibt im Fall der Eingliederungsvereinbarung nach SGB III derzeit fast vollständig. Im Fall der Eingliederungsvereinbarung nach SGB II ist sie zwar gesetzlich angeordnet. Aufgrund der stets bestehenden Möglichkeit, eine einseitige Entscheidung zu erlassen, ist sie trotzdem nicht ausnahmslos erforderlich. Es bleibt ein größerer Spielraum für die Verwaltung, kooperative Instrumente zu verwenden. Dieser Spielraum wirkt sich in Bezug auf die Aktivierung insgesamt und für den Einzelnen im Speziellen nicht positiv aus. Er behindert die Konzentration der Arbeitsverwaltung auf die Eingliederungsdefizite des Einzelnen, und wird, gerade auch von den Leistungsempfängern, oft missverstanden als Mittel der „Gängelei“" und Willkür.

Der Blick ins Ausland war also für die Einführung kooperativer Handlungsinstrumente lohnend, um eine Vorstellung vom „Machbaren“ zu bekommen. Die Umsetzung in das deutsche System folgte aber eigenen Regeln. So wirkt die Einführung eines kooperativen Instrumentes ohne das Bemühen um Innovation und Ausrichtung auf die nationalen Besonderheiten (z.B. Versicherungsstruktur) insbesondere im Arbeitsförderungsrecht kraftlos. Im Hinblick auf die aktivierende Wirkung kooperativer Instrumente bleibt abzuwarten, ob die deutsche Konstruktion im SGB II aufgrund der Orientierung an Handlungsformen und rechtlichen Kontrollen, v.a. innerhalb der Verwaltung, an Ansehen gewinnen wird. Dies hängt insbesondere davon ab, ob es der Verwaltung gelingt, die positive Seite von Verträgen (z.B. die Entstehung von Ansprüchen gegen die Verwaltung) darzustellen und ihr auch im Einzelfall Geltung zu verschaffen.

\section{Bewährungsprobe und Zukunft der Handlungsinstrumente}

Das System der jobseeker's allowance des englischen Rechts steht derzeit vor seiner ersten Bewährungsprobe. Die englische Wirtschaft befindet sich in sog. abflauender Konjunktur. Bei so gut wie nicht vorhandener Arbeitslosigkeit muss sich jetzt zeigen,

102 Vgl. zweites Kapitel A I 3 S. 106 ff.

103 Zur Wirksamkeit verschiedener aktivierender Instrumente aus rechtlicher Sicht vgl. Spellbrink, SGb 2008, S. $445 \mathrm{ff}$. 
ob die Arbeitsverwaltung auch bei wenigen vermittelbaren Arbeitsplätzen effizient arbeitet und das im Gesetz verankerte Wechselspiel von Anreiz und Druck zur Arbeitsaufnahme zum Schutz des Systems der sozialen Sicherung zu nutzen versteht. Es bleibt ferner abzuwarten, ob die enormen Kosten, die die New Deals verursachen, weiterhin politisch getragen werden, auch vor dem Hintergrund eines möglichen Führungswechsels hin zu einer konservativen Regierung. Was heute wohl als ausgewogen $\mathrm{zu}$ bezeichnen ist, das System von Förderung von Arbeitslosen in den New Deals und rigiden Vorgaben der jobseeker's allowance, setzt gerade diese finanziellen Spielräume voraus. Möglicherweise waren bei der Erfolgsgeschichte der neunziger Jahre im Vereinigten Königreich andere Faktoren stärker als die Reform der National Insurance. Oft werden die Verringerung des Einflusses von Gewerkschaften und die Geldpolitik als stärker verantwortlich für den rasanten Abbau der Arbeitslosigkeit im Vereinigten Königreich gesehen, als Änderungen im Leistungsrecht. ${ }^{104}$

Im SGB III leidet die Eingliederungsvereinbarung vor allem an den Rahmenbedingungen der Arbeitslosenversicherung. Wegen der Umsetzung der Reformen in der Bundesagentur für Arbeit und den örtlichen Arbeitsagenturen werden immer noch Kapazitäten in deren Umsetzung gebunden. ${ }^{105}$ Die Eingliederungsvereinbarung kommt in diesen Strukturen bislang nicht zur Entfaltung. Es bleibt aber abzuwarten, ob die im Rahmen der Neuausrichtung der arbeitsmarktpolitischen Instrumente vorgenommenen Änderungen 106 in Bezug auf die Eingliederungsvereinbarung im SGB III Wirkung haben werden.

Die Eingliederungsvereinbarung des SGB II wird noch an Bedeutung zunehmen, weil mit dem gesetzlich verordneten Sanktionsautomatismus der Verwaltung ein effizientes Steuerungsmittel an die Hand gegeben ist, von dem diese gerne Gebrauch machen wird, sobald sie sich damit vertraut gemacht hat. Gerade die vertragliche Einbindung des Einzelnen mit der Möglichkeit, auf gesetzlich eindeutiger Grundlage Leistungen bei Nichteinhaltung des Vereinbarten zu kürzen, bietet noch unausgeschöpfte Spielräume. Es wird nicht nur in der Verwaltungspraxis eine flächendeckende Anwendung geben, sondern auch für Maßnahmeträger Handlungsbedarf entstehen, ihre Angebot an den Anforderungen aus der Eingliederungsvereinbarung auszurichten. Erfolgt dies alles im Sinne einer wirksamen (Wieder-)Eingliederung der Arbeitslosen in den Arbeitsmarkt, handelt es sich um richtig verstandene Hilfe zur Selbsthilfe. Der Bürger muss ernst genommen und die Hilfesituation muss für erwerbsfähige Personen als Übergangssituation erkennbar sein. Dazu ist die Eingliederungsvereinbarung, wie sie im SGB II ausgestaltet ist, ein wesentlicher Schritt.

104 Vgl. Einführung A I 1 S. 28 ff; ausführlich Pissarides, Unemployment in Britain, 2003.

105 Vgl. zweites Kapitel B I 1 S. 140 ff.

106 Gesetz zur Neuausrichtung der arbeitsmarktpolitischen Instrumente v. 21.12.2008 (BGB1. I, S. 2917). 


\section{Abschließende Bemerkung}

Die vorliegende Arbeit zeichnete einen Reformprozess in zwei Rechtsordnungen nach und konzentrierte sich dabei auf ein abgegrenztes Thema, die Vereinbarung zwischen Staat und Bürger. Ausgehend von der Debatte um Aktivierung suchte und fand sie für die Reformen einen gemeinsamen „Aufhänger“, die kooperative Verwaltung, der in ähnlicher Weise in beiden Rechtsordnungen diskutiert und beschrieben wird. So entstand ein verwaltungswissenschaftlicher Überbau für die Länderberichte, der die Auswertung für den Mikro-, aber auch für den Makrovergleich erleichterte.

Im Ergebnis benannte die Arbeit nur am Rande weitergehenden Reformbedarf in der einen oder anderen Rechtsordnung. Sie zeichnete das Funktionieren kooperativer Instrumente nach, ohne sich in Details zu verlieren.

Die in der Einleitung aufgeworfene Diskussion um legal transplantation und die Abgrenzung zur Rechtsrezeption steht deshalb ganz am Ende. Der Reformzusammenhang zwischen dem Vereinigten Königreich und Deutschland ist weder als legal transplantation noch als Rechtsrezeption zu verstehen. Denn der Blick in den Jobseekers Act 1995 führte nicht zur Übernahme des $J S A$ mit all den dort ausführlich beschriebenen Voraussetzungen und Wirkungen, und ist deshalb nicht als Rezeption anzusehen. Die Idee einer vertraglichen (oder vertragsähnlichen) Beziehung zwischen Staat und Bürger ist zudem kein Novum im deutschen Recht, sondern seit der Einführung des verwaltungsrechtlichen Vertrages Rechtsalltag im deutschen Verwaltungsrecht. Der Gesetzgeber bettete die Idee in das bestehende deutsche System ein und ordnete im SGB II, wo er es als passend erachtete, die Form des verwaltungsrechtlichen Vertrags an.

Die Ausgangsformel, das englische Rechts habe dem deutschen Gesetzgeber als Vorbild gedient, muss deshalb relativiert werden. Die beiden Rechtsordnungen zugrunde liegende Idee ist „Allgemeingut“ und kann keiner einzelnen Rechtsordnung exklusiv zugeschrieben werden. Die Vereinbarung zwischen Staat und Bürger ist vielmehr Ausfluss der in vielen Staaten stattfindenden Diskussion um die Einbeziehung des Einzelnen in staatliche Aufgaben und in Verantwortung allgemein. Der Gesetzgeber rückte bei der Ausgestaltung der Eingliederungsvereinbarungen im deutschen Recht in wesentlichen Punkten ab von seinem „Vorbild“. Deshalb steht die Eingliederungsvereinbarung keinesfalls als Fremdkörper im Zweiten oder Dritten Buch Sozialgesetzbuch, sondern entwickelt den verwaltungsrechtlichen Vertrag, jedenfalls den Gedanken kooperativer Verwaltung im Verhältnis Staat-Bürger weiter. 


\section{Literaturverzeichnis}

Abegg, Andreas/Thatcher, Annemarie: Review essay: Freedom of contract in the 19th century, in: GLJ, 5. Jg. (2004), H. 1, S. 101-114.

Achterberg, Norbert: Die Rechtsordnung als Rechtsverhältnisordnung: Grundlegung der Rechtsverhältnistheorie, Berlin (Duncker \& Humblot) 1982.

Adamy, Wilhelm: Das neue SGB II: Der Abstieg von der Arbeitslosenhilfe in die Sozialhilfe: zu den Auswirkungen der Zusammenlegung von Arbeitslosen- und Sozialhilfe, in: SozSich 2003, S. 285-294.

Adamy, Wilhelm: Arbeitslosengeld II: Hick-Hack um Trägerschaft beenden: Bessere Zusammenarbeit vor Ort ist der Schlüssel zum Erfolg, in: SozSich 2004, S. 124-131.

Adamy, Wilhelm: Hartz IV: Schwierige Kooperation zwischen Arbeitsagenturen und Kommunen, in: SozSich 2004, S. 332-338.

Adler, Michael: Decision-making and appeals in social security: in need of reform?, in: PQ, 68. Jg. (1997), S. 388-405.

Adler, Michael: Who is afraid of Sir Andrew Legatt?, in: JSSL, 9. Jg. (2002), H. 4, S. 177-198.

Adomeit, Klaus: Die gestörte Vertragsparität - ein Trugbild, in: NJW 1994, S. 2467-2469.

Alexander, Gregory: The limits of freedom of contract, in: Buckley, F. H. (Hrsg.): The fall and rise of freedom of contract, Durham u.a. (Duke University Press) 1999, S. 103-118.

Allen, Pauline: Contracts in the National Health Service internal market, in: MLR, 58. Jg. (1995), H. 3, S. 321-342.

Allison, J.W.F.: A continental distinction in the common law: A historical and comparative perspective on English public law, Oxford (Oxford University Press) 2000.

Ambs, Friedrich (Hrsg.): Gemeinschaftskommentar zum Arbeitsförderungsrecht (GK-SGB III), Neuwied u.a. (Luchterhand) Loseblattausgabe Stand: Mai 2009.

Annesley, Claire: Americanised and Europeanised UK social policy since 1997, in: BJPIR 2003, H. 5, S. 143-165.

Apelt, Willibalt: Der verwaltungsrechtliche Vertrag: Ein Beitrag zur Lehre von der rechtswirksamen Handlung im öffentlichen Rechte, Leipzig (Meiner) 1920.

Armborst, Christian: Leben auf Pump: Darlehen im SGB II, in: info also 2006, S. 58-60.

Arrowsmith, Sue: Judicial review and the contractual powers of public authorities, in: LQR, 106. Jg. (1990), S. 277-292.

Arts, Wil/Gelissen, John: Three worlds of welfare capitalism or more? A state-of-the-art report, in: JESP 2002, S. 137-159.

Atiyah, Patrick S.: An introduction to the law of contract, Oxford (Clarendon Press) 1995.

Atiyah, Patrick S.: The rise and fall of freedom of contract, Oxford (Clarendon Press) 2003.

Aust, Andreas: Die Europäische Beschäftigungs- und Sozialpolitik nach dem Gipfel von Amsterdam: Versuch einer ersten Bilanz, in: ZSR 2000, S. 13-46.

Bachof, Otto: Beurteilungsspielraum, Ermessen und unbestimmter Rechtsbegriff im Verwaltungsrecht, in: JZ 1955, S. 97-102. 
Bachof, Otto: Über öffentliches Recht, in: Bachof, Otto (Hrsg.), Verwaltungsrecht zwischen Freiheit, Teilhabe und Bindung, Festgabe aus Anlass des 25-jährigen Bestehens des Bundesverwaltungsgerichts, München (C. H. Beck) 1978, S. 1-21.

Bacon, Jo: Moving between sickness and unemployment, in: LMT 2002, H. 4, S. 195-205.

Bader, Peter: Das Arbeitsförderungsreformgesetz, in: AuR 1997, S. 381-390.

Baldwin, Robert: „The next steps“: Ministerial responsibility and government by agency, in: MLR, 51. Jg. (1988), H. 5, S. 622-628.

Baldwin, Robert/Wikeley, Nicholas; Young, Richard: Judging social security: The adjudication of claims for benefit in Britain, Oxford (Clarendon Press) 1992.

Bandemer, Stephan von/Blanke, Bernhard/Hilbert, Josef/Schmid, Josef: Staatsaufgaben - von der „schleichenden Privatisierung“ zum ,aktivierenden Staat", in: Behrens, Fritz u.a. (Hrsg.): Den Staat neu denken: Reformperspektiven für Landesverwaltungen, Berlin (edition sigma) 1995, S. 41-60.

Bandemer, Stephan von/Hilbert, Josef: Vom expandierenden zum aktivierenden Staat, in: Blanke, Bernhard u.a. (Hrsg.): Handbuch zur Verwaltungsreform, Opladen (Leske + Budrich) 2001, S. 17-25.

Baring, Martin: Zur Problematik eines Verwaltungsverfahrensgesetzes, in: DVB1 1965, S. 180192.

Barker, Kit: NHS contracts, restitution and the internal market, in: MLR, 56. Jg. (1993), H. 6, S. 832-843.

Barkhaus, Annette/Hollstein, Bettina: Ein Sozialstaat, der „Sinn macht“? Begründung der Leitidee eines nachhaltig aktivierenden Sozialstaates, in: ZfWU 2003, S. 287-306.

Barron, Anne/Scott, Colin: The Citizen's Charter program, in: MLR, 55. Jg. (1992), S. 526-546.

Bartelheimer, Peter/Hobusch, Tina/Reis, Claus: Case Management in der Sozialhilfe - Anspruch und Realität, in: Dahme, Heinz-Jürgen u.a. (Hrsg.): Soziale Arbeit für den aktivierenden Staat, Opladen (Leske \& Budrich) 2003, S. 309-332.

Bauer, Hartmut: Anpassungsflexibilität im öffentlich-rechtlichen Vertrag, in: Hoffmann-Riem, Wolfgang/Schmidt-Aßmann, Eberhard (Hrsg.): Innovation und Flexibilität des Verwaltungshandelns, Baden-Baden (Nomos) 1994, S. 245-288.

Bauer, Hartmut: Sozialrecht in der Reform: Hartz IV: Die Zusammenführung von Arbeitslosenhilfe und Erwerbsfähigen-Sozialhilfe zu dem neuen Arbeitslosengeld II, in: DÖV 2004, S. 1017-1025.

Baur, Fritz: Leistungsabsprache nach SGB XII, in: SozR akt 2006, S. 51-52.

Beatson, J.: "Public" and "private" in English administrative law, in: LQR, 103. Jg. (1987), H. 1, S. 34-65.

Beatson, J.: Anson's law of contract, Oxford (Oxford University Press) 2002.

Becker, Ulrich: Staat und autonome Träger im Sozialleistungsrecht, Rechtsvergleichende Untersuchung der Risikoabsicherungssysteme in Deutschland, Frankreich, Italien und Großbritannien, Baden-Baden (Nomos) 1996.

Becker, Ulrich: Sozialmodell und Menschenbild in der „Hartz-IV” Gesetzgebung, in: Behrends, Okko (Hrsg.): Gesetzgebung, Menschenbild und Sozialmodell im Familien- und Sozialrecht, Abhandlungen der Akademie der Wissenschaften zu Göttingen, Berlin (de Gruyter) 2008, S. 39-76. 
Becker, Ulrich/Sichert, Markus: Hartz IV in Diensten des Sports: Privilegierung gemeinnütziger und im öffentlichen Interesse liegender Tätigkeiten im SGB II, in: SpuRt 2005, S. 187191.

Beckmann, Petra u.a.: Forschung zum SGB II aus Sicht des IAB: Die neuen Forschungsaufgaben im Überblick, in: IAB-Forschungsbericht 2004, S. 1-26.

Bennet, Chris/Ferlie, Ewan: Contracting in theory and in practice: some evidence from the NHS, in: Pub Admin, 74. Jg. (1996), H. 1, S. 49-66.

Benz, Arthur: Kooperative Verwaltung: Funktionen, Voraussetzungen und Folgen, BadenBaden (Nomos) 1994.

Berlit, Uwe: Verpflichtung zur Selbsthilfe, Verbot der Zwangsarbeit: Verfassungsrechtliche Determinanten der Hilfe zur Arbeit nach dem Bundessozialhilfegesetz, in: RsDE 1996, Bd. 33, S. 30-77.

Berlit, Uwe: Hilfe zur Arbeit im Kontext aktueller sozialpolitischer Entwicklungen, in: Fuchs, Petra/Schulze-Böing, Matthias: Hilfe zur Arbeit und kommunale Beschäftigungspolitik, Zwischenbilanz und Perspektiven, Frankfurt am Main (Deutscher Verein für Öffentliche und Private Fürsorge) 1999, S. 15-58.

Berlit, Uwe: Zusammenlegung von Arbeitslosen- und Sozialhilfe: Bemerkungen zu den Gesetzentwürfen von Bundesregierung und hessischer Landesregierung für ein neues SGB II und SGB XII, in: info also 2003, S. 195-208.

Berlit, Uwe: Das neue Sanktionensystem, in: ZFSH/SGB 2005, S. 707-715, 2006, S. 11-19.

Berlit, Uwe: Eingliederungsvereinbarung nach dem SGB II: Rechtsrahmen und Rechtsschutz, in: SozR akt 2006, S. 41-50.

Bertelsmann Stiftung: Exposé Arbeitsmarktpolitik, Gütersloh (Verlag Bertelsmann Stiftung) 2002

Bettermann, Karl August: Grundfragen des Preisrechts für Mieten und Pachten, Tübingen (Mohr) 1952.

Bewley, Helen/Dorsett, Richard/Thomas, Andrew: Joint claims for JSA: evaluation - synthesis of findings, Research Report Nr. 235, Leeds (Corporate Document Service) 2005.

Bieback, Karl-Jürgen: Fördern und Fordern - Kontinuitäten und Brüche im Arbeitsförderungsrecht der Hartz-Gesetze, in: KJ 2003, S. 25-34.

Bieback, Karl-Jürgen: Reform des Sozialleistungssystems - Zwang oder Vertrag?, in: GMH 2003, S. 22-31.

Bieback, Karl-Jürgen: Umbau der Arbeitsförderung nach den Vorschlägen der HartzKommission: Analyse und Würdigung der Reformen, in: SDSRV 52 (2004), S. 59-83.

Bieback, Karl-Jürgen: Die Reform der Arbeitsförderung (SGB III und II) - Eine Zwischenbilanz, in: SGb 2005, S. 481-490.

Bieback, Karl-Jürgen: Probleme des SGB II: Rechtliche Probleme des Konflikts zwischen Existenzsicherung und Integration in den ersten Arbeitsmarkt, in: NZS 2005, S. 337-343.

Bieback, Karl-Jürgen: Kommunale Sozialpolitik und ihre Koordination mit der Bundesagentur für Arbeit, in: RsDE, Bd. 61 (2006), S. 24-53.

Bieritz-Harder, Renate: Die Arbeitsgelegenheiten des $\S 16$ Abs. 3 Satz 2 SGB II, in: ZfSH/SGB 2005, S. 259-263. 
Birkinshaw, Patrick/Harden, Ian/Lewis, Norman: Government by moonlight: The hybrid parts of the state, London (Unwin Hyman) 1990.

Birks, Peter: An introduction to the law of restitution, Oxford (Clarendon Press) 1989.

Blanke, Bernhard: Aktivierender Staat - aktive Bürgergesellschaft: Eine Analyse für das Bundeskanzleramt, Hannover 2001.

Blanke, Bernhard/Schridde, Henning: Bürgerengagement und aktivierender Staat, in: Heinze, Rolf/Olk, Thomas (Hrsg.): Bürgerengagement in Deutschland: Bestandsaufnahme und Perspektiven, Opladen (Leske \& Budrich) 2001, S. 93-140.

Bleckmann, Albert: Subordinationsrechtlicher Verwaltungsvertrag und Gesetzmäßigkeit der Verwaltung, in: VerwArch, 63. Jg. (1972), S. 404-440.

Blos, Kerstin/Rudolph, Helmut: Simulationsrechnungen zum Arbeitslosengeld II: Verlierer, aber auch Gewinner, in: IAB-Kurzbericht 2005, S. 1-6.

Blüggel, Jens: Die „einheitliche Entscheidung“ der Einigungsstelle nach § 44a SGB II: Zugleich ein Beitrag zur Systemabgrenzung von SGB II und XII, in: SGb 2005, S. 377-384.

Blüggel, Jens: Die Mitwirkungspflichten des Arbeitsuchenden nach dem SGB II: Was fordert das neue Recht?, in: SozSich 2005, S. 12-18.

Blumenwitz, Dieter: Einführung in das anglo-amerikanische Recht, 7. Auflage, München (C. H. Beck) 2003.

Boecken, Winfried: Zusammenführung von Sozialhilfe und Arbeitslosenhilfe, insbesondere zur verfassungsrechtlichen Zulässigkeit einer Abschaffung des Anspruchs auf Arbeitslosenhilfe und einer Beteiligung des Bundes an den Sozialaufwendungen, in: SGb 2002, S. 357-366.

Boecken, Winfried: Eigenverantwortung in der Sozialhilfe: Einführung einer Beweislastumkehr bei der Hilfe zur Arbeit, in: VSSR 2003, S. 45-56.

Bohne, Eberhard: Informales Verwaltungs- und Regierungshandeln als Instrument des Umweltschutzes: Alternativen zu Rechtsnorm, Vertrag, Verwaltungsakt und anderen rechtlich geregelten Handlungsformen, in: VerwArch, 75. Jg. (1984), S. 343-373.

Bolay, Martin/Eisenreich, Albrecht/Isele, Markus: Die neue Arbeitsförderung, 2. Auflage, Baden-Baden (Nomos) 2005.

Braun, Joachim (Hrsg.): Selbsthilfe und Selbsthilfeunterstützung in der Bundesrepublik Deutschland, Stuttgart (Kohlhammer) 1997.

Brennan, Tom: Newstart activity agreements: Are they contracts?, in: Creyke, Robin/Sassella, Michael (Hrsg.): Targeting accountability and review: current issues in income support law, Canberra (Union Offset) 1998, S. 87-103.

Brewer, Mike/Clark, Tom/Wakefield, Matthew: Social Security in the UK under New Labour: What did the third way mean for welfare reform?, in: Fisc Stud, 23. Jg. (2002), H. 4,. S. 505-537.

Brinkmann, Christian u.a.: SGB II-Neue Herausforderungen an Statistik und Forschung, in: IAB-Forschungsbericht 2005, S. 1-26.

Brinktrine, Ralf: Verwaltungsermessen in Deutschland und England: Eine rechtsvergleichende Untersuchung von Entscheidungsspielräumen der Verwaltung im deutschen und englischen Verwaltungsrecht, Heidelberg (C.F. Müller Verlag) 1998. 
Brohm, Winfried: Rechtsstaatliche Vorgaben für informelles Verwaltungshandeln, in: DVBl 1994, S. 133-139.

Brownsword, Roger: Contract law: Themes for the twenty-first century, London (Butterworths) 2000.

Brox, Hans/Walker, Wolf-Dietrich: Allgemeiner Teil des BGB, 32. Auflage, Köln u.a. (Heymanns) 2008.

Brühl, Albrecht/Hofmann, Albert: Sozialgesetzbuch Zweites Buch: Grundsicherung für Arbeitsuchende, Scheßlitz (Rosch Buch-Druckerei) 2004.

Bryson, Alex: The jobseeker's allowance: Help or hindrance for the unemployed?, in: ILJ, 24. Jg. (1995), S. 204-210.

Buchheit, Bernd: Neue Impulse für die Arbeitsmarktpolitik, in: BArbB1 2002, S. 5-10.

Bulling, Manfred: Kooperatives Verwaltungshandeln (Vorverhandlungen, Arrangements, Agreements und Verträge) in der Verwaltungspraxis, in: DÖV 1989, S. 277-289.

Bullinger, Martin: Vertrag und Verwaltungsakt zu den Handlungsformen und Handlungsprinzipien der öffentlichen Verwaltung nach deutschem und englischem Recht, Stuttgart (Kohlhammer) 1962.

Bundesagentur für Arbeit: Jahresbericht 2006, SGB II: Grundsicherung für Arbeitsuchende, Nürnberg 2006.

Bundesagentur für Arbeit: Jahresbericht 2007, SGB II: Grundsicherung für Arbeitsuchende, Nürnberg 2007.

Bundesrechnungshof: Bericht über die Durchführung der Grundsicherung für Arbeitsuchende, Berlin 2006.

Burckhardt, Walther: Der Vertrag im Privatrecht und im öffentlichen Recht, Bern (Stämpfli) 1924.

Buscher, Herbert: Arbeitslosengeld II und das kommunale Optionsgesetz, in: WiW 2004, S. 283-288.

Butcher, Tony: Managing the welfare state, in: Jones, Helen/MacGregor, Susanne: Social issues and party politics, London (Routeledge) 1998.

Bydlinski, Franz: Zu den dogmatischen Grundfragen des Kontrahierungszwanges, in: AcP, 180. Jg. (1980), S. 1-46.

Cane, Peter: Public law and private law: A study of the analysis and use of a legal concept, in: Eekellaar, John (Hrsg.): Oxford essays in jurisprudence: third series, Oxford (Clarendon Press) 1987, S. 57-78.

Cane, Peter: Administrative law, 4. Auflage, Oxford (Clarendon Press) 2004.

Carmel, Emma/Papadopoulos, Theodoros: The new governance of social security in Britain, in: Millar, Jane (Hrsg.), Understanding social security, Bristol (The Policy Press) 2003, S. 31-52.

Carney, Terry/Ramia, Gaby: From rights to management: Contract, new public management and employment services, Den Haag (Kluwer Law International) 2002.

Casey, Bernhard: The OECD Jobs Strategy and the European Employment Strategy: Two views of the labour market and the welfare state, in: EJIR, 10. Jg., 2004, H. 3, S. 329-352.

Castles, Francis G.: Families of nations : patterns of public policy in Western democracies, Dartmouth (Aldershot) 2000. 
Child Poverty Action Group: The Introduction of JSA - what it will mean for claimants and advisers, in: WRB 1996, H. 134, S. 5-15.

Child Poverty Action Group: New Deal for 18/24-year-olds, in: WRB 1998, H. 142, S. 4-11.

Child Poverty Action Group: The New Deal - an update, in: WRB 1998, H. 146, S. 4-5.

Child Poverty Action Group: The New Deal for long-term unemployed people over 25, in: WRB 1998, H. 146, S. 10-17.

Child Poverty Action Group: Welfare benefits and tax credits handbook, London (Child Poverty Action Group) 2005.

Chojetzki, Uwe/Klönne, Margit: Das Vierte Gesetz für moderne Dienstleistungen am Arbeitsmarkt aus Sicht der Rentenversicherung, in: DRV 2004, S. 513-531.

Clasen, Jochen: Motives, Means and Opportunities: Reforming Umemployment Compensation in the 1990s, in: WEP, 23. Jg. (2000), H. 2, S. 89-112.

Clinton, William J.: Acceptance Speech to the Democrativ Convention of July 16, 1992, in: Wolley, John T./Peters, Gerhard: The American Presidency project [online], Santa Barbara, CA, University of California (hosted), Gerhard Peters (database), http://www.presidency.ucsb.edu/ws/?pid=25958.

Collins, Hugh: Regulating contracts, Oxford (Oxford University Press) 2002.

Collins, Hugh: The law of contract, 4. Auflage, London (Nexis Lexis) 2003.

Corden, John: Contracts in Social Work Practice, in: BJSW, 10. Jg. (1980), S. 143-161.

Craig, Paul: Public law and control over private power, in: Taggart, Michael (Hrsg.): The province of administrative law, Oxford (Hart Publishing) 1997, S. 196-216.

Craig, Paul: Contracting out, the Human Rights Act and the scope of judicial review, in: LQR,

118. Jg. (2002), S. 551-568.

Craig, Paul: Administrative law, 5. Auflage, Oxford (Sweet \& Maxwell) 2003.

Daguerre, Anne: Importing welfare policy transfer of social and labour market policies from the

USA to Britain under New Labour, in: SPA, 38. Jg. (2004), H. 1, S. 41-56.

Daguerre, Anne/Taylor-Gooby, Peter: Neglecting Europe: explaining the predominance of American ideas in New Labour's welfare policies since 1997, in: JESP, 14. Jg. 2004, H. (1), S. 25-39.

Dahm, Sabine: Das neue Steuerungsmodell auf Bundes- und Länderebene sowie die Neuordnung der öffentlichen Finanzkontrolle in der Bundesrepublik Deutschland, Berlin (Duncker \& Humblot) 2004.

Damkowski, Wulf/Rösener, Anke: Auf dem Weg zum aktivierenden Staat - Vom Leitbild zum umsetzungsreifen Konzept, Berlin (edition sigma) 2003.

Danwitz, Thomas von: Verwaltungsrechtliches System und europäische Integration, Tübingen (Mohr) 1996.

Darimont, Barbara: Rezeptionen im chinesischen Sozialrecht: Rechtsrezeption und die Suche nach der ,chinesischen Prägung“, in: ZIAS 2003, S. 354-365.

Darimont, Barbara: Rezeption und die Bedeutung für das deutsche Sozialrecht, in: Becker, Ulrich/Gongcheng, Zheng/Darimont, Barbara (Hrsg.), Grundfragen und Organisation der Sozialversicherung in China und Deutschland, Baden-Baden (Nomos) 2005, S. 127-137. 
Dauber, Gerlinde: Möglichkeiten und Grenzen kooperativen Verwaltungshandelns, in: BeckerSchwarze, Kathrin (Hrsg.): Wandel der Handlungsformen im öffentlichen Recht, Stuttgart (Boorberg) 1991, S. 67-98.

Däubler, Wolfgang: Absenkung und Entzug des ALG II - ein Lehrstück zur Verfassungsferne des Gesetzgebers, in: info also 2005, S. 51 - 55.

Däubler, Wolfgang: Das Verbot der Ausgrenzung einzelner Bevölkerungsgruppen - Existenzminimum und Arbeitslosengeld II, in: NZS 2005, S. 225-231.

Däubler, Wolfgang: Einmalbedarf und Arbeitslosengeld II: Zur Darlehensregelung des § 23 I SGB II, in: NJW 2005, S. 1545-1547.

Dauner-Lieb, Barbara: Verbraucherschutz durch Ausbildung eines Sonderprivatrechts für Verbraucher: systemkonforme Weiterentwicklung oder Schrittmacher der Systemveränderung?, Berlin (Duncker \& Humblot) 1983.

Davies, Anne: Accountability: A public law analysis of government by contract, Oxford (Oxford University Press) 2001.

Deacon, Alan: Learning from the US? The influence of American ideas upon "new labour" thinking on welfare reform, in: PP, 28. Jg. (1999), H. 1, S. 5-18.

Deakin, Nicholas/Walsh, Kieron: The enabling state: The role of markets and contracts, in: Pub Admin, 74. Jg. (1996), H. 1, S. 33-48.

Deakin, Simon/Michie, Jonathan (Hrsg.): Contracts, cooperation and competition, Oxford (Oxford University Press) 1997.

Degenhart, Christoph: Vermutungsregeln im Sozialrecht - Verfassungsfrage des $\S 7$ Abs. 3 SGB IV, in: ZfA 1999, S. 531 ff.

Deutscher Verein für öffentliche und private Fürsorge: Stellungnahme des Deutschen Vereins zu dem Entwurf eines Vierten Gesetzes für moderne Dienstleistungen am Arbeitsmarkt, in: NDV 2003, S. 496-506.

Deutscher Verein für öffentliche und private Fürsorge: Empfehlungen des Deutschen Vereins zur Weiterentwicklung der Hilfeplanung nach § 36 SGB VIII, in: NDV 2006, S. 343-354.

Dewitz, Ralf Michael: Der Vertrag in der Lehre Otto Mayers, Berlin (Duncker \& Humblot) 2004.

Diering, Björn/Timme, Hinnerk/Waschull, Dirk: Sozialgesetzbuch X, Lehr- und Praxiskommentar, Baden-Baden (Nomos) 2004.

Di Fabio, Udo: Vertrag statt Gesetz? Gesetzesvertretende und gesetzesausfüllende Verwaltungsverträge im Natur- und Landschaftsschutz, in: DVB1 1990, S. 338-347.

Di Fabio, Udo: Die Ermessensreduzierung - Fallgruppen, Systemüberlegungen und Prüfprogramm, in: VerwArch, 86. Jg. (1995), S. $214 \mathrm{ff}$.

Dicey, A. $V$ : Introduction to the study of the law of the Constitution, London (Macmillan) $1941 / 1889$.

Doern, Bruce G.: The UK Citizen's Charter origins and implementation in three agencies, in: PP, 21. Jg. (1993), H. 1, S. 17-29.

Dolowitz, David P.: Policy transfer and British social policy: learning from the USA?, Buckingham u.a. (Open University Press) 2000.

Dose, Nicolai/Voigt, Rüdiger (Hrsg.): Kooperatives Recht, Baden-Baden (Nomos) 1995. 
Dreas, Susanne/Gerhardt, Michael: Privatvermittlungsagenturen zur beruflichen Eingliederung von Langzeitarbeitslosen- bestehende Barrieren und eine mögliche Konzeption, in: SozFort 2002, S. 2-9.

Drewry, Gavin: Forward from F.M.I.: “The next steps”, in: PL 1988, S. 505-515.

Drewry, Gavin: Whatever happened to the Citizen's Charter?, in: PL 2002, S. 9 ff.

Drewry, Gavin/Giddings, Philip: The origins of the next step programme, in: Giddings, Philip

(Hrsg.): Parliamentary accountability: a study of parliament and executive agencies, Basingstoke u.a. (Macmillan) 1995, S. 3-17.

Dwyer, Peter: Conditional citizens? Welfare rights and responsibilities in the late 1990s, in: CSP, 57. Jg. (1998), H. 18, S. 493-517.

Dwyer, Peter: Understanding social citizenship: Themes and perspectives for policy and practice, Bristol (Policy Press) 2004.

Dyllick, Joachim/Lörincz, Ernö/Neubauer, Reinhard: ARGE - Irrungen und Wirrungen, in: ZFSH/SGB 2007, S. 397-400.

Eberhard, Dietrich: Zivilrecht und Sozialrecht in der Beziehung von Kassenarzt und Kassenpatient, in: AcP, 171. Jg. (1971), S. 289-327.

Ebsen, Ingwer: Die Arbeitslosenhilfe zwischen Sozialversicherung und Fürsorge: Funktionswandel der Arbeitslosenhilfe durch gesetzliche Umgestaltungen?, in: Ebsen, Ingwer (Hrsg.), Stand und Entwicklung des Arbeitsförderungsrechts im Übergang vom AFG zum SGB III, Frankfurt (Eigenverlag des Deutschen Vereins für öffentliche und private Fürsorge) 1997, S. 159-174.

Ebsen, Ingwer: Der Arbeitslose als Sozialbürger und Klient: Der Betroffene im Konzept des aktivierenden Sozialstaates, in: Wulffen, Matthias von (Hrsg.): FS 50 Jahre Bundessozialgericht, Köln (Heymanns) 2004, S. 725-744.

Ebsen, Ingwer: Contracting between social services and their clients in the German concept of "Fördern und Fordern" in: Sol, Els/Westerveld, Mies (Hrsg.): Contractualism in employment services: A new form of welfare state governance, Den Haag (Kluwer International) 2005, S. 231-253.

Efstratiou, Pavlos-Michael: Die Bestandskraft des öffentlich-rechtlichen Vertrags: Eine vergleichende Untersuchung zum griechischen, französischen und insbesondere deutschen Verwaltungsrecht, Berlin (Duncker \& Humblot) 1988.

Eichenhofer, Eberhard: Neue Grundsätze der Arbeitsförderung, in: SGb 2000, 289-295.

Eichenhofer, Eberhard: Die Rolle von öffentlichem und privatem Recht bei der Erbringung sozialer Dienstleistungen, in: SGb 2003, S. 365-370.

Eichenhofer, Eberhard: Eigentum - Verschulden - Vertrag: Privatrechtsbegriffe als Sozialrechtskonstrukte, in: VSSR 2004, S. 93-114.

Eichenhofer, Eberhard: Verträge in der Arbeitsverwaltung, in: SGb 2004, S. 203-209.

Eichenhofer, Eberhard/Westerveld, Mies: Contractualism - A legal perspective, in: Sol, Els/Westerveld, Mies (Hrsg.): Contractualism in employment services: A new form of welfare state governance, Den Haag (Kluwer International) 2005, S. 21-39.

Eicher, Wolfgang/Spellbrink, Wolfgang (Hrsg.): SGB II, Grundsicherung für Arbeitsuchende, München (C. H. Beck) 2005. 
Eicher, Wolfgang,/Schlegel, Roger: SGB III Arbeitsförderung, Kommentar mit Nebenrecht, Neuwied (Luchterhand), Loseblattausgabe Stand: April 2009.

Eichhorst, Werner/Grienberger-Zingerle, Maria/Konle-Seidl, Regina: Activation policies in Germany: From status protection to basic income, in: Eichhorst, Werner/Kaufmann, Otto/Konle-Seidl, Regina (Hrsg.), Bringing the jobless into work?, 2008, S. 17-67.

Eichhorst, Werner/Rhein, Thomas: Die Europäische Beschäftigungsstrategie - Beispiel der Methode der offenen Koordinierung, in: SDSRV 53 (2005), S. 53-66.

Eichhorst, Werner/Kaufmann, Otto/Konle-Seidl, Regina (Hrsg.): Bringing the jobless into work?, Experiences with activation schemes in Europe and the US, Berlin u.a., (Springer) 2008.

Elmeskov, Jorgen: Implementing the OECD Jobs Strategy - Assessing performance and policy, in: $O E C D$, Education at a glance, 2000, S. 39-52.

Empter, Stefan/Esche, Andreas: Eigenverantwortung und Solidarität: Neue Wege in der Sozialund Tarifpolitik, Gütersloh (Bertelsmann Stiftung) 1997.

Enderlein, Wolfgang: Rechtspaternalismus und Vertragsrecht, München (C. H. Beck) 1996.

Engelen-Kefer, Ursula u.a. (Hrsg.): Die Reform der Arbeitsförderung, Fachtagung der BayerStiftung für Deutsches und Internationales Arbeits- und Wirtschaftsrecht am 30. November 1995, München (C. H. Beck) 1997.

Erichsen, Hans-Uwe: Allgemeine Handlungsfreiheit, in: Isensee, Josef/Kirchhof, Paul (Hrsg.): Handbuch des Staatsrechts, Bd. 6, Heidelberg (C. F. Müller Verlag) 2001, § 152.

Erichsen, Hans-Uwe/Ehlers, Dirk (Hrsg.): Allgemeines Verwaltungsrecht, 13. Auflage, Berlin (Walter de Gruyter) 2006.

Esping-Andersen, Gósta: The three worlds of welfare capitalism, Cambridge (Polity Press) 1990.

Estelmann, Martin (Hrsg.): SGB II, Kommentar, Neuwied u.a. (Luchterhand) Loseblattausgabe Stand: Mai 2009

Estelmann, Martin: Die Sperrzeit im Spannungsfeld von Einzelfallgerechtigkeit und Pauschalierung, in: VSSR 1997, S. 313-342.

Evers, Adalbert: Aktivierender Staat: Eine Agenda und ihre möglichen Bedeutungen, in: Mezger, Erika/West, Klaus-W. (Hrsg.): Aktivierender Sozialstaat und politisches Handeln, Marburg (Schüren) 2000, S. 13-29.

Evers, Adalbert: Aktivierender Staat II: Ein Beitrag zur Vitalisierung der Bürgergesellschaft?, in: TuP 2001, S. 83-89.

Faber, Heiko: Verwaltungsrecht, Tübingen (Mohr) 1995.

Faber, Michael: Das neue SGB II: Eine Lösung des Problems der Langzeitarbeitslosigkeit?, in: NZS 2005, S. 75-82.

Fahlbusch, Jonathan/Müller, Imme/Rixen, Stephan: Das SGB II-Fortentwicklungsgesetz, Ein erster Überblick über Auslegungsprobleme und legislativen Korrekturbedarf, in: NDV 2006, S. 411-421.

Fargion, Silvia: Images of contract: An empirical study of the use of theory in practice, in: BJSW, 33. Jg. (2003), H. 4, S. 517-533.

Feldman, David/Birks, Peter: English public law, Oxford (Oxford University Press) 2004. 
Ferge, Zsusza: In defence of messy or multi-principle contracts, in: EJSS, 2. Jg. (2000), H. 1, S. 7-33.

Fichtner, Otto (Hrsg.): Bundessozialhilfegesetz, 2. Auflage, München (Verlag Franz Vahlen) 2003.

Finn, Dan: Uncompelling evidence - The jobseeker's allowance won't help the unemployed, in: NE 1996, S. 60-65.

Finn, Dan: Labour's "New Deal" for the unemployed and the stricter benefit regime, in: SPR 1998, S. 105-122.

Finn, Dan: The role of contracts and the private sector in delivering Britain's "employment first" welfare state, in: Sol, Els/Westerveld, Mies (Hrsg.): Contractualism in employment services: A new form of welfare state governance, Den Haag (Kluwer International) 2005, S. 101-117.

Finn, Dan/Schulte, Bernd: "Employment first": Activating the British welfare state, in: Eichhorst, Werner/Kaufmann, Otto/Konle-Seidl, Regina: Bringing the jobless into work?, 2008, S. 297-343.

Finn, Paul: Public function - private action: a common law dilemma, in: Benn, Stanley/Gaus, Gerald (Hrsg.): Public and private in social life, London (Croom Helm) 1983, S. 93-111.

Fischedick, Hans-Jürgen: Die Wahl der Benutzungsform kommunaler Einrichtungen, Kriterien für die Entscheidung zwischen privatrechtlicher und öffentlich-rechtlicher Benutzungsform, Köln (Dt. Gemeindeverlag) 1986.

Fletcher, Del Roy: Evaluating special measures for the unemployed, in: PP, 25. Jg. (1997), H. 2, S. 173-184.

Flume, Werner: Allgemeiner Teil des Bürgerlichen Rechts, Berlin u.a. (Springer) 1975.

Foulkes, David: Administrative law, 7. Auflage, London u.a. (Butterworths) 1990.

Fraser, Nancy/Gordon, Linda: Civil citizenship against social citizenship? On the ideology of contract-versus-charity, in: Steenbergen, Bart van (Hrsg.): The condition of citizenship, London (Sage) 1994, S. 90-122.

Freedland, Mark: Government by contract and public law, in: PL 1994, S. 86-104.

Freedland, Mark: Public law and private finance: Placing the private finance initiative in a public frame, in: PL 1998, S. 288-307.

Freedland, Mark: The regulation of contractualisation in quasi-markets for public services, in: PL 1999, S. 306-312.

Freedland, Mark: Government by contract re-examined: Some functional issues, in: Craig, Paul/Rawlings, Richard (Hrsg.): Law and administration in Europe - Essays in honour of Carol Harlow, Oxford (Oxford University Press) 2003, S. 123-137.

Freedland, Mark/King, Desmond: Client contractualism between the employment service and jobseekers in the United Kingdom, in: Sol, Els/Westerveld, Mies (Hrsg.): Contractualism in employment services: A new form of welfare state governance, Den Haag (Kluwer International) 2005, S. 119-138.

Friauf, Karl Heinrich/Höfling, Wolfram (Hrsg.): Berliner Kommentar zum Grundgesetz, Berlin (Erich Schmidt Verlag), Loseblattausgabe Stand: April 2008.

Frobisher, Fiona: Joint claims for JSA, in: WRB, 160. Jg. (2001), H. 2, S. 23 ff. 
Frommann, Matthias: Warum nicht 627 Euro? Zur Bemessung des Regelsatzes der Hilfe zum Lebensunterhalt nach dem SGB XII für das Jahr 2005, in: NDV 2004, S. 246-254.

Fuchs, Maximilian: Deutsche Grundsicherung und europäisches Koordinationsrecht, in: NZS 2007, S. 1-6.

Fuchs, Petra: Hilfe zur Arbeit als Integrationsprozess: Anforderungen an eine prozessbegleitende Beratung, in: Fuchs, Petra/Schulze-Böing, Matthias: Hilfe zur Arbeit und kommunale Beschäftigungspolitik, Zwischenbilanz und Perspektiven, Frankfurt am Main (Deutscher Verein für Öffentliche und Private Fürsorge) 1999, S. 87-90.

Fulbrook, Julian: The Jobseeker's Act 1995 Consolidation with a sting of contractual compliance, in: ILJ, 24. Jg. (1995), S. 395-401.

Gagel, Alexander (Hrsg.): Sozialgesetzbuch II/Sozialgesetzbuch III, Grundsicherung und Arbeitsförderung, München (C. H. Beck) Loseblattausgabe Stand: Januar 2009.

Gagel, Alexander: Anmerkung zu BAG, Urteil vom 26.09.2007, -5 AZR 857/06, in: jurisPRArbR 7/2008 Anm. 3.

Gehrmann, Gerd/Müller, Klaus D. (Hrsg.): Aktivierende soziale Arbeit mit nicht-motivierten Klienten, Berlin (Walhalla Fachverlag) 2005.

Geiger, Udo: Neuausrichtung der Arbeitsvermittlung: Mehr Wettbewerb und Kundenservice oder Gängelung im neuen Design?, in: info also 2002, S. 200-204.

Geiger, Udo: Der Zugang Drittstaatsangehöriger zum SGB II: Grundsicherung für Arbeitssuchende, in: Info AuslR 2004, S. 360-361.

Geiger, Udo: Grundsicherung für Arbeitssuchende, in: Plagemann, Hermann/Ehmann, Frank (Hrsg.): Münchener Anwaltshandbuch Sozialrecht, 3. Auflage, München (C. H. Beck) 2009, § 17.

Geis, Max-Emanuel: Die öffentliche Förderung sozialer Selbsthilfe: Verfassungsrechtliche Grundlagen und verwaltungsrechtliche Ausgestaltung, Baden-Baden (Nomos) 1997.

Geis, Max-Emanuel: Die Schuldrechtsreform und das Verwaltungsrecht, in: NVwZ 2002, S. 385-391.

Genn, Hazel: Tribunals and informal justice, in: MLR, 56. Jg. (1993), H. 3, S. 393 - 411.

Gent, Andreas: Der subordinationsrechtliche Vertrag (§ 53 SGB X) als geeignete Handlungsform zur Erbringung von Sozialleistungen: Eine Untersuchung anhand ausgewählter Beispiele aus dem Bereich der Renten- und Unfallversicherung (Rehabilitationsmaßnahmen), der Sozialhilfe (Darlehensgewährung) und der Jugendwohlfahrt, München (Florenz) 1987.

Genz, Herrmann/Schwendy, Arnd: Herzstück der Hartz-Reform: Das Fallmanagement - Werden die Chancen der Arbeitslosen verspielt?, in: TuP 2004, S. 8-13.

Gericke, Thomas (Hrsg.): Jugendliche fördern und fordern: Strategien und Methoden einer aktivierenden Jugendsozialarbeit, München (Verlag Deutsches Jugendinstitut) 2002.

Gerlach, Florian: Das jugendhilferechtliche Hilfeplanverfahren als Modell kooperativer Entscheidungsfindung und seine Umsetzung in der behördlichen Praxis: Beurteilungsspielraum und verwaltungsgerichtliche Kontrolle jugendrechtlicher Entscheidungen, in: ZfJ 1998, S. 134-141.

Gerntke, Axel/Ulber, Jürgen: Der Eingliederungsvertrag: Instrument zur Integration oder zur Entrechtung?, in: AiB 1997, S. 511-520. 
Giddens, Anthony: Der dritte Weg: Die Erneuerung der sozialen Demokratie, Frankfurt a. M. (Suhrkamp) 1999.

Göldner, Detlef: Gesetzmäßigkeit und Vertragsfreiheit im Verwaltungsrecht, in: JZ 1976, S. 352-358.

Gordley, James: The philosophical origins of modern contract law, Oxford (Clarendon Press) 1991.

Graser, Alexander: From the hammock onto the trampoline: Workfare policies in the U.S. and their reception in Germany, in: GLJ, 4. Jg. (2003), H. 3, S. 201-221.

Gray, Anne: Jobseekers and gatekeepers: the role of the private employment agency in the placement of the unemployed, in: WES, 16. Jg. (2002), H. 4, S. 655-674.

Groner-Weber, Sabine: Der aktivierende Staat: Konzepte und Entwicklungsoptionen, in: Mezger, Erika/West, Klaus-W. (Hrsg.): Aktivierender Sozialstaat und politisches Handeln, Marburg (Schüren) 2000, S. 167-172.

Gronwald, Siegfried: Die Mitwirkungspflicht des Leistungsberechtigten nach dem SGB, in: SGb 1989, S. 17-20.

Groth, Andy/Siebel-Huffmann, Heiko: Die Leistungen für die Unterkunft nach $\S 22$ SGB II, in: NZS 2007, S. 69-76.

Grover, Chris/Stewart, John: "Market Workfare": Social security, social regulation and competitiveness in the 1990s, in: JSP 1999, H. (28), S. 73-96.

Grüner, Hans: Sozialgesetzbuch, Kommentar sowie Bundes- und Landesrecht, Percha (Schulz), Loseblattausgabe Stand: Mai 2008.

Grupp, Klaus: Mitwirkungspflichten im Verwaltungsverfahren, in: VerwArch, 80. Jg. (1989), S. 44-73.

Grziwotz, Herbert: Vertragsgestaltung im öffentlichen Recht, München (C. H. Beck) 2002.

Gusy, Christoph: Öffentlich-rechtliche Verträge zwischen Staat und Bürger, in: DVB1 1983, S. 1222-1229.

Hänlein, Andreas: Sozialrechtsvergleich als Rezeptionsforschung?, in: ZIAS 1998, S. 104-108.

Hamisch, Ann-Christine: Der Schutz individueller Rechte bei Rentenreformen -

Deutschland und Großbritannien im Vergleich, Baden-Baden (Nomos) 2001.

Hanau, Peter: Der Eingliederungsvertrag - Ein neues Instrument der Arbeitsförderung, in: DB 1997, S. 1278-1281.

Handler, Joel F.: Myth and ceremony in workfare: Rights, contracts and client satisfaction (September 2002), in: UCLA, School of Law Research Paper No. 02-21).

Handler, Joel F.: Social citizenship and workfare in the US and Western Europe: from status to contract, in: JESP 2003, S. 229-243.

Handler, Joel F.: Social citizenship and workfare in the Unites States and Western Europe: The paradox of inclusion, Cambridge (Cambridge University Press) 2004.

Hannes, Miriam: Was gilt bei Eingliederungsvereinbarungen? Erste Urteile zu den Vereinbarungen zwischen Hartz IV-Empfängern und SGB II-Trägern, in: SGb 2007, 68-72.

Hansen, Eckhard: Mythos und Realität sozialstaatlicher Aktivierungsideologien: Entwicklungstendenzen personenbezogener sozialer Dienstleistungen in England und Deutschland, in: Dahme, Heinz-Jürgen u.a.: Soziale Arbeit für den aktivierenden Staat, Opladen (Leske \& Budrich) 2003, S. 393-418. 
Harden, Ian: The contracting state, Philadelphia (Open University Press) 1992.

Harding, Andrew: Public duties and public law, Oxford (Clarendon Press) 1989.

Harlow, Carol: "Public" and "private" law: Definition without distinction, in: MLR, 43. Jg. (1980), H. 3, S. 241-265.

Harlow, Carol/Rawlings, Richard: Law and administration, London u.a. (Butterworths) 1997.

Hartkamp, Arthur u.a. (Hrsg.): Towards a European civil code, Nijmegen u.a. (Kluwer Law International) 1998.

Hartz, Peter: Moderne Dienstleistungen am Arbeitsmarkt, Berlin (Bundesministerium für Arbeit und Sozialordnung) 2002.

Hassemer, Winfried: Über den Umgang mit der Würde des Menschen, in: EuGRZ 2005, S. 300304.

Hauck, Karl/ Noftz, Wolfgang (Hrsg.): SGB I, Berlin (Erich Schmidt Verlag), Loseblattausgabe Stand: April 2009.

Hauck, Karl/Noftz, Wolfgang (Hrsg.): SGB II, Berlin (Erich Schmidt Verlag), Loseblattausgabe Stand: Juni 2009.

Hauck, Karl/Noftz, Wolfgang (Hrsg.): SGB III, Berlin (Erich Schmidt Verlag), Loseblattausgabe Stand: Juni 2009.

Hauck, Karl/Noftz, Wolfgang (Hrsg.): SGB X, Berlin (Erich Schmidt Verlag), Loseblattausgabe Stand: Juni 2009.

Hebeler, Timo: Die verfassungsrechtliche Einordnung der pauschalierenden Leistungsgestaltung im SGB II, in: SGb 2008, S. 8-13.

Heffernan, Richard: Beyond Euro-scepticism: Exploring the Europeanisation of the Labour Party since 1983, in: PQ, 72. Jg. (2001), S. 180-189.

Heinze, Rolf/Strünck, Christoph: Aktivierender Staat III: Politik zur Entfaltung des bürgerschaftlichen Engagements, in: TuP 2001, S. 163-166.

Heiss, Helmut: Hierarchische Rechtskreiseinteilung: Von der Rechtskreislehre zur Typologie der Rechtskulturen, in: ZfVglRWiss 2001, S. 396-424.

Henneke, Hans-Günter: Das Optionsmodell bei der Grundsicherung für Arbeitssuchende: ein Flexibilitätsmodell mit Zukunft?, in: Henneke, Hans-Günter (Hrsg.): Künftige Funktionen und Aufgaben der Kreise im sozialen Bundesstaat, Stuttgart u.a. (Richard Boorberg Verlag) 2004, S. 95-106.

Henneke, Hans-Günter: Aufgabenwahrnehmung und Finanzlastverteilung im SGB II als Verfassungsproblem, in: DÖV 2005, S. 177-191.

Henneke, Hans-Günter: Hartz IV in der „Überholung“: Die Suche nach klarer Verantwortungszuordnung in den Arbeitsgemeinschaften geht weiter, in: DÖV 2006, S. 726-732.

Henneke, Hans-Günter: Das SGB II dauerhaft sachgerecht und zukunftsfähig organisieren, in: Der Landkreis 2008, 59-62.

Hennig, Werner: Sozialgesetzbuch Drittes Buch (SGB III) - Arbeitsförderung, Neuwied (Luchterhand) Loseblattausgabe Stand: Mai 2008.

Henrich, Dieter/Huber, Peter: Einführung in das englische Privatrecht, Heidelberg (Verlag Recht und Wirtschaft GmbH) 2003. 
Herne Hill, Lester Lord/Clapinska, Lydia: Human rights and the British Constitution, in: Jowel, Jeffrey/Oliver, Dawn (Hrsg.): The changing Constitution, Oxford (Oxford University Press) 2004, S. 62-87.

Heron, Emma: Etzioni's spirit of communitarism community values and welfare realities in Blair's Britain, in: SPR, 13. Jg. (2001), H. 4, S. 63-87.

Hielscher, Volker: Reorganisation der Bundesagentur für Arbeit: „Moderner Dienstleister“ für wen?, in: WSI-Mitteilungen 2006, S. 119-123.

Hill, Hermann: Integratives Verwaltungshandeln: Neue Formen von Kommunikation und Bürgermitwirkung, in: DVB1 1993, S. 973-982.

Hippel, Eike von: Die Kontrolle der Vertragsfreiheit nach anglo-amerikanischem Recht, Zugleich ein Beitrag zur Considerationlehre, Frankfurt a. M. (Vittorio Klostermann) 1963.

HMSO: Social Security Appeal Tribunals: A guide to procedure, London (HMSO) 1988.

HMSO: Efficiency Unit, Improving management in government: The next steps, London [HMSO] 1988.

HMSO: Civil service management reform: the next steps, Cm 524, London [HMSO] 1988.

HMSO: The financing and accountability of next steps agencies, Cm 914, London [HMSO] 1989.

HMSO: The Citizen's Charter: Raising the standards, Cm 1599, London [HMSO] 1991.

HMSO: Jobseeker's allowance, Cm 2687, London [HMSO] 1994.

HMSO: Improving decision making and appeals in social security, Cm 3228, London [HMSO] 1996.

HMSO: New ambitions for our country: A new contract for welfare, Cm 3805, London [HMSO] 1998.

Hochhuth, Martin: Vor schlichthoheitlichem Verwaltungseingriff anhören? Drei Thesen zur Dogmatik des Realhandelns, in: NVwZ 2003, S. 30-35.

Hofacker, Wilhelm: Verwaltungsrechtsordnung für Württemberg, Entwurf eines Gesetzes mit Begründung, Stuttgart (Kohlhammer) 1931.

Höfling, Wolfram: Vertragsfreiheit: Eine grundrechtsdogmatische Studie, Heidelberg (Müller) 1991.

Höfling, Wolfram/Krings, Günter: Der verwaltungsrechtliche Vertrag: Begriff, Typologie, Fehlerlehre, in: JuS 2000, S. 625-632.

Hogg, Peter W.: Liability of the Crown, Toronto u.a. (The Carswell Company Ltd.) 1989.

Hohm, Karl-Heinz: SGB II, Gemeinschaftskommentar zum SGB II, Grundsicherung für Arbeitsuchende, Köln (Luchterhand) Loseblattausgabe Stand: April 2009.

Holzner, Christian/Ochel, Wolfgang/Werding, Martin: Vom OFFENSIV-Gesetz zur „Aktivierenden Sozialhilfe“: Ein Konzept zur Reform der Arbeitsmarkt- und Sozialpolitik als Beitrag zu mehr Beschäftigung und Wachstum, München (ifo-Institut) 2003.

Hombach, Bodo: Aufbruch: Die Politik der Neuen Mitte, München (Econ) 1998.

Hombach, Bodo: Die Balance von Rechten und Pflichten sichern: Der aktivierende Sozialstaat das neue Leitbild, in: SozSich 1999, S. 41-44.

Hönn, Günter: Kompensation gestörter Vertragsparität: ein Beitrag zum inneren System des Vertragsrechts, München (C. H. Beck) 1982. 
Hoppe, Werner: Planung und Pläne in der verwaltungsgerichtlichen Kontrolle, in: Erichsen, Hans-Uwe (Hrsg.): System des verwaltungsgerichtlichen Rechtsschutzes, FS für Christian-Friedrich Menger zum 70. Geburtstag, Köln u.a. (Heymanns) 1985, S. 747-777.

Huber, Hans: Die verfassungsrechtliche Bedeutung der Vertragsfreiheit, in: Schriftenreihe der juristischen Gesellschaft e.V., Berlin 1966, S. 1-31.

Hübner, Heinz: Der öffentlich-rechtliche Vertrag - ein Instrument zur Aushöhlung des Privatrechts?, in: Hofmann, Paul/Meyer-Cording, Ulrich/Wiedermann, Herbert (Hrsg.): FS für Klemens Pleyer zum 65. Geburtstag, Köln u.a. (Heymanns) 1986, S. 497-511.

Hughes, David: The reorganisation of the National Health Service: The rhetoric and reality of the internal market, in: MLR, 54. Jg (1991) H. 1, S. 88-103.

Hughes, David/McHale, Jean V./Griffiths, Lesley: Settling contract disputes in the National Health Service: Formal and informal pathways, in: Flynn, Rob/William, Gareth: Contracting for health: Quasi-markets and the National Health Service, Oxford (Oxford University Press) 1997, S. 99-114.

Hunt, Michael: Accountability, openness and the Citizen's Charter, in: Chandler, J. A.: The Cititzen's Charter, Aldershot u.a. (Dartmouth) 1996, S. 55-66.

Hunt, Murray: Constitutionalism and the contractualisation of government in the United Kingdom, in: Taggart, Michael (Hrsg.): The province of administrative law, Oxford (Hart Publishing) 1997, S. 21-39.

Hüttenbrink, Jost: Sozialhilfe und Arbeitslosengeld II, Hilfe zum Lebensunterhalt, Grundsicherung, sonstige Ansprüche, Verfahren, Verwandtenregress, München (Dt. TaschenbuchVerlag) 2004.

Husmann, Manfred: Leistungen bei Arbeitslosigkeit, in: ZESAR 2009, S. 69-77; 124-129.

Imboden, Max: Der verwaltungsrechtliche Vertrag, Basel (Helbing \& Lichtenhahn) 1958.

Jellinek, Georg: Allgemeine Staatslehre, Darmstadt (Wissenschaftliche Buchgesellschaft) 1914/1959.

Jewell, Michael: An introduction to English contract law for German readers with exercises, Baden-Baden (Nomos) 2002.

Jobcentre Plus: Customer's Charter, o.O., 2005.

Jong, Martin de/Lalenis, Konstantinos/Mamadouh, Virginie (Hrsg.): The theory and practice of institutional transplantation - Experiences with the transfer of policy institutions, Dordrecht u.a. (Kluwer Academic Publishers) 2002.

Judge, Ken: Evaluating welfare to work in the United Kingdom, in: Gilbert, Neil/Van Voorhis, Rebecca A. (Hrsg.): Activating the unemployed: A comparative appraisal of workoriented policies, New Brunswick u.a. (Transaction Publishers) 2001, S. 1-28.

Kahn-Freund, Oliver: On uses and misuses of comparative law, in: MLR 37. Jg. (1974), H. 1, S. 1-27.

Karasch, Jürgen: Zwei Jahre nach dem „Vermittlungsskandal“" Was hat sich bei der Arbeitsvermittlung geändert?, in: SozSich 2004, S. 2-5.

Karl, Beatrix: Offene Methode der Koordinierung als Gestaltungsgrundsatz europäischen Rechts, in: SDSRV 53 (2005), S. 7-57.

Kautz, Steffen: Absprachen im Verwaltungsrecht: Zulässigkeit, Grenzen und Folgen, Berlin (Duncker \& Humblot) 2002. 
Kelsen, Hans: Zur Lehre vom öffentlichen Rechtsgeschäft, in: AöR 1913, Bd. 31, S. 53-98, 190249.

Kenner, Jeff: The EC employment title and the "third way": Making soft law work?, in: IJCLLIR, 15. Jg. (1999), H. 1, S. 33-60.

Kessler, Ronald/Kortmann, Klaus: Die Vertragsstrafe im öffentlich-rechtlichen Vertrag: Ein Beitrag zur Auslegung des $\S 62$ S. 2 VwVfG, in: DVBl 1977, S. 690-692.

Kilian, Wolfgang: Kontrahierungszwang und Zivilrechtssystem, in: AcP, 180. Jg. (1980), S. $47-$ 83.

King, Desmond: In the name of liberalism: illiberal social policy in the USA and Britain, Oxford (Oxford University Press) 1999.

King, Desmond/Wickham-Jones, Mark: From Clinton to Blair: The Democratic (Party) origins of welfare to work, in: PQ, 70. Jg. (1999), S. 62-74.

Kingdom, John: Citizen or state consumer? A fistful of charters, in: Chandler, J. A.: The Citizen's Charter, Aldershot u.a. (Dartmouth) 1996, S. 7-23.

Kingreen, Thorsten: Rechtliche Gehalte sozialpolitischer Schlüsselbegriffe: Vom daseinsvorsorgenden zum aktivierenden Staat, in: SDSRV 52 (2004), S. 7-47.

Kirby, Michael: Towards a grand theory of interpretation: The case of statutes and contracts, in: SLR, 24. Jg. (2003), H. 2, S. 95-111.

Kirchhof, Paul: Mittel staatlichen Handelns, in: Isensee, Josef/Kirchhof, Paul (Hrsg.): Handbuch des Staatsrechts, Bd. 3, Heidelberg (C. F. Müller Verlag) 2005, § 59.

Kirkpatrick, Ian/Lucio, Miguel Martinez: The contract state and the future of public management, in: Pub Admin, 74. Jg. (1996), S. 1-8.

Klaus, Stefan: Anmerkung zu BVerfG 2. Senat, Urteil vom 20.12.2007 - 2 BvR 2433/04, BVerfG 2. Senat, Urteil vom 20.12.2007 - 2 BvR 2434/04, in: jurisPR-SozR 5/2008 Anm. 2.

Klindt, Thomas: Öffentlich-rechtliche Verträge als Instrument der Marktüberwachung, in: NVwZ 2003, S. 307-310.

Kloepfer, Michael: Zu den neuen umweltrechtlichen Handlungsformen des Staates, in: JZ 1991, S. 737-744.

Klückmann, Harald: Zum öffentlich-rechtlichen Vertrag eines X. Buches des SGB, in: SGb 1979, S. 408-415, 444

Klug, Francesca/O'Brien, Claire: The first two years of the Human Rights Act, in: PL 2002, S. 649-662.

Kluth, Winfried: Rechtsfragen der verwaltungsrechtlichen Willenserklärung: Auslegung, Bindung, Widerruf, Anfechtung, in: NVwZ 1990, S. 608-614.

Knack, Hans-Joachim u.a. (Hrsg.): Verwaltungsverfahrensgesetz, 7. Auflage, Köln u.a. (Heymanns) 2000.

Knobel, Ulrike: Wandlungen im Verständnis der Vertragsfreiheit, Berlin (Duncker \& Humblot) 2000

Knoblauch, Dieter/Hübner, Torsten: Die Eingliederungsvereinbarung als Handlungsform im SGB II und SGB III, in: NDV 2005, S. 277-282.

Knoblauch, Dieter/Hübner, Torsten: Hartz IV - ein Vermögensbildungsprogramm für selbständig Erwerbstätige?, in: NDV 2006, S. 375-379. 
Knuth, Matthias/Finn, Dan: Hartz oder Harrods?, Reformen der Arbeitsförderung im Vereinigten Königreich, in: IAT-Report 2004-04, S. 1-12.

Koch, Susanne/Walwei, Ulrich: Was bringt Hartz IV für den Arbeitsmarkt?, in: GSP 2004, S. 923.

Kocialkowski, Christian: Eingliederungsvereinbarungen nach dem SGB II aus Sicht der Bundesagentur für Arbeit, SozR akt 2006, S. 38-40.

Kofner, Stefan: Angemessene Heizkosten im SGB II, in: WuM 2007, S. 310-311.

Köhler, Hajo: Arbeitsförderungsrecht, in: Plagemann, Hermann/Ehmann, Frank (Hrsg.): Münchener Anwaltshandbuch Sozialrecht, 3. Auflage, München (C. H. Beck) 2009, §§ 14-16.

Köhler, Karl Friedrich: Die Bedeutung der Sachverhaltsermittlung im „modernen“ Verwaltungsverfahren, in: WzS 2001, S. 129-137.

Kommunale Gemeinschaftsstelle für Verwaltungsmanagement: Das Neue Steuerungsmodell, in: KGSt-Bericht 5/1993, S. 1-40.

Könemann, Britta: Der verfassungsunmittelbare Anspruch auf das Existenzminimum, Zum Einfluss von Menschenwürde und Sozialstaatsprinzip auf die Sozialhilfe, Hamburg (Verlag Dr. Kovac) 2005.

Konle-Seidl, Regina: Wettbewerbsmechanismen in der Arbeitsmarktpolitik, in: BArbB1 2004, S. 4-14.

Konle-Seidl, Regina: New delivery forms of employment services in Germany: A mixed publicprivate model?, in: Sol, Els/Westerveld, Mies (Hrsg.): Contractualism in employment services: A new form of welfare state governance, Den Haag (Kluwer International) 2005, S. 187-207.

Kopp, Ferdinand/Ramsauer, Ulrich: Verwaltungsverfahrensgesetz, 10. Auflage, München (C. H. Beck) 2008.

Kopp, Ferdinand/Schenke, Wolf-Rüdiger: Verwaltungsgerichtsordnung, 15. Auflage, München (C. H. Beck) 2007.

Korenke, Thomas: Das neue SGB II: Grundsicherung für Arbeitsuchende (Hartz IV): Zur Zusammenlegung von Arbeitslosen- und Sozialhilfe, in: SGb 2004, S. 525-532.

Korioth, Stefan: Leistungsträgerschaft und Kostentragung bei der Grundsicherung für Arbeitssuchende (SGB II) - Aufgabenwahrnehmung aus „einer Hand“ zwischen reformiertem Grundgesetz und Bundesverfassungsgericht, in: DVB1 2008, S. 812-821.

Kormann, Karl: System der rechtsgeschäftlichen Staatsakte: Verwaltungs- und prozessrechtliche Untersuchungen zum allgemeinen Teil des öffentlichen Rechts, Berlin (Julius Springer) 1910 .

Körner, Marita: Die Reform des SGB III durch das Job-Aqtiv-Gesetz: Ein kritischer Überblick, in: NZA 2002, S. 241-246.

Kossens Michael: Neue Impulse durch Job-Aqtiv?, in: AuA 2002, S. 16-19.

Kothe, Wolfhard: Zumutbare Arbeit: Zumutungen und Unzumutbares im SGB II, in: SozSich 2005, S. 146-152.

Kottke, Joachim: System des subordinationsrechtlichen Verwaltungsvertrages, Hamburg 1966.

Kötz, Hein: Abschied von der Rechtskreislehre, in: ZEuP 1998, S. 493 ff.

Kötz, Hein/Flessner, Axel: Europäisches Vertragsrecht, Tübingen (Mohr) 1996. 
Krahmer, Utz: Verfassungsrechtliche Bedenken gegen die Hartz-IV-Gesetze (SGB II und SGB XII), insbesondere das Beispiel ungedeckten Bedarfs der Hilfe zum Lebensunterhalt bei nicht angespartem oder abhanden gekommenem Arbeitslosengeld II - zugleich ein Beitrag zu $§ 5$ Abs. 2 S. 1 SGB II sowie zu $§ 21$ S. 1 SGB XII, in: ZfF 2004, S. 178-182.

Krahmer, Utz: Zur widersprüchlichen Konstruktion der Eingliederungsvereinbarung nach $\S 15$ SGB II aus rechtlicher sowie methodisch-fachlicher Sicht, in: NDV 2006, S. 507-511.

Krahmer, Utz/Spindler, Helga: Rechtliche Maßstäbe für die Erbringung von Arbeitsgelegenheiten für Arbeitssuchende nach $\S 16$ Abs. 3 SGB II, in: NDV 2005, S. 17-24.

Krahmer, Utz: Rechtsmängel bei der praktischen Umsetzung von Arbeitsgelegenheiten, in: SGb 2006, S. 581-589.

Krahmer, Utz: Zur widersprüchlichen Konstruktion der Eingliederungsvereinbarungen nach $\S$ 15 SGB II aus rechtlicher sowie methodisch-fachlicher Sicht, in: NDV 2006, S. 507-511.

Krause, Peter: Die Willenserklärungen des Bürgers im Bereich des öffentlichen Rechts, in: VerwArch, 61. Jg. (1970), S. 297-341.

Krause, Peter: Rechtsformen des Verwaltungshandelns, Berlin (Duncker \& Humblot) 1974.

Krebs, Walter: Verträge und Absprachen zwischen der Verwaltung und Privaten, in: VVDStRL, H. 52 (1993), S. 248-280.

Kreikebohm, Ralf/Koch, Friedrich von: Das Sozialleistungsverhältnis - generelle Rechte und Pflichten zwischen Sozialleistungsempfängern und -trägern, in: Maydell, Bernd von/Ruland, Franz/Becker, Ulrich: Sozialrechtshandbuch, 4. Auflage, Baden-Baden (Nomos) 2008, § 6.

Kretschmer, Kai-Holmger: „Sozialhilfe“ durch Vertrag, Rechtliche Ausgestaltung und verfassungsrechtliche Bewertung der Eingliederungsvereinbarung nach dem „Hartz IV-Gesetz“, in: DÖV 2006, S. 893-901.

Kruse, Jürgen: Zusammenlegung von Arbeitslosenhilfe und Sozialhilfe: Ausgewählte (Rechts-) Fragen zu möglichen Konsequenzen, in: ZIAS 2003, S. 301-307.

Kruse, Jürgen/Lüdtke, Peter-Bernd/Reinhard, Hans-Joachim/Winkler, Jürgen/Zamponi, Irene: Sozialgesetzbuch III, Arbeitsförderung, Lehr- und Praxiskommentar, Baden-Baden (Nomos) 2008.

Kunig, Philip: Verträge und Absprachen zwischen Verwaltung und Privaten, in: DVB1 1992, S. 1193-1203.

Kunig, Philip/Rublack, Susanne: Aushandeln statt Entscheiden: Das Verwaltungsverfahrensrecht vor neuen Herausforderungen, in: Jura 1990, S. 1-11.

Kunkel, Peter-Christian: Schnittstellen zwischen Jugendhilfe (SGB VIII), Grundsicherung (SGB II) und Arbeitsförderung (SGB III), in: ZfSH/SGB 2006, S. 76-85.

Laband, Paul: Zu Otto Mayer, Theorie des französischen Verwaltungsrechts, in: AöR 1887, Bd. 2, S. 149-162.

Labrenz, Christoph: Die Abkehr vom Prinzip der (Hilfe-)Bedürftigkeit - zur verfassungswidrigen Konstruktion der Bedarfsgemeinschaft im SGB II, in: ZfF 2008, S. 217-224.

Lane, J.-E.: Contractualism in the public sector: Some theoretical issues, in: PM 1999, S. 179193.

Lang, Heinrich: Die Eingliederungsvereinbarung zwischen Autonomie und Bevormundung: Rechts- und Verfassungsfragen des SGB II, in: NZS 2006, S. 176-183. 
Larenz, Karl/Wolf, Manfred: Allgemeiner Teil des bürgerlichen Rechts, 9. Auflage, München (C. H. Beck), 2004.

Lauxen-Spangenberg, Marlene: Arbeitsvermittlung als sozialarbeiterisches Handlungsfeld (I) Zielgruppenorientiertes soziales Dienstleistungskonzept, in: TuP 2000, S. 123-126.

Lehmann-Franßen, Nils: Unangemessene Eigenbemühungen und die Nichtigkeit der Eingliederungsvereinbarung nach $\S 15$ SGB II, in: NZS 2005, S. 519-523.

Leisering, Lutz/Hilkert, Bernhard: Von Großbritannien lernen? Wohlfahrtsstaatsreform im Zeichen des Dritten Weges - das Beispiel aktivierender Sozialhilfepolitik unter Blair, London 2000.

Lesch, Hagen: Brauchen wir eine europäische Beschäftigungspolitik?, in: APuZ 2000, B 14-15. Lessenich, Stephan: Der Arme in der Aktivgesellschaft - zum sozialen Sinn des „Förderns und Forderns“, in: WSI-Mitteilungen 2003, S. 214-220.

Leitherer, Stephan (Hrsg.): Kasseler Kommentar Sozialversicherungsrecht, München (C. H. Beck) Loseblattausgabe Stand: Januar 2009.

Levy, Jonah D.: Activation through thick and thin: progressive approaches to labour market activation, in: SPR, $16 \mathrm{Jg}$. (2004), S. 187-208.

Lewis, Clive: Judicial remedies in public law, London (Sweet \& Maxwell) 1992.

Lewis, Norman: The Citizen's Charter and next steps: A new way of governing?, in: PQ, 64. Jg. (1993), H. 3, S. 316-326.

Logie James G./Watchman Paul Q.: Social security appeal tribunals: An excursus on evidential issues, in: CJQ, 8. Jg. (1989), S. 109-128.

Löschau, Martin/Marschner, Andreas: Zusammenlegung von Arbeits- und Sozialhilfe: Hartz

IV: Praxishandbuch zum neu eingeführten Zweiten Buch Sozialgesetzbuch (SGB II), Neuwied (Luchterhand) 2004.

Loughlin, Martin: The state, the Crown and the law, in: Sunkin, Maurice/Payne, Sebastian, The nature of the Crown, Oxford (Oxford University Press) 1999, S. 33-76.

Lühmann, Hans: Verfassungswidrige Zusammenlegung von Arbeitslosen- und Sozialhilfe im SGB II?, Eine organisationsrechtliche Analyse, in: DÖV 2004, S. 677-685.

Luthe, Ernst-Wilhelm: Sozialplanungsrecht, in: ZSR 1994, S. 762-780, 838-855.

Luthe, Ernst-Wilhelm: Rechtsanwendung in den Kernzonen aktiver Arbeitsmarktpolitik des SGB III, in: SGb 2001, S. 345-352.

Luthe, Ernst-Wilhelm: Das Job-Aqtiv-Gesetz, in: SGb 2002, S. 77-84, 136-144.

Luthe, Ernst-Wilhelm: Der aktivierende Sozialstaat im Recht, in: NDV 2003, S. 167-177.

Luthe, Ernst-Wilhelm: Sanktionen, Mindestbedarf und die Sozialpflichtigkeit des Hilfebedürftigen, in: SGb 2006, S. 637-647.

Luthe, Ernst-Wilhelm/Dittmar, Falko: Das Existenzminimum der Gegenwart, in: SGb 2004, S. 272-278.

Luthe, Ernst-Wilhelm/Dittmar, Christa: Das neue Fürsorgerecht in der Praxis, Berlin (Ernst Schmidt Verlag) 2005.

Luthe, Ernst-Wilhelm/Timm, Markus: Die Eingliederungsvereinbarung des SGB II, in: SGb 2005, S. 261-264.

Maluccio, Anthony N./Marlow, Wilma D.: The case for the contract, in: Social Work (USA), 19. Jg. (1974), H. 1, S. 1 ff. 
Mangoldt, Hermann von/Klein, Friedrich/Starck, Christian: Kommentar zum Grundgesetz, 5. Auflage, München (Vahlen) 2005.

Manow, Philip/Seils, Eric: Adjusting badly: The German welfare state, structural change, and the open economy, in: Scharpf, Fritz W./Schmidt, Vivien A. (Hrsg.), Welfare and work in the open economy, Bd. 2, 2000, S. $264 \mathrm{ff}$

Manow, Philip/Seils, Eric: The employment crisis of the German welfare state, in: WES 2000, S. 137-160.

Manssen, Gerrit: Privatrechtsgestaltung durch Hoheitsakt: Verfassungsrechtliche und verwaltungsrechtliche Grundfragen, Tübingen (Mohr) 1994.

Marshall, Geoffrey/Yardley, D.C.M.: Constitutional jurisdiction in the United Kingdom, in: Zeitschrift für ausländisches und öffentliches Recht und Völkerrecht 1962, S. 540-559.

Marshall, Thomas Humphrey: Sociology at the crossroads and other essays, London (Heinemann) 1963.

Martens, Rudolf: Vorschlag für einen spezifischen Preisindex zur Anpassung der Regelsätze, in: SozSich 2008, S. 68-73.

Mather, Graham: Government by contract, in: Vibert, Frank (Hrsg.): Britain's constitutional future, London (Gordon Pro-Print Co. Limited) 1991, S. 73-93.

Matthewman, Jim/Ennals, Simon/Self, Roger: Tolley’s social security and state benefits handbook, A comprehensive guide to state benefits and social security law, Croydon (Surrey Verlag), Loseblattausgabe Stand: November 2007.

Maurer, Hartmut: Der Verwaltungsvertrag - Probleme und Möglichkeiten, in: Hill, Hermann (Hrsg.): Verwaltungshandeln durch Verträge und Absprachen, Baden-Baden (Nomos) 1990, S. 15-38.

Maurer, Hartmut: Allgemeines Verwaltungsrecht, 17. Auflage, München (C. H. Beck) 2009.

Maunz, Theodor/Dürig, Günter u.a. (Hrsg.): Grundgesetz, Kommentar, München (C. H. Beck) Loseblattausgabe Stand: Oktober 2008.

Mayer, Otto: Theorie des französischen Verwaltungsrechts, Goldbach (Keip) 1998 (1886).

Mayer, Otto: Zur Lehre vom öffentlichrechtlichen Vertrage, in: AöR 1888, Bd. 3, S. 1-86.

Mayer, Otto: Deutsches Verwaltungsrecht, Berlin, 1914 (Nachdruck Duncker \& Humblot) 2004.

McDonald, Catherine/ Marston, Greg: Workfare as welfare governing unemployment in the advanced liberal state, in: CSP, 25. Jg. (2005), H. 3, S. 374-401.

McHale, Jean/Hughes, David/Griffiths, Lesley: Conceptualizing contractual disputes in the National Health Service Internal Market, in: Deakin, Simon/Michie, Jonathan (Hrsg.): Contracts, cooperation and competition, Oxford (Oxford University Press) 1997, S. 195-213.

McKay, Stephen/Smith, Alison/Youngs, Rachel/Walker, Robert: Unemployment and jobseeking after the introduction of jobseeker's allowance, Research Report Nr. 199, Leeds (Corporate Document services) 1999.

McLean, Janet: The crown in contract and administrative law, in: OJLS, 24. Jg. (2004), H. 1, S. 129-154.

Mead, Lawrence: Citizenship and social policy: T. H. Marshall and poverty, in: Paul, Ellen F. (Hrsg.): The welfare state, Cambridge (University Press) 1997, S. 197-230. 
Meager, Nigel: New Deal im Vereinigten Königreich, in: Weinert, Patricia u.a.: Beschäftigungsfähigkeit: Von der Theorie zur Praxis, Bern u.a. (Peter Lang Verlag) 2001, S. 167188.

Medicus, Dieter: Schuldrecht I, 14. Auflage, München (C. H. Beck) 2007.

Mehde, Veith: Neues Steuerungsmodell und Demokratieprinzip, Berlin (Duncker \& Humblot) 2000.

Merchel, Joachim: Von der psychosozialen Diagnose zur Hilfeplanung: Aspekte eines Perspektivenwechsels in der Erziehungshilfe, in: Jordan, Erwin/Schrapper, Christian (Hrsg.): Hilfeplanung und Betroffenenbeteiligung, Münster (Votum Verlag) 1994, S. 44-63.

Mergler, Otto/Zink, Günther (Hrsg.): Handbuch für Grundsicherung und Sozialhilfe, Bd. 1: Zweites Buch Sozialgesetzbuch - SGB II, Stuttgart (Kohlhammer) Loseblattausgabe Stand: September 2008.

Merkel, Wolfgang: The third ways of Social Democracy, in: Cuperus, René/Duffek, Karl/Kandel, Johannes (Hrsg.): Multiple third ways: European Social Democracy facing the twin revolution of globalisation and the knowledge society, Amsterdam u.a. (Friedrich-Ebert-Stiftung u.a.) 2001, S. 27-62.

Mesher, John: Social security law, in: Kiralfy, Albert (Hrsg.), The burden of proof, Abingdon (Professional Books Limited) 1987, S. 211-227.

Meurer, Anne: Durch Gestalten Vertrauen stärken, in: DAngVers 2000, S. 361-368.

Michael, Lothar: Rechtsetzende Gewalt im kooperierenden Verfassungsstaat: Normprägende und normersetzende Absprachen zwischen Staat und Wirtschaft, Berlin (Duncker \& Humblot) 2002.

Mills, Liz/Dean, Jacquie/Young, Ken: Entwicklung einer Umweltstrategie durch den Rat der Stadt Cardiff, in: Zilleßen, Horst/Dienel, Peter/Strubelt, Wendelin (Hrsg.): Die Modernisierung der Demokratie: Internationale Ansätze, Opladen (Westdeutscher Verlag) 1993, S. 233-246.

Mitchell, John: The contracts of public authorities: A comparative study, London (Bell in Komm) 1954.

Mitchell, John: The causes and effects of the absence of a system of public law in the United Kingdom, in: PL 1965, S. 95-118.

Möller, Adolf-Martin/Bornholm, Alfred/Stöcken, Gerwin: Nur Zusammenarbeit zwischen Arbeits- und Sozialverwaltung sichert den Erfolg: Kieler Modell, in: TuP 2004, S. 13-19.

Montesquieu, Charles Louis de Secondat de: De l'esprit des lois, Bd. 1, Paris (Garnier) 1749.

Morris, Gillian/Fredman, Sandra: The costs of exclusivity: Public and private re-examined, in: PL 1994, S. 69-85.

Mosley, Hugh/Schmid, Günther: Evaluation der Maßnahmen zur Umsetzung der Vorschläge der Hartz-Kommission, Implementations- und Wirkungsanalyse der Personal-ServiceAgentur, Abschlussbericht, Berlin/Bonn (Eigenverlag) 2006.

Mrozynski, Peter: Grundsicherung für Arbeitssuchende, im Alter, bei voller Erwerbsminderung und die Sozialhilfereform, in: ZfSH/SGB 2004, S. 198-221.

Müller-Thele, Stefan: Hartz IV: Eine datenschutzrechtliche Risikoanalyse, in: NJW 2005, S. 1541-1545. 
Münch, Ingo von/Kunig, Philip: Grundgesetz-Kommentar, 5. Auflage, München (C. H. Beck) 2000.

Münder, Johannes/Armborst, Christian (Hrsg.): Sozialgesetzbuch II: Grundsicherung für Arbeitssuchende, Lehr- und Praxiskommentar, Baden-Baden (Nomos) 2005.

Münder, Johannes: Sozialgesetzbuch XII, Lehr- und Praxiskommentar, 7. Auflage, BadenBaden (Nomos) 2005.

Nachtwey, Oliver/Heise, Arne: Großbritannien: Vom kranken Mann Europas zum Wirtschaftswunderland?, in: WSI-Mitteilungen 2006, S. 131-137.

Nelken, David: The Use of "contracts" as a social work technique, in: CLP, 40. Jg. (1987), S. 207-232.

Nell-Breuning, Oswald von: Soziallehre der Kirche: Erläuterungen der lehramtlichen Dokumente, Wien (Europaverlag) 1977.

Neumann, Volker: Dogmatische und prinzipiengeleitete Argumente bei der Abgrenzung von Verwaltungsverträgen: Zur Rechtsnatur der Pflegesatzvereinbarungen im Sozialhilferecht, in: DÖV 1992, S. 154-161.

Neumann, Volker: Der informelle Sozialstaat, in: VSSR 1993, S. 119-131.

Neumann, Volker: Menschenwürde und Existenzminimum, in: NVwZ 1995, S. 426-432.

Niesel, Klaus: SGB III, Sozialgesetzbuch Arbeitsförderung, Kommentar, 3. Auflage, München (C. H. Beck) 2005.

Nipperdey, Hans Carl: Kontrahierungszwang und diktierter Vertrag, Jena (G. Fischer) 1920.

Ockenga, Edzard: Regelleistung des SGB II und Verfassungswidrigkeit, in: ZfSH/SGB 2006, S. 143-147.

OECD: The OECD jobs strategy: Facts, analysis and strategy, Paris [OECD] 1994.

OECD: Innovations in labour market: The Australian way, Paris [OECD] 2001.

$O E C D$ : Activation strategies and performance of employment services in the Netherlands, Germany and the United Kingdom, Den Haag, 29.3.2006.

Oestreicher, Ernst (Hrsg.): SGB XII/SGB II, Kommentar, München (C. H. Beck) Loseblattausgabe Stand: März 2009.

Oliver, Dawn: Common values in public and private law and the public/private divide, in: PL 1997, S. 630-646.

Oliver, Dawn: The frontiers of the state: Public authorities and public functions under the Human Rights Act, in: PL 2000, S. 476-493.

Oliver, Dawn: Public law procedures and remedies - Do we need them?, in: PL 2002, S. 91-110.

Oliver, Dawn: Constitutional reform in the UK, Oxford (Oxford University Press) 2003.

Oliver, Dawn/Drewry, Gavin: Public service reform: issues of accountability and public law, London u.a. (Pinter) 1996.

Olk, Thomas: Der „aktivierende“ Staat, in: Müller, Siegfried/Sünker, Heinz/Olk, Thomas/Böllert, Karin (Hrsg.): Soziale Arbeit: gesellschaftliche Bedingungen und professionelle Perspektiven, Neuwied/Kriftel (Luchterhand) 2000, S. 99-118.

Ombudsrat - Grundsicherung für Arbeitsuchende: Schlussbericht vom 23.6.2006, Berlin, S. 144.

Oppermann, Dagmar: Verfassungsrechtliche Fragen zur Finanzierung der Grundsicherung für Arbeitsuchende, in: DVB1 2005, S. 1008-1014. 
Osborne, David/Gaebler, Ted: Reinventing government: How the entrepreneurial spirit is transforming the public sector, Reading (Addison-Wesley) 1992.

Oschmiansky, Frank/Kull, Silke/Schmid, Gunther: Faule Arbeitslose? Politische Konjunkturen einer Debatte, in: Discussion Paper FSI 01-206 (August 2001), Wissenschaftszentrum für Sozialforschung, Berlin.

Oschmiansky, Frank: Faule Arbeitslose? Zur Debatte über Arbeitsunwilligkeit und Leistungsmissbrauch, in: APuZ 2003, B 6-7.

Ossenbühl, Fritz: Öffentliches Recht in der Rechtsprechung des BGH, in: NJW 2000, S. 29442953.

Ossenbühl, Fritz: Rechtsquellen und Rechtsbindungen der Verwaltung, in: Erichsen, Hans-Uwe (Hrsg.): Allgemeines Verwaltungsrecht, Berlin (Walter de Gruyter) 2002, S. 111-203.

Ossenbühl, Fritz: Vorrang und Vorbehalt des Gesetzes, in: Isensee, Josef/Kirchhof, Paul (Hrsg.): Handbuch des Staatsrechts, Bd. 3, Heidelberg (C. F. Müller Verlag) 2005, § 62.

O'Sullivan, Daniel: Verfassungsrechtliche Fragen des Leistungsrechts der Grundsicherung für Arbeitsuchende, in: SGb 2005, S. 369-376.

Otto, Sven-Joachim: Die Verpflichtung des Arbeitnehmers zur frühzeitigen Arbeitssuche nach $\S$ 37b SGB III: Inhalt und Rechtsfolgen, in: NZS 2005, S. 288-290.

Page, Alan: The Citizen's Charter and administrative justice, in: Harris, Michael/Partington, Martin (Hrsg.): Administrative justice in the 21st century, Oxford (Hart) 1999, S. 85-98.

Pakeerut, Worachet: Die Entwicklung der Dogmatik des verwaltungsrechtlichen Vertrages, Berlin (Duncker \& Humblot) 2000.

Palandt: Bürgerliches Gesetzbuch, 68. Auflage, München (C. H. Beck) 2009.

Perri, 6/Kendall, Jeremy (Hrsg.): The contract culture in public services: Studies from Britain, Europe and the USA, Aldershot u.a. (Arena) 1997.

Pestalozza, Christian: Der Untersuchungsgrundsatz, in: Schmitt Glaeser, Walter: Verwaltungsverfahren, FS zum 50-jährigen Bestehen des Richard Boorberg Verlags, Stuttgart (Richard-Boorberg-Verlag) 1977, S. 185-204.

Phillips, O. Hood/Jackson, Paul/Leopold, Patricia: Constitutional and administrative law, London (Sweet \& Maxwell) 2001.

Pickel, Harald/Marschner, Andreas: SGB X, Kommentar, Heidelberg (R.v.Decker Verlag) Loseblattausgabe Stand: April 2009.

Pieters, Danny: Reflections on the methodology of social security law comparison, in: Ruland, Franz/Bernd Baron von Maydell/Hans-Jürgen Papier (Hrsg.): Verfassung, Theorie und Praxis des Sozialstaats, FS für Hans F. Zacher zum 70. Geburtstag, Heidelberg (Müller) 1998, S. 715-742.

Pissarides, Christopher: Unemployment in Britain: A European success story, London (London School of Economics and Political Science) 2003.

Pitschas, Rainer: Verwaltungsverantwortung und Verwaltungsverfahren, München (C. H. Beck) 1990.

Pitschas, Rainer: Entwicklung der Handlungsformen im Verwaltungsrecht: Vom Formendualismus des Verwaltungsverfahrens zur Ausdifferenzierung der Handlungsformen, in: Blümel, Willi/Pitschas, Rainer (Hrsg.): Reform des Verwaltungsverfahrensrechts, Berlin (Duncker \& Humblot) 1994, S. 229-256. 
Pitschas, Rainer: Das sozialrechtliche Verwaltungsverfahren im „aktivierenden“ Sozialstaat: Verfahrensrechtliche Konsequenzen der staatlichen Verantwortungspartnerschaft mit der Bürgergesellschaft, in: Wulffen, Matthias von (Hrsg.): FS 50 Jahre Bundessozialgericht, Köln (Heymanns) 2004, S. 765-782.

Pollard, David/Hughes, David: Constitutional and administrative law, London (Butterworth) 1990.

Porte, Caroline de la: The soft open method of co-ordination in social protection, in: European Trade Union Yearbook, Jg. 2001, S. 339-363.

Posner, Eric A.: The decline of formality in contract law, in: Buckley, F. H. (Hrsg.): The fall and rise of freedom of contract, Durham u.a. (Duke University Press) 1999, S. 61-78.

Punke, Jürgen: Verwaltungshandeln durch Vertrag, Kiel (Vorstand des Lorenz-von-SteinInstituts für Verwaltungswissenschaften) 1989.

Pyper, Robert: Ministerial responsibility and next steps agencies, in: Giddings, Philip (Hrsg.): Parliamentary accountability: a study of parliament and executive agencies, Basingstoke u.a. (Macmillan) 1995, S. 19-32.

Quaas, Michael: Der Versorgungsvertrag nach dem Pflege-Versicherungsgesetz, in: NZS 1995, S. $197 \mathrm{ff}$.

Quaas, Michael: Der Versorgungsvertrag nach dem SGB V mit Krankenhäusern und Rehabilitationseinrichtungen, Düsseldorf (Deutsche Krankenhaus Verlagsgesellschaft) 2000.

Quaas, Michael: Die Arbeitsgemeinschaft nach dem neuen SGB II: Ungelöste Rechtsfragen zur Rechtsnatur der Einrichtung, in: SGb 2004, S. 723-729.

Quade, Benno: Verantwortung und ihre Zuschreibung im Recht der Arbeitsförderung - Eine rechtsvergleichende Untersuchung der „Arbeitsförderungssysteme“ der Vereinigten Staaten von Amerika und der Bundesrepublik Deutschland, Baden-Baden (Nomos) [erscheint demnächst].

Quade, Benno/O'Leary, Christopher/Dupper, Ockert: Activation from Income support, in: Eichhorst, Werner/Kaufmann, Otto/Konle-Seidl, Regina, Bringing the jobless into work?, Berlin u.a. (Springer) 2008, S. 345-414.

Raveaud, Gilles: Dynamics of the welfare states regimes and employability (A Study Based on the National Action Plans for Employment, 1998-2000), in: Pieters, Danny (Hrsg.): Confidence and changes: managing social protection in the new millennium, London u.a. (Kluwer Law International) 2001, S. 5-26.

Rayner, Elizabeth u.a.: Evaluating the jobseeker's allowance: A summary of the research findings, Research Report Nr. 116, London (DWP) 2000.

Rebmann, Kurt: Münchener Kommentar, 3. Auflage, München (C. H. Beck) 2006/2007.

Rehbinder, Manfred: Die Rezeption fremden Rechts in soziologischer Sicht, in: Rehbinder, Manfred/Sonn, Ju-Chan (Hrsg.): Zur Rezeption des deutschen Rechts in Korea, BadenBaden (Nomos) 1990, S. 5-16.

Reher, Michael: Modellprojekte zur Kooperation von Arbeits- und Sozialämtern: Ein Überblick, in: BArbB1 2002, S. 8-9.

Renck, Ludwig: Der Rechtsweg im gerichtlichen Verfahrensrecht: Vertragshandeln und Realakte, in: JuS 2000, S. 1001-1006. 
Rengeling, Hans-Werner: Das Kooperationsprinzip im Umweltrecht, Köln u.a. (Heymanns) 1988.

Rexin, Burkhard: Schlussbericht des Ombudsrates: Rat sieht zahlreiche Mängel bei der Grundsicherung und fordert neue Organisationsstruktur, in: SozSich 2006, S. 243-248.

Rixen, Stephan: Das neue Sozialrecht der Arbeitsvermittlung nach der Reform der Bundesanstalt für Arbeit, in: NZS 2002, S. 466-473.

Rixen, Stephan: Personal-Service-Agenturen im Schnittfeld von Sozial-, Haushalts- und Vergaberecht: Strukturen des Arbeitsvermittlungsrechts der $\S \S 37 \mathrm{c}, 434$ V SGB III, in: NZS 2003, S. 401-411.

Rixen, Stephan: „Hartz IV“: Ist jede Arbeit zumutbar? Zum unbenannten Unzumutbarkeitsgrund des $\S 10$ Abs. 1 Nr. 5 SGB II am Beispiel sexualitätsbezogener Dienstleistungen, in: SGb 2005, S. 509-514.

Rixen, Stephan: Unbegrenzte Möglichkeiten im Hartz-IV-Land? Rechtliche Grenzen bei „EinEuro-Jobs“, in: SozSich 2005, S. 152-157.

Rixen, Stephan: Erwerbsfähigkeit als Schlüsselbegriff der Arbeitsmarktreform, insbesondere im SGB II, in: info also 2006, S. 153-161.

Rixen, Stephan/Pananis, Panos: Hartz IV: Welcher Ein-Euro-Job ist „zusätzlich“?, in: NJW 2005, S. 2177-2181.

Rojek, Chris/Collins, Stewart A.: Contract or con trick?, in: BJSW, 17. Jg. (1987), H. 5, S. 199211.

Rolfs, Christian: Arbeitsrechtliche Aspekte des neuen Arbeitsförderungsrechts, in: NZA 1997, S. 17-23.

Roscher, Frank: Vertragsfreiheit als Verfassungsproblem, Dargestellt am Beispiel der Allgemeinen Geschäftsbedindungen, Berlin (Duncker \& Humblot) 1974.

Rothkegel, Ralf: Sozialhilferecht im Umbruch, in: ZfSH/SGB 2004, S. 396-409.

Rothkegel, Ralf (Hrsg.): Sozialhilferecht: Existenzsicherung, Grundsicherung, Baden-Baden (Nomos) 2005.

Rothkegel, Ralf: Ist die Rechtsprechung des Bundesverwaltungsgerichts zur Sozialhilfe durch Hartz IV überholt?, in: SGb 2006, S. 74-78.

Rüfner, Wolfgang: Die Mitwirkungspflichten des Leistungsberechtigten in der Solidargemeinschaft nach $\S \S 60$ ff SGB-AT, in: VSSR 1977, S. 347-365.

Ruge, Kay/Vorholz, Irene: Verfassungs- und verwaltungsrechtliche Fragestellungen bei der Arbeitsgemeinschaft nach $\S 44 b$ SGB II, in: DVB1 2005, 403-415.

Sainsbury, Roy: The reform of social security adjudication, in: Harris, Michael/Partington, Martin (Hrsg.): Administrative justice in the 21st century, Oxford (Hart) 1999, S. 445462.

Sainsbury, Roy/Kennedy, Steven: Contracting welfare? Market testing and social security, in: Lunt, Neil/Coyle, Douglas (Hrsg.): Welfare and policy research, London (Taylor \& Francis) 1996, S. 111-122.

Samuel, Geoffrey: Public and private law: A private lawyer's response, in: MLR, 46. Jg. (1983), S. 558-583.

Sartorius, Ulrich: Das Existenzminimum im Recht, Baden-Baden (Nomos) 2000. 
Sartorius, Ulrich: Höhere Regelleistungen und Regelsätze im Klagewege?, in: info also 2005, S. 56-58.

Scharf, Thomas: Sozialpolitik in Großbritannien: Vom Armengesetz zum „Dritten Weg“, in: Kraus, Katrin/Geisen, Thomas (Hrsg.): Sozialstaat in Europa: Geschichte, Entwicklung, Perspektiven, Opladen (Westdeutscher Verlag) 2001, S. 43-62.

Scherzberg, Arno: Grundfragen des verwaltungsrechtlichen Vertrages, in: JuS 1992, S. 205-212. Schiedermair, Werner: Der Verwaltungsakt auf Unterwerfung: Ein Beitrag zur Lage der öffentlichen Verwaltung und zum Wesen des Grundsatzes des Gesetzmäßigkeit der Verwaltung, Würzburg (Schmidt und Meyer) 1967.

Schilling, Theodor: Die Vertragsstrafe in Verträgen mit der öffentlichen Hand, in: VerwArch, 84. Jg. (1993), S. $226 \mathrm{ff}$.

Schilling, Theodor: Der „unfreiwillige“ Vertrag mit der öffentlichen Hand, in: VerwArch, 87. Jg. (1996), S. 191-211.

Schlette, Volker: Die Verwaltung als Vertragspartner: Empirie und Dogmatik verwaltungsrechtlicher Vereinbarungen zwischen Bürger und Behörde, Tübingen (Mohr Siebeck) 2000.

Schmidt-Aßmann, Eberhard: Das allgemeine Verwaltungsrecht als Ordnungsidee: Grundlagen und Aufgaben der verwaltungsrechtlichen Systembildung, Berlin (Springer) 2004.

Schmidt-Aßmann, Eberhard/Krebs, Walter: Rechtsfragen städtebaulicher Verträge: Vertragstypen und Vertragsrechtslehren, Köln (Kohlhammer) 1992.

Schmidt, Detlef: Die Unterscheidung von privatem und öffentlichem Recht, Baden-Baden (Nomos) 1985.

Schmidt, Reimer: Die Obliegenheiten, Karlsruhe (Verlag Versicherungswirtschaft) 1953.

Schmitt, John/Wadsworth, Jonathan: Is the OECD jobs strategy behind US and British employment and unemployment success in the 1990?, in: CEPA Working Papers $2002-06$ (April 2002).

Schmitz, Heribert: „Die Verträge sollen sicherer werden“: Zur Novellierung der Vorschriften über den öffentlich-rechtlichen Vertrag, in: DVB1 2005, S. 17-24.

Schoch, Dietrich: Die Bedarfsgemeinschaft, die Einsatzgemeinschaft und die Haushaltsgemeinschaft nach dem SGB II und SGB XII, in: ZfF 2004, S. 169-177.

Schoch, Dietrich: Zur Feststellung der Erwerbsfähigkeit nach dem SGB II und der Erwerbsminderung nach dem Vierten Kapitel SGB XII, in: NDV 2006, S. 512-517, 545-551.

Scholz, Rupert: Sachverständigenrat „Schlanker Staat“, Abschlussbericht, Bonn 1998.

Schön, Markus: Forderndes Fördern: Die Eingliederungsvereinbarung des SGB II im Lichte der Rechtsschutzgarantie des Art. 19 Abs. 4 GG, in: SGb 2006, S. 290-297.

Schönig, Werner: Institutionelle Perspektiven der Bundesanstalt für Arbeit - Maßgeschneiderte Betreuung durch das neue Steuerungsmodell?, in: SozFort 2002, S. 9-12.

Schröder, Gerhard: Die zivile Bürgergesellschaft: Anregungen zu einer Neubestimmung der Aufgaben von Staat und Gesellschaft, in: NG/FH 2000, S. 200-207.

Schulin, Bertram/Igl, Gerhard/Welti, Felix: Sozialrecht, 8. Auflage, Düsseldorf (Werner Verlag) 2007.

Schulze-Fielitz, Helmuth: Neue Kriterien für die verwaltungsgerichtliche Kontrolle bei der Anwendung unbestimmter Rechtsbegriffe, in: JZ 1993, S. 772-781. 
Schulze-Fielitz, Helmuth: Kooperatives Recht im Spannungsfeld von Rechtsstaatsprinzip und Verfahrensökonomie, in: Dose, Nicolai/Voigt, Rüdiger (Hrsg.): Kooperatives Recht, Baden-Baden (Nomos) 1995, S. 225-256.

Schütze, Holger/Oschmiansky, Frank: Arbeitsamt war gestern: Neuausrichtung der Vermittlungsprozesse in der Bundesagentur für Arbeit nach den Hartz-Gesetzen, in: ZSR 2006, S. 5-28.

Schwabe, Bernd-Günther: Die Zusammensetzung des Regelsatzes im SGB XII bzw. der Regelleistung im SGB II in Höhe von 351€ ab dem 1.7.2008, in: ZfF 2008, S. 145-151, 204.

Schwarting, Gunnar: Effizienz in der Kommunalverwaltung: Dezentrale Verantwortung, Produkte, Budgets und Controlling, Berlin (Schmidt) 2005.

Schwarze, Jürgen: European Administrative Law, London (Sweet \& Maxwell) 2006.

Schweiger, Maximilian: Rechtliche Einordnung der durch das Job-AQTIV-Gesetz in das Arbeitsförderungsrecht eingefügten Eingliederungsvereinbarung ( 35 Abs. 5 SGB III n. F.), in: NZS 2002, S. 410-415.

Scott, Colin: Privatisation, control and accountability, in: McCahery, Joseph/Picciotto, Sol/Scott, Colin (Hrsg.): Corporate control and accountability: changing structures and the dynamics of regulation, Oxford (Clarendon Press) 1993, S. 231-245.

Seddon, Nick: Activity agreements: Are they contracts?, in: Creyke, Robin/Sassella, Michael (Hrsg.): Targeting accountability and review: Current issues in income support law, Canberra (Union Offset) 1998, S. 82-86.

Seddon, Nick: Government contracts: federal, state and local, Leichardt (Federation Press) 1999. Selznick, Philip: Law, society, and industrial justice: With the collaboration of Philippe Nonet and Howard M. Vollmer, New York (Russell Sage Foundation) 1969.

Sieveking, Klaus: Zur Bedeutung des Arbeitslosengelds II für Ausländer, in: ZAR 2004, S. 283287.

Slapper, Gary/Kelly, David: Sourcebook on the English legal system, London (Cavendish Publishing limited) 2001.

Soergel, Hans Theodor: Bürgerliches Gesetzbuch, 13. Auflage, Stuttgart u.a. (Kohlhammer), $1999 / 2000$.

Sommermann, Karl-Peter: Die Bedeutung der Rechtsvergleichung für die Fortentwicklung des Staats- und Verwaltungsrechts in Europa, in: DÖV 1999, S. 1017-1029.

Soria, José Martínez: Das Recht auf Sicherung des Existenzminimums, in: JZ 2005, S. 644-652. Spanner, Hans: Ein Entwurf eines Verwaltungsverfahrensgesetzes, in: DVB1 1964, S. 845-849.

Spannowsky, Willy: Grenzen des Verwaltungshandelns durch Verträge und Absprachen, Berlin (Duncker \& Humblot) 1994.

Spellbrink, Wolfgang: Ist die Beitragspflicht in der gesetzlichen Arbeitslosenversicherung verfassungsrechtlich noch zu rechtfertigen?, in: JZ 2004, S. 538-545.

Spellbrink, Wolfgang: Wandlungen im Recht der Arbeitsvermittlung - oder: Viel Lärm um wenig, in: SGb 2004, S. 75-83, 153-159.

Spellbrink, Wolfgang: Das Einstiegsgeld nach § 29 II SGB II - oder von den Aporien „moderner" Gesetzgebung, in: NZS 2005, S. 231-237.

Spellbrink, Wolfgang: Eingliederungsvereinbarung nach SGB II und Leistungsabsprache nach SGB XII aus Sicht der Sozialgerichtsbarkeit, in: SozR akt 2006, S. 52-56. 
Spellbrink, Wolfgang: Die Bedarfsgemeinschaft gemäß § 7SGB II eine Fehlkonstruktion?, in: NZS 2007, S. 121-127.

Spellbrink, Wolfgang: Gelingt durch die neuen Instrumente des SGB II die Integration der Langzeitarbeitslosen in den Arbeitsmarkt?, in: SGb 2008, S. 445-451.

Spellbrink, Wolfgang/Eicher, Wolfgang: Kasseler Handbuch des Arbeitsförderungsrechts, München 2003.

Spieß, Gerhard: Inhaltskontrolle von Verträgen: Das Ende privatautonomer Vertragsgestaltung?, in: DVB1 1994, S. 1222-1229.

Spindler, Helga: Rechtliche Rahmenbedingungen der Beratung in der Sozialhilfe: Bestandsaufnahme, Probleme der aktuellen Entwicklung und mögliche Perspektiven, in: NDV 2002, S. 357-363, 386-388.

Spindler, Helga Das neue SGB II: Keine Grundsicherung für Arbeitssuchende, in: SozSich 2003, S. 338-344.

Spindler, Helga: Aktivierende Ansätze in der Sozialhilfe, in: Dahme, Heinz-Jürgen u.a.: Soziale Arbeit für den aktivierenden Staat, Opladen (Leske \& Budrich) 2003, S. 225-246.

Spindler, Helga: Vertragssimulationen bei Ein-Euro-Jobs, in: info also 2006., S. 162-164.

Spitzer, Malte: Bürgeraktivierung und Verwaltungsmodernisierung, in: Blanke, Bernhard u.a. (Hrsg.): Handbuch zur Verwaltungsreform, Opladen (Leske + Budrich) 2001, S. 144-152. Stafford, Bruce: Service delivery and the user, in: Millar, Understanding social policy, 2003, S. 213-234.

Stahlmann, Günther: 1-Euro-Jobs zwischen Arbeitsvermittlung und Abstellgleis, in: ZfSH/SGB 2006, S. 131-143.

Stahlmann, Günther: Sozialdatenschutz bei Eingliederung nach dem SGB II, in: info also 2006, S. 10-17; 61-65.

Staudinger, J. von: Kommentar zum Bürgerlichen Gesetzbuch, 13. Auflage, Berlin (Sellier de Gruyter) 2003.

Steck, Joachim: Die juristische Bedeutung der Mitwirkungspflichten nach den $\S \S 60$ ff SGB I, in: BKK 1992, S. 349-356.

Stein, Eric: Uses, misuses and nonuses of comparative law, in: NwUnivLR, 1977 (72), S. 198216.

Steinmeyer, Horst: Fördern und Fordern-Arbeitsvermittlung und Weiterbildung nach dem JobAQTIV-Gesetz, in: info also 2002, S. 4-11.

Stelkens, Ulrich: Verwaltungsprivatrecht: Zur Privatrechtsbindung der Verwaltung: deren Reichweite und Konsequenzen, Berlin (Duncker \& Humblot) 2005.

Stelkens, Paul/Bonk, Heinz Joachim/Sachs, Michael (Hrsg.): Verwaltungsverfahrensgesetz, 6. Auflage, München (C. H. Beck) 2001.

Stern, Klaus: Zur Problematik eines energiewirtschaftlichen Konzessionsvertrags, in: AöR 1959, Bd. 84, S. 137-184; 273-331.

Stindt, Heinrich Meinhard: Innovative Arbeitsförderung - aus der Sicht der betrieblichen Praxis, in: Engelen-Kefer, Ursula u.a. (Hrsg.): Die Reform der Arbeitsförderung, Fachtagung der Bayer-Stiftung für deutsches und internationales Arbeits- und Wirtschaftsrecht am 30. November 1995, München (C. H. Beck) 1997, S. 37-69. 
Stöbe, Sybille: Verwaltungsreform durch Organisationsentwicklung: Leitbilder als Instrument einer Modernisierungsstrategie, in: Behrens, Fritz u.a. (Hrsg.): Den Staat neu denken: Reformperspektiven für Landesverwaltungen, Berlin (edition sigma) 1995, S. 129-141.

Stöbe-Blossey, Sybille: Kooperation in der lokalen Arbeitsmarktpolitik: Entstehungsbedingungen und Erfolgsfaktoren, Opladen (Leske \& Budrich) 1992.

Stolleis, Michael: Öffentliches Recht und Privatrecht im Prozeß der Entstehung des modernen Staates, in: Hoffmann-Riem, Wolfgang/Schmidt-Aßmann, Eberhard (Hrsg.): Öffentliches Recht und Privatrecht als wechselseitige Auffangordnungen, Baden-Baden (Nomos) 1996, S. 41-61.

Street, Harry: Justice in the welfare state, London (Stevens) 1985.

Streinz, Rudolf: Sozialpolitische Zuständigkeit der EU im Rahmen der offenen Methode der Koordinierung, in: SDSRV 53 (2005), S. 29-51.

Strick, Kerstin: Ansprüche alter und neuer Unionsbürger auf Sozialhilfe und Arbeitslosengeld II, in: NJW 2005, S. 2182-2187.

Strobel, Brigitte: Die Rechtsform der Arbeitsgemeinschaften nach § 44b SGB II, in: NVwZ 2004, S. 1195-1198.

Strobel, Eva: Der Eingliederungsvertrag zur Integration von Arbeitslosen, in: Charbonnier, Volker u.a. (Hrsg.): Beschäftigungsförderung durch neues Arbeits- und Sozialrecht, Fachtagung der Bayer-Stiftung für deutsches und internationales Arbeits- und Wirtschaftsrecht am 7. November 1997, München (C. H. Beck) 1998, S. 145-149.

Struck, Norbert: Vertragsfreiheit - ein Grundrecht?, in: DuR 1988, S. 39 ff.

Strünck, Christoph: Ist mit den Bürgern noch Staat zu machen?, in: Mitb 2000, S. 12-16.

Stüer, Bernhard/König, Claas-Dietrich: Städtebauliche Verträge: strikter Gesetzesvollzug oder grenzenlose Vertragsfreiheit?, in: ZfBauR 2000, S. 528-536.

Sutor, Bernhard: Beteiligungsgerechtigkeit und Sozialstaatsreform, in: Stimmen der Zeit 2005, S. 103-114.

Taggart, Michael: "The peculiarities of the English" resisting the public/private law distinction, in: Craig, Paul/Rawlings, Richard (Hrsg.): Law and administration in Europe, essays in honour of Carol Harlow, Oxford (Oxford University Press) 2003, S. 107-121.

Telò, Mario: The European dimension of the third way, in: Cuperus, René/Duffek, Karl/Kandel, Johannes (Hrsg.): Multiple third ways: European social democracy facing the twin revolution of globalisation and the knowledge society, Amsterdam u.a. (Friedrich-EbertStiftung u.a.) 2001, S. 173-186.

Tettinger, Peter: Rechtsanwendung und gerichtliche Kontrolle im Wirtschaftsverwaltungsrecht, München (Vahlen) 1980.

Tondorf, Karin/Bahnmüller, Reinhard/Klages, Helmut: Steuerung durch Zielvereinbarungen: Anwendungspraxis, Probleme, Gestaltungsüberlegungen, Berlin (Ed. Sigma) 2002.

Trebilcock, Michael: The limits of freedom of contract, Cambridge u.a. (Harvard University Press) 1993.

Treutner, Erhard: Kooperativer Rechtsstaat: Das Beispiel Sozialverwaltung, Baden-Baden (Nomos) 1998. 
Trickey, Heather/Walker, Robert: Steps to compulsion within labour market policies, in: Lodemel, Ivar/Trickey, Heather (Hrsg.): An offer you can't refuse: Workfare in international perspective, Bristol (The Policy Press) 2001, S. 181-213.

Trube, Achim: Aktivierender Sozialstaat: Programmatik, Praxis und Probleme, in: NDV 2003, S. 334-341.

Trube, Achim/Wohlfahrt, Norbert: „Der Aktivierende Sozialstaat“: Sozialpolitik zwischen Individualisierung und einer neuen politischen Ökonomie der inneren Sicherheit, in: WSIMitteilungen 2001, S. 27-35.

TSO: Modernising Government, Cm 4310, London 1999.

Turpin, Colin: Government procurement and contracts, Harlow (Longman) 1989.

Ule, Carl Hermann/Laubinger, Hans-Werner: Verwaltungsverfahrensrecht, 4. Auflage, Köln u.a., (Heymanns), 1995.

Ullrich, Carsten: Die soziale Akzeptanz der Arbeitslosenversicherung, in: WSI-Mitteilungen 2006, S. 200-205.

Urban, Hans-Jürgen: Eigenverantwortung und Aktivierung: Stützpfeiler einer neuen Wohlfahrtsarchitektur, in: WSI-Mitteilungen 2004, S. 467-473.

Vincent, Jill: Jobseeker's allowance evaluation: Qualitative research on disallowed and sanctioned claimants, Research Report Nr. 86, London (DWP) 1998.

Vincent-Jones, Peter: The regulation of contractualisation in quasi-markets for public services, in: PL 1999, S. 304-327.

Vincent-Jones, Peter: Contractual governance: Institutional and organizational analysis, in: OJLS, 20. Jg. (2000), S. 317-351.

Vincent-Jones, Peter: Regulating government by contract: Towards a public law framework?, in: MLR, 65. Jg. (2002), S. 611-628.

Vincent-Jones, Peter: Citizen redress in public contracting for human services, in: MLR, 68. Jg (2005), S. 887-924.

Vincent-Jones, Peter: The new public contracting: regulation, responsiveness, relationality, Oxford (University Press) 2006.

Voelzke, Thomas: Die Herbeiführung des Versicherungsfalls im Sozialversicherungsrecht, Berlin (Erich Schmidt Verlag) 2004.

Voelzke, Thomas: Aktuelle Entwicklungen im Sperrzeitrecht, in: NZS 2005, S. 281-288.

Voelzke, Thomas: Gesetz zur Neuausrichtung der arbeitsmarktpolitischen Instrumente, in: jurisPR-SozR 5/2009 Anm. 4.

Voigt, Rüdiger (Hrsg.): Der kooperative Staat: Krisenbewältigung durch Verhandlung?, Berlin (Nomos) 1995.

Waechter, Kay: Der öffentlich-rechtliche Vertrag: Zur aktuellen Entwicklung der Handlungsformenlehre, in: JZ 2006, S. 166-170.

Wade, Emlyn C./Bradley, Anthony W.: Constitutional and administrative law, 10. Auflage, London (Longman) 1985.

Wade, William/Forsyth, Christopher: Administrative law, 9. Auflage, Oxford (Oxford University Press) 2004.

Wahrendorf, Volker: Wünschenswerte Gesetzgebungsinitiativen zum SGB II, in: SozR akt 2009, S. 1-6. 
Walker, R. J./Ward, Richard: Walker\&Walker's English legal system, 7. Auflage, London u.a. (Butterworths) 1994.

Walsh, Kieron u.a.: Contracts for public services: A comparative perspective, in: Campbell,

David/Vincent-Jones, Peter (Hrsg.): Contracts and economic organisation: socio-legal initiatives, Aldershot (Dartmouth) 1996, S. 212-235.

Wallerath, Maximilian: Zur Dogmatik eines Rechts auf Sicherung des Existenzminimums, Ein

Beitrag zur Schutzdimension des Art. 1 Abs. 1 S. 2 GG, in: JZ 2008, S. 157-208.

Wasmer, Wolfgang: Vertragsfreiheit im UN-Kaufrecht, Hamburg (Verlag Dr. Kovac) 2004.

Watson, Alan: Legal transplants and law reform, in: LQR 1976, H. (92), S. 79-84.

Weihrauch, Oliver: Verwaltungsrechtlicher Vertrag und Urkundeneinheit, in: VerwArch, 82. Jg. (1991), S. 543-564.

Wellenhofer-Klein, Marina: Strukturell ungleiche Verhandlungsmacht und Inhaltskontrolle von Verträgen, in: ZIP 1997, S. 774-781.

White, Stuart: Review article: Social rights and the social contract - Political theory and the new welfare politics, in: BJPS, 30. Jg. (2000), S. 507-532.

Wiesner, Reinhard: Administrative Tribunals in Großbritannien, Berlin (Duncker \& Humblot) 1974.

Wikeley, Nick: Unemployment benefit, the state, and the labour market, in: JLS, 16. Jg. (1989), H. 3, S. 291-309.

Wikeley, Nick: Social security appeals in Great Britain, in: Admin LR, 46. Jg. (1994), S. 183 212.

Wikeley, Nick: Social security tribunals after the social Security Act 1998, in: JSSL, 6. Jg. (1999), H. 4, S. 155-185.

Wikeley, Nick: Burying Bell: Managing the judicialisation of social security tribunals, in: MLR, 63. Jg. (2000), H. 4, S. 475-501.

Wikeley, Nick: Co-ownership of property and entitlement to means-tested benefits, in: JSSL, 8 . Jg. (2001), H. 3, S. 95-121.

Wikeley, Nick/Ogus, Anthony: The Law of Social Security, 5. Auflage, London u.a. (Butterworths Lexis Nexis) 2002.

Wilkinson, David: New Deal for young people: Evaluation of unemployment flows, London (Policy Studies Institute) 2003.

Winkler, Ute: Die Erreichbarkeit in SGB II und III, in: info also 2007, S. 3-9.

Winkler, Werner: Jobcentre Plus - Vorbild für Deutschland?, in: ibv 2004, S. 1-12.

Winkler, Werner: Jobcentre Plus - ein Erfolgsmodell: Vorbild auch für Deutschland, in: BArbB1 2004, S. 18-27.

Wissing, Gerhard (Hrsg.): SGB III, Praxiskommentar, 2. Auflage, Baden-Baden (Nomos) 2005. Wolf, Manfred: Selbstbestimmung durch vertragliches Abschlußrecht, in: JZ 1976, S. 41-45.

Wolff, Birgitta: Public-Private Partnerships, in: Schenk, Karl-Ernst (Hrsg.): Vom Hoheitsstaat zum Konsensualstaat: Neue Formen der Kooperation zwischen Staat und Privaten, Tübingen (J.C.B. Mohr) 1996, S. 243-275.

Wolff, Hans J.: Der Unterschied zwischen öffentlichem und privatem Recht, in: AöR 1950/51, Bd. 76, S. 205-217. 
Wolff, Hans J./Bachof, Otto/Stober, Rolf: Verwaltungsrecht, Bd. 1, 11. Auflage, München (C. H. Beck) 1999.

Wolff, Hans J./Bachof, Otto/Stober, Rolf: Verwaltungsrecht, Bd. 2, 6. Auflage, München (C. H. Beck) 2000.

Wood, Penny u.a.: Social Security Legislation 2006, London (Sweet \& Maxwell) 2006.

Woodhouse, Diana: In pursuit of good administration, Oxford (Oxford University Press) 1997.

Woodhouse, Diana: Ministerial responsibility, in: Bogdanor, Vernon (Hrsg.): The British Constitution in the twentieth century, Oxford (Oxford University Press) 2004, S. 281-333.

Woolf, Harry: Public law - private law: Why the divide? A personal view, in: PL 1986, S. 220238.

Woolf, Harry/Jowell, Jeffrey: Judicial review of administrative action, London (Sweet \& Maxwell) 2005.

Wright, Sharon: Activating the unemployed: the street-level implementation of UK policy, in: Clasen, Jochen (Hrsg.): What future for social security? Debates and reforms in national and cross-national perspective, Den Haag u.a. (Kluwer International) 2001, S. 235-249.

Wulffen, Matthias von (Hrsg.): SGB X, 6. Auflage, München (C. H. Beck) 2008.

Wunder, Annett/Diehm, Alexander: SGB II-Fortentwicklungsgesetz verschärft die Sanktionen:

Sind Kürzungen des Arbeitslosengeldes II um bis zu 100 Prozent verfassungswidrig?, in: SozSich 2006, S. 195-199.

Yardley, David: Principles of administrative law, 2. Auflage, London (Butterworths) 1986.

Zacher, Hans F.: Vorfragen zu den Methoden des Sozialrechtsvergleichs, in: ders. (Hrsg.): Methodische Probleme des Sozialrechtsvergleichs, Colloquium der Projektgruppe für Internationales und Vergleichendes Sozialrecht der Max-Planck-Gesellschaft Tutzing 1976, Berlin (Duncker \& Humblot) 1977, S. 21-74.

Zezschwitz, Friedrich v.: Rechtsstaatliche und prozessuale Fragen des Verwaltungsprivatrechts, in: NJW 1983, S. 1873-1882.

Ziekow, Jan: Verankerung verwaltungsrechtlicher Kooperationsverhältnisse (Public Private Partnership) im Verwaltungsverfahrensgesetz, Berlin 2001.

Ziekow, Jan/Siegel, Thorsten: Entwicklung und Perspektiven des Rechts des öffentlichrechtlichen Vertrages, in: VerwArch, 94. Jg. (2003), S. 593-608; 95. Jg. (2004), S. 133150; 281-299; 573-585.

Zimmermann, Reiner: Konturen eines europäischen Vertragsrechts, in: JZ 1995, S. 477-491.

Zuck, Rüdiger: Hartz IV:Alg II=GG Null?, in: NJW 2005, S. 649-651.

Zwanziger, Bertram: Rechtliche Rahmenbedingungen für „Ein-Euro-Jobs“, in: AuR 2005, S. 815.

Zweigert, Konrad: „Rechtsgeschäft“ und „Vertrag“ heute, in: Caemmerer, Ernst von (Hrsg.): Ius Privatum Gentium, FS Max Rheinstein zum 70. Geburtstag am 5. Juli 1969, Tübingen (J. C. B. Mohr) 1969, S. 493-504.

Zweigert, Konrad/Kötz, Hein: Einführung in die Rechtsvergleichung auf dem Gebiete des Privatrechts, Tübingen (Mohr) 1996. 